CRITICAL PERTURBATIONS OF ELLIPTIC OPERATORS BY LOWER ORDER TERMS

\author{
A Dissertation \\ presented to \\ the Faculty of the Graduate School \\ University of Missouri
}

In Partial Fulfillment

of the Requirements for the Degree

Doctor of Philosophy

by

Jose Luis Luna-Garcia

Dr. Steve Hofmann, Dissertation Supervisor

May 2021 
The undersigned, appointed by the Dean of the Graduate School, have examined the dissertation entitled

\section{CRITICAL PERTURBATIONS OF ELLIPTIC OPERATORS BY LOWER ORDER TERMS}

presented by Jose Luis Luna-Garcia, a candidate for the degree of Doctor of Philosophy of Mathematics, and hereby certify that in their opinion it is worthy of acceptance.

Professor Steve Hofmann

Professor Igor Verbitsky

Professor Samuel Walsh

Professor Steven Neal 


\section{ACKNOWLEDGEMENTS}

I would first like to thank my advisor Steve Hofmann for his patience and support, for always having time to talk about math and freely sharing his ideas.

I'd like to thank Simon Bortz and Bruno Poggi for sharing in the at times unpredictable process of figuring out this project.

Alyssa Genschaw and John Hoffman I thank for sharing in the struggle of research and grad school in general.

Finally I'd like to thank my family: my parents and my brother, for their unconditional support during my studies. 


\section{Contents}

Acknowledgements

ii

Abstract $\quad$ vi

1 Introduction 1

2 Preliminaries $\quad 18$

2.1 Notation . . . . . . . . . . . . . . . . . . . 18

2.2 Function spaces . . . . . . . . . . . . . . . . 22

2.3 Basic properties of $\mathcal{L} \ldots \ldots \ldots \ldots$

2.4 Harmonic Analysis . . . . . . . . . . . . . . . . . 34

2.4.1 Generalized Littlewood-Paley Theory . . . . . . . . . . . . . . 35

2.4.2 Carleson Measures . . . . . . . . . . . . . . . . . 40

2.4.3 Weights and Extrapolation ............... 44

2.4.4 $L^{r}-L^{q}$ Off-diagonal estimates . . . . . . . . . . . . 61

2.4.5 General extrapolation for square functions . . . . . . . . . 63 
2.5 Elliptic theory estimates $\ldots \ldots \ldots \ldots$. . . . . . . . . . 82

2.5.1 Caccioppoli-type inequalities . . . . . . . . . . . . . . 82

2.5.2 Properties of solutions and their gradients on slices . . . . . . . 96

2.5.3 Good Classes of Solutions . . . . . . . . . . . . . . . . . 104

3 Layer potentials: Definitions and basic properties 109

3.1 Basic Definitions . . . . . . . . . . . . . . . . . . . . . . . . . 109

3.2 Green's formula and jump relations $\ldots \ldots \ldots \ldots \ldots$

3.3 Initial $L^{2}$ estimates for the single layer potential $\ldots \ldots \ldots \ldots \ldots$

3.4 Off-diagonal decay and weighted estimates $\ldots \ldots \ldots$. . . . . . 139

$\begin{array}{lll}4 & L^{2} \text { estimates for layer potentials } & 144\end{array}$

$4.1 L^{2}$ square function estimates $\ldots \ldots \ldots \ldots \ldots \ldots \ldots \ldots$

4.2 Reduction to high order $t$-derivatives . . . . . . . . . . . . 145

4.3 Setup for the $T b$ argument and testing functions $\ldots \ldots \ldots \ldots \ldots$

4.4 Properties of the testing functions $\ldots \ldots \ldots \ldots \ldots \ldots \ldots \ldots$

4.5 Control of the auxiliary square functions $\ldots \ldots \ldots \ldots$

4.6 Proof of the square function bound . . . . . . . . . . . . . . . 169

4.7 Control of slices via square function estimates . . . . . . . . . . 179

$5 \quad L^{p}$ estimates for square and nontangential maximal functions 189

5.1 Extrapolation of Square Function Estimates . . . . . . . . . . . . 189 
5.1.1 Estimates for $\nabla \mathcal{S}_{t}^{\mathcal{L}} \ldots \ldots \ldots 19 \ldots \ldots \ldots$

5.1.2 Estimates for $\left(\mathcal{S}_{t}^{\mathcal{L}} \nabla\right) \ldots \ldots . \ldots . \ldots . \ldots 198$

5.2 Control on Slices _. . . . . . . . . . . . . . . . . . 209

5.3 Nontangential Maximal Function Estimates . . . . . . . . . . . . . . 212

5.4 Traveling Down . . . . . . . . . . . . . . . . 223

6 Existence and Uniqueness $\quad 234$

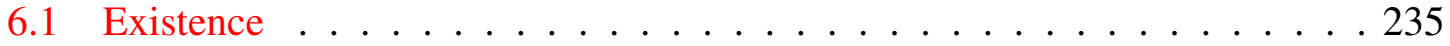

6.2 Uniqueness . . . . . . . . . . . . . . . . . . 252

6.2.1 Neumann and Regularity Problems . . . . . . . . . . . . . 259

6.2.2 The Dirichlet Problem . . . . . . . . . . . . . . . 262

$\begin{array}{ll}\text { Bibliography } & 266\end{array}$

$\begin{array}{ll}\text { Vita } & 283\end{array}$ 


\title{
CRITICAL PERTURBATIONS OF ELLIPTIC OPERATORS BY LOWER ORDER
}

\author{
TERMS \\ Jose Luis Luna Garcia \\ Dr. Steve Hofmann, Dissertation Supervisor
}

\begin{abstract}
In this work we study issues of existence and uniqueness of solutions of certain boundary value problems for elliptic equations in the upper half-space. More specifically we treat the Dirichlet, Neumann, and Regularity problems for the general second order, linear, elliptic operator under a smallness assumption on the coefficients in certain critical Lebesgue spaces.

Our results are perturbative in nature, asserting that if a certain operator $\mathcal{L}_{0}$ has good properties (as far as boundedness and invertibility of certain associated solution operators), then the same is true for $\mathcal{L}_{1}$, whenever the coefficients of these two operators are close in certain $L^{p}$ spaces.
\end{abstract}

Our approach is through the theory of layer potentials, though the lack of good estimates for solutions of $\mathcal{L}=0$ force us to use a more abstract construction of these objects, as opposed to the more classical definition through the fundamental solution. On the other hand, these more general objects suggest a wider range of applications for these techniques. The results contained in this thesis were obtained in collaboration with Simon Bortz, Steve Hofmann, Svitlana Mayboroda, and Bruno Poggi. The resulting publications can be 
found in $\left[\mathrm{BHL}^{+} \mathrm{a}\right]$ and $\left[\mathrm{BHL}^{+} \mathrm{b}\right]$. 


\section{Chapter 1}

\section{Introduction}

In this work, we study the $L^{2}$ Dirichlet, Neumann and Regularity problems for critical perturbations of second order divergence form equations by lower order terms in the upper half-space. In particular, consider operators of the form

$$
\mathcal{L}:=-\operatorname{div}\left(A \nabla+B_{1}\right)+B_{2} \cdot \nabla+V
$$

defined on $\mathbb{R}^{n} \times \mathbb{R}=\{(x, t)\}, n \geq 3$, where $A=A(x)$ is an $(n+1) \times(n+1)$ matrix of $L^{\infty}$ complex coefficients, defined on $\mathbb{R}^{n}$ (independent of $t$ ) and satisfying a uniform ellipticity condition:

$$
\lambda|\xi|^{2} \leq \mathfrak{R e}\langle A(x) \xi, \xi\rangle:=\mathfrak{R e} \sum_{i, j=1}^{n+1} A_{i j}(x) \xi_{j} \bar{\xi}_{i}, \quad\|A\|_{L^{\infty}\left(\mathbb{R}^{n}\right)} \leq \Lambda,
$$

for some $\Lambda, \lambda>0$, and for all $\xi \in \mathbb{C}^{n+1}, x \in \mathbb{R}^{n}$. The first order complex coefficients $B_{1}=B_{1}(x), B_{2}=B_{2}(x) \in\left(L^{n}\left(\mathbb{R}^{n}\right)\right)^{n}$ (independent of $t$ ) and the complex potential $V=$ $V(x) \in L^{\frac{n}{2}}\left(\mathbb{R}^{n}\right)$ (again independent of $t$ ) are such that

$$
\max \left\{\left\|B_{1}\right\|_{L^{n}\left(\mathbb{R}^{n}\right)},\left\|B_{2}\right\|_{L^{n}\left(\mathbb{R}^{n}\right)},\|V\|_{L^{\frac{n}{\left(\mathbb{R}^{n}\right)}}}\right\} \leq \varepsilon_{0}
$$


for some $\varepsilon_{0}$ depending on dimension and the ellipticity of $A$ in order to ensure the accretivity of the form associated to the operator $\mathcal{L}$ on the space

$$
Y^{1,2}\left(\mathbb{R}^{n+1}\right):=\left\{u \in L^{2^{*}}\left(\mathbb{R}^{n+1}\right): \nabla u \in L^{2}\left(\mathbb{R}^{n+1}\right)\right\}
$$

equipped with the norm

$$
\|u\|_{Y^{1,2}\left(\mathbb{R}^{n+1}\right)}:=\|u\|_{L^{2^{*}\left(\mathbb{R}^{n+1}\right)}}+\|\nabla u\|_{L^{2}\left(\mathbb{R}^{n+1}\right)},
$$

where $p^{*}=p_{n+1}^{*}:=\frac{(n+1) p}{n+1-p}$. We interpret solutions of $\mathcal{L} u=0$ in the weak sense; that is, $u \in W_{\text {loc }}^{1,2}\left(\mathbb{R}^{n+1}\right)$ is a solution of $\mathcal{L} u=0$ in $\Omega \subset \mathbb{R}^{n+1}$ if for every $\varphi \in C_{c}^{\infty}(\Omega)$ it holds that

$$
\iint_{\mathbb{R}^{n+1}}\left(\left(A \nabla u+B_{1} u\right) \cdot \overline{\nabla \varphi}+B_{2} \cdot \nabla u \bar{\varphi}\right)=0
$$

We treat the case $n \geq 3$ because the Sobolev spaces we encounter are of the form $\dot{W}^{1,2}\left(\mathbb{R}^{n}\right) \cap L^{s}$ for some $s \geq 1$, and in this case, these spaces embed continuously into Lebesgue spaces. This is not the situation when $n=2$, in which case the Sobolev spaces considered embed continuously into $B M O$. If one were to treat the case $n=2$, it would be natural to assume that $V=0$ and that $B_{i}, i=1,2$ are divergence-free. Under these additional hypotheses, one can use a compensated compactness argument [CLMS93] to obtain the boundedness and invertibility of the form associated to $\mathcal{L}$ (see [GHN16]).

However, there are several considerations in the case $n \geq 3$ that set it qualitatively apart from $n=2$. For instance, when $n=2$, all solutions are locally Hölder continuous and this is certainly not the case when $n \geq 3$. Indeed, let $u(x)=-\ln |x|, x \in \mathbb{R}^{n}$ and build $V(x)$ or 
$B_{1}(x)$ so that either $-\Delta u+V u=0$ or $-\Delta u+\operatorname{div} B_{1} u=0$ in the $n$-dimensional ball $B(0,1 / 2)$. By extending $u$ to be a function on $B(0,1 / 2) \times \mathbb{R}$ by $u(x, t)=u(x)$, we may see that the analogous equations in $n+1$ dimensions are satisfied by $u(x, t)$, and yet $u(x, t)$ fails to be locally bounded despite the fact that $V^{2}, B_{1} \in L^{n}\left(\mathbb{R}^{n}\right)$. Moreover, by considering $u(x, t)$ on a smaller ball and replacing $V$ or $B_{1}$ by $V_{\epsilon}=V \mathbb{1}_{B(0, \epsilon)}$ or $\left(B_{1}\right)_{\epsilon}=B_{1} \mathbb{1}_{B(0, \epsilon)}$ respectively, we may ensure that $V_{\epsilon}^{2}$ or $\left(B_{1}\right)_{\epsilon}$ have arbitrarily small $L^{n}\left(\mathbb{R}^{n}\right)$ norm, provided that we choose $\epsilon>0$ small enough. Therefore, solutions in our perturbative regime fail to be locally bounded and hence fail (miserably) to be locally Hölder continuous.

The lack of local Hölder continuity (or local boundedness) is one reason our results are not at all a straightforward adaptation of related works. For instance, in $\left[\mathrm{AAA}^{+} 11\right]$ the authors are able to treat the fundamental solution as a Calderón-Zygmund-LittlewoodPaley kernel using pointwise estimates on the fundamental solution (and its $t$-derivatives) presented in [HK07]. Additionally, although establishing a Caccioppoli inequality (Proposition 2.5.1) is easy, constants are not necessarily null solutions to our operator and thus this Caccioppoli inequality does not yield the usual "reverse" Poincaré inequality for solutions. We remind the reader that if there are no lower order terms, the Caccioppoli inequality (becomes a "reverse" Poincaré inequality and) improves to an $L^{p}-L^{2}$ version; more precisely, we have that for each ball $B_{r}$ and some $p>2$, the estimate

$$
\left(f_{B_{r}}|\nabla u|^{p} d x\right)^{1 / p} \lesssim \frac{1}{r}\left(f_{B_{2 r}}|u|^{2} d x\right)^{1 / 2}
$$

holds [Mey63, Geh73, Gia83]. We do not manage to obtain the above $L^{p}-L^{2}$ inequality, but 
rather a suitable $L^{p}-L^{p}$ version (Proposition 2.5.9). The unavailability of these desirable estimates makes it far less clear whether constructing the fundamental solution will be useful for us, and so we do not attempt it. We still endeavor to use the method of layer potentials, whence we appeal to (and adapt) the abstract constructions of Barton [Bar17], which avoid the use of fundamental solutions entirely. Fundamental solutions have been constructed in other situations with lower order terms in [DHM18] and [KS19], but they rely on sign conditions (and thus are restricted to real coefficients; in particular this makes it impossible to apply our analytic perturbation arguments as they are).

Our results in this work concern the unique solvability of some classical $L^{2}$ boundary value problems in the upper half space $\mathbb{R}_{+}^{n+1}:=\mathbb{R}^{n} \times \mathbb{R}_{+}$. To state them, we ought to recall the definition of the ( $L^{2}$-averaged) non-tangential maximal operator $N$. Given $x_{0} \in \mathbb{R}^{n}$, define the cone $\gamma\left(x_{0}\right)=\left\{(x, t) \in \mathbb{R}_{+}^{n+1}:\left|x-x_{0}\right|<t\right\}$. Then, for $u: \mathbb{R}_{+}^{n+1} \rightarrow \mathbb{C}$ we write

$$
\widetilde{\mathcal{N}} u\left(x_{0}\right):=\sup _{(x, t) \in \gamma\left(x_{0}\right)}\left(\iint_{|x-y|<t,|s-t|<\frac{t}{2}}|u(y, s)|^{2} d y d s\right)^{\frac{1}{2}}
$$

Given $f: \mathbb{R}^{n} \rightarrow \mathbb{C}$, we say that $u \rightarrow f$ non-tangentially, or $u \rightarrow f$ n.t., if for almost every $x \in \mathbb{R}^{n}, \lim _{(y, t) \rightarrow(x, 0)} u(y, t)=f(x)$, where the limit runs over $(y, t) \in \gamma(x)$.

We consider the Dirichlet problem

$$
\left(\mathrm{D}_{2}\right)\left\{\begin{array}{l}
\mathcal{L} u=0 \quad \text { in } \mathbb{R}^{n+1}+ \\
\lim _{t \rightarrow 0} u(\cdot, t)=f \quad \text { strongly in } L^{2}\left(\mathbb{R}^{n}\right) \text { and non-tangentially, } \\
\widetilde{\mathcal{N}} u \in L^{2}\left(\mathbb{R}^{n}\right), \\
\iint_{\mathbb{R}^{n+1_{+}}} t|\nabla u(x, t)|^{2} d x d t<\infty \\
\lim _{t \rightarrow \infty} u(\cdot, t)=0 \quad \text { in the sense of distributions, }
\end{array}\right.
$$


the Neumann problem

$$
\left(\mathrm{N}_{2}\right)\left\{\begin{array}{l}
\mathcal{L} u=0 \quad \text { in } \mathbb{R}^{n+1}, \\
\frac{\partial u}{\partial v^{\mathcal{L}}}:=-e_{n+1}\left(A \nabla u+B_{1} u\right)(\cdot, 0)=g \in L^{2}\left(\mathbb{R}^{n}\right),{ }^{1} \\
\widetilde{\mathcal{N}}(\nabla u) \in L^{2}\left(\mathbb{R}^{n}\right), \\
\iint_{\mathbb{R}^{n+1}+} t\left|\partial_{t} \nabla u(x, t)\right|^{2} d x d t<\infty, \\
\lim _{t \rightarrow \infty} \nabla u(\cdot, t)=0 \text { in the sense of distributions, }
\end{array}\right.
$$

and the Regularity problem

$$
\left(\mathrm{R}_{2}\right)\left\{\begin{array}{l}
\mathcal{L} u=0 \quad \text { in } \mathbb{R}^{n+1}, \\
u(\cdot, t) \rightarrow f \quad \text { weakly in } Y^{1,2}\left(\mathbb{R}^{n}\right) \text { and non-tangentially, } \\
\widetilde{\mathcal{N}}(\nabla u) \in L^{2}\left(\mathbb{R}^{n}\right), \\
\iint_{\mathbb{R}^{n+1}+} t\left|\partial_{t} \nabla u(x, t)\right|^{2} d x d t<\infty \\
\lim _{t \rightarrow \infty} \nabla u(\cdot, t)=0 \text { in the sense of distributions. }
\end{array}\right.
$$

Uniqueness will be established among the larger class of "good solutions". We refer to subsection 2.5.3 for the relevant precise definitions; here let us just mention that this class contains both the set of solutions satisfying square function estimates, as well as those enjoying nontangential maximal function estimates.

For $\left(\mathrm{N}_{2}\right)$ and $\left(\mathrm{R}_{2}\right)$, if $\mathcal{L} 1=0$, there exists a unique representative of the solution which has the decay property $\lim _{t \rightarrow \infty}\|u(\cdot, t)\|_{\frac{2 n}{n-2}}=0$ (see Corollary 4.7.18). Additionally, since we build our solutions from layer potentials, we will have "compatible well-posedness" (see [HKMP15a]). This means that when the data above is in $C_{c}^{\infty}\left(\mathbb{R}^{n}\right)$, the solutions agree with the solutions constructed via the Lax-Milgram Lemma (see [Axe10, AM19]).

Let us now state the main perturbation result that will be shown in this work.

\footnotetext{
${ }^{1}$ The boundary data is achieved in the distributional sense.
} 
Theorem 1.0.3 (Perturbation result). Suppose that $\mathcal{L}_{0}=-\operatorname{div} A \nabla$ is a divergence form elliptic operator with t-independent coefficients, and that the matrix $A$ is either complex Hermitian, of block form, or constant. Then, there exists $\varepsilon_{0}>0$, depending on dimension and ellipticity, such that if $M \in \mathcal{M}_{n+1}\left(\mathbb{R}^{n}, \mathbb{C}\right), V \in L^{n / 2}\left(\mathbb{R}^{n}\right)$ and $B_{i} \in L^{n}\left(\mathbb{R}^{n}\right)$ are (all) t-independent with

$$
\|M\|_{L^{\infty}\left(\mathbb{R}^{n}\right)}+\left\|B_{1}\right\|_{L^{n}\left(\mathbb{R}^{n}\right)}+\left\|B_{2}\right\|_{L^{n}\left(\mathbb{R}^{n}\right)}+\|V\|_{L^{\frac{n}{2}\left(\mathbb{R}^{n}\right)}}<\varepsilon_{0},
$$

then $\left(D_{2}\right),\left(N_{2}\right)$ and $\left(R_{2}\right)$ are uniquely ${ }^{2}$ solvable for $\mathcal{L}_{1}$, where

$$
\mathcal{L}_{1}=-\operatorname{div}\left([A+M] \nabla+B_{1}\right)+B_{2} \cdot \nabla+V
$$

with the natural square function bounds and non-tangential maximal operator bounds in terms of the norms of the data. Moreover, the solutions to $\left(D_{2}\right),\left(N_{2}\right)$ and $\left(R_{2}\right)$ for $\mathcal{L}_{1}$ can be represented by layer potentials.

Theorem 1.0.3 will be a direct consequence of the current literature and of our second main result, which is

Theorem 1.0.5. Suppose that $\mathcal{L}_{0}=-\operatorname{div} A \nabla$ is a divergence form operator with complex, bounded, elliptic, $t$-independent coefficients. Suppose further that $\pm \frac{1}{2} I+K_{\mathcal{L}}: L^{2}\left(\mathbb{R}^{n}\right) \rightarrow$ $L^{2}\left(\mathbb{R}^{n}\right), \pm \frac{1}{2} I+\widetilde{K}_{\mathcal{L}}: L^{2}\left(\mathbb{R}^{n}\right) \rightarrow L^{2}\left(\mathbb{R}^{n}\right)$, and $\left(S_{0}\right)_{\mathcal{L}}: L^{2}\left(\mathbb{R}^{n}\right) \rightarrow Y^{1,2}\left(\mathbb{R}^{n}\right)$ are all bounded and invertible for $\mathcal{L}=\mathcal{L}_{0}, \mathcal{L}_{0}^{*}$, where $K_{\mathcal{L}}, \widetilde{K}_{\mathcal{L}}$ and $\left(S_{0}\right)_{\mathcal{L}}$ are the "boundary operators" associated to $\mathcal{L}$. Then there exists $\tilde{\varepsilon}_{0}>0$ depending on dimension, ellipticity of $A$ and

\footnotetext{
${ }^{2}$ If $\mathcal{L} 1=0$, then the solutions to $\left(\mathrm{N}_{2}\right)$ and $\left(\mathrm{R}_{2}\right)$ are unique modulo constants. If $\mathcal{L} 1 \neq 0$ then the solutions are unique. See Corollary 4.7.18.
} 
the constants in the operator norms of $\pm \frac{1}{2} I+K_{\mathcal{L}}, \pm \frac{1}{2} I+\widetilde{K}_{\mathcal{L}}$ and $\left(S_{0}\right)_{\mathcal{L}}, \mathcal{L}=\mathcal{L}_{0}, \mathcal{L}_{0}^{*}$ and their inverses, such that if $M \in \mathcal{M} M_{n+1}\left(\mathbb{R}^{n}, \mathbb{C}\right), V \in L^{n / 2}\left(\mathbb{R}^{n}\right)$ and $B_{i} \in L^{n}\left(\mathbb{R}^{n}\right)$ are (all) t-independent with

$$
\|M\|_{L^{\infty}\left(\mathbb{R}^{n}\right)}+\left\|B_{1}\right\|_{L^{n}\left(\mathbb{R}^{n}\right)}+\left\|B_{2}\right\|_{L^{n}\left(\mathbb{R}^{n}\right)}+\|V\|_{L^{\frac{n}{2}\left(\mathbb{R}^{n}\right)}}<\tilde{\varepsilon}_{0}
$$

then $\left(D_{2}\right),\left(N_{2}\right)$ and $\left(R_{2}\right)$ are uniquely solvable for $\mathcal{L}_{1}$, where

$$
\mathcal{L}_{1}:=-\operatorname{div}\left([A+M] \nabla+B_{1}\right)+B_{2} \cdot \nabla+V .
$$

Moreover, the solutions to $\left(D_{2}\right),\left(N_{2}\right)$ and $\left(R_{2}\right)$ for $\mathcal{L}_{1}$ can be represented by layer potentials, and have the natural square function bounds and non-tangential maximal operator bounds in terms of the data.

These results rely on control of certain square functions associated to the single and double layers. For the single layer, we have ${ }^{3}$

Theorem 1.0.6 (Square function bound for the single layer potential). For each $m \in \mathbb{N}$, we have the estimate

$$
\iint_{\mathbb{R}^{n+1}+}\left|t^{m} \partial_{t}^{m+1} \mathcal{S}_{t}^{\mathcal{L}} f(x)\right|^{2} \frac{d x d t}{t} \leq C\|f\|_{L^{2}\left(\mathbb{R}^{n}\right)}^{2}
$$

where $C$ depends on $m, n, \lambda$, and $\Lambda$, provided that $\max \left\{\left\|B_{1}\right\|_{n},\left\|B_{2}\right\|_{n},\|V\|_{\frac{n}{2}}\right\}$ is sufficiently small depending on $n, \lambda$, and $\Lambda$. Under the same hypothesis, the analogous bounds hold for $\mathcal{L}$ replaced by $\mathcal{L}^{*}$, and for $\mathbb{R}_{+}^{n+1}$ replaced by $\mathbb{R}_{-}^{n+1}$.

\footnotetext{
${ }^{3}$ Estimates for $\mathcal{D}$ will be a consequence of this and a formula relating the single and double layer.
} 
We point out that in the previous result, there is no restriction on the matrix $A$, other than that it be $t$-independent and satisfy the complex ellipticity condition (1.2). In the homogeneous, purely second order case (i.e., the case that $B_{1}, B_{2}$, and $V$ are all zero), this result is due to Rosén [Ros13]; an alternative proof, with an extra hypothesis of De Giorgi/Nash/Moser regularity, appears in [GH16]. On the other hand, the smallness of the lower order terms is unavoidable; for one it's required for the coercivity of the form, moreover, our arguments will require some further restrictions in order to obtain the desired control of the square function.

Following this, we obtain uniform control on slices of the first derivatives of solutions. More specifically,

Theorem 1.0.7 (Uniform control of $Y^{1,2}\left(\mathbb{R}^{n}\right)$ norm on each horizontal slice). Suppose that $u \in Y^{1,2}\left(\mathbb{R}_{+}^{n+1}\right)$ and $\mathcal{L} u=0$ in $\mathbb{R}_{+}^{n+1}$ in the weak sense. Then for every $\tau>0$,

$$
\left\|\operatorname{Tr}_{\tau} u\right\|_{L^{\frac{2 n}{n-2}\left(\mathbb{R}^{n}\right)}}+\left\|\operatorname{Tr}_{\tau} \nabla u\right\|_{L^{2}\left(\mathbb{R}^{n}\right)} \lesssim \int_{\tau}^{\infty} \int_{\mathbb{R}^{n}} t\left|D_{n+1}^{2} u\right|^{2} d x d t \leq\left\|t D_{n+1}^{2} u\right\| \|,
$$

where $C$ depends on $m, n, \lambda$, and $\Lambda$, provided that $\max \left\{\left\|B_{1}\right\|_{n},\left\|B_{2}\right\|_{n},\|V\|_{\frac{n}{2}}\right\}$ is sufficiently small depending on $m, n, \lambda$, and $\Lambda$. Under the same hypothesis, the analogous bounds hold for $\mathcal{L}$ replaced by $\mathcal{L}^{*}$, and for $\mathbb{R}_{+}^{n+1}$ replaced by $\mathbb{R}_{-}^{n+1}$.

Theorem 1.0.9. Let

$$
\mathcal{L}:=-\operatorname{div}\left(A \nabla+B_{1}\right)+B_{2} \cdot \nabla+V
$$

where $A, B_{1}, B_{2}, V$ are as above. There exists $\rho_{1} \in\left(0, \tilde{\rho}_{1}\right)$ depending on dimension and the 
ellipticity of A such that if

$$
\max \left\{\left\|B_{1}\right\|_{L^{n}\left(\mathbb{R}^{n}\right)},\left\|B_{2}\right\|_{L^{n}\left(\mathbb{R}^{n}\right)},\|V\|_{L^{\frac{n}{2}\left(\mathbb{R}^{n}\right)}}\right\}<\rho_{1},
$$

then the following estimates hold for the single and double layer potentials.

1. $\|\widetilde{N}(\nabla \mathcal{S} f)\|_{L^{2}\left(\mathbb{R}^{n}\right)} \leq C\|f\|_{L^{2}\left(\mathbb{R}^{n}\right)}$,

2. $\|\widetilde{N}(\mathcal{D} f)\|_{L^{2}\left(\mathbb{R}^{n}\right)} \leq C\|f\|_{L^{2}\left(\mathbb{R}^{n}\right)}$.

Here, the constant $C$ depends only on dimension and ellipticity, and $\widetilde{N}$ is the modified nontangential maximal function (see Definition 2.4 .7 below).

The idea to proving Theorem 1.0.9 starts with proving a weak- $L^{p}$ bound ( $L^{p, \infty}$ bound) for the nontangential maximal function in terms of the $L^{p}$ norms of the vertical and conical square functions (see Lemma 5.3.17). Then, interpolation will show that Theorem 1.0.9 holds provided that the vertical and conical maximal functions are bounded in $L^{p}$ for an open interval (in $p$ ) around 2. The starting point for obtaining such bounds for the square functions is to prove general bounds for operators with sufficient off-diagonal decay which satisfy a local reverse-Hölder inequality using the extrapolation theory from weighted norm inequalities [CMP11]. Arguments similar to ours have been used in [Pri19] to treat square function estimates for operators built out of the heat or Poisson semigroups associated to an elliptic operator; however, in our case we must grapple with the added difficulty of having very mild off-diagonal decay. On the other hand, the local energy inequality for the equation (the Caccioppoli inequality) allows us to obtain the necessary off-diagonal decay 
for related operators with added (transversal) derivatives. Having done so, our remaining task is to "remove" these additional derivatives, a process which we call "traveling down". Due to its definition, this process for the vertical square function is a relatively simple integration by parts computation, relying on the estimates on slices of Theorem 1.0.7. For the conical square function, the additional spatial average impedes the simple integration by parts and our argument for this object requires the boundedness of the non-tangential maximal function with the same number of derivatives. Luckily, our Lemma 5.3.17, when combined with Proposition 5.3.1, gives that the non-tangential maximal function bounds (for this family of operators) depend on square functions with more $e^{4}$ derivatives. This allows us to employ a two-step induction scheme where one alternates between bounding the $L^{p}$ norm for a nontangential maximal function by the $L^{p}$ norm of square functions (with more derivatives) and then bounding the $L^{p}$ norm of the conical square function by the $L^{p}$ norm of a nontangential maximal function (with the same number of derivatives). Thus, in finitely many steps, we remove these additional derivatives. (Recall we can start this process, that is, obtain $L^{p}$ bounds for the vertical and conical square functions, by introducing enough transversal derivatives.)

Once we have these results in hand, we turn to the solvability of $\left(D_{2}\right),\left(N_{2}\right)$, and $\left(R_{2}\right)$. As mentioned before this is done through analytic perturbation, and at this stage, with square and nontangential maximal function estimates available, the process is the same

\footnotetext{
${ }^{4}$ Note that in Lemma 5.3.17, we may use that $\left\|\mathbb{V}\left(\Theta_{t, 1} f\right)\right\|_{L^{p}\left(\mathbb{R}^{n}\right)} \lesssim_{m}\left\|\mathbb{V}\left(\Theta_{t, m+1} f\right)\right\|_{L^{p}\left(\mathbb{R}^{n}\right)}$, by the aforementioned integration by parts argument. The subscript $m$ refers to the number of transversal derivatives.
} 
as in $\left[\mathrm{AAA}^{+} 11\right]$ to obtain boundedness and invertibility of the associated layer potentials. Having this last, existence of solutions with the desired properties follows familiar lines. As mentioned before, uniqueness will be obtained in the larger class of "good" solutions.

The results here may be best thought of as extensions of the results in $\left[\mathrm{AAA}^{+} 11\right]$ to lower-order terms ${ }^{5}$ as well as complex matrices (and not only those arising from perturbations of real symmetric coefficients or constant coefficients), albeit with the important distiction that we do not require DeGiorgi-Nash-Moser [DG57, Nas58, Mos61] estimates; this allows us to consider any complex elliptic matrix for $A$.

We will now review some of the extensive history of boundary value problems for second order divergence form elliptic operators in Lipschitz domains. Unless otherwise stated, the results below will always be results for operators without lower order terms. For Laplace equation $(\mathcal{L}=-\Delta)$ in a Lipschitz domain, solvability of $\left(\mathrm{D}_{2}\right)$ was obtained by Dahlberg [Dah77], and solvability of $\left(\mathrm{N}_{2}\right)$ and $\left(\mathrm{R}_{2}\right)$ was shown by Jerison and Kenig [JK81b]; these were also shown later by Verchota [Ver84] via the method of layer potentials, using the celebrated result of Coifman, McIntosh and Meyer [CMM82]. For real, symmetric and $t$-independent coefficients, the solvability of $\left(\mathrm{D}_{2}\right)$ was shown by Jerison and Kenig [JK81a], and the solvability of $\left(\mathrm{N}_{2}\right)$ and $\left(\mathrm{R}_{2}\right)$ was shown by Kenig and Pipher [KP93]. The solvability via the method of layer potentials in the case of real, symmetric and $t$-independent coefficients was shown in [AAA $\left.{ }^{+} 11\right]$ (and previously by Mitrea,

\footnotetext{
${ }^{5}$ Indeed, this project started out as trying to apply the techniques in the above paper to equations with lower order terms.
} 
Mitrea and Taylor [MMT01] with some additional smoothness assumptions on the coefficients).

Aside from the results in $\left[\mathrm{AAA}^{+} 11, \mathrm{AAH} 08, \mathrm{AAM} 10\right]$, which we describe further below, the known results for non-symmetric, $t$-independent matrices can be split into three categories: complex perturbations of constant coefficient matrices, 'block' form matrices and real t-independent coefficients. In [FJK84], Fabes, Jerison and Kenig showed solvability of $\left(\mathrm{D}_{2}\right)$ for small complex $L^{\infty}$-perturbations of constant coefficient operators, the solvability of $\left(\mathrm{D}_{2}\right),\left(\mathrm{N}_{2}\right)$ and $\left(\mathrm{R}_{2}\right)$ in this setting was shown via layer potentials in [AAA $\left.{ }^{+} 11\right]$ (see $\left[\mathrm{AAA}^{+} 11\right.$, Theorem 1.15]).

Solvability of $L^{2}$ boundary value problems in the case of all 'block' form matrices

$$
A(x)=\left(\begin{array}{ccc|c} 
& & & 0 \\
& B(x) & & \vdots \\
& & & 0 \\
\hline 0 & \cdots & 0 & 1
\end{array}\right),
$$

where $B(x)$ is an $n \times n$ matrix is, in the case of $\left(\mathrm{D}_{2}\right)$, a consequence of the semigroup theory and, in the case of $\left(\mathrm{N}_{2}\right)$ and $\left(\mathrm{R}_{2}\right)$, a consequence of the solution to the Kato problem $\left[\mathrm{AHL}^{+} 02\right]$ on $\mathbb{R}^{n}$. In particular, if we let $J:=-\operatorname{div}_{x} B(x) \nabla_{x}$, then one obtains the solvability of $\left(\mathrm{R}_{2}\right)$ by solving the Kato problem for $J$, and one obtains the solvability of $\left(\mathrm{N}_{2}\right)$ from solving the Kato problem for $a d j(J)$. In fact, for $\left(\mathrm{N}_{2}\right)$, one may equivalently show that the Riesz transforms associated to $J, \nabla J^{-1 / 2}$, are $L^{2}$ bounded, which can be, in turn, be interpreted as a statement about the boundedness of the single layer potential from $L^{2}$ into $\dot{L}_{1}^{2}$. These results were obtained in [CMM82] $(n+1=2)$ and in $\left[\mathrm{AHL}^{+} 02\right](n+1 \geq 3)$; see 
also [HM02, AHLT01, HLM02].

In the case of real, $t$-independent coefficients, the results available are of the form $\left(\mathrm{D}_{p}\right)$ (for some $p<\infty$ sufficiently large), $(\mathrm{N} p)$ and $\left(\mathrm{R}_{p}\right)$ (for some $p>1$, typically dual to the Dirichlet exponent), where $\left(\mathrm{D}_{p}\right),\left(\mathrm{N}_{p}\right)$, and $\left(\mathrm{R}_{p}\right)$ are $L^{p}$ analogues of $\left(\mathrm{D}_{2}\right),\left(\mathrm{N}_{2}\right)$, and $\left(\mathrm{R}_{2}\right)$ respectively. This is the best one can hope for by a counter-example in [KKPT00] (but see also [Axe10]), where the authors show that for any fixed $p<\infty$, there exists a real (non-symmetric) coefficient matrix $A$, such that $\left(\mathrm{D}_{p}\right)$ fails to be solvable for the associated divergence form elliptic operator. In [KKPT00], the authors show that for all real $t$-independent coefficients with $n+1=2$, the problem $\left(\mathrm{D}_{p}\right)$ is solvable for some $p<\infty$. In the same setting, Kenig and Rule [KR09] showed the solvability of $(\mathrm{N} q)$ and $(\mathrm{R} q)$ for $q$ the Hölder conjugate of the exponent $p$ from the aforementioned result [KKPT00]. More recently, Barton [Bar13] perturbed these solvability results to deduce that $\left(\mathrm{D}_{p}\right),\left(\mathrm{N}_{q}\right)$, and $\left(\mathrm{R}_{q}\right)$ remain solvable in the half-plane when the matrix consists of almost-real coefficients; and the methods of [KKPT00] were (nontrivially) extended by Hofmann, Kenig, Mayboroda and Pipher [HKMP15b, HKMP15a] to show the solvability of $\left(\mathrm{D}_{p}\right)$ for some $p<\infty$ for all real $t$-independent coefficients when $n+1 \geq 3$ and solvability of $\left(\mathrm{R}_{q}\right)$, again with $q$ dual to $p$.

As mentioned above, perhaps the closest results to the current exposition are $\left[\mathrm{AAA}^{+} 11\right.$, AAH08, AAM10], where $L^{2}$ solvability of boundary value problems was explored for full complex matrices, either by the method of layer potentials $\left[\mathrm{AAA}^{+} 11\right]$ or the "first-order ap- 
proach" [AAH08, AAM10] (which relies on the functional calculus of Dirac operators associated to divergence form elliptic operators). In [AAA $\left.{ }^{+} 11\right]$, the authors show solvability of $\left(\mathrm{D}_{2}\right),\left(\mathrm{N}_{2}\right)$ and $\left(\mathrm{R}_{2}\right)$ via the method of layer potentials for $L^{\infty}$ perturbations of real, symmetric $t$-independent coefficients, and $L^{\infty}$ perturbations of constant coefficients. In [AAH08], the authors show solvability of $\left(\mathrm{D}_{2}\right),\left(\mathrm{N}_{2}\right)$ and $\left(\mathrm{R}_{2}\right)$ in the same cases as [AAA $\left.{ }^{+} 11\right]$, as well as perturbations from block form matrices. In [AAM10], the authors treat the previous cases of [AAH08] as well as perturbations of Hermitian coefficient matrices.

We mention also the work of Gesztesy, Hofmann and Nichols [GHN16], where the authors studied the $n$-dimensional Kato problem related to our perturbations. These works [AAA ${ }^{+} 11, \mathrm{AAH} 08, \mathrm{AAM} 10, \mathrm{GHN16}$ as well as [Ros13, GH16, HMM15] served as an indication that the present results should hold. The techniques from the solution to the (original) Kato problem are integral to our analysis. In particular, we adapt the methods from [AAA $\left.{ }^{+} 11, \mathrm{GH} 16, \mathrm{HMM} 15\right]$ to prove our square function estimates for the single layer potential (Theorems 1.0.6 and 4.2.5) via the generalized $T b$ theory developed in the resolution of the Kato problem [AHL $\left.{ }^{+} 02\right]$ and since refined in [GH16].

Let us remark on the assumption of $t$-independence. Given a second order divergence form elliptic operator (no lower order terms), define the transverse modulus of continuity $\omega(\tau):(0, \infty) \rightarrow[0, \infty]$ as

$$
\omega(\tau):=\sup _{x \in \mathbb{R}^{n}} \sup _{t \in(0, \tau)}|A(x, t)-A(x, 0)| .
$$

In [CFK81], Caffarelli, Fabes and Kenig showed that given any function $\omega(\tau):(0, \infty) \rightarrow$ 
$[0, \infty]$ such that $\int_{0}^{1}[\omega(\tau)]^{2} d \tau / \tau=\infty$, there exists a real, symmetric elliptic matrix with transverse modulus of continuity $\omega(\tau)$ such that the corresponding elliptic measure and $n$ dimensional Lebesgue measure (on $\mathbb{R}^{n} \times\{0\}$ ) are mutually singular, and hence (D2) (or even $\left(\mathrm{D}_{p}\right)$ for any $p$ ) fails to be solvable. On the other hand, in [FJK84], the authors show that $\left(\mathrm{D}_{2}\right)$ is uniquely solvable provided that $\int_{0}^{1}[\omega(\tau)]^{2} d \tau / \tau<\infty$ and that $A(x, 0)$ is sufficiently close to a constant matrix. Later, refinements of this condition were introduced and investigated; in these refinements one measures some discrepancy on Whitney boxes quantified by a Carleson measure condition; see, for instance, [AA11,FKP91, Dah86, DPP07,DPR17, DP19, HMM15, KP95, KP01].

We end this review of the history of the work on the homogeneous (i.e., no lower order terms) operators by noting that the a priori connections between the different problems $\left(\mathrm{D}_{p}\right),\left(\mathrm{N}_{p^{\prime}}\right)$ and $\left(\mathrm{R}_{p^{\prime}}\right)$ have also been of great interest. In some instances (say, $A$ is real, $t$-independent), one has that the solvability of $\left(\mathrm{R}_{p}\right)$ for $L$ implies solvability of $\left(\mathrm{D}_{p^{\prime}}\right)$ for the adjoint operator $L^{*}$, and viceversa (where $p^{\prime}$ is the Hölder conjugate to $p$ ) (see [Ken94]), but it was found in [May10] that such implications need not hold in the general setting of complex coefficients, even for $t$-independent matrices. We refer to [May10] for a more systematic review of these connections.

The literature in the setting with lower order terms present (that is, not all of $b_{1}, b_{2}, V$ are identically 0) is much more sparse. In [HL01], parabolic operators with singular drift terms $b_{2}$ were studied, and their results would later be applied toward $\left(\mathrm{D}_{p}\right)$ for elliptic 
operators with singular drift terms $b_{2}$ in [KP01] and [DPP07]. When $A \equiv I, b_{1} \equiv b_{2} \equiv 0$ and $V>0$ satisfies certain conditions, Shen proved the solvability of $\left(\mathrm{N}_{p}\right)$ on Lipschitz domains in [She94]. His results were later extended in [Tao12, TW01] to $\left(\mathrm{R}_{p}\right)$ and under weaker assumptions on the potential $V$. It is a critical element of the proof that the leading term of $L \equiv-\Delta+V$ is the Laplacian, and the question of $\left(\mathrm{N}_{p}\right)$-solvability for Schrödinger operators on rough domains in the case that $A \neq I$ remain open, even under generous assumptions on $V$.

More recently, the problems $\left(\mathrm{D}_{2}\right)$ and $\left(\mathrm{R}_{2}\right)$ for equations with lower order terms have been considered in [Sak19] in bounded Lipschitz domains, under some continuity and sign assumptions on the coefficients. Solvability results for the variational Dirichlet problem of equations with lower order terms on unbounded domains have been obtained in [Mou].

The thesis is divided into five main chapters. The first is reserved for a somewhat extensive list of preliminary results, mostly coming from functional analysis and the theory of Muckenhoupt weights. In the second chapter we introduce our main subjects of study: the single and double layer potentials associated to the operator $\mathcal{L}$; it's here that we establish their basic properties, following the approach of Barton in [Bar13]; we also prove useful $L^{2}$ and off-diagonal estimates that we'll need in the next chapters. Chapter 3 contains the proof of the main $L^{2}$ square function estimates for the single and double layer, it's the main result of $\left[\mathrm{BHL}^{+} \mathrm{a}\right]$. The fourth chapter extends the results from chapter 3 to $L^{p}$ spaces, for $p$ in a window around 2, through weighted, restricted extrapolation. Here we have to 
treat in tandem the square and nontangential maximal functions. ${ }^{6}$ Finally, chapter 5 uses all previous results to establish existence and uniqueness for the Dirichlet, Neumann and Regularity problems in $L^{2}{ }^{7}$ The last two chapters contain the main results of $\left[\mathrm{BHL}^{+} \mathrm{b}\right]$.

\footnotetext{
${ }^{6}$ It's actually worth noting that in the absence of $L^{p}$ estimates, our arguments here would only yield weak $L^{2}$ bounds for the nontangential maximal function.

${ }^{7}$ While our arguments seem to give existence of solutions in $L^{p}$ for $p$ in a window around 2 with little effort, uniqueness in the generality that we work in seems to need more work. We do not consider these cases.
} 


\section{Chapter 2}

\section{Preliminaries}

\subsection{Notation}

As stated above, our standing assumption will be that $n \geq 3$, and the ambient space will always be $\mathbb{R}^{n+1}=\left\{(x, t): x \in \mathbb{R}^{n}, t \in \mathbb{R}\right\}$. We employ the following standard notation:

- We will use lower-case $x, y, z$ to denote points in $\mathbb{R}^{n}$ and lower-case $t, s, \tau$ to denote real numbers. By convention, $x=\left(x_{1}, \ldots, x_{n}\right)$, and $x_{n+1}=t$. We will use capital $X, Y, Z$ to denote points in $\mathbb{R}^{n+1}$. The symbols $e_{1}, \ldots, e_{n+1}$ are reserved for the standard basis vectors in $\mathbb{R}^{n+1}$.

- Given the $(n+1) \times(n+1)$ complex-valued matrix $A$, for each $i, j=1, \ldots, n+1$, we denote by $A_{i j}$ the $i j$-th entry of $A$. We denote by $\tilde{A}$ the $(n+1) \times n$ submatrix of $A$ consisting of

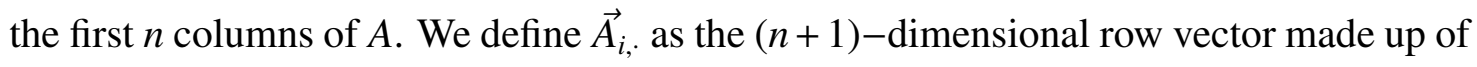
the $i$-th row of $A$; similarly we let $\vec{A}_{\cdot, j}$ be the $(n+1)$-dimensional column vector made up of the $j$-th row of $A$. We also let $A_{\|}$be the $n \times n$ matrix with entries $\left(a_{i j}\right)_{1 \leq i, j \leq n}$. 
- We set $\mathbb{R}^{n+1}{ }_{+}:=\mathbb{R}^{n} \times(0,+\infty)$ and $\partial \mathbb{R}^{n+1}{ }_{+}:=\mathbb{R}^{n} \times\{0\}$. We define $\mathbb{R}^{n+1}$ - similarly and often we write $\mathbb{R}^{n}$ in place of $\partial \mathbb{R}^{n+1}+$ when confusion may not arise. For $t \in \mathbb{R}$, we denote $\mathbb{R}_{t}^{n+1}=\mathbb{R}_{+, t}^{n+1}:=\mathbb{R}^{n} \times(t, \infty)$, and $\mathbb{R}_{-, t}^{n+1}:=\mathbb{R}^{n} \times(-\infty, t)$

- The letter $Q$ will always denote a cube in $\mathbb{R}^{n}$. By $\ell(Q)$ and $x_{Q}$ we denote the side length and center of $Q$, respectively. We write $Q(x, r)$ to denote the cube with center $x$ and sides of length $r$, parallel to the coordinate axes.

- Given a (closed) $n$-dimensional cube $Q=Q(x, r)$, its concentric dilate by a factor of $\kappa>0$ will be denoted $\kappa Q:=Q(x, \kappa r)$. Similar dilations are defined for cubes in $\mathbb{R}^{n+1}$ as well as (open) balls in $\mathbb{R}^{n}$ and $\mathbb{R}^{n+1}$.

- For $a, b \in[-\infty, \infty]$, we set $\Sigma_{a}^{b}:=\left\{X=(x, t) \in \mathbb{R}^{n+1}: t \in(a, b)\right\}$.

- Given a Borel set $E$ and Borel measure $\mu$, for any $\left.\mu\right|_{E}$-measurable function $f$ we define the $\mu$-average of $f$ over $E$ as

$$
f_{E} f d \mu:=\frac{1}{\mu(E)} \int_{E} f d \mu .
$$

- For a Borel set $E \subset \mathbb{R}^{n+1}$, we let $\mathbb{1}_{E}$ denote the usual indicator function of $E$; that is, $\mathbb{1}_{E}(x)=1$ if $x \in E$, and $\mathbb{1}_{E}(x)=0$ if $x \notin E$.

- For a Banach space $X$, we let $\mathcal{B}(X)$ denote the space of bounded linear operators on $X$. Simiarly, if $X$ and $Y$ are Banach spaces, we denote by $\mathcal{B}(X, Y)$ the space of bounded linear operators $X \rightarrow Y$. 
- For $-\infty \leq a<b \leq \infty$ we define the slab $\Sigma_{a}^{b}:=\left\{(x, t) \in \mathbb{R}^{n+1}: a<t<b\right\}$.

- For a vector $\vec{v}=\left(v_{1}, \ldots, v_{n+1}\right) \in \mathbb{R}^{n+1}$, we write $\vec{v}_{\|}:=\left(v_{1}, \ldots, v_{n}\right), \vec{v}_{\perp}:=v_{n+1}$. For vector functions $B: \mathbb{R}^{n} \rightarrow \mathbb{C}^{n+1}$, we define $B_{\|}$and $B_{\perp}$ analogously.

- We label the horizontal component of the $(n+1)$-dimensional gradient operator as

$$
\nabla_{\|}:=\nabla_{x}:=\left(\partial_{x_{1}}, \ldots, \partial_{x_{n}}\right)
$$

and the 'vertical' component as $D_{n+1}$ or $\nabla_{\perp}$.

- For a cube $Q \subset \mathbb{R}^{n}$ we will always denote by $R_{Q}$ the Carleson region above $Q$; that is, $R_{Q}:=Q \times(0, \ell(Q))$

- For $R>0$ we define $I_{R}:=(R, R)^{n+1} \subset \mathbb{R}^{n+1}$, and $I_{R}^{ \pm}:=I_{R} \cap \mathbb{R}^{n+1}{ }_{ \pm}$.

- We denote by $\mathcal{M}$ the (uncentered) Hardy-Littlewood maximal function in $\mathbb{R}^{n}$, and more generally for $r>0$ we define $\mathcal{M}_{r}(f):=\mathcal{M}\left(|f|^{r}\right)^{1 / r}$.

- Given a cube $Q \subset \mathbb{R}^{n}$ we denote by $Q^{*}$ a concentric dilate of $Q$ by a factor that depends only on dimension.

- We will often have use for the fractional integral of order 1 in $\mathbb{R}^{n}$, which we denote by $I_{1}$; that is, for nice enough $f$, we have

$$
I_{1} f(x)=c_{n} \int_{\mathbb{R}^{n}} \frac{f(y)}{|x-y|^{n-1}} d y
$$


- We denote by $\mathbb{D}$ the collection of all dyadic cubes in $\mathbb{R}^{n}$, and for $t>0$ we define $\mathbb{D}_{t}$ to be the cubes in $\mathbb{D}$ which satisfy $\ell(Q)<t \leq 2 \ell(Q)$. Similarly, for a cube $Q \subset \mathbb{R}^{n}$ we denote by $\mathbb{D}(Q)$ the collection of dyadic subcubes of $Q$.

- For $(x, t) \in \mathbb{R}_{+}^{n+1}$ we define the Whitney regions

$$
C_{x, t}:=\left\{(y, s) \in \mathbb{R}_{+}^{n+1}:|x-y|<t / 8,|t-s|<t / 8\right\}
$$

- Let $X$ be a topological space with Borel $\sigma$-algebra $\mathcal{B}$, and let $\mu$ be a non-negative $\sigma$-finite measure on $\mathcal{B}$. If $p \in[1, \infty)$, we denote by $L^{p}(X, \mu)$ the Lebesgue space of $p$-th integrable (complex) functions $f$ on the measure space $(X, \mathcal{B}, \mu)$. We often write $L^{p}\left(\mathbb{R}^{n}\right)=L^{p}\left(\mathbb{R}^{n}, m_{n}\right)$, where $m_{n}$ is the $n$-dimensional Lebesgue measure. If $v \in L_{\text {loc }}^{1}\left(\mathbb{R}^{n}\right)$, we write $L^{p}(v)=L^{p}\left(\mathbb{R}^{n}, v d m_{n}\right)$.

- Given an open set $\Omega \subset \mathbb{R}^{d}, d \geq 3$, we denote by $C_{c}^{\infty}(\Omega)$ the space consisting of all compactly supported smooth complex-valued functions in $\Omega$. As usual, we denote $\mathscr{D}=$ $C_{c}^{\infty}\left(\mathbb{R}^{n+1}\right)$, and we let $\mathscr{D}^{\prime}=\mathscr{D}^{*}$ be the space of distributions on $\mathbb{R}^{n+1}$. The space $\mathscr{S}$ consists of the Schwartz functions on $\mathbb{R}^{n+1}$, and $\mathscr{S}^{\prime}=\mathscr{S}^{*}$ is the space of tempered distributions on $\mathbb{R}^{n+1}$.

- We call a measurable function $v: \mathbb{R}^{n} \rightarrow \mathbb{R}$ a weight if $v>0$ Lebesgue-a.e. on $\mathbb{R}^{n}$ and $v \in L_{\mathrm{loc}}^{1}\left(\mathbb{R}^{n}\right)$. We say that $v$ is doubling if the measure $v(x) d x$ is doubling; that is, if (with a slight abuse of notation) $v(2 Q) \leq C_{0} v(Q)$ for a constant $C_{0}>0$ and all cubes $Q \subset \mathbb{R}^{n}$. 
- For $1<p<m$, the upper and lower Sobolev exponents of order 1 in $m$ dimensions are

$$
p_{m}^{*}:=\frac{m p}{m-p}, \quad p_{*, m}:=\frac{m p}{m+p}
$$

respectively. Sometimes, we will drop $m$ from the subscript when the dimension is clear from the context.

- Given an open set $\Omega \subset \mathbb{R}^{n+1}$, then for $p \in[1, \infty)$, we denote by $W^{1, p}(\Omega)$ the usual Sobolev space of functions in $L^{p}(\Omega)$ whose weak gradients exist and lie in $\left(L^{p}(\Omega)\right)^{n+1}$. We endow this space with the norm

$$
\|u\|_{W^{1, p}(\Omega)}:=\|u\|_{L^{p}(\Omega)}+\|\nabla u\|_{L^{p}(\Omega)} .
$$

We define $W_{0}^{1, p}(\Omega)$ as the completion of $C_{c}^{\infty}(\Omega)$ in the above norm. We shall have occasion to discuss the homogeneous Sobolev spaces as well: by $\dot{W}^{1, p}(\Omega)$ we denote the space of functions in $L_{\text {loc }}^{1}(\Omega)$ whose weak gradients exist and lie in $L^{p}(\Omega)$. We equip this space with the seminorm

$$
|u|_{W^{1, p}(\Omega)}:=\|\nabla u\|_{L^{p}(\Omega)},
$$

and point out that $\dot{W}^{1, p}(\Omega)$ coincides with the completion of the quotient space $C^{\infty}(\Omega) / \mathbb{C}$ in the $|\cdot|_{\dot{W}^{1, p}(\Omega)}$ (quotient) norm.

\subsection{Function spaces}

We will work with several function spaces; let us briefly describe them. For the rest of the paper, we assume that the reader is familiar with the basics of the theory of distribu- 
tions and Fourier Transform (see [Gra14a]) and the basics of the theory of Sobolev spaces (see [Leo17]). We delegate some of the basic definitions and results to these and other introductory texts.

Let $\Omega$ be an open set in $\mathbb{R}^{k}$ for some $k \in \mathbb{N}$. For any $m \in \mathbb{N}$ and any $p \in[1, \infty)$, the space $L^{p}(\Omega)^{m}=L^{p}\left(\Omega, \mathbb{C}^{m}\right)$ consists of the complex-valued $p$-th integrable $m$-dimensional vector functions over $\Omega$. We equip $L^{p}\left(\Omega, \mathbb{C}^{m}\right)$ with the norm

$$
\|\vec{f}\|_{L^{p}\left(\Omega, \mathbb{C}^{m}\right)}=\left(\sum_{i=1}^{m} \int_{\Omega}\left|f_{i}\right|^{p}\right)^{\frac{1}{p}}, \quad \vec{f}=\left(f_{1}, \ldots, f_{m}\right) .
$$

For simplicity of notation, we often write $\|\vec{f}\|_{p}=\|\vec{f}\|_{L^{p}(\Omega)}=\|\vec{f}\|_{L^{p}\left(\Omega, \mathbb{C}^{m}\right)}$ when the domain $\Omega$ and the dimension of the vector function $\vec{f}$ are clear from the context (most often, when $\Omega$ is the ambient space, which for us means either $\Omega=\mathbb{R}^{n}$ or $\Omega=\mathbb{R}^{n+1}$ ).

The space $C_{c}^{\infty}(\Omega)$ consists of all compactly supported smooth complex-valued functions in $\Omega$. As usual, we denote $\mathscr{D}=C_{c}^{\infty}\left(\mathbb{R}^{n+1}\right)$, and we let $\mathscr{D}^{\prime}=\mathscr{D}^{*}$ be the space of distributions on $\mathbb{R}^{n+1}$. The space $\mathscr{S}$ consists of the Schwartz functions on $\mathbb{R}^{n+1}$, and $\mathscr{S}^{\prime}=\mathscr{S}^{*}$ is the space of tempered distributions on $\mathbb{R}^{n+1}$.

For $p \in[1, \infty)$, we denote by $W^{1, p}(\Omega)$ the usual Sobolev space of functions in $L^{p}(\Omega)$ whose weak gradients exist and lie in $\left(L^{p}(\Omega)\right)^{n+1}$. We endow this space with the norm

$$
\|u\|_{W^{1, p}(\Omega)}:=\|u\|_{L^{p}(\Omega)}+\|\nabla u\|_{L^{p}(\Omega)} .
$$

We define $W_{0}^{1, p}(\Omega)$ as the completion of $C_{c}^{\infty}(\Omega)$ in the above norm. We shall have occasion to discuss the homogeneous Sobolev spaces as well: by $\dot{W}^{1, p}(\Omega)$ we denote the space of 
functions in $L_{\text {loc }}^{1}(\Omega)$ whose weak gradients exist and lie in $L^{p}(\Omega)$. We equip this space with the seminorm

$$
|u|_{W^{1, p}(\Omega)}:=\|\nabla u\|_{L^{p}(\Omega)}
$$

and point out that $\dot{W}^{1, p}(\Omega)$ coincides with the completion of the quotient space $C^{\infty}(\Omega) / \mathbb{C}$ in the $|\cdot|_{\dot{W}^{1, p}(\Omega)}$ (quotient) norm. For $p \in(1, n+1)$ and $\Omega \subset \mathbb{R}^{n+1}$ an open set, we define the space $Y^{1, p}(\Omega)$ as

$$
Y^{1, p}(\Omega):=\left\{u \in L^{p^{*}}(\Omega): \nabla u \in L^{p}(\Omega)\right\}
$$

where $p^{*}:=\frac{(n+1) p}{n+1-p}$. We equip this space with the norm

$$
\|u\|_{Y^{1, p}(\Omega)}:=\|u\|_{L^{p^{*}(\Omega)}}+\|\nabla u\|_{L^{p}(\Omega)} .
$$

We define $Y_{0}^{1, p}(\Omega)$ as the completion of $C_{c}^{\infty}(\Omega)$ in this norm. By virtue of the Sobolev embedding, when $p \in(1, n+1)$ we have that $Y_{0}^{1, p}(\Omega)$ coincides with the completion of $C_{c}^{\infty}(\Omega)$ in the $\dot{W}^{1, p}(\Omega)$ seminorm. Moreover, we have that $Y_{0}^{1, p}\left(\mathbb{R}^{n+1}\right)=Y^{1, p}\left(\mathbb{R}^{n+1}\right)$.

The $Y^{1,2}$ spaces exhibit the following useful property.

Lemma 2.2.1 (Integrability up to a constant of a function with square integrable gradient on a half-space). Suppose that $u \in L_{\mathrm{loc}}^{1}\left(\Sigma_{a}^{b}\right)$ for some $a<b, a, b \in[-\infty, \infty]$, either $a=-\infty$ or $b=+\infty$, and that the distributional gradient satisfies $\nabla u \in L^{2}\left(\Sigma_{a}^{b}\right)$. Then there exists $c \in \mathbb{C}$ such that $u-c \in Y^{1,2}\left(\Sigma_{a}^{b}\right)$.

The proof is very similar to that of Theorem 1.78 in [MZ97], thus we omit it. 
Let us present a fact regarding the regularity of functions in $Y^{1,2}\left(\mathbb{R}^{n+1}\right)$ when seen as single-variable vector-valued maps. The proof is omitted as it is straightforward.

Lemma 2.2.2 (Local Hölder continuity in the transversal direction). Suppose that $u, \partial_{t} u \in$ $Y^{1,2}\left(\Sigma_{a}^{b}\right)$ for some $a<b$. Then it holds that $u \in C_{\mathrm{loc}}^{\alpha}\left((a, b) ; L^{2^{*}}\left(\mathbb{R}^{n}\right)\right)$ for some exponent $\alpha>0$. Moreover we also have that $\nabla u \in C_{\mathrm{loc}}^{\beta}\left((a, b) ; L^{2}\left(\mathbb{R}^{n}\right)\right)$ for some $\beta>0$.

Remark 2.2.3. Note that the functions above are representatives of $u(t)$ and $\nabla u(t)$, but that these retain the same properties as their smooth counterparts when acting on functions defined on the slice $\left\{x_{n+1}=t\right\}$. More precisely, for any $\vec{\varphi} \in C_{c}^{\infty}\left(\mathbb{R}^{n} ; \mathbb{C}^{n}\right)$ and any $t \in(a, b)$, we have the identity

$$
\int_{\mathbb{R}^{n}} u(x, t) \operatorname{div}_{\|} \vec{\varphi}(x) d x=-\int_{\mathbb{R}^{n}} \nabla_{\|} u(x, t) \cdot \vec{\varphi}(x) d x
$$

The above identity is already true for a.e. $t \in(a, b)$, and is seen to be true for arbitrary $t \in(a, b)$ by the continuity of $u$ and $\nabla u$.

Analogously, we introduce $Y^{1,2}\left(\mathbb{R}^{n}\right)$ as

$$
Y^{1,2}\left(\mathbb{R}^{n}\right):=\left\{u \in L^{\frac{2 n}{n-2}}\left(\mathbb{R}^{n}\right): \nabla u \in L^{2}\left(\mathbb{R}^{n}\right)\right\}
$$

and equip it with the norm

$$
\|u\|_{Y^{1,2}\left(\mathbb{R}^{n}\right)}:=\|u\|_{L^{\frac{2 n}{n-2}\left(\mathbb{R}^{n}\right)}}+\|\nabla u\|_{L^{2}\left(\mathbb{R}^{n}\right)}
$$

Note carefully that in our convention, $2^{*}=\frac{2(n+1)}{n-1} \neq \frac{2 n}{n-2}$. 
Some fractional Sobolev spaces will be useful for us when discussing trace operators. Let $\mathcal{M} F: L^{2}\left(\mathbb{R}^{n}\right) \rightarrow L^{2}\left(\mathbb{R}^{n}\right)$ be the Fourier transform. Throughout this paper, we shall also denote $\hat{u}:=\mathcal{M} F u$. We write

$$
H^{\frac{1}{2}}\left(\mathbb{R}^{n}\right)=\left\{u \in L^{2}\left(\mathbb{R}^{n}\right): \int_{\mathbb{R}^{n}}(1+|\xi|)|\hat{u}(\xi)|^{2} d \xi<+\infty\right\}
$$

The homogeneous space $\dot{H}^{\frac{1}{2}}\left(\mathbb{R}^{n}\right)$ consists of those functions $u \in L_{\text {loc }}^{1}\left(\mathbb{R}^{n}\right)$ which satisfy that $\left.\int_{\mathbb{R}^{n}}|\xi| \hat{u}(\xi)\right|^{2} d \xi<+\infty$. Naturally, this space comes equipped with the seminorm $|u|_{\dot{H}^{\frac{1}{2}}\left(\mathbb{R}^{n}\right)}=\int_{\mathbb{R}^{n}}|\xi||\hat{u}(\xi)|^{2} d \xi$. We define the space $H_{0}^{\frac{1}{2}}\left(\mathbb{R}^{n}\right)=\dot{H}_{0}^{\frac{1}{2}}\left(\mathbb{R}^{n}\right)$ as the completion of $C_{c}^{\infty}\left(\mathbb{R}^{n}\right)$ under the $\dot{H}^{\frac{1}{2}}\left(\mathbb{R}^{n}\right)$ seminorm. We write $H^{-\frac{1}{2}}\left(\mathbb{R}^{n}\right):=\left(H_{0}^{\frac{1}{2}}\left(\mathbb{R}^{n}\right)\right)^{*}$, and emphasize that we are departing from notation used elsewhere in the literature. Since $H_{0}^{\frac{1}{2}}\left(\mathbb{R}^{n}\right) \supsetneq H^{\frac{1}{2}}\left(\mathbb{R}^{n}\right)$, it follows that $H^{-\frac{1}{2}}\left(\mathbb{R}^{n}\right)$ is contained in the dual space of $H^{\frac{1}{2}}\left(\mathbb{R}^{n}\right)$, which is the usual (inhomogeneous) fractional Sobolev space of order $-1 / 2$ that coincides with the space

$$
\left\{u \in \mathscr{S}^{\prime}\left(\mathbb{R}^{n}\right): \int_{\mathbb{R}^{n}}\left(1+|\xi|^{2}\right)^{-\frac{1}{2}}|\hat{u}(\xi)|^{2} d \xi<+\infty\right\}
$$

For a survey on the properties of fractional Sobolev spaces, see [DNPV12]. We state without proof two easy results which are nevertheless useful.

Proposition 2.2.4 (Sobolev embeddings of the fractional Sobolev spaces). Let $p_{+}:=\frac{2 n}{n-1}$ and $p_{-}:=\frac{2 n}{n+1}$. Then we have the continuous embeddings $H_{0}^{\frac{1}{2}}\left(\mathbb{R}^{n}\right) \hookrightarrow L^{p_{+}}\left(\mathbb{R}^{n}\right), L^{p_{-}}\left(\mathbb{R}^{n}\right) \hookrightarrow$ $H^{-\frac{1}{2}}\left(\mathbb{R}^{n}\right)$.

Proposition 2.2.5. The map $\nabla: H_{0}^{\frac{1}{2}}\left(\mathbb{R}^{n}\right) \rightarrow H^{-\frac{1}{2}}\left(\mathbb{R}^{n}\right)$ is bounded.

For fixed $t \in \mathbb{R}$ and any open set $\Omega \subset \mathbb{R}^{n+1}$ with nice enough (but possibly unbounded) 
boundary such that $\mathbb{R}^{n} \times\{\tau=t\} \subset \Omega$, we define the trace operator $\operatorname{Tr}_{t}: C_{c}^{\infty}(\bar{\Omega}) \rightarrow C_{c}^{\infty}\left(\mathbb{R}^{n}\right)$ by $\operatorname{Tr}_{t} u=u(\cdot, t)$. The relevance of the fractional Sobolev spaces to our theory comes from the following trace result; we cite a paper with the proof for traces of functions in $\dot{W}^{1,2}\left(\mathbb{R}^{2}\right)$, but the result is straightforwardly extended to our situation.

Lemma 2.2.6 (Traces of $Y^{1,2}$ functions; [Str16]). Fix $t>0$. Let $\Omega$ be either $\mathbb{R}^{n+1}$, $\mathbb{R}_{t}^{n+1}$, or $\mathbb{R}_{-, t}^{n+1}$. Then, for each $s \in \mathbb{R}$ such that there exists $x \in \mathbb{R}^{n}$ with $(x, s) \in \Omega$, the trace operator $\operatorname{Tr}_{s}$ extends uniquely to a bounded linear operator $Y^{1,2}(\Omega) \rightarrow H_{0}^{\frac{1}{2}}\left(\mathbb{R}^{n}\right)$.

Definition 2.2.7 (Local weak solutions). Let $f \in L_{\mathrm{loc}}^{1}(\Omega), F \in L_{\mathrm{loc}}^{1}\left(\Omega, \mathbb{C}^{n+1}\right)$, and $u \in$ $W_{\text {loc }}^{1,2}(\Omega)$. We say that $u$ solves the equation $\mathcal{L} u=f-\operatorname{div} F$ in $\Omega$ in the weak sense if, for every $\varphi \in C_{c}^{\infty}(\Omega)$, the identity

$$
\iint_{\mathbb{R}^{n+1}}\left(\left(A \nabla u+B_{1} u\right) \cdot \overline{\nabla \varphi}+B_{2} \cdot \nabla u \bar{\varphi}\right)=\iint_{\mathbb{R}^{n+1}}(f \bar{\varphi}+F \cdot \overline{\nabla \varphi})
$$

holds.

Remark 2.2.9. By a standard density argument, if $u \in Y^{1,2}(\Omega)$ solves $\mathcal{L} u=f+\operatorname{div} F$ in $\Omega$ in the weak sense and either

- $F \in L^{2}(\Omega)$ and $f \in L^{(2 n+2) /(n+3)}(\Omega)$, or

- $\Omega=D \times I$, where $D$ is a domain with nice enough (but possibly unbounded) boundary and $I$ is an interval, and $F \in L^{2}(\Omega), f \in L^{2}\left(I ; L^{(2 n) /(n+2)}(D)\right)+L^{(2 n+2) /(n+3)}(\Omega)$,

then (2.8) holds for all $\varphi \in Y_{0}^{1,2}(\Omega)$. A similar observation to the second item can be made if $\Omega$ is a ball in $\mathbb{R}^{n+1}$. 
For an infinite interval $I \subset \mathbb{R}$ and a Banach space $X$, let $C_{0}^{k}(I ; X)$ be the space of functions $f: I \rightarrow X$ such that all their first $k$ derivatives $f^{(l)}: I \rightarrow X, 0 \leq l \leq k$, exist, are continuous on $I$, and satisfy that $\lim _{t \rightarrow \infty}\left\|f^{(l)}(t)\right\|_{X}=0$ for all $0 \leq l \leq k$. When $k=0$, we will omit the superscript and simply write $C^{0}=C$.

Definition 2.2.10 (Slice Spaces). For $n \geq 3$, we define

$$
D_{+}^{2}:=\left\{v \in C_{0}\left((0, \infty) ; L^{2}\left(\mathbb{R}^{n}\right)\right):\|u\|_{D_{+}^{2}}<\infty\right\}
$$

with norm given by $\|v\|_{D_{+}^{2}}:=\sup _{t>0}\|v(t)\|_{L^{2}\left(\mathbb{R}^{n}\right)}$. We also define

$$
S_{+}^{2}:=\left\{u \in C_{0}^{2}\left((0, \infty) ; Y^{1,2}\left(\mathbb{R}^{n}\right)\right): u^{\prime}(t) \in C_{0}\left((0, \infty) ; L^{2}\left(\mathbb{R}^{n}\right)\right),\|u\|_{S_{+}^{2}}<\infty\right\},
$$

with norm given by

$$
\begin{aligned}
\|u\|_{S_{+}^{2}}:=\sup _{t>0}\|u(t)\|_{Y^{1,2}\left(\mathbb{R}^{n}\right)}+\sup _{t>0}\left\|u^{\prime}(t)\right\|_{L^{2}\left(\mathbb{R}^{n}\right)} & \\
& +\sup _{t>0}\left\|t u^{\prime}(t)\right\|_{Y^{1,2}\left(\mathbb{R}^{n}\right)}+\sup _{t>0}\left\|t^{2} u^{\prime \prime}(t)\right\|_{Y^{1,2}\left(\mathbb{R}^{n}\right)} .
\end{aligned}
$$

In particular, both $D_{+}^{2}$ and $S_{+}^{2}$ are Banach spaces. Similarly, with obvious modifications, we can define the slice spaces $S_{-}^{2}$ and $D_{-}^{2}$ in the negative half line $(-\infty, 0)$.

We also state, without proof, the following criterion for the existence of weak derivatives in $L^{2}(I ; X)$. See [CH98] for further results and definitions.

Theorem 2.2.11 (Vector-valued weak derivatives; [CH98] Theorem 1.4.40). Suppose that $X$ is a reflexive Banach space and let $I \subset \mathbb{R}$ be a (not necessarily bounded) interval. Let 
$f \in L^{2}(I ; X)$. Then $f \in W^{1,2}(I ; X)$ if and only if there exists $\varphi \in L^{2}(I ; \mathbb{R})$ such that for any $t, s \in I$, the estimate

$$
\|f(t)-f(s)\|_{X} \leq\left|\int_{s}^{t} \varphi(r) d r\right|
$$

holds. Moreover, for a.e. $t \in I$, the difference quotients

$$
\Delta^{h} f(t):=\frac{f(t+h)-f(t)}{h}, \quad h \in \mathbb{R},|h| \ll 1,
$$

converge weakly in $X$ to $f^{\prime}(t)$ as $h \rightarrow 0$.

Remark 2.2.12. We will see that if $u \in W_{\text {loc }}^{1,2}\left(\mathbb{R}_{+}^{n+1}\right) \cap S_{+}^{2}$ and $\mathcal{L} u=0$ in $\mathbb{R}_{+}^{n+1}$, then by Caccioppoli's inequality (on slices) we have that

$$
\begin{aligned}
\|u\|_{S_{+}^{2}} \approx \sup _{t>0}\|u(t)\|_{Y^{1,2}\left(\mathbb{R}^{n}\right)}+\sup _{t>0}\left\|u^{\prime}(t)\right\|_{L^{2}\left(\mathbb{R}^{n}\right)} \\
\\
\quad \approx \sup _{t>0}\left\|\nabla_{\|} \operatorname{Tr}_{t} u\right\|_{L^{2}\left(\mathbb{R}^{n}\right)}+\sup _{t>0}\left\|\operatorname{Tr}_{t}\left(D_{n+1} u\right)\right\|_{L^{2}\left(\mathbb{R}^{n}\right)} .
\end{aligned}
$$

We now state a Trace Theorem in cubes. We set

$$
I_{R}^{ \pm}:=(-R, R)^{n} \times(0, \pm R), \quad I_{R}:=(-R, R)^{n+1}, \quad \Delta_{R}:=(-R, R)^{n} \times\{0\} .
$$

Proposition 2.2.13 (Trace operator on a cube). There exists a bounded linear operator $\operatorname{Tr}_{0}{ }^{ \pm}: W^{1,2}\left(I_{R}^{ \pm}\right) \rightarrow H^{\frac{1}{2}}\left(\Delta_{R}\right)$, where the latter space consists of restrictions of functions in $H^{\frac{1}{2}}\left(\mathbb{R}^{n}\right)$ to $\Delta_{R}$. Moreover $\operatorname{Tr}_{0}{ }^{ \pm} u(\cdot)=u(\cdot, 0)$ for the smooth functions $u \in C^{\infty}\left(\overline{I_{R}^{ \pm}}\right)$, and the identity

$$
\int_{\Delta_{R}} \operatorname{Tr}_{0}{ }^{ \pm} u \bar{\phi}=\mp \iint_{I_{R}^{ \pm}}\left(u \overline{D_{n+1} \Phi}+D_{n+1} u \bar{\Phi}\right),
$$


holds for each $\Phi \in C_{c}^{\infty}\left(I_{R}\right)$, where $\phi(\cdot)=\Phi(\cdot, 0)$. In particular the traces are consistent in the sense that for every $R^{\prime}<R$, the restriction to $I_{R^{\prime}}^{ \pm}$of the trace operator associated to $I_{R}^{ \pm}$, agrees with the trace in $I_{R^{\prime}}^{ \pm}$.

Proof. The result follows from the usual Trace Theorem on Lipchitz domains (see, for instance, [Leo17] Theorem 15.23 and the results which follow this theorem) and the fact that $I_{R}^{+}$is an extension domain for $W^{1,2}$ (again, see [Leo17] Theorem 12.15).

\subsection{Basic properties of $\mathcal{L}$}

Our main operator of interest is

$$
\mathcal{L} u=-\operatorname{div}\left(A \nabla u+B_{1} u\right)+B_{2} \cdot \nabla u+V u, \quad u \in Y^{1,2}\left(\mathbb{R}^{n}\right) .
$$

The coefficients are required to satisfy the following conditions:

1. $A: \mathbb{R}^{n} \rightarrow \mathbb{C}^{(n+1)^{2}}$ is a matrix valued function such that there exist $0<\lambda<\Lambda<\infty$ with the property

$$
\lambda|\xi|^{2} \leq \Re e(A(x) \xi \cdot \bar{\xi}), \quad|A(x) \xi \cdot \bar{\zeta}| \leq \Lambda|\xi \| \eta|, \quad \forall \xi, \eta \in \mathbb{C}^{n+1}
$$

2. $B_{1}, B_{2}: \mathbb{R}^{n} \rightarrow \mathbb{C}^{n+1}$ are such that $B_{i} \in L^{n}\left(\mathbb{R}^{n}\right)$.

3. $V: \mathbb{R}^{n} \rightarrow \mathbb{C}$ satisfies $V \in L^{n / 2}\left(\mathbb{R}^{n}\right)$.

We now remark that the zeroth-order term $V$ in our differential equation can be absorbed into the first order terms. 
Lemma 2.3.1 (Zeroth order term absorbed by first order terms). Let $\mathcal{L}$ be as in the beginning of the section with

$$
\max \left\{\left\|B_{1}\right\|_{n},\left\|B_{2}\right\|_{n},\|V\|_{\frac{n}{2}}\right\} \leq \varepsilon_{0} .
$$

Then

$$
\mathcal{L}=-\operatorname{div}\left(A \nabla+\tilde{B}_{1}\right)+\tilde{B}_{2} \cdot \nabla
$$

where

$$
\max \left\{\left\|\tilde{B}_{1}\right\|_{n},\left\|\tilde{B}_{2}\right\|_{n},\right\} \leq C_{n} \varepsilon_{0}
$$

Proof. We write

$$
V(x)=-\operatorname{div}_{x} \nabla_{\|} I_{2} V(x)=c_{n} \operatorname{div}_{x} \vec{R} I_{1} V(x),
$$

where $I_{\alpha}$ is the $\alpha$-order Riesz potential

$$
\left(I_{\alpha} f\right)(x)=\frac{1}{c_{\alpha}} \int_{\mathbb{R}^{n}} \frac{f(y)}{|x-y|^{\alpha}} d y,
$$

and $\vec{R}$ is the Riesz transform on $\mathbb{R}^{n}$. For definitions and properties, see [Ste70]. To conclude the lemma, we note that $I_{1}: L^{n / 2}\left(\mathbb{R}^{n}\right) \rightarrow L^{n}\left(\mathbb{R}^{n}\right)$ and $\vec{R}$ is a bounded operator $L^{n}\left(\mathbb{R}^{n}\right) \rightarrow$ $\left[L^{n}\left(\mathbb{R}^{n}\right)\right]^{n}$. Observe that it suffices that $V \in \dot{L}_{-1}^{n}=\left\{V \in \mathscr{D}^{\prime}: I_{1} V \in L^{n}\right\}$, with small norm. Thus, our results hold under this slightly more general assumption on $V$.

Accordingly, from now on we drop the term $V$ from our operator. We obtain invertibility of the operator $\mathcal{L}$ on the Hilbert space $Y^{1,2}\left(\mathbb{R}^{n+1}\right)$ when the size of the lower order terms is small enough. 
Definition 2.3.2 (Sesquilinear form and associated operator). Define the sesquilinear form $B_{\mathcal{L}}: C_{c}^{\infty}\left(\mathbb{R}^{n+1}\right) \times C_{c}^{\infty}\left(\mathbb{R}^{n+1}\right) \rightarrow \mathbb{C}$ via

$$
B_{\mathcal{L}}[u, v]:=\iint_{\mathbb{R}^{n+1}}\left[A \nabla u \cdot \overline{\nabla v}+u B_{1} \cdot \overline{\nabla v}+\bar{v} B_{2} \cdot \nabla u\right], \quad u, v \in C_{c}^{\infty}\left(\mathbb{R}^{n+1}\right) .
$$

Given $u \in C_{c}^{\infty}\left(\mathbb{R}^{n+1}\right)$, define the operator $\mathcal{L}: \mathscr{D} \rightarrow \mathscr{D}^{\prime}$ via the identity

$$
\langle\mathcal{L} u, v\rangle=B_{\mathcal{L}}[u, v]
$$

It is clear that $\mathcal{L}$ is linear.

In fact, the form $B_{\mathcal{L}}$ extends to a bounded, coercive form on $Y^{1,2}\left(\mathbb{R}^{n+1}\right) \times Y^{1,2}\left(\mathbb{R}^{n+1}\right)$, and the operator $\mathcal{L}$ extends to a bounded, linear, invertible operator on $Y^{1,2}\left(\mathbb{R}^{n+1}\right)$. This is precisely the content of

Proposition 2.3.3 (Extension of operator to $Y^{1,2}$ ). The form $B_{\mathcal{L}}$ extends to a bounded form on $Y^{1,2}\left(\mathbb{R}^{n+1}\right)$; that is,

$$
\left|B_{\mathcal{L}}[u, v]\right| \lesssim\|\nabla u\|_{2}\|\nabla v\|_{2}, \quad \text { for all } u, v \in C_{c}^{\infty}\left(\mathbb{R}^{n+1}\right),
$$

with the implicit constant depending on $n, \lambda, \Lambda$, and $\max \left\{\left\|B_{1}\right\|_{n},\left\|B_{2}\right\|_{n}\right\}$. Hence $\mathcal{L}$ extends to a bounded operator $Y^{1,2}\left(\mathbb{R}^{n+1}\right) \rightarrow\left(Y^{1,2}\left(\mathbb{R}^{n+1}\right)\right)^{*}$.

Moreover, there exists a constant $\varepsilon_{0}=\varepsilon_{0}(n, \lambda, \Lambda)>0$ such that if $\max \left\{\left\|B_{1}\right\|_{n},\left\|B_{2}\right\|_{n}\right\}<\varepsilon_{0}$, then $B_{\mathcal{L}}$ is also coercive in $Y^{1,2}\left(\mathbb{R}^{n+1}\right)$ with lower bound $\lambda / 2$; that is,

$$
\frac{\lambda}{2}\|\nabla u\|_{2}^{2} \lesssim \Re e B_{\mathcal{L}}[u, u], \quad \text { for all } u \in C_{c}^{\infty}\left(\mathbb{R}^{n+1}\right)
$$


In particular, if $\max \left\{\left\|B_{1}\right\|_{n},\left\|B_{2}\right\|_{n}\right\}<\varepsilon_{0}$, then by the Lax-Milgram Theorem the operator $\mathcal{L}^{-1}:\left(Y^{1,2}\left(\mathbb{R}^{n+1}\right)\right)^{*} \rightarrow Y^{1,2}\left(\mathbb{R}^{n+1}\right)$ exists as a bounded linear operator.

Proof. The proof is straightforward, thus omitted.

Remark 2.3.4. We will always assume that $\max \left\{\left\|B_{1}\right\|_{n},\left\|B_{2}\right\|_{n}\right\}<\varepsilon_{0}$, as above. The value of $\varepsilon_{0}$ may be made smaller, but it will always depend only on $n, \lambda$ and $\Lambda$, and we will explicitly state when we impose further smallness.

Definition 2.3.5 (Dual operator). Associated to $\mathcal{L}$ we also have the dual operator, denoted $\mathcal{L}^{*}: Y^{1,2}\left(\mathbb{R}^{n+1}\right) \rightarrow\left(Y^{1,2}\left(\mathbb{R}^{n+1}\right)\right)^{*}$, defined by the relation

$$
\langle\mathcal{L} u, v\rangle=\left\langle u, \mathcal{L}^{*} v\right\rangle
$$

It is a matter of algebra to check that

$$
\mathcal{L}^{*} v=-\operatorname{div}\left(A^{*} \nabla v+\bar{B}_{2} v\right)+\bar{B}_{1} \cdot \nabla v
$$

holds in the weak sense.

In particular, $\mathcal{L}^{*}$ is an operator of the same type as $\mathcal{L}$ and if $\max \left\{\left\|B_{1}\right\|_{n},\left\|B_{2}\right\|_{n}\right\}<\varepsilon_{0}$ so that $\mathcal{L}^{-1}$ is defined, then $\left(\mathcal{L}^{*}\right)^{-1}$ is well-defined, bounded, and satisfies $\left(\mathcal{L}^{*}\right)^{-1}=\left(\mathcal{L}^{-1}\right)^{*}$. 


\subsection{Harmonic Analysis}

Definition 2.4.1 (Vertical and Conical Square Functions). If $F: \mathbb{R}_{+}^{n+1} \rightarrow \mathbb{C}$, we define the conical square function of $F$ as

$$
\mathbb{S} F(x):=\left(\iint_{\Gamma(x)}|F(y, t)|^{2} \frac{d y d t}{t^{n+1}}\right)^{1 / 2},
$$

where $\Gamma(x):=\left\{(y, t) \in \mathbb{R}_{+}^{n+1}:|x-y|<t\right\}$ is the vertical cone with apperture 1 and vertex $x$. Similarly, we define the vertical square function of $F$ as

$$
\mathbb{V} F(x):=\left(\int_{0}^{\infty}|F(x, t)|^{2} \frac{d t}{t}\right)^{1 / 2} .
$$

Remark 2.4.4. In the definition of $\mathbb{S}$, we could have chosen a different aperture; that is, for $\eta>0$, we can set

$$
\mathbb{S}_{\eta} F(x)=\left(\iint_{|x-y|<\eta t}|F(y, t)|^{2} \frac{d y d t}{t^{n+1}}\right)^{1 / 2}
$$

It is well-known that different apertures give rise to objects with equivalent $L^{p}$ norms and even equivalent weighted $L^{p}$ norms (see for instance [CMS85, Proposition 4] for the unweighted case and [CMP20, Proposition 4.9] for the weighted one).

In contrast with the $L^{2}$ case, when $p \neq 2$ the ( $L^{p}$ norms of) conical and vertical square functions are not equivalent.

Proposition 2.4.6 (Comparability of Square Functions [AHM12, Proposition 2.1]). Let F : $\mathbb{R}_{+}^{n+1} \rightarrow \mathbb{C}$ be measurable. 
(i) If $0<p \leq 2$ then $\|\mathbb{V}(F)\|_{L^{p}\left(\mathbb{R}^{n}\right)} \lesssim_{n, p}\|\mathbb{S}(F)\|_{L^{p}\left(\mathbb{R}^{n}\right)}$.

(ii) If $2 \leq p<\infty$ then $\|\mathbb{S}(F)\|_{L^{p}\left(\mathbb{R}^{n}\right)} \lesssim_{n, p}\|\mathbb{V}(F)\|_{L^{p}\left(\mathbb{R}^{n}\right)}$

Definition 2.4.7 (Non-tangential Maximal Functions). For a function $F: \mathbb{R}^{n+1} \rightarrow \mathbb{C}$ and $q>0$ we define $a_{q}(F): \mathbb{R}^{n+1} \rightarrow \mathbb{C}$ as

$$
a_{q}(F)(x, t):=\left(\int_{\mathcal{C}_{x, t}}|F(y, s)|^{q} d y d s\right)^{1 / q} .
$$

We define the non-tangential maximal function and the q-modified non-tangential maximal function of $F$ respectively as

$$
\mathcal{N}(F)(x):=\sup _{\Gamma(x)}|F|
$$

and

$$
\tilde{\mathcal{N}}_{q}(F)(x):=\mathcal{N}\left(a_{q}(F)\right)(x)
$$

We also define the lifted modified non-tangential maximal function, for $\varepsilon>0$, as

$$
\tilde{\mathcal{N}}_{q}^{\varepsilon}(F)(x):=\sup _{\substack{|x-y|<t-\varepsilon \\ t>\varepsilon}} a_{q}(F)(y, t)
$$

Similarly, we define a truncated version of the non-tangential maximal function as $\tilde{\mathcal{N}}_{q}^{(\varepsilon)}(F)(x):=$ $\sup _{\substack{|x-y|<t \\ t>\varepsilon}} a_{q}(F)(y, t)$

\subsubsection{Generalized Littlewood-Paley Theory}

In this subsection, we review some of the known results from the generalized LittlewoodPaley theory. Here, the generalization is that one replaces the classical smoothness assump- 
tion by a so-called quasi-orthogonality condition, and one replaces the classical pointwise decay condition by off-diagonal decay in an $L^{2}$ sense.

First, we introduce the square function norm $\|\cdot|\||$. We define

$$
\left\|\left|F\left\|_{ \pm}:=\left(\iint_{\mathbb{R}_{ \pm}^{n+1}}|F(x, t)|^{2} \frac{d x d t}{t}\right)^{1 / 2} \quad, \quad\right\| F\right|\right\|_{\text {all }}:=\left(\iint_{\mathbb{R}^{n+1}}|F(x, t)|^{2} \frac{d x d t}{t}\right)^{1 / 2}
$$

For a family of operators on $L^{2}\left(\mathbb{R}^{n}\right),\left\{\theta_{t}\right\}_{t>0}$, we define

$$
\left\|\theta_{t}\right\|\left\|_{+, o p}:=\sup _{\|f\|_{2}=1}\right\| \theta_{t} f \|_{+},
$$

and similarly define $\left\|\theta_{t}\right\| \|_{-, \text {op }}$ and $\left\|\theta_{t}\right\|_{\text {all,op }}$. We will often drop the sign in the subscript when in context it is understood that we work in the upper half space.

Recall that $Q$ denotes a cube in $\mathbb{R}^{n}$. Given a measurable function $\Upsilon$ on $\mathbb{R}^{n+1}{ }_{+}$, we define

$$
\|\Upsilon\|_{C}:=\sup _{Q} \frac{1}{|Q|} \int_{0}^{\ell(Q)} \int_{Q}|\Upsilon(x, t)|^{2} \frac{d x d t}{t}
$$

In other words, $\|\Upsilon\|_{C}<\infty$ if and only if $|\Upsilon(x, t)|^{2} \frac{d x d t}{t}$ is a Carleson measure; in this case, we say that $\Upsilon \in C$. There is a deep connection between Carleson measures and square function estimates, as seen in the $T 1$ theorem for square functions of Christ and Journé [CJ87]. Here, we use a generalized version of their result [GH16, Theorem 4.5]. Recall that a Borel measure $\mu$ on $\mathbb{R}_{+}^{n+1}$ is called Carleson if there exists a constant $C$ such that $\mu\left(R_{Q}\right) \leq C|Q|$ for all cubes $Q \subset \mathbb{R}^{n}$, where $R_{Q}=Q \times(0, \ell(Q))$ is the Carleson box above $Q$.

We record several results from $\left[\mathrm{AAA}^{+} 11\right]$, which will be crucial in establishing square function estimates for solutions. 
Definition 2.4.9 (Good off-diagonal decay). We say that a family of operators on $L^{2}\left(\mathbb{R}^{n}\right)$, $\left\{\theta_{t}\right\}_{t>0}$, has good off-diagonal decay if there exists $M \geq 0$ such that the estimate

$$
\left\|\theta_{t}\left(f 1_{2^{k+1} Q \backslash 2^{k} Q}\right)\right\|_{L^{2}(Q)}^{2} \lesssim_{M} 2^{-n k}\left(\frac{t}{2^{k} \ell(Q)}\right)^{2 M+2}\|f\|_{L^{2}\left(2^{k+1} Q \backslash 2^{k} Q\right)}^{2}
$$

holds for every cube $Q \subset \mathbb{R}^{n}$, every $k \geq 2$ and all $0<t \lesssim_{M} \ell(Q)$. Here, the implicit constants may depend only on dimension, $M$, and on the family of operators.

Lemma 2.4.10 (Consequences of off-diagonal decay; [FS72], [AAA ${ }^{+} 11$, Lemma 3.2]). Suppose that $\left\{\theta_{t}\right\}_{t>0}$ is a family of operators on $L^{2}\left(\mathbb{R}^{n}\right)$ with good off-diagonal decay. Then for every simple function $b$, the family $\left\{\theta_{t}\right\}_{t>0}$ satisfies the estimate

$$
\left\|\theta_{t} b\right\|_{C} \lesssim\left(1+\left\|\theta_{t}\right\|_{o p}^{2}\right)\|b\|_{\infty}^{2}
$$

Accordingly, for any $b \in L^{\infty}\left(\mathbb{R}^{n}\right)$, the expression $\theta_{t} b$ makes sense as a measurable function in $\mathbb{R}_{+}^{n+1}$, and it satisfies the above estimate. Moreover, if $\left\|\theta_{t}\right\|_{L^{2} \rightarrow L^{2}} \lesssim 1$ and $\theta_{t} 1=0$ for all $t>0$, then for every $b \in B M O\left(\mathbb{R}^{n}\right)$, we have that

$$
\left\|\theta_{t} b\right\|_{C} \lesssim\left(1+\left\|\theta_{t}\right\|_{o p}^{2}\right)\|b\|_{B M O}^{2}
$$

Lemma 2.4.11 ( $\left[\mathrm{AAA}^{+} 11\right.$, Lemma 3.11]). Suppose that $\left\{\theta_{t}\right\}_{t>0}$ is a family of operators on $L^{2}\left(\mathbb{R}^{n}\right)$ with good off-diagonal decay and which satisfies $\left\|\theta_{t}\right\|_{L^{2} \rightarrow L^{2}} \lesssim 1$ for all $t>0$. For each $t>0$, let $\mathcal{A}_{t}$ denote a self-adjoint averaging operator on $L^{2}\left(\mathbb{R}^{n}\right)$, given as $\mathcal{A}_{t} f=$ $\int_{\mathbb{R}^{n}} f(y) \varphi_{t}(\cdot, y) d y$, whose kernel satisfies

$$
0 \leq \varphi_{t}(x, y) \lesssim t^{-n} \mathbb{1}_{|x-y| \leq C t}, \quad \text { and } \quad \int_{\mathbb{R}^{n}} \varphi_{t}(x, y) d y=1 .
$$


Then for each $t>0$ and any $b \in L^{\infty}\left(\mathbb{R}^{n}\right)$, the function $\theta_{t} b$ is well-defined as an element of $L_{\mathrm{loc}}^{2}\left(\mathbb{R}^{n}\right)$, and we have that

$$
\sup _{t>0}\left\|\left(\theta_{t} b\right) \mathcal{A}_{t} f\right\|_{L^{2}\left(\mathbb{R}^{n}\right)} \lesssim\|b\|_{\infty}\|f\|_{2} .
$$

Lemma 2.4.12 ( $\left[\mathrm{AAA}^{+} 11\right.$, Lemma 3.5]). Suppose that $\left\{R_{t}\right\}_{t>0}$ is a family of operators on $L^{2}\left(\mathbb{R}^{n}\right)$ with good off-diagonal decay, and suppose further that $\left\|R_{t}\right\|_{L^{2} \rightarrow L^{2}} \lesssim 1$ and $R_{t} 1=0$ for all $t>0$ (note that by Lemma 2.4.11, $R_{t} 1$ is defined as an element of $L_{\mathrm{loc}}^{2}\left(\mathbb{R}^{n}\right)$ ). Then for each $h \in \dot{W}^{1,2}\left(\mathbb{R}^{n}\right)$, we have that

$$
\int_{\mathbb{R}^{n}}\left|R_{t} h\right|^{2} \lesssim t^{2} \int_{\mathbb{R}^{n}}\left|\nabla_{x} h\right|^{2} .
$$

If, in addition, $\left\|R_{t} \operatorname{div}_{x}\right\|_{L^{2} \rightarrow L^{2}} \lesssim \frac{1}{t}$, then we also have for each $f \in L^{2}\left(\mathbb{R}^{n}\right)$ that

$$
\int_{\mathbb{R}^{n+1}+}\left|R_{t} f(x)\right|^{2} \frac{d x d t}{t} \lesssim\|f\|_{2}^{2} .
$$

The following definition is important in establishing quasi-orthogonality estimates (compare to the notion of an $\epsilon$-family in [CJ87]).

Definition 2.4.13 (CLP Family). We say that a family of convolution operators on $L^{2}\left(\mathbb{R}^{n}\right)$, $\left\{Q_{s}\right\}_{s>0}$, is a CLP family ("Calderón-Littlewood-Paley" family), if there exist $\sigma>0$ and $\psi \in L^{1}\left(\mathbb{R}^{n}\right)$ satisfying

$$
|\psi(x)| \lesssim(1+|x|)^{-n-\sigma}, \quad \text { and } \quad|\hat{\psi}(\xi)| \lesssim \min \left(|\xi|^{\sigma},|\xi|^{-\sigma}\right)
$$

such that the following four statements hold. 
i) The representation $Q_{s} f=s^{-n} \psi(\cdot / s) * f$ holds.

ii) We have control of the following $L^{2}$ norms uniformly in $s$ :

$$
\sup _{s>0}\left(\left\|Q_{s} f\right\|_{2}+\left\|s \nabla Q_{s} f\right\|_{2}\right) \lesssim\|f\|_{2}
$$

iii) We have the square function estimate

$$
\int_{0}^{\infty} \int_{\mathbb{R}^{n}}\left|Q_{s} f(x)\right|^{2} \frac{d x d s}{s} \lesssim\|f\|_{2}^{2}
$$

iv) The identity

$$
\int_{0}^{\infty} Q_{s}^{2} \frac{d s}{s}=I
$$

holds in the sense that the convergence of the integrals $\int_{\delta}^{R} Q_{s}^{2} \frac{d s}{s}$ to the identity as $\delta \rightarrow 0$ and $R \rightarrow \infty$ occurs in the strong operator topology on $\mathcal{B}\left(L^{2}\left(\mathbb{R}^{n}\right)\right)$.

Proposition 2.4.14 (Qualitative mappings). Let $f \in Y^{1,2}\left(\mathbb{R}^{n}\right)$ and $\left\{\mathcal{M} Q_{s}\right\}_{s>0}$ be either

a) A standard Littlewood-Paley family as in Definition 2.4.13, with kernel $\psi$, with the additional condition that there exists $\sigma>1$ such that $|\hat{\psi}(\xi)| \lesssim \min \left(|\xi|^{\sigma},|\xi|^{-\sigma}\right)$.

b) $\mathcal{M} Q_{s}=\left(I-P_{s}\right)$, where $P_{s}$ is a nice approximate identity.

Then for all $s>0$, we have that $\mathcal{M} Q_{s} f \in W^{1,2}\left(\mathbb{R}^{n}\right)$. 
Proof. In either case, via Plancherel's Theorem, it will suffice to estimate the $L^{2}$ norm of $\widehat{Q_{s} f}$. In case a), by basic properties of the Fourier Transform, we see that

$$
\int_{\mathbb{R}^{n}}\left|\widehat{Q_{s} f}(\xi)\right|^{2} d \xi=\int_{\mathbb{R}^{n}}|\hat{\psi}(s \xi)|^{2}|\hat{f}(\xi)|^{2} d \xi \lesssim \int_{\mathbb{R}^{n}} \min \left(|s \xi|^{\sigma-1},|s \xi|^{-\sigma-1}\right)^{2}|\xi|^{2}|\hat{f}(\xi)|^{2} d \xi
$$

whence the desired conclusion follows in this case. For case b), we similarly compute, using Plancherel's Theorem and the Fundamental Theorem of Calculus, that if $\varphi$ is the radial kernel of the nice approximate identity $P_{s}$,

$$
\begin{aligned}
\int_{\mathbb{R}^{n}}\left|\widehat{Q_{s} f}(\xi)\right|^{2} d \xi & =\int_{\mathbb{R}^{n}}|1-\hat{\varphi}(s|\xi|)|^{2}|\hat{f}(\xi)|^{2} d \xi=\int_{\mathbb{R}^{n}}|\hat{f}(\xi)|^{2}\left|\int_{0}^{s|\xi|} \hat{\varphi}^{\prime}(\tau) d \tau\right|^{2} d \xi \\
& \leq \int_{\mathbb{R}^{n}} s^{2}|\xi|^{2}|\hat{f}(\xi)|^{2} \int_{0}^{s|\xi|}\left|\hat{\varphi}^{\prime}(\tau)\right|^{2} d \tau d \xi \leq s^{2}\left\|\hat{\varphi}^{\prime}\right\|_{L^{\infty}\left(\mathbb{R}^{n}\right)} \int_{\mathbb{R}^{n}}|\xi|^{2}|\hat{f}(\xi)|^{2} d \xi
\end{aligned}
$$

\subsubsection{Carleson Measures}

Lemma 2.4.15 (John-Nirenberg Lemma for Carleson Measures). Let $\mu$ be a non-negative measure on $\mathbb{R}_{+}^{n+1}$. Suppose that there exist $\eta \in(0,1)$ and $C_{0}>0$ such that for every cube $Q \subset \mathbb{R}^{n}$, there exists a disjoint collection of cubes $\left(Q_{j}\right)_{j \in \mathfrak{N}} \subset \mathbb{D}(Q)$ verifying $\sum_{j \geq 1}\left|Q_{j}\right|<$ $\eta|Q|$ and $\mu\left(R_{Q} \backslash\left(\cup_{j} R_{Q_{j}}\right)\right) \leq C_{0}|Q|$. Then $\mu$ is a Carleson measure.

Remark 2.4.16. We may replace the Lebesgue measure on $\mathbb{R}^{n}$ by any other Radon measure, the proof is identical. If we assume that the hypotheses only hold for dyadic cubes, then we require the measure to be doubling. 
Lemma 2.4.17 (John-Nirenberg Lemma for local Square Functions). Suppose that $F$ : $\mathbb{R}_{+}^{n+1} \rightarrow \mathbb{R}, F \geq 0$ and define the local square function $A_{Q, F}: \mathbb{R}^{n} \rightarrow \mathbb{R}$ by

$$
A_{Q, F}:=\left(\iint_{|x-y|<t<\ell(Q)}|F(y, t)|^{2} \frac{d y d t}{t^{n+1}}\right)^{\frac{1}{2}}
$$

If there exists $C_{0}>0$ with the property that for every cube $Q \subset \mathbb{R}^{n}$, the estimate

$$
\int_{Q} A_{Q, F}^{2} d x \leq C_{0}|Q|
$$

holds, then for every $p>1$, there exists a constant $C_{1}$ depending on $p, n$ and $C_{0}$ such that for every cube $Q$, we have that

$$
\int_{Q} A_{Q, F}^{p} d x \leq C_{1}|Q|
$$

Proof. By Jensen's inequality, the result is trivially true, with $C_{1} \leq C_{0}^{p / 2}$, when $p \leq 2$. Therefore we concentrate on the case $p>2$. For ease of notation, we will write $A_{Q}=A_{Q, F}$. Moreover, for $\alpha>0$ we define

$$
A_{Q, \alpha}(x):=\left(\int_{0}^{\ell(Q)} \int_{|x-y|<\alpha t}|F(y, t)|^{2} \frac{d y d t}{t^{n+1}}\right)^{1 / 2}
$$

When $\alpha=1$, we may omit the subscript $\alpha$. We also set $K_{p}:=\sup _{Q \subset \mathbb{R}^{n}} f_{Q} A_{Q}^{p}$. Note first that $K_{\alpha, p} \approx_{\alpha, p} K_{1, p}=: K_{p}$. We defer the proof of this fact to the end, and proceed with the proof of the lemma.

Let us momentarily assume that $K_{p}<\infty$ a priori, and set $\alpha>0$ and $N \gg 1$, both to be specified later. Consider the open set $\Omega_{N}:=\left\{x \in Q: A_{Q, \alpha}(x)>N\right\}$. By the Chebyshev 
inequality and our assumptions, we see that $\left|\Omega_{N}\right| \lesssim_{\alpha} C_{0} N^{-2}|Q|$. In particular, given $\alpha>0$, we may chose $N \gtrsim_{\alpha} \sqrt{C_{0}}$ so that $\Omega_{N} \subsetneq Q$. Observe that

$$
\int_{Q} A_{Q}^{p}=\int_{\Omega_{N}} A_{Q}^{p}+\int_{Q \backslash \Omega_{N}} A_{Q}^{p}=: I+I I .
$$

By definition of $\Omega_{N}$, we have that $I I \leq N^{p}\left|Q \backslash \Omega_{N}\right|$. On the other hand, if $\left(Q_{j}\right)_{j}$ is a Whitney decomposition of $\Omega_{N}$, we can write (exploiting the convexity of $s \mapsto s^{p / 2}$ )

$$
I \lesssim_{p} \sum_{j \geq 1} \int_{Q_{j}} A_{Q_{j}}(x)^{p} d x+\sum_{j \geq 1} \int_{Q_{j}}\left(A_{Q}(x)^{2}-A_{Q_{j}}(x)^{2}\right)^{p / 2} d x .
$$

For the first term, we easily have that

$$
\sum_{j \geq 1} \int_{Q_{j}} A_{Q_{j}}(x)^{p} d x \leq \sum_{j \geq 1} K_{p}\left|Q_{j}\right|=K_{p}\left|\Omega_{N}\right| .
$$

For the second term, by definition of $A_{Q}$ and $A_{Q_{j}}$ we see that

$$
A_{Q}(x)^{2}-A_{Q_{j}}(x)^{2}=\int_{\ell\left(Q_{j}\right)}^{\ell(Q)} \int_{|x-y|<t}|F(y, t)|^{2} \frac{d y d t}{t^{n+1}} .
$$

If $x \in Q_{j}$, then, by properties of a Whitney decomposition, there exists $x_{*} \in Q \backslash \Omega_{N}$ (recall $\left.Q \backslash \Omega_{N} \neq \varnothing\right)$ such that $\left|x-x_{*}\right| \approx \ell\left(Q_{j}\right)$ with implicit constants depending only on $n$. In particular, for some $\alpha=\alpha(n)>0$, we have the inclusion

$$
\begin{aligned}
\left\{(y, t) \in \mathbb{R}_{+}^{n+1}:|x-y|<t,\right. & \left.\ell\left(Q_{j}\right)<t<\ell(Q)\right\} \\
\subseteq & \left\{(y, t) \in \mathbb{R}_{+}^{n+1}:\left|x_{*}-y\right|<\alpha t, \quad 0<t<\ell(Q)\right\},
\end{aligned}
$$

so that $A_{Q}(x)^{2}-A_{Q_{j}}(x)^{2} \leq A_{Q, \alpha}\left(x_{*}\right)^{2} \leq N^{2}$, since $x_{*} \in Q \backslash \Omega_{N}$. Accordingly,

$$
\sum_{j \geq 1} \int_{Q_{j}}\left(A_{Q}(x)^{2}-A_{Q_{j}}(x)^{2}\right)^{p / 2} d x \lesssim_{\alpha} N^{p}\left|\Omega_{N}\right|
$$


Combining these previous estimates, we obtain that $I \lesssim_{p, n} K_{p}\left|\Omega_{N}\right|+N^{p}\left|\Omega_{N}\right|$, and so

$$
\int_{Q} A_{Q}(x)^{p} d x \lesssim_{p, n} K_{p}\left|\Omega_{N}\right|+N^{p}|Q| \leq C_{0} K_{p} N^{-2}|Q|+N^{p}|Q|
$$

Dividing by $|Q|$ and taking supremum over all cubes gives $K_{p} \lesssim_{p, n} C_{0} K_{p} N^{-2}+N^{p}$. Choosing $N=M \sqrt{C}_{0}$ with $M \geq 1$ large enough depending only on $p$ and $n$, we may hide the first term to the left-hand side, and thus obtain $K_{p} \lesssim_{p, n} C_{0}^{p / 2}$.

Finally, to do away with the restriction that $K_{p}<\infty$ a priori, we fix $\eta>0$ and work with $F_{\eta}:=F \mathbb{1}_{\eta<|F|<1 / \eta} \mathbb{1}_{\eta<t<1 / \eta}$, for which $K_{p}<\infty$, and appeal to the monotone convergence theorem in the limit $\eta \rightarrow 0^{+}$.

We now turn to the proof of $K_{\alpha, p} \approx K_{p}$. Notice that we only used this in the case $p=2$, so we will only prove this special case. We will also work only with $\alpha>1$.

By Fubini's theorem, if $\omega_{n}:=|B(0,1)|$ is the volume of the unit ball in $\mathbb{R}^{n}$,

$$
\begin{aligned}
\int_{Q} A_{Q, \alpha}(x)^{2} d x & =\int_{0}^{\ell(Q)} \int_{\mathbb{R}^{n}} \int_{\mathbb{R}^{n}} \mathbb{1}_{Q}(x) \mathbb{1}_{|x-y|<\alpha t}|F(y, t)|^{2} d x d y \frac{d t}{t^{n+1}} \\
& =\alpha^{n} \omega_{n} \int_{0}^{\ell(Q)} \int_{\mathbb{R}^{n}}\left(f_{|x-y|<\alpha t} \mathbb{1}_{Q}(x) d x\right)|F(y, t)|^{2} d y \frac{d t}{t} .
\end{aligned}
$$

We claim that ${ }^{1}$, for some dimensional constants $c, c^{\prime}$ and every $\beta>1$,

$$
c \beta^{-n} \mathbb{1}_{Q}(y) \leq f_{|x-y|<\beta t} \mathbb{1}_{Q}(x) d x \leq \mathbb{1}_{c^{\prime} \beta Q}, \quad \text { whenever } 0<t<\ell(Q) .
$$

This claim follows immediately by noting that

$$
f_{|x-y|<\beta t} \mathbb{1}_{Q}(x) d x=\frac{|Q \cap B(y, \beta t)|}{|B(y, \beta t)|} .
$$

\footnotetext{
${ }^{1}$ We remind the reader that the notation $C Q$ means the concentric dilate of $Q$ by a factor $C>0$.
} 
Using the second inequality with $\beta=\alpha$ and the first with $\beta=1$ and $c^{\prime} \alpha Q$ in place of $Q$, we arrive at

$$
\begin{aligned}
& \int_{Q} A_{Q, \alpha}(x)^{2} d x \lesssim_{\alpha, n} \int_{0}^{\ell(Q)} \int_{c^{\prime} \alpha Q}|F(y, t)|^{2} d y \frac{d t}{t}=\int_{0}^{\ell(Q)} \int_{\mathbb{R}^{n}} \mathbb{1}_{c^{\prime} \alpha Q}(y)|F(y, t)|^{2} d y \frac{d t}{t} \\
& \lesssim_{n} \int_{0}^{\ell(Q)} \int_{\mathbb{R}^{n}}\left(f_{|x-y|<t} \mathbb{1}_{c^{\prime} \alpha Q}(x) d x\right)|F(y, t)|^{2} d y \frac{d t}{t} \lesssim_{n, \alpha} \int_{c^{\prime} \alpha Q} A_{Q}(x)^{2} d x \\
& \leq \int_{c^{\prime} \alpha Q} A_{c^{\prime} \alpha Q}(x)^{2} d x \lesssim_{n, \alpha} K_{p}|Q| .
\end{aligned}
$$

The result now follows from taking the supremum over all cubes.

\subsubsection{Weights and Extrapolation}

Definition 2.4.28 ( $A_{p}$ weights). Let $1<p<\infty$. A weight $v \in L_{\mathrm{loc}}^{1}\left(\mathbb{R}^{n}\right)$ is said to be an $A_{p}$ weight if there exists a constant $C \geq 1$ such that for every cube $Q \subset \mathbb{R}^{n}$, the estimate

$$
\left(f_{Q} v\right)\left(f_{Q} v^{-p^{\prime} / p}\right)^{\frac{p}{p^{\prime}}} \leq C
$$

holds. The infimum over all these constants is denoted $[v]_{A_{p}}$; we refer to it as the $A_{p}$ characteristic of $v$. We say that $v \in A_{1}$ if $(\mathcal{M} v)(x) \leq C v(x)$ for a.e. $x \in \mathbb{R}^{n}$. The infimum over such $C$ is denoted by $[v]_{A_{1}}$.

Closely related to $A_{p}$ weights are the reverse Hölder classes.

Definition 2.4.29 (Reverse Hölder class). Let $1<s<\infty$. A weight $v \in L_{\mathrm{loc}}^{1}\left(\mathbb{R}^{n}\right)$ is said to satisfy a reverse Hölder inequality with exponent $s$, written $v \in R H_{s}$, if there exists a constant $C \geq 1$ such that for every cube $Q \subset \mathbb{R}^{n}$, we have that

$$
\left(f_{Q} v^{s}\right)^{1 / s} \leq C f_{Q} v .
$$


Let us summarize most of the "basic" facts about $A_{p}$ weights which we will need.

Proposition 2.4.30 ( [GR85, Theorem 1.14, Lemma 2.2, Lemma 2.5, Theorem 2.6]). Let $1 \leq p<q<\infty$. The following statements hold.

(i) ( [GR85, Ch. IV Theorem 1.14 (a)]) $A_{p} \subset A_{q}$.

(ii) A weight $v$ belongs to $A_{2}$ if and only if $v^{-1} \in A_{2}$.

(iii) ( [GR85, Ch. IV Theorem 1.14 (b)]) If $v \in A_{p}$ then $v^{\delta} \in A_{p}$ for any $0<\delta<1$.

(iv) ( [GR85, Ch. IV Lemma 2.2]) If $v \in A_{p}$ then $v d x$ is a doubling measure, and the doubling constant depends on $v$ only through $[v]_{A_{p}}($ and $p)$.

(v) ( [GR85, Ch. IV Lemma 2.5]) If $v \in A_{p}$ then $v \in R H_{s}$ for some s that depends on the weight only through $[v]_{A_{p}}($ and $p)$.

(vi) ( [GR85, Ch. IV Theorem 2.6]) If $v \in A_{q}$ then $v \in A_{q-\varepsilon}$ for some $\varepsilon$ depending on $v$ only through $[v]_{A_{q}}($ and $q)$.

(vii) If $v \in A_{q}$ and $s>1$, then $v \in R H_{s}$ if and only if $v^{s} \in A_{s(q-1)+1}$.

(viii) (Coifman-Rochberg [CR80, Proposition 2], [GR85, Ch. II Theorem 3.4]) If $f: \mathbb{R}^{n} \rightarrow$ $\mathbb{C}$ is such that $(\mathcal{M} f)(x)<\infty$ for a.e. $x \in \mathbb{R}^{n}$, then for every $0<\delta<1$ we have that $v_{\delta}:=(\mathcal{M} f)^{\delta} \in A_{1}$ and moreover $\left[v_{\delta}\right]_{A_{1}} \leq C_{\delta}$ depends only on $\delta$.

(ix) (Muckenhoupt's Theorem [Muc72, Theorem 2], [GR85, Ch. IV Theorem 2.8]) For any $1<p<\infty, v \in A_{p}$ and $f \in L^{p}(v),\|\mathcal{M} f\|_{L^{p}(v)} \lesssim_{[v]_{A_{p}}}\|f\|_{L^{p}(v)}$. 
(x) (Coifman-Fefferman [CF74, Theorem III]) Let T be a "regular" singular integral, as defined in [CF74], and $T^{*}$ the associated maximal operator. Then, for every $v \in A_{\infty}$ and $f \in C_{c}^{\infty}\left(\mathbb{R}^{n}\right)$, we have that $\left\|T^{*}\right\|_{L^{p}(v)} \lesssim_{p, n}\|\mathcal{M} f\|_{L^{p}(v)}$. In particular, by Muckenhoupt's Theorem above, we have that $\left\|T^{*} f\right\|_{L^{p}(v)} \lesssim_{[v]_{A}}\|f\|_{L^{p}(v)}$.

The following result was originally proved by Rubio de Francia in [Rub83, Rub84]. We refer to [CMP11, Theorem 1.1] for a simple proof of this fact.

Theorem 2.4.31 (Extrapolation Theorem for $A_{p}$ weights). Let $1<p_{0}<\infty$ and let $T$ be an operator satisfying $\|T f\|_{L^{p_{0}(v)}} \lesssim_{[v]_{A_{p_{0}}}}\|f\|_{L^{p_{0}(v)}}$, for all $v \in A_{p_{0}}$ and all $f \in L^{p_{0}}(v)$. Then, for every $p \in(1, \infty), v \in A_{p}$, and $f \in L^{p}(v)$, we have $\|T f\|_{L^{p}(v)} \lesssim_{[v]_{A_{p}}}\|f\|_{L^{p}(v)}$.

It is important for applications to note that the above theorem does not require any special structure on $T$; it does not need to be linear or sublinear. In fact, we have

Theorem 2.4.32 (Extrapolation Theorem for $A_{p}$ Weights Version 2 [CMP11, Theorem 3.9]). Fix $p_{0} \in(1, \infty)$ and $\mathcal{F}$ a collection of pairs of non-negative measurable functions $(f, g)$. Suppose that $\|f\|_{L^{p_{0}(v)}} \lesssim_{[v]_{A_{p_{0}}}}\|g\|_{L^{p_{0}}(v)}$ for all $v \in A_{p_{0}}$ and all $(f, g) \in \mathcal{F}$. Then for every $p \in(1, \infty), v \in A_{p}$, and $(f, g) \in \mathcal{F}$, we have $\|f\|_{L^{p}(v)} \lesssim_{[v]_{A_{p}}}\|g\|_{L^{p}(v)}$.

In practice, the collection $\mathcal{F}$ often takes the form $\left(\left|S_{1} h\right|,\left|S_{2} h\right|\right)$ for some operators $S_{i}$ and $h$ in some nice class of functions. A corollary of the previous theorem and this observation is the following.

Corollary 2.4.33 ( [CMP11, Corollary 3.14]). Let $r \in(1,2)$, and suppose that $T$ is an 
operator verifying $\|T f\|_{L^{2}(v)} \lessgtr_{[v]_{A_{2 / r}}}\|f\|_{L^{2}(v)}$, for each $f \in C_{c}^{\infty}\left(\mathbb{R}^{n}\right)$ and all $v \in A_{2 / r}$. Then $\|T f\|_{L^{q}\left(\mathbb{R}^{n}\right)} \lesssim_{q}\|f\|_{L^{q}\left(\mathbb{R}^{n}\right)}$ for all $q>r$.

To prove the corollary, one defines $S_{1} f:=|T f|^{r}, S_{2} f:=|f|^{r}$. Then, by hypothesis, $\left\|S_{1} f\right\|_{L^{2 / r(v)}} \lessgtr_{[v]_{A_{2 / r}}}\left\|S_{2} f\right\|_{L^{2 / r}(v)}$, and hence by the previous theorem, $\left\|S_{1} f\right\|_{L^{p}(v)} \lessgtr_{[v]_{A_{p}}}$ $\left\|S_{2} f\right\|_{L^{p}(v)}$ for $p \in(1, \infty)$. Setting $v \equiv 1$ and $p=q / r$ gives the desired result.

Theorem 2.4.34 (Weighted Littlewood-Paley Theorem). Let $\left(Q_{s}\right)_{s}$ be a CLP family (see Definition 2.4.13) and let $v \in A_{2}$. It holds that

$$
\int_{\mathbb{R}^{n}} \int_{0}^{\infty}\left|\left(Q_{t} f\right)(x)\right|^{2} \frac{d t}{t} v(x) d x \lesssim_{n,[v]_{A_{2}}} \int_{\mathbb{R}^{n}}|f(x)|^{2} v(x) d x .
$$

Remark 2.4.36. By Theorem 2.4.31, we obtain that the vertical square function associated to $\left(Q_{s}\right)_{s}$ is bounded on $L^{p}(v)$ for every $v \in A_{p}$ and $1<p<\infty$; that is, $\left\|\mathbb{V}\left(Q_{s} f\right)\right\|_{L^{p}(v)} \lesssim$ $\|f\|_{L^{p}(v)}$ for every $v \in A_{p}$.

Proof of Theorem 2.4.34. The idea is to use Rubio de Francia and Duoandikoetxea's method in [DR86, Theorem B], to interpolate a "good" bound with a plain uniform bound in order to obtain another "good" bound in between. We will combine this with interpolation with change of measures as in [SW58, Theorem 2.11], exploiting the self-improvement property of $A_{p}$ weights. Since this idea will be used quite often throughout the paper we write out this portion of the the argument in full here, and refer back to it when applicable.

We first claim that it is enough to prove the following estimate:

$$
\int_{\mathbb{R}^{n}}\left|Q_{s} \tilde{Q}_{t}^{2} f\right|^{2} v \lesssim_{[v]_{A_{2}}} \min \left(\frac{t}{s}, \frac{s}{t}\right)^{\alpha} \int_{\mathbb{R}^{n}}\left|\tilde{Q}_{t} f\right|^{2} v, \quad \text { for each } s, t>0,
$$


for some $\alpha>0$ and some CLP family $\left(\tilde{Q}_{t}\right)_{t}$. Indeed, once this is shown, the desired result follows from a familiar quasi-orthogonality argument (see for instance the proof of [Gra14b, Theorem 4.6.3]).

To prove (2.37), we first claim that it is enough to prove the following estimates.

(i) (Unweighted quasi-orthogonality) There exists $\beta>0$ such that for any $s, t>0$, we have the estimate

$$
\int_{\mathbb{R}^{n}}\left|Q_{s} \tilde{Q}_{t}^{2} f\right|^{2} \leq C_{1}\left(\frac{s}{t}, \frac{t}{s}\right)^{\beta} \int_{\mathbb{R}^{n}}\left|\tilde{Q}_{t} f\right|^{2}
$$

(ii) (Uniform weighted estimate) For any $s>0$ and $v \in A_{2}$, we have the estimate

$$
\int_{\mathbb{R}^{n}}\left|Q_{s} \tilde{Q}_{t}^{2} f\right|^{2} v \leq C_{2}\left([v]_{A_{2}}\right) \int_{\mathbb{R}^{n}}\left|\tilde{Q}_{t} f\right|^{2} v
$$

Assume that these hold for the moment and fix $v \in A_{2}$. By properties of $A_{2}$ weights, there exist $\delta, C>0$ such that $v^{1+\delta} \in A_{2}$ with $\left[v^{1+\delta}\right]_{A_{2}} \leq C$. In particular, the uniform weighted estimate holds with $v^{1+\delta}$ in place of $v$, with the implicit constants depending only on $[v]_{A_{2}}$. Therefore, if we define the measures $d \mu_{\tau}:=v^{(1+\delta) \tau} d x$, interpolation with change of measure (see [SW58, Theorem 2.11] $]^{2}$ gives

$$
\int_{\mathbb{R}^{n}}\left|Q_{s} \tilde{Q}_{t}^{2} f\right|^{2} d \mu_{\tau} \leq C_{1}^{1-\tau} C_{2}^{\tau}\left(\frac{s}{t}, \frac{t}{s}\right)^{\beta(1-\tau)} \int_{\mathbb{R}^{n}}\left|\tilde{Q}_{t} f\right|^{2} d \mu_{\tau}
$$

The desired estimate (2.37) is exactly the case $\tau=1 /(1+\delta)$ with $\alpha=\beta \delta /(1+\delta)$. This completes the proof, modulo the above pair of estimates.

\footnotetext{
${ }^{2}$ Strictly speaking, the statement of [SW58, Theorem 2.11] explicitly excludes the case under consideration (indeed the proof given does not apply in this case); however as is mentioned immediately after the statement of said Theorem, we may run an argument similar to the standard proof of the Riesz-Thorin Theorem, employing instead the three line lemma for sub-harmonic functions as in [CZ56].
} 
The first estimate, the unweighted quasi-orthogonality, is a consequence of classical Littlewood-Paley theory. On the other hand, the weighted estimate follows from both the fact that $\left|Q_{s} f\right|,\left|\tilde{Q}_{t} f\right| \lesssim \mathcal{M} f$ pointwise in $\mathbb{R}^{n}$ and Muckenhoupt's theorem on the $L^{2}(v)$ boundedness of $\mathcal{M}$ for $v \in A_{2}$ (see Proposition 2.4.30).

Lemma 2.4.39 ( $L^{p}$ inequalities from weighted $L^{2}$ bounds). Suppose that $T: L^{2}\left(\mathbb{R}^{n}\right) \rightarrow$ $L^{2}\left(\mathbb{R}^{n}\right)$ is a bounded (not necessarily linear) operator; that is, $\|T f\|_{L^{2}\left(\mathbb{R}^{n}\right)} \lesssim\|f\|_{L^{2}\left(\mathbb{R}^{n}\right)}$.

(i) Suppose that there exists $M>1$ such that for all $v \in A_{1}$ with the property that $v^{M} \in A_{1}$ it holds that $\|T f\|_{L^{2}(v)} \lesssim_{\left[\nu^{M}\right]_{A_{1}}}\|f\|_{L^{2}(v)}$, for every $f \in C_{c}^{\infty}\left(\mathbb{R}^{n}\right)$. Then for every $p \in(2,2+1 / M)$, it holds that $\|T f\|_{L^{p}\left(\mathbb{R}^{n}\right)} \lessgtr_{p}\|f\|_{L^{p}\left(\mathbb{R}^{n}\right)}$.

(ii) Suppose that there exists $M>1$ such that for all $v$ with the property that $v^{-M} \in A_{1}$ it holds that $\|T f\|_{L^{2}(v)} \lessgtr_{\left[v^{-M}\right]_{A_{1}}}\|f\|_{L^{2}(v)}$ for every $f \in C_{c}^{\infty}\left(\mathbb{R}^{n}\right)$. Then for every $p \in$ $(2-1 / M, 2)$, we have that $\|T f\|_{L^{p}\left(\mathbb{R}^{n}\right)} \lessgtr_{p}\|f\|_{L^{p}\left(\mathbb{R}^{n}\right)}$.

(iii) Suppose that there exists $M>1$ such that for all $v \in A_{2}$ with the property that $v^{M} \in A_{2}$, it holds that $\|T f\|_{L^{2}(v)} \Im_{\left[v^{M}\right]_{A_{2}}}\|f\|_{L^{2}(v)}$ for every $f \in C_{c}^{\infty}\left(\mathbb{R}^{n}\right)$. Then for every $p \in(2-1 / M, 2+1 / M)$, we have $\|T f\|_{L^{p}\left(\mathbb{R}^{n}\right)} \lessgtr_{p}\|f\|_{L^{p}\left(\mathbb{R}^{n}\right)}$.

Proof. This lemma and its proof are contained in [CMP11, Corollary 3.37] for the much more general setting of restricted extrapolation of $A_{p}$ weights. However, since we will later on need to modify the arguments used in the proof a little to fit our needs, it seems appropriate to write the proof down for future reference. The key fact that we will use is 
the Coifman-Rochberg theorem (see Proposition 2.4.30).

We start with (i). Fix $p>2$ such that $M<1 /(p-2)$ and $f \in C_{c}^{\infty}\left(\mathbb{R}^{n}\right)$. Note that $v:=(\mathcal{M}(|T f|))^{p-2} \in A_{1}$, and estimate

$$
\begin{aligned}
& \int_{\mathbb{R}^{n}}|T f|^{p} \leq \int_{\mathbb{R}^{n}}|T f|^{2}(\mathcal{M}(|T f|))^{p-2} \lesssim_{\left[v^{M}\right]_{A_{1}}} \int_{\mathbb{R}^{n}}|f|^{2}(\mathcal{M}(|T f|))^{p-2} \\
& \quad \leq\left(\int_{\mathbb{R}^{n}}|f|^{p}\right)^{2 / p}\left(\int_{\mathbb{R}^{n}}(\mathcal{M}(|T f|))^{p}\right)^{(p-2) / p} \lesssim_{p}\left(\int_{\mathbb{R}^{n}}|f|^{p}\right)^{2 / p}\left(\int_{\mathbb{R}^{n}}|T f|^{p}\right)^{1-\frac{2}{p}} .
\end{aligned}
$$

If we first assume that $\|T f\|_{L^{p}\left(\mathbb{R}^{n}\right)}<\infty$, then the result follows. To get rid of this assumption, we instead consider the sequence of operators $S_{k} f(x):=(T f)(x) \mathbb{1}_{|T f| \leq k}(x)$ on $L^{2}\left(\mathbb{R}^{n}\right)$. Then $\left\{S_{k}\right\}_{k}$ is uniformly bounded on $L^{2}\left(\mathbb{R}^{n}\right)$, and they satisfy the same hypotheses as $T$ with constants independent of $k$. Then, for $f \in C_{c}^{\infty}\left(\mathbb{R}^{n}\right)$, we have that $S_{k} f \in L^{2}\left(\mathbb{R}^{n}\right) \cap L^{\infty}\left(\mathbb{R}^{n}\right)$, and so by our argument above, $\left\|S_{k} f\right\|_{L^{p}\left(\mathbb{R}^{n}\right)} \lesssim_{p}\|f\|_{L^{p}\left(\mathbb{R}^{n}\right)}$. We now let $k \rightarrow \infty$ and use the Monotone Convergence Theorem.

We turn to (ii). Fix $p<2$ with $\frac{1}{2-p}<M$ and $f \in C_{c}^{\infty}\left(\mathbb{R}^{n}\right)$ not identically 0 . Note that $v:=(\mathcal{M}(|T f|+|f|))^{p-2}$ satisfies $v^{-1} \in A_{1} \subset A_{2}$, and hence $v \in A_{2}$. We estimate

$$
\begin{gathered}
\int_{\mathbb{R}^{n}}|T f|^{p} \leq \int_{\mathbb{R}^{n}}(\mathcal{M}(|T f|+|f|))^{p}=\int_{\mathbb{R}^{n}}(\mathcal{M}(|T f|+|f|))^{2}(\mathcal{M}(|T f|+|f|))^{p-2} \\
\lesssim_{[v]_{A_{2}}} \int_{\mathbb{R}^{n}}\left(|T f|^{2}+|f|^{2}\right) v \lesssim_{\left[v^{-M}\right]_{A_{1}}} \int_{\mathbb{R}^{n}}|f|^{2} v \leq \int_{\mathbb{R}^{n}}|f|^{2}(\mathcal{M} f)^{p-2} \leq \int_{\mathbb{R}^{n}}(\mathcal{M} f)^{p},
\end{gathered}
$$

where we have used Muckenhoupt's theorem, yielding the desired result.

The third statement follows from the first two and Jones's factorization theorem of $A_{2}$ weights (see [Jon80]) as quotients of $A_{1}$ weights. 
Sometimes we will not be able to conclude boundedness on all weights $v^{M} \in A_{2}$, but rather only on weights whose characteristic is uniformly bounded by a (large) constant. An inspection of the proof of the above Lemma, together with the Coifman-Rochberg theorem (see Proposition 2.4.30), reveals that this is enough to conclude the unweighted $L^{p}$ estimates. We record this in the following result.

Corollary 2.4.42. Let $M \geq 1,0<\delta<1$ and $T$ be an operator satisfying, for every $v \in A_{2}$ with $\left[v^{M}\right] \leq C_{\delta}$ (where $C_{\delta}$ is as in Proposition 2.4.30), the estimate $\|T f\|_{L^{2}(v)} \lesssim_{\left[v^{M}\right]_{A_{2}}}\|f\|_{L^{2}(v)}$. Then, for every $p \in(2-\delta / M, 2+\delta / M),\|T f\|_{L^{p}\left(\mathbb{R}^{n}\right)} \lesssim_{p}\|f\|_{L^{p}\left(\mathbb{R}^{n}\right)}$. Analogous statements for the one-sided versions of the estimates also hold.

Lemma 2.4.43 (Weighted Carleson's Lemma). Suppose that $\mu$ is a measure in $\mathbb{R}_{+}^{n+1}$ and that $v \in L_{\mathrm{loc}}^{1}\left(\mathbb{R}^{n}\right)$ is a doubling weight. Assume further that for every cube $Q \subset \mathbb{R}^{n}$, it holds that $\mu\left(R_{Q}\right) \lesssim v(Q)$. Then, for every measurable function $F: \mathbb{R}_{+}^{n+1} \rightarrow \mathbb{C}$ and every $p>0$, we have that $\iint_{\mathbb{R}_{+}^{n+1}}|F|^{p} d \mu \lesssim_{n \text {,doub }} \int_{\mathbb{R}^{n}}(\mathcal{N} F)^{p} v$.

The proof is exactly the same as the usual one when $v \equiv 1$, and thus omitted.

We will have need for a version of Carleson's Lemma that introduces the modified nontangential maximal function $\tilde{\mathcal{N}}$ in place of the usual $\mathcal{N}$.

Lemma 2.4.44 (Modified-Weighted Carleson's Lemma). Let $d \mu(x, t)=m(x, t) d x d t$ be $a$ non-negative measure on $\mathbb{R}_{+}^{n+1}$ and $v$ is a doubling weight. For every $(x, t) \in \mathbb{R}_{+}^{n+1}$, suppose that $\left.d \tilde{\mu}(x, t):=\sup _{(y, s) \in C_{x, t}} m(y, s)\right) d x d t$ verifies $\tilde{\mu}\left(R_{Q}\right) \leq C_{0} v(Q)$ for every cube $Q \subset \mathbb{R}^{n}$. 
Then, for every $q>0, \iint_{\mathbb{R}_{+}^{n+1}}|F|^{q} d \mu \lesssim_{\text {doub }} C_{0} \int_{\mathbb{R}^{n}}\left(\tilde{\mathcal{N}}_{q} F\right)^{q} v d x$.

Proof. Introduce an average as follows:

$$
\begin{aligned}
\iint_{\mathbb{R}_{+}^{n+1}}|F(x, t)|^{q} d \mu(x, t)=\iint_{\mathbb{R}_{+}^{n+1}} \iint_{\mathcal{C}_{x, t}}|F(x, t)|^{q} d y d s m(x, t) d x d t \\
=\iint_{\mathbb{R}_{+}^{n+1}} \iint_{C_{y, s}}|F(x, t)|^{q} m(x, t) d x d t d y d s \leq \iint_{\mathbb{R}_{+}^{n+1}} G(y, s)^{q} d \tilde{\mu}(y, s),
\end{aligned}
$$

where we used Fubini's Theorem, and $G(y, s):=\left(\int_{C_{y, s}}|F(x, t)|^{q} d x d t\right)^{1 / q}$. The conclusion now follows from Lemma 2.4.43 and the fact that $\mathcal{N}(G)=\tilde{\mathcal{N}}_{q}(F)$.

Definition 2.4.46 $\left(A_{p, q}\right.$ classes). Let $1<p \leq q<\infty$. We say that a weight $v \in A_{p, q}=$ $A_{p, q}\left(\mathbb{R}^{n}\right)$ if there exists a constant $C>0$ such that for every cube $Q \subset \mathbb{R}^{n}$,

$$
\left(f_{Q} v^{q} d x\right)^{1 / q}\left(f_{Q} v^{-1 / p^{\prime}} d x\right)^{1 / p^{\prime}} \leq C
$$

The infimum over all such $C$ is written $[v]_{A_{p, q}}$.

Theorem 2.4.47 ( [MW74, Theorem 4]). Let $1<p<n$ and set $1 / q:=1 / p-1 / n$. Then $\nu \in A_{p, q}$ if and only if $\left\|I_{1} f\right\|_{L^{q}\left(\nu^{q}\right)} \lesssim_{[v]_{A_{p, q}}}\|f\|_{L^{p}\left(\nu^{p}\right)}$.

Throughout the paper we will encounter instances where multiplication by an $L^{n}\left(\mathbb{R}^{n}\right)$ function is acting as, or rather in place of, a (spatial) gradient. The following proposition should be interpreted as stating that, at least in $L^{p}$ spaces, the two operations are not far from each other. We remind the reader that we assume $n \geq 3$.

Proposition 2.4.48. Let $B \in L^{n}\left(\mathbb{R}^{n}\right)$ and $f \in C_{c}^{\infty}\left(\mathbb{R}^{n}\right)$. Then, for every $v \in A_{2}$, we have

$$
\left\|I_{1}(B \cdot f)\right\|_{L^{2}(v)} \lesssim[v]_{A_{2}}\|B\|_{L^{n}\left(\mathbb{R}^{n}\right)}\|f\|_{L^{2}(v)}
$$


In particular, for every $1<p<\infty$, it holds that $\left\|I_{1}(B \cdot f)\right\|_{L^{p}\left(\mathbb{R}^{n}\right)} \lesssim_{p}\|f\|_{L^{p}\left(\mathbb{R}^{n}\right)}$, where the implicit constants depend on $\|B\|_{L^{n}\left(\mathbb{R}^{n}\right)}$, $p$, and $n$. If in addition we have that $v^{2^{*} / 2} \in A_{2}$ with $2^{*}=2_{n}^{*}$, then

$$
\left\|B \cdot I_{1} f\right\|_{L^{2}(v)} \lesssim_{\left[2^{2^{*}}\right]_{A_{2}}}\|B\|_{L^{n}\left(\mathbb{R}^{n}\right)}\|f\|_{L^{2}(v)} .
$$

Accordingly, $\left\|B \cdot I_{1} f\right\|_{L^{p}\left(\mathbb{R}^{n}\right)} \lesssim_{p}\|f\|_{L^{p}\left(\mathbb{R}^{n}\right)}$, for $1+2 / n<p<3-2 / n$.

Proof. Let $v \in A_{2}$ and set $\omega:=v^{1 / 2}$. We claim that $\omega \in A_{2_{*}, 2}$. Assuming the claim for a moment, we would have

$$
\left\|I_{1}(B \cdot f)\right\|_{L^{2}(v)}=\left\|I_{1}(B \cdot f)\right\|_{L^{2}\left(\omega^{2}\right)} \lesssim_{[\omega]_{A_{2 *}, 2}}\|B \cdot f\|_{L^{2 *}\left(\omega^{2 *}\right)} \leq\|B\|_{L^{n}\left(\mathbb{R}^{n}\right)}\|f\|_{L^{2}\left(\omega^{2}\right)}
$$

where we used Hölder's inequality in the last step. This is the result.

To prove the claim, we use Jensen's inequality to see that $\left(f_{Q} \omega^{-2_{*}}\right)^{2 / 2_{*}} \leq f_{Q} \omega^{-2}$. Using this estimate in the definition of $\omega^{2} \in A_{2}$, we deduce that $[\omega]_{A_{*, 2}} \leq[v]_{A_{2}}^{1 / 2}$. This completes the proof of the first part.

The second part follows the same lines, using instead that

$$
\left(f_{Q} \omega^{2^{*}}\right)\left(f_{Q} \omega^{-2}\right)^{2^{*} / 2} \leq\left(f_{Q} \omega^{2^{*}}\right)\left(f_{Q} \omega^{-2^{*}}\right) \leq\left[v^{2^{*} / 2}\right]_{A_{2}},
$$

so that $[\omega]_{A_{2,2^{*}}} \leq\left[v^{2^{*} / 2}\right]_{A_{2}}^{1 / 2^{*}}$. The $L^{p}$ estimate finally follows from restricted extrapolation (see Lemma 2.4.39), using the fact that $2 / 2^{*}=1-2 / n$.

Proposition 2.4.51. Let $P_{t}$ be an approximate identity with smooth, even, compactly supported kernel. Then, for every $v \in A_{2}$ and $f \in C_{c}^{\infty}\left(\mathbb{R}^{n}\right)$, it holds that

$$
\left\|\mathbb{V}\left(t^{-1}\left(1-P_{t}\right) f\right)\right\|_{L^{2}(v)}^{2}=\int_{0}^{\infty} \int_{\mathbb{R}^{n}}\left|\frac{I-P_{t}}{t} f\right|^{2} \frac{v(x) d x d t}{t} \lesssim_{[v]_{A_{2}}}\left\|\nabla_{\|} f\right\|_{L^{2}(v)}^{2} .
$$


Proof. Recall that $I_{1}$ denotes the fractional integral of order 1 ; hence $\nabla_{\|} I_{1}=I_{1} \nabla_{\|}=R$, where $R$ is the vector-valued Riesz-transform (with symbol $\xi /|\xi|)$. In particular, $\|R f\|_{L^{2}(v)} \approx$ $\|f\|_{L^{2}(v)}$ for all $v \in A_{2}$, allowing us to reduce matters to the estimate

$$
\int_{0}^{\infty} \int_{\mathbb{R}^{n}}\left|I_{1} \frac{I-P_{t}}{t} f\right|^{2} \frac{v(x) d t}{t} \lesssim_{[v]_{A_{2}}}\|f\|_{L^{2}(v)}, \quad f \in C_{c}^{\infty}\left(\mathbb{R}^{n}\right)
$$

We now use a quasi-orthogonality argument, with a change of measure interpolation (see the proof of Theorem 2.4.34), to reduce matters to the pair of estimates: If we denote $T_{t}:=I_{1}\left(1-P_{t}\right) / t$, then for some CLP family $\left(Q_{s}\right)_{s}($ see Definition 2.4.13),

$$
\left\|T_{t} Q_{s}^{2} f\right\|_{L^{2}\left(\mathbb{R}^{n}\right)} \lesssim\left(\frac{s}{t}, \frac{t}{s}\right)^{\alpha}\left\|Q_{s} f\right\|_{L^{2}\left(\mathbb{R}^{n}\right)},
$$

for some $\alpha>0$, and

$$
\left\|T_{t} Q_{s}^{2} f\right\|_{L^{2}(v)} \lesssim[v]_{A_{2}}\left\|Q_{s} f\right\|_{L^{2}(v)}
$$

Indeed, with (2.54) and (2.55) in hand, we may follow the proof of Theorem 2.4.34.

For (2.54), we compute, via the Fourier transform and Plancherel's theorem, and using $\varphi_{t}$ and $\psi_{s}$ for the kernels of $P_{t}$ and $Q_{s}$ respectively,

$$
\left\|T_{t} Q_{s} h\right\|_{L^{2}\left(\mathbb{R}^{n}\right)}^{2}=\left.\left.c_{n} \int_{\mathbb{R}^{n}}|| \xi\right|^{-1} \frac{1-\hat{\varphi}(t|\xi|)}{t} \hat{\psi}(s|\xi|) \hat{h}(\xi)\right|^{2} d \xi
$$

where as usual we have abused notation and written $\varphi, \psi$ for the one-dimensional functions representing them. Consider first the case $t<s$,

$$
\left.\left.\int_{\mathbb{R}^{n}}|| \xi\right|^{-1} \frac{1-\hat{\varphi}(t|\xi|)}{t} \hat{\psi}(s|\xi|) \hat{h}(\xi)\right|^{2} d \xi=\left(\frac{t}{s}\right)^{2} \int_{\mathbb{R}^{n}} \frac{|1-\hat{\varphi}(t|\xi|)|^{2}}{|t \xi|^{4}}|s \xi|^{2}|\hat{\psi}(s|\xi|)|^{2}|\hat{h}(\xi)|^{2} d \xi
$$




$$
\lesssim\left(\frac{t}{S}\right)^{2}\|h\|_{L^{2}\left(\mathbb{R}^{n}\right)}^{2}
$$

where we used the properties of the CLP family and the fact that $|1-\hat{\varphi}(\tau)| \lesssim \tau^{2}$ for $\tau$ near 0 , since $\varphi$ is even. For the case $s<t$, we use instead the Fundamental Theorem of Calculus to obtain

$$
\begin{array}{r}
\left.\int_{\mathbb{R}^{n}}|\xi|^{-1} \frac{1-\hat{\varphi}(t|\xi|)}{t} \hat{\psi}(s|\xi|) \hat{h}(\xi)\right|^{2} d \xi=\left(\frac{s}{t}\right)^{2} \int_{\mathbb{R}^{n}}\left|\int_{0}^{t|\xi|} \hat{\varphi}^{\prime}(\tau) d \tau\right|^{2} \frac{|\hat{\psi}(s|\xi|)|^{2}}{|s \xi|^{2}}|\hat{h}(\xi)|^{2} d \xi \\
\lesssim\left(\frac{s}{t}\right)^{2}\|h\|_{L^{2}\left(\mathbb{R}^{n}\right)}^{2},
\end{array}
$$

where we used that $\hat{\varphi} \in L^{1}(0, \infty)$ and $\hat{\psi}(\tau) / \tau \in L^{\infty}(0, \infty)$. Combining these estimates with $h=Q_{s} f$ gives (2.54).

The weighted estimate (2.55) follows from the pointwise inequality

$$
\left|T_{t} f(x)\right|=\left|t^{-1}\left(1-P_{t}\right) I_{1} f(x)\right| \lesssim \mathcal{M}(R f)(x),
$$

where $R=I_{1} \nabla_{\|}$is as before. We sketch the argument: Write

$$
1-P_{t}=\left(1-E_{t}\right)+\left(E_{t}-P_{t}\right),
$$

where $E_{t}$ is the dyadic averaging operator; that is, $E_{t} f(x)=f_{Q_{x, t}} f$, where $Q_{x, t}$ is the unique dyadic cube $Q_{x, t} \in \mathbb{D}_{t}$ containing $x$. Writing $g=I_{1} f$, we have that

$$
\begin{aligned}
\mid\left(E_{t}-\right. & \left.P_{t}\right) g(x)|\approx| f_{Q_{x, t}} f_{|x-y|<C t} \varphi\left(\frac{x-y}{t}\right)(g(y)-g(z)) d y d z \mid \\
& \lesssim f_{B(x, C t)} f_{B(x, C t)}|g(y)-g(z)| d y d z \lesssim t f_{B(x, C t)}\left|\nabla_{\|} g(y)\right| d y \leq t \mathcal{M}\left(\nabla_{\|} g\right)(x),
\end{aligned}
$$


where we used Poincare's inequality in the second to last step. Since $I_{1} \nabla_{\|} f=R f$, we have the right bound for this term.

To handle the first term in (2.60), we telescope

$$
\left(1-E_{t}\right) g(x)=\sum_{j=0}^{\infty}\left(E_{2^{-j-1} t}-E_{2^{-j} t}\right) g(x)=: \sum_{j=0}^{\infty}\left(E_{t_{j+1}}-E_{t_{j}}\right) g(x),
$$

and we compute that

$$
\begin{array}{r}
\left|\left(E_{t_{j+1}}-E_{t_{j}}\right) g(x)\right|=\left|f_{Q_{x, t_{j+1}}}\left(E_{t_{j}} g(x)-g(y)\right) d y\right| \lesssim f_{Q_{x, t_{j}}}\left|E_{t_{j}} g(x)-g(y)\right| d y \\
\quad \lesssim t_{j} f_{Q_{x, t_{j}}}\left|\nabla_{\|} g(y)\right| d y \leq 2^{-j} t \mathcal{M}\left(\nabla_{\|} g\right)(x) .
\end{array}
$$

The result now follows by summing over $j$.

We will have need for the following properties of the heat semigroup associated to the Laplacian $\Delta$ in $\mathbb{R}^{n}$.

Proposition 2.4.64. Let $P_{t}:=e^{-t^{2} \Delta}$ and $Q_{t}:=t \partial_{t} P_{t}$. We define the measure

$$
d \mu(x, t):=\frac{\left|Q_{t} v(x)\right|^{2}}{\left|P_{t} v(x)\right|^{2}} P_{t} v(x) \frac{d x d t}{t}
$$

This object satisfies the following properties

(i) For any weight $v \in R H_{s}$ for some $s>1$ it holds that $\left|P_{t} v(x)\right| \lesssim f_{|x-y|<t} v(y) d y$, with constants depending on the $R H_{s}$ and doubling constants of $v$.

(ii) The measure du satisfies the hypotheses of the modified Carleson's Lemma 2.4.44, provided $v \in \mathrm{RH}_{2}$. 
Proof. The proof of (i) is a simple computation: the kernel of $P_{t}$ is given by

$$
\varphi_{t}(x-y)=c_{n} t^{-n} e^{-(|x-y| / 2 t)^{2}}, \quad x, y \in \mathbb{R}^{n}, \quad t>0,
$$

and we can write

$$
P_{t} v(x)=\int_{|x-y|<t} \varphi_{t}(x-y) v(y) d y+\sum_{j \geq 0} \int_{2^{j} t \leq|x-y|<2^{j+1} t} \varphi_{t}(x-y) v(y) d y .
$$

Clearly, the first term satisfies the desired estimate; it remains to control the tail. For this, we set $\Delta_{j}:=\left\{y: 2^{j} t \leq|x-y|<2^{j+1} t\right\}$ and employ Hölder's inequality to obtain

$$
\int_{\Delta_{j}} \varphi_{t}(x-y) f(y) d y \leq\left(\int_{\Delta_{j}} \varphi_{t}(x-y)^{s^{\prime}} d y\right)^{1 / s^{\prime}}\left(\int_{\Delta_{j}} v(y)^{s} d y\right)^{1 / s} .
$$

Now we see, using that $v \in R H_{s}$,

$$
\left(\int_{\Delta_{j}} v^{s}\right)^{1 / s} \lesssim\left(2^{j} t\right)^{n\left(\frac{1}{s}-1\right)} \int_{|x-y|<2^{j+1} t} v(y) d y \lesssim\left(C_{d o u b} 2^{n(1 / s-1)}\right)^{j} t^{\frac{n}{s}} f_{|x-y|<t} v(y) d y .
$$

On the other hand, for $y \in \Delta_{j}$ we have that $\varphi_{t}(x-y) \lesssim t^{-n} \exp \left(-2^{j}\right)$, so that

$$
\left(\int_{\Delta_{j}} \varphi_{t}(x-y)^{s^{\prime}} d y\right)^{1 / s^{\prime}} \lesssim\left|\Delta_{j}\right|^{1 / s^{\prime}} t^{-n} \exp \left(-2^{j}\right) \lesssim t^{-n / s} 2^{j n / s^{\prime}} \exp \left(-2^{j}\right)
$$

Combining these estimates, (i) follows.

The proof of (ii) is somewhat more involved. We ought to show that

$$
\sup _{C_{x, t}}\left(\frac{\left|Q_{s} v(y)\right|^{2}}{\left|P_{s} v(y)\right|^{2}} P_{s} v(y) \frac{1}{s}\right) d x d t \approx\left(\sup _{C_{x, t}} \frac{\left|Q_{s} v(y)\right|^{2}}{\left|P_{s} v(y)\right|^{2}} P_{s} v(y)\right) \frac{d x d t}{t}=: d \tilde{\mu}(x, t)
$$

is a Carleson Measure. For this purpose, first note that, using (i) and the doubling property of $v$, it is not hard to show that $P_{s} v(y) \approx P_{t} v(x)$ for all $(y, s) \in C_{x, t}$. In other words,

$$
d \tilde{\mu}(x, y) \approx\left(\sup _{C_{x, t}}\left|Q_{s} v(y)\right|^{2}\right) \frac{1}{P_{t} v(x)} \frac{d x d t}{t}
$$


Now we let $a>0$ be small enough so that $s^{2}-a^{2} t^{2} \approx t^{2}$ whenever $|t-s|<t / 8$ and write

$$
\begin{aligned}
Q_{s} v(y)=s \partial_{s}\left(e^{\left(a^{2} t^{2}-s^{2}\right) \Delta} e^{-a^{2} t^{2} \Delta} v(y)\right)=-2 s^{2} \Delta e^{\left(a^{2} t^{2}-s^{2}\right) \Delta} e^{-a^{2} t^{2} \Delta} v(y) & \\
& =-\frac{s^{2}}{a^{2} t^{2}} e^{-\left(s^{2}-a^{2} t^{2}\right) \Delta} Q_{a t} v(y) .
\end{aligned}
$$

Therefore, there exists a universal constant $c>0$ such that $\left|Q_{s} v(y)\right| \lesssim P_{c t}\left|Q_{a t} v\right|(y)$, for all $|s-t|<t / 8$. Setting $g_{t}(z):=\left|Q_{a t} v(z)\right|$, we see that

$$
\begin{aligned}
P_{c t} g_{t}(y)= & c_{n} \int_{\mathbb{R}^{n}}(c t)^{-n} e^{-\frac{|y-z|^{2}}{4(c t)^{2}}} g_{t}(z) d z \\
& =\int_{|x-z|<t}(c t)^{-n} e^{-\frac{|y-z|^{2}}{4(c t)^{2}}} g_{t}(z) d z+\sum_{j \geq 0} \int_{\Delta_{j}}(c t)^{-n} e^{-\frac{|y-z|^{2}}{4(c t)^{2}}} g_{t}(z) d z=: I+I I .
\end{aligned}
$$

For $I$, we simply note that

$$
I \lesssim f_{|x-z|<t} g_{t}(z) d z \lesssim P_{t} g_{t}(x) .
$$

For the tails, we use that $|y-z| \geq \frac{7}{8}|x-z|$ for any $z \in \Delta_{j}$, to obtain the bound $I I \lesssim P_{c^{\prime} t} g_{t}(x)$. We conclude that $\left|Q_{s} v(y)\right| \lesssim P_{c t}\left|Q_{a t} v\right|(x)$ for $(y, s) \in C_{x, t}$.

We have thus reduced matters to proving a (weighted) Carleson Measure estimate for $d \mu^{\prime}(x, t):=\frac{\left(P_{c t}\left|Q_{a t} v\right|(x)\right)^{2}}{P_{t} v(x)} \frac{d x d t}{t}$; that is, we want to show that $\mu^{\prime}\left(R_{Q}\right) \lesssim v(Q)$, for all $Q \subset \mathbb{R}^{n}$. So fix a cube $Q \subset \mathbb{R}^{n}$. We run a stopping time argument to obtain a collection of maximal (dyadic) subcubes $\left(Q_{j}\right)_{j \geq 1}$ of $Q$ with respect to the properties

$$
\text { either (a) } f_{Q_{j}} v \geq A f_{Q} v, \quad \text { or } \quad \text { (b) } f_{Q_{j}} v \leq A^{-1} f_{Q} v \text {, }
$$

for some $A>1$ large. We call $\mathcal{F}_{1}$ the collection of $Q_{j}$ satisfying the property (a), and $\mathcal{F}_{2}$ the collection of $Q_{j}$ verifying (b). 
Notice that, by construction, if $Q_{j} \in \mathcal{F}_{1}$ we have that $\left|Q_{j}\right| \leq \frac{|Q|}{A v(Q)} \int_{Q_{j}} v$, so, after summing over $j, \sum_{Q_{j} \in \mathcal{F}_{1}}\left|Q_{j}\right| \leq \frac{|Q|}{A}$. By the $A_{\infty}$ property of $v$, if $A$ is large enough, we may write $\sum_{Q_{j} \in \mathcal{F}_{1}} v\left(Q_{j}\right) \leq \frac{v(Q)}{4}$. On the other hand, if $Q_{j} \in \mathcal{F}_{2}$ we obtain directly that $v\left(Q_{j}\right) \leq A^{-1}\left|Q_{j}\right| f_{Q} v$, so that $\sum_{Q_{j} \in \mathcal{F}_{2}} v\left(Q_{j}\right) \leq \frac{v(Q)}{4}$, if we choose $A>1 / 4$.

By the John-Nirenberg Lemma for Carleson Measures (Lemma 2.4.15) it is enough obtain a bound $\mu^{\prime}\left(R_{Q} \backslash\left(\cup_{\mathcal{F}_{1} \cup \mathcal{F}_{2}} R_{Q_{j}}\right)\right) \lesssim v(Q)$. Moreover, notice that for $(x, t) \in R_{Q} \backslash\left(\cup_{\mathcal{F}_{1} \cup \mathcal{F}_{2}} R_{Q_{j}}\right)$, we have that $f_{Q} v \lesssim P_{t} v(x)$, by construction of the $Q_{j}$. Accordingly, it is enough to show the estimate

$$
\iint_{R_{Q}}\left(P_{c t}\left|Q_{a t} v\right|\right)(x)^{2} \frac{d x d t}{t} \lesssim \frac{v(Q)^{2}}{|Q|}
$$

To do this, we use Minkowski's inequality to write

$$
\left(\iint_{R_{Q}}\left(P_{c t}\left|Q_{a t} v\right|\right)(x)^{2} \frac{d x d t}{t}\right)^{\frac{1}{2}} \leq \sum_{j=0}^{\infty}\left(\iint_{R_{Q}}\left(P_{c t}\left|Q_{a t}\left(\mathbb{1}_{R_{j}(Q)} v\right)\right|\right)(x)^{2} \frac{d x d t}{t}\right)^{\frac{1}{2}}:=\sum_{j=0}^{\infty} T_{j}
$$

where we denote $R_{0}=2 Q$ and $R_{j}=2^{j+1} Q \backslash 2^{j} Q$ for $j \geq 1$. For the first term $T_{0}$, we employ the fact that $P_{t}$ is uniformly $L^{2}\left(\mathbb{R}^{n}\right)$-bounded and that $Q_{t}$ satisfies an $L^{2}$ square function estimate to obtain

$$
T_{0}^{2} \leq \int_{0}^{\infty} \int_{\mathbb{R}^{n}}\left(P_{c t}\left|Q_{a t}\left(\mathbb{1}_{R_{0}} v\right)\right|\right)(x)^{2} \frac{d x d t}{t} \lesssim \int_{0}^{\infty} \int_{\mathbb{R}^{n}}\left|Q_{a t}\left(\mathbb{1}_{R_{0}} v\right)(x)\right|^{2} \frac{d x d t}{t} \lesssim \int_{2 Q} v^{2}
$$

We now use the Reverse Hölder property of $v$ to see that $\int_{2 Q} v^{2} \lesssim|Q|^{-1} v(Q)^{2}$, which gives the desired estimate for $T_{0}$. For the others, we use the kernel representations; first recall that if $\varphi_{t}$ is the kernel for $P_{t}$ and $\psi_{t}$ the one for $Q_{t}$ then there exist constants such that 
$\left|\varphi_{t}(z)\right|,\left|\psi_{t}(z)\right| \leq c_{1} t^{-n} e^{-c_{2}|z|^{2} / t^{2}}$. Calling $v_{j}=\mathbb{1}_{R_{j}} v$, we compute

$$
\begin{aligned}
\int_{Q}\left(P_{c t}\left|Q_{a t} v_{j}\right|\right)(x)^{2} d x=\int_{Q}\left(\int_{\mathbb{R}^{n}}\right. & \left.\left|\int_{\mathbb{R}^{n}} \varphi_{c t}(x-y) \psi_{a t}(y-z) v_{j}(z) d z\right| d y\right)^{2} d x \\
& \lesssim \int_{Q}\left(\int_{R_{j}} v(z) \int_{\mathbb{R}^{n}} t^{-2 n} e^{-c \frac{|x-y|^{2}+|y-z|^{2}}{t^{2}}} d y d z\right)^{2} d x .
\end{aligned}
$$

It is easy to verify that $|x-y|^{2}+|y-z|^{2} \geq\left(|x-y|^{2}+|x-z|^{2}\right) / 4$, and hence

$$
\int_{Q}\left(P_{c t}\left|Q_{a t} v_{j}\right|\right)(x)^{2} d x \lesssim \int_{Q}\left(\int_{R_{j}} v(z) t^{-n} e^{-c \frac{|x-z|^{2}}{t^{2}}} d z\right)^{2} d x \lesssim e^{-c \frac{\left(2^{j} \ell(Q)\right)^{2}}{2 t^{2}}} \int_{Q}\left(\tilde{P}_{t} v\right)^{2}
$$

where we define $\tilde{P}_{t}$ the convolution operator with kernel $t^{-n} e^{-c|z|^{2} /\left(2 t^{2}\right)}$. Now we see, from the proof of part (i), that $\tilde{P}_{t} v(x) \lesssim f_{|x-y|<t} v(y) d y \lesssim v(Q) / t^{n}$. Therefore,

$$
\int_{Q}\left(P_{c t}\left|Q_{a t} v_{j}\right|\right)(x)^{2} d x \lesssim e^{-c^{\prime} \frac{\left(2 e^{j}(Q)\right)^{2}}{t^{2}}} \frac{v(Q)^{2}|Q|}{t^{2 n}} .
$$

The desired estimate for $T_{j}$ now follows by integrating in $t$ over $(0, \ell(Q))$.

We record here also a weighted version of the Riesz transform estimates for $\mathcal{L}_{\|}$and, more importantly for us, estimates for the Hodge decomposition associated to $\mathcal{L}_{\|}$.

Theorem 2.4.80 ( [CMR18, Proposition 9.1]). Let $\mathcal{L}_{\|}:=-\operatorname{div}_{\|} A_{\|} \nabla_{\|}$, then there exist $M>0$ (depending only on dimension and the ellipticity of $A_{\|}$) such that if $v \in A_{2}$ is such that $v^{M} \in A_{2}$, then

$$
\left\|\nabla_{\|} \mathcal{L}_{\|}^{-1 / 2}\right\|_{L^{2}(v) \rightarrow L^{2}(v)},\left\|\mathcal{L}_{\|}^{-1 / 2} \operatorname{div}_{\|}\right\|_{L^{2}(v) \rightarrow L^{2}(v)} \lesssim_{\left[v^{M}\right]_{A_{2}}} 1 .
$$

In particular, if for $f \in L^{2}\left(\mathbb{R}^{n} ; \mathbb{C}^{n}\right)$ we denote $f=A_{\|} \nabla_{\|} F+H$ with $F \in \dot{W}^{1,2}\left(\mathbb{R}^{n}\right)$ and $\operatorname{div}_{\|} H=0$ the Hodge Decomposition of $f$, then for $v$ as above

$$
\left\|\nabla_{\|} F\right\|_{L^{2}(v)} \lesssim_{\left[v^{M}\right]_{A_{2}}}\|f\|_{L^{2}(v)}
$$




\subsection{4 $L^{r}-L^{q}$ Off-diagonal estimates}

Throughout this section we denote by $T_{t}$, with $t \neq 0$, an operator mapping functions $C_{c}^{\infty}\left(\mathbb{R}^{n} ; \mathbb{C}^{d_{1}}\right)$ to measurable functions in $\mathbb{R}^{n}$ with values in $\mathbb{C}^{d_{2}}$ for some integers $d_{1}, d_{2}$.

Definition 2.4.83 ( $L^{r} \rightarrow L^{q}$ Off-diagonal estimates). Let $1 \leq r \leq q \leq \infty$. We say that a family of operators $\left(T_{t}\right)_{t \neq 0}$ satisfies $L^{r}-L^{q}$ off-diagonal estimates if there exist $C_{0}>0$ and numbers $\gamma_{1} \in \mathbb{R}, \gamma_{2}>0$ such that for every cube $Q \subset \mathbb{R}^{n}$, the following estimates hold with $\gamma:=\gamma_{1}+\gamma_{2}$

(i) If $|t| \approx \ell(Q)$, then

$$
\left\|T_{t}\left(f \mathbb{1}_{R_{0}(Q)}\right)\right\|_{L^{q}(Q)} \leq C_{0}|Q|^{1 / q-1 / r}\|f\|_{L^{r}(Q)}
$$

(ii) If $t \in \mathbb{R}$ and we set $R_{j}(Q):=2^{j+1} Q \backslash 2^{j} Q$ for $j \geq 1$, then

$$
\left\|T_{t}\left(f \mathbb{1}_{R_{j}(Q)}\right)\right\|_{L^{q}(Q)} \leq C_{0} 2^{-n j \gamma_{1}}\left(\frac{|t|}{2^{j} \ell(Q)}\right)^{n j \gamma_{2}}|Q|^{1 / q-1 / r}\|f\|_{L^{r}\left(R_{j}(Q)\right)}
$$

(iii) If $t \in \mathbb{R}$ and supp $f \subset Q$ then

$$
\left\|T_{t}(f)\right\|_{L^{q}\left(R_{j}(Q)\right)} \leq C_{0} 2^{-n j \gamma_{1}}\left(\frac{|t|}{2^{j} \ell(Q)}\right)^{n j \gamma_{2}}|Q|^{1 / q-1 / r}\|f\|_{L^{r}(Q)}
$$

Proposition 2.4.86 (Weighted estimates from off-diagonal decay). Suppose $\left(T_{t}\right)_{t>0}$ is sublinear and satisfies $L^{r}-L^{2}$ off diagonal estimates for some $1<r<2$ and $\gamma>1 / r$. Then for all $v \in A_{2 / r}$, and every $t>0$,

$$
\left\|\left(f_{|x-y|<t}\left|T_{t} f(y)\right|^{2} d y\right)^{1 / 2}\right\|_{L^{2}(v d x)} \lesssim_{[v]_{A_{2} / r}}\|f\|_{L^{2}(v)} .
$$


Proof. This proposition is contained within [GH17], but we provide the proof for completeness.

We first decompose $\mathbb{R}^{n}$ into cubes in the dyadic grid $\mathbb{D}_{t}$ of sidelength $\approx t$ to obtain

$$
\begin{aligned}
& \left\|\left(f_{|x-y|<t}\left|T_{t} f(y)\right|^{2} d y\right)^{1 / 2}\right\|_{L^{2}(v d x)}=\left(\sum_{Q \in \mathbb{D}_{t}} \int_{Q} f_{|x-y|<t}\left|T_{t} f(y)\right|^{2} d y v(x) d x\right)^{1 / 2} \\
& \lesssim\left(\sum_{Q \in \mathbb{D}_{t}} f_{Q^{*}} \int_{Q}\left|T_{t} f(y)\right|^{2} d y v(x) d x\right)^{1 / 2} \\
& \quad \lesssim \sum_{j \geq 0}\left(\sum_{Q \in \mathbb{D}_{t}} f_{Q^{*}} \int_{Q}\left|T_{t}\left(\mathbb{1}_{R_{j}(Q)} f(y)\right)\right|^{2} d y v(x) d x\right)^{1 / 2}=: I,
\end{aligned}
$$

where as usual we define $R_{0}(Q):=2 Q$ and $R_{j}(Q):=2^{j+1} Q \backslash 2^{j} Q$ for $j \geq 1$, and we used Minkowski's inequality in the last line. We now exploit the off-diagonal decay of $T_{t}$ to get,

$$
\left(\int_{Q}\left|T_{t}\left(\mathbb{1}_{R_{j}(Q)} f(y)\right)\right|^{2} d y\right)^{1 / 2} \lesssim 2^{-j n \gamma} t^{n(1 / 2-1 / r)}\left(\int_{R_{j}(Q)}|f(y)|^{r} d y\right)^{1 / r} .
$$

Going back to $I$, we see that

$$
\begin{aligned}
& I \lesssim \sum_{j \geq 0} 2^{-j n \gamma} t^{n(1 / 2-1 / r)}\left(\sum_{Q \in \mathbb{D}_{t}} f_{Q^{*}}\left(\int_{R_{j}(Q)}|f(y)|^{r} d y\right)^{2 / r} v(x) d x\right)^{1 / 2} \\
& \lesssim \sum_{j \geq 0} 2^{-j n \gamma} t^{n(1 / 2-1 / r)}\left(2^{j} t\right)^{n / r}\left(\sum_{Q \in \mathbb{D}_{t}} f_{Q^{*}}\left(f_{R_{j}(Q)}|f(y)|^{r} d y\right)^{2 / r} v(x) d x\right)^{1 / 2} \\
& \lesssim \sum_{j \geq 0} 2^{-j n(\gamma-1 / r)} t^{n / 2}\left(\sum_{Q \in \mathbb{D}_{t}} f_{Q^{*}} \mathcal{M}_{r}(f)(x)^{2} v(x) d x\right)^{1 / 2} \\
& \lesssim \sum_{j \geq 0} 2^{-j n(\gamma-1 / r)}\left\|\mathcal{M}_{r}(f)\right\|_{L^{2}(v)} \lesssim\left\|\mathcal{M}_{r}(f)\right\|_{L^{2}(v)},
\end{aligned}
$$

since $\gamma>1 / r$. Since $r<2$ and $A_{2 / r} \subset A_{2}, \mathcal{M}_{r}$ is bounded on $L^{2}(v)$ and so

$$
I \lesssim_{[v]_{A_{2 / r}}}\left\|\mathcal{M}_{r}(f)\right\|_{L^{2}(v)} \lesssim\|f\|_{L^{2}(v)}
$$




\subsubsection{General extrapolation for square functions}

In this section we prove two extrapolation theorems for conical and vertical square functions. The takeaway from these considerations seems to be that conical square functions have good estimates in the range $(r, \infty)$ in the presence of $L^{r}-L^{2}$ off-diagonal estimates plus an $L^{2}$ square function bound. The vertical square function on the other hand requires (for our argument) that the operator satisfies a reverse Hölder inequality (and in fact, in this case we see that the vertical square function is controlled by the conical on an interval around $p=2$; this should be compared with Proposition 2.4.6 which is optimal for general functions $F$, see [AHM12, Proposition 2.1 (c)]).

Lemma 2.4.92 (Extrapolation for Conical Square Functions). Suppose $T_{t}$ is an operator satisfying, for $q=2$ and some $r<2$, the off-diagonal estimates ${ }^{3}$ in Definition 2.4 .83 with $\gamma>1 / r$. (Notice this allows us to define $T_{t} 1$ as an element of $L_{\mathrm{loc}}^{2}$.) Set $R_{t}:=T_{t}-T_{t} 1 \cdot P_{t}$ for a given approximate identity $P_{t}$ with compactly supported kernel of the form $P_{t}=\tilde{P}_{t} \tilde{P}_{t}$ for another approximate identity $\tilde{P}_{t}$. Finally assume that for every $f \in L^{2}\left(\mathbb{R}^{n}\right)$,

$$
\left\|\mathbb{S}\left(T_{t} f\right)\right\|_{L^{2}\left(\mathbb{R}^{n}\right)} \lesssim\|f\|_{L^{2}\left(\mathbb{R}^{n}\right)},
$$

and

$$
\left\|R_{t} Q_{s}^{2} f\right\|_{L^{2}\left(\mathbb{R}^{n}\right)} \lesssim\left(\frac{s}{t}\right)^{\beta}\left\|Q_{s} f\right\|_{L^{2}\left(\mathbb{R}^{n}\right)}, \quad \text { for } s \leq t,
$$

\footnotetext{
${ }^{3}$ In fact we will only need the first and second estimates in Definition 2.4 .83 for $T_{t}$, in the range $|t| \approx \ell(Q)$.
} 
for some (and therefore any) CLP family $Q_{s}$ (see Definition 2.4.13) and some $\beta>0$.

Then

$$
\left\|\mathbb{S}\left(T_{t} f\right)\right\|_{L^{2}(v)} \lesssim\|f\|_{L^{2}(v)}, \quad \text { for each } v \in A_{2 / r} .
$$

In particular,

$$
\left\|\mathbb{S}\left(T_{t} f\right)\right\|_{L^{p}\left(\mathbb{R}^{n}\right)} \lesssim\|f\|_{L^{p}\left(\mathbb{R}^{n}\right)}, \quad \text { for each } p \in(r, \infty)
$$

The above lemma can be thought of as a Calderón-Zygmund-type theorem. In this case the off-diagonal decay plays the role of the usual size condition while the quasiorthogonality estimate for $R_{t}$ plays the role of Hölder continuity of the kernel.

Note also that the case $t \leq s$ in the quasi-orthogonality estimate (2.94) is a consequence of the off-diagonal decay of $R_{t}$ and [AAA ${ }^{+} 11$, Lemma 3.5]. Therefore, with the off-diagonal decay of $R_{t}$ as a background assumption, (2.94) is equivalent to

$$
\left\|R_{t} Q_{s}^{2} f\right\|_{L^{2}\left(\mathbb{R}^{n}\right)} \lesssim \min \left(\frac{t}{s}, \frac{s}{t}\right)^{\beta}\left\|Q_{s} f\right\|_{L^{2}\left(\mathbb{R}^{n}\right)} .
$$

Proof. Let $f \in C_{c}^{\infty}\left(\mathbb{R}^{n}\right)$

We begin by writing

$$
T_{t} f(x)=R_{t} f(x)+\left[T_{t} 1(x)\right] \cdot P_{t} f(x),
$$

where $R_{t}$ and $P_{t}$ are as in the hypotheses. To handle the first term we use interpolation with change of measure (see the proof of Theorem 2.4.34) to reduce the weighted estimate of 
$\mathbb{S}\left(R_{t}\right)$ to the pair of estimates

$$
\left\|\left(f_{|x-y|<t}\left|R_{t} Q_{s}^{2} f(y)\right|^{2} d y\right)^{1 / 2}\right\|_{L^{2}\left(\mathbb{R}^{n}\right)} \lesssim \min \left(\frac{s}{t}, \frac{t}{s}\right)^{\beta}\left\|Q_{s} f\right\| L^{2}\left(\mathbb{R}^{n}\right)
$$

for some $\beta>0$, and

$$
\left\|\left(f_{|x-y|<t}\left|R_{t} Q_{s}^{2} f(y)\right|^{2} d y\right)^{1 / 2}\right\|_{L^{2}(v)} \lesssim_{[v]_{A_{2} / r}}\left\|Q_{s} f\right\|_{L^{2}(v)},
$$

for $r$ as in the statement of the lemma.

The unweighted quasi-orthogonality estimate (2.99) follows from Fubini's Theorem and the good off-diagonal decay.

The uniform weighted estimate follows from Proposition 2.4.86 and the fact that $\left|Q_{s} h(x)\right| \lesssim$ $\mathcal{M} h(x)$ and $\mathcal{M}$ is bounded on $L^{2}(v)$ (because $\left.A_{2 / r} \subset A_{2}\right)$. This shows the desired weighted estimate, and so by interpolation with change of measure,

$$
\left\|\left(f_{|x-y|<t}\left|R_{t} Q_{s}^{2} f(y)\right|^{2} d y\right)^{1 / 2}\right\|_{L^{2}(v)} \lesssim \min \left(\frac{s}{t}, \frac{t}{s}\right)^{\beta}\left\|Q_{s} f\right\|_{L^{2}(v)},
$$

(for a possibly smaller $\beta$ than the one for (2.99)). The estimate

$$
\left\|\mathbb{S}\left(R_{t} f\right)\right\|_{L^{2}(v)} \lesssim\|f\|_{L^{2}(v)}
$$

now follows from a standard quasi-orthogonality argument, once one realizes that if

$$
\tilde{R}_{t} h(x):=\left(f_{|x-y|<t}\left|R_{t} h(y)\right|^{2} d y\right)^{1 / 2}
$$

then $\mathbb{S}\left(R_{t}\right)=\mathbb{V}\left(\tilde{R}_{t}\right)$. 
Now it remains to establish the square function bound for $T_{t} 1(x) \cdot P_{t}$. For this we first claim that the measure

$$
d \mu(x, t):=\left(f_{|x-y|<t}\left|T_{t} 1(y)\right|^{2} d y\right) d v(x) \frac{d x d t}{t}
$$

is a $v$-Carleson measure, i.e. for every cube $Q$

$$
\mu\left(R_{Q}\right) \lesssim v(Q), \quad R_{Q}:=Q \times(0, \ell(Q)) .
$$

Assuming the claim we would have, by a weighted version of Carleson's lemma (Lemma 2.4.43), and using the fact that $P_{t}=\tilde{P}_{t} \tilde{P}_{t}$ implies $\left|P_{t} f(y)\right| \lesssim \tilde{P}_{t}(\mathcal{M} f)(x)$ whenever $|x-y|<t$,

$$
\begin{aligned}
\int_{\mathbb{R}^{n}} \iint_{\Gamma(x)}\left|\left(T_{t} 1(y)\right)\right|^{2}\left|P_{t} f(y)\right|^{2} \frac{d y d t}{t^{n+1}} v(x) d x & \lesssim \int_{\mathbb{R}^{n}} \int_{0}^{\infty}\left|\tilde{P}_{t}(\mathcal{M} f)(x)\right|^{2} d \mu(x, t) \\
& \lesssim \int_{\mathbb{R}^{n}} \mathcal{N}\left(\tilde{P}_{t}(\mathcal{M} f)\right)(x)^{2} v(x) d x \\
& \lesssim \int_{\mathbb{R}^{n}} \mathcal{M}(\mathcal{M} f)(x)^{2} v(x) d x \\
& \lesssim \int_{\mathbb{R}^{n}}|f(x)|^{2} v(x) d x
\end{aligned}
$$

where we used the fact that $\mathcal{M}: L^{2}(v) \rightarrow L^{2}(v)$ since $r>1$. This accounts for the contribution of the second term in (2.98), using Theorem 2.4.34.

To prove the claim we invoke a version of the John-Nirenberg lemma for Carleson measures (see Lemma 2.4.17) and the reverse Hölder inequality for $A_{2 / r}$ weights (see Proposition 2.4.30) in the following way: For a fixed cube $Q \subset \mathbb{R}^{n}$, using Hölder's inequality 


$$
\begin{aligned}
\mu\left(R_{Q}\right) & =\int_{Q} \int_{0}^{\ell(Q)} f_{|x-y|<t<\ell(Q)}\left|T_{t} 1(y)\right|^{2} \frac{d y d t}{t} v(x) d x \\
& =\int_{Q}\left(\iint_{|x-y|<t<\ell(Q)}\left|T_{t} 1(y)\right|^{2} \frac{d y d t}{t^{n+1}}\right) v(x) d x \\
& =: \int_{Q} A_{Q}^{2}(x) v(x) d x \\
& \leq\left(\int_{Q} A_{Q}^{2\left(1+\delta_{1}\right)} d x\right)^{1 /\left(1+\delta_{1}\right)}\left(\int_{Q} v^{1+\delta_{2}} d x\right)^{1 /\left(1+\delta_{2}\right)},
\end{aligned}
$$

where $\delta_{1}=1 / \delta_{2}$ and $1+\delta_{2}$ is the exponent corresponding to the reverse Hölder inequality for $v$ so that

$$
\begin{aligned}
\mu\left(R_{Q}\right) & \lesssim\left(\int_{Q} A_{Q}^{2\left(1+\delta_{1}\right)} d x\right)^{1 /\left(1+\delta_{1}\right)}|Q|^{-1+1 /\left(1+\delta_{2}\right)} v(Q) \\
& \lesssim|Q|^{1 /\left(1+\delta_{2}\right)}|Q|^{-1+1 /\left(1+\delta_{1}\right)} v(Q) \\
& =v(Q),
\end{aligned}
$$

where we used the John-Nirenberg Lemma for local square functions (see Lemma 2.4.17) in the second to last line.

We should remark here that the implicit constant depends on $\delta_{1}$ and the constant in the reverse Hölder inequality for $v$, but these in turn depend only on $[v]_{A_{2 / r}}$ (see for instance [Ste93]).

This finishes the proof of the weighted estimate (2.95). The unweighted result now follows from Corollary 2.4.33.

We now proceed to the extrapolation result for vertical square functions. The idea will be the same, which is to reduce matters to a weighted $L^{2}$ estimate. However, notice that before we used crucially the properties of cones in both the weighted estimates for $R_{t}$ and 
the Carleson measure estimate for $T_{t}$ in Lemma 2.4.92. In order to handle this issue we will transform $\mathbb{V}\left(T_{t}\right)$ into $\mathbb{S}\left(\tilde{T}_{t}\right)$ for an appropriate $\tilde{T}$ involving the weight; this makes the analysis more involved than in Lemma 2.4.92.

Lemma 2.4.109 (A General Extrapolation Result for Vertical Square Functions). Let $T_{t}$ be an operator satisfying, for some $r<2<q$ and $\delta \in(0,1)$, the $L^{r}-L^{q}$ off-diagonal estimates in Definition 2.4.83 for some $\gamma>-1 / n+2 / r+\log _{2}\left(C_{\delta}\right) / n$ (here $C_{\delta}$ is as in (viii) of Proposition 2.4.30). We will also require that, for every cube $Q \subset \mathbb{R}^{n}$,

$$
\left(\int_{I(Q)}\left|T_{t} f(x)\right|^{q} d x d t\right)^{1 / q} \lesssim\left(\int_{I\left(Q^{*}\right)}\left|S_{t} f(x)\right|^{2} d x d t\right)^{1 / 2},
$$

where as usual $I(Q)=Q \times(\ell(Q) / 2, \ell(Q))$ and $S_{t}$ is an operator satisfying that $\left\|\mathbb{S}\left(S_{t}\right)\right\|_{L^{2}\left(\mathbb{R}^{n}\right) \rightarrow L^{2}\left(\mathbb{R}^{n}\right)}<$ $\infty$. We refer to (2.110) as the reverse Hölder assumption.

The assumption on $\gamma$ allows us to define $T_{t} 1$ as an element of $L_{\mathrm{loc}}^{2}$, and we set

$$
R_{t} f(x):=\left[T_{t}-T_{t} 1(x) \cdot P_{t}\right](f)(x)
$$

for some approximate identity $P_{t}$ with compactly supported kernel. Suppose further that $R_{t}$ satisfies the quasi-orthogonality estimate

$$
\left\|R_{t} Q_{s}^{2} f\right\|_{L^{2}\left(\mathbb{R}^{n}\right)} \lesssim\left(\frac{s}{t}\right)^{\beta}\|f\|_{L^{2}\left(\mathbb{R}^{n}\right)}, \quad s<t
$$

for all $f \in L^{2}\left(\mathbb{R}^{n}\right)$ and some $\beta>0$, and that $T_{t}$ satisfies the $L^{2}$ square function estimate

$$
\left\|\mathbb{V}\left(T_{t} f\right)\right\|_{L^{2}\left(\mathbb{R}^{n}\right)} \lesssim\|f\|_{L^{2}\left(\mathbb{R}^{n}\right)}
$$


Then, if $v \in R H_{M} \cap A_{1}$ for $M>\max \left(2 r /(r-2),(q / 2)^{\prime}\right)$ and $[v]_{A_{1}} \leq C_{\delta}$, we have that

$$
\left\|\mathbb{V}\left(T_{t} f\right)\right\|_{L^{2}(v)} \lesssim\|f\|_{L^{2}(v)} \cdot
$$

In particular, for any $p \in(2-\delta / M, 2+\delta / M)$, it holds that

$$
\left\|\mathbb{V}\left(T_{t} f\right)\right\|_{L^{p}\left(\mathbb{R}^{n}\right)} \lesssim\|f\|_{L^{p}\left(\mathbb{R}^{n}\right)}
$$

If $T_{t} 1=0$, that is, if $T_{t}=R_{t}$, then we can dispense of the reverse Hölder assumption.

Remark 2.4.116. As will be seen from the proof of the above result, we can weaken the Reverse Hölder condition on $\Theta_{t, m}$ to

$$
\left(\int_{I(Q)}\left|T_{t} f(x)\right|^{\bar{q}} d x d t\right)^{1 / \bar{q}} \lesssim\left(\int_{I\left(Q^{*}\right)}\left|S_{t} f(x)\right|^{\bar{q}} d x d t\right)^{1 / \bar{q}},
$$

for every $r \leq \bar{q} \leq q$, and where the operator $S_{t}$ satisfies $\left\|\mathbb{S}\left(S_{t} f\right)\right\|_{L^{2}\left(\mathbb{R}^{n}\right)} \lesssim\|f\|_{L^{2}\left(\mathbb{R}^{n}\right)}$, and a reverse Hölder inequality (what one should keep in mind here is that, in our intended application, where $T_{t}=t^{m} \partial_{t}^{m} \nabla \mathcal{S}_{t}^{\mathcal{L}}$, we don't have a reverse Hölder inequality for gradients of solutions, but we do have them for solutions, say $S_{t}=t^{m-1} \partial_{t}^{m} \mathcal{S}_{t}^{\mathcal{L}}$ ).

Proof. We first note that, by Proposition 2.4.6, in the range $p<2$ we have that

$$
\left\|\mathbb{V}\left(T_{t} f\right)\right\|_{L^{p}\left(\mathbb{R}^{n}\right)} \lesssim\left\|\mathbb{S}\left(T_{t} f\right)\right\|_{L^{p}\left(\mathbb{R}^{n}\right)}
$$

and for $r<p<2$, by Lemma 2.4 .92 (recall that vertical and conical square functions coincide on $L^{2}$ ) and Corollary 2.4.33, we have

$$
\left\|\mathbb{S}\left(T_{t} f\right)\right\|_{L^{p}\left(\mathbb{R}^{n}\right)} \lesssim\|f\|_{L^{p}\left(\mathbb{R}^{n}\right)}
$$


Therefore, it's enough to consider the case $p>2$.

We now proceed to write our vertical square function into a conical square function by introducing an average adapted to $v$. For this purpose we set, for $x \in \mathbb{R}^{n}$ and $t>0$ fixed,

$$
v_{x, t}:=f_{|x-y|<t} v(y) d y .
$$

We now write, using Fubini's theorem

$$
\begin{aligned}
\int_{\mathbb{R}^{n}}\left|\mathbb{V}\left(T_{t} f\right)(x)\right|^{2} v(x) d x & =\int_{\mathbb{R}^{n}} \int_{0}^{\infty}\left|T_{t} f(x)\right|^{2} \frac{d t}{t} v(x) d x \\
& =\int_{\mathbb{R}^{n}} \int_{0}^{\infty} f_{|x-y|<t} \frac{v(y)}{v_{x, t}}\left|T_{t} f(x)\right|^{2} d y \frac{d t}{t^{n+1}} v(x) d x \\
& =\int_{\mathbb{R}^{n}} \iint_{|x-y|<t} \frac{v(x)}{v_{x, t}}\left|T_{t} f(x)\right|^{2} \frac{d x d t}{t^{n+1}} v(y) d y \\
& =: \int_{\mathbb{R}^{n}} \iint_{|x-y|<t}\left|\tilde{T}_{t} f(x)\right|^{2} \frac{d x d t}{t^{n+1}} v(y) d y \\
& =\left\|\mathbb{S}\left(\tilde{T}_{t} f\right)\right\|_{L^{2}(v)} .
\end{aligned}
$$

We are now in a position to try and mimic the proof of Lemma 2.4.92. Unfortunately the process is quite a bit more involved and, rather than proving a full weighted estimate, we will use the specific form of our weight $v$. To simplify notation we introduce the operators:

$$
\tilde{R}_{t} f(x)=\sqrt{\frac{v(x)}{v_{x, t}}} R_{t}
$$

where $R_{t}$ is as in the statement of the Lemma. With this in hand we write

$$
\tilde{T}_{t} f(x)=\tilde{R}_{t} f(x)+\tilde{T}_{t} 1(x) \cdot P_{t} f(x) .
$$


As was done in the case of the conical square function, to handle the second term it's enough to show the $v$-Carleson measure estimate (see Lemma 2.4.43)

$$
\mu\left(R_{Q}\right) \lesssim v(Q),
$$

for every cube $Q \subset \mathbb{R}^{n}$, where $R_{Q}:=Q \times(0, \ell(Q))$ and the measure $\mu$ is defined as

$$
d \mu(x, t):=\left(f_{|x-y|<t}\left|\tilde{T}_{t} 1(y)\right|^{2} d x\right) v(x) \frac{d x d t}{t}
$$

For this we first reduce matters to an unweighted estimate via the John-Nirenberg lemma for local square functions (see Lemma 2.4.17) as follows: Notice that, for any $\bar{q}>1$,

$$
\begin{aligned}
& \mu\left(R_{Q}\right)=\int_{Q}\left(\iint_{|x-y|<t<\ell(Q)}\left|\tilde{T}_{t} 1(x)\right|^{2} \frac{d x d t}{t^{n+1}}\right) v(y) d y \\
& =: \int_{Q} A_{Q}^{2}(y) v(y) d y \\
& \leq\left(\int_{Q} A_{Q}^{2 \bar{q}} d y\right)^{1 / \bar{q}}\left(\int_{Q} v(y)^{\bar{q}^{\prime}} d y\right)^{1 / \bar{q}^{\prime}}
\end{aligned}
$$

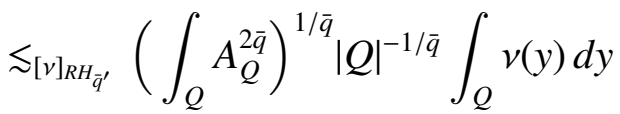

$$
\begin{aligned}
& =\left(f_{Q} A_{Q}^{2 \bar{q}} d y\right)^{1 / \bar{q}} v(Q),
\end{aligned}
$$

where as before the quantity $[v]_{R H_{\bar{q}^{\prime}}}$ is admissible if say $M>\bar{q}^{\prime}$ (see Proposition 2.4.30). Therefore it is enough to show that

$$
\left(f_{Q} A_{Q}^{2 \bar{q}} d y\right)^{1 / \bar{q}} \lesssim 1 .
$$

By the John-Nirenberg lemma for local square functions (see Lemma 2.4.17), it's enough to show

$$
f_{Q} A_{Q}^{2} d y \lesssim 1
$$


Using Fubini's theorem, we see that this last estimate is equivalent to the unweighted Carleson measure estimate

$$
\int_{0}^{\ell\left(Q^{*}\right)} \int_{Q^{*}}\left|\tilde{T}_{t} 1(x)\right|^{2} \frac{d x d t}{t} \lesssim\left|Q^{*}\right|
$$

where as before $Q^{*}=c_{n} Q$ is a dilate of $Q$. Since the above has to hold for every cube, we write $Q$ in place of $Q^{*}$ in what follows. Moreover, since the quantity $v / v_{x, t}$ is invariant under scalar multiplication of $v$ by a positive constant, for a fixed cube $Q$ we may assume that $v(Q) /|Q|=1$.

First we use a stopping time argument to deal with $v_{x, t}$ : For a fixed constant $A^{-1}<1 / 4$, to be selected later, we let $\left\{Q_{j}\right\}_{j \in \mathfrak{N}}$ be the collection of maximal dyadic sub-cubes of $Q$ with respect to the conditions

$$
f_{Q_{j}} v(x) d x>A, \quad \text { or } \quad f_{Q_{j}} v(x) d x<A^{-1}
$$

We say $j \in I_{1}$ if the first condition holds, and $j \in I_{2}$ if the second does.

By the first condition we have

$$
\sum_{j \in I_{1}}\left|Q_{j}\right|<\sum_{j \in I_{1}} A^{-1} \int_{Q_{j}} v(x) d x \leq A^{-1} \int_{Q} v(x) d x=A^{-1}|Q|,
$$

by our condition $v(Q) /|Q|=1$. On the other hand if $j \in I_{2}$

$$
\int_{Q_{j}} v(x) d x<A^{-1}\left|Q_{j}\right|, \quad \text { and } \quad \int_{Q_{j}^{*}} v(x) d x \geq A^{-1}\left|Q_{j}^{*}\right|,
$$

where $Q_{j}^{*}$ is the dyadic parent of $Q_{j}$. Therefore

$$
\sum_{j \in I_{2}} v\left(Q_{j}\right) \leq \sum_{j \in I_{2}} A^{-1}\left|Q_{j}\right| \leq A^{-1}|Q|=A^{-1} v(Q) .
$$


By the $A_{\infty}$ property of $v$ we can choose $A$, depending only on the $A_{1}$ characteristic of $v$, small enough such that the above inequality implies

$$
\left|\cup_{j \in I_{2}} Q_{j}\right|<\frac{1}{2}|Q|
$$

Combining this with the corresponding estimate for $I_{1}$, and using the fact that the cubes $Q_{j}$ are pairwise disjoint,

$$
\sum_{j \geq 0}\left|Q_{j}\right|<B|Q|
$$

for $B=1 / 2+A^{-1}<1$. By the John-Nirenberg lemma for Carleson measures (see Lemma 2.4.15), the above implies that it's enough to show the estimate

$$
\iint_{E_{Q}} \frac{v(x)}{v_{x, t}}\left|T_{t} 1(x)\right|^{2} d x \frac{d x d t}{t} \leq C_{0}|Q|
$$

where we define the sawtooth region

$$
E_{Q}:=R_{Q} \backslash\left(\cup_{j \geq 0} R_{Q_{j}}\right)
$$

To handle (2.136) we first claim the following:

$$
v_{x, t} \gtrsim 1, \quad \forall(x, t) \in E_{Q},
$$

with implicit constants depending only on the doubling constant of $v$. To see this fix $(x, t) \in$ $E_{Q}$ and consider first the case $x \notin \cup_{j \geq 0} Q_{j}$ so that

$$
A^{-1} \leq f_{Q^{\prime}} v(y) d y \leq A,
$$


for any dyadic subcube $Q^{\prime} \in \mathbb{D}(Q)$ containing $x$. In particular, choosing $Q_{t}^{\prime} \in \mathbb{D}_{t}(Q)$ with this property, and using the doubling property of $v$ we see

$$
A^{-1} \leq f_{Q_{t}^{\prime}} v(y) d y \approx f_{|x-y|<t} v(y) d y=v_{x, t} .
$$

On the other hand if $x \in Q_{j}$ for some $j \geq 0$ we proceed as follows: If $t>4 \ell\left(Q_{j}\right)$, it means that, if as before $Q_{t}^{\prime} \in \mathbb{D}_{t}(Q)$ is the unique dyadic subcube of $Q$ containing $x$, then $Q_{t}^{\prime}$ is not in the collection $\left\{Q_{j}\right\}_{j}$ so by definition

$$
A^{-1} \leq f_{Q_{t}^{\prime}} v(y) d y \leq A
$$

and we conclude as before since this average is comparable, by doubling of $v$, to $v_{x, t}$. If $\ell\left(Q_{j}\right) \leq t \leq 4 \ell\left(Q_{j}\right)$ (the first inequality owing to the definition of $\left.E_{Q}\right)$ then by definition the dyadic parent $\tilde{Q}_{j}$ of $Q_{j}$ satisfies

$$
A^{-1} \leq f_{\tilde{Q}_{j}} v(y) d y \leq A
$$

so that, again by doubling of $v$, the claim follows.

We conclude, using (2.136) and (2.138), that it's enough to establish (recall $v(Q)=|Q|$ )

$$
\iint_{E_{Q}}\left|T_{t} 1(x)\right|^{2} v(x) \frac{d x d t}{t} \leq C_{0}|Q| .
$$

To show this we first fix $\psi=\psi_{Q} \in C_{c}^{\infty}(4 Q)$ with the property that $\psi \equiv 1$ in $2 Q$ and 
$0 \leq \psi \leq 1$, so that

$$
\begin{aligned}
\iint_{E_{Q}}\left|T_{t} 1(x)\right|^{2} v(x) \frac{d x d t}{t} \lesssim & \iint_{E_{Q}}\left|T_{t} \psi(x)\right|^{2} v(x) \frac{d x d t}{t} \\
& +\iint_{E_{Q}}\left|T_{t}(1-\psi)(x)\right|^{2} v(x) \frac{d x d t}{t} \\
= & I I+I I I .
\end{aligned}
$$

We first handle III: Using Hölder's inequality, with $q / 2>1$ as in the hypotheses, we recall that we have chosen $M>(q / 2)^{\prime}$ so that $v \in R H_{(q / 2)^{\prime}}$ (see Proposition 2.4.30),

$$
\begin{aligned}
I I I & \leq \int_{0}^{\ell(Q)} \int_{Q}\left|T_{t}(1-\psi)\right|^{2} v(x) \frac{d x d t}{t} \\
& \leq \int_{0}^{\ell(Q)}\left(\int_{Q} v^{(q / 2)^{\prime}}(x) d x\right)^{1 /(q / 2)^{\prime}}\left(\int_{Q}\left|T_{t}(1-\psi)\right|^{q} d x\right)^{2 / q} \frac{d t}{t} \\
& \lesssim \int_{0}^{\ell(Q)}|Q|^{2 / q} v(Q)\left(\int_{Q}\left|T_{t}(1-\psi)\right|^{q} d x\right)^{2 / q} \frac{d t}{t} \\
& =\int_{0}^{\ell(Q)}|Q|^{1-2 / q}\left(\int_{Q}\left|T_{t}(1-\psi)\right|^{q} d x\right)^{2 / q} \frac{d t}{t}
\end{aligned}
$$

where we used the normalization $v(Q) /|Q|=1$ in the last line. Now, since $T_{t}$ satisfies $L^{2}-L^{q}$ off-diagonal estimates (see Definition 2.4.83), using as usual $R_{j}=R_{j}(Q)=2^{j+1} Q \backslash 2^{j} Q$ for $j \geq 1$ and recalling that $1-\psi \equiv 1$ outside of $4 Q$,

$$
\begin{aligned}
\left(\int_{Q}\left|T_{t}(1-\psi)\right|^{q} d x\right)^{1 / q} & \leq \sum_{j \geq 1}\left(\int_{Q}\left|T_{t}\left[(1-\psi) \mathbb{1}_{R_{j}}\right]\right|^{q} d x\right)^{1 / q} \\
& \leq \sum_{j \geq 1} 2^{-n j \gamma_{1}}\left(\frac{t}{\left(2^{j} \ell(Q)\right)}\right)^{n \gamma_{2}} \ell(Q)^{n(1 / q-1 / 2)}\left(\int_{R_{j}}|1-\psi|^{2} d x\right)^{1 / 2} \\
& \lesssim \sum_{j \geq 1} 2^{-n j \gamma}\left(\frac{t}{\ell(Q)}\right)^{n \gamma_{2}} \ell(Q)^{n(1 / q-1 / 2)}\left|R_{j}\right|^{1 / 2} \\
& \lesssim \sum_{j \geq 1} 2^{-n j(\gamma-1 / 2)}\left(\frac{t}{\ell(Q)}\right)^{n \gamma_{2}}|Q|^{1 / q} \\
& \lesssim\left(\frac{t}{\ell(Q)}\right)^{n \gamma_{2}}|Q|^{1 / q}
\end{aligned}
$$


since $\gamma>1 / 2$. Plugging this into the estimate for $I I I$ above we see, since $\gamma_{2}>0$,

$$
I I I \lesssim \int_{0}^{\ell(Q)}|Q|\left(\frac{t}{\ell(Q)}\right)^{2 n \gamma_{2}} \frac{d t}{t} \lesssim|Q| .
$$

This is the desired estimate for III.

To handle $I I$ we first define, for $Q^{\prime} \in \mathbb{D}(Q)$,

$$
I\left(Q^{\prime}\right):=\left\{(x, t) \in R_{Q}: x \in Q^{\prime}, \ell\left(Q^{\prime}\right) / 2<t \leq \ell\left(Q^{\prime}\right)\right\}
$$

the Whitney region in $\mathbb{R}_{+}^{n+1}$ associated to $Q^{\prime}$. We see that

$$
I I=\sum_{\substack{Q^{\prime} \in \mathbb{D}(Q) \\ Q^{\prime} \cap Q_{j}=\emptyset, \forall j}} \iint_{I\left(Q^{\prime}\right)}\left|T_{t} \psi(x)\right|^{2} v(x) \frac{d x d t}{t}
$$

We now use Hölder's Inequality with $q>2$ so that the $L^{q}$ reverse Hölder inequality for $T_{t}$ holds, again noting that we have chosen $M$ large enough to guarantee $v \in R H_{(q / 2)^{\prime}}$, to conclude

$$
\begin{aligned}
I I & \leq \sum_{\substack{Q^{\prime} \in \mathbb{D}(Q) \\
Q^{\prime} \not \subset Q_{j}, \forall j}}\left(\iint_{I\left(Q^{\prime}\right)}\left|T_{t} \psi\right|^{q} \frac{d x d t}{t}\right)^{2 / q}\left(\iint_{I\left(Q^{\prime}\right)} v^{(q / 2)^{\prime}} \frac{d x d t}{t}\right)^{1 /(q / 2)^{\prime}} \\
& \lesssim \sum_{\substack{Q^{\prime} \in \mathbb{D}(Q) \\
Q^{\prime} \not \subset Q_{j}, \forall j}}\left|Q^{\prime}\right|^{1 /(q / 2)^{\prime}}\left(\iint_{I\left(Q^{\prime}\right)}\left|T_{t} \psi\right|^{q} \frac{d x d t}{t}\right)^{2 / q},
\end{aligned}
$$

where we used that for $Q^{\prime}$ satisfying $Q^{\prime} \not \subset Q_{j}$ for all $j$ (i.e. for $Q^{\prime}$ not contained in any of the $Q_{j}$ ) we have, by construction of the $Q_{j}$,

$$
f_{Q^{\prime}} v(x) d x \approx 1 .
$$


We now use reverse Hölder assumption on $T_{t}$ to obtain

$$
\left(\int_{I\left(Q^{\prime}\right)}\left|T_{t} \psi\right|^{q} \frac{d x d t}{t}\right)^{2 / q} \lesssim \iint_{I\left(\left[Q^{\prime}\right]^{*}\right)}\left|S_{t} \psi\right|^{2} \frac{d x d t}{t},
$$

Therefore, using this in the estimate for $I I$,

$$
\begin{aligned}
I I & \lesssim \sum_{\substack{Q^{\prime} \in \mathbb{D}(Q) \\
Q^{\prime} \not Q_{j}, \forall j}}\left|Q^{\prime}\right|^{1 / p^{\prime}}\left|Q^{\prime}\right|^{1 / p} \iint_{I\left(\left[Q^{\prime}\right]^{*}\right)}\left|S_{t} \psi\right|^{2} d x d t \\
& \lesssim \sum_{\substack{Q^{\prime} \in \mathbb{D}(Q) \\
Q^{\prime} \not \subset Q_{j}, \forall j}} \iint_{I\left(\left[Q^{\prime}\right]^{*}\right)}\left|S_{t} \psi\right|^{2} \frac{d x d t}{t} \\
& \lesssim \iint_{R_{Q^{*}}}\left|S_{t} \psi\right|^{2} \frac{d x d t}{t} .
\end{aligned}
$$

We conclude from the fact that $T_{t}$ satisfies an $L^{2}\left(\mathbb{R}^{n}\right)$ square function estimate and $\|\psi\|_{L^{2}\left(\mathbb{R}^{n}\right)} \lesssim$ $|Q|^{1 / 2}$ by construction.

Combining the estimates for $I I$ and $I I I,(2.143)$ follows and thus, by our previous reductions, we have shown

$$
\left\|\mathbb{S}\left(T_{t} 1 \cdot P_{t} f\right)\right\|_{L^{2}(v)} \lesssim\|f\|_{L^{2}(v)} .
$$

It remains to handle the contribution of $\tilde{R}_{t}$. Notice that so far, we've only required that $v \in A_{2 / r}$ and $\gamma>1 / r$. The extra assumptions will be needed in order to handle $\tilde{R}_{t}$.

Again as in the proof of Lemma 2.4.92 we will appeal to interpolation with change of measure (see Theorem 2.4.34). For this it's enough to prove the following pair of estimates:

$$
\left\|\left(f_{|x-y|<t}\left|\tilde{R}_{t} Q_{s}^{2} f(y)\right|^{2} d y\right)^{1 / 2}\right\|_{L^{2}\left(\mathbb{R}^{n}\right)} \lesssim_{\left[v^{M}\right]_{A_{1}}} \min \left(\frac{t}{s}, \frac{s}{t}\right)^{\beta}\left\|Q_{s} f\right\|_{L^{2}\left(\mathbb{R}^{n}\right)}
$$


valid for some (and therefore all) Littlewood-Paley family $\left(Q_{s}\right)_{s}$ and some $\beta>0$; and

$$
\left\|\left(f_{|x-y|<t}\left|\tilde{R}_{t} Q_{s}^{2} f(y)\right|^{2} d y\right)^{1 / 2}\right\|_{L^{2}(v)} \lesssim_{\left[v^{M}\right]_{A_{1}}}\left\|Q_{s} f\right\|_{L^{2}(v)} .
$$

We remark that in the first quasi-orthogonality estimate (2.155), even though the estimate itself is unweighted, $\tilde{R}_{t}$ still has a dependence on $v$.

The uniform $L^{2}(v)$ estimate is handled the same way it was done for the conical; setting $h:=Q_{s}^{2} f$ we see

$$
\begin{aligned}
\left(\int_{\mathbb{R}^{n}} f_{|x-y|<t}\left|\tilde{R}_{t} h(y)\right|^{2} d y v(x) d x\right)^{1 / 2} & \lesssim\left(\sum_{Q \in \mathbb{D}_{t}} \int_{Q} \int_{|x-y|<t}\left|\tilde{R}_{t} h(y)\right|^{2} d y v(x) d x\right)^{1 / 2} \\
& \lesssim\left(\sum_{Q \in \mathbb{D}_{t}} f_{Q^{*}} \int_{Q} \frac{v(y)}{v_{y, t}}\left|R_{t} h(y)\right|^{2} d y v(x) d x\right)^{1 / 2} .
\end{aligned}
$$

By Hölder's Inequality with exponent $q / 2>1$ and $M>(2 / q)^{\prime}$, we see

$$
\begin{aligned}
\int_{Q} \frac{v(y)}{v_{y, t}}\left|R_{t} h(y)\right|^{2} d y v(x) d x & \leq\left(\int_{Q}\left|\frac{v(y)}{v_{y, t}}\right|^{(q / 2)^{\prime}} d y\right)^{1 /(q / 2)^{\prime}}\left(\int_{Q}\left|R_{t} h(y)\right|^{q} d y\right)^{2 / q} \\
& \lesssim_{\left[v^{M}\right]_{A_{1}}}|Q|^{1-2 / q}\left(\int_{Q}\left|R_{t} h(y)\right|^{q} d y\right)^{2 / q} .
\end{aligned}
$$

Plugging this into the first estimate, we can now proceed as in the conical case (see Lemma 2.4.92), exploiting the $L^{r}-L^{q}$ off-diagonal decay in place of the $L^{r}-L^{2}$.

For the quasi-orthogonality estimate we proceed as follows: For this we will exploit the off-diagonal decay that $\tilde{R}_{t}$ inherits from $R_{t}$. More explicitly we have, for fixed $t, s>0$ using 
Fubini's Theorem and duality

$$
\begin{aligned}
\int_{\mathbb{R}^{n}} f_{|x-y|<t}\left|\tilde{R}_{t} Q_{s}^{2} f(x)\right|^{2} d x d y & =\int_{\mathbb{R}^{n}} f_{|x-y|<t} \frac{v(x)}{v_{x, t}}\left|R_{t} Q_{s}^{2} f(x)\right|^{2} d x d y \\
& =\int_{\mathbb{R}^{n}} \frac{v(x)}{v_{x, t}}\left|R_{t} Q_{s}^{2} f(x)\right|^{2} d x \\
& =\int_{\mathbb{R}^{n}} \frac{v(x)}{v_{x, t}} R_{t} Q_{s}^{2} f(x) \cdot \overline{R_{t} Q_{s}^{2} f(x)} d x d y \\
& =\int_{\mathbb{R}^{n}} R_{t}^{*}\left(\frac{v(x)}{v_{x, t}} R_{t} Q_{s}^{2} f\right)(x) \cdot \overline{Q_{s}^{2} f(x)} d x \\
& \leq \| R_{t}^{*}\left(\left(v(x) / v_{x, t} R_{t} Q_{s}^{2} f\right)\left\|_{L^{2}\left(\mathbb{R}^{n}\right)}\right\| Q_{s}^{2} f \|_{L^{2}\left(\mathbb{R}^{n}\right)},\right.
\end{aligned}
$$

where $R_{t}^{*}$ is the adjoint of $R_{t}$, for fixed $t>0$, in $L^{2}\left(\mathbb{R}^{n}\right)$. Since $\left\|Q_{s}^{2} f\right\|_{L^{2}\left(\mathbb{R}^{n}\right)} \lesssim\left\|Q_{s} f\right\|_{L^{2}\left(\mathbb{R}^{n}\right)}$, we have reduced matters to showing

$$
\int_{\mathbb{R}^{n}}\left|R_{t}^{*}\left(\frac{v(x)}{v_{x, t}} R_{t} Q_{s}^{2} f\right)(x)\right|^{2} d x \lesssim \min \left(\frac{s}{t}, \frac{t}{s}\right)^{\alpha} \int_{\mathbb{R}^{n}}\left|Q_{s} f(x)\right|^{2} d x,
$$

for some $\alpha>0$. To save space we denote by $I$ the left-hand-side of this last inequality. Recall that we denote by $\mathbb{D}_{t}$ the collection of dyadic cubes of scale $2^{-k}$ where $t / 2<2^{-k} \leq t$. We compute, denoting by $Q^{*}=c_{n} Q$ for any cube $Q \subset \mathbb{R}^{n}$ where $c_{n}$ is a dimensional constant,

$$
\begin{aligned}
I^{1 / 2} & =\left(\sum_{Q \in \mathbb{D}_{t}} \int_{Q}\left|R_{t}^{*}\left(\frac{v(x)}{v_{x, t}} R_{t} Q_{s} f\right)\right|^{2} d x\right)^{1 / 2} \\
& =\left(\sum_{Q \in \mathbb{D}_{t}} \int_{Q} f_{|x-y|<t}\left|R_{t}^{*}\left(\frac{v(x)}{v_{x, t}} R_{t} Q_{s} f\right)\right|^{2} d y d x\right)^{1 / 2} \\
& \lesssim\left(\sum_{Q \in \mathbb{D}_{t}} f_{Q^{*}} \int_{Q}\left|R_{t}^{*}\left(\frac{v(x)}{v_{x, t}} R_{t} Q_{s} f\right)\right|^{2} d x d y\right)^{1 / 2} \\
& \lesssim \sum_{j \geq 0}\left(\sum_{Q \in \mathbb{D}_{t}} f_{Q^{*}} \int_{Q}\left|R_{t}^{*}\left(\mathbb{1}_{R_{j}(Q)} \frac{v(x)}{v_{x, t}} R_{t} Q_{s} f\right)\right|^{2} d x d y\right)^{1 / 2},
\end{aligned}
$$

where we define $R_{0}(Q):=2 Q$ and for $j \geq 1, R_{j}(Q):=2^{j+1} Q \backslash 2^{j} Q$ and we used the triangle inequality in the last line, together with the $L^{2}\left(\mathbb{R}^{n}\right)$-boundedness of $R_{t}^{*}$. We now use the 
off-diagonal decay for $R_{t}^{*}$ to write,

$$
\begin{aligned}
& \int_{Q} \mid R_{t}^{*}\left(\mathbb{1}_{\left.R_{j}(Q) \frac{v(x)}{v_{x, t}} R_{t} Q_{s} f\right)\left.\right|^{2} d x \leq} 2^{-n j \gamma}|Q|^{1-2 / r}\right. \\
& \times\left(\int_{R_{j}(Q)}\left|\frac{v(x)}{v_{x, t}} R_{t} Q_{s}^{2} f(x)\right|^{r} d x\right)^{2 / r} \\
& \approx 2^{-n j(\gamma-2 / r)}|Q|\left(f_{R_{j}(Q)}\left|\frac{v(x)}{v_{x, t}} R_{t} Q_{s}^{2} f(x)\right|^{r} d x\right)^{2 / r} \\
& \leq 2^{-n j(\gamma-2 / r)}|Q|\left(f_{R_{j}(Q)}\left|R_{t} Q_{s}^{2} f(x)\right|^{2} d x\right) \\
& \times\left(f_{R_{j}(Q)}\left|\frac{v(x)}{v_{x, t}}\right|^{\tilde{r}} d x\right)^{2 / \tilde{r}},
\end{aligned}
$$

where $\tilde{r}^{-1}=1 / r-1 / 2$ by Hölder's inequality. Plugging this estimate into (2.161), we see that

$$
I^{1 / 2} \lesssim \sum_{j \geq 0}\left(\sum_{Q \in \mathbb{D}_{t}} f_{Q^{*}} C_{j}^{2}|Q|\left(f_{R_{j}(Q)}\left|R_{t} Q_{s}^{2} f(x)\right|^{2} d x\right)\left(f_{R_{j}(Q)}\left|\frac{v(x)}{v_{x, t}}\right|^{\tilde{r}} d x\right)^{2 / \tilde{r}} d y\right)^{1 / 2},
$$

where we have defined $C_{j}:=2^{-n j(\gamma-2 / r)}$. Since $M>\tilde{r}$, so that $v \in R H_{\tilde{r}}$ (see Proposition 2.4.30). Moreover, using the doubling property of $v$ and denoting $C_{d o u b}$ to be the doubling constant, we have

$$
v_{x, t} \geq 2^{j} C_{d o u b}^{-j} v_{x, 2^{j} t} \approx C_{d o u b}^{-j} 2^{j} v\left(Q_{j}^{\prime}\right)
$$

where $Q^{\prime}$ is any cube with $\ell\left(Q_{j}^{\prime}\right) \approx 2^{j} t$ containing $x$. Therefore decomposing $R_{j}(Q)$ into 
$N=N(n)$ cubes $Q^{\prime}$ of sidelenght $2^{j} t$ we compute

$$
\begin{aligned}
\left(f_{R_{j}(Q)}\left|\frac{v(x)}{v_{x, t}}\right|^{\tilde{r}} d x\right)^{1 / \tilde{r}} & \lesssim\left(\sum_{Q^{\prime}} f_{Q^{\prime}}\left|\frac{v(x)}{v_{x, t}}\right|^{\tilde{r}} d x\right)^{1 / \tilde{r}} \\
& \lesssim_{\left[v^{M}\right]_{A_{2}}}\left(\sum_{Q^{\prime}}\left(f_{Q^{\prime}} \frac{v(x)}{v_{x, t}} d x\right)^{\tilde{r}}\right)^{1 / \tilde{r}} \\
& \lesssim C_{d o u b}^{j} 2^{-j}\left(\sum_{Q^{\prime}}\left(\frac{v\left(Q^{\prime}\right)\left|Q^{\prime}\right|}{\nu\left(Q^{\prime}\right)\left|Q^{\prime}\right|}\right)^{\tilde{r}}\right)^{1 / \tilde{r}} \\
& \lesssim C_{\text {doub }}^{j} 2^{-j},
\end{aligned}
$$

where we used (2.164) in the second to last line. In what follows, we absorb this constant into $C_{j}$, now writing $\widetilde{C}_{j}:=2^{-n j\left(\gamma+1 / n-2 / r-\log _{2}\left(C_{d o u b}\right) / n\right)}$.

Plugging this into the estimate for I, appearing in (2.163), and using Fubini's Theorem,

$$
\begin{aligned}
I^{1 / 2} & \lesssim \sum_{j \geq 0}\left(\sum_{Q \in \mathbb{D}_{t}} f_{Q^{*}} \widetilde{C}_{j}^{2}|Q| f_{R_{j}(Q)}\left|R_{t} Q_{s}^{2} f(x)\right|^{2} d x d y\right)^{1 / 2} \\
& \approx \sum_{j \geq 0}\left(\sum_{Q \in \mathbb{D}_{t}} \int_{Q^{*}} \widetilde{C}_{j}^{2} f_{|x-y|<2^{k+1} t}\left|R_{t} Q_{s}^{2} f(x)\right|^{2} d x d y\right)^{1 / 2} \\
& \approx \sum_{j \geq 0}\left(\widetilde{C}_{j}^{2} \int_{\mathbb{R}^{n}} f_{|x-y|<2^{(k+1) t} t}\left|R_{t} Q_{s}^{2} f(x)\right|^{2} d x d y\right)^{1 / 2} \\
& \lesssim\left(\int_{\mathbb{R}^{n}}\left|R_{t} Q_{s}^{2} f(x)\right|^{2} d x\right)^{1 / 2}\left(\sum_{j \geq 0} \widetilde{C}_{j}^{2}\right)^{1 / 2} \\
& \lesssim\left(\int_{\mathbb{R}^{n}}\left|R_{t} Q_{s}^{2} f(x)\right|^{2} d x\right)^{1 / 2},
\end{aligned}
$$

where in the last step we used that $C_{d o u b} \lesssim_{n}[v]_{A_{1}} \leq C_{\delta}$. This gives the desired estimate (2.160), since we have good quasi-orthogonality estimates for this object (see the proof of Theorem 5.1.10).

This concludes the proof. 


\subsection{Elliptic theory estimates}

In this section, we establish several estimates for the operators under consideration, which are 'standard' in the elliptic theory. We begin with Caccioppoli-type estimates.

\subsubsection{Caccioppoli-type inequalities}

Let us first show

Proposition 2.5.1 (Caccioppoli inequality). Let $\Omega \subset \mathbb{R}^{n+1}$ be an open set. Suppose that $u \in W_{\mathrm{loc}}^{1,2}(\Omega), f \in L_{\mathrm{loc}}^{2}(\Omega), \vec{F} \in L_{\mathrm{loc}}^{2}(\Omega)^{n+1}$, and that $\mathcal{L} u=f-\operatorname{div} \vec{F}$ in $\Omega$ in the weak sense. Then, for every ball $B \subset 2 B \subset \Omega$, the estimate

$$
\iint_{B}|\nabla u|^{2} \lesssim \iint_{2 B}\left(\frac{1}{r(B)^{2}}|u|^{2}+|\vec{F}|^{2}+r(B)^{2}|f|^{2}\right)
$$

holds, with the implicit constant depending only on $n, \lambda, \Lambda$.

Proof. Consider $\eta \in C_{c}^{\infty}(2 B)$ such that $0 \leq \eta \leq 1, \eta \equiv 1$ in $B$ and $|\nabla \eta| \lesssim r(B)^{-1}$. Note that $u \eta^{2}$ is a valid testing function in (2.8), and therefore we obtain that

$$
\begin{aligned}
& \iint_{\mathbb{R}^{n+1}} \lambda|\nabla u|^{2} \eta^{2} \leq \iint_{\mathbb{R}^{n+1}} A \nabla u \cdot \overline{\nabla u} \eta^{2} \\
& =\iint_{\mathbb{R}^{n+1}}\left(-2(A \nabla u \cdot \nabla \eta) \eta \bar{u}+B_{1} u \cdot \overline{\nabla\left(u \eta^{2}\right)}-B_{2} \cdot \nabla u \overline{u \eta^{2}}\right) \\
& \quad+\iint_{\mathbb{R}^{n+1}}\left(\vec{F} \cdot \overline{\nabla\left(u \eta^{2}\right)}+f \overline{u \eta^{2}}\right) \\
& =: I+I I+I I I+I V+V .
\end{aligned}
$$

To handle the term $I$, we use Cauchy's inequality with $\varepsilon>0$ and the boundedness of $A$ to 
obtain that

$$
|I| \leq 2 \Lambda \iint_{\mathbb{R}^{n+1}}|\nabla u| \eta|\nabla \eta||u| \leq \Lambda \varepsilon \iint_{\mathbb{R}^{n+1}}|\nabla u|^{2} \eta^{2}+\frac{\Lambda}{\varepsilon} \iint_{\mathbb{R}^{n+1}}|u|^{2}|\nabla \eta|^{2} .
$$

with $\varepsilon$ small enough (depending only on $\lambda, \Lambda$ ) that we can hide the first term. The second term is seen to be of a desired form after using the bound on $|\nabla \eta|$.

To handle the term III, we use the Hölder and Sobolev inequalities in $\mathbb{R}^{n}$ coupled with the $t$-independence of $B_{2}$, as follows:

$$
\begin{aligned}
& |I I I| \leq \int_{-\infty}^{\infty} \int_{\mathbb{R}^{n}}\left|B_{2}\right|(|\nabla u| \eta)|u| \eta d x d t \\
& \leq \int_{-\infty}^{\infty}\left\|B_{2}\right\|_{L^{n}\left(\mathbb{R}^{n}\right)}\|\eta \nabla u\|_{L^{2}\left(\mathbb{R}^{n}\right)}\|u \eta\|_{L^{\frac{2 n}{n-2}\left(\mathbb{R}^{n}\right)}} d t \\
& \leq\left\|B_{2}\right\|_{L^{n}\left(\mathbb{R}^{n}\right)} \int_{-\infty}^{\infty}\|\eta \nabla u\|_{L^{2}\left(\mathbb{R}^{n}\right)}\left\|\nabla_{\|}(u \eta)\right\|_{L^{2}\left(\mathbb{R}^{n}\right)} d t \\
& \leq\left\|B_{2}\right\|_{L^{n}\left(\mathbb{R}^{n}\right)} \int_{-\infty}^{\infty}\left(\|\eta \nabla u\|_{L^{2}\left(\mathbb{R}^{n}\right)}^{2}+\|\eta \nabla u\|_{L^{2}\left(\mathbb{R}^{n}\right)}\|u \nabla \eta\|_{L^{2}\left(\mathbb{R}^{n}\right)}\right) d t .
\end{aligned}
$$

Using the Cauchy inequality on the second term, we arrive at the estimate

$$
|I I I| \lesssim\left\|B_{2}\right\|_{n} \iint_{\mathbb{R}^{n+1}}\left(|\nabla u|^{2} \eta^{2}+|u|^{2}|\nabla \eta|^{2}\right)
$$

If we choose $\left\|B_{2}\right\|_{n}<\varepsilon_{0}$ (see Proposition 2.3.3) with $\varepsilon_{0}$ small enough (depending only on $n, \lambda, \Lambda)$, we can hide the first term, while the second term is of a desired form.

To handle the term $I I$, notice that the product rule allows us to write the estimate

$$
|I I| \leq \iint_{\mathbb{R}^{n+1}}\left(\left|B_{1}\|u\| \nabla u\right| \eta^{2}+2\left|B_{1} \| u\right|^{2} \eta|\nabla \eta|\right)=: I I_{1}+I I_{2}
$$

The first term is handled similarly as $I I I$. As for $I I_{2}$, we appeal again to the Hölder and Sobolev inequalities, together with the $t$-independence of $B_{1}$, to see that 


$$
\begin{aligned}
|I I| \lesssim \int_{-\infty}^{\infty}\left\|B_{1}\right\|_{L^{n}\left(\mathbb{R}^{n}\right)}\|u \nabla \eta\|_{L^{2}\left(\mathbb{R}^{n}\right)}\|u \eta\|_{L^{2 n / n-2}\left(\mathbb{R}^{n}\right)} d t & \\
& \lesssim\left\|B_{1}\right\|_{L^{n}\left(\mathbb{R}^{n}\right)} \int_{-\infty}^{\infty}\|u \nabla \eta\|_{L^{2}\left(\mathbb{R}^{n}\right)}\left\|\nabla_{\|}(u \eta)\right\|_{L^{2}\left(\mathbb{R}^{n}\right)} d t,
\end{aligned}
$$

and this last expression may be handled in the same way as in $I I$.

For the term $I V$, we use the product rule to obtain that

$$
|I V| \leq \iint_{\mathbb{R}^{n+1}}(|\vec{F}| \eta|\nabla u| \eta+2|\vec{F}| \eta|u||\nabla \eta|)=: I V_{1}+I V_{2}
$$

The first term may be estimated with the Cauchy's inequality with $\varepsilon$ :

$$
I V_{1} \leq \iint_{2 B}\left(\frac{1}{\varepsilon}|\vec{F}|^{2}+\varepsilon|\nabla u|^{2} \eta^{2}\right)
$$

and we can hide the second term. The term $I V_{2}$, since after using the Cauchy inequality, both terms are of a desired form:

$$
I V_{2} \leq \iint_{2 B}\left(|\vec{F}|^{2}+|u|^{2}|\nabla \eta|^{2}\right) .
$$

Combining these estimates gives

$$
\iint_{B}|\nabla u|^{2} \leq \iint_{\mathbb{R}^{n+1}}|\nabla u|^{2} \eta^{2} \lesssim \frac{1}{r(B)^{2}} \iint_{2 B}\left(|u|^{2}+|\vec{F}|^{2}\right)+|V| .
$$

To handle the term $V$, we use the Cauchy inequality to obtain that

$$
|V| \leq \iint_{\mathbb{R}^{n+1}}|f||u| \eta^{2} \leq \iint_{2 B}\left(r(B)^{2}|f|^{2}+\frac{1}{r(B)^{2}}|u|^{2}\right) .
$$

This completes the proof. 
Remark 2.5.2 ( $Y^{1, p}$ form a complex interpolation scale). In the case of purely second order operators (that is, $B_{1}=B_{2}=0$ ), we may exploit the fact that constants are always nullsolutions. Applying the Poincaré inequality, we obtain a weak reverse Hölder inequality for $\nabla u$, which in particular implies $L^{p}$ integrability for the gradient, for some $p>2$. We do not obtain the analogous estimate here, but rather a suitable substitute. More precisely, we shall muster an $L^{p}$ version of the Caccioppoli inequality. In order to prove this result, we remark that the spaces $Y^{1, p}\left(\mathbb{R}^{n+1}\right)$ and their dual spaces, $\left(Y^{1, p}\right)^{*}$, form a complex interpolation scale, with

$$
\left[Y^{1, p_{1}}, Y^{1, p_{2}}\right]_{\theta}=Y^{1, p_{\theta}}, \quad \frac{1}{p_{\theta}}=\frac{1-\theta}{p_{1}}+\frac{\theta}{p_{2}}
$$

for $\theta \in(0,1)$ and $1<p_{1}<p_{2}<n$. We may show this fact by gathering the following two ingredients. First, the homogeneous spaces $\dot{W}^{1, p}$ form a complex interpolation scale (see [Tri95]). Next, one uses that the map that sends an element in $\dot{W}^{1, p}$ to its unique representative in $Y^{1, p}$ is a 'retract' (see [KMM07, Lemma 7.11] and the discussion preceding it). Thus, we employ [KMM07, Lemma 7.11] and conclude that the spaces $Y^{1, p}$ form a complex interpolation scale. The fact that $\left(Y^{1, p}\right)^{*}$ form a complex interpolation scale is a general consequence of the interpolation scale for $Y^{1, p}$; see, for instance, [BL76, Theorem 4.5.1].

The $L^{p}$ Caccioppoli inequality will also make use of the well-known lemma of Snerberg [Sne74]. The (explicitly) quantitative version stated here appears in [ABES19]. 
Theorem 2.5.3 (Sneı̌berg's Lemma [ABES19, Theorem A.1], [Sne74]). Let

$\bar{X}=\left(X_{0}, X_{1}\right)$ and $\bar{Y}=\left(Y_{0}, Y_{1}\right)$ be interpolation couples, and $T \in \mathcal{B}(\bar{X}, \bar{Y})$. Suppose that for some $\theta^{*} \in(0,1)$ and some $\kappa>0$, the lower bound $\|T x\|_{Y_{\theta^{*}}} \geq \kappa\|x\|_{X_{\theta^{*}}}$ holds for all $x \in X_{\theta^{*}}$. Then the following statements are true.

i) Given $0<\varepsilon<1 / 4$, the lower bound $\|T x\|_{Y_{\theta}} \geq \varepsilon \kappa\|x\|_{X_{\theta}}$ holds for all $x \in X_{\theta}$, provided that $\left|\theta-\theta^{*}\right| \leq \frac{\kappa(1-4 \varepsilon) \min \left\{\theta^{*}, 1-\theta^{*}\right\}}{3 \kappa+6 M}$, where $M=\max _{j=0,1}\|T\|_{X_{j} \rightarrow Y_{j}}$.

ii) If $T: X_{\theta^{*}} \rightarrow Y_{\theta^{*}}$ is invertible, then the same is true for $T: X_{\theta} \rightarrow Y_{\theta}$ if $\theta$ is as in (i). The inverse mappings agree on $X_{\theta} \cap X_{\theta^{*}}$ and their norms are bounded by $\frac{1}{\varepsilon \kappa}$.

Using the above result, we can easily obtain

Lemma 2.5.4 (Invertibility of $\mathcal{L}$ in a window around 2). Let $p \in(1, n)$ be such that $p^{\prime}<n$, where $p^{\prime}$ is the Hölder conjugate of $p$. The operator $\mathcal{L}$ extends to a bounded operator $Y^{1, p}\left(\mathbb{R}^{n+1}\right) \rightarrow\left(Y^{1, p^{\prime}}\left(\mathbb{R}^{n+1}\right)\right)^{*}$. Moreover, the operator is invertible if $|p-2|$ is small enough depending on $n, \lambda$, and $\Lambda$.

Remark 2.5.5. Here and throughout, we assume that the range of $p$ near 2 in Lemma 2.5.4 is such that $p_{*}=\frac{(n+1) p}{n+1+p}<2$.

The following lemma details the modification to the operator output upon multiplying a solution by a cut-off function.

Lemma 2.5.6. Let $\Omega \subset \mathbb{R}^{n+1}$ be an open set. Suppose that $u \in W_{\text {loc }}^{1,2}(\Omega)$ satisfies $\mathcal{L} u=0$ in 
$\Omega$ in the weak sense. Then for any $\chi \in C_{c}^{\infty}(\Omega, \mathbb{R})$, we have that

$$
\mathcal{L}(\chi u)=\operatorname{div} \vec{F}+f
$$

in $\mathbb{R}^{n+1}$ in the weak sense, where $\vec{F}=A(\nabla \chi) u$, and $f=-A \nabla u \cdot \nabla \chi-B_{1} u \nabla \chi+B_{2} u \nabla \chi$.

Proof. We apply the operator $\mathcal{L}$ to $u \chi$ and test against $\varphi \in C_{c}^{\infty}\left(\mathbb{R}^{n+1}\right)$ with the goal in mind of extracting a term of the form $\langle\mathcal{L} u, \varphi \chi\rangle=0$. Observe that

$$
\begin{aligned}
\int_{\mathbb{R}^{n+1}} A \nabla(u \chi) \cdot \overline{\nabla \varphi} & =\int_{\mathbb{R}^{n+1}} A \nabla u \cdot \overline{\nabla(\chi \varphi)}+\int_{\mathbb{R}^{n+1}} u A \nabla \chi \cdot \overline{\nabla \varphi}-\int_{\mathbb{R}^{n+1}}[A \nabla u \cdot \nabla \chi] \bar{\varphi}, \\
\int_{\mathbb{R}^{n+1}}\left(B_{1} u \chi\right) \cdot \overline{\nabla \varphi} & =+\int_{\mathbb{R}^{n+1}} B_{1} u \overline{\nabla(\chi \varphi)}-\int_{\mathbb{R}^{n+1}}\left[B_{1} u \nabla \chi\right] \bar{\varphi}, \\
\int_{\mathbb{R}^{n+1}} B_{2} \nabla(u \chi) \bar{\varphi} & =\int_{\mathbb{R}^{n+1}} B_{2} \nabla u \overline{\chi \varphi}+\int_{\mathbb{R}^{n+1}}\left[B_{2} u \nabla \chi\right] \bar{\varphi},
\end{aligned}
$$

where we use that $\chi$ is real-valued. Collecting the first terms in each inequality and noting that $\varphi \chi \in C_{c}^{\infty}(\Omega)$, we realize that the contribution of these terms is $\langle\mathcal{L} u, \varphi \chi\rangle=0$. Then we have that $\langle\mathcal{L}(\chi u), \varphi\rangle=\langle\operatorname{div} \vec{F}+f, \varphi\rangle$, as desired.

We are now ready to combine the past few results and obtain the local high integrability of the gradient.

Lemma 2.5.8 (Local high integrability of the gradient of a solution). Let $\Omega$ be an open set. Suppose that $u \in W_{\mathrm{loc}}^{1,2}(\Omega)$ solves $\mathcal{L} u=0$ in $\Omega$ in the weak sense. Then $u \in W_{\mathrm{loc}}^{1, p}(\Omega)$, where p depends only on $n, \lambda, \Lambda$, and $\varepsilon_{0}$. Moreover, for any $\chi \in C_{c}^{\infty}(\Omega, \mathbb{R})$ we have the estimate

$$
\|\chi u\|_{Y^{1, p}\left(\mathbb{R}^{n+1}\right)} \leq\left\|\mathcal{L}^{-1}(\operatorname{div} \vec{F}+f)\right\|_{Y^{1, p}\left(\mathbb{R}^{n+1}\right)} \lesssim\|\vec{F}\|_{p}+\|f\|_{p_{*}}
$$

where $\vec{F}$ and $f$ are as in Lemma 2.5.6. 
Proof. Let $\vec{F}$ and $f$ be as in the previous lemma. One may verify, using the Sobolev embedding and the fact that $\chi$ is smooth and compactly supported, that $\vec{F} \in L^{1}\left(\mathbb{R}^{n+1}\right) \cap$ $L^{2^{*}}\left(\mathbb{R}^{n+1}\right)$ and that $f \in L^{1}\left(\mathbb{R}^{n+1}\right) \cap L^{2}\left(\mathbb{R}^{n+1}\right)$. Choosing $p>2$ with $|p-2|$ sufficiently small, we may apply Lemma 2.5.4 to show that the operator $\mathcal{L}$ extends to a bounded and invertible operator $Y^{1, p}\left(\mathbb{R}^{n+1}\right) \rightarrow\left(Y^{1, p^{\prime}}\left(\mathbb{R}^{n+1}\right)\right)^{*}$. Hence $\mathcal{L}^{-1}$ is bounded. Applying $\mathcal{L}^{-1}$ to each side of (2.7), we obtain that

$$
\|\chi u\|_{Y^{1, p}} \leq\left\|\mathcal{L}^{-1}(\operatorname{div} \vec{F}+f)\right\|_{Y^{1, p}} \lesssim\|\vec{F}\|_{p}+\|f\|_{p_{*}}
$$

Here, we note that $L^{p_{*}}$ embeds continuously into $\left(Y^{1, p^{\prime}}\right)^{*}$, and $\operatorname{div} \vec{F} \in\left(Y^{1, p^{\prime}}\right)^{*}$ since $\vec{F} \in L^{p}$. This observation uses the identity $\left[\left(p^{\prime}\right)^{*}\right]^{\prime}=p_{*}$ and the continuous embedding $Y^{1, p^{\prime}}\left(\mathbb{R}^{n+1}\right) \hookrightarrow$ $L^{\left(p^{\prime}\right)^{*}}\left(\mathbb{R}^{n+1}\right)$

Finally, we provide a more precise version of the above Lemma, namely the $L^{p}$-Caccioppoli inequality.

Proposition 2.5.9 ( $L^{p}$-Caccioppoli inequality). Let $\Omega \subset \mathbb{R}^{n+1}$ be an open set and let $u \in$ $W_{\text {loc }}^{1,2}(\Omega)$ solve $\mathcal{L} u=0$ in $\Omega$ in the weak sense. Suppose that $B$ is a ball such that $\kappa B \subset \Omega$ for some $\kappa>1$. Then, for every $p>0$ such that $|p-2|$ is small enough that the conditions of Lemma 2.5.8 are satisfied, the estimate

$$
\|\nabla u\|_{L^{p}(B)} \lesssim \frac{1}{r(B)}\|u\|_{L^{p}(\kappa B)}
$$

holds, where the implicit constants depend on $\kappa, p, n, \lambda, \Lambda$, and $\varepsilon_{0}$. 
Proof. Set $r:=r(B)$ and let $\chi=\eta^{2}$ with $\eta \in C_{c}^{\infty}\left(\frac{1+\kappa}{2} B, \mathbb{R}\right), 0 \leq \eta \leq 1,|\nabla \eta| \lesssim \frac{1}{r}$. Note that $\chi$ has the same properties as $\eta$. The estimate (2.10) will follow immediately from the estimate

$$
\|u \chi\|_{Y^{1, p}\left(\mathbb{R}^{n+1}\right)} \lesssim \frac{1}{r}\|u\|_{L^{p}(\kappa B)}
$$

since $\|\nabla u\|_{L^{p}(B)} \lesssim\|(\nabla u) \chi\|_{p}$ and (the reverse triangle inequality yields)

$$
\|(\nabla u) \chi\|_{p}-\|(\nabla \chi) u\|_{p} \lesssim\|\nabla(u \chi)\|_{p} \leq\|u \chi\|_{Y^{1, p}\left(\mathbb{R}^{n+1}\right)}
$$

We immediately note that we have already established (2.11) in the case $p=2$; this is the classical Caccioppoli inequality. Applying Lemma 2.5.8, we have that

$$
\|\chi u\|_{Y^{1, p}\left(\mathbb{R}^{n+1}\right)} \lesssim\|\vec{F}\|_{p}+\|f\|_{p_{*}}
$$

where $\vec{F}$ and $f$ are as in Lemma 2.5.6. The bound

$$
\|\vec{F}\|_{p}=\|A \nabla \chi u\|_{p} \lesssim \frac{1}{r}\|u\|_{L^{p}(\kappa B)}
$$

is trivial from the properties of $A$ and $\chi$ and desirable from the standpoint of (2.11). It remains to find appropriate bounds for the terms appearing in the expression for $f$. To this end, we have by Minkowski's inequality that

$$
\|f\|_{p_{*}} \leq\|A \nabla u \cdot \nabla \chi\|_{p_{*}}+\left\|B_{1} u \nabla \chi\right\|_{p_{*}}+\left\|B_{2} u \nabla \chi\right\|_{p_{*}}=I+I I+I I I .
$$

Before continuing, we remark that the relation $\frac{n+1}{p_{*}}=\frac{n+1}{(n+1) p}[(n+1)+p]=\frac{n+1}{p}+1$ 
holds. Using the $L^{2}$ Caccioppoli inequality, Jensen's inequality and the fact that $p>2$, we have that

$$
\begin{aligned}
I=\|A \nabla u \cdot \nabla \chi\|_{p_{*}} \lesssim r^{\frac{n+1}{p}}\left(f_{\frac{1+\kappa}{2} B}|\nabla u|^{2}\right)^{\frac{1}{2}} & \lesssim \frac{1}{r} r^{\frac{n+1}{p}}\left(f_{\kappa B}|u|^{2}\right)^{\frac{1}{2}} \\
& \lesssim \frac{1}{r} r^{\frac{n+1}{p}}\left(f_{\kappa B}|u|^{p}\right)^{\frac{1}{p}} \lesssim \frac{1}{r}\left(\int_{\kappa B}|u|^{p}\right)^{\frac{1}{p}} .
\end{aligned}
$$

Next we bound $I I$ and $I I I$. The Sobolev embedding on $\mathbb{R}^{n}$ and the Caccioppoli inequality ${ }^{4}$ yield for $i=1,2$ the estimate

$$
\begin{aligned}
&\left\|B_{i} u(\nabla \chi)\right\|_{p_{*}} \lesssim \frac{1}{r}\left\|B_{i}(u \eta)\right\|_{p_{*}} \lesssim \frac{1}{r} r^{\frac{n+1}{p_{*}}}\left(f_{\frac{1+\kappa}{2} B}\left|B_{i}(u \eta)\right|^{p_{*}}\right)^{\frac{1}{p_{*}}} \\
& \lesssim \frac{1}{r} r^{\frac{n+1}{p_{*}}} r^{-\frac{n+1}{2}}\left(\int_{\frac{1+\kappa}{2} B}\left|B_{i}(u \eta)\right|^{2}\right)^{\frac{1}{2}} \lesssim \frac{1}{r} r^{\frac{n+1}{p_{*}}} r^{-\frac{n+1}{2}}\left(\int_{\mathbb{R}^{n+1}}|\nabla(u \eta)|^{2}\right)^{\frac{1}{2}} \\
& \lesssim \frac{1}{r} r^{\frac{n+1}{p}}\left(f_{\kappa B}|u|^{2}\right)^{\frac{1}{2}} \lesssim \frac{1}{r}\left(\int_{\kappa B}|u|^{p}\right)^{\frac{1}{p}} .
\end{aligned}
$$

Combining (2.13), (2.14) and (2.15) with (2.12) and the definitions of $\vec{F}$ and $f$, we obtain (2.11). As we had reduced the proof of the statement of the Proposition to (2.11), we have thus shown our claim.

Proposition 2.5.16 (Weak Reverse Hölder Inequality for Solutions). Let $u \in W_{\mathrm{loc}}^{1,2}(\Omega)$ be $a$ solution to $\mathcal{L} u=\operatorname{div} F$ in $\Omega \subset \mathbb{R}^{n+1}$, with $F \in L_{\mathrm{loc}}^{2}(\Omega)$. Let $B$ be an $(n+1)$-dimensional ball in $\mathbb{R}^{n+1}$ with $2 B \subset \Omega$. Then, for any $q \geq 1$ we have that

$$
\left(\oiiint_{B}|u|^{2_{n+1}^{*}}\right)^{1 / 2_{n+1}^{*}} \lesssim\left(\int_{2 B}|u|^{q}\right)^{1 / q}+r(B)\left(\int_{2 B}|F|^{2}\right)^{1 / 2},
$$

${ }^{4}$ More precisely, we use (2.11) with $p=2$. 
with implicit constants depending only on $q, n$ and ellipticity, and where we define

$$
\frac{1}{2_{n+1}^{*}}=\frac{1}{2}-\frac{1}{n+1}, \quad \frac{1}{2_{n+1}^{*}}+\frac{1}{2_{*, n+1}}=1 .
$$

Proof. We first prove the result for a ball $B$ with $r(B)=1$. To simplify notation, during this proof we will write $2^{*}=2_{n+1}^{*}$.

Fix $1 \leq t<s \leq 2$, then, from the proof of the Caccioppoli inequality (see $\left[\mathrm{BHL}^{+} \mathrm{a}\right.$, Proposition 3.1], and note that $f=0$ for us), we have

$$
\begin{aligned}
\|\nabla u\|_{L^{2}\left(B_{t}\right)} & \lesssim \frac{1}{s-t}\|u\|_{L^{2}\left(B_{s}\right)}+\|F\|_{L^{2}\left(B_{s}\right)} \\
& \leq \frac{1}{s-t}\|u\|_{L^{2}\left(B_{s}\right)}+\|F\|_{L^{2}(2 B)},
\end{aligned}
$$

where $B_{t}$ denotes the concentric dilate of $B$ by a factor of $t$.

On the other hand, if as usual we denote by $u_{B}$ the average of $u$ over $B$, by the PoincaréSobolev inequality

$$
\begin{aligned}
\|u\|_{L^{2^{*}\left(B_{t}\right)}} & \lesssim t \cdot t^{(n+1)\left(1 / 2^{*}-1 / 2\right)}\|\nabla u\|_{L^{2}\left(B_{t}\right)}+t^{(n+1) / 2^{*}}\left|u_{B_{t}}\right| \\
& \lesssim\|\nabla u\|_{L^{2}\left(B_{t}\right)}+\|u\|_{L^{1}(2 B)},
\end{aligned}
$$

where we use that $t \geq 1 / 2$ to control the last term in the last line. Combining these two inequalities we obtain

$$
\begin{aligned}
\|u\|_{L^{2^{*}\left(B_{t}\right)}} \lesssim & \frac{1}{s-t}\|u\|_{L^{2}\left(B_{s}\right)}+\|F\|_{L^{2}(2 B)} \\
& +\|u\|_{L^{1}(2 B)} .
\end{aligned}
$$

Note that if here we set $t=1$ and $s=2$ the result follows for $q \geq 2$. It thus remains to treat the case $q<2$. 
Recall, from interpolation of $L^{p}$ norms (here we use $q<2$ ) and the Cauchy inequality with a parameter,

$$
\|u\|_{L^{2}} \leq\|u\|_{L^{2^{*}}}^{1-\theta}\|u\|_{L^{q}}^{\theta} \lesssim_{\theta} \eta^{1 /(1-\theta)}\|u\|_{L^{2^{*}}}+\frac{1}{\eta^{1 / \theta}}\|u\|_{L^{2 *}}
$$

valid for any $\eta>0$ and where $\theta$ satisfies

$$
\frac{1}{2}=\frac{1-\theta}{2^{*}}+\frac{\theta}{q} .
$$

Choosing $\eta^{1 /(1-\theta)} \approx t-s$ and setting

$$
A:=\|F\|_{L^{2}(2 B)}+\|u\|_{L^{1}(2 B)},
$$

we arrive at

$$
\|u\|_{L^{2^{*}\left(B_{t}\right)}} \leq \frac{1}{2}\|u\|_{L^{2^{*}\left(B_{s}\right)}}+\frac{C}{(s-t)^{(1-\theta) / \theta}}\|u\|_{L^{q}\left(B_{s}\right)}+A
$$

the above being valid for any $1 \leq t<s \leq 2$. We are now in a position to apply the result in [HL97, Lemma 4.3] and conclude

$$
\|u\|_{L^{2^{*}\left(B_{t}\right)}} \lesssim \frac{1}{(s-t)^{(1-\theta) / \theta}}\|u\|_{L^{q}}+A
$$

Setting now $t=1$ and $s=2$ we arrive at

$$
\|u\|_{L^{2^{*}(B)}} \lesssim\|u\|_{L^{q}(2 B)}+\|u\|_{L^{1}(2 B)}+\|F\|_{L^{2}(2 B)} .
$$

This is the desired inequality, since $q \geq 1$ and $r(B)=1$. 
To obtain the result for a general $B$ we simply note that for $r>0 u_{r}(X)=u(r X)$ solves $\mathcal{L}_{r} u_{r}=\operatorname{div} F_{r}$ in $r \Omega$, where the coefficients of $\mathcal{L}_{r}$ are given by

$$
A_{r}(X)=A(r X), \quad B_{i, r}(X)=r B_{i}(r X), \quad F_{r}(X)=r F(r X) .
$$

It can be checked that these coefficients satisfy the same conditions as the originals, with the same relevant norms except for $F_{r}$ which satisfies $\left\|F_{r}\right\|_{L^{2}(1 / r B)}=r^{(-n+1) / 2}\|F\|_{L^{2}(B)}$. The result now follows from this.

We now prove a result on the boundary behavior of solutions, under the assumption that we have good control of a modified nontangential maximal function.

Proposition 2.5.29. Let $u \in W_{\text {loc }}^{1,2}\left(\mathbb{R}^{n}\right)$ be a solution to $\mathcal{L} u=0$ in $\mathbb{R}_{+}^{n+1}$. Define

$$
\tilde{u}(z, t):=f_{t / 2}^{3 t / 2} f_{|z-y|_{\infty}<t} u(y, s) d y d s .
$$

Then $\tilde{u}$ converges non-tangentially at every point where $\tilde{\mathcal{N}}_{1}(\nabla)(x)<\infty$, i.e. for any such $x \in \mathbb{R}^{n}$

$$
\lim _{\substack{Y \rightarrow x \\ Y \in \Gamma(x)}} \tilde{u}(Y)=g(x)
$$

where $\Gamma(x)$ is the non-tangential cone with vertex $x$.

Proof. We will follow the arguments in [KP93, Theorem 3.1 (a)], with modifications due to the lack of pointwise estimates for $u$.

Let $x \in \mathbb{R}^{n}$ be such that $\tilde{\mathcal{N}}_{1}(\nabla u)(x)<\infty$, where we recall that

$$
\tilde{\mathcal{N}}_{1}(F):=\sup _{(z, t) \in \Gamma(x)} f_{t / 2}^{3 t / 2} f_{|z-y|_{\infty}<t}|F| d y d s
$$


and that $\tilde{\mathcal{N}}_{1}(\nabla u)(x)<\infty$ for almost every $x \in \mathbb{R}^{n}$. Following [KP93] it is our goal to show for $Y, Z \in \Gamma(x) \cap B(x, r)$ we have

$$
|\tilde{u}(Y)-\tilde{u}(Z)| \leq C r \tilde{\mathcal{N}}_{1}(\nabla u)(x)
$$

from which we may easily establish (via the Cauchy criterion) that

$$
\lim _{\substack{Y \rightarrow x \\ Y \in \Gamma(x)}} \tilde{u}(Y)
$$

exists and define $g_{0}(x)$ to be the limit when it does so. Write $Y=\left(y, t_{1}\right)$ and $Z=\left(z, t_{2}\right)$ then to establish (2.30) it is clearly enough to establish

$$
\left|\tilde{u}(Y)-\tilde{u}\left(x, t_{1}\right)\right|,\left|\tilde{u}(Z)-\tilde{u}\left(x, t_{2}\right)\right| \leq C r \tilde{\mathcal{N}}_{1}(\nabla u)(x)
$$

and

$$
\left|\tilde{u}\left(x, t_{2}\right)-\tilde{u}\left(x, t_{1}\right)\right| \leq C r \tilde{\mathcal{N}}_{1}(\nabla u)(x) .
$$

To prove (2.31) and (2.32) we need the following fact.

Claim 2.5.33. For $X \in \mathbb{R}^{n+1}$ and $r>0$ let $I(X, r):=\left\{W \in \mathbb{R}^{n+1}:|X-W|_{\infty}<r\right\}$ be the open cube with center $X$ and side length $2 r$. Let $I_{i}=I\left(X_{i}, r_{i}\right), i=1,2, \Omega \subset \mathbb{R}^{n+1}$ open with $I_{i} \subset \Omega$ $i=1,2$ and $\varphi \in W^{1,2}(\Omega)$. If $\alpha \in[0,2)$ and $\left|X_{1}-X_{2}\right| \leq \alpha \min \left\{r_{1}, r_{2}\right\}$ then

$$
\left|f_{I_{1}} \varphi d x d t-f_{I_{2}} \varphi d x d t\right| \leq C_{\alpha} \max \left\{r_{1}, r_{2}\right\}\left(\max \left\{\frac{r_{1}}{r_{2}}, \frac{r_{2}}{r_{1}}\right\}\right)^{\frac{n+1}{2}} \max _{i=1,2}\left(f_{I_{i}}|\nabla \varphi|^{2}\right)^{1 / 2}
$$

where $C_{\alpha}=C(n, \alpha)$. In particular, if $r_{1} \approx r_{2} \approx r$ then

$$
\left|f_{I_{1}} \varphi d x d t-f_{I_{2}} \varphi d x d t\right| \leq C_{\alpha} r \max _{i=1,2}\left(f_{I_{i}}|\nabla \varphi|^{2}\right)^{1 / 2}
$$


where $C_{\alpha}$ depends on the implicit constants in the expression $r_{1} \approx r_{2} \approx r$.

proof of Claim 2.5.33. Let $X_{3}=\frac{X_{1}+X_{2}}{2}$, then

$$
\left|X_{3}-X_{i}\right|<\frac{\alpha r_{i}}{2}, \quad i=1,2
$$

and hence $I_{3}=I\left(x_{3}, r\right) \subset I_{i}, i=1,2$ for $r=(1-\alpha / 2) \min \left\{r_{1}, r_{2}\right\}$. It follows from the triangle inequality and the Poincaré inequality

$$
\begin{aligned}
\left|f_{I_{1}} \varphi-f_{I_{2}} \varphi\right| & \leq\left|f_{I_{1}} \varphi-f_{I_{3}} \varphi\right|+\left|f_{I_{1}} \varphi-f_{I_{3}} \varphi\right| \\
& \leq 2 \max _{i=1,2}\left(f_{I_{3}}\left|\varphi-f_{I_{i}} \varphi\right|^{2}\right)^{1 / 2} \\
& \leq C_{\alpha}\left(\max \left\{\frac{r_{1}}{r_{2}}, \frac{r_{2}}{r_{1}}\right\}\right)^{\frac{n+1}{2}} \max _{i=1,2}\left(f_{I_{i}}\left|\varphi-f_{I_{i}} \varphi\right|^{2}\right)^{1 / 2} \\
& \leq C_{\alpha} \max \left\{r_{1}, r_{2}\right\}\left(\max \left\{\frac{r_{1}}{r_{2}}, \frac{r_{2}}{r_{1}}\right\}\right)^{\frac{n+1}{2}} \max _{i=1,2}\left(f_{I_{i}}|\nabla \varphi|^{2}\right)^{1 / 2}
\end{aligned}
$$

as desired.

Now let us prove (2.31) for the term with $Y$ (the proof for the term with $Z$ is identical). Note that $|x-y| \leq t_{1}<r$ since $Y \in \Gamma(x) \cap B(x, r)$. Let $I_{2}=I\left(z, t_{1} / 2\right)$, for $z=(x+y) / 2$, then $|z-x|=|z-y| \leq t_{1} / 2$. This allows us to apply (2.34) with $\varphi=u, I_{1}=I\left(x, t_{1} / 2\right)$ and then $I_{1}=I\left(y, t_{1} / 2\right)$ and use the triangle inequality to obtain (2.31).

We turn our attention to (2.32) and we assume, without loss of generality, that $t_{1} \leq t_{2}$. Let $a=2 / 3, s_{k}=a^{k} t_{2}$ for $k=0,1, \ldots, K$, where $K=\max \left\{k: a^{k} t_{2} \geq t_{1}\right\}$. Notice that for $k=0, \ldots K-1$

$$
\left|s_{k}-s_{k+1}\right|=\frac{s_{k+1}}{2}=\min \left\{\frac{s_{k}}{2}, \frac{s_{k+1}}{2}\right\}
$$


Defining $s_{K+1}=t_{1}$ we see that the choice of $K$ guarantees

$$
\left|s_{K}-s_{K+1}\right| \leq \min \left\{\frac{s_{K}}{2}, \frac{t_{1}}{2}\right\}
$$

Set $I_{k}:=I\left(\left(x, s_{k}\right), \frac{s_{k}}{2}\right), k=0, \ldots K+1$, then the previous two inequalities allow us to apply (2.34) with $\varphi=u$ and the consecutive cubes $I_{k}$ and $I_{k+1}, k=0,1, \ldots, K$ (in place of $I_{1}$ and $I_{2}$ therein). One then obtains

$$
\begin{aligned}
\left|\tilde{u}\left(x, t_{1}\right)-\tilde{u}\left(x, t_{2}\right)\right| & \leq\left|\tilde{u}\left(x, s_{K+1}\right)-\tilde{u}\left(x, s_{K}\right)\right|+\sum_{k=0}^{K-1}\left|\tilde{u}\left(x, s_{k}\right)-\tilde{u}\left(x, s_{k+1}\right)\right| \\
& \lesssim t_{1} \tilde{\mathcal{N}}_{1}(\nabla u)(x)+t_{2} \sum_{k=0}^{K-1} a^{k} \tilde{\mathcal{N}}_{1}(\nabla u)(x) \\
& \lesssim r \tilde{\mathcal{N}}_{1}(\nabla u)(x),
\end{aligned}
$$

as desired (since $\sum_{k \geq 0} a^{k}=3$ ).

\subsubsection{Properties of solutions and their gradients on slices}

Our next goal is to study the $t$-regularity of our solutions as well as their properties on 'slices', which are sets of the form $\left\{(x, t): t=t_{0}\right\}$. Let us first note that $t$-derivatives of solutions are solutions.

Proposition 2.5.35 (The $t$-derivatives of solutions are solutions). Let $\Omega \subset \mathbb{R}^{n+1}$ be an open set, let $f, \vec{F} \in L_{\mathrm{loc}}^{2}(\Omega)$, and suppose that $u \in W_{\mathrm{loc}}^{1,2}(\Omega)$ satisfies $\mathcal{L} u=f-\operatorname{div} \vec{F}$ in $\Omega$ in the weak sense. Assume further that $f_{t}:=\partial_{t} f \in L_{\mathrm{loc}}^{2}(\Omega)$ and $\vec{F}_{t}:=\partial_{t} \vec{F} \in L_{\mathrm{loc}}^{2}(\Omega)$. Then the function $v=\partial_{t} u$ lies in $W_{\text {loc }}^{1,2}(\Omega)$ and satisfies $\mathcal{L} v=f_{t}-\operatorname{div} F_{t}$ in $\Omega$ in the weak sense. 
Proof. Fix a ball $B \subset 2 B \subset \Omega$ and consider the difference quotients

$$
u_{h}:=\frac{u\left(\cdot+h e_{n+1}\right)-u(\cdot)}{|h|}, \quad|h|<\operatorname{dist}(B, \partial \Omega)
$$

We define $f_{h}$ and $\vec{F}_{h}$ similarly. By $t$-independence of the coefficients, we have that $\mathcal{L} u_{h}=$ $f_{h}-\operatorname{div} \vec{F}_{h}$ in $B$ for any such $h$. By the Caccioppoli inequality (Proposition 2.5.1), we obtain that for any $h$ as above,

$$
\begin{aligned}
\iint_{B}\left|\nabla u_{h}\right|^{2} \lesssim \iint_{2 B}\left(\frac{1}{r(B)^{2}}\left|u_{h}\right|^{2}+\left|\vec{F}_{h}\right|^{2}+\right. & \left.r(B)^{2}\left|f_{h}\right|^{2}\right) \\
& \lesssim \iint_{2 B}\left(\frac{1}{r(B)^{2}}\left|\partial_{t} u\right|^{2}+\left|\vec{F}_{t}\right|^{2}+r(B)^{2}\left|f_{t}\right|^{2}\right) .
\end{aligned}
$$

In particular, the difference quotients of $\nabla u$ are bounded, which implies that $\partial_{t} u \in W_{\text {loc }}^{1,2}(\Omega)$. Consequently, we must have that the difference quotients $u_{h}$ converge weakly (in $W_{\text {loc }}^{1,2}(\Omega)$ ) to $v=\partial_{t} u$ (and similarly for $f_{h}$ and $\vec{F}_{h}$ ). From (2.8) and the fact that $\mathcal{L} u_{h}=f_{h}-\operatorname{div} \vec{F}_{h}$, we conclude that $\mathcal{L} v=f_{t}-\operatorname{div} \vec{F}_{t}$, as desired.

We now check that $t$-derivatives of solutions are well-behaved on horizontal strips.

Lemma 2.5.36 (Good integrability of the $t$-derivative of a solution on a strip). Denote $\Sigma_{a}^{b}:=$ $\left\{(x, t) \in \mathbb{R}^{n+1}: a<t<b\right\}$. Suppose that $u$ and $v:=\partial_{t} u$ are as in Proposition 2.5.35 with $\Omega=\Sigma_{a}^{b}$, and suppose further that $v \in L^{2}\left(\Sigma_{a}^{b}\right)$. Then $\nabla v \in L^{2}\left(\sum_{a^{\prime}}^{b^{\prime}}\right)$ for each $a<a^{\prime}<b^{\prime}<b$.

Proof. Let $\chi_{R}=\phi(x) \psi(t)$ be a product of infinitely smooth cut-off functions with $0 \leq$ $\phi_{R}, \psi \leq 1, \psi \equiv 1$ on $\left(a^{\prime}, b^{\prime}\right), \psi \in C_{c}^{\infty}(a, b)$, and $\phi_{R} \equiv 1$ on $B_{R}, \phi_{R} \in C_{c}^{\infty}\left(B_{2 R}\right)$. Then, for all $R \gg \min \left\{a^{\prime}-a, b-b^{\prime}\right\}$, we claim that 


$$
\begin{aligned}
& \int_{a^{\prime}}^{b^{\prime}} \int_{B_{R}}|\nabla v|^{2} d x d t \lesssim \iint_{\mathbb{R}^{n+1}} \chi_{R}^{2}|\nabla v|^{2} \\
& \lesssim \iint_{\mathbb{R}^{n+1}}\left(|v|^{2}+\left|\vec{F}_{t}\right|^{2}+\left|f_{t}\right|^{2}\right)\left(\left|\nabla \chi_{R}\right|^{2}+1\right) \\
& \quad \lesssim \frac{1}{\left(\min \left\{a^{\prime}-a, b-b^{\prime}, 1\right\}\right)^{2}} \int_{a}^{b} \int_{\mathbb{R}^{n}}\left(|v|^{2}+\left|\vec{F}_{t}\right|^{2}+\left|f_{t}\right|^{2}\right) .
\end{aligned}
$$

We provide the details of the second line in a moment; note that in the third line we used that the dominant contribution for the gradient of $\chi_{R}$ is its $t$ component when $R$ is large. Sending $R \rightarrow \infty$ finishes the proof modulo the aforementioned line.

To see the computation above, denote $\chi:=\chi_{R}$ and observe that

$$
\begin{aligned}
\iint_{\mathbb{R}^{n+1}} \chi^{2}|\nabla v|^{2} \lesssim \iint_{\mathbb{R}^{n+1}} \chi^{2} \mathfrak{R} e(A \nabla v \overline{\nabla v}) \\
\quad \leq \Re e\left[\iint_{\mathbb{R}^{n+1}} A \nabla v \overline{\nabla\left(v \chi^{2}\right)}-2 \iint_{\mathbb{R}^{n+1}} \chi \bar{v} A \nabla v \nabla \chi\right]=: \Re e[I+I I] .
\end{aligned}
$$

Clearly,

$$
|I I| \lesssim \epsilon \iint_{\mathbb{R}^{n+1}}|\chi \nabla v|^{2}+\frac{1}{\epsilon} \iint_{\mathbb{R}^{n+1}}|\nabla \chi v|^{2},
$$

and the first term can be absorbed to the left-hand side. It remains to handle $I$. We use the equation $\mathcal{L} v=f_{t}-\operatorname{div} \vec{F}_{t}$ to write $I=I_{1}+I_{2}+I_{3}+I_{4}$, where each $I_{j}$ is a term of the equation and each will be given explicitly below. First, note that

$$
\left|I_{4}\right|:=\left|\iint_{\mathbb{R}^{n+1}} f_{t} \bar{v} \chi^{2}\right| \lesssim \iint_{\mathbb{R}^{n+1}}|\nu \chi|^{2}+\iint_{\mathbb{R}^{n+1}}\left|f_{t} \chi\right|^{2},
$$

which handles this term. Next, we have that

$$
\left|I_{3}\right|:=\left|\iint_{\mathbb{R}^{n+1}} \vec{F}_{t} \overline{\nabla\left(\nu \chi^{2}\right)}\right| \lesssim \iint_{\mathbb{R}^{n+1}}\left|\vec{F}_{t} \nabla v \chi\right|^{2}+\iint_{\mathbb{R}^{n+1}}\left|\vec{F}_{t} \chi \nabla \chi v\right| .
$$


We handle the first term as in $I I$, and we handle the second term as $I_{4}$. Moving on, we see that

$$
\left|I_{1}\right|:=\left|\iint_{\mathbb{R}^{n+1}} B_{1} v \overline{\nabla\left(v \chi^{2}\right)}\right| \lesssim \iint_{\mathbb{R}^{n+1}}\left|\left(B_{1} v \chi\right) \nabla v \chi\right|+\iint_{\mathbb{R}^{n+1}}\left|\left(B_{1} v \chi\right) \nabla \chi v\right| .
$$

Both of the terms above are handled by using the smallness of $B_{1}$ as in the proof of the Caccioppoli inequality. Now, for the last term, we have that

$$
\left|I_{2}\right|:=\left|\iint_{\mathbb{R}^{n+1}} B_{2} \nabla v \chi^{2} \bar{v}\right| \lesssim \iint_{\mathbb{R}^{n+1}}\left|\left(B_{2} \chi v\right) \nabla v \chi\right|,
$$

so that we may handle this term exactly as we did $I_{1}$

Remark 2.5.37. We may bring the above lemma and Lemma 2.2.2 together to conclude that if $u$ solves $\mathcal{L} u=0$ in $\Sigma_{a}^{b}$, then automatically we have the transversal Hölder continuity of its gradient, and $u \in C_{\text {loc }}^{\alpha^{\prime}}\left((a, b), L^{\frac{2 n}{n-2}}\left(\mathbb{R}^{n}\right)\right)$ for some $\alpha>0$.

Next, we present a formula for our equation on a slice. Recall that $\tilde{A}$ denotes the $(n+$ 1) $\times n$ submatrix of $A$ consisting of the first $n$ columns of $A$.

Proposition 2.5.38 (Integration by parts on slices for $\mathcal{L}$ ). Let $u \in Y^{1,2}\left(\Sigma_{a}^{b}\right)$ and suppose that $\mathcal{L} u=g$ in $\Sigma_{a}^{b}$ for some $g \in C_{c}^{\infty}\left(\mathbb{R}^{n+1}\right)$. Then, for every $t \in(a, b)$ and $\varphi \in W^{1,2}\left(\mathbb{R}^{n}\right)$, the identity

$$
\begin{aligned}
\int_{\mathbb{R}^{n}}\left((A(x) \nabla u(x, t))_{\|}+\left(B_{1}\right)_{\|} u(x, t)\right) \cdot \overline{\nabla_{\|} \varphi(x)} d x+\int_{\mathbb{R}^{n}} B_{2}(x) \cdot \nabla u(x, t) \overline{\varphi(x)} d x \\
=\int_{\mathbb{R}^{n}}\left(\vec{A}_{n+1, \cdot}(x) \cdot \partial_{t} \nabla u(x, t)+\left(B_{1}(x)\right)_{\perp} \partial_{t} u(x, t)\right) \overline{\varphi(x)} d x+\int_{\mathbb{R}^{n}} g(x, t) \overline{\varphi(x)} d x
\end{aligned}
$$


holds. If $v, \partial_{t} v \in Y^{1,2}\left(\Sigma_{a}^{b}\right)$, and $\mathcal{L}^{*} v=0$ in $\Sigma_{a}^{b}$ for some $g \in C_{c}^{\infty}\left(\mathbb{R}^{n}\right)$, then for every $t \in(a, b)$ and $\varphi \in W^{1,2}\left(\mathbb{R}^{n}\right)$, the identity

$$
\begin{aligned}
\int_{\mathbb{R}^{n}}\left[\nabla_{\|} \varphi \cdot \overline{\left(\left(\bar{B}_{2}\right)_{\|} v(t)\right)}+\tilde{A} \nabla_{\|} \varphi \cdot \overline{\nabla v(t)}+\right. & \left.B_{1} \varphi \cdot \overline{\nabla v(t)}\right] \\
& =\int_{\mathbb{R}^{n}}\left[\varphi \overline{\varphi\left(\bar{B}_{2}\right)_{\perp} D_{n+1} v(t)}+\varphi \vec{A}_{, n+1} \overline{\nabla D_{n+1} v(t)}\right]
\end{aligned}
$$

holds. Finally, for $v$ and $\varphi$ as above, we also have the identity

$$
\begin{aligned}
\int_{\mathbb{R}^{n}} \nabla_{\|} \varphi \cdot \overline{\left(A^{*} \nabla v(t)\right)_{\|}}=\int_{\mathbb{R}^{n}} \varphi \cdot \overline{A_{n+1,}^{*} D_{n+1} \nabla v(t)} & -\int_{\mathbb{R}^{n}} \nabla_{\|} \varphi \cdot \overline{\left(\bar{B}_{2}\right)_{\|} v(t)} \\
& +\int_{\mathbb{R}^{n}} \varphi \overline{\left(\bar{B}_{2}\right)_{\perp} v(t)}-\int_{\mathbb{R}^{n}} \varphi \overline{\bar{B}_{1} \cdot \nabla v(t)} .
\end{aligned}
$$

Proof. Fix $\varphi \in C_{c}^{\infty}\left(\mathbb{R}^{n}\right)$ and $t \in(a, b)$. Let $\varphi_{\varepsilon}(x, s):=\varphi(x) \eta_{\varepsilon}(t-s)$ with $\varepsilon<\min \{b-t, t-a\}$, and where $\eta_{\varepsilon}(\cdot)=\varepsilon^{-1} \eta(\cdot / \varepsilon), \eta \in C_{c}^{\infty}(-1,1), \int_{\mathbb{R}} \eta=1$. In particular, $\varphi_{\varepsilon} \in C_{c}^{\infty}\left(\Sigma_{a}^{b}\right)$ is an admissible test function in the definition of the weak solution. Thus, from the definition of $\mathcal{L} u=g$, we have that

$$
\begin{gathered}
\iint_{\mathbb{R}^{n+1}}\left\{\left((A(x) \nabla u(x, s))_{\|}+\left(B_{1}\right)_{\|} u(x, s)\right) \cdot \overline{\nabla_{\|} \varphi_{\varepsilon}(x, s)}+B_{2}(x) \cdot \nabla u(x, s) \overline{\varphi_{\varepsilon}(x, s)}\right\} d x d s \\
=\iint_{\mathbb{R}^{n+1}}\left(\vec{A}_{n+1, \cdot}(x) \partial_{s} \nabla u(x, s)+\left(B_{1}(x)\right)_{\perp} \partial_{s} u(x, s)+g(x, s)\right) \overline{\varphi_{\varepsilon}(x, s)} d x d s .
\end{gathered}
$$

Notice, for instance, that the map

$$
t \mapsto \int_{\mathbb{R}^{n}}\left((A(x) \nabla u(x, t))_{\|}+\left(B_{1}\right)_{\|}(x) u(x, t)\right) \cdot \overline{\nabla_{\|} \varphi(x)} d x
$$

is continuous in $(a, b)$, owing to Lemma 2.2.2 and the continuity of the duality pairings in each of its entries. A similar statement holds for all the other integrals. The desired 
conclusion now follows from the fact that for any continuous function $h:(a, b) \rightarrow \mathbb{C}$, we have that $\lim _{\varepsilon \rightarrow 0} \int_{\mathbb{R}} \eta_{\varepsilon}(t-\cdot) h=h(t)$, for each $t \in(a, b)$

As in [AAA $\left.{ }^{+} 11\right]$, the $t$-independence of our coefficients allows us to obtain $L^{p}$ estimates on cubes lying in horizontal slices.

Lemma 2.5.39 ( $L^{p}$ estimates on slices; $\left[\mathrm{AAA}^{+} 11\right.$, Proposition 2.1]). Let $t \in \mathbb{R}, Q \subset \mathbb{R}^{n}$ be a cube, and $I_{Q}$ be the box $I_{Q}=4 Q \times(t-\ell(Q), t+\ell(Q))$. Let $p \geq 2$ with $|p-2|$ small enough that the conclusion of Lemma 2.5.4 holds. Suppose that $u \in W^{1,2}\left(I_{Q}\right)$ satisfies $\mathcal{L} u=0$ in $I_{Q}$. Then the estimates

$$
\left(\frac{1}{|Q|} \int_{Q}|\nabla u(x, t)|^{p}\right)^{1 / p} \lesssim\left(\frac{1}{\left|Q^{*}\right|} \iint_{Q^{*}}|\nabla u(x, t)|^{p}\right)^{1 / p},
$$

and

$$
\left(\frac{1}{|Q|} \int_{Q}|\nabla u(x, t)|^{p}\right)^{1 / p} \lesssim_{p} \frac{1}{\ell(Q)}\left(\frac{1}{\left|Q^{* *}\right|} \iint_{Q^{* *}}|u(x, t)|^{p}\right)^{1 / p}
$$

hold, where $Q^{*}:=2 Q \times(t-\ell(Q) / 4, t+\ell(Q) / 4)$ is an $(n+1)$-dimensional rectangle, and $Q^{* *}:=3 Q \times(t-\ell(Q) / 2, t+\ell(Q) / 2)$ is a slight dilation of $Q^{*}$.

In $\left[\mathrm{AAA}^{+} 11\right]$, the analogue of the preceding lemma is proved in the purely second order case. However, the argument there extends almost verbatim to the present situation, given Lemma 2.5.9. We omit the details.

Let us consider how the shift operator acts on $\mathcal{L}^{-1}$. For each $\tau \in \mathbb{R}$, denote by $\mathscr{T}^{\tau}$ the (positive) shift by $\tau$ in the $t$-direction: If $u \in C_{c}^{\infty}\left(\mathbb{R}^{n+1}\right)$, then $\left(\mathscr{T}^{\tau} u\right)=u(\cdot, \cdot+\tau)$. 
More generally, if $f \in \mathscr{D}^{\prime}$ is a distribution, we define the distribution $\mathscr{T}^{\tau} f$ by $\left\langle\mathscr{T}^{\tau} f, \varphi\right\rangle=$ $\left\langle f, \mathscr{T}^{-\tau} \varphi\right\rangle$, for each $\varphi \in \mathscr{D}$.

Proposition 2.5.42. Suppose that $u \in W_{\text {loc }}^{1,2}\left(\mathbb{R}_{+}^{n+1}\right)$ solves $\mathcal{L} u=0$ in $\mathbb{R}_{+}^{n+1}$. Then

i) Let $f \in\left(Y^{1,2}\left(\mathbb{R}^{n+1}\right)\right)^{*}$ and fix $s \in \mathbb{R}$. Then $\mathscr{T}^{s} \mathcal{L}^{-1} f \in Y^{1,2}\left(\mathbb{R}^{n+1}\right)$ and satisfies $\mathscr{T}^{s} \mathcal{L}^{-1} f=$ $\mathcal{L}^{-1} \mathscr{T}^{s} f$

ii) Let $s>0$. Then $\mathscr{T}^{s} u \in W_{\text {loc }}^{1,2}\left(\mathbb{R}_{+}^{n+1}\right)$ and $\mathcal{L} \mathscr{T}^{s} u=0$ in $\mathbb{R}_{+}^{n+1}$.

iii) We have that $D_{n+1} u \in W_{\mathrm{loc}}^{1,2}\left(\mathbb{R}_{+}^{n+1}\right)$ and $\mathcal{L} D_{n+1} u=0$ in $\mathbb{R}_{+}^{n+1}$.

iv) For any $s>0$, we have that $D_{n+1} \mathscr{T}^{s} u \in Y^{1,2}\left(\mathbb{R}_{+}^{n+1}\right) \cap L^{2}\left(\mathbb{R}_{+}^{n+1}\right)=W^{1,2}\left(\mathbb{R}_{+}^{n+1}\right)$. In particular, for any $t>0$, the trace $\operatorname{Tr}_{t} D_{n+1} u$ is an element of $H^{\frac{1}{2}}\left(\mathbb{R}^{n}\right)=L^{2}\left(\mathbb{R}^{n}\right) \cap H_{0}^{\frac{1}{2}}\left(\mathbb{R}^{n}\right)$. Moreover, for each $t>0$, the estimate

$$
\left\|t \operatorname{Tr}_{t} \nabla \partial_{t} u\right\|_{L^{2}\left(\mathbb{R}^{n}\right)} \lesssim\|u\|_{Y^{1,2}\left(\mathbb{R}_{t / 2}^{n+1}\right)}
$$

holds. In particular, for each $s>0$ we have that

$$
\sup _{t \geq 0}\left\|(t+s) \operatorname{Tr}_{t} \nabla \partial_{t} \mathscr{T}^{s} u\right\|_{L^{2}\left(\mathbb{R}^{n}\right)} \lesssim\|u\|_{Y^{1,2}\left(\mathbb{R}_{+}^{n+1}\right)}
$$

Finally, for each $t>0$ and $\zeta \in H^{-\frac{1}{2}}\left(\mathbb{R}^{n}\right)$, we have the identity

$$
\left(\operatorname{Tr}_{t} D_{n+1} u, \zeta\right)=\frac{d}{d t}\left(\operatorname{Tr}_{t} u, \zeta\right)
$$

Proof. The proofs of i), ii), and iii) are very similar to the proof of Proposition 2.5.35, and are thus omitted. We prove iv), and to this end fix $s>0$. By assumption, it is clear that 
$\mathscr{T}^{s} u \in Y^{1,2}\left(\mathbb{R}_{+}^{n+1}\right)$, and by ii), we have that $\mathcal{L} \mathscr{T}^{s} u=0$ in $\mathbb{R}_{+}^{n+1}$. Hence, by iii), we have that $D_{n+1} \mathscr{T}^{s} u \in W_{\text {loc }}^{1,2}\left(\mathbb{R}_{+}^{n+1}\right)$ and $\mathcal{L} D_{n+1} \mathscr{T}^{s} u=0$ in $\mathbb{R}_{+}^{n+1}$. Let $\mathbb{G}(s / 2)$ be a grid of pairwise disjoint cubes $R \subset \mathbb{R}_{s}^{n+1}$ such that $\mathbb{R}_{s}^{n+1}=\cup_{R \in \mathbb{G}(s / 2)} R$ and $\ell(R)=\frac{s}{2}$. Consider the estimate

$$
\begin{aligned}
& \iint_{\mathbb{R}_{+}^{n+1}}\left|\nabla D_{n+1} \mathscr{T}^{s} u\right|^{2}=\iint_{\mathbb{R}_{s}^{n+1}}\left|\nabla D_{n+1} u\right|^{2}=\sum_{R \in \mathbb{G}(s / 2)} \iint_{R}\left|\nabla D_{n+1} u\right|^{2} \\
& \lesssim \sum_{R \in \mathbb{G}(s / 2)} \frac{1}{s^{2}} \iint_{\tilde{R}}\left|D_{n+1} u\right|^{2} \lesssim \frac{1}{s^{2}}\left\|D_{n+1} u\right\|_{L^{2}\left(\mathbb{R}_{s / 2}^{n+1}\right)}^{2} \leq \frac{1}{s^{2}}\|u\|_{Y^{1,2}\left(\mathbb{R}_{+}^{n+1}\right)}^{2},
\end{aligned}
$$

which proves that $\nabla D_{n+1} \mathscr{T}^{s} u \in L^{2}\left(\mathbb{R}_{+}^{n+1}\right)$. Since $D_{n+1} \mathscr{T}^{s} u \in L^{2}\left(\mathbb{R}_{+}^{n+1}\right)$ by the assumption that $u \in Y^{1,2}\left(\mathbb{R}_{+}^{n+1}\right)$, it is proven that $D_{n+1} \mathscr{T}^{s} u \in W^{1,2}\left(\mathbb{R}_{+}^{n+1}\right)$. Hence, for each $t \geq 0$, $\operatorname{Tr}_{t} D_{n+1} \mathscr{T}^{s} u \in H^{\frac{1}{2}}\left(\mathbb{R}^{n}\right)$. But $\operatorname{Tr}_{t} D_{n+1} \mathscr{T}^{s} u=\operatorname{Tr}_{t+s} D_{n+1} u$. The estimate (2.43) is true by Caccioppoli on slices (Proposition 2.5.39), as follows: break $\mathbb{R}^{n}$ into a grid $\mathbb{G}_{n}(t / 2)$ of cubes $Q \subset \mathbb{R}^{n}, \ell(Q)=t / 2$, and use Caccioppoli on slices in each cube.

It remains to check the identity (2.45), so fix $t>0$. We have seen that $\operatorname{Tr}_{\tau} D_{n+1} u \in$ $H_{0}^{\frac{1}{2}}\left(\mathbb{R}^{n}\right)$ for each $\tau>0$. Fix $\zeta \in H^{-\frac{1}{2}}\left(\mathbb{R}^{n}\right)$, and define $g(\tau):=\left(\operatorname{Tr}_{\tau} u, \zeta\right)$ for each $\tau>0$. We will show that $g$ is differentiable at $t$, and compute its derivative. To this end, observe the calculation

$$
\frac{g(t+h)-g(t)}{h}=\frac{\left(\operatorname{Tr}_{t+h} u, \zeta\right)-\left(\operatorname{Tr}_{t} u, \zeta\right)}{h}=\left(\operatorname{Tr}_{t} \frac{\mathscr{T}^{h} u-u}{h}, \zeta\right)=\left(\operatorname{Tr}_{0} \frac{\mathscr{T}^{h} \mathscr{T}^{t} u-\mathscr{T}^{t} u}{h}, \zeta\right)
$$

By our previous computations, we have that $\frac{\mathscr{T}^{h} \mathscr{T}^{t} u-\mathscr{T}^{t} u}{h} \longrightarrow D_{n+1} \mathscr{T}^{t} u$ in $Y^{1,2}\left(\mathbb{R}_{+}^{n+1}\right)$ as $h \rightarrow$ 0, which implies that $\operatorname{Tr}_{0}\left(\frac{\mathscr{T}^{h} \mathscr{T}^{t} u-\mathscr{T}^{t} u}{h}\right) \longrightarrow \operatorname{Tr}_{0} D_{n+1} \mathscr{T}^{t} u$ in $H_{0}^{\frac{1}{2}}\left(\mathbb{R}^{n}\right)$ as $h \rightarrow 0$, and hence we have that $\frac{g(t+h)-g(t)}{h} \longrightarrow\left(\operatorname{Tr}_{0} D_{n+1} \mathscr{T}^{t} u, \zeta\right)=\left(\operatorname{Tr}_{t} D_{n+1} u, \zeta\right)$ as $h \rightarrow 0$. This finishes the proof. 


\subsubsection{Good Classes of Solutions}

Definition 2.5.46 (Slice Spaces). For $n \geq 3$, we define

$$
D_{+}^{2}:=\left\{v \in C_{0}\left((0, \infty) ; L^{2}\left(\mathbb{R}^{n}\right)\right):\|u\|_{D_{+}^{2}}<\infty\right\}
$$

with norm given by $\|v\|_{D_{+}^{2}}:=\sup _{t>0}\|v(t)\|_{L^{2}\left(\mathbb{R}^{n}\right)}$. We also define

$$
S_{+}^{2}:=\left\{u \in C_{0}^{2}\left((0, \infty) ; Y^{1,2}\left(\mathbb{R}^{n}\right)\right): u^{\prime}(t) \in C_{0}\left((0, \infty) ; L^{2}\left(\mathbb{R}^{n}\right)\right),\|u\|_{S_{+}^{2}}<\infty\right\},
$$

with norm given by

$$
\begin{aligned}
\|u\|_{S_{+}^{2}}:=\sup _{t>0}\|u(t)\|_{Y^{1,2}\left(\mathbb{R}^{n}\right)}+\sup _{t>0}\left\|u^{\prime}(t)\right\|_{L^{2}\left(\mathbb{R}^{n}\right)} & \\
& +\sup _{t>0}\left\|t u^{\prime}(t)\right\|_{Y^{1,2}\left(\mathbb{R}^{n}\right)}+\sup _{t>0}\left\|t^{2} u^{\prime \prime}(t)\right\|_{Y^{1,2}\left(\mathbb{R}^{n}\right)} .
\end{aligned}
$$

In particular, both $D_{+}^{2}$ and $S_{+}^{2}$ are Banach spaces. Similarly, with obvious modifications, we can define the slice spaces $S_{-}^{2}$ and $D_{-}^{2}$ in the negative half line $(-\infty, 0)$.

Definition 2.5.47 (Good $\mathcal{D}$ Solutions). We say that $u \in W_{\text {loc }}^{1,2}\left(\mathbb{R}_{+}^{n+1}\right)$ is a good $\mathcal{D}$ solution if $\mathcal{L} u=0$ in $\mathbb{R}_{+}^{n+1}$ in the weak sense, $u \in D_{+}^{2}$, and $u_{\tau}:=u(\cdot, \cdot+\tau) \in Y^{1,2}\left(\mathbb{R}_{+}^{n+1}\right)$ for any $\tau>0$

Definition 2.5.48 $\left(\operatorname{Good} \mathcal{N} / \mathcal{R}\right.$ Solutions). We say that $u \in W_{\mathrm{loc}}^{1,2}\left(\mathbb{R}_{+}^{n+1}\right)$ is a $\operatorname{good} \mathcal{N} / \mathcal{R}$ solution if $\mathcal{L} u=0$ in $\mathbb{R}_{+}^{n+1}$ in the weak sense, $u \in S_{+}^{2}$, and $\partial_{t} u_{\tau} \in Y^{1,2}\left(\mathbb{R}_{+}^{n+1}\right)$ for every $\tau>0$.

The following result is a companion to Corollary 4.7.18. Together they will imply that our uniqueness statement holds among the two most commonly used classes of solutions (those with either square or nontangential maximal function estimates). 
Lemma 2.5.49. Let $u \in W_{\mathrm{loc}}^{1,2}\left(\mathbb{R}_{+}^{n+1}\right)$ be a solution of $\mathcal{L} u=0$ in $\mathbb{R}_{+}^{n+1}$. The following holds

1. If $\tilde{\mathcal{N}}_{2}(u) \in L^{2}\left(\mathbb{R}^{n}\right)$, then $u$ is a good $\mathcal{D}$ solution (see Definition 2.5.47).

2. If $\tilde{\mathcal{N}}_{2}(\nabla u) \in L^{2}\left(\mathbb{R}^{n}\right)$ then either $u$ is a good $\mathcal{N} / \mathcal{R}$ solution (see Definition 2.5.48) if $\mathcal{L} 1 \neq 0$, or there exists a constant $c \in \mathbb{C}$ such that $u-c$ is a good $\mathcal{N} / \mathcal{R}$ solution if $\mathcal{L} 1=0$.

Proof. As will be seen from the proof, (1) will follow the same outline as (2), and is a bit easier. We first prove that

$$
\sup _{t>0}\|\nabla u(\cdot, t)\|_{L^{2}\left(\mathbb{R}^{n}\right)} \lesssim\left\|\tilde{\mathcal{N}}_{2}(\nabla u)\right\|_{L^{2}\left(\mathbb{R}^{n}\right)}
$$

Fix $t>0$ and let $\psi: \mathbb{R} \rightarrow \mathbb{R}$ be a nonnegative Lipschitz cutoff function such that $\psi(t)=1, \psi(3 t / 4)=0$, and $\left|\psi^{\prime}(s)\right| \leq 4 / t$ for each $s \in \mathbb{R}$. We make the computation

$$
\begin{aligned}
& \|\nabla u(\cdot, t)\|_{2}^{2}=\int_{\mathbb{R}^{n}}|\nabla u(\cdot, t)|^{2} \psi(t)=\int_{\mathbb{R}^{n}}|\nabla u(\cdot, t)|^{2} \psi(t)-\int_{\mathbb{R}^{n}}|\nabla u(\cdot, 3 t / 4)|^{2} \psi(3 t / 4) \\
& =\int_{\mathbb{R}^{n}} \int_{3 t / 4}^{t} \partial_{s}\left[|\nabla u(x, s)|^{2} \psi(s)\right] d s d x \\
& \leq \int_{\mathbb{R}^{n}} \int_{3 t / 4}^{t}\left[2|\nabla u(x, s)|\left|\nabla \partial_{s} u(x, s)\right| \psi(s)+|\nabla u(x, s)|^{2}\left|\psi^{\prime}(s)\right|\right] d s d x \\
& \leq 2 \int_{\mathbb{R}^{n}} f_{3 t / 4}^{t}|\nabla u(x, s)|^{2} d s d x+\frac{t^{2}}{16} \int_{\mathbb{R}^{n}} f_{3 t / 4}^{t}\left|\nabla \partial_{s} u(x, s)\right|^{2} d s d x=: I+I I,
\end{aligned}
$$

where in the third equality we used the fundamental theorem of calculus and in the last line we used the Cauchy inequality with $\varepsilon>0$. We now use Fubini's theorem to see that

$$
I=2 \int_{\mathbb{R}^{n}} f_{\left|y-x_{0}\right|<t} f_{3 t / 4}^{t}|\nabla u(y, s)|^{2} d s d x_{0} d y \leq 8 \int_{\mathbb{R}^{n}} \int_{\substack{\left|x_{0}-y\right|<t \\|s-t|<t / 2}}|\nabla u(y, s)|^{2} d s d y d x_{0}
$$




$$
\leq 8 \int_{\mathbb{R}^{n}} \sup _{(x, \tau) \in \gamma\left(x_{0}\right)}\left(\iint_{\substack{|x-y|<\tau \\|s-\tau|<\tau / 2}}|\nabla u(y, s)|^{2} d s d y\right) d x_{0}=8\left\|\tilde{\mathcal{N}}_{2}(\nabla u)\right\|_{2}^{2} .
$$

It remains to control $I I$; for this we will use the Caccioppoli inequality as follows:

$$
\begin{aligned}
& I I \leq \frac{t^{2}}{16} \int_{\mathbb{R}^{n}} f_{\left|y-x_{0}\right|<t / 2} f_{3 t / 4}^{t}\left|\nabla \partial_{s} u(y, s)\right|^{2} d s d x_{0} d y \lesssim \\
& \qquad \int_{\mathbb{R}^{n}} f_{\left|x_{0}-y\right|<t} f_{t / 2}^{5 t / 4}\left|\partial_{s} u(x, s)\right|^{2} d s d y d x_{0},
\end{aligned}
$$

and thus it is clear that we may handle $I I$ as above. We have obtained that

$$
\|\nabla u(\cdot, t)\|_{2} \lesssim\left\|\tilde{\mathcal{N}}_{2}(\nabla u)\right\|_{2}, \quad \text { for each } t>0 .
$$

Taking supremum over $t>0$ yields the desired result.

We now improve this to

$$
\lim _{t \rightarrow \infty}\|\nabla u(\cdot, t)\|_{L^{2}\left(\mathbb{R}^{n}\right)}=0,
$$

where $\nabla=\left(\nabla_{\|}, \partial_{t}\right)$ is the full gradient in $n+1$ variables. This follows from the above estimate on slices: Notice that the proof actually gives that

$$
\|\nabla u(\cdot, t)\|_{L^{2}\left(\mathbb{R}^{n}\right)} \lesssim\left\|\tilde{\mathcal{N}}_{2}^{(t)}(\nabla u)\right\|_{L^{2}\left(\mathbb{R}^{n}\right)},
$$

where we use the truncated nontangential maximal function (see Definition 2.4.7) on the right hand side.

We claim now that $\tilde{\mathcal{N}}_{2}^{(t)}(\nabla u)(x) \rightarrow 0$ for every $x \in \mathbb{R}^{n}$ as $t \rightarrow \infty$. To see this, assume to the contrary that

$$
\liminf _{t \rightarrow \infty} \tilde{\mathcal{N}}_{2}^{(t)}(\nabla u)(x)>\eta>0
$$


for some $x \in \mathbb{R}^{n}$. This means there exists a sequence $t_{k} \rightarrow \infty$ and points $x_{k}$ with $\left|x-x_{k}\right|<t$ such that

$$
\iint_{C_{x_{k}, t_{k}}}|\nabla u(y, s)|^{2} d y d s>\eta^{2} .
$$

By the definition of the nontangential maximal function we then have

$$
\tilde{\mathcal{N}}_{2}(\nabla u)(z)^{2} \geq \iint_{C_{x_{k}, t_{k}}}|\nabla u(y, s)|^{2} d y d s>\eta^{2},
$$

for every $z \in \mathbb{R}^{n}$ such that $\left|z-x_{k}\right|<t_{k}$. Integrating over this set gives

$$
\left\|\tilde{\mathcal{N}}_{2}(\nabla u)\right\|_{L^{2}\left(\mathbb{R}^{n}\right)}^{2} \geq \int_{\left|z-x_{k}\right|<t_{k}} \tilde{\mathcal{N}}_{2}(\nabla u)(z)^{2} d z \geq c_{n} \eta^{2} t_{k}^{n}
$$

Since $t_{k} \rightarrow \infty$, this contradicts our assumption that $\tilde{\mathcal{N}}_{2}(\nabla u) \in L^{2}\left(\mathbb{R}^{n}\right)$.

The claim now proved, and since $\tilde{\mathcal{N}}_{2}^{(t)}(\nabla u) \leq \tilde{\mathcal{N}}_{2}(\nabla u)$ by definition, the dominated convergence theorem gives

$$
\|\nabla u(\cdot, t)\|_{L^{2}\left(\mathbb{R}^{n}\right)} \lesssim\left\|\tilde{\mathcal{N}}_{2}^{(t)}(\nabla u)\right\|_{L^{2}\left(\mathbb{R}^{n}\right)} \rightarrow 0,
$$

as $t \rightarrow \infty$.

Appealing to Caccioppoli's inequality and the above, together with Proposition 4.7.14, we see that $u \in S_{+}^{2}$ when $\mathcal{L} 1 \neq 0$. If $\mathcal{L} 1=0$, we proceed as follows: First, by the sup on slices estimate above and Caccioppoli's inequality on slices we see that $\partial_{t} u(\cdot, t) \in W^{1,2}\left(\mathbb{R}^{n}\right)$ for every $t>0$; in particular

$$
\int_{s}^{t} \partial_{\tau} u(\cdot, \tau) d \tau \in W^{1,2}\left(\mathbb{R}^{n}\right) \subset Y^{1,2}\left(\mathbb{R}^{n}\right), \quad \forall 0<s<t<\infty
$$


On the other hand, again by the sup on slices and Lemma 2.2.1, we have that for every $t>0$ there exists a constant $c_{t} \in \mathbb{C}$ such that $u(\cdot, t)-c_{t} \in Y^{1,2}\left(\mathbb{R}^{n}\right)$. Therefore, by the fundamental theorem of calculus, for any $0<s<t<\infty$,

$$
\int_{s}^{t} \partial_{\tau} u(\cdot, \tau) d \tau-\left(c_{t}-c_{s}\right)=u(\cdot, t)-c_{t}-\left[u(\cdot, s)-c_{s}\right] \in Y^{1,2}\left(\mathbb{R}^{n}\right)
$$

We conclude $c_{t}=c_{s}=c$ as desired, and so $u-c \in S_{+}^{2}$.

Finally we show that $\partial_{t} u_{\tau}:=\partial_{t} u(\cdot, \cdot+\tau) \in Y^{1,2}\left(\mathbb{R}_{+}^{n+1}\right)$ for every $\tau>0$. For this we simply compute, decomposing $\mathbb{R}^{n}$ into cubes in $\mathbb{D}_{s}$ and using Caccioppoli's inequality on slices together with Fubini's Theorem

$$
\iint_{\mathbb{R}_{+}^{n+1}}\left|\nabla \partial_{t} u(y, s+\tau)\right|^{2} d y d s=\int_{\tau}^{\infty} \int_{\mathbb{R}^{n}}\left|\nabla \partial_{t} u(y, s)\right|^{2} d y d s \lesssim \sup _{t>0}\|\nabla u(\cdot, t)\|_{L^{2}\left(\mathbb{R}^{n}\right)} \int_{\tau}^{\infty} \frac{d s}{s^{2}}<\infty
$$

For (1) we run the same argument with $u$ in place of $\nabla u$. 


\section{Chapter 3}

\section{Layer potentials: Definitions and basic properties}

\subsection{Basic Definitions}

In this section, we develop the abstract layer potential theory. Our methods often closely follow the constructions of Ariel Barton [Bar17]; but see also [Ros13].

Definition 3.1.1 (Single layer potential). Define the single layer potential of $\mathcal{L}$ as the operator $\mathcal{S}^{\mathcal{L}}: H^{-\frac{1}{2}}\left(\mathbb{R}^{n}\right) \rightarrow Y^{1,2}\left(\mathbb{R}^{n+1}\right)$ given by $\mathcal{S}^{\mathcal{L}}:=\left(\operatorname{Tr}_{0} \circ\left(\mathcal{L}^{-1}\right)^{*}\right)^{*}$, which is well-defined by virtue of Lemma 2.2.6 and Proposition 2.3.3. For $t \in \mathbb{R}$, we denote $\mathcal{S}_{t}^{\mathcal{L}}:=\operatorname{Tr}_{t} \circ \mathcal{S}^{\mathcal{L}}$. When the operator under consideration is clear from the context, we will sometimes drop the superscript, so that we write $\mathcal{S}=\mathcal{S}^{\mathcal{L}}$. For each $t \in \mathbb{R}, \mathbf{f}: \mathbb{R}^{n} \rightarrow \mathbb{C}^{n+1}$ and $\vec{f}: \mathbb{R}^{n} \rightarrow \mathbb{C}^{n}$, define $\left(\mathcal{S}_{t}^{\mathcal{L}} \nabla_{\|}\right) \vec{f}:=-\mathcal{S}_{t}^{\mathcal{L}}(\operatorname{div} \vec{f}), \mathcal{S}_{t}^{\mathcal{L}} D_{n+1}:=-\partial_{t} \mathcal{S}_{t}^{\mathcal{L}}$, and $\left(\mathcal{S}_{t}^{\mathcal{L}} \nabla\right) \mathbf{f}=\left(\mathcal{S}_{t}^{\mathcal{L}} \nabla_{\|}\right) \mathbf{f}_{\|}+\mathcal{S}_{t}^{\mathcal{L}} D_{n+1} \mathbf{f}_{n+1}$.

Let us elucidate a few properties of the single layer potential.

Proposition 3.1.2 (Properties of the single layer potential). Fix $\gamma \in H^{-\frac{1}{2}}\left(\mathbb{R}^{n}\right)$. The following statements hold. 
i) The function $\mathcal{S}^{\mathcal{L}} \gamma \in Y^{1,2}\left(\mathbb{R}^{n+1}\right)$ is the unique element in $Y^{1,2}\left(\mathbb{R}^{n+1}\right)$ such that

$$
B_{\mathcal{L}}\left[\mathcal{S}^{\mathcal{L}} \gamma, \Phi\right]=\left\langle\gamma, \operatorname{Tr}_{0} \Phi\right\rangle, \quad \text { for all } \Phi \in Y^{1,2}\left(\mathbb{R}^{n+1}\right)
$$

Accordingly, $\mathcal{S}^{L}$ is a bounded linear operator.

ii) The function $\mathcal{S}^{\mathcal{L}} \gamma$ satisfies $\mathcal{L} \mathcal{S}^{\mathcal{L}} \gamma=0$ in $\Omega$, where $\Omega=\mathbb{R}_{+}^{n+1}, \mathbb{R}_{-}^{n+1}$.

iii) Suppose that $\gamma$ has compact support. Then $\mathcal{L S}^{\mathcal{L}} \gamma=0$ in $\mathbb{R}^{n+1} \backslash \operatorname{supp} \gamma$.

iv) Define $p_{-}, p_{+}$as in Proposition 2.2.4 and suppose that $\gamma \in L^{p_{-}}\left(\mathbb{R}^{n}\right)$. Then the bound $\left\|\operatorname{Tr}_{t} \mathcal{S}^{\mathcal{L}} \gamma\right\|_{L^{p+}\left(\mathbb{R}^{n}\right)} \lesssim\|\gamma\|_{L^{p-\left(\mathbb{R}^{n}\right)}}$ holds for each $t \in \mathbb{R}$

v) For each $t \in \mathbb{R}$, the operators $\mathcal{S}_{t}^{\mathcal{L}}$ and $\mathcal{S}_{-t}^{\mathcal{L}^{*}}$ are adjoint. That is, for each $\gamma, \psi \in H^{-\frac{1}{2}}\left(\mathbb{R}^{n}\right)$, the identity $\left\langle\mathcal{S}_{t}^{\mathcal{L}} \gamma, \psi\right\rangle=\left\langle\gamma, \mathcal{S}_{-t}^{\mathcal{L}^{*}} \psi\right\rangle$ holds.

vi) For each $t \in \mathbb{R}$, we have the characterization

$$
\mathscr{T}^{-t} \mathcal{S}^{\mathcal{L}} \gamma=\left(\operatorname{Tr}_{t} \circ\left(\mathcal{L}^{-1}\right)^{*}\right)^{*}
$$

vii) For each $t \in \mathbb{R} \backslash\{0\}$, we have that $\operatorname{Tr}_{t} D_{n+1} \mathcal{S}^{\mathcal{L}} \gamma \in H_{0}^{\frac{1}{2}}\left(\mathbb{R}^{n}\right)$. Moreover, for each $t \in \mathbb{R} \backslash\{0\}$ and each $\zeta \in H^{-\frac{1}{2}}\left(\mathbb{R}^{n}\right)$, we have that $\left\langle\operatorname{Tr}_{t} D_{n+1} \mathcal{S}^{\mathcal{L}} \gamma, \zeta\right\rangle=\frac{d}{d t}\left\langle\mathcal{S}_{t}^{\mathcal{L}} \gamma, \zeta\right\rangle=-\left\langle\gamma, \operatorname{Tr}_{-t} D_{n+1} \mathcal{S}^{\mathcal{L}^{*}} \zeta\right\rangle$.

viii) Let $t \in \mathbb{R} \backslash\{0\}$. Let $\mathbf{g}=\left(\overrightarrow{g_{\|}}, g_{\perp}\right): \mathbb{R}^{n} \rightarrow \mathbb{C}^{n+1}$ be such that $g_{\|} \in H_{0}^{\frac{1}{2}}\left(\mathbb{R}^{n}\right)$ (so that in particular $\operatorname{div}_{\|} \overrightarrow{g_{\|}} \in H^{-\frac{1}{2}}\left(\mathbb{R}^{n}\right)$ by Proposition 2.2.5) and $g_{\perp} \in H^{-\frac{1}{2}}\left(\mathbb{R}^{n}\right)$. In the sense of distributions, we have the adjoint relation

$$
\left\langle\nabla \mathcal{S}_{t}^{\mathcal{L}} \gamma, \mathbf{g}\right\rangle_{\mathscr{D}^{\prime}, \mathscr{D}}=\left\langle\gamma,\left(\mathcal{S}_{-t}^{\mathcal{L}^{*}} \nabla\right) \mathbf{g}\right\rangle_{H^{-\frac{1}{2}}\left(\mathbb{R}^{n}\right), H_{0}^{\frac{1}{2}}\left(\mathbb{R}^{n}\right)}
$$


Proof. Fix $\gamma \in H^{-\frac{1}{2}}\left(\mathbb{R}^{n}\right)$. Proof of i). Since $\operatorname{Tr}_{0}: Y^{1,2}\left(\mathbb{R}^{n+1}\right) \rightarrow H_{0}^{\frac{1}{2}}\left(\mathbb{R}^{n}\right)$ is a bounded linear operator, then $T_{\gamma}:=\left\langle\gamma, \operatorname{Tr}_{0} \cdot\right\rangle$ is a bounded linear functional on $Y^{1,2}\left(\mathbb{R}^{n+1}\right)$. By the LaxMilgram theorem, there exists a unique $u_{\gamma} \in Y^{1,2}\left(\mathbb{R}^{n+1}\right)$ such that $B_{\mathcal{L}}\left[u_{\gamma}, \Phi\right]=\left\langle T_{\gamma}, \Phi\right\rangle=$ $\left\langle\gamma, \operatorname{Tr}_{0} \Phi\right\rangle$, for all $\Phi \in Y^{1,2}\left(\mathbb{R}^{n+1}\right)$. Now let $\Psi \in\left(Y^{1,2}\left(\mathbb{R}^{n+1}\right)\right)^{*}$ be arbitrary, and observe that

$$
\begin{aligned}
&\left\langle\Psi, \mathcal{S}^{\mathcal{L}} \gamma\right\rangle=\left\langle\Psi,\left(\operatorname{Tr}_{0} \circ\left(\mathcal{L}^{-1}\right)^{*}\right)^{*} \gamma\right\rangle=\left\langle\operatorname{Tr}_{0} \circ\left(\mathcal{L}^{-1}\right)^{*} \Psi, \gamma\right\rangle=\overline{\left\langle T_{\gamma},\left(\mathcal{L}^{*}\right)^{-1} \Psi\right\rangle} \\
&=\overline{B_{\mathcal{L}}\left[u_{\gamma},\left(\mathcal{L}^{*}\right)^{-1} \Psi\right]}=\overline{\left\langle\mathcal{L} u_{\gamma},\left(\mathcal{L}^{*}\right)^{-1} \Psi\right\rangle}=\overline{\left\langle u_{\gamma}, \Psi\right\rangle}=\left\langle\Psi, u_{\gamma}\right\rangle .
\end{aligned}
$$

Proof of ii). Let $\Phi \in C_{c}^{\infty}\left(\mathbb{R}_{+}^{n+1}\right)$, and let $\tilde{\Phi}$ be an extension of $\Phi$ to $C_{c}^{\infty}\left(\mathbb{R}^{n+1}\right)$ with $\tilde{\Phi} \equiv 0$ on $\mathbb{R}^{n+1} \backslash \operatorname{supp} \Phi$. In particular, $\operatorname{Tr}_{0} \tilde{\Phi} \equiv 0$. Then (3.3) gives that $B_{\mathcal{L}}\left[\mathcal{S}^{\mathcal{L}} \gamma, \Phi\right]=$ $B_{\mathcal{L}}\left[\mathcal{S}^{\mathcal{L}} \gamma, \tilde{\Phi}\right]=0$. Since $\Phi$ was arbitrary, the claim follows.

Proof of iii). Let $\Omega:=\mathbb{R}^{n+1} \backslash \operatorname{supp} \gamma$, and let $\Phi \in C_{c}^{\infty}(\Omega)$. Let $\tilde{\Phi}$ be an extension of $\Phi$ to $C_{c}^{\infty}\left(\mathbb{R}^{n+1}\right)$ with $\tilde{\Phi} \equiv 0$ on $\mathbb{R}^{n+1} \backslash \operatorname{supp} \gamma$. In particular, the supports of $\tilde{\Phi}$ and $\gamma$ are disjoint. It follows that $\left\langle\gamma, \operatorname{Tr}_{0} \tilde{\Phi}\right\rangle=0$. Using (3.3) now yields the result.

Proof of iv). By the boundedness of $\mathcal{S}^{\mathcal{L}}$ and the Sobolev embeddings, we have that

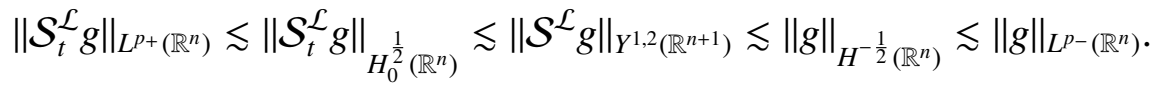

Proof of v). Fix $t \in \mathbb{R}$ and $\gamma, \zeta \in H^{-\frac{1}{2}}\left(\mathbb{R}^{n}\right)$. By the Lax-Milgram theorem, there exists a unique $v^{\zeta, t} \in Y^{1,2}\left(\mathbb{R}^{n+1}\right)$ such that $B_{\mathcal{L}^{*}}\left[\nu^{\zeta, t}, \Phi\right]=\left\langle\zeta, \operatorname{Tr}_{t} \Phi\right\rangle$, for all $\Phi \in Y^{1,2}\left(\mathbb{R}^{n+1}\right)$. Observe that

$$
\left\langle\operatorname{Tr}_{t} \mathcal{S}^{\mathcal{L}} \gamma, \zeta\right\rangle=\overline{\left\langle\zeta, \operatorname{Tr}_{t} \mathcal{S}^{\mathcal{L}} \gamma\right\rangle}=\overline{B_{\mathcal{L}^{*}}\left[v^{\zeta, t}, \mathcal{S}^{\mathcal{L}} \gamma\right]}=B_{\mathcal{L}}\left[\mathcal{S}^{\mathcal{L}} \gamma, \nu^{\zeta, t}\right]=\left\langle\gamma, \operatorname{Tr}_{0} v^{\zeta, t}\right\rangle
$$


Thus it suffices to show that $\operatorname{Tr}_{0} v^{\zeta, t}$ and $\mathcal{S}_{-t}^{\mathcal{L}^{*}} \zeta$ coincide as elements in $H_{0}^{\frac{1}{2}}\left(\mathbb{R}^{n}\right)$. In turn, this will follow if we prove that $\mathcal{S}^{\mathcal{L}^{*}} \zeta=\mathscr{T}^{t} v^{\zeta, t}=v^{\zeta, t}(\cdot, \cdot+t)$, in $Y^{1,2}\left(\mathbb{R}^{n+1}\right)$. Let $\Phi \in Y^{1,2}\left(\mathbb{R}^{n+1}\right)$ be arbitrary. Note then that $\mathscr{T}^{t} \Phi$ also lies in $Y^{1,2}\left(\mathbb{R}^{n+1}\right)$. By the $t$-independence of the coefficients of $\mathcal{L}$ and a change of variables we have that

$$
B_{\mathcal{L}^{*}}\left[\mathscr{T}^{t} v^{\zeta, t}, \mathscr{T}^{t} \Phi\right]=B_{\mathcal{L}^{*}}\left[v^{\zeta, t}, \Phi\right]=\left\langle\gamma, \operatorname{Tr}_{t} \Phi\right\rangle=\left\langle\gamma, \operatorname{Tr}_{0} \mathscr{T}^{t} \Phi\right\rangle
$$

By (3.3) with $\mathcal{L}$ replaced by $\mathcal{L}^{*}$ throughout, $\mathcal{S}^{\mathcal{L}^{*} \zeta}$ is the unique element of $Y^{1,2}\left(\mathbb{R}^{n+1}\right)$ for which the above identity can hold for all $\Phi \in Y^{1,2}\left(\mathbb{R}^{n+1}\right)$, as desired.

Proof of vi). In v), we proved that for each $\gamma \in H^{-\frac{1}{2}}\left(\mathbb{R}^{n}\right), \mathcal{S}^{\mathcal{L}} \gamma=\mathscr{T}^{t} \mathcal{L}^{-1}\left(T_{\gamma}^{t}\right)$, where $T_{\gamma}^{t} \in\left(Y^{1,2}\left(\mathbb{R}^{n+1}\right)\right)^{*}$ is given by $\left\langle T_{\gamma}^{t}, \Phi\right\rangle=\left\langle\gamma, \operatorname{Tr}_{t} \Phi\right\rangle$ for $\Phi \in Y^{1,2}\left(\mathbb{R}^{n+1}\right)$. Hence $\mathscr{T}^{-t} \mathcal{S}^{\mathcal{L}} \gamma=$ $\mathcal{L}^{-1}\left(T_{\gamma}^{t}\right)$. Reproduce the proof of i) in reverse to obtain the claim.

Proof of vii). Let $t>0$ (the case $t<0$ is analogous). By ii) we have that $\mathcal{L S}^{\mathcal{L}} \gamma=0$ in $\mathbb{R}_{+}^{n+1}$. Therefore, using Proposition 2.5.42 iv) we have that $\operatorname{Tr}_{\tau} D_{n+1} \mathcal{S}^{\mathcal{L}} \gamma \in H_{0}^{\frac{1}{2}}\left(\mathbb{R}^{n}\right)$ for each $\tau>0$. Using (2.45) and v), we calculate that

$$
\begin{aligned}
& \left.\frac{d}{d \tau}\left\langle\operatorname{Tr}_{\tau} \mathcal{S}^{\mathcal{L}} \gamma, \zeta\right\rangle\right|_{\tau=t}=\left.\overline{\frac{d}{d \tau}\left\langle\zeta, \operatorname{Tr}_{\tau} \mathcal{S}^{\mathcal{L}} \gamma\right\rangle}\right|_{\tau=t}=\left.\overline{\frac{d}{d \tau}\left\langle\operatorname{Tr}_{-\tau} \mathcal{S}^{\mathcal{L}} \zeta, \gamma\right\rangle}\right|_{\tau=t} \\
& =-\left.\overline{\frac{d}{d(-\tau)}\left\langle\operatorname{Tr}_{-\tau} \mathcal{S}^{\left.\mathcal{L}^{*} \zeta, \gamma\right\rangle}\right.}\right|_{-\tau=-t}=-\overline{\left\langle\operatorname{Tr}_{-t} D_{n+1} \mathcal{S}^{\left.\mathcal{L}^{*} \zeta, \gamma\right\rangle}\right.}=-\left\langle\gamma, \operatorname{Tr}_{-t} D_{n+1} \mathcal{S}^{\mathcal{L}^{*}} \zeta\right\rangle .
\end{aligned}
$$

Proof of viii). It is clear by an easy induction procedure that (vii) holds for higher $t$-derivatives in the expected manner. Now observe the calculation

$$
\left\langle\nabla \mathcal{S}_{t}^{\mathcal{L}} \gamma, \mathbf{g}\right\rangle_{\mathscr{D}^{\prime}, \mathscr{D}}=\left\langle\nabla_{\|} \mathcal{S}_{t}^{\mathcal{L}} \gamma, \overrightarrow{g_{\|}}\right\rangle_{\mathscr{D}^{\prime}, \mathscr{D}}+\left\langle\operatorname{Tr}_{t} D_{n+1} \mathcal{S}^{\mathcal{L}} \gamma, g_{\perp}\right\rangle_{\mathscr{D}^{\prime}, \mathscr{D}}
$$




$$
\begin{aligned}
=- & \left\langle\mathcal{S}_{t}^{\mathcal{L}} \gamma, \operatorname{div} \overrightarrow{g_{\|}}\right\rangle_{\mathscr{D}^{\prime}, \mathscr{D}}-\left\langle\gamma, \operatorname{Tr}_{-t} D_{n+1} \mathcal{S}^{\mathcal{L}^{*}} g_{\perp}\right\rangle_{H^{-\frac{1}{2}}\left(\mathbb{R}^{n}\right), H_{0}^{\frac{1}{2}}\left(\mathbb{R}^{n}\right)} \\
& =-\left\langle\gamma, \mathcal{S}_{-t}^{\mathcal{L}^{*}} \operatorname{div} \overrightarrow{g_{\|}}\right\rangle_{H^{-\frac{1}{2}}\left(\mathbb{R}^{n}\right), H_{0}^{\frac{1}{2}}\left(\mathbb{R}^{n}\right)}+\left\langle\gamma,\left(\mathcal{S}_{-t}^{\mathcal{L}^{*}} D_{n+1}\right) g_{\perp}\right\rangle_{H^{-\frac{1}{2}}\left(\mathbb{R}^{n}\right), H_{0}^{\frac{1}{2}}\left(\mathbb{R}^{n}\right)}=\left\langle\gamma,\left(\mathcal{S}_{-t}^{\mathcal{L}^{*}} \nabla\right) \mathbf{g}\right\rangle .
\end{aligned}
$$

In preparation for defining the double layer potential, let us make the following remark.

Remark. Given $\varphi \in H_{0}^{\frac{1}{2}}\left(\mathbb{R}^{n}\right)$, there exists $\Phi \in Y^{1,2}\left(\mathbb{R}^{n+1}\right)$ with $\operatorname{Tr}_{0} \Phi=\varphi$ and

$$
\|\Phi\|_{Y^{1,2}\left(\mathbb{R}^{n+1}\right)} \lesssim\|\varphi\|_{H_{0}^{\frac{1}{2}\left(\mathbb{R}^{n}\right)}}
$$

For a fixed $u \in Y^{1,2}\left(\mathbb{R}_{+}^{n+1}\right)$, let $\mathscr{F}_{u}^{+}$be the functional on $Y^{1,2}\left(\mathbb{R}^{n+1}\right)$ defined by

$$
\left\langle\mathscr{F}_{u}^{+}, v\right\rangle:=B_{\mathcal{L}, \mathbb{R}_{+}^{n+1}}[u, v]=\iint_{\mathbb{R}_{+}^{n+1}}\left[A \nabla u \cdot \overline{\nabla v}+B_{1} u \cdot \overline{\nabla v}+B_{2} \cdot \nabla u \bar{v}\right],
$$

for each $v \in Y^{1,2}\left(\mathbb{R}^{n+1}\right)$. Then $\mathscr{F}_{u}^{+}$is clearly bounded on $Y^{1,2}\left(\mathbb{R}^{n+1}\right)$. We define $B_{\mathcal{L}_{,} \mathbb{R}_{-}^{n+1}}$ and $\mathscr{F}_{u}^{-}$in a similar way (using $\mathbb{R}_{-}^{n+1}$ instead of $\mathbb{R}_{+}^{n+1}$ ), and we note that if $u \in Y^{1,2}\left(\mathbb{R}^{n+1}\right)$, then $\mathcal{L} u=\mathscr{F}_{u}^{+}+\mathscr{F}_{u}^{-}$.

Definition 3.1.6 (Double layer potential). Given $\varphi \in H_{0}^{\frac{1}{2}}\left(\mathbb{R}^{n}\right)$, let $\Phi \in Y^{1,2}\left(\mathbb{R}^{n+1}\right)$ be any extension of $\varphi$ to $\mathbb{R}^{n+1}$. Define $\mathcal{D}^{\mathcal{L},+}(\varphi):=-\left.\Phi\right|_{\mathbb{R}_{+}^{n+1}}+\left.\mathcal{L}^{-1}\left(\mathscr{F}_{\Phi}^{+}\right)\right|_{\mathbb{R}_{+}^{n+1}}$ (see below for a proof that this is well-defined). We call the operator $\mathcal{D}^{\mathcal{L},+}: H_{0}^{\frac{1}{2}}\left(\mathbb{R}^{n}\right) \rightarrow Y^{1,2}\left(\mathbb{R}_{+}^{n+1}\right)$ the double layer potential associated to operator $\mathcal{L}$ on the upper half-space. Analogously, we define $\mathcal{D}^{\mathcal{L},-}$, the double-layer potential associated to operator $\mathcal{L}$ on the lower half-space, by extending $\varphi$ to $\mathbb{R}_{-}^{n+1}$. We define $\mathcal{D}^{\mathcal{L}^{*}, \pm}$ similarly, by replacing $\mathcal{L}$ with $\mathcal{L}^{*}$.

Proposition 3.1.7 (Properties of the double layer). Fix $\varphi \in H_{0}^{\frac{1}{2}}\left(\mathbb{R}^{n}\right)$ and let $\Phi$ be any $Y^{1,2}\left(\mathbb{R}^{n+1}\right)$-extension of $\varphi$ to $\mathbb{R}^{n+1}$ with $\operatorname{Tr}_{0} \Phi=\varphi$. The following statements hold. 
i) The double layer potential $\mathcal{D}^{\mathcal{L},+}$ is well-defined.

ii) We have the characterizations

$$
\mathcal{D}^{\mathcal{L},+} \varphi=-\left.\mathcal{L}^{-1}\left(\mathscr{F}_{\Phi}^{-}\right)\right|_{\mathbb{R}_{+}^{n+1}}, \quad \mathcal{D}^{\mathcal{L},-} \varphi=-\left.\mathcal{L}^{-1}\left(\mathscr{F}_{\Phi}^{+}\right)\right|_{\mathbb{R}_{-}^{n+1}} .
$$

iii) The bound $\left\|\mathcal{D}^{\mathcal{L},+} \varphi\right\|_{Y^{1,2\left(\mathbb{R}_{+}^{n+1}\right)}} \lesssim\|\varphi\|_{H_{0}^{\frac{1}{2}\left(\mathbb{R}^{n}\right)}}$ holds.

iv) The function $\mathcal{D}^{\mathcal{L},+} \varphi$ satisfies $\mathcal{L} \mathcal{D}^{\mathcal{L},+} \varphi=0$ in the weak sense in $\mathbb{R}_{+}^{n+1}$.

Proof. Proof of i). Let $\Phi, \Phi^{\prime} \in Y^{1,2}\left(\mathbb{R}^{n+1}\right)$ be any two extensions of $\varphi$ to $\mathbb{R}^{n+1}$. Then $\left(\Phi-\Phi^{\prime}\right)(\cdot, 0)=0$. If $w$ is defined as $\left.w\right|_{\mathbb{R}_{+}^{n+1}}=\Phi-\Phi^{\prime}$ with $w_{\mathbb{R}_{+}^{n+1}} \equiv 0$, then $w \in Y^{1,2}\left(\mathbb{R}^{n+1}\right)$. Thus observe that $\langle\mathcal{L} w, \Psi\rangle=B_{\mathcal{L}}[w, \Psi]=\left\langle\mathscr{F}_{\Phi-\Phi^{\prime}}^{+}, \Psi\right\rangle$, for all $\Psi \in Y^{1,2}\left(\mathbb{R}^{n+1}\right)$, whence we conclude that $w=\mathcal{L}^{-1}\left(\mathscr{F}_{\Phi-\Phi^{\prime}}^{+}\right)$. Hence

$$
\begin{aligned}
& {\left[-\Phi+\mathcal{L}^{-1}\left(\mathscr{F}_{\Phi}^{+}\right)\right]_{\mathbb{R}_{+}^{n+1}}-\left[-\Phi^{\prime}+\mathcal{L}^{-1}\left(\mathscr{F}_{\Phi^{\prime}}^{+}\right)\right]_{\mathbb{R}_{+}^{n+1}}} \\
& =\left[\Phi^{\prime}-\Phi+\mathcal{L}^{-1}\left(\mathscr{F}_{\Phi}^{+}-\mathscr{F}_{\Phi^{\prime}}^{+}\right)\right]_{\mathbb{R}_{+}^{n+1}}=\left[\Phi^{\prime}-\Phi+\mathcal{L}^{-1}\left(\mathscr{F}_{\Phi-\Phi^{\prime}}^{+}\right)\right]_{\mathbb{R}_{+}^{n+1}} \equiv 0 .
\end{aligned}
$$

Proof of ii). Observe the computation

$$
\mathcal{D}^{\mathcal{L},+} \varphi=\left[-\Phi+\mathcal{L}^{-1}\left(\mathscr{F}_{\Phi}^{+}\right)\right]_{\mathbb{R}_{+}^{n+1}}=\left[\mathcal{L}^{-1}\left(-\mathcal{L} \Phi+\mathscr{F}_{\Phi}^{+}\right)\right]_{\mathbb{R}_{+}^{n+1}}=\left[\mathcal{L}^{-1}\left(-\mathscr{F}_{\Phi}^{-}\right)\right]_{\mathbb{R}_{+}^{n+1}} .
$$

Proof of iii). Owing to (3.8) we write

$$
\left\|\mathcal{D}^{\mathcal{L},+} \varphi\right\|_{Y^{1,2}\left(\mathbb{R}_{+}^{n+1}\right)}=\left\|\mathcal{L}^{-1}\left(\mathscr{F}_{\Phi}^{-}\right)\right\|_{Y^{1,2}\left(\mathbb{R}_{+}^{n+1}\right)} \lesssim\left\|\mathscr{F}_{\Phi}^{-}\right\|_{\left(Y^{1,2}\left(\mathbb{R}^{n+1}\right)\right)^{*}}
$$

Let $0 \neq \Psi \in Y^{1,2}\left(\mathbb{R}^{n+1}\right)$. We have

$$
\left|\left(\mathscr{F}_{\Phi}^{-}, \Psi\right)\right|=\left|B_{\mathcal{L}, \mathbb{R}_{-}^{n+1}}[\Phi, \Psi]\right| \lesssim\|\Phi\|_{Y^{1,2}\left(\mathbb{R}_{-}^{n+1}\right)}|| \Psi \|_{Y^{1,2}\left(\mathbb{R}^{n+1}\right)},
$$


whence we deduce that $\left\|\mathscr{F}_{\Phi}^{-}\right\|_{\left(Y^{1,2}\left(\mathbb{R}^{n+1}\right)\right)^{*}} \lesssim\|\Phi\|_{Y^{1,2}\left(\mathbb{R}_{-}^{n+1}\right)} \lesssim\|\varphi\|_{H_{0}^{\frac{1}{2}}\left(\mathbb{R}^{n}\right)}$. Putting these estimates together we obtain the desired result.

Proof of iv). Let $\Psi \in C_{c}^{\infty}\left(\mathbb{R}_{+}^{n+1}\right)$ and extend it as a function in $\Psi \in C_{c}^{\infty}\left(\mathbb{R}^{n+1}\right)$ so that $\Psi \equiv 0$ in $\mathbb{R}_{-}^{n+1}$. Observe that

$$
\begin{aligned}
B_{\mathcal{L}, \mathbb{R}_{+}^{n+1}}\left[\mathcal{D}^{\mathcal{L},+} \varphi, \Psi\right]=B_{\mathcal{L}, \mathbb{R}_{+}^{n+1}}\left[-\mathcal{L}^{-1}\left(\mathscr{F}_{\Phi}^{-}\right), \Psi\right]= & B_{\mathcal{L}}\left[-\mathcal{L}^{-1}\left(\mathscr{F}_{\Phi}^{-}\right), \Psi\right] \\
& =-\left\langle\mathscr{F}_{\Phi}^{-}, \Psi\right\rangle=-B_{\mathcal{L}, \mathbb{R}_{-}^{n+1}}[\Phi, \Psi] \equiv 0 .
\end{aligned}
$$

We may now introduce the definition of the conormal derivative. First let us make the quick observation that since $Y_{0}^{1,2}\left(\mathbb{R}_{+}^{n+1}\right) \hookrightarrow Y^{1,2}\left(\mathbb{R}_{+}^{n+1}\right)$, then we have a surjection $\left(Y^{1,2}\left(\mathbb{R}_{+}^{n+1}\right)\right)^{*} \rightarrow$ $\left(Y_{0}^{1,2}\left(\mathbb{R}_{+}^{n+1}\right)\right)^{*}$ given by restricting the functionals. In particular, if $f \in\left(Y^{1,2}\left(\mathbb{R}_{+}^{n+1}\right)\right)^{*}$, then we can also think of $f \in\left(Y_{0}^{1,2}\left(\mathbb{R}_{+}^{n+1}\right)\right)^{*}$.

Definition 3.1.9 (Conormal derivative). Suppose that $u \in Y^{1,2}\left(\mathbb{R}_{+}^{n+1}\right), f \in\left(Y^{1,2}\left(\mathbb{R}_{+}^{n+1}\right)\right)^{*}$ (note carefully that this space is not $\left.\left(Y_{0}^{1,2}\left(\mathbb{R}_{+}^{n+1}\right)\right)^{*}\right)$, and that $\mathcal{L} u=f$ in $\mathbb{R}_{+}^{n+1}$ in the sense of Definition 2.2.7. Define the conormal derivative of $u$ associated to $\mathcal{L}$ with respect to $\mathbb{R}_{+}^{n+1}$, $\partial_{v}^{\mathcal{L},+} u \in H^{-\frac{1}{2}}\left(\mathbb{R}^{n}\right)$, by

$$
\left\langle\partial_{v}^{\mathcal{L},+} u, \varphi\right\rangle=B_{\mathcal{L}, \mathbb{R}_{+}^{n+1}}[u, \Phi]-\langle f, \Phi\rangle_{\left.\left(Y^{1,2}\left(\mathbb{R}_{+}^{n+1}\right)\right)^{*}, Y^{1,2}\left(\mathbb{R}_{+}^{n+1}\right)\right)}, \quad \varphi \in H_{0}^{\frac{1}{2}}\left(\mathbb{R}^{n}\right)
$$

where $\Phi \in Y^{1,2}\left(\mathbb{R}_{+}^{n+1}\right)$ is any bounded extension of $\varphi$ to $\mathbb{R}_{+}^{n+1}$ with $\operatorname{Tr}_{0} \Phi=\varphi$. Note that we also define the objects $\partial_{v}^{\mathcal{L}^{*},+} u, \partial_{v}^{\mathcal{L},-} u, \partial_{v}^{\mathcal{L}^{*},-} u$ similarly. 
Let us show that $\partial_{v}^{\mathcal{L},+} u$ is well-defined. Let $\Phi, \Phi^{\prime}$ be $Y^{1,2}\left(\mathbb{R}_{+}^{n+1}\right)$-extensions of $\varphi$ with $\operatorname{Tr}_{0} \Phi=\operatorname{Tr}_{0} \Phi^{\prime}=\varphi$. Then $\Phi-\Phi^{\prime} \in Y_{0}^{1,2}\left(\mathbb{R}_{+}^{n+1}\right)$, and so

$$
B_{\mathcal{L}, \mathbb{R}_{+}^{n+1}}[u, \Phi]-B_{\mathcal{L}, \mathbb{R}_{+}^{n+1}}\left[u, \Phi^{\prime}\right]=B_{\mathcal{L}, \mathbb{R}_{+}^{n+1}}\left[u, \Phi-\Phi^{\prime}\right]=\left\langle f, \Phi-\Phi^{\prime}\right\rangle_{\left.\left(Y_{0}^{1,2}\left(\mathbb{R}_{+}^{n+1}\right)\right)^{*}, Y_{0}^{1,2}\left(\mathbb{R}_{+}^{n+1}\right)\right)}
$$

since $u$ solves $\mathcal{L} u=f$ in $\mathbb{R}_{+}^{n+1}$ in the sense of Definition 2.2.7. Finally, observe that

$$
\begin{aligned}
&\langle f, \Phi\rangle_{\left.\left(Y^{1,2}\left(\mathbb{R}_{+}^{n+1}\right)\right)^{*}, Y^{1,2}\left(\mathbb{R}_{+}^{n+1}\right)\right)}-\left\langle f, \Phi^{\prime}\right\rangle_{\left.\left(Y^{1,2}\left(\mathbb{R}_{+}^{n+1}\right)\right)^{*}, Y^{1,2}\left(\mathbb{R}_{+}^{n+1}\right)\right)} \\
& \quad=\left\langle f, \Phi-\Phi^{\prime}\right\rangle_{\left.\left(Y^{1,2}\left(\mathbb{R}_{+}^{n+1}\right)\right)^{*}, Y^{1,2}\left(\mathbb{R}_{+}^{n+1}\right)\right)}=\left\langle f, \Phi-\Phi^{\prime}\right\rangle_{\left.\left(Y_{0}^{1,2}\left(\mathbb{R}_{+}^{n+1}\right)\right)^{*}, Y_{0}^{1,2}\left(\mathbb{R}_{+}^{n+1}\right)\right)},
\end{aligned}
$$

so that, upon subtracting these two identities, we see that $\partial_{v}^{\mathcal{L},+} u$ does not depend on the particular extension $\Phi$ taken. It remains to show that $\partial_{v}^{\mathcal{L},+}: H_{0}^{\frac{1}{2}}\left(\mathbb{R}^{n}\right) \rightarrow H^{-\frac{1}{2}}\left(\mathbb{R}^{n}\right)$ is bounded. Observe that

$$
\begin{aligned}
\left|\left\langle\partial_{v}^{\mathcal{L},+} u, \varphi\right\rangle\right| \leq\left|B_{\mathcal{L}_{,} \mathbb{R}_{+}^{n+1}}[u, \Phi]\right|+\left|\langle f, \Phi\rangle_{\left(Y^{1,2}\left(\mathbb{R}_{+}^{n+1}\right)\right)^{*}, Y^{1,2}\left(\mathbb{R}_{+}^{n+1}\right)}\right| & \\
\lesssim\left(\|u\|_{Y^{1,2}\left(\mathbb{R}_{+}^{n+1}\right)}+\|f\|_{\left(Y^{1,2}\left(\mathbb{R}_{+}^{n+1}\right)\right)^{*}}\right)\|\Phi\|_{Y^{1,2}\left(\mathbb{R}_{+}^{n+1}\right)} & \\
& \lesssim\left(\|u\|_{Y^{1,2}\left(\mathbb{R}_{+}^{n+1}\right)}+\|f\|_{\left(Y^{1,2}\left(\mathbb{R}_{+}^{n+1}\right)\right)^{*}}\right)\|\varphi\|_{H_{0}^{\frac{1}{2}}\left(\mathbb{R}^{n}\right)} .
\end{aligned}
$$

It will also be helpful to consider conormal derivatives on slices other than $t=0$, denoted $\partial_{v, t}^{\mathcal{L}, \pm}$. The definition is entirely analogous.

The following identities tie these definitions of the conormal derivatives together.

Lemma 3.1.10. Let $\gamma \in H^{-\frac{1}{2}}\left(\mathbb{R}^{n}\right)$. The following statements are true.

i) Suppose that $u \in Y^{1,2}\left(\mathbb{R}_{+}^{n+1}\right)$ solves $\mathcal{L} u=0$ in $\mathbb{R}_{+}^{n+1}$ in the weak sense. Then for any $t>0, \partial_{v}^{\mathcal{L},+} \mathscr{T}^{t} u=\partial_{v, t}^{\mathcal{L},+} u$. Moreover, for any $t>0, \partial_{v, t}^{\mathcal{L},+} u \in L^{2}\left(\mathbb{R}^{n}\right)$, and we have the 
identity

$$
\partial_{v, t}^{\mathcal{L},+} u=-e_{n+1} \cdot \operatorname{Tr}_{t}\left[A \nabla u+B_{1} u\right] \quad \text { in } L^{2}\left(\mathbb{R}^{n}\right)
$$

ii) Suppose that $u \in Y^{1,2}\left(\mathbb{R}_{-}^{n+1}\right)$ solves $\mathcal{L} u=0$ in $\mathbb{R}_{-}^{n+1}$. Then for any $t>0, \partial_{v}^{\mathcal{L},-} \mathscr{T}^{-t} u=$ $\partial_{\nu,-t}^{\mathcal{L},-} u$

iii) Let $t>0$. Then for each $\gamma \in H^{-\frac{1}{2}}\left(\mathbb{R}^{n}\right)$, the identity $-\partial_{v,-}^{\mathcal{L},-} \mathcal{S}^{\mathcal{L}} \gamma=\partial_{v,-t}^{\mathcal{L},+} \mathcal{S}^{\mathcal{L}} \gamma$ holds in the space $H^{-\frac{1}{2}}\left(\mathbb{R}^{n}\right)$.

Proof. Proof of i) and ii). Let $\varphi \in H_{0}^{\frac{1}{2}}\left(\mathbb{R}^{n}\right)$, and $\Phi \in Y^{1,2}\left(\mathbb{R}_{+}^{n+1}\right)$ is any extension of $\varphi$ such that $\operatorname{Tr}_{0} \Phi=\varphi$. Observe the calculation

$$
\begin{aligned}
\left\langle\partial_{v}^{\mathcal{L},+} \mathscr{T}^{t} u, \varphi\right\rangle=B_{{\mathcal{L}, \mathbb{R}_{+}^{n+1}}\left[\mathscr{T}^{t} u, \Phi\right]=B_{\mathcal{L}, \mathbb{R}_{t}^{n+1}}\left[u, \mathscr{T}^{-t} \Phi\right]} & =\left\langle\partial_{v, t}^{\mathcal{L},+} u, \operatorname{Tr}_{t} \mathscr{T}^{-t} \Phi\right\rangle=\left\langle\partial_{v, t}^{\mathcal{L},+} u, \varphi\right\rangle .
\end{aligned}
$$

We turn our attention now to (3.11). By Remark 2.5.37, we have that $F(x, t)=-e_{n+1}$. $\operatorname{Tr}_{t}\left[A \nabla u+B_{1} u\right]$ is continuous in $t$ taking values in $L^{2}\left(\mathbb{R}^{n}\right)$. In order to prove the lemma we will regularize our coefficients and solution simultaneously.

Let $P_{\varepsilon}$ be an $(n+1)$-dimensional approximate identity; that is, $P_{\varepsilon}(f)=\eta_{\varepsilon} * f$, where $\eta_{\varepsilon}(X)=\frac{1}{\varepsilon^{n+1}} \eta(X / \varepsilon)\left(X \in \mathbb{R}^{n+1}\right), \eta \in C_{c}^{\infty}(B(0,1)), \eta$ non-negative and radially decreasing with $\int_{\mathbb{R}^{n+1}} \eta=1$. We claim that

$$
-e_{n+1} \cdot P_{\varepsilon}\left(A \nabla u+B_{1} u\right)\left(x, t_{0}\right) \longrightarrow-e_{n+1} \cdot\left(A \nabla u+B_{1} u\right)\left(x, t_{0}\right)
$$


strongly in $L^{2}\left(\mathbb{R}^{n}\right)$. Assume (3.12) for a moment. Then to show the lemma it is enough to show that for every $\Phi \in C_{c}^{\infty}\left(\mathbb{R}^{n+1}\right)$ with $\Phi\left(x, t_{0}\right)=\varphi(x)$, we have that

$$
\lim _{\varepsilon \rightarrow 0} \int_{\mathbb{R}^{n}}-e_{n+1} \cdot P_{\varepsilon}\left(A \nabla u+B_{1} u\right)\left(x, t_{0}\right) \overline{\varphi(x)} d x=\iint_{\mathbb{R}_{t_{0}^{n+1}}^{n+1}} A \nabla u \overline{\nabla \Phi}+B_{1} u \overline{\nabla \Phi}+B_{2} \cdot \nabla u \bar{\Phi}
$$

To prove the above equality, first define for any cube $Q \subset \mathbb{R}^{n}, R_{Q}^{t_{0}}:=Q \times\left[t_{0}, t_{0}+\ell(Q)\right]$. Now choose any cube $Q \subset \mathbb{R}^{n}$ such that supp $\Phi \cap\left\{t \geq t_{0}\right\} \subset \overline{R_{\frac{1}{2}}^{t_{0}}}$. Integrating by parts, we have for $0<\varepsilon \ll \min \left\{\ell(Q), t_{0}\right\}$ the identity

$$
\begin{aligned}
& \int_{\mathbb{R}^{n}}-e_{n+1} \cdot P_{\varepsilon}(A\left.\nabla u+B_{1} u\right)\left(x, t_{0}\right) \overline{\varphi(x)} d x=\iint_{R_{Q}^{t_{0}}} \operatorname{div}\left[P_{\varepsilon}\left(A \nabla u+B_{1} u\right) \bar{\Phi}\right] \\
&=\iint_{R_{Q}^{t_{0}}} \operatorname{div}\left[P_{\varepsilon}\left(A \nabla u+B_{1} u\right)\right] \bar{\Phi}+\iint_{R_{Q}^{t_{0}}} P_{\varepsilon}\left(A \nabla u+B_{1} u\right) \cdot \overline{\nabla \Phi} .
\end{aligned}
$$

Now let $X=(x, t) \in \operatorname{supp} \Phi \cap\left\{t \geq t_{0}\right\}$, and $\varepsilon<\frac{t_{0}}{2}$. Then, since $\mathcal{L} u=0$ in $\mathbb{R}_{+}^{n+1}$, we have that

$$
\begin{aligned}
& \operatorname{div}_{X}\left[P_{\varepsilon}\left(A \nabla u+B_{1} u\right)\right](X)=\operatorname{div}_{X} \iint_{\mathbb{R}^{n+1}} \eta_{\varepsilon}(X-Y)\left(A \nabla_{Y} u+B_{1} u\right)(Y) d Y \\
& =-\iint_{\mathbb{R}^{n+1}} \nabla_{Y} \eta_{\varepsilon}(X-Y)\left(A \nabla_{Y} u+B_{1} u\right)(Y) d Y \\
& \quad=\iint_{\mathbb{R}_{+}^{n+1}} \eta_{\varepsilon}(X-Y) B_{2} \nabla_{Y} u(Y) d Y=P_{\varepsilon}\left(B_{2} \nabla u\right)(X),
\end{aligned}
$$

and therefore the identity

$$
\iint_{R_{Q}^{t_{0}}} \operatorname{div}\left[P_{\varepsilon}\left(A \nabla u+B_{1} u\right)\right] \bar{\Phi}=\iint_{R_{Q}^{t_{0}}} P_{\varepsilon}\left(B_{2} \nabla u\right) \bar{\Phi}
$$

holds. Finally, we want to pass in the limit as $\varepsilon \rightarrow 0$ the identity (3.13) while using (3.14), so we use the Lebesgue dominated convergence theorem. Observe that for some $p>1$, 
$|A \nabla u|+\left|B_{1} u\right|+\left|B_{2} \nabla u\right| \in L^{p}\left(U_{Q}^{t_{0}}\right)$, where $U_{Q}^{t_{0}}:=R_{Q}^{t_{0}}+B\left(0, \frac{t_{0}}{4}\right)$ (the $\frac{t_{0}}{4}$-neighborhood of $\left.R_{Q}^{t_{0}}\right)$. It follows that for $\varepsilon \in\left(0, \frac{t_{0}}{4}\right)$,

$$
P_{\varepsilon}\left(A \nabla u+B_{1} u\right)(x, t)+P_{\varepsilon}\left(B_{2} \nabla u\right)(x, t) \leq \mathscr{M}\left(\left[|A \nabla u|+\left|B_{1} u\right|+\left|B_{2} \nabla u\right|\right] \mathbb{1}_{U_{Q}^{t_{0}}}\right)(x, t)
$$

for all $(x, t) \in R_{Q}^{t_{0}}$, where $\mathscr{M}$ is the usual Hardy-Littlewood maximal function in $\mathbb{R}^{n+1}$. Hence we have that

$$
\begin{aligned}
& \lim _{\varepsilon \rightarrow 0} \int_{\mathbb{R}^{n}}-e_{n+1} \cdot P_{\varepsilon}\left(A \nabla u+B_{1} u\right)\left(x, t_{0}\right) \overline{\varphi(x)} d x \\
& =\lim _{\varepsilon \rightarrow 0} \iint_{R_{Q}^{t_{0}}} P_{\varepsilon}\left(A \nabla u+B_{1} u\right) \overline{\nabla \Phi}+P_{\varepsilon}\left(B_{2} \nabla u\right) \bar{\Phi} \\
& \quad=\iint_{R_{Q}^{t_{0}}} A \nabla u+B_{1} u \overline{\nabla \Phi}+B_{2} \nabla u \bar{\Phi} .
\end{aligned}
$$

Thus it remains to prove (3.12). Set $F_{\varepsilon}(x, t):=-e_{n+1} \cdot P_{\varepsilon}\left(A \nabla u+B_{1} u\right)(x, t), F(x, t):=$ $-e_{n+1} \cdot\left(A \nabla u+B_{1} u\right)(x, t)$. For $\varepsilon<\frac{t_{0}}{2}$, we have that

$$
\begin{aligned}
& \limsup _{\varepsilon \rightarrow 0}\left\|F_{\varepsilon}\left(\cdot, t_{0}\right)-F_{0}\left(\cdot, t_{0}\right)\right\|_{2} \\
& =\limsup _{\varepsilon \rightarrow 0}\left(\int_{\mathbb{R}^{n}}\left|\iint_{\mathbb{R}^{n+1}}\left[F_{0}\left(x-\varepsilon y, t_{0}-\varepsilon s\right)-F_{0}\left(x, t_{0}\right)\right] \eta_{\varepsilon}(y, s) d y d s\right|^{2} d x\right)^{\frac{1}{2}} \\
& \leq \limsup _{\varepsilon \rightarrow 0} \iint_{\mathbb{R}^{n+1}} \eta(y, s)\left[\int_{\mathbb{R}^{n}}\left|F_{0}\left(x-\varepsilon y, t_{0}-\varepsilon s\right)-F_{0}\left(x, t_{0}\right)\right|^{2} d x\right]^{\frac{1}{2}} d y d s \\
& \leq \limsup _{\varepsilon \rightarrow 0} \sup _{|y|,|s|<1}\left\|F_{0}\left(\cdot-\varepsilon y, t_{0}-\varepsilon s\right)-F_{0}\left(\cdot, t_{0}\right)\right\|_{2} \\
& \leq \lim \sup _{\varepsilon \rightarrow 0} \sup _{|\hat{y},| \hat{s} \mid<\varepsilon}\left\|F_{0}\left(\cdot-\hat{y}, t_{0}-\hat{s}\right)-F_{0}\left(\cdot-\hat{y}, t_{0}\right)\right\|_{2}+\left\|F_{0}\left(\cdot-\hat{y}, t_{0}\right)-F_{0}\left(\cdot, t_{0}\right)\right\|_{2},
\end{aligned}
$$

which drops to 0 as $\varepsilon \rightarrow 0$, finishing the proof.

Proof of iii). Let $\varphi \in H_{0}^{\frac{1}{2}}\left(\mathbb{R}^{n}\right)$ and let $\Phi \in Y^{1,2}\left(\mathbb{R}^{n+1}\right)$ be any extension of $\varphi$ such that $\operatorname{Tr}_{0} \Phi=\varphi$. Note that $\mathcal{L} \mathcal{S}^{\mathcal{L}} \gamma=0$ in $\mathbb{R}_{-,-t}^{n+1}$, while $\mathcal{L} \mathcal{S}^{\mathcal{L}} \gamma=T_{\gamma}$ in $\mathbb{R}_{+,-t}^{n+1}$, where $T_{\gamma} \in$ 
$\left(Y^{1,2}\left(\mathbb{R}^{n+1}\right)\right)^{*}$ is the distribution given by $\left\langle T_{\gamma}, \Psi\right\rangle=\left\langle\gamma, \operatorname{Tr}_{0} \Psi\right\rangle$, for $\Psi \in Y^{1,2}\left(\mathbb{R}^{n+1}\right)$. Observe the calculation

$$
\begin{aligned}
& \left\langle\partial_{v,-t}^{\mathcal{L},+} \mathcal{S}^{\mathcal{L}} \gamma, \varphi\right\rangle=B_{\mathcal{L}, \mathbb{R}_{+,-t}^{n+t}}\left[\mathcal{S}^{\mathcal{L}} \gamma, \Phi\right]-\left\langle\gamma, \operatorname{Tr}_{0} \Phi\right\rangle \\
& =-B_{\mathcal{L}, \mathbb{R}_{-,-t}^{n+1}}\left[\mathcal{S}^{\mathcal{L}} \gamma, \Phi\right]+B_{\mathcal{L}}\left[\mathcal{S}^{\mathcal{L}} \gamma, \Phi\right]-\left\langle\gamma, \operatorname{Tr}_{0} \Phi\right\rangle \\
& \quad=-B_{\mathcal{L}, \mathbb{R}_{-,-t}^{n+1}}\left[\mathcal{S}^{\mathcal{L}} \gamma, \Phi\right]+\left\langle\gamma, \operatorname{Tr}_{0} \Phi\right\rangle-\left\langle\gamma, \operatorname{Tr}_{0} \Phi\right\rangle=-\left\langle\partial_{v,-t}^{\mathcal{L},-} \mathcal{S}^{\mathcal{L}} \gamma, \varphi\right\rangle .
\end{aligned}
$$

\subsection{Green's formula and jump relations}

Let us remark that the functional $\mathscr{F}_{u}^{+}$makes sense even if we only have that $u \in Y^{1,2}\left(\mathbb{R}_{+}^{n+1}\right)$ and $u \notin Y^{1,2}\left(\mathbb{R}^{n+1}\right)$. Also, if $\Omega \subset \mathbb{R}^{n+1}$ and $f \in\left(Y^{1,2}(\Omega)\right)^{*}$, define the functional $\mathbb{1}_{\Omega} f$ by $\left\langle\mathbb{1}_{\Omega} f, \Psi\right\rangle:=\left\langle f, \mathbb{1}_{\Omega} \Psi\right\rangle$.

Theorem 3.2.1 (Green's formula). Suppose that $u \in Y^{1,2}\left(\mathbb{R}_{+}^{n+1}\right)$ solves $\mathcal{L} u=f$ in $\mathbb{R}_{+}^{n+1}$ for some $f \in\left(Y^{1,2}\left(\mathbb{R}_{+}^{n+1}\right)\right)^{*}$. Then the following statements hold.

i) We have the identity

$$
\mathcal{S}^{\mathcal{L}}\left(\partial_{v}^{\mathcal{L},+} u\right)=\mathcal{L}^{-1}\left(\mathscr{F}_{u}^{+}\right)-\mathcal{L}^{-1}\left(\mathbb{1}_{\mathbb{R}_{+}^{n+1}} f\right) \quad \text { in } Y^{1,2}\left(\mathbb{R}^{n+1}\right)
$$

ii) The identity $u=-\mathcal{D}^{\mathcal{L},+}\left(\operatorname{Tr}_{0} u\right)+\left.\mathcal{S}^{\mathcal{L}}\left(\partial_{v}^{\mathcal{L},+} u\right)\right|_{\mathbb{R}_{+}^{n+1}}+\left.\mathcal{L}^{-1}\left(\mathbb{1}_{\mathbb{R}_{+}^{n+1}} f\right)\right|_{\mathbb{R}_{+}^{n+1}}$ holds in $Y^{1,2}\left(\mathbb{R}_{+}^{n+1}\right)$.

iii) We have that $-\left.\mathcal{L}^{-1}\left(\mathbb{1}_{\mathbb{R}_{+}^{n+1}} f\right)\right|_{\mathbb{R}_{-}^{n+1}}=\mathcal{D}^{\mathcal{L},-}\left(\operatorname{Tr}_{0} u\right)+\left.\mathcal{S}^{\mathcal{L}}\left(\partial_{v}^{\mathcal{L},+} u\right)\right|_{\mathbb{R}_{-}^{n+1}}$ in $Y^{1,2}\left(\mathbb{R}_{-}^{n+1}\right)$.

iv) Suppose that $\mathcal{L} u=0$ in $\mathbb{R}^{n+1}$. Then $\mathcal{D}^{\mathcal{L},+}\left(\operatorname{Tr}_{0} u\right)=-\mathcal{S}\left(\partial_{v}^{\mathcal{L},-} u\right)$ holds in $\mathbb{R}_{+}^{n+1}$. 
Proof. Proof of i). Let $\Psi \in\left(Y^{1,2}\left(\mathbb{R}^{n+1}\right)\right)^{*}$. Then

$$
\begin{aligned}
& \left\langle\Psi, \mathcal{S}^{\mathcal{L}} \partial_{v}^{\mathcal{L},+} u\right\rangle=\left\langle\operatorname{Tr}_{0}\left(\mathcal{L}^{*}\right)^{-1} \Psi, \partial_{v}^{\mathcal{L},+} u\right\rangle \\
& =\overline{B_{\mathcal{L}, \mathbb{R}_{+}^{n+1}}\left[u,\left(\mathcal{L}^{*}\right)^{-1} \Psi\right]}-\overline{\left\langle f,\left(\mathcal{L}^{*}\right)^{-1} \Psi\right\rangle} \\
& =\overline{\left\langle\mathscr{F}_{u}^{+},\left(Y^{1,2}\left(\mathbb{R}_{+}^{n+1}\right)\right)^{*}, Y^{1,2}\left(\mathbb{R}_{+}^{n+1}\right)\right.} \\
& \\
& =\left\langle\overline{\left\langle\mathbb{1}_{\mathbb{R}_{+}^{n+1}} f,\left(\mathcal{L}^{-1}\right)^{*} \Psi\right\rangle}=\overline{\left\langle\mathcal{L}^{-1}\left(\mathscr{F}_{u}^{+}\right), \Psi\right\rangle}-\overline{\left\langle\mathcal{L}^{-1}\left(\mathbb{1}_{\mathbb{R}_{+}^{n+1}} f\right), \Psi\right\rangle}\right. \\
& =\left\langle\Psi, \mathcal{L}^{-1}\left(\mathscr{F}_{u}^{+}\right)-\mathcal{L}^{-1}\left(\mathbb{1}_{\mathbb{R}_{+}^{n+1}} f\right)\right\rangle .
\end{aligned}
$$

Proof of ii). Let $\Psi \in\left(Y^{1,2}\left(\mathbb{R}^{n+1}\right)\right)^{*}$ have compact support within $\mathbb{R}_{+}^{n+1}$. Using (3.2), we have that

$$
\begin{aligned}
& \left\langle\Psi, \mathcal{S}^{\mathcal{L}} \partial_{\nu} u-\mathcal{D}^{\mathcal{L},+} \operatorname{Tr}_{0} u\right\rangle \\
& \begin{aligned}
\left\langle\Psi, \mathcal{L}^{-1}\left(\mathscr{F}_{u}^{+}\right)\right\rangle-\left\langle\Psi, \mathcal{L}^{-1}\left(\mathbb{1}_{\mathbb{R}_{+}^{n+1}} f\right)\right\rangle-\left[-\left\langle\Psi,\left.u\right|_{\mathbb{R}_{+}^{n+1}}\right\rangle+\left\langle\Psi,\left.\mathcal{L}^{-1}\left(\mathscr{F}_{u}^{+}\right)\right|_{\mathbb{R}_{+}^{n+1}}\right\rangle\right] \\
\quad=\left\langle\Psi, u-\mathcal{L}^{-1}\left(\mathbb{1}_{\mathbb{R}_{+}^{n+1}} f\right)\right\rangle .
\end{aligned}
\end{aligned}
$$

Proof of iii). Let $\Psi \in\left(Y^{1,2}\left(\mathbb{R}^{n+1}\right)\right)^{*}$ have compact support within $\mathbb{R}_{-}^{n+1}$. Using (3.8) and (3.2), we have that

$$
\begin{aligned}
\left\langle\Psi, \mathcal{D}^{\mathcal{L},-}\left(\operatorname{Tr}_{0} u\right)+\mathcal{S}^{\mathcal{L}}\left(\partial_{v}^{\mathcal{L},+} u\right)\right\rangle=\left\langle\Psi,-\left.\mathcal{L}^{-1}\left(\mathscr{F}_{u}^{+}\right)\right|_{\mathbb{R}_{-}^{n+1}}+\mathcal{L}^{-1}\left(\mathscr{F}_{u}^{+}\right)-\mathcal{L}^{-1}\left(\mathbb{1}_{\mathbb{R}_{+}^{n+1}} f\right)\right\rangle \\
=-\left\langle\Psi, \mathcal{L}^{-1}\left(\mathbb{1}_{\mathbb{R}_{+}^{n+1}} f\right)\right\rangle .
\end{aligned}
$$

The proof of iv) is the same as iii), and is thus omitted.

Let us now consider some adjoint relations for the double layer potential. First, for any $u \in Y^{1,2}\left(\mathbb{R}^{n+1}\right)$, denote by $\mathscr{F}_{u}^{*+} \in\left(Y^{1,2}\left(\mathbb{R}^{n+1}\right)\right)^{*}$ the functional given by $\left\langle\mathscr{F}_{u}^{*+}, v\right\rangle:=$ $B_{\mathcal{L}^{*}, \mathbb{R}_{+}^{n+1}}[u, v]$ for $v \in Y^{1,2}\left(\mathbb{R}^{n+1}\right)$. 
Proposition 3.2.3. We have the following identities.

i) For each $\varphi, \psi \in H_{0}^{\frac{1}{2}}\left(\mathbb{R}^{n}\right)$, the identity $\left\langle\partial_{v}^{\mathcal{L},+} \mathcal{D}^{\mathcal{L},+} \varphi, \psi\right\rangle=\left\langle\varphi, \partial_{v}^{\mathcal{L}^{*},+} \mathcal{D}^{\mathcal{L}^{*},+} \psi\right\rangle$ holds.

ii) For each $\gamma \in H^{-\frac{1}{2}}\left(\mathbb{R}^{n}\right), \varphi \in H_{0}^{\frac{1}{2}}\left(\mathbb{R}^{n}\right)$, $t \geq 0$, the adjoint relation

$$
\left\langle\gamma, \operatorname{Tr}_{t} \mathcal{D}^{\mathcal{L},+} \varphi\right\rangle=-\left\langle\partial_{v}^{\mathcal{L}^{*},-} \mathscr{T}^{-t} \mathcal{S}^{\mathcal{L}^{*}} \gamma, \varphi\right\rangle=-\left\langle\partial_{v,-t}^{\mathcal{L}^{*},-} \mathcal{S}^{\mathcal{L}^{*}} \gamma, \varphi\right\rangle=\left\langle\partial_{v,-t}^{\mathcal{L}^{*},+} \mathcal{S}^{\mathcal{L}^{*}} \gamma, \varphi\right\rangle
$$

holds. In the case that $t=0$, we may write

$$
\left\langle\gamma, \operatorname{Tr}_{0} \mathcal{D}^{\mathcal{L},+} \varphi\right\rangle=-\langle\gamma, \varphi\rangle+\left\langle\partial_{v}^{\mathcal{L}^{*},+} \mathcal{S}^{\mathcal{L}^{*}} \gamma, \varphi\right\rangle
$$

iii) Fix $\varphi \in H^{\frac{1}{2}}\left(\mathbb{R}^{n}\right)$. For each $t>0$, and every $\zeta \in H^{-\frac{1}{2}}\left(\mathbb{R}^{n}\right)$, we have the identity

$$
\left\langle\operatorname{Tr}_{t} D_{n+1} \mathcal{D}^{\mathcal{L},+} \varphi, \zeta\right\rangle=\frac{d}{d t}\left\langle\operatorname{Tr}_{t} \mathcal{D}^{\mathcal{L},+} \varphi, \zeta\right\rangle=\left\langle\varphi, \partial_{\nu,-t}^{\mathcal{L}^{*},-} D_{n+1} \mathcal{S}^{\mathcal{L}^{*}} \zeta\right\rangle_{L^{2}, L^{2}}
$$

iv) Fix $t>0$. Let $\mathbf{g}=\left(\overrightarrow{g_{\|}}, g_{\perp}\right): \mathbb{R}^{n} \rightarrow \mathbb{C}^{n+1}$ be such that $g_{\|} \in H_{0}^{\frac{1}{2}}\left(\mathbb{R}^{n}\right)$ and $g_{\perp} \in H^{-\frac{1}{2}}\left(\mathbb{R}^{n}\right)$.

In the sense of distributions, we have the adjoint relation

$$
\left\langle\nabla \operatorname{Tr}_{t} D_{n+1} \mathcal{D}^{\mathcal{L},+} \varphi, \mathbf{g}\right\rangle_{\mathscr{D}^{\prime}, \mathscr{D}}=\left\langle\varphi, D_{n+1} \partial_{v,-t}^{\mathcal{L}^{*},-}\left(\mathcal{S}^{\mathcal{L}^{*}} \nabla\right) \zeta\right\rangle_{L^{2}, L^{2}}
$$

Proof. Proof of i). Let $\Phi, \Psi$ be extensions of $\varphi, \psi$ respectively to $Y^{1,2}\left(\mathbb{R}^{n+1}\right)$. Observe the computation

$$
\begin{aligned}
& \left\langle\partial_{v}^{\mathcal{L},+} \mathcal{D}^{\mathcal{L},+} \varphi, \psi\right\rangle=B_{\mathcal{L}, \mathbb{R}_{+}^{n+1}}\left[\mathcal{D}^{\mathcal{L},+} \varphi, \Psi\right]=-B_{\mathcal{L}, \mathbb{R}_{+}^{n+1}}[\Phi, \Psi]+B_{\mathcal{L}, \mathbb{R}_{+}^{n+1}}\left[\mathcal{L}^{-1}\left(\mathscr{F}_{\Phi}^{+}\right), \Psi\right] \\
& =-\overline{B_{\mathcal{L}^{*}, \mathbb{R}_{+}^{n+1}}[\Psi, \Phi]}+\overline{B_{\mathcal{L}^{*}, \mathbb{R}_{+}^{n+1}}\left[\Psi, \mathcal{L}^{-1}\left(\mathscr{F}_{\Phi}^{+}\right)\right]} \\
& =\overline{B_{\mathcal{L}^{*}, \mathbb{R}_{+}^{n+1}}\left[\mathcal{D}^{\mathcal{L}^{*},+} \psi, \Phi\right]}-\overline{B_{\mathcal{L}^{*}, \mathbb{R}_{+}^{n+1}}\left[\left(\mathcal{L}^{*}\right)^{-1}\left(\mathscr{F}_{\Psi}^{*+}\right), \Phi\right]}+\overline{B_{\mathcal{L}^{*}, \mathbb{R}_{+}^{n+1}}\left[\Psi, \mathcal{L}^{-1}\left(\mathscr{F}_{\Phi}^{+}\right)\right]}
\end{aligned}
$$




$$
=\left\langle\varphi, \partial_{v}^{\mathcal{L}^{*},+} \mathcal{D}^{\mathcal{L}^{*},+} \psi\right\rangle+\overline{\left[-B_{\mathcal{L}^{*}, \mathbb{R}_{+}^{n+1}}\left[\left(\mathcal{L}^{*}\right)^{-1}\left(\mathscr{F}_{\Psi}^{*+}\right), \Phi\right]+B_{\mathcal{L}^{*}, \mathbb{R}_{+}^{n+1}}\left[\Psi, \mathcal{L}^{-1}\left(\mathscr{F}_{\Phi}^{+}\right)\right]\right]},
$$

where in the first equality we used the definition of the conormal derivative, in the second equality we used the definition of the double layer potential. Hence it suffices to show that $B_{\mathcal{L}^{*}, \mathbb{R}_{+}^{n+1}}\left[\Psi, \mathcal{L}^{-1}\left(\mathscr{F}_{\Phi}^{+}\right)\right]=B_{\mathcal{L}^{*}, \mathbb{R}_{+}^{n+1}}\left[\left(\mathcal{L}^{*}\right)^{-1}\left(\mathscr{F}_{\Psi}^{*+}\right), \Phi\right]$. Observe the computation

$$
\begin{aligned}
B_{\mathcal{L}^{*}, \mathbb{R}_{+}^{n+1}}\left[\Psi, \mathcal{L}^{-1}\left(\mathscr{F}_{\Phi}^{+}\right)\right]=\left\langle\mathscr{F}_{\Psi}^{*+}, \mathcal{L}^{-1}\left(\mathscr{F}_{\Phi}^{+}\right)\right\rangle=\left\langle\left(\mathcal{L}^{-1}\right)^{*}\left(\mathscr{F}_{\Psi}^{*+}\right), \mathscr{F}_{\Phi}^{+}\right\rangle \\
=\overline{B_{\mathcal{L}, \mathbb{R}_{+}^{n+1}}\left[\Phi,\left(\mathcal{L}^{-1}\right)^{*}\left(\mathscr{F}_{\Psi}^{*+}\right)\right]}=B_{\mathcal{L}^{*}, \mathbb{R}_{+}^{n+1}}\left[\left(\mathcal{L}^{*}\right)^{-1}\left(\mathscr{F}_{\Psi}^{*+}\right), \Phi\right],
\end{aligned}
$$

where in the first equality we used the definition of the functional $\mathscr{F}_{\Psi}^{*+}$, and in the third equality we used the definition of $\mathscr{F}_{\Phi}^{+}$. The desired identity follows.

Proof of ii). Let $\gamma \in H^{-\frac{1}{2}}\left(\mathbb{R}^{n}\right), \varphi \in H_{0}^{\frac{1}{2}}\left(\mathbb{R}^{n}\right)$, and let $\Phi \in Y^{1,2}\left(\mathbb{R}^{n+1}\right)$ be an extension of $\varphi$ such that $\operatorname{Tr}_{0} \Phi=\varphi$. By the definition of $\mathcal{D}^{\mathcal{L},+} \varphi$, we have that $\left\langle\gamma, \operatorname{Tr}_{t} \mathcal{D}^{\mathcal{L},+} \varphi\right\rangle=-\left\langle\gamma, \operatorname{Tr}_{t} \Phi\right\rangle+$ $\left\langle\gamma, \operatorname{Tr}_{t} \mathcal{L}^{-1}\left(\mathscr{F}_{\Phi}^{+}\right)\right\rangle$. By (3.4), we have that

$$
\begin{aligned}
& \left\langle\gamma, \operatorname{Tr}_{t} \mathcal{L}^{-1}\left(\mathscr{F}_{\Phi}^{+}\right)\right\rangle=\left\langle\left(\operatorname{Tr}_{t} \circ \mathcal{L}^{-1}\right)^{*} \gamma, \mathscr{F}_{\Phi}^{+}\right\rangle=\left\langle\mathscr{T}^{-t} \mathcal{S}^{\mathcal{L}^{*}} \gamma, \mathscr{F}_{\Phi}^{+}\right\rangle \\
& =\overline{\left\langle\mathscr{F}_{\Phi}^{+}, \mathscr{T}^{-t} \mathcal{S}^{\mathcal{L}^{*}} \gamma\right\rangle}=\overline{B_{\mathcal{L}, \mathbb{R}_{+}^{n+1}}\left[\Phi, \mathscr{T}^{-t} \mathcal{S}^{\mathcal{L}^{*}} \gamma\right]}=B_{\mathcal{L}^{*}, \mathbb{R}_{+}^{n+1}}\left[\mathscr{T}^{-t} \mathcal{S}^{\mathcal{L}^{*}} \gamma, \Phi\right] \\
& \quad=B_{\mathcal{L}^{*}}\left[\mathscr{T}^{-t} \mathcal{S}^{\mathcal{L}^{*}} \gamma, \Phi\right]-B_{\mathcal{L}^{*}, \mathbb{R}_{+}^{n+1}}\left[\mathscr{T}^{-t} \mathcal{S}^{\mathcal{L}^{*}} \gamma, \Phi\right]=\left\langle\gamma, \operatorname{Tr}_{t} \Phi\right\rangle-\left\langle\partial_{\nu}^{\mathcal{L}^{*},-} \mathscr{T}^{-t} \mathcal{S}^{\mathcal{L}^{*}} \gamma, \varphi\right\rangle,
\end{aligned}
$$

where in the last equality we used (3.4) combined with (3.3) for the first term, and for the second term we used the definition of the conormal derivative and the fact that $\mathcal{L} \mathscr{T}^{-t} \mathcal{S}^{\mathcal{L}^{*}}=$ 0 in $\mathbb{R}_{-}^{n+1}$. From this calculation, the first equality in (3.4) follows. The second and third equalities are straightforward consequences of Lemma 3.1.10. To see that (3.5) is true, 
simply observe that when $t=0$, we have that $\mathcal{L}^{*} \mathcal{S}^{\mathcal{L}^{*}} \gamma=0$ in $\mathbb{R}_{+}^{n+1}$ and in $\mathbb{R}_{-}^{n+1}$, whence we deduce that $\left\langle\partial_{v}^{\mathcal{L}^{*},-} \mathscr{T}^{-0} \mathcal{S}^{\mathcal{L}^{*}} \gamma+\partial_{v}^{\mathcal{L}^{*},+} \mathscr{T}^{-0} \mathcal{S}^{\mathcal{L}^{*}} \gamma, \varphi\right\rangle=B_{\mathcal{L}^{*}}\left[\mathcal{S}^{\mathcal{L}^{*}} \gamma, \Phi\right]=\left\langle\gamma, \operatorname{Tr}_{0} \Phi\right\rangle$. Adding and subtracting $\left\langle\partial_{v}^{\mathcal{L}^{*},+} \mathscr{T}^{-t} \mathcal{S}^{\mathcal{L}^{*}} \gamma, \varphi\right\rangle$ to the right-hand side of (3.4) now proves the claim.

Proof of iii). Let $t>0$. By Proposition 3.1.7 iv), we have that $\mathcal{L} \mathcal{D}^{\mathcal{L},+} \varphi=0$ in $\mathbb{R}_{+}^{n+1}$. Therefore, using Proposition 2.5.42 iv) we see that $\operatorname{Tr}_{\tau} D_{n+1} \mathcal{D}^{\mathcal{L},+} \varphi \in H^{\frac{1}{2}}\left(\mathbb{R}^{n}\right)$ for each $\tau>0$. Similarly, we have that $\operatorname{Tr}_{-\tau} \nabla D_{n+1} \mathcal{S}^{\mathcal{L}^{*}} \zeta \in L^{2}\left(\mathbb{R}^{n}\right)$ for each $\tau>0$. Using (2.45) and ii), we calculate that $\left.\frac{d}{d \tau}\left(\operatorname{Tr}_{t} \mathcal{D}^{\mathcal{L},+} \varphi, \zeta\right)\right|_{\tau=t}=-\left.\frac{d}{d \tau}\left(\varphi, \partial_{v,-t}^{\mathcal{L}^{*},-} \mathcal{S}^{\mathcal{L}^{*}} \zeta\right)\right|_{\tau=t}$. Now we use the characterization of the conormal derivative, (3.11), to obtain

$$
\begin{aligned}
-\left.\frac{d}{d \tau}\left(\varphi, \partial_{v,-t}^{\mathcal{L}^{*}--} \mathcal{S}^{\mathcal{L}^{*}} \zeta\right)\right|_{\tau=t}=-\left.\frac{d}{d \tau}\left(\varphi,\left[e_{n+1} \cdot \operatorname{Tr}_{-\tau}\left(A^{*} \nabla+\overline{B_{2}}\right) \mathcal{S}^{\mathcal{L}^{*} \zeta}\right]\right)_{2,2}\right|_{\tau=t} \\
=\left(\varphi,\left[e_{n+1} \cdot \operatorname{Tr}_{-t}\left(A^{*} \nabla+\overline{B_{2}}\right) D_{n+1} \mathcal{S}^{\left.\left.\mathcal{L}^{*} \zeta\right]\right)_{2,2}}\right.\right.
\end{aligned}
$$

Finally, iv) follows from iii) similarly as in Proposition 3.1.2 viii).

Let us now establish the standard

Proposition 3.2.7 (Jump relations). Let $\varphi \in H_{0}^{\frac{1}{2}}\left(\mathbb{R}^{n}\right)$ and $\gamma \in H^{-\frac{1}{2}}\left(\mathbb{R}^{n}\right)$.

i) The identity $\operatorname{Tr}_{0} \mathcal{D}^{\mathcal{L},+} \varphi+\operatorname{Tr}_{0} \mathcal{D}^{\mathcal{L},+} \varphi=-\varphi$ holds in $H_{0}^{\frac{1}{2}}\left(\mathbb{R}^{n}\right)$.

ii) The identity $\partial_{v}^{\mathcal{L},+} \mathcal{S}^{\mathcal{L}} \gamma+\partial_{v}^{\mathcal{L},-} \mathcal{S}^{\mathcal{L}} \gamma=\gamma$ holds in $H^{-\frac{1}{2}}\left(\mathbb{R}^{n}\right)$.

iii) The identity $\partial_{v}^{\mathcal{L},+} \mathcal{D}^{\mathcal{L},+} \varphi=\partial_{v}^{\mathcal{L},-} \mathcal{D}^{\mathcal{L},-} \varphi$ holds in $H^{-\frac{1}{2}}\left(\mathbb{R}^{n}\right)$.

iv) The identity $\operatorname{Tr}_{0}\left(\left.\mathcal{S}^{\mathcal{L}} \gamma\right|_{\mathbb{R}_{+}^{n+1}}\right)=\operatorname{Tr}_{0}\left(\left.\mathcal{S}^{\mathcal{L}} \gamma\right|_{\mathbb{R}_{-}^{n+1}}\right)$ holds in $H_{0}^{\frac{1}{2}}\left(\mathbb{R}^{n}\right)$. 
Proof. The statement iv) is immediate from the fact that $\mathcal{S}^{\mathcal{L}} \gamma \in Y^{1,2}\left(\mathbb{R}^{n+1}\right)$. The statement ii) follows from the definition of the conormal derivative and the fact that $\mathcal{L S}^{\mathcal{L}} \gamma=0$ in $\mathbb{R}^{n+1} \backslash\{t=0\}$.

Proof of i). Let $\gamma \in H^{-\frac{1}{2}}\left(\mathbb{R}^{n}\right)$, and let $\Phi \in Y^{1,2}\left(\mathbb{R}^{n+1}\right)$ be any extension of $\varphi$ such that $\operatorname{Tr}_{0} \Phi=\varphi$. Using (3.5) we, observe the calculation

$$
\begin{aligned}
\left\langle\gamma, \operatorname{Tr}_{0}\left[\mathcal{D}^{\mathcal{L},+} \varphi+\mathcal{D}^{\mathcal{L},-} \varphi\right]\right\rangle & =-2\langle\gamma, \varphi\rangle+\left\langle\partial_{v}^{\mathcal{L}^{*},-} \mathcal{S}^{\mathcal{L}^{*}} \gamma+\partial_{v}^{\mathcal{L}^{*},+} \mathcal{S}^{\mathcal{L}^{*}} \gamma, \varphi\right\rangle \\
& =-2\langle\gamma, \varphi\rangle+B_{\mathcal{L}^{*}}\left[\mathcal{S}^{\mathcal{L}^{*}} \gamma, \Phi\right]=-2\langle\gamma, \varphi\rangle+\left\langle\gamma, \operatorname{Tr}_{0} \Phi\right\rangle=-\langle\gamma, \varphi\rangle .
\end{aligned}
$$

Proof of iii). Let $\psi \in H_{0}^{\frac{1}{2}}\left(\mathbb{R}^{n}\right)$, and let $\Phi, \Psi \in Y^{1,2}\left(\mathbb{R}^{n+1}\right)$ be extensions of $\varphi, \psi$ respectively such that $\operatorname{Tr}_{0} \Phi=\varphi, \operatorname{Tr}_{0} \Psi=\psi$. Also recall that $\mathcal{L} \mathcal{D}^{\mathcal{L},+} \varphi=0$ in $\mathbb{R}_{+}^{n+1}$, and $\mathcal{L} \mathcal{D}^{\mathcal{L},-} \varphi=0$ in $\mathbb{R}_{-}^{n+1}$. Observe the calculation

$$
\begin{aligned}
& \left\langle\partial_{v}^{\mathcal{L},+} \mathcal{D}^{\mathcal{L},+} \varphi, \psi\right\rangle=B_{\mathcal{L}, \mathbb{R}_{+}^{n+1}}\left[\mathcal{D}^{\mathcal{L},+} \varphi, \Psi\right]=-B_{\mathcal{L}, \mathbb{R}_{+}^{n+1}}[\Phi, \Psi]+B_{\mathcal{L}, \mathbb{R}_{+}^{n+1}}\left[\mathcal{L}^{-1}\left(\mathscr{F}_{\Phi}^{+}\right), \Psi\right] \\
& =-B_{\mathcal{L}, \mathbb{R}_{+}^{n+1}}[\Phi, \Psi]+B_{\mathcal{L}}\left[\mathcal{L}^{-1}\left(\mathscr{F}_{\Phi}^{+}\right), \Psi\right]-B_{{\mathcal{L}, \mathbb{R}_{-}^{n+1}}}\left[\mathcal{L}^{-1}\left(\mathscr{F}_{\Phi}^{+}\right), \Psi\right]
\end{aligned}
$$

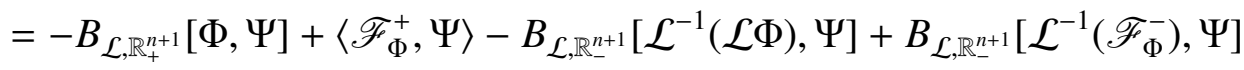

$$
\begin{aligned}
& =-B_{\mathcal{L}, \mathbb{R}_{-}^{n+1}}[\Phi, \Psi]+B_{\mathcal{L}, \mathbb{R}_{-}^{n+1}}\left[\mathcal{L}^{-1}\left(\mathscr{F}_{\Phi}^{-}\right), \Psi\right]=B_{\mathcal{L}, \mathbb{R}_{-}^{n+1}}\left[\mathcal{D}^{\mathcal{L},-} \varphi, \Psi\right]=\left\langle\partial_{v}^{\mathcal{L},-} \mathcal{D}^{\mathcal{L},-} \varphi, \psi\right\rangle
\end{aligned}
$$

\subsection{Initial $L^{2}$ estimates for the single layer potential}

We now establish several estimates for the single layer potential. This will allow us to prove the square function estimates, via a $T b$ theorem, in the next section. We begin with a 
perturbation result.

Proposition 3.3.1 (Initial slice estimates). The following statements hold provided that $\max \left\{\left\|B_{1}\right\|_{n},\left\|B_{2}\right\|_{n}\right\}$ is small enough, depending only on $n, \lambda$, and $\Lambda$.

i) For each $f \in C_{c}^{\infty}\left(\mathbb{R}^{n}\right)$, each $a>0$, and each $m \geq 1$, we have the estimate

$$
f_{a}^{2 a} \int_{\mathbb{R}^{n}}\left|t^{m} \nabla \partial_{t}^{m} \mathcal{S}^{\mathcal{L}} f\right|^{2} d t \lesssim_{m}\|f\|_{2}^{2}
$$

ii) For each $f \in C_{c}^{\infty}\left(\mathbb{R}^{n}\right)$, each $t \geq 0$, and each $m \geq 2$, we have the estimate

$$
\left\|t^{m} \nabla \partial_{t}^{m} \mathcal{S}_{t}^{\mathcal{L}} f\right\|_{L^{2}\left(\mathbb{R}^{n}\right)} \lesssim_{m}\|f\|_{2}
$$

Proof. First we see that the second estimate is a consequence of the first by the Caccioppoli inequality on slices (2.41). In particular, we have that

$$
\left\|t^{m} \nabla \partial_{t}^{m} \mathcal{S}_{t}^{\mathcal{L}} f\right\|_{2}^{2}=\sum_{Q \in \mathbb{D}_{t}} \int_{Q}\left|t^{m} \nabla \partial_{t}^{m} \mathcal{S}_{t}^{\mathcal{L}} f\right|^{2} d x \lesssim f_{t}^{2 t} \int_{\mathbb{R}^{n}}\left|s^{m} \nabla \partial_{s}^{m-1} \mathcal{S}_{s}^{\mathcal{L}} f\right|^{2} d x d s,
$$

where $\mathbb{D}_{t}$ is a grid of $n$-dimensional cubes of side length $t$. Thus it suffices to show i).

To this end, we know from $\left[\mathrm{AAA}^{+} 11\right]$ that i) holds with $\mathcal{S}^{\mathcal{L}}$ replaced by $\mathcal{S}^{\mathcal{L}_{0}}$, where $\mathcal{L}_{0}=-\Delta$. Thus, to prove i), we show that $f_{a}^{2 a} \int_{\mathbb{R}^{n}}\left|t^{m} \nabla \partial_{t}^{m}\left(\mathcal{S}^{\mathcal{L}}-\mathcal{S}^{\mathcal{L}_{0}}\right) f\right|^{2} d t \lesssim\|f\|_{2}$. Observe that

$$
\begin{aligned}
\mathcal{S}^{\mathcal{L}}-\mathcal{S}^{\mathcal{L}_{0}}=\left(\operatorname{Tr}_{0} \circ\left(\left(\mathcal{L}^{*}\right)^{-1}-\left(\mathcal{L}_{0}^{*}\right)^{-1}\right)\right)^{*}=\left(\operatorname{Tr}_{0} \circ\left(\left(\mathcal{L}_{0}^{*}\right)^{-1}\left(\mathcal{L}_{0}^{*}-\mathcal{L}^{*}\right)\left(\mathcal{L}^{*}\right)^{-1}\right)\right)^{*} \\
=\left(\left(\mathcal{L}_{0}^{*}-\mathcal{L}^{*}\right)\left(\mathcal{L}^{*}\right)^{-1}\right)^{*} \mathcal{S}^{\mathcal{L}_{0}}=\mathcal{L}^{-1}\left(\mathcal{L}_{0}-\mathcal{L}\right) \mathcal{S}^{\mathcal{L}_{0}} \\
=-\operatorname{div}(I-A) \nabla \mathcal{S}^{\mathcal{L}_{0}}-\mathcal{L}^{-1} \operatorname{div}\left(B_{1} \mathcal{S}^{\mathcal{L}_{0}}\right)-\mathcal{L}^{-1} B_{2} \cdot \nabla \mathcal{S}^{\mathcal{L}_{0}} .
\end{aligned}
$$


Now let $f \in C_{c}^{\infty}\left(\mathbb{R}^{n}\right)$. Then we have that

$$
\begin{gathered}
\left.f_{a}^{2 a} \int_{\mathbb{R}^{n}}\left|t^{m} \nabla D_{n+1}^{m}\left(\mathcal{S}^{\mathcal{L}}-\mathcal{S}^{\mathcal{L}_{0}}\right) f\right|^{2} d t \lesssim f_{a}^{2 a} \int_{\mathbb{R}^{n}} \mid t^{m} \nabla D_{n+1}^{m} \mathcal{L}^{-1} \operatorname{div}(I-A) \nabla \mathcal{S}^{\mathcal{L}_{0}} f\right)\left.\right|^{2} d t \\
+f_{a}^{2 a} \int_{\mathbb{R}^{n}}\left|t^{m} \nabla D_{n+1}^{m} \mathcal{L}^{-1} \operatorname{div}\left(B_{1} \mathcal{S}^{\mathcal{L}_{0}} f\right)\right|^{2} d t \\
+f_{a}^{2 a} \int_{\mathbb{R}^{n}}\left|t^{m} \nabla D_{n+1}^{m} \mathcal{L}^{-1} B_{2} \cdot \nabla \mathcal{S}^{\mathcal{L}_{0}} f\right|^{2} d t \quad=: I+I I+I I I .
\end{gathered}
$$

We prove only the bound $I I \lesssim\|f\|_{2}^{2}$ as the bounds for $I$ and $I I I$ are entirely analogous, and we will indicate the small differences after we bound II. Let $\psi=\psi(t)$ be such that $\psi \in$ $C_{c}^{\infty}(-a / 5, a / 5), \psi \equiv 1$ on $(-a / 10, a / 10), 0 \leq \psi \leq 1, \frac{d^{k}}{d t^{k}} \psi \lesssim_{k}(1 / a)^{k}$. Writing $1=\psi+(1-\psi)$ we have that

$$
\begin{aligned}
& I I \leq \int_{a}^{2 a} \int_{\mathbb{R}^{n}}\left|t^{m} \nabla D_{n+1}^{m} \mathcal{L}^{-1} \operatorname{div}\left(B_{1} \mathcal{S}^{\mathcal{L}_{0}} f\right)\right|^{2} d t \\
& \leq \int_{a}^{2 a} \int_{\mathbb{R}^{n}}\left|t^{m} \nabla D_{n+1}^{m} \mathcal{L}^{-1} \operatorname{div}\left(\psi B_{1} \mathcal{S}^{\mathcal{L}_{0}} f\right)\right|^{2} d t \\
& \quad+\int_{a}^{2 a} \int_{\mathbb{R}^{n}}\left|t^{m} \nabla D_{n+1}^{m} \mathcal{L}^{-1} \operatorname{div}\left((1-\psi) B_{1} \mathcal{S}^{\mathcal{L}_{0}} f\right)\right|^{2} d t=: I I_{1}+I I_{2} .
\end{aligned}
$$

To bound $I I_{1}$, we notice that if $g=\operatorname{div}\left(\psi B_{1} \mathcal{S}^{\mathcal{L}_{0}} f\right)$, then $g \equiv 0$ on $\mathbb{R}^{n} \times(a / 5, \infty)$. It follows that each $D_{n+1}^{k} \mathcal{L}^{-1} g=\mathcal{L}^{-1} D_{n+1}^{k} g, k=0,1, \ldots m$ is a (null) solution in $\mathbb{R}^{n} \times(a / 5, \infty)$. Let $\mathbb{D}_{a}$ be a grid of $n$-dimensional cubes with side length $a$. Applying the Caccioppoli inequality $m$ times and using that $t \approx a$ on $(a, 2 a)$, we see that

$$
\begin{aligned}
& I I_{1} \lesssim a^{2 m-1} \int_{a}^{2 a} \int_{\mathbb{R}^{n}}\left|\nabla D_{n+1}^{m} \mathcal{L}^{-1} \operatorname{div}\left(\psi B_{1} \mathcal{S}^{\mathcal{L}_{0}} f\right)\right|^{2} \\
& \lesssim a^{2 m-1} \sum_{Q \in \mathbb{D}_{a}} \int_{a}^{2 a} \int_{Q}\left|\nabla D_{n+1}^{m} \mathcal{L}^{-1} \operatorname{div}\left(\psi B_{1} \mathcal{S}^{\mathcal{L}_{0}} f\right)\right|^{2}
\end{aligned}
$$




$$
\begin{aligned}
\lesssim a^{-1} \sum_{Q \in \mathbb{D}_{a}} \int_{a / 2}^{4 a} \int_{2 Q} \mid D_{n+1} \mathcal{L}^{-1} & \left.\operatorname{div}\left(\psi B_{1} \mathcal{S}^{\mathcal{L}_{0}} f\right)\right|^{2} \lesssim a^{-1} \int_{\mathbb{R}} \int_{\mathbb{R}^{n}}\left|\nabla \mathcal{L}^{-1} \operatorname{div}\left(\psi B_{1} \mathcal{S}^{\mathcal{L}_{0}} f\right)\right|^{2} \\
& \lesssim a^{-1} \int_{\mathbb{R}} \int_{\mathbb{R}^{n}}\left|\psi B_{1} \mathcal{S}^{\mathcal{L}_{0}} f\right|^{2} \lesssim f_{-a / 5}^{a / 5} \int_{\mathbb{R}^{n}}\left|B_{1} \mathcal{S}^{\mathcal{L}_{0}} f\right|^{2} \lesssim\|f\|_{2}^{2},
\end{aligned}
$$

where we used that $\sup _{t \neq 0}\left\|B_{1} \mathcal{S}_{t}^{\mathcal{L}_{0}}\right\|_{L^{2}\left(\mathbb{R}^{n}\right) \rightarrow L^{2}\left(\mathbb{R}^{n}\right)} \lesssim \sup _{t \neq 0}\left\|\nabla \mathcal{S}_{t}^{\mathcal{L}_{0}}\right\|_{L^{2}\left(\mathbb{R}^{n}\right) \rightarrow L^{2}\left(\mathbb{R}^{n}\right)} \leq C$ (see $\left[\mathrm{AAA}^{+} 11\right.$, Lemma 4.18]) and that $\nabla \mathcal{L}^{-1} \operatorname{div}: L^{2}\left(\mathbb{R}^{n+1}\right) \rightarrow L^{2}\left(\mathbb{R}^{n+1}\right)$.

Now we deal with $I I_{2}$. Set $g=(1-\psi) B_{1} \mathcal{S}^{\mathcal{L}_{0}} f$. Then, we have that

$$
D_{n+1}^{m} g=(1-\psi) B_{1} D_{n+1}^{m} \mathcal{S}^{\mathcal{L}_{0}} f+\sum_{k=1}^{m} \psi^{(k)} B_{1} D_{n+1}^{m-k} \mathcal{S}^{\mathcal{L}_{0}} f=: F_{0}+\sum_{k=1}^{m} F_{k} .
$$

where $\psi^{(k)}=\frac{d^{k}}{d t^{k}} \psi$. The triangle inequality yields that

$$
I I_{2} \leq \sum_{k=0}^{m} f_{a}^{2 a} \int_{\mathbb{R}^{n}}\left|t^{m} \nabla D_{n+1}^{m} \mathcal{L}^{-1} \operatorname{div}\left(F_{k}\right)\right|^{2} d t=: \sum_{k=0}^{m} I I_{2, k} .
$$

For $I I_{2, k}, k=1,2 \ldots m$, we use that $t \approx a$ in the region of integration, the properties of $\psi$, and that $\nabla \mathcal{L}^{-1} \operatorname{div}: L^{2} \rightarrow L^{2}$ to obtain that

$$
\begin{aligned}
& I I_{2, k} \lesssim a^{2 m-1} \int_{\mathbb{R}} \int_{\mathbb{R}^{n}}\left|\psi^{(k)} B_{1} \partial_{t}^{m-k} \mathcal{S}^{\mathcal{L}_{0}} f\right|^{2} d t \\
& \lesssim a^{2 m-2 k-1} \int_{a / 10 \leq|t| \leq a / 5} \int_{\mathbb{R}^{n}}\left|B_{1} \partial_{t}^{m-k} \mathcal{S}^{\mathcal{L}_{0}} f\right|^{2} d t \\
& \lesssim \int_{-a / 5}^{a / 5} \int_{\mathbb{R}^{n}}\left|t^{m-k} B_{1} \partial_{t}^{m-k} \mathcal{S}^{\mathcal{L}_{0}} f\right|^{2} d t \lesssim\|f\|_{2}^{2},
\end{aligned}
$$

where we used [AAA ${ }^{+} 11$, Lemma 2.10] in the last line. Finally, to handle $I I_{2,0}$, we use that $(1-\psi)=0$ if $|t|<a / 10$, and that $\nabla \mathcal{L} \operatorname{div}: L^{2} \rightarrow L^{2}$ to obtain that

$$
I I_{2,0} \lesssim a^{2 m-1} \int_{\mathbb{R}} \int_{\mathbb{R}^{n}}\left|(1-\psi) B_{1} \partial_{t}^{m} \mathcal{S}^{\mathcal{L}_{0}} f\right|^{2} d t \lesssim a^{2 m-1} \int_{|t|>a / 10} \int_{\mathbb{R}^{n}}\left|B_{1} \partial_{t}^{m} \mathcal{S}^{\mathcal{L}_{0}} f\right|^{2} d t
$$




$$
\lesssim \int_{|t|>a / 10} \int_{\mathbb{R}^{n}}\left|t^{m+1} B_{1} \partial_{t}^{m} \mathcal{S}^{\mathcal{L}_{0}} f\right|^{2} \frac{d t}{t} \lesssim\|f\|_{2}^{2}
$$

where we used the estimate $\left\|t^{m+1} B_{1} \partial_{t}^{m} \mathcal{S}^{\mathcal{L}_{0}} f\right\|_{2}^{2} \lesssim\left\|t^{m+1} \partial_{t}^{m} \nabla \mathcal{S}^{\mathcal{L}_{0}} f\right\|_{2}^{2} \lesssim\|f\|_{2}^{2}$ in the last line. To see this last estimate, we simply use the "travelling up" procedure for square functions (see Proposition 4.2.2 below) and that $\mathcal{L}_{0}=\Delta$ has good square function estimates. We now observe that handling the term $I I I$ amounts to replacing the use of the mapping property $\nabla \mathcal{L}^{-1} \operatorname{div}: L^{2} \rightarrow L^{2}$ by the fact that $\nabla \mathcal{L}^{-1} B_{2}: L^{2} \rightarrow L^{2}$. The term $I$ is handled exactly the same way, using the $L^{\infty}$ bound for $(I-A)$, without appealing to the mapping properties of multiplication by $B_{1}$.

Remark 3.3.4. Note that, from now on, it makes sense to write the objects appearing in (3.2) and (3.3) for $f$ in $L^{2}\left(\mathbb{R}^{n}\right)$ after we have made extensions by continuity.

Before proceeding, we will need some identities improving on the duality results in the previous subsections for the single and double layers. To ease the notation, we will use $(G)_{t}$ to denote the trace at $t$ of a function $G$ defined in $\mathbb{R}_{+}^{n+1}$.

Proposition 3.3.5 (Further distributional identities of the layer potentials). For any $t \neq 0$ and $m \geq 1$, the following statements are true.

i) For any $f \in C_{c}^{\infty}\left(\mathbb{R}^{n}\right)$ and any $\vec{g} \in L^{2}\left(\mathbb{R}^{n} ; \mathbb{C}^{n+1}\right)$, we have that

$$
\frac{d^{m}}{d t^{m}}\left\langle\nabla \mathcal{S}_{t}^{\mathcal{L}} f, \vec{g}\right\rangle=\left\langle\left(D_{n+1}^{m} \nabla \mathcal{S}^{\mathcal{L}}[f]\right)_{t}, \vec{g}\right\rangle
$$


ii) For any $f \in L^{2}\left(\mathbb{R}^{n}\right)$ and any $\vec{g} \in C_{c}^{\infty}\left(\mathbb{R}^{n} ; \mathbb{C}^{n+1}\right)$, we have that

$$
\frac{d^{m}}{d t^{m}}\left\langle f,\left(\left(\mathcal{S}^{\mathcal{L}^{*}} \nabla\right)[\vec{g}]\right)_{-t}\right\rangle=(-1)^{m}\left\langle f,\left(D_{n+1}^{m}\left(\mathcal{S}^{\mathcal{L}^{*}} \nabla\right)[\vec{g}]\right)_{-t}\right\rangle
$$

iii) If $m \geq 2$, then for every $f \in L^{2}\left(\mathbb{R}^{n}\right)$ and $\left.\vec{g} \in L^{2}\left(\mathbb{R}^{n}, \mathbb{C}^{n+1}\right)\right)$, we have the identity

$$
\left\langle\left(D_{n+1}^{m} \nabla \mathcal{S}^{\mathcal{L}}[f]\right)_{t}, \vec{g}\right\rangle=(-1)^{m}\left\langle f,\left(D_{n+1}^{m}\left(\mathcal{S}^{\mathcal{L}^{*}} \nabla\right)[\vec{g}]\right)_{-t}\right\rangle
$$

Proof. Let us first show the identities with $f \in C_{c}^{\infty}\left(\mathbb{R}^{n}\right)$ and $\vec{g} \in C_{c}^{\infty}\left(\mathbb{R}^{n} ; \mathbb{C}^{n+1}\right)$. For the first equality, note that $u:=\mathcal{S}^{\mathcal{L}}[f] \in Y^{1,2}\left(\mathbb{R}^{n+1}\right)$ and $\mathcal{L} u=0$ in $\mathbb{R}^{n+1} \backslash\left\{x_{n+1}=0\right\}$. In particular, $\partial_{t} u \in W^{1,2}\left(\Sigma_{a}^{b}\right)$ for any $a<b$ such that $0 \notin[a, b]$, by Lemma 2.5.36. By iteration we have that $\partial_{t}^{m} \nabla u \in L^{2}\left(\Sigma_{a}^{b}\right)$. In particular, arguing as in Lemma 2.2.2, we realize that the map $t \mapsto \nabla u(\cdot, t)$ is smooth (with values in $L^{2}\left(\mathbb{R}^{n} ; \mathbb{C}^{n+1}\right)$ ). The first equality for $m=1$ then boils down to proving the weak convergence of the difference quotients to the derivative in $L^{2}\left(\mathbb{R}^{n}\right)$; that is, showing that

$$
\lim _{h \rightarrow 0} \frac{\nabla u(t+h)-\nabla u(t)}{h}=\partial_{t} \nabla u(t), \quad \text { weakly in } L^{2}\left(\mathbb{R}^{n}\right) .
$$

But this follows from the smoothness of our map. The case of general $m$ now follows by induction.

For the second equality, by definition we have that

$$
\left(\left(\mathcal{S}^{\mathcal{L}^{*}} \nabla\right)[\vec{g}]\right)_{s}=-\left(\mathcal{S}^{\mathcal{L}^{*}}\left[\operatorname{div}_{\|} g_{\|}\right]\right)_{s}-\left(D_{n+1} \mathcal{S}^{\mathcal{L}^{*}}\left[g_{\perp}\right]\right)_{s}
$$

and since $\vec{g} \in C_{c}^{\infty}\left(\mathbb{R}^{n} ; \mathbb{C}^{n+1}\right)$, we can apply the same argument as above to conclude that

$$
\frac{d^{m}}{d t^{m}}\left\langle f,\left(\left(\mathcal{S}^{\mathcal{L}^{*}} \nabla\right)[g]\right)_{-t}\right\rangle=(-1)^{m}\left\langle f,\left(D_{n+1}^{m}\left(\mathcal{S}^{\mathcal{L}^{*}} \nabla\right)\left[g_{\perp}\right]\right)_{-t}\right\rangle
$$


The third equality now follows by duality: For $f \in C_{c}^{\infty}\left(\mathbb{R}^{n}\right)$ and $g \in C_{c}^{\infty}\left(\mathbb{R}^{n} ; \mathbb{C}^{n+1}\right)$, we have that

$$
\begin{aligned}
\left\langle\left(D_{n+1}^{m} \nabla \mathcal{S}^{\mathcal{L}}[f]\right)_{t}, \vec{g}\right\rangle=\frac{d^{m}}{d t^{m}}\left\langle\nabla \mathcal{S}_{t}^{\mathcal{L}} f, \vec{g}\right\rangle=\frac{d^{m}}{d t^{m}}\left\langle f,\left(\left(\mathcal{S}^{\mathcal{L}} \nabla\right)[\vec{g}]\right)_{-t}\right\rangle & \\
= & (-1)^{m}\left\langle f,\left(D_{n+1}^{m}\left(\mathcal{S}^{\mathcal{L}} \nabla\right)[\vec{g}]\right)_{-t}\right\rangle
\end{aligned}
$$

Finally, the identities are extended to the respective $L^{2}$ spaces via a straightforward density argument using Proposition 3.3.1.

We now present an off-diagonal decay result.

Proposition 3.3.6 (Good off-diagonal decay). Let $Q \subset \mathbb{R}^{n}$ be a cube and $g \in L^{2}(Q)$ with $\operatorname{supp} g \subseteq Q$. If $p \in\left[2, p_{+}\right]$is such that $|p-2|$ is small enough that Lemma 2.5.4 holds, we have that

$$
\left(\int_{R_{0}}\left|t^{m}\left(\partial_{t}\right)^{m} \nabla \mathcal{S}_{t}^{\mathcal{L}} g(x)\right|^{p} d x\right)^{\frac{1}{p}} \lesssim 2^{-(m+1)} t^{m} \ell(Q)^{-n(1 / 2-1 / p)} \ell(Q)^{-m}\|g\|_{L^{2}(Q)},
$$

provided $t \approx \ell(Q)$. Moreover, for any $k \geq 1$ and any $t \in \mathbb{R}$, the estimate

$$
\left(\int_{R_{k}}\left|t^{m}\left(\partial_{t}\right)^{m} \nabla \mathcal{S}_{t}^{\mathcal{L}} g(x)\right|^{p} d x\right)^{\frac{1}{p}} \lesssim 2^{n k \alpha} 2^{-k(m+1)} t^{m} \ell(Q)^{-n(1 / 2-1 / p)} \ell(Q)^{-m}\|g\|_{L^{2}(Q)},
$$

where $\alpha=\alpha(p)=\frac{1}{p}\left(1-\frac{p}{p_{+}}\right)$and the annular regions $R_{k}=R_{k}(Q)$ are defined by $R_{0}:=2 Q$, $R_{k}:=2^{k+1} Q \backslash 2^{k} Q$, for all $k \geq 1$. In particular, if $t \approx \ell(Q)$ we have that

$$
\left(\int_{R_{k}}\left|t^{m}\left(\partial_{t}\right)^{m} \nabla \mathcal{S}_{t}^{\mathcal{L}} g(x)\right|^{p} d x\right)^{\frac{1}{p}} \lesssim 2^{n k \alpha} 2^{-k(m+1)} \ell(Q)^{-n(1 / 2-1 / p)}\|g\|_{L^{2}(Q)} .
$$

By a straightforward duality argument, from the above proposition we deduce 
Corollary 3.3.7. Define $\Theta_{t, m}:=t^{m} \partial_{t}^{m}\left(\mathcal{S}_{t}^{\mathcal{L}} \nabla\right)$. Let $g \in L^{2}(Q)$ and suppose that $p \in\left[2, p_{+}\right]$is such that $|p-2|$ is small enough so that Lemma 2.5.4 holds. Then for $q=\frac{p}{p-1}$ and $k \geq 1$, we have that

$$
\left\|\Theta_{t, m}\left(f 1_{R_{k}}\right)\right\|_{L^{2}(Q)} \lesssim 2^{n k \alpha} 2^{-k(m+1)} t^{m} \ell(Q)^{-n(1 / q-1 / 2)} \ell(Q)^{-m}\|f\|_{L^{q}\left(R_{k}\right)},
$$

where $\alpha=\alpha(p)$ is as in Proposition 3.3.6. Moreover, if $t \approx \ell(Q)$, then for all $k \geq 0$,

$$
\begin{aligned}
\left\|\Theta_{t, m}\left(f 1_{R_{k}}\right)\right\|_{L^{2}(Q)} \lesssim 2^{n k \alpha} 2^{-k(m+1)} \ell(Q)^{-n(1 / q-1 / 2)}\|f\|_{L^{q}\left(R_{k}\right)} & \\
& \approx 2^{n k \alpha} 2^{-k(m+1)} t^{-n(1 / q-1 / 2)}\|f\|_{L^{q}\left(R_{k}\right)} .
\end{aligned}
$$

Proof of Proposition 3.3.6. Notice that $g \in L^{2}(Q) \subset L^{2 n /(n+1)}(Q) \subset H^{-\frac{1}{2}}\left(\mathbb{R}^{n}\right)$, so that $\mathcal{S}_{t}^{\mathcal{L}} g$ is well-defined.

We treat first the case $k \geq 1$. Fix a small parameter $\delta=\delta(m)>0$ and set $\tilde{R}_{k}=$ $(2+\delta)^{k+1} Q \backslash(2-\delta)^{k} Q$ be a small (but fixed) dilation of $R_{k}$. We may use that $\partial_{t}^{m} u$ is a solution (see Proposition 2.5.35), a slight variant of Lemma 2.5.39 adapted to annular regions, and Proposition 2.5.9 to see that

$$
\left(\int_{R_{k}}\left|t^{m}\left(\partial_{t}\right)^{m} \nabla \mathcal{S}_{t}^{\mathcal{L}} g\right|^{p}\right)^{\frac{1}{p}} \lesssim \frac{t^{m}}{\left(2^{k} \ell(Q)\right)^{1+1 / p}}\left(\iint_{I_{k, 1}}\left|\partial_{t}^{m} \mathcal{S}_{t}^{\mathcal{L}} g\right|^{p}\right)^{1 / p},
$$

where $I_{k}:=\left\{(y, s) \in \mathbb{R}^{n+1}: y \in \tilde{R}_{k, 1}, s \in\left(t-2^{k} \ell(Q), t+2^{k} \ell(Q)\right\}\right.$ and $\tilde{R}_{k, j}$ is defined as $\tilde{R}_{k}$ but with $\delta /(m+2-j)$ instead of $\delta$ (so that, in particular, $\left.\tilde{R}_{k, m+1}=\tilde{R}_{k}\right)$. Now, applying the $(n+1)$-dimensional $L^{p}$ Caccioppoli $m$ times (see Proposition 2.5.9), we further obtain that

$$
\left(\int_{R_{k}}\left|t^{m} \partial_{t}^{m} \nabla \mathcal{S}_{t}^{\mathcal{L}} g\right|^{p}\right)^{\frac{1}{p}} \lesssim \frac{t^{m}}{\left(2^{k} \ell(Q)\right)^{m+1+1 / p}}\left(\iint_{I_{k, m+1}}\left|\mathcal{S}_{t}^{\mathcal{L}} g\right|^{p}\right)^{1 / p}
$$


Now, using Hölder's inequality in $t$ and the mapping properties of $\mathcal{S}_{t}^{\mathcal{L}}$ we see that

$$
\begin{aligned}
& \left(\int_{R_{k}}\left|t^{m} \partial_{t}^{m} \nabla \mathcal{S}_{t}^{\mathcal{L}} g\right|^{p}\right)^{\frac{1}{p}} \lesssim \frac{t^{m}}{\left[2^{k} \ell(Q)\right]^{m+1}} \sup _{t \in\left(-2^{k} \ell(Q), 2^{k} \ell(Q)\right)}\left\|\mathcal{S}_{t}^{\mathcal{L}} g\right\|_{L^{p}\left(\tilde{R_{k}}\right)} \\
& \lesssim \frac{t^{m}\left[2^{k} \ell(Q)\right]^{n \alpha}}{\left[2^{k} \ell(Q)\right]^{m+1}} \sup _{t \in\left(-2^{k} \ell(Q), 2^{k} \ell(Q)\right)}\left\|\mathcal{S}_{t}^{\mathcal{L}} g\right\|_{L^{p+}\left(\tilde{R_{k}}\right)} \lesssim \frac{t^{m}\left[2^{k} \ell(Q)\right]^{n \alpha}}{\left[2^{k} \ell(Q)\right]^{m+1}}\|g\|_{L^{p-}(Q)} \\
& \lesssim \frac{t^{m}\left[2^{k} \ell(Q)\right]^{n \alpha}}{\left[2^{k} \ell(Q)\right]^{m+1}}|Q|^{\frac{1}{2^{n}}}\|g\|_{L^{2}(Q)} .
\end{aligned}
$$

The case $k=0$ is treated similarly, except that we impose the restriction $t \approx \ell(Q)$ to guarantee that we are far away from the support of $g$.

For the most part, the case $q=p=2$ in the above proposition will be enough for our purposes; however, the introduction of error terms in the $T b$ theorem below will necessitate a certain quasi-orthogonality result for which we use the case $p>2>q$.

Lemma 3.3.8 (Quasi-orthogonality). Let $m \geq n$ and let $Q_{s}$ be a CLP family (see Definition 2.4.13). Then there exist $\gamma, C>0$ such that for all $s<t$, we have that

$$
\left\|\Theta_{t, m} B_{1} I_{1} Q_{s}^{2} g\right\|_{2} \leq C\left(\frac{s}{t}\right)^{\gamma}\left\|Q_{s} g\right\|_{2}
$$

for all $g \in L^{2}\left(\mathbb{R}^{n}\right)$, where $I_{1}$ is the standard fractional integral operator of order 1 . Here, $C$ and $\gamma$ depend on $m, n, \lambda, \Lambda$, and the constants in the definition of $Q_{s}$.

Proof. Let us first note that if $\alpha(p)$ is given as in Proposition 3.3.6, then $\alpha(p) \leq 1 /(2 n)$. Therefore, for all $k \geq 0$ and $Q$ with $\ell(Q) \approx t$, we have that

$$
\left\|\Theta_{t, m}\left(f 1_{R_{k}}\right)\right\|_{L^{2}(Q)} \lesssim 2^{n k \alpha} 2^{-k(m+1)} t^{-n(1 / q-1 / 2)}\|f\|_{L^{q}\left(R_{k}\right)}
$$




$$
\lesssim 2^{-k \beta} t^{-n(1 / q-1 / 2)}\|f\|_{L^{q}\left(R_{k}\right)},
$$

for some $\beta \geq n / 2+1$, where we use that $m \geq n$.

We first establish a variant of (3.9) with a collection of CLP families. Let $\zeta \in C_{c}^{\infty}\left(B\left(0, \frac{1}{100}\right)\right)$ be real, radial and have zero average. Define $Q_{s}^{(1)} f(x):=\left(\zeta_{s} * f\right)(x)$, where $\zeta_{s}(x)=s^{-n} \zeta\left(\frac{x}{s}\right)$. Set $Q_{s}^{(2)} f:=s^{2} \Delta e^{s^{2} \Delta} f$. By re-normalizing $\zeta$ (multiplying by a constant) we may assume that

$$
\int_{0}^{\infty} Q_{s}^{(1)} Q_{s}^{(2)} \frac{d s}{s}=I
$$

in the strong operator topology of $L^{2}$. Indeed,

$$
\begin{aligned}
\mathscr{F}\left(\int_{0}^{\infty} Q_{s}^{(1)} Q_{s}^{(2)} f \frac{d s}{s}\right)=-\int_{0}^{\infty} \hat{\zeta}(s|\xi|) s^{2}|\xi|^{2} e^{-s^{2}|\xi|^{2}} \hat{f}(\xi) & \frac{d s}{s} \\
& =-\hat{f}(\xi) \int_{0}^{\infty} \hat{\zeta}(s) s^{2} e^{-s^{2}} \frac{d s}{s},
\end{aligned}
$$

where $\hat{\zeta}$ is the Fourier transform of $\zeta$ and we abused notation by regarding $\zeta$ and hence $\hat{\zeta}$ as a function of the radial variable. Then, to achieve the desired reproducing formula, (3.11), we may renormalize $\zeta$ so that $\int_{0}^{\infty} \hat{\zeta}(s) s^{2} e^{-s^{2}} \frac{d s}{s}=-1$. Let $q<2$ be such that the conclusion of Corollary 3.3.7 holds. We will show that for all $s<t$,

$$
\left\|\Theta_{t, m} B_{1} I_{1} Q_{s}^{(1)} Q_{s}^{(2)} g\right\|_{2} \lesssim\left(\frac{s}{t}\right)^{n(1 / q-1 / 2)}\left\|Q_{s}^{(3)} \vec{R} g\right\|_{2},
$$

where $\vec{R}=I_{1} \nabla_{\|}$is the vector valued Riesz transform on $\mathbb{R}^{n}$ and $Q_{s}^{(3)} \vec{f}:=s e^{s^{2} \Delta} \operatorname{div}_{\|} \vec{f}$. Before proving (3.12), we establish a "local hypercontractivity" estimate. For $Q \subset \mathbb{R}^{n}$ a cube and 
$s<\ell(Q)$, we have that

$$
\left\|Q_{s}^{(1)} h\right\|_{L^{n-q}\left(R_{k}(Q)\right)} \lesssim s^{-n\left(\frac{1}{2}-\frac{n-q}{n q}\right)}\|h\|_{L^{2}\left(B_{k}\right)}
$$

for all $k \geq 0$, where $R_{0}(Q)=2 Q, R_{k}(Q)=2^{k+1} Q \backslash 2^{k} Q$ for $k \geq 1$, and $B^{k}(Q)=$ $B\left(x_{Q}, 2^{k+2} \ell(Q) \sqrt{n}\right)$. To verify (3.13), we use that $s<\ell(Q)$, Young's convolution inequality, and the properties of $\zeta_{s}$.

Now we are ready to prove (3.12). Let $\mathbb{D}_{t}$ be a grid of cubes on $\mathbb{R}^{n}$ with side length $t$ and set $F=I_{1} g$. Consider the estimate

$$
\begin{aligned}
& \left\|\Theta_{t, m} B_{1} I_{1} Q_{s}^{(1)} Q_{s}^{(2)} g\right\|_{2}=\left\|\Theta_{t, m} B_{1} Q_{s}^{(1)} Q_{s}^{(2)} F\right\|_{2}=\left(\sum_{Q \in \mathbb{D}_{t}} \int_{Q}\left|\Theta_{t, m} B_{1} Q_{s}^{(1)} Q_{s}^{(2)} F\right|^{2}\right)^{1 / 2} \\
& \leq \sum_{k \geq 0}\left(\sum_{Q \in \mathbb{D}_{t}} \int_{Q}\left|\Theta_{t, m}\left(\left[B_{1} Q_{s}^{(1)} Q_{s}^{(2)} F\right] \mathbb{1}_{R_{k}(Q)}\right)(x)\right|^{2} d x\right)^{1 / 2} \\
& \lesssim \sum_{k \geq 0} 2^{-\beta k} t^{-n(1 / q-1 / 2)}\left(\sum_{Q \in \mathbb{D}_{t}}\left(\int_{R_{k}(Q)}\left|B_{1} Q_{s}^{(1)} Q_{s}^{(2)} F\right|^{q}\right)^{2 / q}\right)^{1 / 2} \\
& \lesssim\left\|B_{1}\right\|_{n} \sum_{k \geq 0} 2^{-\beta k} t^{-n(1 / q-1 / 2)}\left(\sum_{Q \in \mathbb{D}_{t}}\left(\int_{R_{k}(Q)}\left|Q_{s}^{(1)} Q_{s}^{(2)} F\right|^{\frac{n q}{n-q}}\right)^{\frac{2(n-q)}{n q}}\right)^{1 / 2} \\
& \lesssim \sum_{k \geq 0} 2^{-\beta k} t^{-n(1 / q-1 / 2)} s^{-n\left(\frac{1}{2}-\frac{n-q}{n q}\right)}\left(\sum_{Q \in \mathbb{D}_{t}} \int_{B_{k}(Q)}\left|Q_{s}^{(2)} F\right|^{2}\right)^{1 / 2} \\
& \lesssim \sum_{k \geq 0} 2^{-\beta k} t^{-n(1 / q-1 / 2)} s^{-n\left(\frac{1}{2}-\frac{n-q}{n q}\right)} s\left(\sum_{Q \in \mathbb{D}_{t}} \int_{B_{k}(Q)}\left|Q_{s}^{(3)} \nabla_{\|} F\right|^{2}\right)^{1 / 2} \\
& \lesssim\left(\frac{s}{t}\right)^{n(1 / q-1 / 2)} \sum_{k \geq 0} 2^{-\beta k+\frac{n k}{2}}\left(\sum_{Q \in \mathbb{D}_{t}} \int_{Q} f_{B_{k}(Q)}\left|Q_{s}^{(3)} \nabla_{\|} F(x)\right|^{2} d x d y\right)^{1 / 2} \\
& \vdots\left(\frac{s}{t}\right)^{n(1 / q-1 / 2)} \sum_{k \geq 0} 2^{-\beta k+\frac{n k}{2}}\left(\int_{\mathbb{R}^{n}} f_{|x-y|<2^{k} t}\left|Q_{s}^{(3)} \nabla_{\|} F(x)\right|^{2} d x d y\right)^{1 / 2} \\
& \quad \leq\left(\frac{s}{t}\right)^{n(1 / q-1 / 2)}\left\|Q_{s}^{(3)} \nabla_{\|} F\right\|_{2}=\left(\frac{s}{t}\right)^{n(1 / q-1 / 2)}\left\|Q_{s}^{(3)} \vec{R} g\right\|_{2},
\end{aligned}
$$


where first we used that $I_{1} g=F$, then Minkowski's inequality in the second line, (3.10) in the third line, Hölder's inequality in the fourth line, (3.13) in the fifth line, and the mapping properties of the Hardy-Littlewood maximal function in the last line. The above estimate proves (3.12).

Now we are ready to pass to an arbitrary CLP family $Q_{s}$. We may obtain, using the Cauchy-Schwarz inequality and (3.11), that

$$
\begin{aligned}
& \left\|\Theta_{t, m} B_{1} I_{1} Q_{s}^{2} g\right\|_{2}=\int_{\mathbb{R}^{n}}\left|\int_{0}^{\infty} \Theta_{t, m} B_{1} I_{1} Q_{\tau}^{(1)} Q_{\tau}^{(2)} Q_{s}^{2} g(x) \frac{d \tau}{\tau}\right| d x \\
& \quad \leq C_{\gamma} \int_{\mathbb{R}^{n}} \int_{0}^{\infty} \max \left(\frac{s}{\tau}, \frac{\tau}{s}\right)^{\gamma}\left|\Theta_{t, m} B_{1} I_{1} Q_{\tau}^{(1)} Q_{\tau}^{(2)} Q_{s}^{2} g(x)\right|^{2} \frac{d \tau}{\tau} d x=: I+I I+I I I,
\end{aligned}
$$

where $I, I I, I I I$ are, respectively, the integrals over the intervals $\tau<s<t, s \leq \tau \leq t$, and $s<t<\tau$. On the other hand, note that the kernel of $Q_{s}^{(3)} \vec{R}$ is, up to a constant multiple, the inverse Fourier transform of $s|\xi| e^{-s^{2}|\xi|^{2}}$. Therefore, if we set $Q_{s}^{(4)}=Q_{s}^{(3)} \vec{R}$, then we have that

$$
\max \left\{\left\|Q_{\tau}^{(4)} Q_{s} f\right\|_{2},\left\|Q_{\tau}^{(2)} Q_{s} f\right\|_{2}\right\} \lesssim \min \left(\frac{\tau}{s}, \frac{s}{\tau}\right)^{2 \gamma}\|f\|_{2}
$$

for some $\gamma>0$ (and hence all smaller $\gamma$ ). For convenience, set $\sigma=n(1 / q-1 / 2)$ and we assume that $\gamma$ above is such that $\gamma<2 \sigma$. By (3.12) and (3.14), we have that

$$
I \lesssim \int_{0}^{s}\left(\frac{s}{\tau}\right)^{\gamma}\left(\frac{\tau}{t}\right)^{2 \sigma}\left\|Q_{\tau}^{(4)} Q_{s}^{2} h\right\|_{2}^{2} \frac{d \tau}{\tau} \lesssim\left(\frac{s}{t}\right)^{2 \sigma}\left\|Q_{s} h\right\|_{2}^{2}
$$

and observe that $\tau<s$ in the present scenario. Similarly, we have that

$$
I I \lesssim \int_{s}^{t}\left(\frac{\tau}{s}\right)^{\gamma}\left(\frac{\tau}{t}\right)^{2 \sigma}\left(\frac{s}{\tau}\right)^{2 \gamma}\left\|Q_{s} h\right\|_{2}^{2} \frac{d \tau}{\tau} \lesssim\left(\frac{s}{t}\right)^{\gamma}\left\|Q_{s} h\right\|_{2}^{2}
$$


since in particular, $\gamma<2 \sigma$. Finally, by (3.3) and the mapping $B_{1} I_{1}: L^{2}\left(\mathbb{R}^{n}\right) \rightarrow L^{2}\left(\mathbb{R}^{n}\right)$, we have that $\Theta_{t, m} B_{1} I_{1} Q_{\tau}^{(1)}: L^{2}\left(\mathbb{R}^{n}\right) \rightarrow L^{2}\left(\mathbb{R}^{n}\right)$ uniformly in $t$ and $\tau$, and thus it follows that

$$
I I I \lesssim \int_{t}^{\infty}\left(\frac{\tau}{s}\right)^{\gamma}\left\|Q_{\tau}^{(2)} Q_{s} h(x)\right\|_{2}^{2} \frac{d \tau}{\tau} \lesssim \int_{t}^{\infty}\left(\frac{\tau}{s}\right)^{\gamma}\left(\frac{s}{\tau}\right)^{2 \gamma}\left\|Q_{s} h\right\|_{2}^{2} \frac{d \tau}{\tau} \lesssim\left(\frac{s}{t}\right)^{\gamma}\left\|Q_{s} h\right\|_{2}^{2}
$$

where we used (3.14).

We conclude this section with the following proposition, which summarizes the offdiagonal decay given by Proposition 3.3.6 and Corollary 3.3.7.

Proposition 3.3.15. For $m \in \mathbb{N}, m \geq \frac{n+1}{2}+2$, the operators $t^{m} \partial_{t}^{m}\left(\mathcal{S}_{t} \nabla\right), t^{m} \partial_{t}^{m+1} \mathcal{S}_{t}$ and $\Theta_{t}^{\prime}$ defined by

$$
\Theta_{t}^{\prime} \vec{g}(x):=\left(t^{m} \partial_{t}^{m} \mathcal{S}_{t} \nabla \widetilde{A} \vec{g}+t^{m} \partial_{t}^{m} \mathcal{S}_{t}\left[B_{2 \|} g\right]\right)(x)
$$

have good off diagonal-decay in the sense of Definition 2.4 .9 with the implicit constants depending on $n, m, \lambda$, and $\Lambda$, provided that $\max \left\{\left\|B_{1}\right\|_{n},\left\|B_{2}\right\|_{n}<\varepsilon_{0}\right.$, where $\varepsilon_{0}$ depends on $n$, $\lambda$, and $\Lambda$.

Proof. By Corollary 3.3.7 with $p=2$, for any cube $Q \subset \mathbb{R}^{n}$ and $k \geq 2$ we have that

$$
\left\|\Theta_{t, m}\left(f 1_{R_{k}}\right)\right\|_{L^{2}(Q)}^{2} \lesssim 2^{-k}\left(\frac{t}{2^{k} \ell(Q)}\right)^{2 m}\|f\|_{L^{2}\left(R_{k}\right)}^{2}
$$

where $R_{k}=R_{k}(Q)=2^{k+1} Q \backslash 2^{k} Q$. Thus, for all $t \in(0, C \ell(Q))$, it follows that

$$
\left\|\Theta_{t, m}\left(f 1_{R_{k}}\right)\right\|_{L^{2}(Q)}^{2} \lesssim 2^{-k n}\left(\frac{t}{2^{k} \ell(Q)}\right)^{2 m-(n-1)}\|f\|_{L^{2}\left(R_{k}\right)}^{2}
$$


so that if $m \geq \frac{n+1}{2}$, we obtain the estimate

$$
\left\|\Theta_{t, m}\left(f 1_{R_{k}}\right)\right\|_{L^{2}(Q)}^{2} \lesssim 2^{-k n}\left(\frac{t}{2^{k} \ell(Q)}\right)^{2}\|f\|_{L^{2}\left(R_{k}\right)}^{2}
$$

This bound provides the desired good off-diagonal decay for $t^{m} \partial_{t}^{m}\left(\mathcal{S}_{t}^{\mathcal{L}} \nabla\right), t^{m} \partial_{t}^{m+1} \mathcal{S}_{t}^{\mathcal{L}}$ and $t^{m} \partial_{t}^{m} \mathcal{S}_{t}^{\mathcal{L}} \nabla \widetilde{A} \vec{g}$ in the sense of Definition 2.4.9. To obtain the good off-diagonal decay for the remainder of $\Theta_{t}^{\prime}, t^{m} \partial_{t}^{m}\left(\mathcal{S}_{t}^{\mathcal{L}} B_{2 \|}\right)$, we return to the proof of Proposition 3.3.6 and make a slight modification. Let $\eta$ be a smooth cut-off adapted to $R_{k}$; that is, $\eta \equiv 1$ on $R_{k}, \eta \in C_{c}^{\infty}\left(\widetilde{R}_{k}\right)$ and $|\nabla \eta| \lesssim \frac{1}{\ell(Q)}$, where $\widetilde{R}_{k}$ is as in Proposition 3.3.6. Then for $g \in L^{2}(Q)$ with supp $g \subseteq Q$, from Hölder's inequality and the Sobolev embedding on $\mathbb{R}^{n}$ we have that

$$
\begin{aligned}
& \left\|t^{m} \partial_{t}^{m} B_{2 \|} \mathcal{S}_{t}^{\mathcal{L}} g\right\|_{L^{2}\left(R_{k}\right)} \lesssim\left\|\eta t^{m} \partial_{t}^{m} \mathcal{S}_{t}^{\mathcal{L}} g\right\|_{L^{\frac{2 n}{n-2}\left(\mathbb{R}^{n}\right)}}^{2} \\
& \lesssim\left\|(\nabla \eta) t^{m} \partial_{t}^{m} \mathcal{S}_{t}^{\mathcal{L}} g\right\|_{L^{2}\left(\widetilde{R}_{k}\right)}^{2}+\left\|t^{m} \partial_{t}^{m} \nabla \mathcal{S}_{t}^{\mathcal{L}} g\right\|_{L^{2}\left(\widetilde{R}_{k}\right)}^{2} \lesssim\left\|t^{m-1} \partial_{t}^{m} \mathcal{S}_{t}^{\mathcal{L}} g\right\|_{L^{2}\left(\widetilde{R}_{k}\right)}^{2}+\left\|t^{m} \partial_{t}^{m} \nabla \mathcal{S}_{t}^{\mathcal{L}} g\right\|_{L^{2}\left(\widetilde{R}_{k}\right)}^{2} \\
& \lesssim\left\|\left(\Theta_{t, m-1}\right)^{*} g\right\|_{L^{2}\left(\widetilde{R}_{k}\right)}^{2}+\left\|\left(\Theta_{t, m}\right)^{*} g\right\|_{L^{2}\left(\widetilde{R}_{k}\right)}^{2} .
\end{aligned}
$$

Dualizing these estimates, the off-diagonal decay for $t^{m} \partial_{t}^{m}\left(\mathcal{S}_{t}^{\mathcal{L}} B_{2 \|}\right)$ follows from the offdiagonal decay in (3.16), provided that $m \geq \frac{n+1}{2}+1$.

Before continuing on to the next section we make two remarks.

Remarks 3.3.17. i) In the next section, we will use the off diagonal decay of the operators in Proposition 3.3.15 or similar ones. The proof of good off-diagonal decay for these operators is entirely analogous to those above.

ii) As seen above there, may be some loss of $t$-derivatives (and hence decay) in our operators when we obtain certain estimates. Therefore, when proving the first square function 
estimate (Theorem 4.2.1), we ensure that $m \geq n+10>\frac{n+1}{2}+10$ so that Lemma 3.3.8 and Proposition 3.3.15 hold.

\subsection{Off-diagonal decay and weighted estimates}

Definition 3.4.1. We define the interval $\left(2_{-}, 2_{+}\right)$as the largest open interval, symmetric around 2 with the following two properties:

1. $2 n /(n+1)=2_{\#}<2_{-}<2<2_{+}<2^{\#}=2 n /(n-1)$.

2. If $p \in\left(2_{-}, 2_{+}\right)$, then for every weak solution $u \in W_{\text {loc }}^{1,2}\left(\mathbb{R}_{+}^{n+1}\right)$ of $\mathcal{L} u=0$ in $\mathbb{R}_{+}^{n+1}$, the $L^{p}$ Caccioppoli inequalities in Proposition 2.5.9 and Lemma 2.5.39 hold, with implicit constants depending only on $n, p, \alpha$, ellipticity of $\mathcal{L}$, and $\rho$.

Proposition 3.4.2 (Off-diagonal Estimates. Part 1). For this Proposition let $\Theta_{t, m}$ denote either of the following operators:

$$
t^{m} \partial_{t}^{m} \nabla \mathcal{S}_{t}^{\mathcal{L}}, \quad t^{m} \partial_{t}^{m-1} \nabla\left(\mathcal{S}_{t}^{\mathcal{L}} \nabla\right), \quad\left(t^{m} \partial_{t}^{m} \nabla \mathcal{S}_{t}^{\mathcal{L}} 1\right) \cdot P_{t},
$$

where $P_{t} i$ is an approximate identity with smooth, even, compactly supported kernel.

Let $2_{-}<q \leq p<2_{+}$and $Q \subset \mathbb{R}^{n}$ a cube. For every $h \in L^{q}\left(\mathbb{R}^{n}\right),|t| \approx \ell(Q)$, and $k \geq 0$ it holds

$$
\left\|\Theta_{t, m}\left(h \mathbb{1}_{Q}\right)\right\|_{L^{p}(2 Q)} \lesssim_{n, p, q}|Q|^{\frac{1}{p}-\frac{1}{q}}\|h\|_{L^{q}(Q)},
$$

Moreover for any $j \geq 1$ and $t \in \mathbb{R}$, if we define as usual $R_{j}(Q):=2^{j+1} Q \backslash 2^{j} Q$,

$$
\left\|\Theta_{t, m} g\right\|_{L^{p}\left(R_{j}(Q)\right)} \lesssim_{n, p, q, m} 2^{-n \gamma_{1}}\left(\frac{|t|}{2^{j} \ell(Q)}\right)^{m}|Q|^{\frac{1}{p}-\frac{1}{q}}\|g\|_{L^{q}(Q)},
$$


and

$$
\left\|\Theta_{t, m} g\right\|_{L^{p}(Q)} \lesssim_{n, p, q, m} 2^{-n \gamma_{1}}\left(\frac{|t|}{2^{j} \ell(Q)}\right)^{m}|Q|^{\frac{1}{p^{-}-\frac{1}{q}}}\|g\|_{L^{q}\left(R_{j}(Q)\right)},
$$

where $\gamma_{1}=1 / 2^{\#}-1 / p$, for the case of $\nabla \mathcal{S}_{t}^{\mathcal{L}}$, and $\gamma_{1}=1 / 2^{\#}-1 / p-1$ in the case of $\nabla\left(\mathcal{S}_{t}^{\mathcal{L}} \nabla\right)$.

Proof. The proof of this result is essentially contained in Proposition 3.3.6. We sketch some of the modifications needed.

We start with the case of $\Theta_{t, m}=t^{m} \partial_{t}^{m} \nabla \mathcal{S}_{t}^{\mathcal{L}}$. Here estimate (3.5) was obtained in Proposition 3.3.6. By duality estimate (3.6) is equivalent to (3.5) for $t^{m} \partial_{t}^{m}\left(\mathcal{S}_{t}^{\mathcal{L}} \nabla\right)$.

Let $\widetilde{R}_{j}:=(3 / 2) R_{j} \times\left(t-2^{j} \ell(Q), t+2^{j} \ell(Q)\right)$ for some $\delta>0$ small enough so that if $f \in C_{c}^{\infty}(Q)$ then $\left(\mathcal{S}_{t}^{\mathcal{L}} \nabla_{\|} g\right)=\mathcal{S}_{t}^{\mathcal{L}} \operatorname{div}_{\|} g$ is a solution in $\widetilde{R}_{j}$. By a careful application of Caccioppoli's inequality on slices, followed by the standard Caccioppoli inequality in $L^{p}$ $m-1$ times we obtain

$$
\begin{aligned}
\left\|t^{m} \partial_{t}^{m}\left(\mathcal{S}_{t}^{\mathcal{L}} \nabla_{\|}\right) \cdot g\right\|_{L^{p}\left(R_{j}(Q)\right)} & =|t|^{m}\left\|\partial_{t}^{m} \mathcal{S}_{t}^{\mathcal{L}} \operatorname{div}_{\|} g\right\|_{L^{p}\left(R_{j}(Q)\right)} \\
& \lesssim\left(\frac{|t|}{2^{j} \ell(Q)}\right)^{m}\left(\frac{1}{2^{j} \ell(Q)} \iint_{\widetilde{R}_{j}}\left|\mathcal{S}_{s}^{\mathcal{L}} \operatorname{div}_{\|} g(x)\right|^{p} d x d s\right)^{1 / p} .
\end{aligned}
$$

By duality again, it's enough to prove that

$$
\left\|\nabla_{\|} \mathcal{S}_{S}^{\mathcal{L}} h\right\|_{L^{q^{\prime}}(Q)} \lesssim 2^{-n \gamma_{1}}|Q|^{1 / p-1 / q}\|h\|_{L^{p^{\prime}\left(3 / 2 R_{j}\right)}},
$$

uniformly for $s \in \mathbb{R}$. For this, if we define $\widetilde{Q}:=3 / 2 Q \times(s-\ell(Q), s+\ell(Q))$, by Caccioppoli's 
inequality

$$
\begin{aligned}
\left\|\nabla_{\|} \mathcal{S}_{S}^{\mathcal{L}} h\right\|_{L^{q^{\prime}}(Q)} & \lesssim \frac{1}{\ell(Q)}\left(\frac{1}{\ell(Q)} \iint_{\widetilde{Q}}\left|\mathcal{S}_{\tau}^{\mathcal{L}} h(x)\right|^{q^{\prime}} d x d \tau\right)^{1 / q^{\prime}} \\
& \lesssim|Q|^{1 / q^{\prime}-1 / 2^{\#}-1 / n}\left(\frac{1}{\ell(Q)} \int_{s-\ell(Q)}^{s+\ell(Q)}\left|R_{j}\right|^{q^{\prime}\left(1 / 2 \#-1 / p^{\prime}\right)}\|h\|_{L^{p^{\prime}\left(3 / 2 R_{j}\right)}}^{q^{\prime}} d \tau\right)^{1 / q^{\prime}} \\
& \lesssim 2^{n\left(1 / p-1 / 2^{\sharp}\right)}|Q|^{1 / q^{\prime}-1 / p^{\prime}}\|h\|_{L^{p^{\prime}\left(3 / 2 R_{j}\right)}},
\end{aligned}
$$

where we used the mapping property $\mathcal{S}_{s}^{\mathcal{L}}: L^{2 \#}\left(\mathbb{R}^{n}\right) \rightarrow L^{2^{\#}}\left(\mathbb{R}^{n}\right)$ uniformly in $s \in \mathbb{R}$, and Hölder's inequality.

The above proof works the same, with straightforward modifications, in the case $\Theta_{t, m}=$ $t^{m} \partial_{t}^{m-1} \nabla\left(\mathcal{S}_{t}^{\mathcal{L}} \nabla\right)$. The case of $\Theta_{t, m}=t^{m} \partial_{t}^{m} \nabla \mathcal{S}_{t}^{\mathcal{L}} 1 \cdot P_{t}$ is handled with the previous estimates and $\left[\mathrm{AAA}^{+} 11\right.$, Lemma 3.11].

The following Proposition follows the same lines as the above, the appropriate modifications being outlined in the proof of Proposition 3.3.15.

Proposition 3.4.10 (Off-diagonal Estimates. Part 2). Let $B \in L^{n}\left(\mathbb{R}^{n} ; \mathbb{C}^{n+1}\right)$ and set $\Theta_{t, m}^{B}:=$ $t^{m} \partial_{t}^{m} \mathcal{S}_{t}^{\mathcal{L}} B \cdot$ acting o functions $C_{c}^{\infty}\left(\mathbb{R}^{n} ; \mathbb{C}^{n+1}\right)$. Then for $2_{-}<r<2<q<2_{+} \Theta_{t, m}^{B}$ satisfies the $L^{r}-L^{q}$ off-diagonal estimates of Definition 2.4 .83 with $\gamma=m / n-\alpha$ for some $\alpha>0$ depending only on dimension, $r$ and $q$.

Proposition 3.4.11. Let $\Theta_{t, m}:=t^{m} \partial_{t}^{m}\left(\mathcal{S}_{t}^{\mathcal{L}} \nabla\right), B \in L^{n}\left(\mathbb{R}^{n} ; \mathbb{C}^{n}\right)$, and let $Q_{s}$ be a standard Littlewood-Paley family. There exists $m_{0}$ such that if $m \geq m_{0}$ and $v \in A_{2 / r}$ (here $r$ is as in the $L^{r}-L^{2}$ off-diagonal estimates for $\Theta_{t, m}$ in Proposition 3.4.2)

$$
\left\|\left(f_{|x-y|<t}\left|\Theta_{t, m} B I_{1} Q_{s}^{2} g(y)\right|^{2} d y\right)^{1 / 2}\right\|_{L^{2}(v)} \lesssim_{[v]_{A_{2} / r}}\left(\frac{s}{t}\right)^{\beta}\left\|Q_{s} g\right\|_{L^{2}(v)}, \quad s<t,
$$


for some $\beta>0$ (possibly depending on $v$ only through $[v]_{A_{2 / r}}$ ).

Proof. The unweighted case is proved in Lemma 3.3.8. The idea is to use interpolation with change of measure to reduce matters to proving a uniform weighted bound of the form

$$
\left\|\left(f_{|x-y|<t}\left|\Theta_{t, m} B I_{1} Q_{s}^{2} g(y)\right|^{2} d y\right)^{1 / 2}\right\|_{L^{2}(v)} \lesssim_{[v]_{A_{2 / r}}}\|g\|_{L^{2}(v)}
$$

This in turn follows from the $L^{r}-L^{2}$ off-diagonal estimates of $\Theta_{t, m}$ in Proposition 3.4.2 and Proposition 2.4.86, together with the bounds for $I_{1} B$ from Proposition 2.4.48; we omit the details.

The computation that follows appears, for example, in [BES19].

Proposition 3.4.14. Let $m \geq 1$ and $\Theta_{t, m}:=t^{m} \partial_{t}^{m} \nabla\left(\mathcal{S}_{t}^{\mathcal{L}} \nabla\right)$ or $t^{m} \partial_{t}^{m} \nabla \mathcal{D}_{t}^{\mathcal{L}}$, then for a.e. $x \in \mathbb{R}^{n}$

$$
\sup _{t>0}\left|\Theta_{t, m} f(x)\right| \lesssim_{m} \mathbb{V}\left(\Theta_{t, m} f\right)(x)+\mathbb{V}\left(\Theta_{t, m+1} f\right)(x)+\left|\Theta_{1, m} f(x)\right|
$$

Proof. First we note that, owing to Lemma 2.2.2, the function $t \mapsto \Theta_{t, m} f(x)=: g_{t}(x)$ is absolutely continuous for a.e. $x \in \mathbb{R}^{n}$. Therefore, by the fundamental theorem of calculus, for such an $x \in \mathbb{R}^{n}$ and every $0<s<t$,

$$
\left|g_{t}(x)\right|^{2}=\left|g_{s}(x)\right|^{2}+2 \int_{s}^{t} \operatorname{Re}\left(g_{t}(x) \overline{\partial_{\tau} g_{\tau}(x)}\right) d \tau .
$$

Notice

$$
\left|\int_{s}^{t} \operatorname{Re}\left(g_{t}(x) \overline{\partial_{\tau} g_{\tau}(x)}\right) d \tau\right| \leq \int_{s}^{t}\left|g_{t}(x)\right|\left|\tau \partial_{\tau} g_{\tau}(x)\right| \frac{d \tau}{\tau} \leq \mathbb{V}\left(g_{t}\right)(x) \mathbb{V}\left(\tau \partial_{\tau} g_{\tau}\right)(x)
$$


by the Cauchy-Schwarz inequality. The result now follows by setting $s=1$ and using Cauchy's inequality with a parameter. 


\section{Chapter 4}

\section{$L^{2}$ estimates for layer potentials}

The main result of this chapter is the following:

Theorem 4.0.1 ( $\left.\left[\mathrm{BHL}^{+} \mathrm{a}\right]\right)$. Let

$$
\mathcal{L}:=-\operatorname{div}\left(A \nabla+B_{1}\right)+B_{2} \cdot \nabla+V
$$

where $A, B_{1}, B_{2}, V$ are as above. There exists $\tilde{\rho}_{1}>0$ depending on dimension and the ellipticity of A such that if

$$
\max \left\{\left\|B_{1}\right\|_{L^{n}\left(\mathbb{R}^{n}\right)},\left\|B_{2}\right\|_{L^{n}\left(\mathbb{R}^{n}\right)},\|V\|_{L^{n / 2}\left(\mathbb{R}^{n}\right)}\right\}<\tilde{\rho}_{1}
$$

then the following estimates hold for the single and double layer potentials.

(i)

$$
\iint_{\mathbb{R}^{n+1}+}\left|t^{m} \partial_{t}^{m} \nabla \mathcal{S}_{t}^{\mathcal{L}} f(x)\right|^{2} \frac{d x d t}{t} \leq C_{m}\|f\|_{L^{2}\left(\mathbb{R}^{n}\right)}^{2}, \quad \text { for each } m \in \mathbb{N},
$$

(ii)

$$
\left\|\operatorname{Tr}_{\tau} \mathcal{S} f\right\|_{L^{\frac{2 n}{n-2}\left(\mathbb{R}^{n}\right)}}+\left\|\operatorname{Tr}_{\tau} \nabla \mathcal{S} f\right\|_{L^{2}\left(\mathbb{R}^{n}\right)} \leq C \int_{\tau}^{\infty} \int_{\mathbb{R}^{n}}\left|t \partial_{t} \nabla \mathcal{S}_{t}^{\mathcal{L}} f(x)\right|^{2} \frac{d x d t}{t}
$$


(iii)

$$
\iint_{\mathbb{R}^{n+1}+}\left|t^{m} \partial_{t}^{m-1} \nabla \mathcal{D}_{t}^{\mathcal{L}} f(x)\right|^{2} \frac{d x d t}{t} \leq C_{m}\|f\|_{L^{2}\left(\mathbb{R}^{n}\right)}^{2} \quad \text { for each } m \in \mathbb{N}
$$

(iv)

$$
\sup _{\tau>0}\left\|\operatorname{Tr}_{\tau} \mathcal{D} f\right\|_{L^{2}\left(\mathbb{R}^{n}\right)} \leq C\|f\|_{L^{2}\left(\mathbb{R}^{n}\right)}
$$

Here, $C$ depends on dimension and ellipticity, while $C_{m}$ depends on $m$, dimension, and ellipticity.

Most of the work is devoted to (i), which we treat via a local $T b$ theorem modeled after the works of [GHN16], with several modifications due to the lower order terms.

Part (ii) we deal with through an integration by parts argument and Green's formula, in order to obtain the control by the square function.

The estimates for the double layer are obtained through an identity relating the single and double layer.

\section{1 $\quad L^{2}$ square function estimates}

The goal of this section is to prove Theorem 1.0.6.

\subsection{Reduction to high order $t$-derivatives}

We will adapt the methods of [GH16, HMM15] to prove the square function bound in Theorem 1.0.6 for $m$ large: 
Theorem 4.2.1 (Square function bound for high $t$-derivatives). For each $m \in \mathbb{N}$ with $m \geq$ $n+10$, we have the estimate

$$
\iint_{\mathbb{R}^{n+1}+}\left|t^{m}\left(\partial_{t}\right)^{m+1} \mathcal{S}_{t}^{\mathcal{L}} f(x)\right|^{2} \frac{d x d t}{t} \leq C\|f\|_{L^{2}\left(\mathbb{R}^{n}\right)}^{2}
$$

where $C$ depends on $m, n, \lambda$, and $\Lambda$, provided that $\max \left\{\left\|B_{1}\right\|_{n},\left\|B_{2}\right\|_{n}\right\}$ is sufficiently small depending on $n, \lambda$, and $\Lambda$. Under the same hypotheses, the analogous bounds hold for $\mathcal{L}$ replaced by $\mathcal{L}^{*}$, and for $\mathbb{R}_{+}^{n+1}$ replaced by $\mathbb{R}_{-}^{n+1}$.

Let us see that we may reduce the proof of Theorem 1.0.6 to that of Theorem 4.2.1. First, it is a fact awarded by using the Caccioppoli inequality on Whitney cubes that square function estimates for solutions $u$ of $\mathcal{L} u=0$ "travel up" the $t$-derivatives:

Lemma 4.2.2 (Square function bound "travels up" $t$-derivatives). Fix $m, k \in \mathbb{N}$ with $m>$ $k \geq 1$. Suppose that $u \in W_{\text {loc }}^{1,2}\left(\mathbb{R}_{+}^{n+1}\right)$ solves $\mathcal{L} u=0$ in $\mathbb{R}_{+}^{n+1}$ in the weak sense. Then there exists a constant $C$ depending only on $m, n, \lambda, \Lambda$, and $\max \left\{\left\|B_{1}\right\|_{n},\left\|B_{2}\right\|_{n}\right\}$, such that $\left\|t^{m} \partial_{t}^{m-1} \nabla u\right\|\left|\leq C\|\| t^{k} \partial_{t}^{k} u \|\right|$

Now, the following proposition (and Lemma 4.2.2 ${ }^{1}$ ) immediately allow us to reduce proof of Theorem 1.0.6 to that of Theorem 4.2.1, and is a partial converse to Lemma 4.2.2. Recall that $L^{2}\left(\mathbb{R}^{n}\right) \subset H^{-\frac{1}{2}}\left(\mathbb{R}^{n}\right)$.

Proposition 4.2.3 (Square function bound "travels down" $t$-derivatives). The following estimates hold, where the implicit constants depend on $m, k, \lambda$, and $\Lambda$.

\footnotetext{
${ }^{1}$ Lemma 4.2.2 is used to show that $\varepsilon_{0}$ can be chosen independently of $m$.
} 
i) For each $f \in L^{2}\left(\mathbb{R}^{n}\right)$ and each $m \geq 1$, $\left\|t^{m} \partial_{t}^{m} \nabla \mathcal{S} f\right\|\left\|\lesssim_{m}\right\| t^{m+1} \partial_{t}^{m+1} \nabla \mathcal{S} f\|+\| f \|_{2}$.

ii) For each $f \in L^{2}\left(\mathbb{R}^{n}\right)$ and each $m>k \geq 1$,

$$
\|\| t^{k} \partial_{t}^{k} \nabla \mathcal{S} f\left\|\left|\lesssim_{m}\left\|t^{m} \partial_{t}^{m+1} \mathcal{S} f\right\|\right|+\right\| f \|_{2}
$$

Proof. One may obtain $i i$ ) as a consequence of $i$ ) via induction on $m$, using Caccioppoli's inequality on Whitney boxes after increasing the number of $t$ derivatives appropriately. So it suffices to prove $i$ ). Fix $m \in \mathbb{N}, N>0$ large, $\epsilon>0$ small and let $f \in L^{2}\left(\mathbb{R}^{n}\right)$. Let $\psi \in C_{c}^{\infty}(0, \infty)$ be a non-negative function which satisfies

$$
\begin{array}{rlrl}
\psi \equiv 1 \quad \text { on }\left(\epsilon, \frac{1}{\epsilon}\right), & \psi \equiv 0 & \text { on }\left(0, \frac{\epsilon}{2}\right) \cup\left(\frac{2}{\epsilon}, \infty\right), \\
\left|\psi^{\prime}\right| \leq \frac{4}{\epsilon} & \text { on }\left(\frac{\epsilon}{2}, \epsilon\right), & \left|\psi^{\prime}\right| \leq 2 \epsilon & \text { on }\left(\frac{1}{\epsilon}, \frac{2}{\epsilon}\right) .
\end{array}
$$

Since $\mathcal{S} f \in Y^{1,2}\left(\mathbb{R}^{n+1}\right)$ and $\mathcal{L} \mathcal{S} f=0$ in $\mathbb{R}_{+}^{n+1}$ in the weak sense, then $\partial_{t}^{m} \mathcal{S} f \in W_{\mathrm{loc}}^{1,2}\left(\mathbb{R}^{n+1}\right)$ and $\mathcal{L} \partial_{t}^{m} \mathcal{S} f=0$ in $\mathbb{R}_{+}^{n+1}$ in the weak sense. Observe that

$$
\int_{B(0, N)} \int_{\epsilon}^{1 / \epsilon} t^{2 m-1}\left|\partial_{t}^{m} \nabla \mathcal{S} f\right|^{2} d t \leq \int_{B(0, N)} \int_{\epsilon / 2}^{2 / \epsilon} t^{2 m-1}\left|\partial_{t}^{m} \nabla \mathcal{S} f\right|^{2} \psi d t
$$

and notice per our observations in Proposition 3.3.1 that the right-hand side above is finite. Now,

$$
\begin{aligned}
& \int_{B(0, N)} \int_{\epsilon / 2}^{2 / \epsilon} t^{2 m-1}\left|\partial_{t}^{m} \nabla \mathcal{S} f\right|^{2} \psi d t=\int_{B(0, N)} \int_{\epsilon / 2}^{2 / \epsilon} t^{2 m-1} \partial_{t}^{m} \nabla \mathcal{S} f \overline{\partial_{t}^{m} \nabla \mathcal{S} f} \psi d t \\
& =-\frac{1}{2 m} \int_{B(0, N)} \int_{\epsilon / 2}^{2 / \epsilon} t^{2 m} \partial_{t}\left(\partial_{t}^{m} \nabla \mathcal{S} f \overline{\partial_{t}^{m} \nabla \mathcal{S} f} \psi\right) d t
\end{aligned}
$$




$$
\begin{aligned}
\leq \frac{1}{m} \int_{B(0, N)} \int_{\epsilon / 2}^{2 / \epsilon} t^{2 m} \mid \partial_{t}^{m+1} \nabla & \mathcal{S} f \| \partial_{t}^{m} \nabla \mathcal{S} f \mid \psi d t \\
& +\frac{1}{m} \int_{B(0, N)}\left[f_{\epsilon / 2}^{\epsilon} t^{2 m}\left|\partial_{t}^{m} \nabla \mathcal{S} f\right|^{2} d t+\int_{1 / \epsilon}^{2 / \epsilon} t^{2 m}\left|\partial_{t}^{m} \nabla \mathcal{S} f\right|^{2} d t\right] .
\end{aligned}
$$

The last two terms are controlled by (3.2). As for the first term, note that $2 m=\frac{2 m-1}{2}+\frac{2 m+1}{2}$, and we use Cauchy's inequality and absorb one of the resulting summands to the left-hand side. Sending $N \rightarrow \infty$ and $\epsilon \searrow 0$ yields the desired result.

Combining Lemma 4.5.5 below and Theorem 4.2.1, we will also obtain the following result.

Theorem 4.2.5 (Square function bound for $\mathcal{S} \nabla$ ). For each $m \in \mathbb{N}$, with $m \geq n+10$,

$$
\iint_{\mathbb{R}^{n+1}+}\left|t^{m}\left(\partial_{t}\right)^{m}\left(\mathcal{S}_{t}^{\mathcal{L}} \nabla\right) \vec{f}(x)\right|^{2} \frac{d x d t}{t} \lesssim\|\vec{f}\|_{L^{2}\left(\mathbb{R}^{n}\right)},
$$

where $C$ depends on $m, n, \lambda, \Lambda$, provided that $\max \left\{\left\|B_{1}\right\|_{n},\left\|B_{2}\right\|_{n}\right\}$ is sufficiently small depending on $m, n, \lambda, \Lambda$. These results hold for $\mathcal{L}^{*}$ and in the lower half space as the hypotheses are symmetric.

\subsection{Setup for the $T b$ argument and testing functions}

Having reduced matters to proving Theorem 4.2.1, we fix $m \in \mathbb{N}$ with $m \geq n+10$. We define the space $H$ to be the subspace of $L^{2}\left(\mathbb{R}^{n}\right)^{n}$ consisting of the gradients of $Y^{1,2}\left(\mathbb{R}^{n}\right)$ functions. That is, $H=\left\{h^{\prime}: h^{\prime}=\nabla F, F \in Y^{1,2}\left(\mathbb{R}^{n}\right)\right\}$. For $h^{\prime} \in H$ and $h^{0} \in L^{2}\left(\mathbb{R}^{n}\right)$, we set $h=\left(h^{\prime}, h^{0}\right)$ and define for each $t \in \mathbb{R} \backslash\{0\}$,

$$
\Theta_{t}^{0} h^{0}:=t^{m} \partial_{t}^{m+1} \mathcal{S}_{t}^{\mathcal{L}} h^{0}, \quad \text { and }
$$




$$
\Theta_{t}^{\prime} h^{\prime}:=t^{m} \partial_{t}^{m}\left(\mathcal{S}_{t}^{\mathcal{L}} \nabla\right) \tilde{A} h^{\prime}+t^{m}\left(\partial_{t}\right)^{m} \mathcal{S}_{t}^{\mathcal{L}}\left(B_{2 \|} \cdot h^{\prime}\right)
$$

where we recall that $\tilde{A}$ is the $(n+1) \times n$ submatrix of $A$ consisting of the first $n$ columns of $A$. We let $\Theta_{t}:=\left(\Theta_{t}^{\prime}, \Theta_{t}^{0}\right): H \times L^{2}\left(\mathbb{R}^{n}\right) \rightarrow L^{2}\left(\mathbb{R}^{n}\right)$, which acts on $h=\left(h^{\prime}, h^{0}\right)$ via the identity $\Theta_{t} h=\Theta_{t}^{\prime} h^{\prime}+\Theta_{t}^{0} h^{0}$.

For each $t>0$, we also define an auxiliary operator $\Theta_{t}^{(a)}: L^{2}\left(\mathbb{R}^{n}, \mathbb{C}^{n+1}\right) \rightarrow L^{2}\left(\mathbb{R}^{n}\right)$ which acts on $g=\left(g^{\prime}, g^{0}\right)$ via $\Theta_{t}^{(a)} g=t^{m}\left(\partial_{t}\right)^{m}\left(\mathcal{S}_{t}^{\mathcal{L}} \nabla\right)\left(g^{\prime}, g^{0}\right)$. This auxiliary operator will play the role of an error term that allows us to integrate by parts. Accordingly, define $\widehat{\Theta}_{t}$ acting on functions $h=\left(h^{\prime}, h^{0}, h^{\prime \prime}\right) \in H \times L^{2}\left(\mathbb{R}^{n}, \mathbb{C}\right) \times L^{2}\left(\mathbb{R}^{n}, \mathbb{C}^{n+1}\right)$ via

$$
\widehat{\Theta}_{t} h(x)=\Theta_{t}\left(h^{\prime}, h_{0}\right)(x)+\Theta_{t}^{(a)} h^{\prime \prime}(x) .
$$

We need to define appropriate testing functions for our family $\left\{\Theta_{t}\right\}$. Let $\tau \in(0,1 / 40)$ be a small parameter to be chosen later, and let $\widetilde{\Psi}$ be a smooth cut-off function in $\mathbb{R}^{n+1}$ with the following properties:

$$
\begin{aligned}
& \widetilde{\Psi} \in C_{c}^{\infty}\left(\left[-\frac{1}{1000}, \frac{1}{1000}\right]^{n} \times\left[-\frac{\tau}{2}, \frac{\tau}{2}\right]\right), \quad \widetilde{\Psi} \equiv 1 \text { on }\left[-\frac{1}{2000}, \frac{1}{2000}\right]^{n} \times\left[-\frac{\tau}{4}, \frac{\tau}{4}\right] \\
& 0 \leq \widetilde{\Psi} \leq 1, \quad|\nabla \widetilde{\Psi}| \lesssim 1 / \tau .
\end{aligned}
$$

Let $\Psi:=c_{n, \tau} \widetilde{\Psi}$ where $c_{n, \tau}$ is chosen so that $\|\Psi\|_{1}=1$. Hence $\Psi$ is a normalization of $\widetilde{\Psi}$. For any cube $Q \subset \mathbb{R}^{n}$, we define the measurable functions

$$
\begin{gathered}
\Psi_{Q}(X):=\frac{1}{\ell(Q)^{n+1}} \Psi\left(\frac{1}{\ell(Q)}\left[X-\left(x_{Q}, 0\right)\right]\right), \quad\left(\text { note that }\left\|\Psi_{Q}\right\|_{1}=1\right), \\
\Psi_{Q}^{ \pm}(y, s):=\Psi_{Q}\left(y, s \mp \frac{3}{2} \tau \ell(Q)\right)
\end{gathered}
$$


and $\Psi_{Q}^{s^{\prime}}(y, s):=\Psi_{Q}\left(y, s+s^{\prime}\right)$, for each $s^{\prime} \in \mathbb{R}$. Let us make a few observations about $\widetilde{\Psi}$ and $\Psi$. The fact that

$$
\mathbb{1}_{\left[-\frac{1}{2000}, \frac{1}{2000}\right]^{n} \times\left[-\frac{\tau}{4}, \frac{\tau}{4}\right]} \leq \widetilde{\Psi} \leq \mathbb{1}_{\left[-\frac{1}{1000}, \frac{1}{1000}\right]^{n} \times\left[-\frac{\tau}{2}, \frac{\tau}{2}\right]}
$$

forces that $c_{n, \tau} \approx \frac{1}{\tau}$ and that $\|\widetilde{\Psi}\|_{2_{*}} \approx \tau^{\frac{1}{2_{*}}}$. Consequently, $\|\Psi\|_{2_{*}} \approx \tau^{-1+1 / 2_{*}}$, and

$$
\left\|\Psi_{Q}\right\|_{2_{*}} \approx \tau^{-1+1 / 2_{*}}\left[\ell(Q)^{n+1}\right]^{-1+1 / 2_{*}}=\left[\tau \ell(Q)^{n+1}\right]^{-1 / 2+1 /(n+1)} .
$$

Of course, the same $L^{2_{*}}$ estimate holds for $\Psi_{Q}^{ \pm}$and $\Psi_{Q}^{s^{\prime}}$. Now, we define for any cube and $s^{\prime} \in \mathbb{R}$ the quantities

$$
F_{Q}^{ \pm}:=\mathcal{L}^{-1}\left(\Psi_{Q}^{ \pm}\right), \quad F_{Q}:=F_{Q}^{+}-F_{Q}^{-}, \quad F_{Q}^{s^{\prime}}:=\mathcal{L}^{-1}\left(\Psi_{Q}^{s^{\prime}}\right)
$$

By our previous observations and the fact that $L^{2 *}\left(\mathbb{R}^{n+1}\right)$ embeds continuously into $\left(Y^{1,2}\left(\mathbb{R}^{n+1}\right)\right)^{*}$, we easily see that for any cube $Q$ and any $s^{\prime} \in \mathbb{R}$, the estimate

$$
\max \left\{\left\|\nabla F_{Q}\right\|_{2},\left\|\nabla F_{Q}^{ \pm}\right\|_{2},\left\|\nabla F_{Q}^{s^{\prime}}\right\|_{2}\right\} \lesssim\left[\tau \ell(Q)^{n+1}\right]^{-1 / 2+1 /(n+1)}
$$

holds. Notice that we have

$$
\Psi_{Q}^{+}(y, s)-\Psi_{Q}^{-}(y, s)=-\int_{-\frac{3}{2} \tau \ell(Q)}^{\frac{3}{2} \tau \ell(Q)} \partial_{s^{\prime}} \Psi\left(y, s+s^{\prime}\right) d s^{\prime}=-\int_{-\frac{3}{2} \tau \ell(Q)}^{\frac{3}{2} \tau \ell(Q)} \partial_{s} \Psi_{Q}^{s^{\prime}}(y, s) d s^{\prime} .
$$

Therefore, the identity

$$
F_{Q}=-\int_{-\frac{3}{2} \tau \ell(Q)}^{\frac{3}{2} \tau \ell(Q)} \mathcal{L}^{-1}\left(D_{n+1} \Psi_{Q}^{s^{\prime}}\right) d s^{\prime}=-\int_{-\frac{3}{2} \tau \ell(Q)}^{\frac{3}{2} \tau \ell(Q)} \partial_{t} \mathcal{L}^{-1}\left(\Psi_{Q}^{s^{\prime}}\right) d s^{\prime}
$$




$$
=-\int_{-\frac{3}{2} \tau \ell(Q)}^{\frac{3}{2} \tau \ell(Q)} \partial_{t} F_{Q}^{s^{\prime}} d s^{\prime}
$$

is valid in $Y^{1,2}\left(\mathbb{R}^{n+1}\right)$. For convenience, we write $\left(\nabla_{y, s} u\right)(y, 0):=\left.\left(\nabla_{y, s} u(y, s)\right)\right|_{s=0}$. We are now ready to define our testing functions $b_{Q}=\left(b_{Q}^{\prime}, b_{Q}^{0}\right)$. Let $b_{Q}^{0}$ be defined via $b_{Q}^{0}(y):=$ $|Q|\left(\partial_{v}^{\mathcal{L},-} F_{Q}\right)(y, 0)$, where

$$
\begin{aligned}
\partial_{v}^{\mathcal{L},-} u(y, 0)=e_{n+1} \cdot\left[A\left(\nabla_{y, s} u\right)(y, 0)-B_{1} u(y, 0)\right] & \\
& =e_{n+1} \cdot\left[A\left(\nabla_{y, s} u\right)(y, 0)\right]-\left(B_{1}\right)_{\perp} u(y, 0) .
\end{aligned}
$$

We define $b_{Q}^{\prime}$ via $b_{Q}^{\prime}:=|Q| \nabla_{\|} F_{Q}(y, 0)$, while we define the auxiliary testing function $b_{Q}^{(a)}$ $\operatorname{via} b_{Q}^{(a)}:=|Q| B_{1} F_{Q}(y, 0)$.

We will define a measure for each cube $Q$ that corresponds to a smoothened characteristic function. We do this exactly as in [GH16]. Let $\omega>0$ to be chosen. For each cube, we let $d \mu_{Q}=\phi_{Q} d x$, where $\phi_{Q}: \mathbb{R}^{n} \rightarrow[0,1]$ is a smooth bump function supported in $(1+\omega) Q$ with $\phi_{Q} \equiv 1$ on $(1 / 2) Q$. Clearly, we can choose $\phi_{Q}$ so that $\phi_{Q} \gtrsim \omega$ on $Q$ and $\left\|\nabla \phi_{Q}\right\|_{L^{\infty}} \lesssim 1 / \ell(Q)$. We also let $\Phi_{Q}: \mathbb{R}^{n+1} \rightarrow[0,1]$ be a smooth extension of $\phi_{Q}$; that is, $\Phi_{Q}(y, 0)=\phi_{Q}(y)$, with $\Phi_{Q}$ supported in $I_{(1+\omega) Q}$ and $\Phi_{Q} \equiv 1$ on $I_{(1 / 2) Q}$, where for any cube $Q \subset \mathbb{R}^{n}$, we let $I_{Q}=Q \times(-\ell(Q), \ell(Q))$ denote the "double Carleson box" associated to $Q$. We may also ensure that $\left\|\nabla \Phi_{Q}\right\|_{L^{\infty}\left(\mathbb{R}^{n+1}\right)} \lesssim 1 / \ell(Q)$. 


\subsection{Properties of the testing functions}

The testing functions defined above enjoy the following essential properties which justify their use in the $T b$ argument.

Proposition 4.4.1 (Properties of the testing functions). Let $b_{Q}=\left(b_{Q}^{\prime}, b_{Q}^{0}\right), \hat{b}_{Q}$, and $\widehat{\Theta}_{t}$ be as above. For any $\eta>0$, there exists $\tau \in(0,1)$ depending on $n, \lambda, \Lambda, \eta$, and $C_{0}=C_{0}(m, \tau)$, and there exists a measure $\mu_{Q}$ as described above, such that for each cube $Q$, the estimates

$$
\begin{aligned}
& \int_{\mathbb{R}^{n}}\left|b_{Q}\right|^{2} \leq C_{0}|Q| \\
& \int_{0}^{\ell(Q)} \int_{Q}\left|\widehat{\Theta}_{t} \hat{b}_{Q}(x)\right|^{2} \frac{d x d t}{t} \leq C_{0}|Q| \\
& \frac{1}{2} \leq \Re e\left(\frac{1}{\mu_{Q}(Q)} \int_{Q} b_{Q}^{0} d \mu\right) \\
& \left|\frac{1}{\mu_{Q}(Q)} \int_{Q} b_{Q}^{\prime} d \mu_{Q}\right| \leq \frac{\eta}{2},
\end{aligned}
$$

hold, provided that $\max \left\{\left\|B_{1}\right\|_{n},\left\|B_{2}\right\|_{n}\right\}=\varepsilon_{m}<\tau$.

We note that while the smallness of $\varepsilon_{m}=\max \left\{\left\|B_{1}\right\|_{n},\left\|B_{2}\right\|_{n}\right\}$ apparently depends on $m$ at this point, we may prove Theorem 4.2.1 for a fixed sufficiently large $m$, and then 
use Lemma 4.2.2 and Proposition 4.2.3 to remove any dependence on $m$ in the bound for $\max \left\{\left\|B_{1}\right\|_{n},\left\|B_{2}\right\|_{n}\right\}$. For now, throughout the $T b$ argument, we shall continue to use $\varepsilon_{m}$ to denote this quantity.

We will establish several preliminary lemmas in anticipation of the proof of the above proposition.

Lemma 4.4.6 (Estimate of the $L^{2}$ norm of $b_{Q}$ ). The estimate

$$
\int_{\mathbb{R}^{n}}\left|b_{Q}\right|^{2} \lesssim \tau^{-2+2 /(n+1)}|Q|
$$

holds, where the implicit constant depends on $n, \lambda$, and $\Lambda$.

Proof. Set $a:=\frac{\tau \ell(Q)}{1000}$ and observe that $F_{Q}$ solves $\mathcal{L} F_{Q}=0$ in the strip $\{(x, t):|t|<50 a\}$. Let $\mathbb{G}_{a}$ be the grid of pairwise disjoint $n$-dimensional cubes with sides of length $a$ parallel to the coordinate axes, and for each $P \in \mathbb{G}_{a}$, define the $(n+1)$-dimensional box $P^{*}:=$ $2 P \times[-2 \ell(P), 2 \ell(P)]$. Applying Lemma 2.5.39 and the estimate (4.1), we obtain that

$$
\begin{aligned}
\int_{\mathbb{R}^{n}}\left|\nabla F_{Q}(\cdot, 0)\right|^{2}=\sum_{P \in \mathbb{G}_{a}} \int_{P}\left|\nabla F_{Q}(\cdot, 0)\right|^{2} & \lesssim \frac{1}{a} \sum_{P \in \mathbb{G}_{a}} \iint_{P^{*}}\left|\nabla F_{Q}\right|^{2} \\
& \lesssim \frac{1}{a}\left\|\nabla F_{Q}\right\|_{2}^{2} \lesssim \frac{1}{a}\left[\tau \ell(Q)^{n+1}\right]^{-1+2 /(n+1)} \lesssim \tau^{-2+2 /(n+1)}|Q|^{-1},
\end{aligned}
$$

where we used that $a \approx \tau \ell(Q)$ and the bounded overlap of $\left\{P^{*}\right\}_{P \in \mathbb{G}_{a}}$. Upon multiplying the above inequality by $|Q|^{2}$, we have the desired estimate up to controlling $\left\||Q|\left(B_{1}\right)_{\perp} F_{Q}(\cdot, 0)\right\|_{L^{2}\left(\mathbb{R}^{n}\right)}^{2}$. We have already shown that $\left\|\nabla_{\|} F_{Q}(\cdot, 0)\right\|_{L^{2}\left(\mathbb{R}^{n}\right)}<\infty$, and from Lemma 2.2.2 and Lemma 2.5.36, we have that $F_{Q}(\cdot, 0) \in L^{2^{*}}\left(\mathbb{R}^{n}\right)$, so that $F(\cdot, 0) \in Y^{1,2}\left(\mathbb{R}^{n}\right)$. From this, we can deduce 
the estimate $\left\|F_{Q}(\cdot, 0)\right\|_{L^{\frac{2 n}{n-2}\left(\mathbb{R}^{n}\right)}} \lesssim\left\|\nabla_{\|} F_{Q}(\cdot, 0)\right\|_{L^{2}\left(\mathbb{R}^{n}\right)}$. Consequently, we may use the estimate for $\left\|\nabla F_{Q}(\cdot, 0)\right\|_{L^{2}\left(\mathbb{R}^{n}\right)}^{2}$ obtained above and Hölder's inequality to show that

$$
\begin{array}{r}
\int_{\mathbb{R}^{n}}\left|\left(B_{1}\right)_{\perp} F_{Q}(\cdot, 0)\right|^{2} \leq\left\|B_{1}\right\|_{n}^{2}\left\|F_{Q}(\cdot, 0)\right\|_{L^{\frac{2 n}{n-2}\left(\mathbb{R}^{n}\right)}}^{2} \lesssim \varepsilon_{m}^{2}\left\|\nabla F_{Q}(\cdot, 0)\right\|_{L^{2}\left(\mathbb{R}^{n}\right)}^{2} \\
\\
\quad \lesssim \varepsilon_{m}^{2} \tau^{-2+2 /(n+1)}|Q|^{-1} .
\end{array}
$$

Upon multiplying the previous estimates by $|Q|^{2}$, we easily obtain the claimed inequality from the ellipticity of $A$.

The next lemma says that we have a Carleson estimate by including the error term.

Lemma 4.4.7 (Good behavior of $\hat{b}_{Q}$ vis-à-vis Carleson norm of $\left.\hat{\Theta}_{t}\right)$. Let $b_{Q}^{\prime}$, $b_{Q}^{0}$, and $b_{Q}^{(a)}$ be as above. Then, if $\hat{b}_{Q}=\left(b_{Q}^{\prime}, b_{Q}^{0}, b_{Q}^{(a)}\right)$, we have the estimate

$$
\int_{0}^{\ell(Q)} \int_{Q}\left|\widehat{\Theta}_{t} \hat{b}_{Q}(x)\right|^{2} \frac{d x d t}{t} \leq C|Q| \tau^{-\beta}
$$

where $\beta=2+2 m-2 /(n+1)>0$, and $C$ depends on $m, n, \lambda$, and $\Lambda$.

Proof. First, let us show the identity

$$
\widehat{\Theta}_{t} \hat{b}_{Q}(x)=|Q| t^{m}\left(\partial_{t}\right)^{m+1} F_{Q}^{-}, \quad \text { on } \mathbb{R}_{+}^{n+1} \text {. }
$$

By (an analogue of) Theorem 3.2.1 iii), to show the above identity, it suffices to show that for each $t>0$, the representation

$$
\hat{\Theta}_{t} \hat{b}_{Q}=|Q| t^{m} \partial_{t}^{m+1}\left(\mathcal{S}_{t}^{\mathcal{L}}\left(\partial_{v}^{\mathcal{L},-} F_{Q}\right)+\mathcal{D}_{t}^{\mathcal{L},+}\left(\operatorname{Tr}_{0} F_{Q}\right)\right)
$$


holds in $L^{2}\left(\mathbb{R}^{n}\right)$. For notational convenience, we will write $F_{Q}^{0}:=\operatorname{Tr}_{0} F_{Q}$. By definition, we have that for any $f \in C_{c}^{\infty}\left(\mathbb{R}^{n}\right)$,

$$
\begin{aligned}
& \left\langle\hat{\Theta}_{t} \hat{b}_{Q}, f\right\rangle=\left\langle|Q| t^{m}\left(D_{n+1}^{m+1} \mathcal{S}^{\mathcal{L}}\right)_{t}\left[\partial_{v}^{\mathcal{L},-} F_{Q}\right], f\right\rangle \\
& +\left\langle|Q| t^{m}\left(D_{n+1}^{m}\left(\mathcal{S}^{\mathcal{L}} \nabla\right)\left[\tilde{A} \nabla_{\|} F_{Q}^{0}+B_{1} F_{Q}^{0}\right]\right)_{t}, f\right\rangle+\left\langle|Q| t^{m}\left(D_{n+1}^{m} \mathcal{S}^{\mathcal{L}}\left[B_{2 \|} \cdot \nabla_{\|} F_{Q}^{0}\right]\right)_{t}, f\right\rangle \\
& =\left\langle|Q| t^{m}\left(D_{n+1}^{m+1} \mathcal{S}^{\mathcal{L}}\right)_{t}\left[\partial_{v}^{\mathcal{L},-} F_{Q}\right], f\right\rangle \\
& +(-1)^{m}\left\langle\tilde{A} \nabla_{\|} F_{Q}^{0}+B_{1} F_{Q}^{0},|Q| t^{m}\left(D_{n+1}^{m} \nabla \mathcal{S}^{\mathcal{L}^{*}}[f]\right)_{-t}\right\rangle \\
& +(-1)^{m}\left\langle B_{2 \|} \cdot \nabla_{\|} F_{Q}^{0},|Q| t^{m}\left(D_{n+1}^{m} \mathcal{S}^{\mathcal{L}^{*}}[f]\right)_{-t}\right\rangle .
\end{aligned}
$$

Therefore, it suffices to show that

$$
\begin{aligned}
\left\langle|Q| t^{m}\left(D_{n+1}^{m+1} \mathcal{D}^{\mathcal{L},+}\left[F_{Q}^{0}\right]\right)_{t}, f\right\rangle=(-1)^{m}\left\langle\tilde{A} \nabla_{\|} F_{Q}^{0}+B_{1} F_{Q}^{0},|Q| t^{m}\left(D_{n+1}^{m} \nabla \mathcal{S}^{\mathcal{L}^{*}}[f]\right)_{-t}\right\rangle & \\
+(-1)^{m}\left\langle B_{2 \|} \cdot \nabla_{\|} F_{Q}^{0},|Q| t^{m}\left(D_{n+1}^{m} \mathcal{S}^{\mathcal{L}^{*}}[f]\right)_{-t}\right\rangle & =:(-1)^{m}|Q| t^{m} I_{t} .
\end{aligned}
$$

We rewrite $I_{t}$ as follows, using Proposition 2.5.38, and the fact that $F_{Q}^{0} \in W^{1,2}\left(\mathbb{R}^{n}\right)$,

$$
\begin{aligned}
I_{t}= & \left\langle\tilde{A} \nabla_{\|} F_{Q}^{0}+B_{1} F_{Q}^{0},\left(\nabla D_{n+1}^{m} \mathcal{S}^{\mathcal{L}^{*}}[f]\right)_{-t}\right\rangle+\left\langle B_{2 \|} \cdot \nabla_{\|} F_{Q}^{0},\left(D_{n+1}^{m} \mathcal{S}^{\mathcal{L}^{*}}[f]\right)_{-t}\right\rangle \\
= & \left\langle\nabla_{\|} F_{Q}^{0},\left(\left(A^{*} \nabla D_{n+1}^{m} \mathcal{S}^{\mathcal{L}^{*}}[f]\right)_{\|}\right)_{-t}+\overline{B_{2 \|}}\left(D_{n+1}^{m} \mathcal{S}^{\mathcal{L}^{*}}[f]\right)_{-t}\right\rangle \\
& +\left\langle F_{Q}^{0}, \overline{B_{1}}\left(\nabla D_{n+1}^{m} \mathcal{S}^{\mathcal{L}^{*}}[f]\right)_{-t}\right\rangle \\
= & (-1)^{m+1}\left\langle F_{Q}^{0}, D_{n+1}^{m+1}\left(\vec{A}_{n+1, \cdot}^{*} \nabla\left(\mathcal{S}^{\mathcal{L}^{*}}[f]\right)_{-s}\right)_{s=t}+D_{n+1}^{m+1}\left(\overline{B_{2 \perp}}\left(\mathcal{S}^{\mathcal{L}^{*}}[f]\right)_{-s}\right)_{s=t}\right\rangle \\
= & \left.(-1)^{m+1} \frac{d^{m+1}}{d s^{m+1}}\right|_{s=t}\left\langle F_{Q}^{0}, \vec{A}_{n+1, \cdot}^{*} \nabla\left(\mathcal{S}^{\mathcal{L}^{*}}[f]\right)_{-s}+\overline{B_{2 \perp}}\left(\mathcal{S}^{\mathcal{L}^{*}}[f]\right)_{-s}\right\rangle
\end{aligned}
$$




$$
\begin{aligned}
=\left.(-1)^{m+1} \frac{d^{m+1}}{d t^{m+1}}\right|_{s=t}\left\langle F_{Q}^{0}, \partial_{v,-s}^{\mathcal{L}^{*},-}\left(\mathcal{S}^{\mathcal{L}^{*}}[f]\right)\right\rangle=\left.(-1)^{m+2} \frac{d^{m+1}}{d t^{m+1}}\right|_{s=t}\left\langle\mathcal{D}_{s}^{\mathcal{L},+}\left[F_{Q}^{0}\right], f\right\rangle \\
=(-1)^{m+2}\left\langle\left(D_{n+1}^{m+1} \mathcal{D}^{\mathcal{L},+}\left[F_{Q}^{0}\right]\right)_{t}, f\right\rangle
\end{aligned}
$$

where we used (i) in Lemma 3.1.10 in the fifth equality, we used (ii) of Proposition 3.2.3 in the sixth equality, and we justify the handling of the $t$-derivatives via Proposition 3.3.5. This concludes the proof of the identity (4.8).

Now, we let $a=\frac{\tau \ell(Q)}{1000}$ as before, and note that $\left(\partial_{t}\right)^{m+2} F_{Q}^{-}$is a solution in the half space $\{(x, t): t>50 a\}$. For $P \in \mathbb{G}_{a}$ and $t \geq 0$, we set

$$
P_{t}^{*}=2 P \times\left(t-\frac{a}{20}, t+\frac{a}{20}\right), \quad \text { and } \quad P_{t}^{* *}=4 P \times\left(t-\frac{a}{5}, t+\frac{a}{5}\right) .
$$

Then using (2.40) and then Proposition 2.5.9 repeatedly $(m+1$ times), we obtain for $t \in(0, \ell(Q)]$

$$
\begin{aligned}
& \int_{Q}\left|\widehat{\Theta}_{t} \hat{b}_{Q}\right|^{2} \leq \int_{\mathbb{R}^{n}}|| Q\left|t^{m}\left(\partial_{t}\right)^{m+1} F_{Q}^{-}(\cdot, t)\right|^{2}=t^{2 m}|Q|^{2} \sum_{P \in \mathbb{G}_{a}} \int_{P}\left|\left(\partial_{t}\right)^{m+1} F_{Q}^{-}(\cdot, t)\right|^{2} \\
& \lesssim t^{2 m}|Q|^{2} a^{-1} \sum_{P \in \mathbb{G}_{a}} \iint_{P_{t}^{*}}\left|\left(\partial_{t}\right)^{m+1} F_{Q}^{-}\right|^{2} \lesssim t^{2 m}|Q|^{2} a^{-1-2 m} \sum_{P \in \mathbb{G}_{a}} \iint_{P_{t}^{* *}}\left|\partial_{t} F_{Q}^{-}\right|^{2} \\
& \lesssim t^{2 m}|Q|^{2} a^{-1-2 m}\left\|\nabla F_{Q}^{-}\right\|_{2}^{2} \lesssim t^{2 m}|Q|^{2} a^{-1-2 m}\left[\tau \ell(Q)^{n+1}\right]^{-1+2 /(n+1)} \lesssim|Q| \tau^{-\beta}\left(\frac{t}{\ell(Q)}\right)^{2 m},
\end{aligned}
$$

where we used the bounded overlap of $\left\{P_{t}^{* *}\right\}_{P \in \mathbb{G}_{a}}$. Hence, we see that

$$
\int_{0}^{\ell(Q)} \int_{Q}\left|\widehat{\Theta}_{t} \hat{b}_{Q}(x)\right|^{2} \frac{d x d t}{t} \lesssim|Q| \tau^{-\beta} \int_{0}^{\ell(Q)}\left(\frac{t}{\ell(Q)}\right)^{2 m} \frac{d x d t}{t} \lesssim|Q| \tau^{-\beta}
$$


Observe that Lemma 4.4.6 and the properties of $\mu_{Q}$ allow us to establish that

$$
\int_{\mathbb{R}^{n} \backslash Q}\left|b_{Q}\right| d \mu_{Q} \leq|(1+\omega) Q \backslash Q|^{1 / 2}\left\|b_{Q}\right\|_{L^{2}\left(\mathbb{R}^{n}\right)} \lesssim \omega^{1 / 2} \tau^{-1+1 /(n+1)}|Q|
$$

Let us furnish a smallness estimate for $b_{Q}^{\prime}$.

Lemma 4.4.10 (Almost atomic behavior of $b_{Q}^{\prime}$ ). Let $b_{Q}^{\prime}$ and $\mu_{Q}$ be as above. Then

$$
\left|\int_{\mathbb{R}^{n}} b_{Q}^{\prime} d \mu_{Q}\right| \lesssim|Q| \tau^{1 / 2+1 /(n+1)}
$$

where the implicit constant depends on $n, \lambda$, and $\Lambda$. In particular,

$$
\left|\frac{1}{\mu_{Q}(Q)} \int_{Q} b_{Q}^{\prime} d \mu_{Q}\right| \lesssim \tau^{1 / 2+1 /(n+1)}+\omega^{1 / 2} \tau^{-1+1 /(n+1)} .
$$

Proof. We first show how to derive (4.12) from the first inequality. We have that

$$
\left|\int_{Q} b_{Q}^{\prime} d \mu_{Q}\right| \leq\left|\int_{\mathbb{R}^{n}} b_{Q}^{\prime} d \mu_{Q}\right|+\int_{\mathbb{R}^{n} \backslash Q}\left|b_{Q}\right| d \mu_{Q}
$$

so that (4.12) readily follows from (4.11), (4.9), and the fact that $\mu_{Q}(Q) \geq(1 / 2)^{n}|Q|$. It remains to show (4.11). To this end, we utilize the properties of $\phi_{Q},(4.2),(2.40)$ and Hölder's inequality to see that

$$
\begin{aligned}
& \left|\int_{\mathbb{R}^{n}} b_{Q}^{\prime} d \mu_{Q}\right|=|Q|\left|\int_{\mathbb{R}^{n}} \nabla_{\|} F_{Q}(\cdot, 0) \phi_{Q}\right|=|Q|\left|\int_{\mathbb{R}^{n}} F_{Q}(\cdot, 0) \nabla \phi_{Q}\right| \\
& \lesssim \ell(Q)^{n-1} \int_{(1+\omega) Q \backslash(1 / 2) Q}\left|F_{Q}(\cdot, 0)\right| \\
& \lesssim \ell(Q)^{n-1} \int_{(1+\omega) Q \backslash(1 / 2) Q}\left|\int_{-\frac{3}{2} \tau \ell(Q)}^{\frac{3}{2} \tau \ell(Q)} \partial_{t} F_{Q}^{s^{\prime}}(y, 0) d s^{\prime}\right| d y \\
& \lesssim \ell(Q)^{n-1} \int_{-\frac{3}{2} \tau \ell(Q)}^{\frac{3}{2} \tau \ell(Q)} \int_{(1+\omega) Q \backslash(1 / 2) Q}\left|\partial_{t} F_{Q}^{s^{\prime}}(y, 0)\right| d y d s^{\prime}
\end{aligned}
$$




$$
\lesssim \ell(Q)^{n-1} \frac{\ell(Q)^{n / 2}}{\ell(Q)^{1 / 2}} \int_{-\frac{3}{2} \tau \ell(Q)}^{\frac{3}{2} \tau \ell(Q)}\left(\iint_{I_{2} \ell I_{(1 / 4)}}\left|\nabla F_{Q}^{s^{\prime}}(Y)\right|^{2} d Y\right)^{1 / 2} d s^{\prime}
$$$$
\lesssim|Q| \tau^{1 / 2+1 /(n+1)},
$$

where we used (4.1) in the last line and, in order to use (2.40), we used that for $s \in$ $\left(-\frac{3}{2} \tau \ell(Q), \frac{3}{2} \tau \ell(Q)\right)$ each $F_{Q}^{s^{\prime}}$ is a solution in $I_{2 Q} \backslash I_{(1 / 4) Q}$.

The last preliminary lemma we will need establishes a coercivity estimate for $b_{Q}^{0}$.

Lemma 4.4.13 (Coercivity of $\left.b_{Q}^{0}\right)$. Let $b_{Q}^{0}$ and $d \mu_{Q}=\phi_{Q} d x$ as above. Suppose that $\varepsilon_{m}>0$ is a small number depending on $m$. Then, if $\max \left\{\left\|B_{1}\right\|_{n},\left\|B_{2}\right\|_{n}\right\} \leq \varepsilon_{m}$, the estimate

$$
\Re e\left(\frac{1}{\mu_{Q}(Q)} \int_{Q} b_{Q}^{0} d \mu_{Q}\right) \geq\left(1-C\left[\tau^{1 / 2+1 /(n+1)}+\varepsilon_{m} \tau^{-1 / 2+1 /(n+1)}+\omega^{1 / 2} \tau^{-1+1 /(n+1)}\right]\right),
$$

holds, where $C$ depends on $m, n, \lambda$, and $\Lambda$.

Proof. By the definitions of $\mu_{Q}, b_{Q}^{0}$, and the conormal derivative, we observe that

$$
\begin{aligned}
& \int_{\mathbb{R}^{n}} b_{Q}^{0} d \mu_{Q}=\int_{\mathbb{R}^{n}} b_{Q}^{0} \phi_{Q}=|Q| \int_{\mathbb{R}^{n}}\left(\partial_{v}^{\mathcal{L},-} F_{Q}\right)(y, 0) \phi_{Q}(y) d y \\
& =|Q|\left(-\left\langle\Phi_{Q}, \mathcal{L} F_{Q}\right\rangle_{\mathbb{R}^{n+1}-}+\iint_{\mathbb{R}^{n+1}} A \nabla F_{Q} \cdot \nabla \Phi_{Q}+\left(B_{1} F_{Q}\right) \cdot \nabla \Phi_{Q}+\left(B_{2} \cdot \nabla F_{Q}\right) \Phi_{Q}\right) \\
& =|Q|(I+I I) .
\end{aligned}
$$

Since supp $\Psi_{Q}^{+} \cap \mathbb{R}_{-}^{n+1}=\varnothing, \Phi_{Q} \equiv 1$ on supp $\Psi_{Q}^{-}$, and $\iint_{\mathbb{R}^{n+1}-} \Psi_{Q}^{-}=1$, we have that

$$
I=-\left\langle\Phi_{Q}, \mathcal{L} F_{Q}\right\rangle_{\mathbb{R}^{n+1}-}=-\iint_{\mathbb{R}^{n+1}-}\left(-\Psi_{Q}^{-}\right)=1 .
$$

To bound $I I$, we write $I I=I I_{1}+I I_{2}+I I_{3}$, where the $I I_{i}$ correspond to each of the summands in the integral defining $I I$. For the term, $I I_{1}$, we use essentially the same estimates 
as in the previous lemma. In particular we use the properties of $\Phi_{Q}$, Hölder's inequality, the Caccioppoli inequality, and (4.2) to obtain that

$$
\begin{aligned}
& \left|I I_{1}\right| \leq \iint_{\mathbb{R}^{n+1}}\left|A \nabla F_{Q} \cdot \nabla \Phi_{Q}\right| \lesssim \frac{1}{\ell(Q)} \iint_{I_{(1+\omega) Q} \backslash I_{(1 / 2) Q}}|\nabla F| \\
& \lesssim \ell(Q)^{(n-1) / 2}\left(\iint_{I_{(1+\omega) Q} \backslash I_{(1 / 2)}}|\nabla F|^{2}\right)^{1 / 2} \lesssim \ell(Q)^{\frac{(n-3)}{2}}\left(\iint_{I_{(1+\omega) Q} \backslash I_{(1 / 2)}}|F|^{2}\right)^{1 / 2} \\
& \lesssim \ell(Q)^{\frac{(n-3)}{2}}\left(\iint_{I_{(1+\omega) Q} \backslash I_{(1 / 2) Q}}\left|\int_{-\frac{3}{2} \tau \ell(Q)}^{\frac{3}{2} \tau \ell(Q)} \partial_{t} F_{Q}^{s^{\prime}}(Y) d s^{\prime}\right|^{2} d Y\right)^{1 / 2} \lesssim \tau^{1 / 2+1 /(n+1)} .
\end{aligned}
$$

To bound $I I_{2}$, we use the estimate $\left\|B_{1} F_{Q}\right\|_{2} \lesssim \varepsilon_{m}\left\|\nabla F_{Q}\right\|_{2}$ and (4.1) to see that

$$
\begin{aligned}
\left|I I_{2}\right| \leq \iint_{I_{2} Q}\left|\left(B_{1} F\right) \cdot \nabla \Phi_{Q}\right| & \lesssim \frac{1}{\ell(Q)} \iint_{I_{2 Q}}\left|B_{1} F_{Q}\right| \\
& \lesssim \frac{\ell(Q)^{\frac{n+1}{2}}}{\ell(Q)}\left(\iint_{I_{2}}\left|B_{1} F_{Q}\right|^{2}\right)^{1 / 2} \lesssim \varepsilon_{m} \ell(Q)^{\frac{n-1}{2}}\left\|\nabla F_{Q}\right\|_{2} \lesssim \varepsilon_{m} \tau^{-1 / 2+1 /(n+1)} .
\end{aligned}
$$

To bound $I I_{3}$ we use Hölder's inequality, $\left\|B_{2}\right\|_{n} \leq \varepsilon_{m}$, and (4.1) as follows:

$$
\begin{aligned}
& \left|I I_{3}\right| \leq \int_{-2 \ell(Q)}^{2 \ell(Q)} \int_{2 Q}\left|\nabla F_{Q} B_{2}\right| \leq \varepsilon_{m} \int_{-2 \ell(Q)}^{2 \ell(Q)}\left(\int_{2 Q}\left|\nabla F_{Q}\right|^{\frac{n}{n-1}}\right)^{\frac{n-1}{n}} \\
& \lesssim \varepsilon_{m} \ell(Q)^{\frac{n-2}{2}} \int_{-2 \ell(Q)}^{2 \ell(Q)}\left(\int_{2 Q}\left|\nabla F_{Q}\right|^{2}\right)^{\frac{1}{2}} \lesssim \varepsilon_{m} \ell(Q)^{\frac{n-1}{2}}\left(\int_{-2 \ell(Q)}^{2 \ell(Q)} \int_{2 Q}\left|\nabla F_{Q}\right|^{2}\right)^{\frac{1}{2}} \\
& \lesssim \varepsilon_{m} \ell(Q)^{\frac{n-1}{2}}\left\|\nabla F_{Q}\right\|_{2} \lesssim \varepsilon_{m} \tau^{-1 / 2+1 /(n+1)} .
\end{aligned}
$$

Combining the previous estimates gives that

$$
\Re e\left(\int_{\mathbb{R}^{n}} b_{Q}^{0} d \mu_{Q}\right) \geq|Q|\left(1-C\left[\tau^{1 / 2+1 /(n+1)}+\varepsilon_{m} \tau^{-1 / 2+1 /(n+1)}\right]\right) .
$$

This estimate, in concert with (4.9) and the fact that $\mu_{Q}(Q) \leq 1$, ends the proof. 
With $\varepsilon_{m}$ and $\omega$ at our disposal, we collapse the dependence of parameters to only $\tau$, leaving freedom to take $\varepsilon_{m}$ even smaller. We ensure that $\varepsilon_{m}<\tau$ and set $\omega=\tau^{3}$. Under these choices, we are ready to present the

Proof of Proposition 4.4.1. When the choices $\varepsilon_{m}<\tau$ and $\omega=\tau^{3}$ are used in Lemma 4.4.13, we have that

$$
\mathfrak{R} e\left(\frac{1}{\mu_{Q}(Q)} \int_{Q} b_{Q}^{0} d \mu\right) \geq 1-C \tau^{1 / 2+1 /(n+1)}
$$

where $C$ depends on $n, \lambda, \Lambda$. Accordingly, we may pick $\tau$ small enough so that (4.4) holds. The choice $\omega=\tau^{3}$ used in (4.12) gives that

$$
\left|\frac{1}{\mu_{Q}(Q)} \int_{Q} b_{Q}^{\prime} d \mu_{Q}\right| \leq C \tau^{1 / 2+1 /(n+1)}
$$

where $C$ depends on $n, \lambda$, and $\Lambda$. Hence, we may guarantee that (4.5) holds by choosing $\tau$ small depending on $C$ and $\eta$. Having chosen $\tau$ so that (4.4) and (4.5) hold, (4.2) and (4.3) follow from Lemma 4.4.6 and Lemma 4.4.7 respectively.

\subsection{Control of the auxiliary square functions}

As a last preliminary step to presenting the proof of the square function bound, we elucidate how to control the error terms involving $\Theta_{t}^{(a)}$ and $\Theta_{t}^{\prime}$.

Proposition 4.5.1 (Control of error terms). Let $T_{t}$ be either $\Theta_{t}^{\prime}$ or $\Theta_{t}^{(a)}$. Then, for each fixed $t>0, T_{t} 1$ is well-defined as an element of $L_{\mathrm{loc}}^{2}\left(\mathbb{R}^{n}\right)$. Moreover, we have the estimates

$$
\left\|T_{t}\right\|\left\|_{o p} \leq C\right\|\left\|\Theta_{t}^{0}\right\| \|_{o p}+1
$$


and

$$
\left\|T_{t} 1\right\|_{C} \leq C\left\|\Theta_{t}^{0} 1\right\|_{C}+1
$$

where $C$ depends on $m, n, \lambda$, and $\Lambda$, provided that $\max \left\{\left\|B_{1}\right\|_{n},\left\|B_{2}\right\|_{n}\right\}$ is sufficiently small depending on $m, n, \lambda$, and $\Lambda$.

Remark 4.5.4. We will operate under the assumption that $T_{t} 1$ and $\Theta_{t}^{0} 1$ have finite $\|\cdot\|_{C}$ norm. Indeed, otherwise for $\gamma>0$, we replace $T_{t} 1$ by $\left(T_{t} 1\right)_{\gamma}=\left(T_{t} 1\right) \mathbb{1}_{\gamma<t \leq 1 / \gamma}$ and analogously for $\Theta_{t}^{0} 1$, and we observe that these truncated versions will always have finite $\|\cdot\|_{C}$ norm under our hypotheses.

Proposition 4.5.1 will be a direct consequence of the following lemma.

Lemma 4.5.5 (Control of gradient field terms). Let $\widetilde{\Theta}_{t}:=t^{m} \partial_{t}^{m} \mathcal{S}_{t}^{\mathcal{L}} \nabla_{\|}$for $m \in \mathbb{N}, m \geq n+10$. Then

$$
\left\|\widetilde{\Theta}_{t}\right\|\left\|_{o p} \lesssim\right\|\left\|\Theta_{t}^{0} \mid\right\|_{o p}+1
$$

and

$$
\left\|\widetilde{\Theta}_{t} 1\right\|_{C} \lesssim\left\|\Theta_{t}^{0} 1\right\|_{C}+1
$$

where the constants depends on $m, n, \lambda$, and $\Lambda$, provided that $\max \left\{\left\|B_{1}\right\|_{n},\left\|B_{2}\right\|_{n}\right\}$ is sufficiently small depending on $m, n, \lambda, \Lambda$.

Proof. We note that (4.7) follows from Lemma 2.4.10, (4.6) and Proposition 3.3.15. The proof will follow the general scheme of [HMM15, Lemma 3.1], with modifications due 
to the first order terms. Write $L_{\|}:=\operatorname{div}_{x} A_{\|} \nabla_{\|}$where $A_{\|}=\left(A_{i, j}\right)_{1 \leq i, j \leq n}$. By the Hodge decomposition for the operator $L_{\|}$, to prove (4.6) it is enough to show that

$$
\iint_{\mathbb{R}^{n+1}+}\left|t^{m} \partial_{t}^{m} \mathcal{S}_{t}^{\mathcal{L}}\left(\nabla_{\|} \cdot A_{\|} \nabla_{\|} F\right)(x)\right|^{2} \frac{d x d t}{t} \lesssim\left(1+\left\|\Theta_{t}^{0}\right\| \|_{o p}^{2}\right),
$$

for all $F \in Y^{1,2}\left(\mathbb{R}^{n}\right)$ with $\left\|\nabla_{\|} F\right\|_{L^{2}} \lesssim 1$ (dependence on $\lambda$ and $\Lambda$ ). We write

$$
\begin{array}{r}
t^{m} \partial_{t}^{m} \mathcal{S}_{t}^{\mathcal{L}} \nabla_{\|} A_{\|} \nabla_{\|} F \\
=\left\{t^{m} \partial_{t}^{m} \mathcal{S}_{t}^{\mathcal{L}} \nabla_{\|} A_{\|}-t^{m}\left(\partial_{t}^{m} \mathcal{S}_{t}^{\mathcal{L}} \nabla_{\|} A_{\|}\right) P_{t}\right\} \nabla_{\|} F+t^{m}\left(\partial_{t}^{m} \mathcal{S}_{t}^{\mathcal{L}} \nabla_{\|} A_{\|}\right) P_{t} \nabla_{\|} F \\
=: R_{t}\left(\nabla_{\|} F\right)+t^{m}\left(\partial_{t}^{m} \mathcal{S}_{t}^{\mathcal{L}} \nabla_{\|} \cdot A_{\|}\right) P_{t} \nabla_{\|} F,
\end{array}
$$

where $t^{m}\left(\partial_{t}^{m} \mathcal{S}_{t}^{\mathcal{L}} \nabla_{\|} A_{\|}\right)$is the (vector-valued) operator $t^{m} \partial_{t}^{m} \mathcal{S}_{t}^{\mathcal{L}} \nabla_{\|}$applied to $A_{\|}$, the latter understood as a vector function with components in $L_{l o c}^{2}\left(\mathbb{R}^{n} ; \mathbb{C}^{n}\right)$, and $P_{t}$ is a nice approximate identity constructed as follows. Let $\zeta_{t}(x)=t^{-n} \zeta\left(\frac{|x|}{t}\right)$, where $\zeta \in C_{c}^{\infty}(B(0,1 / 2))$ is radial with $\int_{\mathbb{R}^{n}} \zeta=0$ and $Q_{t} f(x)=\left(\zeta_{t} * f\right)(x)$ satisfies the Calderón reproducing formula

$$
\int_{0}^{\infty} Q_{t}^{2} \frac{d t}{t}=I, \quad \text { in the strong operator topology on } L^{2}
$$

Then $Q_{s}$ is a CLP family (see Definition 2.4.13) and we set $P_{t}:=\int_{t}^{\infty} Q_{s}^{2} \frac{d s}{s}$. Then $P_{t}$ is a nice approximate identity; that is, $P_{t}=\left(\varphi_{t} * f\right)(x)$ where $\varphi_{t}=t^{-n} \varphi\left(\frac{|\cdot|}{t}\right)$ and $\varphi \in C_{c}^{\infty}(B(0,1))$ is a radial function with $\int_{\mathbb{R}^{n}} \varphi=1$.

The term $t^{m} \partial_{t}^{m} \mathcal{S}_{t}^{\mathcal{L}} \nabla_{\|} \cdot A_{\|} P_{t} \nabla_{\|} F$ is the 'main term' and we will apply the techniques of the solution to the Kato problem [AHL $\left.{ }^{+} 02\right]$ to handle its contribution. For now, we focus on the remainder term $R_{t}\left(\nabla_{\|} F\right)$, which takes a bit of exposition due to the number of terms 
arising from the lower order terms in the differential operator $\mathcal{L}$. To this end, we write

$$
\begin{aligned}
R_{t}= & t^{m} \partial_{t}^{m} \mathcal{S}_{t}^{\mathcal{L}} \nabla_{\|} A_{\|}-t^{m}\left(\partial_{t}^{m} \mathcal{S}_{t}^{\mathcal{L}} \nabla_{\|} A_{\|}\right) P_{t} \\
& =\left\{t^{m} \partial_{t}^{m} \mathcal{S}_{t}^{\mathcal{L}} \nabla_{\|} A_{\|} P_{t}-t^{m}\left(\partial_{t}^{m} \mathcal{S}_{t}^{\mathcal{L}} \nabla_{\|} A_{\|}\right) P_{t}\right\}+t^{m} \partial_{t}^{m} \mathcal{S}_{t}^{\mathcal{L}} \nabla_{\|} A_{\|}\left(I-P_{t}\right)=: R_{t}^{[1]}+R_{t}^{[2]}
\end{aligned}
$$

Observe that $R_{t}^{[1]} 1=0, R_{t}^{[1]}$ has sufficient off-diagonal decay (Proposition 3.3.15) and uniform $L^{2}$ boundedness (Proposition 3.3.1), and $\left\|R_{t}^{[1]} \nabla_{x}\right\|_{2 \rightarrow 2} \leq C / t$. Then the square function bound

$$
\iint_{\mathbb{R}^{n+1}+}\left|R_{t}^{[1]} \nabla_{\|} F\right|^{2} \frac{d x d t}{t} \lesssim\left\|\nabla_{\|} F\right\|_{2}^{2}
$$

follows from Lemma 2.4.12 as desired. To control $R_{t}$ it remains to control $R_{t}^{[2]}$. Set $Z_{t}:=I-$ $P_{t}$ and define $\vec{b}:=\left(A_{n+1,1}, \ldots, A_{n+1, n}\right)$. By using integration by parts on slices (Proposition 2.5.38) and Proposition 2.4.14, we obtain that

$$
\begin{aligned}
& t^{m} \partial_{t}^{m} \mathcal{S}_{t}^{\mathcal{L}} \nabla_{\|} A_{\|} Z_{t} \nabla_{\|} F=t^{m} \partial_{t}^{m} \mathcal{S}_{t}^{\mathcal{L}} \nabla_{\|} A_{\|} \nabla_{\|} Z_{t} F \\
& =t^{m} \partial_{t}^{m+1}\left(\mathcal{S}_{t}^{\mathcal{L}} \nabla\right) \cdot \vec{A}_{, n+1} Z_{t} F \quad-t^{m} \partial_{t}^{m+1} \mathcal{S}_{t}^{\mathcal{L}}\left(\vec{b} \nabla Z_{t} F\right) \quad+t^{m} \partial_{t}^{m}\left(\mathcal{S}_{t}^{\mathcal{L}} \nabla\right) B_{1} Z_{t} F \\
& -t^{m} \partial_{t}^{m} \mathcal{S}_{t}^{\mathcal{L}}\left(B_{2 \|} \nabla_{\|} Z_{t} F\right) \quad+t^{m} \partial_{t}^{m+1} \mathcal{S}_{t}^{\mathcal{L}}\left(B_{2 \perp} Z_{t} F\right) \quad=: J_{1}+J_{2}+J_{3}+J_{4}+J_{5} .
\end{aligned}
$$

Note that, using Plancherel's theorem, we have that

$$
\iint_{\mathbb{R}^{n+1}+}\left|t^{-1}\left(I-P_{t}\right) F(x)\right|^{2} \frac{d x d t}{t} \lesssim\left\|\nabla_{\|} F\right\|_{2}^{2}
$$

Since $t^{m+1} \partial_{t}^{m+1}\left(\mathcal{S}_{t}^{\mathcal{L}} \nabla\right): L^{2} \rightarrow L^{2}$ uniformly in $t$, we easily obtain the associated square function bound for $J_{1}$. To bound $J_{2}$, we write 


$$
\begin{array}{r}
J_{2}=-t^{m} \partial_{t}^{m+1} \mathcal{S}_{t}^{\mathcal{L}}\left(\vec{b} \cdot \nabla_{\|}\left(I-P_{t}\right) F\right) \\
=-t^{m} \partial_{t}^{m+1} \mathcal{S}_{t}^{\mathcal{L}} \vec{b} \cdot \nabla_{\|} F+\left\{t^{m} \partial_{t}^{m+1} \mathcal{S}_{t}^{\mathcal{L}} \vec{b} P_{t}-\left(t^{m} \partial_{t}^{m+1} \mathcal{S}_{t}^{\mathcal{L}} \vec{b}\right) P_{t}\right\} \nabla_{\|} F+\left(t^{m} \partial_{t}^{m+1} \mathcal{S}_{t}^{\mathcal{L}} \vec{b}\right) P_{t} \nabla_{\|} F \\
=: J_{2,1}+J_{2,2}+J_{2,3} .
\end{array}
$$

For $J_{2,1}$, we see that $J_{2,1}=\Theta_{t}^{0} \vec{b} \nabla_{\|} F$, whence

$$
\iint_{\mathbb{R}^{n+1}+}\left|t^{m} \partial_{t}^{m+1} \mathcal{S}_{t}^{\mathcal{L}} \vec{b} \nabla_{\|} F\right|^{2} \frac{d x d t}{t} \lesssim\|\| \Theta_{t}^{0}\|\|_{o p}^{2}\left\|\nabla_{\|} F\right\|_{2}^{2}
$$

Similarly, by Lemma 2.4.10 and Carleson's Lemma, we have that

$$
\iint_{\mathbb{R}^{n+1}+}\left|\left(t^{m} \partial_{t}^{m+1} \mathcal{S}_{t}^{\mathcal{L}} \vec{b}\right) P_{t} \nabla_{\|} F\right|^{2} \frac{d x d t}{t} \lesssim\|\| \Theta_{t}^{0}\|\|_{o p}^{2}\left\|\nabla_{\|} F\right\|_{2}^{2}
$$

so that the contribution from $J_{2,3}$ has the desired control. Notice that $J_{2,2}$ is of the form $R_{t} \nabla_{\|} F$ where $R_{t} 1=0, R_{t}: L^{2} \rightarrow L^{2}$ and $\left\|R_{t} \nabla_{x}\right\|_{L^{2} \rightarrow L^{2}} \leq C / t$ and $R_{t}$ good off-diagonal decay.

Thus, the desired square function bound for term $J_{2,2}$, follows immediately from Lemma 2.4.12.

For term $J_{3}$, let $g$ be such that $I_{1} g=F$ and $\|g\|_{2} \approx\left\|\nabla_{\|} F\right\|_{2}$. Then using $t^{m} \partial_{t}^{m}\left(\mathcal{S}_{t}^{\mathcal{L}} \nabla\right)=$ $\Theta_{t}^{(a)}$, we have by Proposition 3.3.8 that

$$
\left\|\Theta_{t}^{(a)} B_{1} I_{1} Q_{s}^{2} g\right\|_{L^{2}\left(\mathbb{R}^{n}\right)} \lesssim\left(\frac{s}{t}\right)^{\gamma}\left\|Q_{s} g\right\|_{L^{2}\left(\mathbb{R}^{n}\right)}
$$

for some $\gamma>0$ independent of $g$. Then by standard estimates we obtain

$$
\begin{aligned}
& \iint_{\mathbb{R}^{n+1}+}\left|t^{m} \partial_{t}^{m}\left(\mathcal{S}_{t}^{\mathcal{L}} \nabla\right) B_{1}\left(I-P_{t}\right) F\right|^{2} \frac{d x d t}{t}=\iint_{\mathbb{R}^{n+1}+}\left|t^{m} \partial_{t}^{m}\left(\mathcal{S}_{t}^{\mathcal{L}} \nabla\right) B_{1} I_{1}\left(I-P_{t}\right) g\right|^{2} \frac{d x d t}{t} \\
& \lesssim \iint_{\mathbb{R}^{n+1}+}\left|t^{m} \partial_{t}^{m}\left(\mathcal{S}_{t}^{\mathcal{L}} \nabla\right) B_{1} I_{1}\left(\int_{0}^{t} Q_{s}^{2} g \frac{d s}{s}\right)\right|^{2} \frac{d x d t}{t}
\end{aligned}
$$




$$
\begin{aligned}
\lesssim_{\gamma} \iint_{\mathbb{R}^{n+1}+} \int_{0}^{t}\left(\frac{t}{s}\right)^{\gamma / 2}\left|t^{m} \partial_{t}^{m}\left(\mathcal{S}_{t}^{\mathcal{L}} \nabla\right) B_{1} I_{1} Q_{s}^{2} g\right|^{2} \frac{d s}{s} \frac{d x d t}{t} \\
\quad \lesssim \int_{0}^{\infty} \int_{s}^{\infty}\left(\frac{s}{t}\right)^{\gamma / 2}\left\|Q_{s} g\right\|_{2}^{2} \frac{d t}{t} \frac{d s}{s} \lesssim_{\gamma} \int_{0}^{\infty}\left\|Q_{s} g\right\|_{2}^{2} \frac{d s}{s} \lesssim\|g\|_{2}^{2} \approx\left\|\nabla_{\|} F\right\|_{2}^{2},
\end{aligned}
$$

where in the fourth inequality we used Cauchy's inequality in the $\frac{d s}{s}$ integral noting that $\int_{0}^{t}(s / t)^{\gamma} \frac{d s}{s} \lesssim C_{\gamma}$, and we used the square function estimate for the CLP family $Q_{s}$ (see Definition 2.4.13). This takes care of the contribution from $J_{3}$.

Next, we handle $J_{4}$. We write $J_{4}$ as the sum of its pieces, as follows:

$$
\begin{aligned}
J_{4}=-t^{m} \partial_{t}^{m} \mathcal{S}_{t}^{\mathcal{L}} B_{2 \|} \nabla_{\|}\left(I-P_{t}\right) F & \\
& =-t^{m} \partial_{t}^{m} \mathcal{S}_{t}^{\mathcal{L}} B_{2 \|} \nabla_{\|} F+t^{m} \partial_{t}^{m} \mathcal{S}_{t}^{\mathcal{L}} B_{2 \|} \nabla_{\|} P_{t} F=J_{4,1}+J_{4,2} .
\end{aligned}
$$

For $J_{4,1}$, we observe that

$$
J_{4,1}=-t^{m} \partial_{t}^{m} \mathcal{S}_{t}^{\mathcal{L}} B_{2 \|} \nabla_{\|} F=-t^{m} \partial_{t}^{m} \mathcal{S}_{t}^{\mathcal{L}} \operatorname{div}_{\|} \nabla_{\|} I_{2} B_{2 \|} \nabla_{\|} F=-\widetilde{\Theta}\left(\nabla_{\|} I_{2} B_{2 \|} F\right)
$$

and notice that $\left\|\nabla_{\|} I_{2} B_{2 \|} F\right\|_{2} \lesssim\left\|B_{2}\right\|_{n}\left\|\nabla_{\|} F\right\|_{2}$. Therefore,

$$
\iint_{\mathbb{R}^{n+1}+}\left|t^{m} \partial_{t}^{m} \mathcal{S}_{t}^{\mathcal{L}} B_{2 \|} \nabla_{\|} F\right|^{2} \frac{d x d t}{t} \lesssim\left\|\widetilde{\Theta}_{t}\right\|\left\|_{o p}^{2}\right\| B_{2}\left\|_{n}^{2}\right\| \nabla_{\|} F \|_{2}^{2}
$$

and hence $J_{4,1}$ can be hidden in (4.8) when $\left\|B_{2}\right\|_{n}$ is small. For $J_{4,2}$, we write

$$
\begin{aligned}
J_{4,2}=\left\{t^{m} \partial_{t}^{m} \mathcal{S}_{t}^{\mathcal{L}} B_{2 \|} P_{t}-\left(t^{m} \partial_{t}^{m} \mathcal{S}_{t}^{\mathcal{L}} B_{2 \|}\right) P_{t}\right\} \nabla_{\|} F+\left(t^{m} \partial_{t}^{m} \mathcal{S}_{t}^{\mathcal{L}} B_{2 \|}\right) P_{t} \nabla_{\|} F \\
=\widetilde{R}_{t} \nabla_{\|} F+\left(t^{m} \partial_{t}^{m} \mathcal{S}_{t}^{\mathcal{L}} B_{2 \|}\right) P_{t} \nabla_{\|} F .
\end{aligned}
$$

We may handle $\widetilde{R}_{t} \nabla_{\|} F$ using Lemma 2.4.12, as $\widetilde{R}_{t}$ satisfies the required hypotheses (see Propositions 3.3.1 and 3.3.15). We see, in a similar fashion to $J_{4,1}$, that $t^{m} \partial_{t}^{m} \mathcal{S}_{t}^{\mathcal{L}} B_{2 \|}=$ 
$\widetilde{\Theta}_{t} \nabla_{\|} I_{2} B_{2 \|}$, and $\left\|\nabla_{\|} I_{2} B_{2 \|}\right\|_{B M O} \lesssim\left\|B_{2}\right\|_{n}^{2}$. Noting that $\widetilde{\Theta}_{t} 1=0$, it follows from Lemma 2.4.10 and Carleson's Lemma that

$$
\iint_{\mathbb{R}^{n+1}+}\left|\left(t^{m} \partial_{t}^{m} \mathcal{S}_{t}^{\mathcal{L}} B_{2 \|}\right) P_{t} F\right|^{2} \frac{d x d t}{t} \lesssim\left(1+\left\|\widetilde{\Theta}_{t}\right\|_{o p}^{2}\right)\left\|B_{2}\right\|_{n}^{2}\left\|\nabla_{\|} F\right\|_{2}^{2}
$$

which can be hidden in (4.8) when $\left\|B_{2}\right\|_{n}$ is sufficiently small.

Finally, to handle $J_{5}$, rewrite it as $J_{5}=t^{m+1} \partial_{t}^{m+1} \mathcal{S}_{t}^{\mathcal{L}} B_{2 \perp}\left(\frac{1}{t}\left[I-P_{t}\right] F\right)$. Since $t^{m+1} \partial_{t}^{m+1} \mathcal{S}_{t}^{\mathcal{L}} B_{2 \perp}: L^{2} \rightarrow L^{2}$ uniformly in $t$, we may handle this term exactly as $J_{1}$ by using (4.9).

Having handled the remainder $R_{t}$, we have reduced matters to showing that the square function bound

$$
\iint_{\mathbb{R}^{n+1}+}\left|t^{m}\left(\partial_{t}^{m} \mathcal{S}_{t}^{\mathcal{L}} \nabla_{\|} \cdot A_{\|}\right)(x) P_{t} \nabla_{\|} F(x)\right|^{2} \frac{d x d t}{t} \lesssim\left\|\nabla_{\|} F\right\|_{2}^{2}
$$

holds for all $F \in Y^{1,2}\left(\mathbb{R}^{n}\right)$ with $\left\|\nabla_{\|} F\right\|_{2} \leq 1$. By Carleson's Lemma, it is enough to show that

$$
\sup _{Q} \frac{1}{|Q|} \int_{0}^{\ell(Q)} \int_{\mathbb{R}^{n}}\left|t^{m}\left(\partial_{t}^{m} \mathcal{S}_{t}^{\mathcal{L}} \nabla_{\|} \cdot A_{\|}\right)(x)\right|^{2} \frac{d x d t}{t} \leq C .
$$

In order to obtain (4.10), we appeal to the technology of the solution of the Kato problem $\left[\mathrm{AHL}^{+} 02\right]$, and follow the argument of [HMM15]. By [AHL $\left.{ }^{+} 02\right]$, for each dyadic cube $Q$ there exists a mapping $F_{Q}: \mathbb{R}^{n} \rightarrow \mathbb{C}^{n}$ such that

i) $\int_{\mathbb{R}^{n}}\left|\nabla_{\|} F_{Q}\right|^{2} \leq C|Q|$ 
ii) $\int_{\mathbb{R}^{n}}\left|L_{\|} F_{Q}\right|^{2} \leq \frac{|Q|}{\ell(Q)^{2}}$

iii) $\sup _{Q} \int_{0}^{\ell(Q)} f_{Q}|\vec{\zeta}(x, t)|^{2} \frac{d x d t}{t} \lesssim C \sup _{Q} \int_{0}^{\ell(Q)} f_{Q}\left|\vec{\zeta}(x, t) E_{t} \nabla_{\|} F_{Q}\right|^{2} \frac{d x d t}{t}$

for each $\vec{\zeta}: \mathbb{R}^{n+1}{ }_{+} \rightarrow \mathbb{C}^{n}$, where $E_{t}$ denotes the dyadic averaging operator; that is, if $Q(x, t)$ is the minimal dyadic cube containing $x \in \mathbb{R}^{n}$ with side length at least $t$, then $E_{t} g(x)=f_{Q(x, t)} g$. Here, we note that $\nabla_{\|} F_{Q}$ is the Jacobian of $F_{Q}$ and $\vec{\zeta} E_{t} \nabla_{\|} F_{Q}$ is a vector. Given such a family $\left\{F_{Q}\right\}_{Q}$, we see that by applying property $\left.i i i\right)$ with $\vec{\zeta}(x, t)=T_{t} A_{\|}$, where $T_{t}:=t^{m} \partial_{t}^{m}\left(\mathcal{S}_{t}^{\mathcal{L}} \nabla_{\|}\right)$it is enough to show that

$$
\int_{0}^{\ell(Q)} \int_{Q}\left|\left(T_{t} A_{\|}\right)(x) E_{t} \nabla_{\|} F_{Q}(x)\right|^{2} \frac{d x d t}{t} \lesssim\left(1+\left\|\left|\Theta_{t}^{0}\right|\right\|_{o p}^{2}\right)|Q| .
$$

Following [AT98, CM86], we write that

$$
\begin{gathered}
\left(T_{t} A_{\|}\right) E_{t} \nabla_{\|} F_{Q}=\left\{\left(T_{t} A_{\|}\right) E_{t}-T_{t} A_{\|}\right\} \nabla_{\|} F_{Q}+T_{t} A_{\|} \nabla_{\|} F_{Q} \\
=T_{t} A_{\|}\left(E_{t}-P_{t}\right) \nabla_{\|} F_{Q}+\left\{\left(T_{t} A_{\|}\right) P_{t}-T_{t} A_{\|}\right\} \nabla_{\|} F_{Q}+T_{t} A_{\|} \nabla_{\|} F_{Q} \\
=: R_{t}^{(1)} \nabla_{\|} F_{Q}+R_{t}^{(2)} \nabla_{\|} F_{Q}+T_{t} A_{\|} \nabla_{\|} F_{Q} .
\end{gathered}
$$

Observe that $R_{t}^{(2)}=-R_{t}$ from above, and we have already shown that $\left\|R_{t}\right\|_{o p} \lesssim(1+$ \|\|$\left.\Theta_{t}^{0}\|\|_{o p}\right)^{2}$, so that the desired bound holds from property $i$ ) of $F_{Q}$. For the last term, we have that $T_{t} A_{\|} \nabla_{\|} F_{Q}=t^{m} \partial_{t}^{m} \mathcal{S}_{t}^{\mathcal{L}} L_{\|} F_{Q}$, and we know that $t^{m-1} \partial_{t}^{m} \mathcal{S}_{t}^{\mathcal{L}}: L^{2} \rightarrow L^{2}$ uniformly in $t$. Thus, by property $i$ ) of $F_{Q}$, we have that

\footnotetext{
${ }^{2}$ We have shown that $\left.\left\|R_{t}\right\|\right|_{o p} \lesssim\left(1+\|\| \Theta_{t}^{0} \mid \|_{o p}\right)+\epsilon\left\|\widetilde{\Theta}_{t}\right\| \|_{o p}$, where $\epsilon$ is at our disposal by the smallness of $\max \left\{\left\|B_{1}\right\|_{n},\left\|B_{2}\right\|_{n}\right\}$, and this is enough for our purposes.
} 


$$
\begin{aligned}
\int_{0}^{\ell(Q)} \int_{Q}\left|\left(T_{t} A_{\|} F_{Q}\right)(x)\right|^{2} \frac{d x d t}{t} \leq\left.\int_{0}^{\ell(Q)} \int_{\mathbb{R}^{n}} t^{m-1} \partial_{t}^{m} \mathcal{S}_{t}^{\mathcal{L}} L_{\|} F(x)\right|^{2} t d x d t & \\
& \lesssim \frac{|Q|}{\ell(Q)^{2}} \int_{0}^{\ell(Q)} t d t \lesssim|Q|,
\end{aligned}
$$

which shows the desired bound for this term.

To bound the contribution from $R_{t}^{(1)}$, we note that $T_{t}: L^{2} \rightarrow L^{2}$ uniformly in $t$ and

$$
\iint_{\mathbb{R}^{n+1}+}\left|\left(E_{t}-P_{t}\right) g(x)\right|^{2} \frac{d x d t}{t} \lesssim\|g\|_{2}^{2}
$$

for $g \in L^{2}\left(\mathbb{R}^{n}\right)$. Therefore,

$$
\begin{aligned}
& \int_{0}^{\ell(Q)} \int_{Q}\left|R_{t}^{(1)} \nabla_{\|} F_{Q}\right|^{2} \frac{d x d t}{t} \leq \int_{0}^{\ell(Q)} \int_{\mathbb{R}^{n}}\left|T_{t} A_{\|}\left(E_{t}-P_{t}\right) \nabla_{\|} F_{Q}\right|^{2} \frac{d x d t}{t} \\
& \lesssim \int_{0}^{\ell(Q)} \int_{\mathbb{R}^{n}}\left|\left(E_{t}-P_{t}\right) \nabla_{\|} F_{Q}\right|^{2} \frac{d x d t}{t}
\end{aligned}
$$

$$
\lesssim\left\|\nabla_{\|} F\right\|_{2}^{2} \lesssim C|Q|,
$$

where we used the ellipticity of $A$ in the second inequality, and property i) of $F_{Q}$ in the last inequality. This controls the contribution from $R_{t}^{(1)}$ and finishes the proof of the Lemma.t.

We move on to the

Proof of Proposition 4.5.1. To see that $\left\|\Theta_{t}^{(a)}\right\|\left\|_{o p} \lesssim 1+\right\|\left\|\Theta_{t}^{0}\right\| \|_{o p}$, and that $\left\|\Theta_{t}^{(a)} 1\right\|_{C} \lesssim 1+$ $\left\|\Theta_{t}^{0}\right\|_{C}$, we simply notice that $\Theta_{t}^{(a)}=\left(\Theta_{t}^{0}, t^{m} \partial_{t}^{m}\left(\mathcal{S}_{t}^{\mathcal{L}} \nabla_{\|}\right)\right)$so that the desired bounds follow directly from the previous lemma.

We are left with showing the bounds in Proposition 4.5.1 for $T_{t}=\Theta_{t}^{\prime}$. We note immediately that (4.3) will follow from (4.2) and Lemma 2.4.10. Therefore, it is enough to show 
(4.2). In fact, by Lemma 4.5.5, it suffices to show that $\left\|\mid \Theta_{t}^{\prime}\right\|\left\|_{o p} \lesssim\right\| \widetilde{\Theta}_{t}\|\|_{o p}+\|\| \Theta_{t}^{0}\|\|_{o p}$. For $g \in L^{2}\left(\mathbb{R}^{n}, \mathbb{C}^{n}\right)$, we have that

$$
\begin{aligned}
\Theta_{t}^{\prime} g=t^{m} \partial_{t}^{m} \mathcal{S}_{t}^{\mathcal{L}}\left(B_{2 \|} g\right)+t^{m} \partial_{t}^{m}\left(\mathcal{S}_{t}^{\mathcal{L}} \nabla\right) \cdot \tilde{A} g & \\
& =t^{m} \partial_{t}^{m} \mathcal{S}_{t}^{\mathcal{L}}\left(B_{2 \|} g\right)+t^{m} \partial_{t}^{m}\left(\mathcal{S}_{t}^{\mathcal{L}} \nabla_{\|}\right) \cdot A_{\|} g-t^{m} \partial_{t}^{m+1} \mathcal{S}_{t}^{\mathcal{L}} \vec{b} g,
\end{aligned}
$$

where $\vec{b}=\left(A_{n+1, j}\right)_{1 \leq j \leq n}$. The ellipticity of $A$ gives immediately that $\left\|t^{m} \partial_{t}^{m}\left(\mathcal{S}_{t}^{\mathcal{L}} \nabla_{\|}\right) A_{\|}\right\|\left\|_{o p} \lesssim\right\| \widetilde{\Theta}\|\|_{o p}$, and $\left\|t^{m} \partial_{t}^{m+1} \mathcal{S}_{t}^{\mathcal{L}} \vec{b}\right\|\left\|_{o p} \lesssim\right\| \widetilde{\Theta} \|_{o p}$. It remains to handle the first term. Observe that $B_{2 \|} g=\operatorname{div}_{\|} \nabla_{\|} I_{2} B_{2 \|} g=\operatorname{div}_{\|} \vec{R} I_{1} B_{2 \|} g$, where $\vec{R}$ is the vector-valued Riesz tranform. It follows that $B_{2 \|} g=\operatorname{div}_{\|} \vec{G}$ with $\|\vec{G}\|_{2} \lesssim\left\|B_{2}\right\|_{n}\|g\|_{2}$, and hence

$$
\left\|t^{m} \partial_{t}^{m} S_{t} B_{2}\right\|\left\|_{o p} \lesssim\right\| \widetilde{\Theta}_{t}\left\|_{o p}\right\| B_{2} \|_{n}
$$

which yields the desired bound.

\subsection{Proof of the square function bound}

We finally turn to the proof of Theorem 4.2.1 (and hence, by our reduction, the proof of Theorem 1.0.6). Our method follows the lines of [GH16], circumventing some difficulties by introducing $\Theta_{t}^{(a)}$ and $b_{Q}^{(a)}$.

Proof of Theorem 4.2.1. Let $C_{1}$ be a constant, depending on $m, n, \lambda$ and $\Lambda$, for which the inequalities (4.2) and (4.3) hold. We choose $\eta$ in Proposition 4.4.1 as $\eta:=1 /\left(2 C_{1}+4\right)$. By the generalized Christ-Journé $T 1$ theorem for square functions, (see [GH16, Theorem 4.5]) 
to prove the theorem it is enough ${ }^{3}$ to show that

$$
\left\|\Theta_{t}^{0} 1\right\|_{C} \leq C
$$

As in [GH16], we want to reduce the above estimate to one of the form

$$
\iint_{R_{Q}}\left|\left(\Theta_{t} 1\right) A_{t}^{\mu_{Q}} b_{Q}\right|^{2} \frac{d x d t}{t} \leq C|Q|,
$$

where $A_{t}^{\mu_{Q}}$ is an averaging operator adapted to $\mu_{Q}$ (and hence $Q$ ) we will introduce later and $R_{Q}$ is the Carleson region $Q \times(0, \ell(Q))$. The argument up until this reduction, namely (4.9), is almost exactly as in [GH16]. Define $\zeta(x, t):=\Theta_{t} 1(x), \zeta^{0}(x, t):=\Theta_{t}^{0} 1(x)$, and $\zeta^{\prime}(x, t):=\Theta_{t}^{\prime} 1(x)$, where these objects make sense as elements of $L_{\mathrm{loc}}^{2}\left(\mathbb{R}_{+}^{n+1}\right)$ by Lemma 2.4.11 and Proposition 3.3.15. Consider the cut-off surfaces

$$
\begin{aligned}
& F_{1}:=\left\{(x, t) \in \mathbb{R}^{n+1}{ }_{+}:\left|\zeta^{0}(x, t)\right| \leq \sqrt{\eta}\left|\zeta^{\prime}(x, t)\right|\right\}, \\
& F_{2}:=\left\{(x, t) \in \mathbb{R}^{n+1}{ }_{+}:\left|\zeta^{0}(x, t)\right|>\sqrt{\eta}\left|\zeta^{\prime}(x, t)\right|\right\} .
\end{aligned}
$$

We easily have that $\left\|\zeta^{0}\right\|_{C} \leq\left\|\zeta^{0} \mathbb{1}_{F_{1}}\right\|_{C}+\left\|\zeta^{0} \mathbb{1}_{F_{2}}\right\|_{C}$. By definition of $F_{1}$, Proposition 4.5.1, and the fact that $\eta<1 /\left(2 C_{1}\right)$, we realize that

$$
\left\|\zeta^{0} \mathbb{1}_{F_{1}}\right\|_{C} \leq \eta\left\|\zeta^{1}\right\|_{C} \leq C_{1} \eta\left(1+\left\|\zeta^{0}\right\|_{C}\right) \leq \frac{1}{2}\left(1+\left\|\zeta^{0}\right\|_{C}\right)
$$

Consequently, $\left\|\zeta^{0}\right\|_{C} \leq 1+2\left\|\zeta^{0} \mathbb{1}_{F_{2}}\right\|_{C}$, and recall that we may work with truncated versions of each of $\zeta, \zeta^{0}, \zeta^{\prime}$ so that all quantities are finite. Accordingly, we have reduced the proof

\footnotetext{
${ }^{3}$ The careful reader will notice that we have verified the hypotheses of [GH16, Theorem 4.5] above aside from the quasiorthogonality estimate [GH16, equation (4.4)]. This estimate is slightly misstated in [GH16], where $h$ should be replaced by $Q_{s} h$ and we verify this below when dealing with the term labeled $J_{1}$.
} 
of (4.1) to showing that

$$
\left\|\zeta^{0} \mathbb{1}_{F_{2}}\right\|_{C} \leq C
$$

By (4.4) and (4.5) we have that

$$
\begin{array}{r}
\frac{1}{2}\left|\zeta^{0}\right| \leq\left|\zeta^{0} \cdot \frac{1}{\mu_{Q}(Q)} \int_{Q} b_{Q}^{0} d \mu_{Q}\right| \leq\left|\zeta \cdot \frac{1}{\mu_{Q}(Q)} \int_{Q} b_{Q} d \mu_{Q}\right|+\left|\zeta^{\prime} \cdot \frac{1}{\mu_{Q}(Q)} \int_{Q} b_{Q}^{\prime} d \mu_{Q}\right| \\
\leq\left|\zeta \cdot \frac{1}{\mu_{Q}(Q)} \int_{Q} b_{Q} d \mu_{Q}\right|+\frac{\eta}{2}\left|\zeta^{\prime}\right|
\end{array}
$$

for every dyadic cube $Q \subset \mathbb{R}^{n}$. Therefore, for every such $Q \subset \mathbb{R}^{n}$, the estimates

$$
\begin{gathered}
\frac{1}{2}\left|\zeta^{0}\right| \leq\left|\zeta \cdot \frac{1}{\mu_{Q}(Q)} \int_{Q} b_{Q} d \mu_{Q}\right|+\frac{\sqrt{\eta}}{2}\left|\zeta^{0}\right|, \quad \text { and } \\
|\zeta| \leq\left|\zeta^{0}\right|+\left|\zeta^{\prime}\right| \leq\left(1+\eta^{-\frac{1}{2}}\right)\left|\zeta^{0}\right| \leq 2 \eta^{-1 / 2}\left|\zeta^{0}\right|
\end{gathered}
$$

hold in $F_{2}$. Combining the previous three estimates, we have that for $(x, t) \in F_{2}$ and every dyadic cube $Q$

$$
\frac{\sqrt{\eta}}{2}(1-\sqrt{\eta}) \frac{1}{2}|\zeta(x, t)| \leq(1-\sqrt{\eta}) \frac{1}{2}\left|\zeta^{0}(x, t)\right| \leq\left|\zeta \cdot \frac{1}{\mu_{Q}(Q)} \int_{Q} b_{Q} d \mu_{Q}\right|
$$

At this juncture, we make the observation that, in order to obtain (4.2), it suffices to show that for some $\alpha>0$ chosen small enough, we have that

$$
\left\|\zeta^{0} \mathbb{1}_{F_{2}} \mathbb{1}_{\Gamma_{v}^{\alpha}}(\zeta)\right\|_{C} \leq C
$$

with $C$ independent of $v$, where $\Gamma_{v}^{\alpha}$ is an arbitrary cone of aperture $\alpha$; that is,

$$
\left.\Gamma_{v}^{\alpha}:=\left\{z \in \mathbb{C}^{2}: \mid(z /|z|)-v\right) \mid<\alpha\right\}
$$


for $v \in \mathbb{C}^{2}$ a unit vector. It is clear that if we establish (4.4), then (4.2) follows by summing over a collection of cones covering $\mathbb{C}^{2}$. In light of this, we fix such a cone $\Gamma_{v}^{\alpha}$ with $\alpha$ to be chosen. By (4.3) and the fact that $\eta<1 / 4$ we have that for each $(x, t) \in F_{2}$ with $\zeta(x, t) \in \Gamma_{v}^{\alpha}$ and every dyadic cube $Q \subset \mathbb{R}^{n}$,

$$
\begin{aligned}
& \frac{\sqrt{\eta}}{8} \leq\left|\frac{\zeta(x, t)}{|\zeta(x, t)|} \cdot \frac{1}{\mu_{Q}(Q)} \int_{Q} b_{Q} d \mu\right| \\
& \leq\left|\left(\frac{\zeta(x, t)}{|\zeta(x, t)|}-v\right) \cdot \frac{1}{\mu_{Q}(Q)} \int_{Q} b_{Q} d \mu\right|+\left|v \cdot \frac{1}{\mu_{Q}(Q)} \int_{Q} b_{Q} d \mu\right| \\
& \quad \leq C_{0} \alpha+\left|v \cdot \frac{1}{\mu_{Q}(Q)} \int_{Q} b_{Q} d \mu\right|,
\end{aligned}
$$

where in the last step, we used Schwarz's inequality, the fact that

$$
1 / C_{0} \leq d \mu / d x=\phi_{Q} \leq 1 \text { on } Q
$$

and (4.2). Since $\alpha$ is at our disposal, we may choose $\alpha<\frac{\sqrt{\eta}}{16 C_{0}}$, so that

$$
\frac{\sqrt{\eta}}{16}=: \theta \leq\left|v \cdot \frac{1}{\mu_{Q}(Q)} \int_{Q} b_{Q} d \mu\right| .
$$

Next, we observe that in order to obtain (4.5) we needed $(x, t) \in F_{2}$ with $\zeta(x, t) \in \Gamma_{v}^{\alpha}$. This means that (4.5) holds whenever

$$
\iint_{R_{Q}}\left|\zeta^{0}(x, t)\right|^{2} \mathbb{1}_{F_{2}}(x, t) \mathbb{1}_{\Gamma_{\nu}^{\alpha}}(\zeta(x, t)) \frac{d x d t}{t} \neq 0
$$

Consequently, when proving (4.4) we can always assume that (4.5) holds.

Now, fix any dyadic cube $Q$ such that (4.5) holds and, following [GH16], use a stopping time procedure to extract a family $\mathcal{F}=\left\{Q_{j}\right\}$ of non-overlapping dyadic subcubes of $Q$ 
which are maximal with respect to the property that at least one of the following conditions holds:

$$
\begin{aligned}
& \left.\frac{1}{\mu_{Q}\left(Q_{j}\right)} \int_{Q_{j}}\left|b_{Q}\right| d \mu_{Q}>\frac{\theta}{4 \alpha} \quad \text { (type } I\right) \\
& \left.\left|v \cdot \frac{1}{\mu_{Q}\left(Q_{j}\right)} \int_{Q_{j}} b_{Q} d \mu_{Q}\right| \leq \frac{\theta}{2} \quad \text { (type } I I\right) .
\end{aligned}
$$

If some $Q_{j}$ happens to satisfy both the type $I$ and type $I I$ conditions we (arbitrarily) assign it to be of type $I I$. We will write $Q_{j} \in \mathcal{F}_{I}$ or $Q_{j} \in \mathcal{F}_{I I}$ to mean that a cube is of type $I$ or of type II respectively. This stopping time argument produces an 'ample sawtooth' with desirable bounds in the following sense.

Claim 4.6.6 (Ample sawtooth). There exists $\beta>0$, uniform in $Q$, such that

$$
\sum_{Q_{j} \in \mathcal{F}}\left|Q_{j}\right| \leq(1-\beta)|Q|
$$

provided that $\alpha>0$ is small enough (depending on allowable constants). Moreover,

$$
|\zeta(x, t)|^{2} \mathbb{1}_{\Gamma^{\alpha}}(\zeta(x, t)) \leq C_{\theta}\left|\zeta(x, t) A_{t}^{\mu_{Q}} b_{Q}(x)\right|^{2}, \quad \text { for }(x, t) \in E_{Q}^{*}
$$

where $E_{Q}^{*}:=R_{Q} \backslash\left(\cup_{Q_{j} \in \mathcal{F}} R_{Q_{j}}\right)$. Here $A_{t}^{\mu_{Q}}$ is the 'dyadic averaging operator adapted to the measure $\mu_{Q}$, that is, $A_{t}^{\mu} f(x)=\frac{1}{\mu_{Q}(Q(x, t))} \int_{Q(x, t)} f d \mu_{Q}$, where $Q(x, t)$ denotes the smallest dyadic cube, of side length at least $t$, that contains $x$.

We postpone the proof of the claim for a bit. The ampleness condition (4.7) allows us to use the "John-Nirenberg lemma for Carleson measures" to replace $R_{Q}$ in the definition of $\|\cdot\|_{C}$ by $E_{Q}^{*}$. This is done via an induction argument; see for instance, [Hof10, Lemma 
1.37]. Thus, we have by (4.8) that

$$
\begin{aligned}
&\left\|\zeta^{0} \mathbb{1}_{F_{2}} \mathbb{1}_{\Gamma_{v}^{\alpha}}(\zeta)\right\|_{C} \lesssim_{\beta} \sup _{Q} \frac{1}{|Q|} \iint_{E_{Q}^{*}}\left|\zeta^{0}(x, t)\right|^{2} \mathbb{1}_{F_{2}}(x, t) \mathbb{1}_{\Gamma_{v}^{\alpha}}(\zeta(x, t)) \frac{d x d t}{t} \\
& \lesssim \sup _{Q} \frac{1}{|Q|} \iint_{R_{Q}}\left|\zeta(x, t) A_{t}^{\mu_{Q}} b_{Q}(x)\right|^{2} \frac{d x d t}{t}
\end{aligned}
$$

where we used that $\left|\zeta^{0}\right| \leq|\zeta|$ in the first line and replaced $E_{Q}^{*}$ by the larger set $R_{Q}$ after using (4.8) in the second line. As we had reduced the proof of the theorem to showing the estimate (4.4), it is enough to show that

$$
\sup _{Q} \frac{1}{|Q|} \iint_{R_{Q}}\left|\zeta(x, t) A_{t}^{\mu_{Q}} b_{Q}(x)\right|^{2} \frac{d x d t}{t} \leq C .
$$

To this end, we fix a dyadic cube $Q$ and write

$$
\zeta A_{t}^{\mu_{Q}} b_{Q}=\left[\left(\Theta_{t} 1\right) A_{t}^{\mu}-\Theta_{t}\right] b_{Q}+\Theta_{t} b_{Q}=: R_{t} b_{Q}+\Theta_{t} b_{Q}=I+I I
$$

First we handle term $I I$, which is (almost) good by design. We write

$$
I I=\Theta_{t} b_{Q}=\hat{\Theta}_{t} \hat{b}_{Q}-\Theta_{t}^{(a)} b_{Q}^{(a)}=: I I_{1}+I I_{2}
$$

By (4.3), the contribution from the term $I_{1}$ in (4.9) is controlled by $C_{0}$. Moreover, by Proposition 4.5.1 we have that

$$
\begin{array}{r}
\iint_{R_{Q}}\left|\Theta_{t}^{(a)} b_{Q}^{(a)}\right|^{2} \frac{d x d t}{t} \leq C_{1}\left\|b_{Q}^{(a)}\right\|_{L^{2}\left(\mathbb{R}^{n}\right)}^{2}\left(1+\left\|\Theta_{t}^{0} 1\right\|_{C}\right) \\
\quad \leq C_{1} C_{0}|Q|\left\|B_{1}\right\|_{n}^{2}\left(1+\left\|\Theta_{t}^{0} 1\right\|_{C}\right),
\end{array}
$$


so that the contribution of $I I_{2}$ can be hidden in (4.1), provided that $\left\|B_{1}\right\|_{n}$ is sufficiently small (depending on $\eta, \alpha)$. Here, we used that $b_{Q}^{(a)}(y)=|Q| B_{1} F_{Q}(y, 0)$, so that

$$
\left\|b_{Q}^{(a)}\right\|_{L^{2}\left(\mathbb{R}^{n}\right)}^{2}=\int_{\mathbb{R}^{n}}|Q|^{2}\left|B_{1} F_{Q}(\cdot, 0)\right|^{2} \leq\left\|B_{1}\right\|_{n}^{2}|Q|^{2} \int_{\mathbb{R}^{n}}\left|\nabla F_{Q}(\cdot, 0)\right|^{2} \leq C_{0}\left\|B_{1}\right\|_{n}^{2}|Q|
$$

It remains to obtain a desirable bound for $I$. Let $\left\{Q_{s}\right\}_{s>0}$ be a CLP family (see Definition 2.4.13). By a standard orthogonality argument and (4.2), it is enough to show that for some $\beta_{0}>0$ and all $t \in(0, \ell(Q))$, the estimate

$$
\int_{Q}\left|R_{t} Q_{s}^{2} h\right|^{2} \lesssim \min \left(\frac{s}{t}, \frac{t}{s}\right)^{\beta_{0}} \int_{\mathbb{R}^{n}}\left|Q_{s} h\right|^{2}
$$

holds for all $h \in H \times L^{2}\left(\mathbb{R}^{n}\right)$.

We remind the reader that $H:=\left\{h^{\prime}: h^{\prime}=\nabla F, F \in Y^{1,2}\left(\mathbb{R}^{n}\right)\right\}$ and that $b_{Q} \in H \times L^{2}\left(\mathbb{R}^{n}\right)$. Before proving (4.10), we make a small technical point. Having fixed $Q$, we let $\tilde{\mu}_{Q}$ be a measure on $\mathbb{R}^{n}$ defined by $\tilde{\mu}_{Q}:=\left.\mu_{Q}\right|_{Q}+\left.\frac{1}{\tilde{C}_{0}} d x\right|_{\mathbb{R}^{n} \backslash Q}$, and set $E_{t}=A_{t}^{\tilde{\mu}_{Q}}$. Notice that for $(x, t) \in Q \times(0, \ell(Q)), A_{t}^{\tilde{\mu}_{Q}}$ acts exactly as $A_{t}^{\mu_{Q}}$. Thus, in order to prove (4.10), we may replace $R_{t}$ by $\widetilde{R}_{t}$, where $\widetilde{R}_{t}:=\left[\left(\Theta_{t} 1\right) E_{t}-\Theta_{t}\right]$. Notice that we may apply Lemma 2.4 .11 to $\Theta_{t}$, since $\Theta_{t}$ has good off-diagonal decay (see Proposition 3.3.15) and satisfies uniform $L^{2}$ bounds on slices (see Proposition 3.3.1). Thus, $\left(\Theta_{t} 1\right)$ is well defined as an element of $L_{\mathrm{loc}}^{2}$ and, since $E_{t}$ is a self-adjoint averaging operator, we have that

$$
\sup _{t>0}\left\|\left(\Theta_{t} 1\right) E_{t}\right\|_{L^{2} \rightarrow L^{2}} \leq C .
$$

We break (4.10) into cases. 
Case 1: $t \leq s$. In this case, we see by (4.11) and properties of $\Theta_{t}$ that $\widetilde{R}_{t} 1=0$, $\left\|\widetilde{R}_{t}\right\|_{L^{2} \rightarrow L^{2}} \leq C$ and $\widetilde{R}_{t}$ has good off-diagonal decay. Hence, it follows from Lemma 2.4.12 that

$$
\left\|\widetilde{R}_{t} Q_{s}^{2} h\right\|_{L^{2}\left(\mathbb{R}^{n}\right)} \lesssim t\left\|\nabla Q_{s}^{2} h\right\|_{L^{2}\left(\mathbb{R}^{n}\right)} \lesssim \frac{t}{s}\left\|s \nabla Q_{s} Q_{s} h\right\|_{L^{2}\left(\mathbb{R}^{n}\right)} \lesssim \frac{t}{s}\left\|Q_{s} h\right\|_{L^{2}\left(\mathbb{R}^{n}\right)}
$$

which shows (4.10) with $\beta_{0}=2$ in this case.

Case 2: $t>s$. In this case, we break $\widetilde{R}_{t}$ into its two separate operators. One can verify that $\left\|E_{t} Q_{s}\right\|_{L^{2} \rightarrow L^{2}} \lesssim\left(\frac{s}{t}\right)^{\gamma}$ for some $\gamma>0$. Since $E_{t}$ is a projection operator, we have that $E_{t}=E_{t}^{2}$ and hence by (4.11), we see that

$$
\left\|\left(\Theta_{t} 1\right) E_{t} Q_{s}^{2} h\right\|_{2}=\left\|\left(\Theta_{t} 1\right) E_{t}\left[E_{t} Q_{s}^{2} h\right]\right\|_{2} \lesssim\left\|E_{t} Q_{s}^{2} h\right\|_{2} \lesssim\left(\frac{s}{t}\right)^{\gamma}\left\|Q_{s} h\right\|_{2}
$$

which shows that the contribution of $\left(\Theta_{t} 1\right) E_{t} Q_{s}^{2}$ to (4.10) when $t>s$ is as desired with $\beta_{0}=2 \gamma$

We are left with handling $\Theta_{t} Q_{s}^{2} h$. Since $h=\left(h^{\prime}, h^{0}\right) \in H \times L^{2}\left(\mathbb{R}^{n}\right)$, we write $h=\left(\nabla_{\|} F, h^{0}\right)$, with $F \in Y^{1,2}\left(\mathbb{R}^{n}\right)$ (note $\nabla_{\|}=\nabla$ here). Then we may write

$$
\begin{aligned}
& \Theta_{t} Q_{s} h=\Theta_{t}^{0} Q_{s}^{2} h^{0}+\Theta_{t}^{\prime} Q_{s}^{2} \nabla_{\|} F \\
& =\Theta_{t}^{0} Q_{s}^{2} h^{0}+\left[\Theta_{t}^{\prime} Q_{s}^{2} \nabla_{\|} F+\Theta_{t}^{(a)} B_{1} Q_{s}^{2} F\right]-\Theta_{t}^{(a)} B_{1} Q_{s}^{2} F
\end{aligned}
$$

$$
=J_{1}+J_{2}+J_{3}
$$

To handle $J_{1}$, we write $Q_{s}=s \operatorname{div}_{\|} s \nabla_{\|} e^{s^{2} \Delta}$, so that

$$
J_{1}=\Theta_{t}^{0} Q_{s}^{2} h^{0}=t^{m}\left(\partial_{t}\right)^{m+1} \mathcal{S}_{t}^{\mathcal{L}} Q_{s} Q_{s} h^{0}=\frac{s}{t} t^{m+1}\left(\partial_{t}\right)^{m+1} \mathcal{S}_{t}^{\mathcal{L}} \operatorname{div}_{\|} s \nabla_{\|} e^{s^{2} \Delta} Q_{s} h^{0}
$$


Note that by (3.3) we have that $t^{m+1}\left(\partial_{t}\right)^{m+1} \mathcal{S}_{t}^{\mathcal{L}} \operatorname{div}_{\|}$and $s \nabla_{\|} e^{s^{2} \Delta}$ are bounded operators on $L^{2}\left(\mathbb{R}^{n}\right)$. Therefore, we have that $\left\|\Theta_{t}^{0} Q_{s}^{2} h^{0}\right\|_{2} \lesssim \frac{s}{t}\left\|Q_{s} h^{0}\right\|_{2}$, and the contribution of $J_{1}$ to (4.10) when $t>s$ is as desired with $\beta_{0}=2$.

For the term $J_{2}$, first we use Proposition 2.4.14 to justify that there exists $g \in L^{2}\left(\mathbb{R}^{n}\right)$ such that $Q_{s} F=I_{1} g$, where $I_{1}=(-\Delta)^{-1 / 2}$ is the Riesz potential of order 1 , and satisfying $\|g\|_{2} \approx\left\|\nabla_{\|} Q_{s} F\right\|_{2}=\left\|Q_{s} \nabla_{\|} F\right\|_{2}=\left\|Q_{s} h^{\prime}\right\|_{2}$ (every $F \in Y^{1,2}\left(\mathbb{R}^{n}\right)$ arises as the Riesz potential of a function $g$ in $L^{2}\left(\mathbb{R}^{n}\right)$ ). Then, we may use integration by parts on slices (Proposition 2.5.38) to compute that

$$
\begin{aligned}
J_{2}=t^{m}\left(\partial_{t}\right)^{m} \mathcal{S}_{t}^{\mathcal{L}} & \left(B_{2 \|} \nabla_{\|} Q_{s}^{2} F\right)+t^{m}\left(\partial_{t}\right)^{m}\left(\mathcal{S}_{t}^{\mathcal{L}} \nabla\right) \tilde{A} \nabla_{\|} Q_{s}^{2} F+t^{m}\left(\partial_{t}\right)^{m}\left(\mathcal{S}_{t}^{\mathcal{L}} \nabla\right) B_{1} Q_{s}^{2} F \\
& =-t^{m}\left(\partial_{t}\right)^{m+1}\left(\mathcal{S}_{t}^{\mathcal{L}} \nabla\right) \vec{A}_{\cdot, n+1} Q_{s} I_{1} g+t^{m}\left(\partial_{t}\right)^{m+1} \mathcal{S}_{t}^{\mathcal{L}} B_{2 \perp} Q_{s} I_{1} g=J_{2,1}+J_{2,2}
\end{aligned}
$$

Since $\left\|s^{-1} Q_{s} I_{1}\right\|_{L^{2} \rightarrow L^{2}} \leq C$ and $t^{m+1}\left(\partial_{t}\right)^{m+1}\left(\mathcal{S}_{t}^{\mathcal{L}} \nabla\right): L^{2} \rightarrow L^{2}$, we obtain that the contribution of $J_{2,1}$ to (4.10) when $t>s$ is as desired with $\beta_{0}=2$. Similarly, $t^{m}\left(\partial_{t}\right)^{m+1} \mathcal{S}_{t}^{\mathcal{L}} B_{2 \perp}: L^{2} \rightarrow L^{2}$, so that the contribution of $J_{2,2}$ to (4.10) when $t>s$ is as desired with $\beta_{0}=2$.

We are left with controlling the contribution of

$$
J_{3}=\Theta_{t}^{(a)} B_{1} Q_{s}^{2} F=t^{m} \partial_{t}^{m}\left(\mathcal{S}_{t}^{\mathcal{L}} \nabla\right) B_{1} Q_{s} F=\Theta_{t, m} B_{1} I_{1} g
$$

where $F=I_{1} g, F \in Y^{1,2}$ and $g \in L^{2}$ with $\|g\|_{2} \approx\left\|\nabla_{\|} F\right\|_{2}$. By Proposition 3.3.8, for all $s<t$ we have that

$$
\left\|\Theta_{t}^{(a)} B_{1} Q_{s}^{2} F\right\|_{L^{2}\left(\mathbb{R}^{n}\right)} \lesssim\left(\frac{s}{t}\right)^{\gamma}\left\|Q_{s} g\right\|_{L^{2}\left(\mathbb{R}^{n}\right)}
$$


Then we may control this term in (4.10) with $g$ in place of $h=\nabla_{\|} F$, which is sufficient as $\|g\|_{2} \lesssim\left\|\nabla_{\|} F\right\|_{2}$.

The proof of the theorem is finished modulo the

Proof of Claim 4.6.6. We first verify (4.8). Observe, by the maximality of the family $Q_{j}$, that for any dyadic subcube $Q^{\prime}$ of $Q$ which is not contained in any $Q_{j}$, we have the inequalities opposite to the type $I$ and type $I I$ inequalities, with $Q^{\prime}$ in place of $Q_{j}$. Thus,

$$
\frac{\theta}{2} \leq\left|v \cdot A_{t}^{\mu_{Q}} b_{Q}(x)\right| \quad \text { and } \quad\left|A_{t}^{\mu_{Q}} b_{Q}(x)\right| \leq \frac{\theta}{4 \alpha}
$$

for all $(x, t) \in E_{Q}^{*}$. It follows that if $z \in \Gamma_{v}^{\alpha}$ and $(x, t) \in E_{Q}^{*}$, we have the bound

$$
\begin{aligned}
\frac{\theta}{2} \leq\left|v \cdot A_{t}^{\mu_{Q}} b_{Q}(x)\right| \leq\left|(z /|z|) \cdot A_{t}^{\mu_{Q}} b_{Q}(x)\right|+\left|(z /|z|-v) \cdot A_{t}^{\mu_{Q}} b_{Q}(x)\right| & \\
& \leq\left|(z /|z|) \cdot A_{t}^{\mu_{Q}} b_{Q}(x)\right|+\frac{\theta}{4}
\end{aligned}
$$

where we used the definition of $\Gamma_{v}^{\alpha}$ in the last line. The above estimate yields (4.8) with $C_{\theta}=\left(\frac{4}{\theta}\right)^{2}$ by setting $z=\zeta(x, t)$.

Now we establish (4.7). Set $E:=Q \backslash\left(\cup_{Q_{j} \in \mathcal{F}} Q_{j}\right)$ and $B_{I}:=\cup_{Q_{j} \in \mathcal{F}_{I}} Q_{j}$. By definition of $\mathcal{F}_{I}$ and the fact that $1 / C_{0} \leq d \mu / d x \leq 1$ on $Q$, we have that $B_{I} \subset\left\{\mathscr{M}\left(b_{Q}\right)>\frac{\theta}{4 C_{0} \alpha}\right\}$ where $\mathscr{M}$ is the uncentered Hardy-Littlewood maximal function on $\mathbb{R}^{n}$ (taken over cubes). The weak-type $(2,2)$ inequality for the Hardy-Littlewood maximal function and (4.2) yield the estimate

$$
\left|B_{I}\right| \leq C C_{0}^{2}\left(\frac{\alpha}{\theta}\right)^{2} \int_{\mathbb{R}^{n}}\left|b_{Q}\right|^{2} \leq C C_{0}^{3}\left(\frac{\alpha}{\theta}\right)^{2}|Q| .
$$


From this estimate, (4.2), (4.5), the definition of type $I I$ cubes, and Hölder's inequality we obtain

$$
\begin{aligned}
& \theta \mu_{Q}(Q) \leq\left|v \cdot \int_{Q} b_{Q} d \mu_{Q}\right| \\
& \leq\left|v \cdot \int_{E} b_{Q} d \mu_{Q}\right|+\int_{B_{I}}\left|b_{Q}\right| d \mu+\sum_{Q_{j} \in \mathcal{F}_{I I}}\left|v \cdot \int_{Q_{j}} b_{Q} d \mu_{Q}\right| \\
& \leq|E|^{1 / 2}|| b_{Q}\left\|_{L^{2}\left(\mathbb{R}^{n}\right)}+\left|B_{I}\right|^{1 / 2}|| b_{Q}\right\|_{L^{2}\left(\mathbb{R}^{n}\right)}+\frac{\theta}{2} \sum_{Q_{j} \in \mathcal{F}_{I I}} \mu_{Q}\left(Q_{j}\right) \\
& \leq C|E|^{1 / 2}|Q|^{1 / 2}+C_{\theta} \alpha|Q|+\frac{\theta}{2} \mu_{Q}(Q) .
\end{aligned}
$$

Choosing $\alpha>0$ small enough and using the fact that $(1 / 2)^{n}|Q| \leq \mu_{Q}(Q) \leq|Q|$, the above estimate implies that $|Q| \leq C_{\theta}|E|$, which yields the claim with $\beta=1 / C_{\theta}$.

Thus we conclude the proof of Theorem 4.2.1

\subsection{Control of slices via square function estimates}

We are able to use the square function estimate obtained in the previous section to immediately improve our $L^{2} \rightarrow L^{2}$ boundedness results of $t$-derivatives of the single layer potential. More precisely, in the following lemma, we extend estimate (3.3) (previously valid for $m \geq 2$ ), to the case $m=1$, given sufficient smallness of $\max \left\{\left\|B_{1}\right\|_{n},\left\|B_{2}\right\|_{n}\right\}$.

Lemma 4.7.1 (Stronger $L^{2} \rightarrow L^{2}$ estimate). The estimate

$$
\left\|t \nabla \partial_{t} \mathcal{S}_{t}^{\mathcal{L}} f\right\|_{L^{2}\left(\mathbb{R}^{n}\right)} \lesssim\|f\|_{L^{2}\left(\mathbb{R}^{n}\right)}
$$


holds, provided that $\max \left\{\left\|B_{1}\right\|_{n},\left\|B_{2}\right\|_{n}<\varepsilon_{0}\right.$ and $\varepsilon_{0}>0$ is small enough that (4.6) holds for $m=n+10$.

We may use Lemma 4.7.1 to obtain the "travel down" procedure for $\nabla \mathcal{S}^{\mathcal{L}} \nabla$.

Lemma 4.7.2 $\left(L^{2} \rightarrow L^{2}\right.$ estimates for $\left.S_{t} \nabla\right)$. The following statements are true.

i) For each $\mathbf{f} \in L^{2}\left(\mathbb{R}^{n}, \mathbb{C}^{n+1}\right)$ and each $t \neq 0$ we have that

$$
\left\|t^{k} \partial_{t}^{k}\left(\mathcal{S}_{t}^{\mathcal{L}} \nabla\right) \mathbf{f}\right\|_{2} \lesssim\|\mathbf{f}\|_{2}, \quad k \geq 1
$$

$$
\left\|t^{k} \partial_{t}^{k-1} \nabla\left(\mathcal{S}_{t}^{\mathcal{L}} \nabla\right) \mathbf{f}\right\|_{2} \lesssim\|\mathbf{f}\|_{2}, \quad k \geq 2
$$

provided that $\max \left\{\left\|B_{1}\right\|_{n},\left\|B_{2}\right\|_{n}<\varepsilon_{0}\right.$ is small. Therefore, for each $m>k \geq 2$,

$$
\left\|\left|t^{k} \partial_{t}^{k-1} \nabla\left(\mathcal{S}_{t}^{\mathcal{L}} \nabla\right) \mathbf{f}\right|\right\| \lesssim_{m}\left\|t^{m} \partial_{t}^{m}\left(\mathcal{S}_{t}^{\mathcal{L}} \nabla\right) \mathbf{f} \mid\right\|+\|\mathbf{f}\|_{2}
$$

provided that $\max \left\{\left\|B_{1}\right\|_{L^{n}\left(\mathbb{R}^{n}\right)},\left\|B_{2}\right\|_{L^{n}\left(\mathbb{R}^{n}\right)}\right\}$ is small.

ii) The estimate (4.5) holds for $k=1$ if the operator $\nabla$ acting on $\left(\mathcal{S}_{t}^{\mathcal{L}} \nabla\right)$ is replaced by $\partial_{t}$.

We proceed with the

Proof of Theorem 1.0.7. Let $\mathbf{h} \in C_{c}^{\infty}\left(\mathbb{R}^{n}\right)^{n+1}$ and fix $\tau>0$. Notice that by Lemma 2.2.2, the pairing $\left(\mathbf{h}, \operatorname{Tr}_{t} \nabla u\right)_{2,2}$ is meaningful. Let $R \gg \tau, \psi \in C_{c}^{\infty}(\mathbb{R})$ satisfy $\psi \equiv 1$ on $[\tau, R], \psi \equiv 0$ on $[2 R, \infty),|\psi| \leq 1$ and $\left|\psi^{\prime}\right| \leq \frac{2}{R}$. Observe the estimates: 


$$
\begin{aligned}
& |I|:=\left|\int_{\mathbb{R}^{n}} \mathbf{h} \cdot \int_{R}^{2 R} \psi^{\prime} \nabla u\right| \leq \int_{\mathbb{R}^{n}} \int_{R}^{2 R}\left|\mathbf{h} \| \psi^{\prime}\right||\nabla u| \\
& \leq \frac{2}{\sqrt{R}}\|\mathbf{h}\|_{L^{2}\left(\mathbb{R}^{n}\right)}\|\nabla u\|_{L^{2}\left(\mathbb{R}_{+}^{n+1}\right)} \longrightarrow 0 \quad \text { as } R \rightarrow \infty, \\
& \left|\int_{\mathbb{R}^{n}} \mathbf{h} \cdot t \operatorname{Tr}_{t+\tau} \nabla \partial_{t} u\right| \leq\|\mathbf{h}\|_{L^{2}\left(\mathbb{R}^{n}\right)} \frac{t}{t+\tau}\left\|(t+\tau) \operatorname{Tr}_{t+\tau} \nabla \partial_{t} u\right\|_{L^{2}\left(\mathbb{R}^{n}\right)} \\
& \leq \frac{t}{\tau}\|\mathbf{h}\|_{L^{2}\left(\mathbb{R}^{n}\right)}\|\nabla u\|_{L^{2}\left(\mathbb{R}_{+}^{n+1}\right)} \longrightarrow 0 \quad \text { as } t \searrow 0, \\
& |I I|:=\left|\int_{\mathbb{R}^{n}} \int_{R-\tau}^{2 R-\tau} \mathbf{h} \cdot t \psi^{\prime}(t+\tau) \operatorname{Tr}_{t+\tau} \partial_{t} \nabla u d t\right| \leq 2 f_{R}^{2 R} \int_{\mathbb{R}^{n}} t|\mathbf{h}|\left|\partial_{t} \nabla u\right| d t \\
& \leq 2\|\mathbf{h}\|_{L^{2}\left(\mathbb{R}^{n}\right)} \sup _{t \in(R, 2 R)}\left\|t \operatorname{Tr}_{t} \partial_{t} \nabla u\right\|_{L^{2}\left(\mathbb{R}^{n}\right)} \lesssim\|\mathbf{h}\|_{L^{2}\left(\mathbb{R}^{n}\right)}\|\nabla u\|_{L^{2}\left(\mathbb{R}_{R / 2}^{n+1}\right)} \longrightarrow 0 \quad \text { as } R \rightarrow \infty,
\end{aligned}
$$

where in (4.7) we used (2.44), and in (4.8) we used (2.43) and the absolute continuity of the integral. We now perform two integration by parts in the following calculation, recalling that $\psi(2 R)=0$ so that the arising boundary terms vanish.

$$
\begin{aligned}
& \int_{\mathbb{R}^{n}} \mathbf{h} \cdot \operatorname{Tr}_{\tau} \nabla u=\int_{\mathbb{R}^{n}} \mathbf{h} \cdot \psi(\tau) \operatorname{Tr}_{\tau} \nabla u-\int_{\mathbb{R}^{n}} \mathbf{h} \cdot \psi(2 R) \operatorname{Tr}_{2 R} \nabla u \\
& =-\int_{\mathbb{R}^{n}} \mathbf{h} \cdot \int_{\tau}^{2 R} \psi \partial_{t} \nabla u-\int_{\mathbb{R}^{n}} \mathbf{h} \cdot \int_{R}^{2 R} \psi^{\prime} \nabla u \\
& =\int_{\mathbb{R}^{n}} \int_{0}^{2 R-\tau} \mathbf{h} \cdot t \operatorname{Tr}_{t} \mathscr{T}^{\tau} \psi \partial_{t}^{2} \nabla \mathscr{T}^{\tau} u d t+\int_{\mathbb{R}^{n}} \int_{R-\tau}^{2 R-\tau} \mathbf{h} \cdot t \operatorname{Tr}_{t} \mathscr{T}^{\tau} \psi^{\prime} \partial_{t} \nabla \mathscr{T}^{\tau} u d t-I \\
& =\int_{\mathbb{R}^{n}} \int_{0}^{2 R-\tau} \mathbf{h} \cdot t \operatorname{Tr}_{t} \mathscr{T}^{\tau} \psi \partial_{t}^{2} \nabla \mathscr{T}^{\tau} u d t+I I-I
\end{aligned}
$$

where in the third equality we used (4.7) already. Note that the terms $I, I I$ drop to 0 as $R \rightarrow \infty$ by the estimates (4.6) and (4.8). For technical reasons, let us integrate by parts one more time. The boundary term that is introduced is again controlled as in (4.7) and (4.8) because we may apply the results of Proposition 2.5.42 to $\partial_{t}^{2} \mathscr{T}^{\tau} u$. Hence we have that 


$$
\begin{aligned}
\int_{\mathbb{R}^{n}} \int_{0}^{2 R-\tau} \mathbf{h} \cdot t \operatorname{Tr}_{t} \mathscr{T}^{\tau} \psi \partial_{t}^{2} \nabla \mathscr{T}^{\tau} u d t & \\
= & -\frac{1}{2} \int_{\mathbb{R}^{n}} \int_{0}^{2 R-\tau} \mathbf{h} \cdot t^{2} \operatorname{Tr}_{t} \mathscr{T}^{\tau} \psi \partial_{t}^{3} \nabla \mathscr{T}^{\tau} u d t+I I I,
\end{aligned}
$$

where $|I I I| \rightarrow 0$ as $R \rightarrow \infty$. Intuitively, we would like to introduce Green's formula at this point, but we want the "input" in the layer potentials to still depend on $t$ for when we later dualize to control our integral by square function estimates. Let us now do a change of variables $t \mapsto 2 t$, and carefully track the use of the chain rule:

$$
\begin{aligned}
& \frac{1}{2} \int_{\mathbb{R}^{n}} \int_{0}^{2 R-\tau} \mathbf{h} \cdot t^{2} \operatorname{Tr}_{t} \mathscr{T}^{\tau} \psi \partial_{t}^{3} \nabla \mathscr{T}^{\tau} u d t \\
& =4 \int_{\mathbb{R}^{n}} \int_{0}^{R-\frac{\tau}{2}} \mathbf{h} \cdot t^{2} \mathscr{T}^{\tau} \psi(2 t) \partial_{2 t}^{3} \nabla_{x, 2 t} \mathscr{T}^{\tau} u(\cdot, 2 t) d t \\
& =\frac{1}{2} \int_{\mathbb{R}^{n}}\left[\int_{0}^{R-\frac{\tau}{2}} \overrightarrow{h_{\|}} \cdot t^{2} \mathscr{T}^{\tau} \psi(2 t) \partial_{t}^{3} \nabla_{\|} \mathscr{T}^{\tau} u(\cdot, 2 t) d t\right. \\
& \left.\quad+\frac{1}{2} h_{\perp} \int_{0}^{R-\frac{\tau}{2}} t^{2} \mathscr{T}^{\tau} \psi(2 t) \partial_{t}^{4} \mathscr{T}^{\tau} u(x, 2 t) d t\right] .
\end{aligned}
$$

We now consider $s \in \mathbb{R}$ and write $2 t=t+\left.s\right|_{s=t}$. If $F$ is a differentiable function in $t$, the chain rule tells us that $\partial_{t} F(t+s)=\partial_{s} F(t+s)$. By this change of variables, and the above identity, we compute that

$$
\begin{aligned}
& \frac{1}{2} \int_{\mathbb{R}^{n}} \int_{0}^{2 R-\tau} \mathbf{h} \cdot t^{2} \operatorname{Tr}_{t} \mathscr{T}^{\tau} \psi \partial_{t}^{3} \nabla \mathscr{T}^{\tau} u d t \\
& =4 \int_{0}^{R-\frac{\tau}{2}} t^{2} \mathscr{T}^{t+\tau} \psi(t)\left[\int_{\mathbb{R}^{n}} \mathbf{h} \cdot \operatorname{Tr}_{t} \nabla_{x, t} D_{n+1}^{3} \mathscr{T}^{s} \mathscr{T}^{\tau} u(x, t)\right]_{s=t} d t \\
& =4 \int_{0}^{R-\frac{\tau}{2}} t^{2} \mathscr{T}^{t+\tau} \psi(t)\left[\int_{\mathbb{R}^{n}} \mathbf{h} \cdot \operatorname{Tr}_{t} \nabla D_{n+1} \mathscr{T}^{\tau}\left(D_{n+1}^{2} \mathscr{T}^{s} u\right)\right]_{s=t} d t .
\end{aligned}
$$

We now apply Green's formula, Theorem 3.2.1 ii). The function $v:=D_{n+1}^{2} \mathscr{T}^{s} u$ belongs 
to $W^{1,2}\left(\mathbb{R}_{+}^{n+1}\right) \subset Y^{1,2}\left(\mathbb{R}_{+}^{n+1}\right)$ and solves $L v=0$ in $\mathbb{R}_{+}^{n+1}$ in the weak sense. Therefore the identity $v=-\mathcal{D}^{L,+}\left(\operatorname{Tr}_{0} v\right)+\mathcal{S}^{L}\left(\partial_{v}^{L,+} v\right)$ holds in $Y^{1,2}\left(\mathbb{R}_{+}^{n+1}\right)$, for any $s>0$. But by the results of Proposition 2.5.42, for each $t>0$ we have the identity

$$
\operatorname{Tr}_{t} \nabla D_{n+1} \mathscr{T}^{\tau} v=\operatorname{Tr}_{t} \nabla D_{n+1} \mathscr{T}^{\tau}\left(-\mathcal{D}^{L,+}\left(\operatorname{Tr}_{0} v\right)+\mathcal{S}^{L}\left(\partial_{v}^{L,+} v\right)\right)
$$

in $L^{2}\left(\mathbb{R}^{n}\right)$, for any $s>0$ and $t>0$. As such, per our calculations we have the identity

$$
\begin{aligned}
& \frac{1}{2} \int_{\mathbb{R}^{n}} \int_{0}^{2 R-\tau} \mathbf{h} \cdot t^{2} \operatorname{Tr}_{t} \mathscr{T}^{\tau} \psi \partial_{t}^{3} \nabla \mathscr{T}^{\tau} u d t \\
& =-4 \int_{0}^{R-\frac{\tau}{2}} t^{2} \mathscr{T}^{t+\tau} \psi(t)\left[\int_{\mathbb{R}^{n}} \mathbf{h} \cdot \operatorname{Tr}_{t} \nabla D_{n+1} \mathscr{T}^{\tau} \mathcal{D}^{L,+}\left(\operatorname{Tr}_{0} v\right)\right]_{s=t} d t \\
& \quad+4 \int_{0}^{R-\frac{\tau}{2}} t^{2} \mathscr{T}^{t+\tau} \psi(t)\left[\int_{\mathbb{R}^{n}} \mathbf{h} \cdot \operatorname{Tr}_{t} \nabla D_{n+1} \mathscr{T}^{\tau} \mathcal{S}^{L}\left(\partial_{v}^{L,+} v\right)\right]_{s=t} d t=I V+V .
\end{aligned}
$$

Now we make use of the adjoint relations (3.5), (3.6) and (2.45) to dualize $I V$ and $V$. Indeed, we see that

$$
\begin{aligned}
\int_{\mathbb{R}^{n}} \mathbf{h} \cdot \operatorname{Tr}_{t} \nabla D_{n+1} \mathscr{T}^{\tau} \mathcal{D}^{L,+}\left(\operatorname{Tr}_{0} v\right) & =\overline{\left(D_{n+1} \partial_{v,-t-\tau}^{L^{*},-}\left(\mathcal{S}^{L^{*}} \nabla\right) \overline{\mathbf{h}}, \operatorname{Tr}_{0} v\right)_{2,2}} \\
= & \overline{\left(D_{n+1} e_{n+1} \cdot \operatorname{Tr}_{-t-\tau}\left[A^{*} \nabla+\overline{B_{2}}\right]\left(\mathcal{S}^{L^{*}} \nabla\right) \overline{\mathbf{h}}, \operatorname{Tr}_{s} D_{n+1}^{2} u\right)_{2,2}}, \\
\int_{\mathbb{R}^{n}} \mathbf{h} \cdot \operatorname{Tr}_{t} \nabla D_{n+1} \mathscr{T}^{\tau} \mathcal{S}^{L}\left(\partial_{v}^{L,+} v\right) & =\overline{\left(\operatorname{Tr}_{-t-\tau} D_{n+1}\left(\mathcal{S}^{L^{*}} \nabla\right) \overline{\mathbf{h}}, \partial_{v}^{L,+} v\right)_{2,2}} \\
= & \overline{\left(\operatorname{Tr}_{-t-\tau} D_{n+1}\left(\mathcal{S}^{L^{*}} \nabla\right) \overline{\mathbf{h}},-e_{n+1} \cdot \operatorname{Tr}_{s}\left[A \nabla+B_{1}\right] D_{n+1}^{2} u\right)_{2,2}} .
\end{aligned}
$$

Therefore, using the Cauchy-Schwartz inequality, we estimate that

$$
|I V| \leq 4 \int_{0}^{R-\frac{\tau}{2}} t^{2} \int_{\mathbb{R}^{n}}\left|\operatorname{Tr}_{-t-\tau} D_{n+1}\left[A^{*} \nabla+\overline{B_{2}}\right]\left(\mathcal{S}^{L^{*}} \nabla\right) \overline{\mathbf{h}}\right|\left|\operatorname{Tr}_{t} D_{n+1}^{2} u\right| d t
$$




$$
\begin{array}{r}
\left.\lesssim\left\|t^{2} \partial_{t} \nabla \mathcal{M}\left(S^{L^{*}} \nabla\right) \overline{\mathbf{h}}\right\|\right|_{-}\left\|t \partial_{t}^{2} u\right\| \| \\
|V| \leq 4 \int_{0}^{R-\frac{\tau}{2}} t^{2} \int_{\mathbb{R}^{n}}\left|\operatorname{Tr}_{-t-\tau} D_{n+1}\left(\mathcal{S}^{L^{*}} \nabla\right) \overline{\mathbf{h}}\right||| \partial_{t}^{2} u \|, \\
\lesssim \| \operatorname{Tr}_{t}\left[A \nabla+\partial_{t} \mathcal{M}\left(S^{L^{*}} \nabla\right) \overline{\mathbf{h}}\left\|\left.\right|_{-}\right\| t_{n+1}^{2} \partial_{t}^{2} \nabla u|d t| \lesssim\|\mathbf{h}\|_{2}\left\|t^{2} \partial_{t}^{2} \nabla u\right\|,\right.
\end{array}
$$

where we used the square function estimate (4.6) and the "travel-down" procedure (4.5). Now send $R \rightarrow \infty$, which sends $|I|,|I I|,|I I I| \rightarrow 0$. By the bounds (4.10), (4.11), and Lemma 4.2.2, the desired bound for the gradient follows.

To obtain the bound for the $L^{\frac{2 n}{n-2}}\left(\mathbb{R}^{n}\right)$ norm, we use Lemma 2.2.2 to ensure that at each horizontal slice, the $L^{\frac{2 n}{n-2}}\left(\mathbb{R}^{n}\right)$ norm of a $Y^{1,2}\left(\mathbb{R}_{+}^{n+1}\right)$ solution is finite. Then we may apply the Sobolev embedding, whence the desired result follows.

The method of proof of Theorem 1.0.7 is robust, in the sense that we may loosen the condition that $u \in Y^{1,2}\left(\mathbb{R}_{+}^{n+1}\right)$, provided that $u$ satisfies that the square function in the righthand side of (1.8) is finite, and that the gradient of $u$ decays to 0 in the sense of distributions for large $t$. More precisely, we have

Theorem 4.7.12 (A more general $\operatorname{Tr}<S$ result). Suppose that $u \in W_{\text {loc }}^{1,2}\left(\mathbb{R}_{+}^{n+1}\right), \mathcal{L} u=0$ in $\mathbb{R}_{+}^{n+1}$ in the weak sense, and $\nabla u(\cdot, t)$ converges to 0 in the sense of distributions as $t \rightarrow$ $\infty$ (we refer to this last condition as the decaying condition). Furthermore, assume that $\left\|t \nabla D_{n+1} u\right\| \mid<\infty$. Then, for every $\tau>0$, the following statements are true.

i) If $\mathcal{L} 1 \neq 0$ in $\mathbb{R}_{+}^{n+1}$, then

$$
\left\|\operatorname{Tr}_{\tau} u\right\|_{L^{\frac{2 n}{n-2}\left(\mathbb{R}^{n}\right)}}+\left\|\operatorname{Tr}_{\tau} \nabla u\right\|_{L^{2}\left(\mathbb{R}^{n}\right)} \lesssim \int_{\tau}^{\infty} \int_{\mathbb{R}^{n}} t\left|D_{n+1}^{2} u\right|^{2} d x d t \lesssim\left\|t D_{n+1}^{2} u\right\| \| .
$$


ii) If $\mathcal{L} 1=0$ in $\mathbb{R}_{+}^{n+1}$, then there exists a constant $c \in \mathbb{C}$ such that $v:=u-c$ (which is again a solution) satisfies estimate (4.13).

The proof of this theorem is omitted as it is very similar to the proof of Theorem 1.0.7 as soon as we have the following technical result.

Proposition 4.7.14 (Solutions with gradient decay). Suppose that $u \in W_{\mathrm{loc}}^{1,2}\left(\mathbb{R}_{+}^{n+1}\right)$ is a solution of $\mathcal{L} u=0$ in $\mathbb{R}_{+}^{n+1}$ and that $\mathcal{L} 1 \neq 0$ on some box $I=Q \times\left(t_{1}, t_{2}\right) \subset \mathbb{R}_{+}^{n+1}$. Further, assume that $\sup _{t>0}\|\nabla u(t)\|_{L^{2}\left(\mathbb{R}^{n}\right)}<\infty$, and that $\lim _{t \rightarrow \infty}\|\nabla u(t)\|_{L^{2}\left(\mathbb{R}^{n}\right)}=0$. Then $u(t) \in Y^{1,2}\left(\mathbb{R}^{n}\right)$ for every $t>0$.

Proof. Step 1. There exists a constant $c \in \mathbb{C}$ such that for all $t>0, u(\cdot, t)-c \in Y^{1,2}\left(\mathbb{R}^{n}\right)$.

To see this, first note that by the Sobolev embedding, there exists a function $f:(0,+\infty) \rightarrow$ $\mathbb{C}$ such that for each $t>0, u(\cdot, t)-f(t) \in Y^{1,2}\left(\mathbb{R}^{n}\right)$. We must show that $f$ is identically a constant. Since (see the proof of Theorem 1.78 in [MZ97]) for each $t>0$ we have that $f(t)=\lim _{R \rightarrow \infty} f_{B(0, R)} u(\cdot, t)$, it can be shown by the Sobolev embedding and considering the difference quotient $\frac{u(\cdot, t+h)-u(\cdot, t)}{h}$ that $f$ is differentiable and that $f^{\prime}(t) \equiv 0$ for all $t>0$. It follows that $f$ is a constant, as desired.

Step 2. For the box $I \subset \mathbb{R}_{+}^{n+1}$ as in the hypotheses, it holds that

$$
\iint_{I}\left|u^{R}\right|^{2^{*}} \rightarrow 0 \quad \text { as } R \rightarrow \infty
$$

where $u^{R}(\cdot, \cdot)=u(\cdot, \cdot+R)$.

This is the crucial step. We set $p=2^{*}$ and $u_{I}^{R}=|I|^{-1} \iint_{I} u^{R}$ for ease of notation. By the 
Poincaré-Sobolev inequality, we see that

$$
\left\|u^{R}-u_{I}^{R}\right\|_{L^{p}(I)} \lesssim\left\|\nabla u^{R}\right\|_{L^{2}(I)} \rightarrow 0, \quad \text { as } R \rightarrow \infty,
$$

where we used the definition of $u^{R}$ and the decaying condition of the gradient. In particular, we have that $u^{R}-u_{I}^{R} \rightarrow 0$ in $Y^{1,2}(I)$, so that $\mathcal{L}\left(u^{R}-u_{I}^{R}\right) \rightarrow 0$ in $I$, which implies that for every $\varphi \in C_{c}^{\infty}(I)$, the limit

$$
-u_{I}^{R} \iint_{I} B_{1} \cdot \overline{\nabla \varphi}=\iint_{I}\left[\left(A \nabla\left(u^{R}-u_{I}^{R}\right)+B_{1}\left(u^{R}-u_{I}^{R}\right)\right) \cdot \overline{\nabla \varphi}+B_{2} \cdot \nabla\left(u^{R}-u_{I}^{R}\right) \bar{\varphi}\right] \rightarrow 0
$$

holds. Since $\mathcal{L} 1 \neq 0$ in $I$, for some $\varphi_{0} \in C_{c}^{\infty}(I)$ we have that $\iint_{I} B_{1} \cdot \overline{\nabla \varphi_{0}} \neq 0$, whence $u_{I}^{R} \rightarrow 0$ as $R \rightarrow \infty$. The claim now follows by using this result in (4.15). Notice that this argument holds just as well for any box $J$ containing $I$, in particular it holds for $\frac{3}{2} I$.

Step 3. For $Q \subset \mathbb{R}^{n}, t \in\left(t_{1}, t_{2}\right)$ as in the hypotheses, we have that

$$
\int_{Q}\left|\operatorname{Tr}_{t} u^{R}\right|^{p} \rightarrow 0, \quad \text { as } R \rightarrow \infty .
$$

This is a consequence of Step 2 and the definition of the trace: For any $\phi \in C_{c}^{\infty}(Q)$ and $\eta \in C_{c}^{\infty}\left(t_{1}, t_{2}\right)$ with $\eta(s)=1$ near $t$, we set $\Phi:=\phi \eta \in C_{c}^{\infty}(I)$ and estimate

$$
\begin{aligned}
& \left|\left(\operatorname{Tr}_{t} u^{R}, \phi\right)\right|=\left|\iint_{\mathbb{R}^{n+1}+}\left(D_{n+1} u^{R} \Phi+u^{R} D_{n+1} \Phi\right)\right| \\
& \leq\left\|D_{n+1} u^{R}\right\|_{Y^{1,2}(I)}\|\Phi\|_{L^{p^{\prime}(I)}}+\left\|u^{R}\right\|_{Y^{1,2}(I)}\left\|D_{n+1} \Phi\right\|_{L^{p^{\prime}(I)}} \\
& \quad \varliminf_{\eta, \eta^{\prime}}\left(\left\|D_{n+1} u^{R}\right\|_{Y^{1,2}(I)}+\left\|u^{R}\right\|_{Y^{1,2}(I)}\right)\|\phi\|_{L^{p^{\prime}(I)}} .
\end{aligned}
$$

The claim now follows by the Caccioppoli inequality; to wit,

$$
\left\|D_{n+1} u^{R}\right\|_{L^{p}(I)}+\left\|\nabla D_{n+1} u^{R}\right\|_{L^{2}(I)} \lesssim_{|I|} \sup _{s>t_{2}+R}\|\nabla u(s)\|_{L^{2}\left(\mathbb{R}^{n}\right)} \rightarrow 0 \quad \text { as } R \rightarrow \infty
$$


using the fact that $p<\frac{2 n}{n-2}$.

We now conclude the proof: By Step 1 , we can place $\operatorname{Tr}_{s}(u-c) \in Y^{1,2}\left(\mathbb{R}^{n}\right)$ for all $s>0$. By Sobolev's inequality and the hypotheses, $\left\|\operatorname{Tr}_{s} u-c\right\|_{Y^{1,2}\left(\mathbb{R}^{n}\right)} \rightarrow 0$ as $s \rightarrow \infty$. On the other hand, by Step 3, we have that $\operatorname{Tr}_{s} u \rightarrow 0$ in $L^{p}(Q)$, so that $c=0$ and the desired result follows.

A quick application of Theorem 4.7.12 to the improvement of (4.4) will be useful for the Dirichlet problem:

Corollary 4.7.16 (Improvement to slice estimate). The estimate (4.4) holds true for $k=1$. In particular, (4.5) holds true for $k=1$ as well.

We can also, very similarly, prove

Theorem 4.7.17 ( $L^{2}$-sup on slices). Suppose that $u \in W_{\text {loc }}^{1,2}\left(\mathbb{R}_{+}^{n+1}\right), \mathcal{L} u=0$ in $\mathbb{R}_{+}^{n+1}$, and that $u$ converges to 0 in the sense of distributions. Furthermore, assume that $\|t \nabla u\| \mid<\infty$. Then, for every $\tau>0$,

$$
\left\|\operatorname{Tr}_{\tau} u\right\|_{L^{2}\left(\mathbb{R}^{n}\right)} \lesssim \int_{\tau}^{\infty} \int_{\mathbb{R}^{n}} t|\nabla u|^{2} d x d t \lesssim\|\| t \nabla u\|\|
$$

where the implicit constant is independent of $\tau$ and $u$.

As an immediate consequence of Theorems 4.7.12 and 4.7.17 we exhibit

Corollary 4.7.18. Let $u \in W_{\text {loc }}^{1,2}\left(\mathbb{R}_{+}^{n+1}\right)$ satisfy $\mathcal{L} u=0$ in $\mathbb{R}_{+}^{n+1}$.

i) If $\left\|t \nabla \partial_{t} u\right\| \mid<\infty$ and $\lim _{t \rightarrow \infty} \nabla u(t)=0$ in the sense of distributions, then either $u$ is $a$ 
$\operatorname{good} \mathcal{N} / \mathcal{R}$ solution (in the case that $\mathcal{L} 1 \neq 0$ in $\mathbb{R}_{+}^{n+1}$ ), or $u-c$ is a good $\mathcal{N} / \mathcal{R}$ solution for some constant c (in the case that $\mathcal{L}=0$ in $\mathbb{R}_{+}^{n+1}$ ).

ii) If $\|t \nabla u\| \mid<\infty$ and $\lim _{t \rightarrow \infty} u(t)=0$ in the sense of distributions, then $u$ is a good $\mathcal{D}$ solution. 


\section{Chapter 5}

\section{$L^{p}$ estimates for square and nontangential maximal functions}

\subsection{Extrapolation of Square Function Estimates}

In this section, we obtain weighted and $L^{p}$ estimates for operators of the form $t^{m} \partial_{t}^{m} \nabla\left(\mathcal{S}_{t}^{\mathcal{L}} \nabla\right)$, for some $m \in \mathfrak{N}$ large. The main ingredients for these estimates are the $L^{r}-L^{q}$ off-diagonal diagonal decay estimates for our operators (see Propositions 3.4.2 and 3.4.10) for $r<2<q$, used implicitly through the extrapolation results of the previous sections.

At this stage we also mention the work [Pri19], where the vertical and conical square functions for objects associated to the heat and Poisson semigroups of $\mathcal{L}$ (without lower order terms) are considered. We remark that our objects are a bit more technically involved to handle, in part due to the mild off-diagonal decay that they enjoy. Nevertheless, the basic idea of extrapolation and control of the vertical square function by a conical square function is the same.

In order to simplify the statement of our results, we make use of the following definition 
which encapsulates the assumptions that $\mathcal{L}$ must verify.

Definition 5.1.1 (Hypothesis A). We say that the operator $\mathcal{L}$ satisfies hypothesis $A$ if the following conditions holds.

1. $\mathcal{L}$ has the form

$$
\mathcal{L}=-\operatorname{div}\left(A \nabla+B_{1}\right)+B_{2} \cdot \nabla
$$

for some $B_{i} \in L^{n}\left(\mathbb{R}^{n} ; \mathbb{C}^{n+1}\right)$ and a complex elliptic, $t$-independent, matrix $A$, that is, for some $\lambda, \Lambda>0$, a.e. $x \in \mathbb{R}^{n}$ and every $\xi, \zeta \in \mathbb{C}^{n+1}$ it holds that

$$
\lambda|\xi|^{2} \leq \operatorname{Re}(A(x) \xi \cdot \bar{\xi}), \quad|A(x) \xi \cdot \bar{\zeta}| \leq \Lambda|\xi||\zeta|
$$

2. With $\tilde{\rho}_{1}>0$ as in Theorem 4.0.1, we have

$$
\max \left\{\left\|B_{1}\right\|_{L^{n}\left(\mathbb{R}^{n}\right)},\left\|B_{2}\right\|_{L^{n}\left(\mathbb{R}^{n}\right)}\right\}<\tilde{\rho}_{1}
$$

We will say a quantity depends on ellipticity if it depends only on $\lambda, \Lambda$ and $\tilde{\rho}_{1}$.

Next, we state the main result of this section.

Theorem 5.1.5 ( $L^{p}$ extrapolation of square function estimates). Suppose that $\mathcal{L}$ satisfies Hypothesis A (see Definition 5.1.1), and let $\Theta_{t, m}$ be any one of the operators

$$
t^{m} \partial_{t}^{m-1} \nabla\left(\mathcal{S}_{t}^{\mathcal{L}} \nabla\right), \quad t^{m} \partial_{t}^{m-1} \nabla\left(\mathcal{S}_{t}^{\mathcal{L}} B_{i}\right), \quad t^{m} \partial_{t}^{m-1} B_{i}\left(\mathcal{S}_{t}^{\mathcal{L}} \nabla\right), \quad i=1,2
$$

Then there exist $\varepsilon_{0}>0, m_{0} \in \mathfrak{N}$, and $\rho_{0}>0$ depending on dimension and ellipticity, such that for every $m \geq m_{0}$ and $p \in\left(2-\varepsilon_{0}, 2+\varepsilon_{0}\right)$, we have the estimate

$$
\left\|\mathbb{S}\left(\Theta_{t, m} f\right)\right\|_{L^{p}\left(\mathbb{R}^{n}\right)}+\left\|\mathbb{V}\left(\Theta_{t, m} f\right)\right\|_{L^{p}\left(\mathbb{R}^{n}\right)} \lesssim_{p}\|f\|_{L^{p}\left(\mathbb{R}^{n}\right)},
$$


provided that

$$
\max \left\{\left\|B_{1}\right\|_{L^{n}\left(\mathbb{R}^{n}\right)},\left\|B_{2}\right\|_{L^{n}\left(\mathbb{R}^{n}\right)}\right\}<\rho_{0} .
$$

Let us give a quick roadmap to the location of the proofs of the various estimates summarized in the previous theorem.

- The conical square function estimate for $t^{m} \partial_{t}^{m-1} \nabla\left(\mathcal{S}_{t}^{\mathcal{L}} \nabla\right)$ is obtained in Theorem 5.1.64, while the vertical square function estimate is given in Theorem 5.1.69.

- The estimates for $t^{m} \partial_{t}^{m-1} \nabla\left(\mathcal{S}_{t}^{\mathcal{L}} B\right)$ are contained in Corollary 5.1.72.

- The results for $t^{m} \partial_{t}^{m-1} B\left(\mathcal{S}_{t}^{\mathcal{L}} \nabla\right)$ are obtained in Lemma 5.1.22. There the results are obtained for the operator with the gradient replaced by a $t$ derivative. A careful inspection of the proof though shows that, as long as we have good estimates for the operator $t^{m} \partial_{t}^{m-1} \nabla\left(\mathcal{S}_{t}^{\mathcal{L}} \nabla\right)$, the same argument applies.

- Estimates for the double layer potential are obtained in Theorem 5.1.77.

\subsubsection{Estimates for $\nabla \mathcal{S}_{t}^{\mathcal{L}}$}

In this subsection we prove the relevant estimates for operators of the form $t^{m} \partial_{t}^{m} \nabla \mathcal{S}_{t}^{\mathcal{L}}$. These will follow immediately from the extrapolation results from the previous section; together with the off-diagonal estimates obtained in Propositions 3.4.2 and 3.4.10.

Remark 5.1.9. We would like to be able to apply Lemmas 2.4.92 and 2.4.109 to $\Theta_{t, m}=$ $t^{m} \partial_{t}^{m}\left(\mathcal{S}_{t}^{\mathcal{L}} \nabla_{\|}\right)$to handle the double layer potential; it is not a simple matter however to obtain 
the necessary quasi-orthogonality condition in those (one reason is that in the regime $s<t$ we need to "add" derivatives to $\Theta_{t, m}$, while taking them away from $Q_{s}$; however adding derivatives to $\Theta_{t, m}$ is tricky since we already have a $\nabla_{\|}$in front. We will have to use the equation to circumvent this issue). We will treat this operator separately, in Section 5.1.2.

Theorem 5.1.10. Suppose $\mathcal{L}$ satisfies Hypothesis $A$ (see Definition 5.1.1). Let $\Theta_{t, m}=$ $t^{m} \partial_{t}^{m} \nabla \mathcal{S}_{t}^{\mathcal{L}}$, then there exist $\varepsilon_{1}>0$ and $m_{1} \in \mathfrak{N}$, depending on dimension and ellipticity, such that if $m \geq m_{1}$ and $2-\varepsilon_{1}<p<\infty$ then

$$
\left\|\mathbb{S}\left(\Theta_{t, m} f\right)\right\|_{L^{p}\left(\mathbb{R}^{n}\right)} \lesssim_{p, m}\|f\|_{L^{p}\left(\mathbb{R}^{n}\right)}
$$

Proof. This follows immediately from Lemma 2.4.92.

The off-diagonal decay is contained in Proposition 3.4.2, while the quasi-orthogonality estimate (2.94) for $R_{t}$ is obtained in the proof of the $L^{2}$ square function bound for $\Theta_{t, m}$ (see Theorem 4.2.1); we sketch it here for completeness. Fix $0<s<t$ and we choose $Q_{s}=s \operatorname{div}_{\|} \widetilde{Q}_{s}$, so that

$$
\Theta_{t, m} Q_{s} h(x)=t^{m} \partial_{t}^{m} \nabla \mathcal{S}_{t}^{\mathcal{L}}\left(s \operatorname{div}_{\|} \widetilde{Q}_{s} h\right)(x)=\frac{s}{t} t^{m+1} \partial_{t}^{m} \nabla\left(\mathcal{S}_{t}^{\mathcal{L}} \nabla_{\|}\right)\left(\widetilde{Q}_{s} h\right),
$$

and we appeal to Lemma 4.7.2, which shows that the operator $t^{m+1} \partial_{t}^{m} \nabla\left(\mathcal{S}_{t}^{\mathcal{L}} \nabla_{\|}\right)$is uniformly bounded in $L^{2}\left(\mathbb{R}^{n}\right)$, moreover so is $\widetilde{Q}_{s}$. This takes care of the contribution of $\Theta_{t, m}$ to $R_{t}$. To handle the other term we further choose $P_{t}=\widetilde{P}_{t} \widetilde{P}_{t}$ for an approximate identity $\widetilde{P}_{t}$ and note that $\left|\Theta_{t, m} 1(x)\right| \widetilde{P}_{t}$ is uniformly bounded in $L^{2}\left(\mathbb{R}^{n}\right)$ while $\widetilde{P}_{t} Q_{s}$ satisfies good quasiorthogonality estimates when $s<t$. Finally, the $L^{2}$ square function bound is obtained in 
Theorem 4.7.2 and Lemma 4.2.2.

We now turn to the appropriate vertical square function bounds.

Theorem 5.1.13 ( $L^{p}$ Bounds for Vertical Square Function). Suppose $\mathcal{L}$ satisfies Hypothesis $A$ (see Definition 5.1.1). Let $\Theta_{t, m}:=t^{m} \partial_{t}^{m} \nabla \mathcal{S}_{t}^{\mathcal{L}}$. There exists $\varepsilon_{2}>0$ and $m_{2} \in \mathbb{N}$, depending on dimension and ellipticity, such that if $p \in\left(2-\varepsilon_{2}, 2+\varepsilon_{2}\right)$ and $m \geq m_{2}$ then

$$
\left\|\mathbb{V}\left(\Theta_{t, m} f\right)\right\|_{L^{p}\left(\mathbb{R}^{n}\right)} \lesssim\|f\|_{L^{p}\left(\mathbb{R}^{n}\right)}
$$

Proof. We use Remark 2.4.116, with $T_{t}=t^{m} \partial_{t}^{m} \nabla \mathcal{S}_{t}^{\mathcal{L}}$ and $S_{t}=t^{m-1} \partial_{t}^{m} \mathcal{S}_{t}^{\mathcal{L}}$. Then the square function bound for $T_{t}$ follow from Theorem 4.7.2 and Lemma 4.2.2, while the square function bound $S_{t}$ follows from Theorem 4.7.2. The comparability of $T$ and $S$, as in Remark 2.4.116, follows from Caccioppoli's inequality (Proposition 2.5.9), and the Reverse Hölder inequality for $S_{t}$ is contained in Proposition 2.5.16, recalling that $S_{t} f(x)$ is a solution of $\mathcal{L} u=0$ in $\mathbb{R}_{+}^{n+1}$ (see for instance Proposition 2.5.35). The necessary off-diagonal decay for both $S$ and $T$ is in Proposition 3.4.2, choosing $m$ large enough. The conclusion now follows from Lemma 2.4.109.

While the extrapolation result in Lemma 2.4.109 is interesting on its own, it turns out that in our context, exploiting Caccioppoli's inequality, it's easy to get a much stronger bound (in fact the moral of the proof seems to be that, if $T_{t}$ enjoys a reverse Hölder inequality on slices, then we can always control the vertical square function by the conical in an interval around $p=2$ ). We state this in the following 
Theorem 5.1.15 (Weighted Bounds for Vertical Square Function). Suppose $\mathcal{L}$ satisfies $\mathrm{Hy}$ pothesis A (see Definition 5.1.1). Let $\Theta_{t, m}:=t^{m} \partial_{t}^{m} \nabla \mathcal{S}_{t}^{\mathcal{L}}$. There exist $m_{2}^{\prime} \in \mathfrak{N}$ and $M_{2} \geq 1$, depending on dimension and ellipticity, such that for every $m \geq m_{2}^{\prime}, M \geq M_{2}$ and every $v \in A_{2}$ with the property $v^{M} \in A_{2}$ it holds

$$
\left\|\mathbb{V}\left(\Theta_{t, m}\right)\right\|_{L^{2}(v)} \approx\left\|\mathbb{S}\left(\tilde{\Theta}_{t, m} f\right)\right\|_{L^{2}(v)} \lesssim_{\left[v^{M}\right]_{A_{2}}}\|f\|_{L^{2}(v)},
$$

where we define

$$
\tilde{\Theta}_{t, m} f(x):=\sqrt{\frac{v(x)}{f_{|x-y|<t} v(y) d y}} \Theta_{t, m} f(x)=\sqrt{\frac{v(x)}{v_{x, t}}} \Theta_{t, m} f(x) .
$$

Proof. We note, from the beginning of the proof of Lemma 2.4.109, the comparability

$$
\left\|\mathbb{V}\left(\Theta_{t, m} f\right)(x)\right\|_{L^{2}(v)} \approx\left\|\mathbb{S}\left(\tilde{\Theta}_{t, m} f\right)\right\|_{L^{2}(v)}
$$

holds for any weight $0<v \in L_{\text {loc }}^{1}\left(\mathbb{R}^{n}\right)$. Therefore it remains to estimate the conical square function associated to $\tilde{\Theta}_{t, m}$. First writing

$$
\left\|\mathbb{S}\left(\tilde{\Theta}_{t, m} f\right)\right\|_{L^{2}(v)}^{2}=\int_{\mathbb{R}^{n}} \int_{0}^{\infty} f_{|x-y|<t} \frac{v(y)}{v_{y, t}}\left|\Theta_{t, m} f(y)\right|^{2} d y \frac{d t}{t} v(x) d x,
$$

and using, by Hölder's and Caccioppoli's Inequalities,

$$
\begin{aligned}
f_{|x-y|<t} \frac{v(y)}{v_{y, t}}\left|\Theta_{t, m} f(y)\right|^{2} d y & \leq\left(f_{|x-y|<t}\left|\frac{v(y)}{v_{y, t}}\right|^{q^{\prime}} d y\right)^{1 / q^{\prime}}\left(f_{|x-y|<t}\left|\Theta_{t, m} f(y)\right|^{2 q} d y\right)^{1 / q} \\
& \lesssim\left[y^{M}\right]_{A_{2}}\left(f_{|x-y|<t}\left|\Theta_{t, m} f(y)\right|^{2 q} d y\right)^{1 / q} \\
& \lesssim\left(f_{t / 2}^{3 t / 2} f_{|x-y|<2 t}\left|\theta_{s, m-1} f(y)\right|^{2 q} d y d s\right)^{1 / q}
\end{aligned}
$$


where we have defined $\theta_{s, m-1}:=t^{m-1} \partial_{t}^{m} \mathcal{S}_{t}^{\mathcal{L}}$, and chosen $q \in(1,2)$ such that our operators satisfy a $2 q$ Caccioppoli Inequality on slices (see Proposition 2.5.39) and then chosen $M>$ $q^{\prime}$. Now since $\theta_{s, m-1}$ satisfies a Reverse Hölder Inequality (see Proposition 2.5.16) we see that

$$
\begin{aligned}
\left(f_{t / 2}^{3 t / 2} f_{|x-y|<2 t}\left|\theta_{s, m-1} f(y)\right|^{2 q} d y d s\right)^{1 / q} & \lesssim f_{t / 4}^{7 t / 4} f_{|x-y|<3 t}\left|\theta_{s, m-1} f(y)\right|^{2} d y d s \\
& \lesssim f_{t / 4}^{7 t / 4} f_{|x-y|<4 s}\left|\theta_{s, m-1} f(y)\right|^{2} d y d s .
\end{aligned}
$$

The desired result follows now from Fubini's theorem and the fact that conical square functions with different cone appertures are comparable (see the comments after Definition 2.4.1).

In what follows we will need square function estimates for the operators $t^{m} \partial_{t}^{m} B \mathcal{S}_{t}^{\mathcal{L}}$, where $B \in L^{n}\left(\mathbb{R}^{n}\right)$ is independent of the transversal variable. The $L^{2}$ case follows from the bounds for $t^{m} \partial_{t}^{m} \nabla \mathcal{S}_{t}^{\mathcal{L}}$ and Sobolev's inequality, the case $p \neq 2$ requires a bit of an argument both in the case of the vertical and conical square functions.

Lemma 5.1.22. Suppose $\mathcal{L}$ satisfies Hypothesis A (see Definition 5.1.1). For a function $B \in L^{n}\left(\mathbb{R}^{n}\right)$, independent of the $t$ variable and $m \in \mathfrak{N}$ consider the operators

$$
\Theta_{t, m}^{B} f(x):=t^{m} \partial_{t}^{m} B \mathcal{S}_{t}^{\mathcal{L}} f(x), \quad \Theta_{t, m} f(x):=t^{m} \partial_{t}^{m} \nabla_{\|} \mathcal{S}_{t}^{\mathcal{L}} f(x) .
$$

For every $1<p<n$ and $f \in C_{c}^{\infty}\left(\mathbb{R}^{n}\right)$ it holds

$$
\left\|\mathbb{V}\left(\Theta_{t, m}^{B} f\right)\right\|_{L^{p}\left(\mathbb{R}^{n}\right)} \lesssim\left\|\mathbb{V}\left(\Theta_{t, m} f\right)\right\|_{L^{p}\left(\mathbb{R}^{n}\right)}
$$


Moreover, if

$$
\theta_{t, m} f:=t^{m} \partial_{t}^{m+1} \mathcal{S}_{t}^{\mathcal{L}} f
$$

then for any $1<p<\infty$

$$
\left\|\mathbb{S}\left(\Theta_{t, m}^{B} f\right)\right\|_{L^{p}\left(\mathbb{R}^{n}\right)} \lesssim\left\|\mathbb{S}\left(\Theta_{t, m} f\right)\right\|_{L^{p}\left(\mathbb{R}^{n}\right)}+\left\|\mathbb{S}\left(\theta_{t, m-1} f\right)\right\|_{L^{p}\left(\mathbb{R}^{n}\right)} \lesssim\left\|\mathbb{S}\left(\theta_{t, m-1} f\right)\right\|_{L^{p}\left(\mathbb{R}^{n}\right)} .
$$

Proof. We begin with the bound for the conical versions. First note that the second inequality follows from the fact that conical square functions, in our setting, always "travel up" by the $L^{2}$ Caccioppoli inequality. To handle the first inequality we note that for fixed $x \in \mathbb{R}^{n}$ and $t>0$ we have, by Hölder's and Poincaré-Sobolev Inequalities,

$$
\begin{aligned}
\left(f_{|x-y|<t}\left|\Theta_{t, m}^{B} f(y)\right|^{2} d y\right)^{1 / 2} & \lesssim \frac{\|B\|_{L^{n}\left(\mathbb{R}^{n}\right)}}{t}\left(f_{|x-y|<t}\left|t^{m} \partial_{t}^{m} \mathcal{S}_{t}^{\mathcal{L}} f(y)\right|^{2^{*}} d y\right)^{1 / 2^{*}} \\
& \lesssim\|B\|_{L^{n}\left(\mathbb{R}^{n}\right)}\left[\left(f_{|x-y|<t}\left|\Theta_{t, m} f(y)\right|^{2} d y\right)^{1 / 2}+\left|\left(\theta_{t, m-1} f\right)_{x, t}\right|\right],
\end{aligned}
$$

where $\left(\theta_{t, m-1} f\right)_{x, t}$ denotes the average of $\theta_{t, m-1} f$ on the $n$-ball $|x-y|<t$. The result now follows from Jensen's inequality and the definition of $\mathbb{S}$.

The vertical square function is a bit more involved. The idea is to write

$$
\Theta_{t, m}^{B} f(x)=B I_{1} R \nabla_{\|} t^{m} \partial_{t}^{m} \mathcal{S}_{t}^{\mathcal{L}} f(x)=B I_{1} R \Theta_{t, m} f(x)
$$

where $I_{1}$ is the fractional integral of order 1 and $R$ is a vector valued Riesz Transform (note that the above makes sense in $L^{2}\left(\mathbb{R}^{n}\right)$ owing to the slices estimates of Theorem 4.7.12 and the mapping properties of $I_{1}$ and $R$ ). Therefore, by Hölder's Inequality

$$
\left\|\mathbb{V}\left(\Theta_{t, m}^{B} f\right)\right\|_{L^{p}\left(\mathbb{R}^{n}\right)} \leq\|B\|_{L^{n}\left(\mathbb{R}^{n}\right)}\left\|\mathbb{V}\left(I_{1} R \Theta_{t, m} f\right)\right\|_{L^{p^{*}}\left(\mathbb{R}^{n}\right)}
$$


where $1 / p^{*}=1 / p-1 / n$ is the Sobolev exponent in dimension $n$.

The desired result follows from the following estimate: Let $F: \mathbb{R}_{+}^{n+1} \rightarrow \mathbb{C}$, then for every $1<p<n$

$$
\left\|\mathbb{V}\left(I_{1} R F\right)\right\|_{L^{p^{*}}\left(\mathbb{R}^{n}\right)} \lesssim\|\mathbb{V}(F)\|_{L^{p}\left(\mathbb{R}^{n}\right)}
$$

To show this first note that for every $1<p<\infty$ we have

$$
\|\mathbb{V}(R F)\|_{L^{p}\left(\mathbb{R}^{n}\right)} \lesssim\|\mathbb{V}(F)\|_{L^{p}\left(\mathbb{R}^{n}\right)}
$$

This is a consequence of the weighted estimate

$$
\int_{\mathbb{R}^{n}} \int_{0}^{\infty}|R F(x, t)|^{2} \frac{d t}{t} v(x) d x \lesssim \int_{\mathbb{R}^{n}} \int_{0}^{\infty}|F(x, t)|^{2} \frac{d t}{t} v(x) d x, \quad v \in A_{2},
$$

and the extrapolation theorem for $A_{p}$ weights (see Theorem 2.4.31). Therefore it's enough to prove the estimate for $I_{1}$ alone. For this we will need an off-diagonal extrapolation result (see [CMP11, Theorem 3.23]) to reduce matters to proving

$$
\left(\int_{\mathbb{R}^{n}} \int_{0}^{\infty}\left|I_{1} F(x, t)\right|^{2} \frac{d t}{t} v^{2}(x) d x\right)^{1 / 2} \lesssim\left(\int_{\mathbb{R}^{n}}\left(\int_{0}^{\infty}\left|I_{1} F(x, t)\right|^{2} \frac{d t}{t}\right)^{2_{*} / 2} v(x)^{2_{*}} d x\right)^{1 / 2_{*}}
$$

where $1 / 2_{*}=1 / 2+1 / n$, and the above holds for every $v \in A_{2_{*}, 2}$ (see Definition 2.4.46). To prove the above inequality we appeal to Theorem 2.4.47 to obtain, for a weight $v$ as above,

$$
\left\|I_{1} g\right\|_{L^{2}\left(v^{2}\right)} \lesssim\|g\|_{L^{2 *}\left(v^{2 *}\right)}
$$


Therefore

$$
\left(\int_{\mathbb{R}^{n}} \int_{0}^{\infty}\left|I_{1} F(x, t)\right|^{2} \frac{d t}{t} v^{2}(x) d x\right)^{1 / 2} \lesssim\left(\int_{0}^{\infty}\left(\int_{\mathbb{R}^{n}}|F(x, t)|^{2_{*}} v(x)^{2_{*}} d x\right)^{2 / 2_{*}} \frac{d t}{t}\right)^{1 / 2}
$$

The desired bound now follows from Minkowski's inequality (in $L^{2 / 2_{*}}$ ).

Remark 5.1.35. More generally, the proof above gives weighted inequalities and, in fact, shows the following: Weighted bounds $T: L^{2}(v) \rightarrow L^{2}(v)$ imply that $\|\mathbb{V}(T F)\|_{L^{2}(v)} \lesssim$ $\|\mathbb{V}(F)\|_{L^{2}(v)}$. The same is true for the conical square function if $T$ in addition has good local estimates, we refer to [AP17].

\subsubsection{Estimates for $\left(\mathcal{S}_{t}^{\mathcal{L}} \nabla\right)$}

We will need the analogue of Lemma 5.1.22 for the dual (in $L^{2}\left(\mathbb{R}^{n}\right)$ ) operator.

Lemma 5.1.36. Suppose $\mathcal{L}$ satisfies Hypothesis A (see Definition 5.1.1). Let $B \in L^{n}\left(\mathbb{R}^{n} ; \mathbb{C}^{n}\right)$ and set $\Theta_{t, m}:=t^{m} \partial_{t}^{m}\left(\mathcal{S}_{t}^{\mathcal{L}} \nabla_{\|}\right)$and $\Theta_{t, m}^{B}:=t^{m} \partial_{t}^{m} \mathcal{S}_{t}^{\mathcal{L}} B$. then for any weight $v \in A_{2}$ we have

$$
\left\|\mathbb{S}\left(\Theta_{t, m}^{B} f\right)\right\|_{L^{2}(v)} \lesssim[v]_{A_{2}}\|B\|_{L^{n}\left(\mathbb{R}^{n}\right)}\left\|\mathbb{S}\left(\Theta_{t, m}\right)\right\|_{L^{2}(v) \rightarrow L^{2}(v)}\|f\|_{L^{2}(v)}
$$

In fact the constant can be shown to be at most a dimensional constant times $[v]_{A_{2}}^{1+\alpha}$ for some $\alpha<1$ (see for instance [Pet08] and [LMPT10])

Proof. We begin by writing, for $f \in C_{c}^{\infty}\left(\mathbb{R}^{n} ; \mathbb{C}^{n}\right), B \cdot f=\operatorname{div}_{\|} I_{1} I_{1} \nabla_{\|}(B \cdot f)=\operatorname{div}_{\|} I_{1} R(B \cdot f)$, where $I_{1}$ is the fractional integral of order 1 . Therefore

$$
\Theta_{t, m}^{B} f=\Theta_{t, m}\left(I_{1} R(B \cdot f)\right),
$$


and so

$$
\left\|\mathbb{S}\left(\Theta_{t, m}^{B} f\right)\right\|_{L^{2}(v)} \lesssim\left\|\mathbb{S}\left(\Theta_{t, m}\right)\right\|_{L^{2}(v) \rightarrow L^{2}(v)}\left\|R I_{1}(B \cdot f)\right\|_{L^{2}(v)}
$$

Since $R: L^{2}(v) \rightarrow L^{2}(v)$ by the Coifman-Fefferman maximal inequality (see Proposition 2.4.30), the result follows from Proposition 2.4.48.

Theorem 5.1.40 (Square Function bounds for $\left(\mathcal{S}_{t}^{\mathcal{L}} \nabla\right)$ ). Suppose $\mathcal{L}$ satisfies Hypothesis $A$ (see Definition 5.1.1). Let $\Theta_{t, m}:=t^{m} \partial_{t}^{m}\left(\mathcal{S}_{t}^{\mathcal{L}} \nabla\right)$ and $\Theta_{t, m}^{\|}:=t^{m} \partial_{t}^{m}\left(\mathcal{S}_{t}^{\mathcal{L}} \nabla_{\|}\right)$and $\delta \in(0,1)$. Then there exist $M>0, m_{3} \in \mathfrak{N}$ (depending only on dimension, ellipticity, and for $m_{3}$ also $\delta$ ) such that for every $m \geq m_{3}$ and if $v^{M} \in A_{2}$ is such that $\left[v^{M}\right]_{A_{2}} \leq C_{\delta}$ (with $C_{\delta}$ as in 7 of Proposition 2.4.30) then

$$
\left\|\mathbb{S}\left(\Theta_{t, m}^{\|} f\right)\right\|_{L^{2}(v)} \lesssim_{C_{\delta}, m}\|f\|_{L^{2}(v)},
$$

provided ${ }^{1}\left\|B_{2 \|}\right\|_{L^{n}\left(\mathbb{R}^{n}\right)} \leq \rho_{3}$, for some $\rho_{3}$ depending only on dimension, ellipticity of $\mathcal{L}_{\|}^{2}$ and $C_{\delta}$.

In particular, for $p \in(2-1 / 2 M, 2+1 / 2 M)$, it holds

$$
\left\|\mathbb{S}\left(\Theta_{t, m} f\right)\right\|_{L^{p}\left(\mathbb{R}^{n}\right)} \lesssim\|f\|_{L^{p}\left(\mathbb{R}^{n}\right)}
$$

Proof. We will follow the same outline as in the proof the corresponding unweighted $L^{2}$ bound for this object (see Lemma 4.5.1, which in turn is based on the method in [HMM15]).

\footnotetext{
${ }^{1}$ This is one of the few places we may require additional smallness in addition to that imposed in $\left[\mathrm{BHL}^{+} \mathrm{a}\right]$, prior to discussing existence and uniqueness for boundary value problems.

${ }^{2}$ More specifically on the constants appearing in Theorem 2.4.80.
} 
Throughout we will fix $Q_{s}$ a CLP family (see Definition 2.4.13) with smooth compactly supported kernel, and set

$$
P_{t}:=-\int_{t}^{\infty} Q_{s}^{2} \frac{d s}{s} .
$$

By the Hodge decomposition and the weighted estimates in Theorem 2.4.80, we see that it's enough to show

$$
\left\|\mathbb{S}\left(\Theta_{t, m} A_{\|} \nabla_{\|} F\right)\right\|_{L^{2}(v)} \lesssim_{\left[\nu^{M}\right]_{A_{2}}}\left\|\nabla_{\|} F\right\|_{L^{2}(v)} .
$$

We start by writing, via the Coifman-Meyer technique [CM86],

$$
\begin{aligned}
\Theta_{t, m}^{\|} A_{\|} \nabla_{\|} F(x) & =\left(\Theta_{t, m}^{\|}\left(A_{\|} \nabla_{\|} F\right)(x)-\left[\Theta_{t, m}^{\|} A_{\|}(x)\right] \cdot P_{t} \nabla_{\|} F(x)\right)+\left[\Theta_{t, m}^{\|} A_{\|}(x)\right] \cdot P_{t} \nabla_{\|} F(x) \\
& =: R_{t}\left(\nabla_{\|} F\right)(x)+\left[\Theta_{t, m}^{\|} A_{\|}(x)\right] \cdot P_{t} \nabla_{\|} F(x) .
\end{aligned}
$$

Since these objects already satisfy good (unweighted) $L^{2}$ estimates, the difficulties now shift to the "error" term $R_{t}$; indeed, using the weighted version of Carleson's lemma (see Lemma 2.4.43) to handle the second term it's enough to show that

$$
\mu\left(R_{Q}\right) \lesssim v(Q), \quad \forall Q \subset \mathbb{R}^{n},
$$

where we have defined the measure $\mu$ as (recall that we're trying to control a conical square function)

$$
d \mu(x, t):=\left(f_{|x-y|<t}\left|\Theta_{t, m}^{\|} A_{\|}(y)\right|^{2} d y\right) \frac{v(x) d x d t}{t} .
$$

To obtain (5.46), owing to the off-diagonal decay of $\Theta_{t, m}^{\|}$in Proposition 3.4.2 and the fact that $d \mu$ is a Carelson measure when $v=1$ by Lemma 4.5.1, we can mimic the argument used in the proof of the extrapolation theorem for conical square functions (Theorem 
2.4.92) involving the John-Nirenberg lemma for local square functions (Lemma 2.4.17); we omit the details.

It now remains to show that $R_{t}$ has good square function bounds. This is the main part of the proof; we will follow almost verbatim the proof of Lemma 4.5.1, replacing weighted bounds where appropriate.

We start by rewriting $R_{t}$ in the following way:

$$
\begin{aligned}
R_{t} & =\Theta_{t, m}^{\|} A_{\|}-\left[\Theta_{t, m}^{\|} A_{\|}\right] P_{t} \\
& =\left(\Theta_{t, m}^{\|} A_{\|} P_{t}-\left[\Theta_{t, m}^{\|} A_{\|}\right] P_{t}\right)+\Theta_{t, m}^{\|} A_{\|}\left(I-P_{t}\right)=: R_{t}^{[1]}+R_{t}^{[2]} .
\end{aligned}
$$

Since $\Theta_{t, m}^{\|}$has good off-diagonal decay by Proposition 3.4.2 (see also [AAA ${ }^{+}$11, Lemma 3.3]), so does $R_{t}$ and satisfies the quasi orthogonality estimate (2.94), thanks to the presence of the $P_{t}$ term. We can then apply the extrapolation lemma for conical square functions (Lemma 2.4.92) to conclude that

$$
\left\|\mathbb{S}\left(R_{t}^{[1]} \nabla_{\|} F\right)\right\|_{L^{2}(v)} \lesssim\left\|\nabla_{\|} F\right\|_{L^{2}(v)}, \quad v \in A_{2 / r}
$$

for some $1<r<2$.

For the term $R_{t}^{[2]}$ we'll use the equation in the form of the identities on slices (see Proposition 2.5.38). For notational convenience, we will denote $Z_{t}:=\left(1-P_{t}\right)$, and also

$$
\vec{b}:=\left(A_{n+1,1}, \ldots, A_{n+1, n}\right), \quad \vec{a}:=\left(A_{1, n+1}, \ldots, A_{n+1, n+1}\right) .
$$


We write

$$
\begin{aligned}
R_{t}^{[2]}\left(\nabla_{\|} F\right) & =\Theta_{t, m}^{\|} A_{\|} Z_{t} \nabla_{\|} F=\Theta_{t, m}^{\|} A_{\|} \nabla_{\|} Z_{t} F \\
& =\partial_{t} \Theta_{t, m} \vec{a} Z_{t} F-\theta_{t, m}\left(\vec{b} \nabla Z_{t} F\right)+\Theta_{t, m} B_{1} Z_{t} F \\
& -t \theta_{t, m-1}\left(B_{2 \|} \nabla_{\|} Z_{t} F\right)+\theta_{t, m}\left(B_{2 \perp} Z_{t} F\right) \\
& =: J_{1}+J_{2}+J_{3}+J_{4}+J_{5},
\end{aligned}
$$

where as usual we have defined

$$
\theta_{t, m}:=t^{m} \partial_{t}^{m+1} \mathcal{S}_{t}^{\mathcal{L}}
$$

To handle $J_{1}$ we note that, owing to the $L^{r}-L^{2}$ off-diagonal decay of $\Theta_{t, m}$ (Proposition 3.4.2) and the average weighted estimates on slices of Proposition 2.4.86, we see that

$$
\begin{aligned}
\left\|\mathbb{S}\left(J_{1}\right)\right\|_{L^{2}(v)}^{2} & =\int_{0}^{\infty} \int_{\mathbb{R}^{n}} f_{|x-y|<t}\left|\Theta_{t, m+1} \vec{a} t^{-1} Z_{t} F(y)\right|^{2} d y v(x) d x \frac{d t}{t} \\
& \lesssim[v]_{A_{2 / r}} \int_{0}^{\infty} \int_{\mathbb{R}^{n}}\left|\vec{a} t^{-1} Z_{t} F(x)\right|^{2} v(x) d x \frac{d t}{t} \\
& \lesssim\left\|\nabla_{\|} F\right\|_{L^{2}(v)},
\end{aligned}
$$

where we have used Proposition 2.4.51 to handle the square function associated to $Z_{t}$ in the last line.

$J_{2}$ we rewrite as follows:

$$
\begin{aligned}
J_{2} & =\theta_{t, m}\left(\vec{b} \cdot \nabla_{\|} F\right)+\left(\theta_{t, m}\left(\vec{b} \cdot P_{t} \nabla_{\|} F\right)-\left[\theta_{t, m} \vec{b}\right] \cdot P_{t} \nabla_{\|} F\right)+\left[\theta_{t, m} \vec{b}\right] \cdot P_{t} \nabla_{\|} F \\
& =: J_{2,1}+J_{2,2}+J_{2,3} .
\end{aligned}
$$

Again appealing to the John-Nirenberg lemma for local square functions (Lemma 2.4.17, see also the proof of Lemma 2.4.92) we see that the contribution of $J_{2,3}$ is under control by 
the weighted version of Carleson's lemma (Lemma 2.4.43). The term $J_{2,2}$ we can handle the same way we did $R_{t}^{[1]}$; we omit the details. Finally, by Theorem 5.1.15, we have good weighted conical square function bounds for $\theta_{t, m}$ and $\vec{b} \in L^{\infty}\left(\mathbb{R}^{n}\right)$, so the contribution of $J_{2,1}$ is also under control.

For $J_{3}$ we appeal to Proposition 3.4.11, which, for $s<t$, gives the bound (for $I_{1} g=F$ )

$$
\left\|\left(f_{|x-y|<t}\left|\Theta_{t, m} B_{1} I_{1} Q_{s}^{2} g(y)\right|^{2} d y\right)^{1 / 2}\right\|_{L^{2}(v)} \lesssim\left(\frac{s}{t}\right)^{\beta}\left\|Q_{s} g\right\|_{L^{2}(v)} .
$$

Therefore

$$
\begin{aligned}
\left\|\mathbb{S}\left(J_{3}\right)\right\|_{L^{2}(v)} & =\int_{0}^{\infty} \int_{\mathbb{R}^{n}} f_{|x-y|<t}\left|\Theta_{t, m} B_{1}\left(1-P_{t}\right) F(y)\right|^{2} d y v(x) d x \frac{d t}{t} \\
& =\int_{0}^{\infty} \int_{\mathbb{R}^{n}} f_{\mid x-y<t}\left|\Theta_{t, m} B_{1} I_{1} \int_{0}^{t} Q_{s}^{2} g(y) \frac{d s}{s}\right|^{2} d y v(x) d x \frac{d t}{t} \\
& \lesssim_{\beta} \int_{0}^{\infty} \int_{\mathbb{R}^{n}} f_{|x-y|<t} \int_{0}^{t}\left(\frac{t}{s}\right)^{\beta / 2}\left|\Theta_{t, m} B_{1} I_{1} Q_{s}^{2} g(y)\right|^{2} \frac{d s}{s} d y v(x) d x \frac{d t}{t} \\
& \lesssim \int_{0}^{\infty} \int_{s}^{\infty}\left(\frac{s}{t}\right)^{\beta / 2}\left\|Q_{s} g\right\|_{L^{2}(v)}^{2} \frac{s t}{t} \frac{d s}{s} \\
& \lesssim\left\|\mathbb{V}\left(Q_{s} g\right)\right\|_{L^{2}(v)} \\
& \lesssim\|g\|_{L^{2}(v)},
\end{aligned}
$$

where we invoked Theorem 2.4.34 in the last line. To conclude we note that $I_{1} g=F$ and so $R g=\nabla_{\|} F$, where $R$ is the vector-valued Riesz transform (with symbol $\xi /|\xi|$ ) and we know $\|R g\|_{L^{2}(v)} \approx\|g\|_{L^{2}(v)}$ for every $v \in A_{2}$; the desired bound follows from this since $A_{2 / r} \subset A_{2}$.

To handle $J_{4}$ we write it as

$$
J_{4}=-t \theta_{t, m-1} B_{2 \|} \nabla_{\|} F+t \theta_{t, m-1} B_{2 \|} \nabla_{\|} P_{t} F=: J_{4,1}+J_{4,2}
$$


For $J_{4,1}$ we appeal to Lemma 5.1.36 to bound

$$
\left\|\mathbb{S}\left(J_{4,1}\right)\right\|_{L^{2}(v)} \lesssim_{[v]_{A_{2}}}\left\|B_{2 \|}\right\|_{L^{n}\left(\mathbb{R}^{n}\right)}\left\|\nabla_{\|} F\right\|_{L^{2}(v)}\left\|\Theta_{t, m}^{\|}\right\|_{L^{2}(v) \rightarrow L^{2}(v)}
$$

Therefore, if $\left\|B_{2}\right\|_{L^{n}\left(\mathbb{R}^{n}\right)}$ is small enough we may hide this term on the left hand side.

We rewrite $J_{4,2}$ in the following way:

$$
\begin{aligned}
J_{4,2} & =\left(t \theta_{t, m-1}\left(B_{2 \|} \cdot P_{t} \nabla_{\|} F\right)-\left[t \theta_{t, m-1} B_{2 \|}\right] \cdot P_{t} \nabla_{\|} F\right)+\left[t \theta_{t, m-1} B_{2 \|}\right] \cdot P_{t} \nabla_{\|} F \\
& =: R_{t}^{[3]}+\left[t \theta_{t, m-1} B_{2 \|}\right] \cdot P_{t} \nabla_{\|} F .
\end{aligned}
$$

$R_{t}^{[3]}$ may be handled the same way as $R_{t}^{[1]}$, using Proposition 3.4.10 to obtain the right $L^{r}-L^{2}$

off-diagonal estimates. It remains to show, by an application of the weighted version of Carelson's lemma, a $v$-Carleson measure estimate for

$$
d \mu(x, t):=\left(f_{|x-y|<t}\left|t^{m} \partial_{t}^{m} \mathcal{S}_{t}^{\mathcal{L}} B_{2 \|}(y)\right|^{2} d y\right) v(x) \frac{d x d t}{t} .
$$

This follows, once again, by an application of the John-Nirenberg lemma for local square functions (Lemma 2.4.17, see also the proof of Lemma 2.4.92) ${ }^{3}$.

Finally, to handle $J_{5}$, we appeal again to the $L^{r}-L^{2}$ off-diagonal estimates of $t^{m+1} \partial_{t}^{m+1} \mathcal{S}_{t}^{\mathcal{L}} B_{2 \perp}$ (Proposition 3.4.10) and Proposition 2.4.86 (which give that $t^{m+1} \partial_{t}^{m+1} \mathcal{S}_{t}^{\mathcal{L}} B_{2 \perp}$ satisfies good averaged weighted bounds on slices) to conclude

$$
\left\|\mathbb{S}\left(J_{5}\right)\right\|_{L^{2}(v)} \lesssim \int_{0}^{\infty} \int_{\mathbb{R}^{n}}\left|\frac{Z_{t}}{t} F\right|^{2} v(x) \frac{d t}{t} .
$$

\footnotetext{
${ }^{3}$ Notice that, since we already have good unweighted $L^{2}$ square function estimates for $\Theta_{t, m}$, the JohnNirenberg lemma gives us that this object is under control; as opposed to the unweighted case, where we were forced to hide this term.
} 
We conclude by the square function estimates of Proposition 2.4.51, the same as we did for $J_{1}$.

Combining all the above, we see that we've shown:

$$
\left\|\mathbb{S}\left(\Theta_{t, m}^{\|}\right)\right\|_{L^{2}(v) \rightarrow L^{2}(v)} \lesssim_{\left[v^{M}\right]_{A_{2}}} 1+\left\|B_{2 \|}\right\|_{L^{n}\left(\mathbb{R}^{n}\right)}\left\|\mathbb{S}\left(\Theta_{t, m}^{\|}\right)\right\|_{L^{2}(v) \rightarrow L^{2}(v)}
$$

this gives the desired bound if the left hand side is finite and $\|B\|_{L^{n}\left(\mathbb{R}^{n}\right)}$ is small enough. To achieve the former we may work with the truncated square functions given by

$$
\mathbb{S}_{\eta}\left(\Theta_{t, m}^{\|} f\right)(x):=\int_{\eta}^{1 / \eta} f_{|x-y|<t}\left|\Theta_{t, m}^{\|} f(y)\right|^{2} d y \frac{d t}{t}
$$

which satisfy $\left\|\mathbb{S}_{\eta}\left(\Theta_{t, m}^{\|}\right)\right\|_{L^{2}(v) \rightarrow L^{2}(v)}<\infty$, owing to the estimates on slices of Proposition 2.4.86. Fix now $C_{\delta}$ as in the assumptions, i.e. $\left[v^{M}\right] \leq C_{\delta}$, then our estimates read (see also Theorem 2.4.80)

$$
\left\|\mathbb{S}\left(\Theta_{t, m}^{\|}\right)\right\|_{L^{2}(v) \rightarrow L^{2}(v)} \lesssim_{C_{0}} 1+\left\|B_{2 \|}\right\|_{L^{n}\left(\mathbb{R}^{n}\right)}\left\|\mathbb{S}\left(\Theta_{t, m}^{\|}\right)\right\|_{L^{2}(v) \rightarrow L^{2}(v)} .
$$

Thus, choosing $\left\|B_{2 \|}\right\|_{L^{n}\left(\mathbb{R}^{n}\right)}<\rho=\rho\left(C_{\delta}\right)$ we can hide the second term on the right hand side and conclude the result.

The $L^{p}$ bounds are a consequence of this and Corollary 2.4.42 if we choose $\delta=1 / 2$, where recall $C_{1 / 2}$ is defined as in 7 of Proposition 2.4.30.

Theorem 5.1.64. Suppose $\mathcal{L}$ satisfies Hypothesis $A$ (see Definition 5.1.1). Let $\Theta_{t, m}:=$ $t^{m} \partial_{t}^{m-1} \nabla\left(\mathcal{S}_{t}^{\mathcal{L}} \nabla\right)$ and $\delta \in(0,1)$. There exist $m_{4} \in \mathfrak{N}$ and $M>0$ (depending only on dimension and ellipticity, and for $m_{4}$ also on $\delta$ ) such that if $m \geq m_{4}$ and $v \in A_{2}$ is such that 
$\left[v^{M}\right]_{A_{2}} \leq C_{\delta}$, then

$$
\left\|\mathbb{S}\left(\Theta_{t, m} f\right)\right\|_{L^{2}(v)} \lesssim\left[v^{M}\right]_{A_{2}}\|f\|_{L^{2}(v)},
$$

provided $\left\|B_{2 \|}\right\|_{L^{n}\left(\mathbb{R}^{n}\right)}<\rho_{4}$, for some $\rho_{4}$ depending on dimension, ellipticity of $\mathcal{L}_{\|}$, and $C_{\delta}$ only.

In particular there exists $\varepsilon_{4}>0$ (depending on dimension and ellipticity) such that if $p \in\left(2-\varepsilon_{4}, 2+\varepsilon_{4}\right)$ and $m \geq m_{4}$ then

$$
\left\|\mathbb{S}\left(\Theta_{t, m} f\right)\right\|_{L^{p}\left(\mathbb{R}^{n}\right)} \lesssim_{p}\|f\|_{L^{p}\left(\mathbb{R}^{n}\right)} .
$$

Proof. Notice that it's enough to consider $\nabla_{\|}$instead of $\nabla$ in both instances; otherwise we're in the situation of Theorem 5.1.10 or Theorem 5.1.40. Therefore, without loss of generality, $\Theta_{t, m}=t^{m} \partial_{t}^{m-1} \nabla_{\|}\left(\mathcal{S}_{t}^{\mathcal{L}} \nabla_{\|}\right)$. In this case, for $f \in C_{c}^{\infty}\left(\mathbb{R}^{n} ; \mathbb{C}^{n}\right)$ we can write

$$
\Theta_{t, m} f(x)=t^{m} \partial_{t}^{m-1} \nabla_{\|} \mathcal{S}_{t}^{\mathcal{L}}\left(\operatorname{div}_{\|} f\right)(x)=: t^{m} \partial_{t}^{m-1} \nabla_{\|} \mathcal{S}_{t}^{\mathcal{L}} g(x)
$$

By the Caccioppoli inequality on slices (Proposition 2.5.39) we see that, for fixed $x \in \mathbb{R}^{n}$ and $t>0$,

$$
\begin{aligned}
\int_{0}^{\infty} f_{|x-y|<t}\left|t^{m} \partial_{t}^{m-1} \nabla_{\|} \mathcal{S}_{t}^{\mathcal{L}} f(y)\right|^{2} d y \frac{d t}{t} & \lesssim \int_{0}^{\infty} f_{t / 4}^{5 t / 4} f_{|x-y|<2 t}\left|t^{m-1} \partial_{t}^{m-1} \mathcal{S}_{t}^{\mathcal{L}} g(y)\right|^{2} d y \frac{d t}{t} \\
& =\int_{0}^{\infty} f_{t / 4}^{5 t / 4} f_{|x-y|<2 t}\left|s^{m-1} \partial_{s}^{m-1}\left(\mathcal{S}_{s}^{\mathcal{L}} \nabla_{\|}\right) f(y)\right|^{2} d y d s \frac{d t}{t} \\
& \lesssim \int_{0}^{\infty} f_{|x-y|<2 t}\left|s^{m-1} \partial_{s}^{m-1}\left(\mathcal{S}_{s}^{\mathcal{L}} \nabla_{\|}\right) f(y)\right|^{2} d y \frac{d s}{s}
\end{aligned}
$$


The result now follows from Theorem 5.1.40 and the change of angle formula for square functions (see the comments after Definition 2.4.1).

The following is the weighted version of Theorem 5.1.5 for the vertical square function. Theorem 5.1.69. Suppose $\mathcal{L}$ satisfies Hypothesis $A$ (see Definition 5.1.1). Let $\Theta_{t, m}:=$ $t^{m} \partial_{t}^{m-1} \nabla\left(\mathcal{S}_{t}^{\mathcal{L}} \nabla\right)$ and $\delta \in(0,1)$. There exists $m_{5} \in \mathfrak{N}$ and $M>0$ (depending only on dimension and ellipticity, and in the case of $m_{5}$ also on $\delta$ ) such that if $m \geq m_{5}$ and $v \in A_{2}$ satisfies $\left[v^{M}\right]_{A_{2}} \leq C_{\delta}$, then

$$
\left\|\mathbb{V}\left(\Theta_{t, m} f\right)\right\|_{L^{2}(v)} \lesssim C_{0}\|f\|_{L^{2}(v)},
$$

provided $\left\|B_{2 \|}\right\|<\rho_{5}$, for some $\rho_{5}>0$ depending only on dimension, ellipticity of $\mathcal{L}_{\|}$, and $C_{\delta}$.

In particular there exists $\varepsilon_{5}>0$ (depending on dimension and ellipticity) such that for $p \in\left(2-\varepsilon_{5}, 2+\varepsilon_{5}\right)$

$$
\left\|\mathbb{V}\left(\Theta_{t, m} f\right)\right\|_{L^{p}\left(\mathbb{R}^{n}\right)} \lesssim\|f\|_{L^{p}\left(\mathbb{R}^{n}\right)} .
$$

Proof. By Theorem 5.1.15 and $t$-independence, it's enough to consider $\Theta_{t, m}:=t^{m} \partial_{t}^{m-1} \nabla\left(\mathcal{S}_{t}^{\mathcal{L}} \nabla_{\|}\right)$. Now the idea is to repeat the proof of the weighted bound for $\mathbb{V}\left(t^{m} \partial_{t}^{m} \nabla \mathcal{S}_{t}^{\mathcal{L}} g\right)$, with $g=$ $\operatorname{div}_{\|} f$, in Theorem 5.1.15, using now instead Theorem 5.1.64; we omit the details.

The following Corollary will be useful when dealing with square functions involving the double layer potential. 
Corollary 5.1.72. Suppose $\mathcal{L}$ satisfies Hypothesis $A$ (see Definition 5.1.1). Let $\varepsilon_{0}>0$ and $M_{0}>0$ be as in Theorem 5.1.5. Let $\Theta_{t, m}^{B}:=t^{m} \partial_{t}^{m-1} \nabla\left(\mathcal{S}_{t}^{\mathcal{L}} B\right)$ for some $B \in L^{n}\left(\mathbb{R}^{n} ; \mathbb{C}^{n+1}\right)$. Then for every $f \in C_{c}^{\infty}\left(\mathbb{R}^{n} ; \mathbb{C}^{n+1}\right)$

$$
\left\|\mathbb{S}\left(\Theta_{t, m}^{B} f\right)\right\|_{L^{2}(v)},\left\|\mathbb{V}\left(\Theta_{t, m}^{B} f\right)\right\|_{L^{2}(v)} \lesssim\left[v^{m}\right]_{A_{2}}\|f\|_{L^{2}(v)}
$$

Proof. Write $B_{\|} \cdot f_{\|}=\operatorname{div}_{\|} \nabla_{\|} I_{1} I_{1}\left(B_{\|} \cdot f_{\|}\right)=\operatorname{div}_{\|} R I_{1}\left(B_{\|} \cdot f_{\|}\right)=\operatorname{div}_{\|} g_{\|}$. Notice that, by the proof of Proposition 2.4.48, we know

$$
\left\|g_{\|}\right\|_{L^{p}\left(\mathbb{R}^{n}\right)} \lesssim\left\|f_{\|}\right\|_{L^{p}\left(\mathbb{R}^{n}\right)}
$$

for every $1<p<\infty$. On the other hand we can also write $B_{\perp} f_{\perp}=\operatorname{div}_{\|} R I_{1}\left(B_{\perp} f_{\perp}\right)=\operatorname{div}_{\|} g_{\perp}$ where

$$
\left\|g_{\perp}\right\|_{L^{p}\left(\mathbb{R}^{n}\right)} \lesssim\left\|f_{\perp}\right\|_{L^{p}\left(\mathbb{R}^{n}\right)}
$$

Combining these two observations the result follows from either Theorem 5.1.64 for the conical version or Theorem 5.1.69 for the vertical.

Recall that for $\vec{N}=-e_{n+1}$ the exterior normal to $\partial \mathbb{R}_{+}^{n+1}$ we have the following representation formula for the double layer:

$$
\mathcal{D}_{t}^{\mathcal{L}} f=\left(\mathcal{S}_{t}^{\mathcal{L}} \nabla\right) \cdot A \vec{N} f+\left(\mathcal{S}_{t}^{\mathcal{L}} \overline{B_{2}}\right) \cdot \vec{N} f
$$

for $f \in C_{c}^{\infty}\left(\mathbb{R}^{n}\right)$. As an immediate consequence of this and the previous results we have 
Theorem 5.1.77 (Square Function Bounds for $\mathcal{D}_{t}^{\mathcal{L}}$. Part 1). Suppose $\mathcal{L}$ satisfies Hypothesis A (see Definition 5.1.1). Let $\varepsilon_{0}>0, m_{0} \in \mathfrak{N}$, and $\rho_{0}>0$ as in Theorem 5.1.5. Suppose $\Theta_{t, m}=t^{m} \partial_{t}^{m} \nabla \mathcal{D}_{t}^{\mathcal{L}}$. Then for $m \geq m_{0}, p \in\left(2-\varepsilon_{0}, 2+\varepsilon_{0}\right)$ and $f \in C_{c}^{\infty}\left(\mathbb{R}^{n}\right)$

$$
\left\|\mathbb{S}\left(\Theta_{t, m} f\right)\right\|_{L^{p}\left(\mathbb{R}^{n}\right)},\left\|\mathbb{V}\left(\Theta_{t, m} f\right)\right\|_{L^{p}\left(\mathbb{R}^{n}\right)} \lesssim\|f\|_{L^{p}\left(\mathbb{R}^{n}\right)}
$$

\subsection{Control on Slices}

Theorem 5.2.1 (Estimates on Slices for $\nabla \mathcal{S}_{t}^{\mathcal{L}}$ ). Suppose $\mathcal{L}$ satisfies the hypotheses of Theorem 5.1.5. If $\varepsilon_{0}>0$ is as in Theorem 5.1.5 and $p \in\left(2-\varepsilon_{0}, 2+\varepsilon_{0}\right)$, then for $f \in C_{c}^{\infty}\left(\mathbb{R}^{n}\right)$

$$
\left\|\mathcal{S}_{t}^{\mathcal{L}} f\right\|_{L^{p^{*}}\left(\mathbb{R}^{n}\right)}+\left\|\nabla \mathcal{S}_{t}^{\mathcal{L}} f\right\|_{L^{p}\left(\mathbb{R}^{n}\right)} \lesssim_{m, p}\|f\|_{L^{p}\left(\mathbb{R}^{n}\right)}, \quad t>0
$$

Proof. The proof of this result is essentially contained in Theorem 1.0.7 (see the previous chapter), using the $L^{p}$ square function estimates from the previous section instead. We omit the details.

As a consequence of this result we obtain the necessary boundedness on slices of our operators.

Corollary 5.2.3. Suppose $\mathcal{L}$ satisfies the hypotheses of Theorem 5.1.5. If $\varepsilon_{0}$ is as in Theorem 5.1 .5$, then for every $m \geq 1, p \in\left(2-\varepsilon_{0}, 2+\varepsilon_{0}\right)$ and $f \in C_{c}^{\infty}\left(\mathbb{R}^{n} ; \mathbb{C}^{n+1}\right)$

$$
\left\|t^{m} \partial_{t}^{m-1}\left(\mathcal{S}_{t}^{\mathcal{L}} \nabla\right) \cdot f\right\|_{L^{p^{*}}\left(\mathbb{R}^{n}\right)}+\left\|t^{m} \partial_{t}^{m-1} \nabla\left(\mathcal{S}_{t}^{\mathcal{L}} \nabla\right) \cdot f\right\|_{L^{p}\left(\mathbb{R}^{n}\right)} \lesssim_{m, p}\|f\|_{L^{p}\left(\mathbb{R}^{n}\right)}, \quad t>0
$$

If either of the gradients is replaced by $\partial_{t}$, then the above remains true for $m=0$. 
Proof. It's enough to treat $\nabla\left(\mathcal{S}_{t}^{\mathcal{L}} \nabla_{\|}\right)$by $t$-independence. The idea is to write $\nabla\left(\mathcal{S}_{t}^{\mathcal{L}} \nabla_{\|} f\right)=$ $\nabla \mathcal{S}_{t}^{\mathcal{L}} \operatorname{div}_{\|} f$ and apply the $L^{p}$ Caccioppoli's inequality on slices (Proposition 2.5.39) once, and then use induction (recall that the off-diagonal decay already gives uniform $L^{p}$ bounds for $m$ large enough). Details can be found in [AAA ${ }^{+} 11$, Lemma 2.11].

Similarly we have the result for $B_{i}$ in place of the gradient, the proof is a simple application of Sobolev's inequality for $m=0$, Caccioppoli's inequality on slices, and duality.

Corollary 5.2.5. Suppose $\mathcal{L}$ satisfies the hypotheses of Theorem 5.1.5. If $\varepsilon_{0}>0$ is as in Theorem 5.1.5, $m \geq 0$, and $p \in\left(2-\varepsilon_{0}, 2+\varepsilon_{0}\right)$, then for $f \in C_{c}^{\infty}\left(\mathbb{R}^{n}\right)$ and $g \in C_{c}^{\infty}\left(\mathbb{R}^{n} ; \mathbb{C}^{n+1}\right)$

$$
\left\|t^{m} \partial_{t}^{m} B_{i} \mathcal{S}_{t}^{\mathcal{L}} f\right\|_{L^{p}\left(\mathbb{R}^{n}\right)} \lesssim\|f\|_{L^{p}\left(\mathbb{R}^{n}\right)}
$$

and

$$
\left\|t^{m} \partial_{t}^{m}\left(\mathcal{S}_{t}^{\mathcal{L}} B_{i}\right) \cdot g\right\|_{L^{p}\left(\mathbb{R}^{n}\right)} \lesssim\|g\|_{L^{p}\left(\mathbb{R}^{n}\right)} .
$$

The following result is really a Corollary of the above estimates; we state it on its own for future reference.

Theorem 5.2.8 (Estimates on Slices for $\mathcal{D}^{\mathcal{L}}$ ). Suppose $\mathcal{L}$ satisfies the hypotheses of Theorem 5.1.5. If $\varepsilon_{0}$ is as in Theorem 5.1.5, then for $m \geq 0$ and $p \in\left(2-\varepsilon_{0}, 2+\varepsilon_{0}\right)$ and $f \in C_{c}^{\infty}\left(\mathbb{R}^{n}\right)$

$$
\left\|t^{m} \partial_{t}^{m} \mathcal{D}_{t}^{\mathcal{L}} f\right\|_{L^{p}\left(\mathbb{R}^{n}\right)} \lesssim\|f\|_{L^{p}\left(\mathbb{R}^{n}\right)}, \quad t>0
$$

Proof. Again by Caccioppoli's inequality on slices it's enough to treat the case $m=0$. 
The result is an immediate consequence of the following representation formula for the double layer given earlier: For $f \in C_{c}^{\infty}\left(\mathbb{R}^{n}\right)$ we have

$$
\mathcal{D}_{t}^{\mathcal{L}} f=\left(\mathcal{S}_{t}^{\mathcal{L}} \nabla\right) \cdot(A \vec{N} f)+\left(\mathcal{S}_{t}^{\mathcal{L}} \bar{B}_{2}\right) \cdot \vec{N} f
$$

where $\vec{N}=-e_{n+1}$ is the exterior normal to $\partial \mathbb{R}_{+}^{n+1}$.

The following result will be used in the proof of the nontangential maximal function estimate.

Lemma 5.2.11. Suppose that $u \in W_{\text {loc }}^{1,2}\left(\mathbb{R}_{+}^{n+1}\right)$ is a solution of $\mathcal{L} u=0$ in $\mathbb{R}_{+}^{n+1}$, given by either $u=\mathcal{S}_{t}^{\mathcal{L}} f$ or $u=\mathcal{D}^{\mathcal{L}} g$ for some $f, g \in C_{c}^{\infty}$. For any positive Lipschitz function $\varphi: \mathbb{R}^{n} \rightarrow \mathbb{R}$ with $\|\nabla \varphi\|_{L^{\infty}\left(\mathbb{R}^{n}\right)} \leq 1$, if we define the function

$$
u_{\varphi}(x, t):=u(x, t+\varphi(x)), \quad(x, t) \in \mathbb{R}_{+}^{n+1},
$$

then we have

$$
\sup _{t>0}\left\|u_{\varphi}(\cdot, t)\right\|_{L^{p}\left(\mathbb{R}^{n}\right)} \lesssim\|\mathbb{V}(t \nabla u)\|_{L^{p}\left(\mathbb{R}^{n}\right)}
$$

for $p \in\left(2-\varepsilon_{0}, 2+\varepsilon_{0}\right)$ as in Theorem 5.4 .1 (one has to keep in mind here that the vertical square function "travels down" as long as we have good estimates on slices).

Proof. We sketch the proof: The function $u_{\varphi}$ solves an elliptic equation $\mathcal{L}_{\varphi} u_{\varphi}=0$ of the same type as $\mathcal{L}$, with the corresponding norms of the operator $\mathcal{L}_{\varphi}$ controlled in terms of those for $\mathcal{L}$ and $\|\nabla \varphi\|_{L^{\infty}\left(\mathbb{R}^{n}\right)}$. Next, we apply Theorem 4.7 .12 (or, more precisely, its proof of the sup on slices estimate). 


\subsection{Nontangential Maximal Function Estimates}

Proposition 5.3.1. Let $u \in W_{\mathrm{loc}}^{1,2}\left(\mathbb{R}_{+}^{n+1}\right)$ be a solution of $\mathcal{L} u=0$ in $\mathbb{R}_{+}^{n+1}$. For all $q \geq 1$ and $\varepsilon>0$ it holds ${ }^{4}$

$$
\tilde{\mathcal{N}}_{2}^{(\varepsilon)}(\nabla u) \lesssim \mathcal{M}\left(\tilde{\mathcal{N}}_{q}\left(\partial_{t} u\right)\right)+\mathcal{M}\left(\nabla_{\|} u(\cdot, \varepsilon)\right)+\mathcal{M}(u(\cdot, \varepsilon)) \cdot \mathcal{M}_{2}\left(B_{1}\right)
$$

with implicit constants depending on dimension, ellipticity and $q$. Here we recall that we have defined, for any $r>0$ and $g \in L_{\mathrm{loc}}^{r}\left(\mathbb{R}^{n}\right)$,

$$
\mathcal{M}_{r}(g):=\mathcal{M}\left(|g|^{r}\right)^{1 / r}
$$

In particular, if $1<p<n$ and $u(\cdot, \varepsilon) \in Y^{1, p}\left(\mathbb{R}^{n}\right)$ for every $\varepsilon>0$,

$$
\left\|\tilde{\mathcal{N}}_{2}(\nabla u)\right\|_{L^{p}\left(\mathbb{R}^{n}\right)} \lesssim\left\|\tilde{\mathcal{N}}_{1}\left(\partial_{t} u\right)\right\|_{L^{p}\left(\mathbb{R}^{n}\right)}+\sup _{\varepsilon>0}\left\|\nabla_{\|} u(\cdot, \varepsilon)\right\|_{L^{p}\left(\mathbb{R}^{n}\right)}
$$

whenever the right hand side is finite.

\section{Moreover for $u$ we have the estimate}

$$
\left\|\tilde{\mathcal{N}}_{2}(u)\right\|_{L^{p}\left(\mathbb{R}^{n}\right)} \lesssim\left\|\tilde{\mathcal{N}}_{1}(u)\right\|_{L^{p}\left(\mathbb{R}^{n}\right)},
$$

for any $p>1$.

Proof. The statement for $u$ follows from the reverse Hölder inequality for solutions (Proposition 2.5.16) and the comparability of $\tilde{\mathcal{N}}$ defined with different parameters for $C_{x, t}$.

\footnotetext{
${ }^{4}$ See Definition 2.4.7 for the truncated maximal function $\tilde{\mathcal{N}}_{2}^{(\varepsilon)}$.
} 
Fix $\varepsilon>0$ and set $u_{\varepsilon}(x)=u(x, \varepsilon)$. Fix $z \in \mathbb{R}^{n}$ and $(x, t) \in \Gamma(z)$. Recall that we defined the cylinders

$$
C_{x, t}:=\left\{(y, s) \in \mathbb{R}_{+}^{n+1}:|x-y|<t / 8,|t-s|<t / 8\right\}
$$

and we set, for $F \in L_{\text {loc }}^{2}\left(\mathbb{R}_{+}^{n+1}\right)$,

$$
\tilde{\mathcal{N}}_{2}(F)(z):=\sup _{(x, t) \in \Gamma(z)} a_{2}(u)(x, t):=\sup _{(x, t) \in \Gamma(z)}\left(\int_{C_{x, t}}|F(w, \tau)|^{2} d w d \tau\right)^{1 / 2} .
$$

We denote by $C_{x, t}^{*}$ the concentric dilate $2 C_{x, t}$ and $a_{1}^{*}$ the corresponding $L^{1}$ average built with $C_{x, t}^{*}$ instead of $C_{x, t}$. By the reverse Hölder inequality in Proposition 2.5.16, for any $c \in \mathbb{C}$

$$
a_{2}(\nabla u)(x, t) \lesssim \frac{1}{t} a_{1}^{*}(u-c)(x, t)+|c| a_{2}^{*}\left(B_{1}\right)(x, t)=: I+I I .
$$

If we choose

$$
c:=\int_{\Delta_{x, t}} u_{\varepsilon} d z
$$

then we immediately see, exploiting the $t$-independence of $B_{1}$,

$$
I I \leq \mathcal{M}\left(u_{\varepsilon}\right)(z) \cdot a_{2}^{*}\left(B_{1}\right)(x, t) \lesssim \mathcal{M}\left(u_{\varepsilon}\right)(z) \cdot \mathcal{M}_{2}\left(B_{1}\right)(z) .
$$

It remains to estimate $I$. For this purpose we compute

$$
a_{1}^{*}(u-c)(x, t) \leq a_{1}^{*}\left(u-u_{\varepsilon}\right)(x, t)+a_{1}^{*}\left(u_{\varepsilon}-c\right)(x, t) .
$$

From the definition of $c$ we see that

$$
\begin{aligned}
a_{1}^{*}\left(u_{\varepsilon}-c\right)(x, t) & =\int_{C_{x, t}^{*}}\left|u_{\varepsilon}(y)-f_{\Delta_{x, t}} u_{\varepsilon}(w) d w\right| d y d s \\
& \lesssim t f_{\Delta_{x, t}}\left|\nabla_{\|} u_{\varepsilon}(y)\right| d y \\
& \leq t \mathcal{M}\left(\nabla_{\|} u_{\varepsilon}\right)(z),
\end{aligned}
$$


where we used the Poincaré inequality in $L^{1}$ for the second line.

On the other hand, by the fundamental theorem of calculus, and introducing an average in space we have

$$
\begin{aligned}
a_{1}^{*}\left(u-u_{\varepsilon}\right)(x, t) & =\int_{\mathcal{C}_{x, t}^{*}}|u(y, s)-u(y, \varepsilon)| d y d s \\
& \leq \iint_{\mathcal{C}_{x, t}^{*}} \int_{\varepsilon}^{s}\left|\partial_{\tau} u(y, \tau)\right| d \tau d y d s \\
& \leq \int_{\mathcal{C}_{x, t}^{*}} \int_{0}^{s}\left|\partial_{\tau} u(y, \tau)\right| d \tau d y d s \\
& =f_{|t-s|<t / 8} \int_{0}^{s} f_{|x-y|<t / 8} f_{|y-w|<\tau / 8}\left|\partial_{\tau} u(y, \tau)\right| d w d y d \tau d s \\
& \lesssim f_{|t-s|<t / 8} f_{|x-w|<t / 2} \int_{0}^{s} f_{|w-y|<\tau / 8}\left|\partial_{\tau} u(y, \tau)\right| d y d \tau d w d s .
\end{aligned}
$$

Now we notice, for fixed $s>0$,

$$
\begin{aligned}
\int_{0}^{s} f_{|w-y|<\tau / 8}\left|\partial_{\tau} u(y, \tau)\right| d y d \tau & \lesssim \sum_{k \geq 0} 2^{-k} s f_{2^{-k-1} s}^{2^{-k} s} f_{|w-y|<2^{-k-3} s}\left|\partial_{\tau} u(y, \tau)\right| d y d \tau \\
& \lesssim \sum_{k \geq 0} 2^{-k} s a_{1}^{*}\left(\partial_{\tau} u\right)\left(y, 2^{-k-1 / 2} s\right) \\
& \lesssim \sum_{k \geq 0} 2^{-k}{ }_{s} \tilde{\mathcal{N}}_{1}\left(\partial_{\tau} u\right)(y) \\
& \lesssim s \tilde{\mathcal{N}}_{1}\left(\partial_{\tau} u\right)(y) .
\end{aligned}
$$

Plugging this estimate into the inequality preceding it we arrive at

$$
a_{1}^{*}\left(u-u_{\varepsilon}\right)(x, t) \lesssim f_{|t-s|<t / 8} f_{|x-w|<t / 2} s \tilde{\mathcal{N}}_{1}\left(\partial_{\tau} u\right)(y) d y d s \lesssim t \mathcal{M}\left(\tilde{\mathcal{N}}_{1}\left(\partial_{\tau} u\right)\right)(z),
$$

where we used the fact that $t \approx s$ for the last inequality. We conclude

$$
I \lesssim \mathcal{M}\left(\tilde{\mathcal{N}}_{1}\left(\partial_{\tau} u\right)\right)(z),
$$

which combined with the estimate for $I I$ yields the desired result in the case $q=1$. 
Lemma 5.3.17. Suppose $\mathcal{L}$ satisfies Hypothesis $A$ (see Definition 5.1.1). Let $u(x, t)=$ $\partial_{t} \mathcal{S}_{t}^{\mathcal{L}} f(x)$ for some $f \in C_{c}^{\infty}\left(\mathbb{R}^{n}\right)$ or $u(x, t)=\mathcal{D}_{t}^{\mathcal{L}} g$ for some $g \in C_{c}^{\infty}\left(\mathbb{R}^{n}\right)$. There exists $m_{6} \in \mathfrak{N}$ and $\varepsilon_{6}>0$ such that if $m \geq m_{6}$ and $p \in\left(2-\varepsilon_{6}, 2+\varepsilon_{6}\right)$ then for every $q<p$

$$
\left\|\tilde{\mathcal{N}}_{q}\left(\theta_{t, m} f\right)\right\|_{L^{p, \infty}\left(\mathbb{R}^{n}\right)} \lesssim\left\|\mathbb{S}\left(\Theta_{t, m+1} f\right)\right\|_{L^{p}\left(\mathbb{R}^{n}\right)}+\left\|\mathbb{V}\left(\Theta_{t, 1} f\right)\right\|_{L^{p}\left(\mathbb{R}^{n}\right)}
$$

with implicit constants depending on $p, m, n$ and ellipticity; and where we have defined, in the case of $u(x, t)=\partial_{t} \mathcal{S}_{t}^{\mathcal{L}} f(x)$,

$$
\Theta_{t, m} f:=t^{m} \partial_{t}^{m} \nabla \mathcal{S}_{t}^{\mathcal{L}} f=t^{m} \partial_{t}^{m-1} \nabla u
$$

and

$$
\theta_{t, m} f:=t^{m} \partial_{t}^{m} \partial_{t} \mathcal{S}_{t}^{\mathcal{L}} f=t^{m} \partial_{t}^{m} u
$$

and in the case of $u(x, t)=\mathcal{D}_{t}^{\mathcal{L}} g$

$$
\Theta_{t, m} f=t^{m} \partial_{t}^{m_{1}} \nabla \mathcal{D}^{\mathcal{L},+} f
$$

and

$$
\theta_{t, m} f=t^{m} \partial_{t}^{m} \mathcal{D}^{\mathcal{L},+} f
$$

Therefore the conclusion can be rewritten, in terms of $u$, as

$$
\left\|\tilde{\mathcal{N}}_{q}\left(t^{m} \partial_{t}^{m} u\right)\right\|_{L^{p, \infty}\left(\mathbb{R}^{n}\right)} \lesssim\left\|\mathbb{S}\left(t^{m+1} \partial_{t}^{m} \nabla u\right)\right\|_{L^{p}\left(\mathbb{R}^{n}\right)}+\|\mathbb{V}(t \nabla u)\|_{L^{p}\left(\mathbb{R}^{n}\right)}
$$


Proof. For $m \geq 0$ let us define a modified version of the averages $a_{q}$ given by

$$
a_{q, m}(u)(x, t):=\left(\int_{\mathcal{C}_{x, t}}\left|t^{m} \partial_{s}^{m} u(y, s)\right|^{q} d y d s\right)^{1 / q} \approx\left(\int_{C_{x, t}}\left|s^{m} \partial_{s}^{m} u(y, s)\right|^{q} d y d s\right)^{1 / q}
$$

In particular, for $z \in \mathbb{R}^{n}$,

$$
\tilde{\mathcal{N}}_{q}\left(\theta_{t, m}\right)(z) \approx \sup _{(x, t) \in \Gamma(z)} a_{q, m}(u)(x, t)
$$

Writing for $\lambda>0$,

$$
\begin{aligned}
\left|\left\{z \in \mathbb{R}^{n}: \tilde{\mathcal{N}}_{q}\left(\theta_{t, m} f\right)(z)>\lambda\right\}\right| \leq & \left|\left\{z \in \mathbb{R}^{n}: \tilde{\mathcal{N}}_{q}\left(\theta_{t, m} f\right)(z)>\lambda, \mathbb{S}\left(\Theta_{t, m+1} f\right)(z) \leq \gamma \lambda\right\}\right| \\
& +\left|\left\{z \in \mathbb{R}^{n}: \mathbb{S}\left(\Theta_{t, m+1} f\right)(z)>\gamma \lambda\right\}\right|
\end{aligned}
$$

we see that it's enough to prove that, for $\gamma>0$,

$$
\left|E_{\lambda, \varepsilon}\right|:=\left|\left\{z \in \mathbb{R}^{n}: \tilde{\mathcal{N}}_{q}^{\varepsilon}\left(\theta_{t, m} f\right)(z)>\lambda, \mathbb{S}\left(\Theta_{t, m+1} f\right)(z) \leq \gamma \lambda\right\}\right| \lesssim \lambda^{-p}\left\|\mathbb{V}\left(\Theta_{t, 1} f\right)\right\|_{L^{p}\left(\mathbb{R}^{n}\right)},
$$

with constants uniform in $\varepsilon$ and $\lambda$. Define the function $\varphi_{\varepsilon}$ as follows:

$$
\varphi_{\varepsilon}(x):=\inf \left\{t>\varepsilon: \sup _{(y, s) \in(x, t)+\Gamma(0)} a_{q, m}(u)(y, s) \leq \lambda\right\}
$$

Recall that we have, from the control on slices in Corollary 5.2.3, if $p \in\left(2-\varepsilon_{0}, 2+\varepsilon_{0}\right)$ (here $\varepsilon_{0}$ is as in Theorem 5.1.5 and Corollary 5.2.3)

$$
\sup _{t>0}\left\|t^{m} \partial_{t}^{m} u(\cdot, t)\right\|_{L^{p}\left(\mathbb{R}^{n}\right)}<\infty
$$

so in particular

$$
a_{q, m}(u)(y, s) \lesssim s^{-n / q} \sup _{t>0}\left\|t^{m} \partial_{t}^{m} u\right\|_{L^{p}\left(\mathbb{R}^{n}\right)}
$$


We conclude from this

$$
\lim _{s \rightarrow \infty} \sup _{y \in \mathbb{R}^{n}} a_{q, m}(u)(y, s)=0,
$$

and so $\varepsilon \leq \varphi_{\varepsilon}(x)<\infty$ for every $x \in \mathbb{R}^{n}$. Moreover $\varphi_{\varepsilon}$ is a Lipschitz function with constant 1 , since it satisfies the appropriate uniform cone condition. We set

$$
\Gamma_{\varphi_{\varepsilon}}:=\left\{(x, t) \in \mathbb{R}_{+}^{n+1}: t=\varphi_{\varepsilon}(x)\right\}
$$

the graph of $\varphi_{\varepsilon}$. Finally recall that we denote $(u)_{A}$ the average of $u$ over a set $A \subset \mathbb{R}_{+}^{n+1}$ of finite measure.

We first claim that, for every $z \in E_{\lambda, \varepsilon}$ and if we denote $Z_{\varepsilon}:=\left(z, \varphi_{\varepsilon}(z)\right)$,

$$
\lambda \lesssim \mathcal{M}_{\Gamma_{\varphi_{\varepsilon}}}\left(a_{q, m}(u)\right)\left(Z_{\varepsilon}\right),
$$

for some implicit constant independent of $\lambda$ and $\varepsilon$, and where $\mathcal{M}_{\Gamma_{\varphi_{\varepsilon}}}$ denotes the maximal function on $\Gamma_{\varphi_{\varepsilon}}$ with its natural surface measure, which we denote by $\sigma$.

To see this fix $z \in E_{\lambda, \varepsilon}$ and $(x, t) \in Z_{\varepsilon}+\Gamma(0)$ and note that, owing to (5.28) there exists $R>0$ such that $a_{q, m}(u)(x, t) \leq \lambda / 2$ if $t>R$. Moreover using that $\tilde{\mathcal{N}}_{q}^{\varepsilon}\left(\theta_{t, m} f\right)(z)>\lambda$, so that $\varphi_{\varepsilon}(z)>\varepsilon$, there exists $(y, s) \in \overline{Z_{\varepsilon}+\Gamma(0)}$ satisfying $a_{q, m}(u)(x, t)>\lambda$. By continuity of $a_{q, m}$ in $\mathbb{R}_{+}^{n+1}$ we conclude from the above that there exists a point $(x, t) \in \overline{Z_{\varepsilon}+\Gamma(0)}$ such that $a_{q, m}(u)(x, t)=\lambda$, and $(x, t) \in \overline{\left\{a_{q, m}(u)>\lambda\right\}}$.

Note also that the above implies $(x, t) \in \Gamma_{\varphi_{\varepsilon}}$, i.e. $t=\varphi_{\varepsilon}(x)$ : For every $\delta>0$ there exists $(y, s) \in B((x, t), \delta)$ such that $a_{q, m}(y, s)>\lambda$, and so, since $B((x, t), \delta) \subset(x, t-\sqrt{2} \delta)+\Gamma(0)$, 
we have $\varphi_{\varepsilon}(x)>t-\sqrt{2} \delta$. Since $\delta$ was arbitrary we conclude $\varphi_{\varepsilon}(x) \geq t$. On the other hand, by the Lipschitz condition on $\varphi_{\varepsilon}$ it can't happen that $\Gamma_{\varphi_{\varepsilon}}$ intersects the interior of the cone $Z_{\varepsilon}+\Gamma(0)$, therefore $\varphi_{\varepsilon}(x) \leq t$. Notice that, in fact, the above shows that $(x, t)=\left(x, \varphi_{\varepsilon}(x)\right) \in$ $\partial\left(Z_{\varepsilon}+\Gamma(0)\right)$.

Given such a point $(x, t)=\left(x, \varphi_{\varepsilon}(x)\right):=X_{\varepsilon}$, and for any $(y, s) \in B\left(X_{\varepsilon}, t / 100\right)$ we have, by the Poincaré-Sobolev inequality and writing $v_{m}(w, \tau):=\partial_{\tau}^{m} u(w, \tau)$,

$$
\begin{aligned}
\lambda & =a_{q, m}(u)(x, t) \leq\left(\int_{\mathcal{C}_{x, t}} t^{2 m}\left|v_{m}-\left(v_{m}\right)_{\mathcal{C}_{x, t}}\right|^{q} d w d \tau\right)^{1 / q}+t^{m}\left|\left(v_{m}\right)_{C_{x, t}}-\left(v_{m}\right)_{C_{y, s}}\right|+t^{m}\left|\left(v_{m}\right)_{C_{y, s}}\right| \\
& \lesssim\left(\iint_{C_{x, t}}\left|\tau^{m} \nabla v_{m}\right|^{2} \tau^{1-n} d w d \tau\right)^{1 / 2}+t^{m}\left|\left(v_{m}\right)_{C_{y, s}}\right| \lesssim \mathbb{S}\left(\Theta_{t, m+1} f\right)(x)+t^{m}\left|\left(v_{m}\right)_{C_{y, s}}\right| \\
& \leq \gamma \lambda+t^{m}\left|\left(v_{m}\right)_{C_{y, s}}\right| \leq \gamma \lambda+a_{q, m}(u)(y, s),
\end{aligned}
$$

where we also used the fact that $x \in E_{\lambda, \varepsilon}$ and that $\tau \approx s \approx t$. Choosing $\gamma<1$ small enough above we can write

$$
\lambda \lesssim a_{q, m}(u)(y, s), \quad \forall(y, s) \in B\left(X_{\varepsilon}, t / 100\right) .
$$

From here (5.28) follows easily: Integrating the above inequality on $\Gamma_{\varphi_{\varepsilon}}$ we have

$$
\lambda \lesssim f_{B\left(X_{\varepsilon}, t / 100\right) \cap \Gamma_{\varphi_{\varepsilon}}} a_{q, m}(u)(W) d \sigma(W) \lesssim f_{B\left(X_{\varepsilon}, t\right) \cap \Gamma_{\varphi_{\varepsilon}}} a_{q, m}(u)(W) d \sigma(W)
$$

where we have used that $\sigma(B) \approx r(B)$ for any ball centered on $\Gamma_{\varphi_{\varepsilon}}$. Moreover, since $X_{\varepsilon} \in$ $\partial\left(Z_{\varepsilon}+\Gamma(0)\right)$ we have

$$
|z-x|=\left|t-\varphi_{\varepsilon}(z)\right|=t-\varphi_{\varepsilon}(x)<t,
$$


and so we conclude $Z_{\varepsilon} \in B\left(X_{\varepsilon}, t\right)$ and (5.28) follows.

Since (5.28) holds for any $z \in E_{\lambda, \varepsilon}$ we see that

$$
\left|E_{\lambda, \varepsilon}\right| \lesssim\left|\left\{W \in \Gamma_{\varphi_{\varepsilon}}: \mathcal{M}_{\Gamma_{\varphi_{\varepsilon}}}\left(a_{q, m}(u)\right)(W)\right\}\right| \lesssim \lambda^{-p} \int_{\Gamma_{\varphi_{\varepsilon}}} a_{q, m}(u)^{p}(W) d \sigma(W) .
$$

Therefore, it's enough to prove

$$
\int_{\Gamma_{\varphi_{\varepsilon}}} a_{q, m}(u)^{p}(W) d \sigma(W) \lesssim\|\mathbb{V}(t \nabla u)\|_{L^{p}\left(\mathbb{R}^{n}\right)}^{p}
$$

We make a further reduction as follows: Note that, by the $L^{q}$ Caccioppoli's inequality applied $m$ times, for any $(x, t) \in \mathbb{R}_{+}^{n+1}$

$$
\begin{aligned}
a_{q, m}(u)(x, t) & =\left(\int_{C_{x, t}}\left|t^{m} \partial_{t}^{m} u(y, s)\right|^{q} d y d s\right)^{1 / q} \\
& \lesssim\left(\int_{\mathcal{C}_{x, t}^{*}}|u(y, s)|^{q} d y d s\right)^{1 / q} \\
& \approx a_{q}^{*}(u)(x, t) .
\end{aligned}
$$

Therefore the result would follow from

$$
\int_{\mathbb{R}^{n}}\left|a_{q}^{*}(u)\left(x, \varphi_{\varepsilon}(x)\right)\right|^{p} d y \approx \int_{\Gamma_{\varphi_{\varepsilon}}}\left|a_{q}^{*}(W)\right|^{p} d \sigma(W) \lesssim\|\mathbb{V}(t \nabla u)\|_{L^{p}\left(\mathbb{R}^{n}\right)}
$$

To simplify notation in what follows we set

$$
g(x):=a_{q}^{*}(u)\left(x, \varphi_{\varepsilon}(x)\right), \quad v(x)=\mathcal{M}(g)(x)^{p-q} .
$$

We then have

$$
\int_{\mathbb{R}^{n}}\left|a_{q}^{*}(u)\left(x, \varphi_{\varepsilon}(x)\right)\right|^{p} d y=\int_{\mathbb{R}^{n}} g^{p}(x) d x \leq \int_{\mathbb{R}^{n}} g^{q}(x) v(x) d x .
$$


Note that

$$
\frac{4}{5} \varphi_{\varepsilon}(y) \leq \varphi_{\varepsilon}(x) \leq \frac{4}{3} \varphi_{\varepsilon}(y), \quad \text { whenever }|x-y|<\frac{\varphi_{\varepsilon}(x)}{4}
$$

owing to the fact $\varphi_{\varepsilon}$ is Lipschitz with constant one.

We now go back to the definition of $g$ and $a_{q}^{*}$ to compute,

$$
\begin{aligned}
\int_{\mathbb{R}^{n}} g^{q}(x) v(x) d x & \approx \int_{\mathbb{R}^{n}} f_{|x-y|<\varphi_{\varepsilon} / 4} f_{3 \varphi_{\varepsilon}(x) / 4}^{5 \varphi_{\varepsilon}(x) / 4}|u(y, s)|^{q} d s d y v(x) d x \\
& \lesssim \int_{4 / 5}^{5 / 3} \int_{\mathbb{R}^{n}} f_{|x-y|<\varphi_{\varepsilon}(x) / 8}\left|u\left(y, \tau \varphi_{\varepsilon}(y)\right)\right|^{q} d y v(x) d x d \tau \\
& \lesssim \int_{4 / 5}^{5 / 3} \int_{\mathbb{R}^{n}} \mathcal{M}\left(\left|u\left(\cdot, \tau \varphi_{\varepsilon}(\cdot)\right)\right|^{q}\right)(x) v(x) d x d \tau .
\end{aligned}
$$

By Hölder's Inequality with exponents $p / q$ and $p /(p-q)$ we see

$$
\int_{\mathbb{R}^{n}} \mathcal{M}\left(\left|u\left(\cdot, \tau \varphi_{\varepsilon}(\cdot)\right)\right|^{q}\right)(x) v(x) d x \leq\left\|\mathcal{M}_{q}\left(u\left(\cdot, \tau \varphi_{\varepsilon}(\cdot)\right)\right)\right\|_{L^{p}\left(\mathbb{R}^{n}\right)}^{q}\|v\|_{L^{p /(p-q)}\left(\mathbb{R}^{n}\right)},
$$

and, since $q<p$, by the boundedness of $\mathcal{M}_{q}$ in $L^{p}$

$$
\left\|\mathcal{M}_{q}\left(u\left(\cdot, \tau \varphi_{\varepsilon}(\cdot)\right)\right)\right\|_{L^{p}\left(\mathbb{R}^{n}\right)}^{q} \lesssim\left(\int_{\mathbb{R}^{n}}\left|u\left(x, \tau \varphi_{\varepsilon}(x)\right)\right|^{p} d x\right)^{q / p} .
$$

Similarly,

$$
\|v\|_{L^{p /(p-q)}\left(\mathbb{R}^{n}\right)}=\|\mathcal{M}(g)\|_{L^{p}\left(\mathbb{R}^{n}\right)}^{p-q} \lesssim\left(\int_{\mathbb{R}^{n}}|g(x)|^{p} d x\right)^{(p-q) / p}
$$

Combining the above estimates, we obtain

$$
\int_{\mathbb{R}^{n}} g^{p}(x) d x \lesssim \int_{4 / 5}^{5 / 3}\left(\int_{\mathbb{R}^{n}}\left|u\left(x, \tau \varphi_{\varepsilon}(x)\right)\right|^{p} d x\right)^{q / p}\left(\int_{\mathbb{R}^{n}} g^{p}(x) d x\right)^{(p-q) / p} d \tau
$$


Using Hölder's inequality again (perhaps working with $g(x) \mathbb{1}_{g<M}$ if necessary in order to divide by $\left.\|g\|_{L^{p}\left(\mathbb{R}^{n}\right)}\right)$ we arrive at

$$
\int_{\mathbb{R}^{n}} g^{p}(x) d x \lesssim \int_{4 / 5}^{5 / 3} \int_{\mathbb{R}^{n}}\left|u\left(x, \tau \varphi_{\varepsilon}(x)\right)\right|^{p} d x d \tau .
$$

We now note that $\tau \varphi_{\varepsilon}$ is a Lipschitz function with constant $\tau$. Therefore the function $v(x, t)=u\left(x, t+\varphi_{\varepsilon}(x)\right)$ solves $\mathcal{L}_{\tau \varphi_{\varepsilon}} v=0$ in $\mathbb{R}_{+}^{n+1}$, where the operator $\mathcal{L}_{\tau \varphi_{\varepsilon}}$ is of the same type as $\mathcal{L}$ and moreover its coefficients are controlled (in the appropriate norms) by those of $\mathcal{L}$. Therefore, by the control on slices by the vertical square function in Theorem 5.2.1 (see also Lemma 5.2.11)

$$
\begin{aligned}
\int_{\mathbb{R}^{n}}\left|u\left(x, \tau \varphi_{\varepsilon}(x)\right)\right|^{p} d x & =\int_{\mathbb{R}^{n}}|v(x, 0)|^{p} d x \lesssim \int_{\mathbb{R}^{n}}\left(\int_{0}^{\infty}|t \nabla v(x, t)|^{2} \frac{d t}{t}\right)^{p / 2} d x \\
& \lesssim \int_{\mathbb{R}^{n}}\left(\int_{\tau \varphi_{\varepsilon}(x)}^{\infty}|\nabla u(x, t)|^{2} t d t\right)^{p / 2} d x \\
& \leq\|\mathbb{V}(t \nabla u)\|_{L^{p}\left(\mathbb{R}^{n}\right)}^{p} .
\end{aligned}
$$

This yields (5.37) and the result is proved.

Remark 5.3.50. Notice that in the above lemma, the only things required for its proof were:

1. $\theta_{t, m}$ satisfies good estimates on slices (as in (5.27)).

2. We already have, for all operators of the form $\mathcal{L}=-\operatorname{div}\left(A \nabla+B_{1}\right)+B_{2} \cdot \nabla$ (with sufficient smallness of the first order coefficients), the control on slices by the vertical square function

$$
\|u(\cdot, t)\|_{L^{p}\left(\mathbb{R}^{n}\right)} \lesssim\|\mathbb{V}(t \nabla u)\|_{L^{p}\left(\mathbb{R}^{n}\right)}
$$


The following result uses the fact that $\mathbb{V}\left(t^{m} \partial_{t}^{m-1} \nabla\left(\mathcal{S}_{t}^{\mathcal{L}} \nabla\right)\right)$ satisfies $L^{p}$ bounds for all $m \geq 1$, however it seems appropriate to state it at this point; it will be proved in the next section.

Corollary 5.3.52. Suppose $\mathcal{L}$ satisfies the hypotheses of Theorem 5.1.5. If $\varepsilon_{0}>0$ and $m_{0}$ are as in Theorem 5.1.5, and if $p \in\left(2-\varepsilon_{0}, 2+\varepsilon_{0}\right)$ and $m \geq m_{0} \geq 1$ then

$$
\left\|\tilde{\mathcal{N}}_{2}\left(\Theta_{t, m} f\right)\right\|_{L^{p}\left(\mathbb{R}^{n}\right)} \lesssim\|f\|_{L^{p}\left(\mathbb{R}^{n}\right)}
$$

where $\Theta_{t, m}$ is either $t^{m} \partial_{t}^{m} \nabla\left(\mathcal{S}_{t}^{\mathcal{L}} \nabla\right)$ or $t^{m} \partial_{t}^{m-1} \nabla \mathcal{D}_{t}^{\mathcal{L}}$.

Proof. We treat only the single layer. The double layer argument is identical. Also, notice by $t$ independence it's enough to treat the operator with the inside gradient replaced by $\nabla_{\|}$.

First, from the pointwise inequality in Proposition 5.3.1 and the dominated convergence theorem, we see that for any $q \geq 1$ and $p$ in the above range, and setting $\theta_{t, m}=$

$$
\begin{aligned}
t^{m} \partial_{t}^{m}\left(\mathcal{S}_{t}^{\mathcal{L}} \nabla_{\|}\right)=-t^{m} \partial_{t}^{m} \mathcal{S}_{t}^{\mathcal{L}} \operatorname{div}_{\|} \\
\quad\left\|\tilde{\mathcal{N}}_{2}\left(\Theta_{t, m} f\right)\right\|_{L^{p, \infty}\left(\mathbb{R}^{n}\right)} \lesssim\left\|\tilde{\mathcal{N}}_{q}\left(\theta_{t, m} f\right)\right\|_{L^{p, \infty}\left(\mathbb{R}^{n}\right)}+\sup _{t>0}\left\|\Theta_{t, m} f\right\|_{L^{p}\left(\mathbb{R}^{n}\right)} .
\end{aligned}
$$

In particular by the slices estimates in Corollary 5.2.3 and Theorem 5.2.8, and choosing $q$ as in the above Lemma,

$$
\left\|\tilde{\mathcal{N}}_{2}\left(\Theta_{t, m} f\right)\right\|_{L^{p, \infty}\left(\mathbb{R}^{n}\right)} \lesssim\left\|\mathbb{S}\left(\Theta_{t, m} f\right)\right\|_{L^{p}\left(\mathbb{R}^{n}\right)}+\left\|\mathbb{V}\left(\Theta_{t, 1} f\right)\right\|_{L^{p}\left(\mathbb{R}^{n}\right)} \lesssim\|f\|_{L^{p}\left(\mathbb{R}^{n}\right)},
$$

where we have used Theorem 5.4.1 for the last step; ensuring that $\mathbb{V}\left(\Theta_{t, 1} f\right)$ is under control. The result now follows from real interpolation. 


\subsection{Traveling Down}

We first establish the vertical square function estimates, since there's little difficulty there. The discrepancy between these so-called traveling down procedures for the vertical and conical square functions should be contrasted with the situation in the extrapolation arguments.

Theorem 5.4.1. Suppose $\mathcal{L}$ satisfies the hypotheses of Theorem 5.1.5. Let $\varepsilon_{0}$ be as in Theorem 5.1.5, then for $p \in\left(2-\varepsilon_{0}, 2+\varepsilon_{0}\right)$ and every $m \geq 1$ it holds

$$
\left\|\mathbb{V}\left(\Theta_{t, m} f\right)\right\|_{L^{p}\left(\mathbb{R}^{n}\right)} \lesssim_{m}\|f\|_{L^{p}\left(\mathbb{R}^{n}\right)}
$$

where $\Theta_{t, m}$ is either $t^{m} \partial_{t}^{m-1} \nabla\left(\mathcal{S}_{t}^{\mathcal{L}} \nabla\right)$ or $t^{m} \partial_{t}^{m-1} \nabla \mathcal{D}_{t}^{\mathcal{L}}$. In the case of the single layer and $m=1$, we replace one of the gradients with a $t$-derivative.

Proof of Theorem 5.4.1. We employ the same idea as in the $L^{2}$ case from $\left[\mathrm{BHL}^{+} \mathrm{a}\right]$; integrating by parts in $t$ to control the square function of $\Theta_{t, m}$ in terms of $\Theta_{t, m+1}$ plus terms that are bounded in $L^{p}$. Notice that for $m \geq m_{0}$ large enough the desired bound is a consequence of Theorem 5.1.5 and Lemma 5.1.36 for the case of the double layer.

We start by defining, for $\eta>0$,

$$
\int_{\mathbb{R}^{n}}\left(\int_{0}^{\infty}\left|\Theta_{t, m} f(x)\right|^{2} \frac{d t}{t}\right)^{p / 2} d x=\lim _{\eta \rightarrow 0^{+}} \int_{\mathbb{R}^{n}}\left(\int_{\eta}^{1 / \eta}\left|\Theta_{t, m} f(x)\right|^{2} \frac{d t}{t}\right)^{p / 2} d x=: I_{\eta}
$$

In particular, owing to the estimates on slices from Corollary 5.2.3, we see that $I_{\eta}<\infty$ for all such $\eta$. 
We now carry out the integration by parts

$$
\begin{aligned}
I_{\eta}= & \int_{\mathbb{R}^{n}}\left(\int_{\eta}^{1 / \eta} t^{2 m-1}\left|\partial_{t}^{m-1} \nabla \mathcal{S}_{t}^{\mathcal{L}} \operatorname{div}_{\|} f(x)\right|^{2} d t\right)^{p / 2} d x \\
\leq & \int_{\mathbb{R}^{n}}\left(\left.\left|t^{m} \partial_{t}^{m-1} \nabla \mathcal{S}_{t}^{\mathcal{L}} \operatorname{div}_{\|} f(x)\right|^{2}\right|_{t=\eta} ^{1 / \eta}\right)^{p / 2} d x \\
& +\int_{\mathbb{R}^{n}}\left(\int_{\eta}^{1 / \eta}\left|\Theta_{t, m} f(x) \| \Theta_{t, m+1} f(x)\right| \frac{d t}{t}\right)^{p / 2} d x \\
\leq & C_{m}\|f\|_{L^{p}\left(\mathbb{R}^{n}\right)}^{p}+\int_{\mathbb{R}^{n}}\left(\int_{\eta}^{1 / \eta}\left|\Theta_{t, m} f(x) \| \Theta_{t, m+1} f(x)\right| \frac{d t}{t}\right)^{p / 2} d x,
\end{aligned}
$$

where we used the estimates on slices in Corollary 5.2.3 for the single layer and Theorem 5.2.8 for the double layer for the last line. Finally we use Cauchy's inequality with a parameter to obtain

$$
\int_{\mathbb{R}^{n}}\left(\int_{\eta}^{1 / \eta}\left|\Theta_{t, m} f(x)\right|^{2} \frac{d t}{t}\right)^{p / 2} d x \lesssim\|f\|_{L^{p}\left(\mathbb{R}^{n}\right)}^{p}+\int_{\mathbb{R}^{n}}\left(\int_{\eta}^{1 / \eta}\left|\Theta_{t, m+1} f(x)\right|^{2} \frac{d t}{t}\right)^{p / 2} d x
$$

Letting $\eta \rightarrow 0$ we can write

$$
\left\|\mathbb{V}\left(\Theta_{t, m} f\right)\right\|_{L^{p}\left(\mathbb{R}^{n}\right)} \lesssim\|f\|_{L^{p}\left(\mathbb{R}^{n}\right)}+\left\|\mathbb{V}\left(\Theta_{t, m+1} f\right)\right\|_{L^{p}\left(\mathbb{R}^{n}\right)}
$$

The result now follows by induction and Theorem 5.1.5. The restriction, for this argument, in the case of the single layer and $m=1$ comes from the lack of $L^{p}$ (even $L^{2}$ ) estimates on slices for $\nabla\left(\mathcal{S}^{\mathcal{L}} \nabla\right)$.

We now turn to the much harder task of traveling down with the conical square function. Here, although the idea is the same basic integration by parts technique, the arguments become much more elaborate due to the 'space averaging' happening alongside the integration over the transversal variable. To handle this we will make use of the nontangential 
maximal function estimates from the previous section (see Lemma 5.3.17) through a modified version of the classical Carleson embedding lemma (see Lemma 2.4.44). However, the use of the nontangential maximal function makes the traveling down procedure for either this object or the conical square function a bit subtle. It's our hope that the following lemma (which should be read with the results of the previous section in mind) and Theorem 5.4.36 clarifies the intertwining of these two procedures.

We also note that for the range $p>2$ we already have the conical square function bounded by the vertical (Proposition 2.4.6) by general facts about square functions, so it's only the case $p<2$ that's of interest.

Lemma 5.4.7. Let $\Theta_{t, m}$ be either $t^{m} \partial_{t}^{m-1} \nabla\left(\mathcal{S}_{t}^{\mathcal{L}} \nabla\right)$ or $t^{m} \partial_{t}^{m-1} \nabla \mathcal{D}_{t}^{\mathcal{L}}$. Suppose $m \geq 1$ is given such that it holds

$$
\mathbb{S}\left(\Theta_{t, m} f\right), \mathbb{S}\left(\Theta_{t, m+1} f\right), \mathbb{V}\left(\Theta_{t, m+1} f\right), \mathbb{V}\left(\Theta_{t, m} f\right), \tilde{\mathcal{N}}\left(\Theta_{t, m} f\right) \in L^{2}\left(\mathbb{R}^{n}\right)
$$

and

$$
\sup _{t>0}\left\|\Theta_{t, m} f\right\|_{L^{2}\left(\mathbb{R}^{n}\right)}<\infty, \quad \lim _{t \rightarrow \infty}\left\|\Theta_{t, m} f\right\|_{L^{2}\left(\mathbb{R}^{n}\right)}=0
$$

for every $f \in C_{c}^{\infty}\left(\mathbb{R}^{n}\right)$. Then for every $1<p<2$

$$
\begin{aligned}
\left\|\mathbb{S}\left(\Theta_{t, m} f\right)\right\|_{L^{p}\left(\mathbb{R}^{n}\right)} \lesssim & \left\|\mathbb{S}\left(\Theta_{t, m+1} f\right)\right\|_{L^{p}\left(\mathbb{R}^{n}\right)}+\left\|\mathbb{V}\left(\Theta_{t, m+1} f\right)\right\|_{L^{p}\left(\mathbb{R}^{n}\right)} \\
& +\left\|\mathbb{V}\left(\Theta_{t, m} f\right)\right\|_{L^{p}\left(\mathbb{R}^{n}\right)}+\left\|\tilde{\mathcal{N}}\left(\Theta_{t, m} f\right)\right\|_{L^{p}\left(\mathbb{R}^{n}\right)} \\
& +\sup _{t>0}\left\|\Theta_{t, m} f\right\|_{L^{p}\left(\mathbb{R}^{n}\right)},
\end{aligned}
$$

for every $f \in C_{c}^{\infty}\left(\mathbb{R}^{n}\right)$. 
Proof. We fix $m \geq 1$ as in the hypotheses and define

$$
g_{m}:=\sup _{t>0}\left|\Theta_{t, m} f\right|, \quad h_{m}=\tilde{\mathcal{N}}\left(\Theta_{t, m}\right)
$$

and set

$$
H_{m}:=\mathbb{S}\left(\Theta_{t, m+1} f\right)+g_{m}+h_{m}
$$

Recall from Proposition 3.4.14

$$
\left\|g_{m}\right\|_{L^{2}\left(\mathbb{R}^{n}\right)} \lesssim\left\|\mathbb{V}\left(\Theta_{t, m} f\right)\right\|_{L^{2}\left(\mathbb{R}^{n}\right)}+\left\|\mathbb{V}\left(\Theta_{t, m+1} f\right)\right\|_{L^{2}\left(\mathbb{R}^{n}\right)}+\sup _{t>0}\left\|\Theta_{t, m} f\right\|_{L^{2}\left(\mathbb{R}^{n}\right)}
$$

Therefore $H_{m} \in L^{2}\left(\mathbb{R}^{n}\right)$ and so, by the Coifman-Rochberg theorem, we see that if we define $v(x):=\mathcal{M}\left(\mathbb{S}\left(\Theta_{t, m} f\right)+H_{m}\right)(x)^{p-2}$ for some $1<p<2$, then $v^{M} \in A_{2}$ for any $M>1$ satisfying $M(2-p)<1$ and moreover $\left[v^{M}\right]_{A_{2}}$ depends only on the quantity $M(2-p)$.

We now mimick the proof of the extrapolation lemma 2.4.39 and write, for fixed $1<$ $p<2$,

$$
\begin{aligned}
\int_{\mathbb{R}^{n}}\left|\mathbb{S}\left(\Theta_{t, m} f\right)(x)\right|^{p} d x & \leq \int_{\mathbb{R}^{n}} \mathcal{M}\left(\mathbb{S}\left(\Theta_{t, m} f\right)+H_{m}\right)(x)^{p} d x \\
& =\int_{\mathbb{R}^{n}} \mathcal{M}\left(\mathbb{S}\left(\Theta_{t, m} f\right)+H_{m}\right)(x)^{2} v(x) d x \\
& \lesssim \int_{\mathbb{R}^{n}} \mathcal{M}\left(\mathbb{S}\left(\Theta_{t, m} f\right)\right)(x)^{2} v(x) d x+\int_{\mathbb{R}^{n}} \mathcal{M}\left(H_{m}\right)(x)^{2} v(x) d x \\
& \lesssim[v]_{A_{2}} \int_{\mathbb{R}^{n}} \mathbb{S}\left(\Theta_{t, m} f\right)(x)^{2} v(x) d x+\int_{\mathbb{R}^{n}} \mathcal{M}\left(H_{m}\right)(x)^{p} d x \\
& \lesssim \int_{\mathbb{R}^{n}} \mathbb{S}\left(\Theta_{t, m} f\right)(x)^{2} v(x) d x+\int_{\mathbb{R}^{n}} H_{m}(x)^{p} d x,
\end{aligned}
$$

where we used the boundedness of $\mathcal{M}$ in $L^{2}(v)$, by the above discussion, and in $L^{p}\left(\mathbb{R}^{n}\right)$. By 
definition of $H_{m}$, and Proposition 3.4.14, we have

$$
\begin{gathered}
\left\|H_{m}\right\|_{L^{p}\left(\mathbb{R}^{n}\right)} \lesssim_{p}\left\|\mathbb{V}\left(\Theta_{t, m} f\right)\right\|_{L^{p}\left(\mathbb{R}^{n}\right)}+\left\|\mathbb{V}\left(\Theta_{t, m+1} f\right)\right\|_{L^{p}\left(\mathbb{R}^{n}\right)}+\sup _{t>0}\left\|\Theta_{t, m} f\right\|_{L^{p}\left(\mathbb{R}^{n}\right)} \\
+\left\|\mathbb{S}\left(\Theta_{t, m+1} f\right)\right\|_{L^{p}\left(\mathbb{R}^{n}\right)}+\left\|\tilde{\mathcal{N}}\left(\Theta_{t, m} f\right)\right\|_{L^{p}\left(\mathbb{R}^{n}\right)} .
\end{gathered}
$$

It thus remains to estimate the first term in (5.14). For this we will try to emulate the procedure for the vertical square function, introducing an approximate identity $P_{t}$ to smooth-out the averaging implicit in the definition of $\mathbb{S}$. We will first fix the approximate identity: For $t>0$ we define

$$
P_{t}:=e^{t^{2} \Delta}, \quad Q_{t}:=t \partial_{t} e^{t^{2} \Delta}=t \partial_{t} P_{t}
$$

We will also need to truncate our weight to formally justify our computations so we define, for $N>0$,

$$
v_{N}:=\min (v, N)
$$

We first compute, using Fubini's theorem

$$
\begin{aligned}
\int_{\mathbb{R}^{n}} \mathbb{S}\left(\Theta_{t, m} f\right)(x)^{2} v(x) d x & =\int_{\mathbb{R}^{n}} \int_{0}^{\infty} f_{|x-y|<t}\left|\Theta_{t, m} f(y)\right|^{2} d y \frac{d t}{t} v(x) d x \\
& =\int_{\mathbb{R}^{n}} \int_{0}^{\infty}\left|\Theta_{t, m} f(y)\right|^{2} f_{|x-y|<t} v(x) d x \frac{d t}{t} d y \\
& \lesssim \int_{\mathbb{R}^{n}} \int_{0}^{\infty}\left|\Theta_{t, m} f(y)\right|^{2} P_{t} v(y) \frac{d y d t}{t} \\
& =: I
\end{aligned}
$$

Now, by the Monotone convergence theorem,

$$
\begin{aligned}
I & =\lim _{N \rightarrow \infty} \lim _{\varepsilon \rightarrow 0} I_{\varepsilon, N} \\
& :=\lim _{N \rightarrow \infty} \lim _{\varepsilon \rightarrow 0} \int_{\mathbb{R}^{n}} \int_{\varepsilon}^{1 / \varepsilon}\left|\Theta_{t, m} f(y)\right|^{2} P_{t} v_{N}(y) \frac{d y d t}{t} .
\end{aligned}
$$


Recalling the definition of $\Theta_{t, m}$ and integrating by parts in $t$ we obtain, recalling that $Q_{t}:=$ $t \partial_{t} P_{t}$,

$$
\begin{aligned}
I_{\varepsilon, N}= & -\frac{1}{2 m} \int_{\mathbb{R}^{n}} \int_{\varepsilon}^{1 / \varepsilon} \partial_{t}\left(\left|\partial_{t}^{m} \mathcal{S}_{t}^{\mathcal{L}} f\right|^{2} P_{t} v_{N}\right) t^{2 m} d y d t \\
& +\left.\frac{1}{2 m} \int_{\mathbb{R}^{n}}\left|\Theta_{t, m} f\right|^{2} P_{t} v_{N} d y\right|_{t=\varepsilon} ^{1 / \varepsilon} \\
\leq & \frac{1}{m} \int_{\mathbb{R}^{n}} \int_{\varepsilon}^{1 / \varepsilon}\left|\Theta_{t, m} f\right|\left|\Theta_{t, m+1} f\right| P_{t} \nu_{N} \frac{d y d t}{t} \\
& +\frac{1}{2 m} \int_{\mathbb{R}^{n}} \int_{\varepsilon}^{1 / \varepsilon}\left|\Theta_{t, m} f\right|^{2}\left|Q_{t} v_{N}\right| \frac{d y d t}{t} \\
& +\frac{1}{2 m} \int_{\mathbb{R}^{n}}\left|\Theta_{1 / \varepsilon, m} f\right|^{2} P_{1 / \varepsilon} v_{N} d y \\
& -\frac{1}{2 m} \int_{\mathbb{R}^{n}}\left|\Theta_{\varepsilon, m} f\right|^{2} P_{\varepsilon} v_{N} d y \\
= & : I I_{\varepsilon, N}+I I I_{\varepsilon, N}+I V_{\varepsilon, N}+V_{\varepsilon, N} .
\end{aligned}
$$

We handle the boundary terms first. To start we note that by Theorem 4.7.12 we have

$$
\lim _{t \rightarrow \infty}\left\|\Theta_{t, m} f\right\|_{L^{2}\left(\mathbb{R}^{n}\right)} \rightarrow 0
$$

so that, using $\left|P_{t} v_{N}\right| \lesssim N$ pointwise in $\mathbb{R}^{n}$, we have

$$
I V_{\varepsilon, N} \lesssim N \int_{\mathbb{R}^{n}}\left|\Theta_{1 / \varepsilon, m} f\right|^{2} d y \rightarrow 0 \quad \text { as } \quad \varepsilon \rightarrow 0 .
$$

Therefore

$$
\lim _{N \rightarrow \infty} \lim _{\varepsilon \rightarrow 0} I V_{\varepsilon, N}=0 .
$$

On the other hand, as $\varepsilon \rightarrow 0$, we have $P_{\varepsilon} v_{N} \rightarrow v_{N}$ pointwise a.e. and $\left|\Theta_{\varepsilon, m} f\right|^{2} \leq g_{m}^{2}$, by definition of $g_{m}$. By the Dominated Convergence Theorem (recall $g_{m} \in L^{2}\left(\mathbb{R}^{n}\right)$ and 
$\left.P_{\varepsilon} v_{N} \lesssim N\right)$ we have

$$
\begin{aligned}
V_{\varepsilon, N} & \lesssim \int_{\mathbb{R}^{n}} g_{m}^{2} P_{\varepsilon} v_{N} d y \\
& \stackrel{\varepsilon \rightarrow 0}{\longrightarrow} \int_{\mathbb{R}^{n}} g_{m}^{2} \nu_{N} d y \\
& \leq \int_{\mathbb{R}^{n}} g_{m}^{2} v d y \\
& \lesssim \int_{\mathbb{R}^{n}} g_{m}^{p} d y,
\end{aligned}
$$

where we used the definition of $v$ in the last line to conclude $v(y) \leq \mathcal{M}\left(g_{m}\right)(y)^{p-2}$ and the Hardy-Littlewood maximal theorem. We conclude

$$
\limsup _{N \rightarrow \infty} \limsup _{\varepsilon \rightarrow 0} V_{\varepsilon, N} \lesssim \int_{\mathbb{R}^{n}} g_{m}^{p} d x
$$

The first term $I I_{\varepsilon, N}$ we can treat as usual; using Cauchy's inequality with a parameter we see

$$
\begin{aligned}
I I_{\varepsilon, N} & \lesssim \delta \int_{\mathbb{R}^{n}} \int_{\varepsilon}^{1 / \varepsilon}\left|\Theta_{t, m} f\right|^{2} P_{t} v_{N} \frac{d y d t}{t}+C(\delta) \int_{\mathbb{R}^{n}} \int_{\varepsilon}^{1 / \varepsilon}\left|\Theta_{t, m+1} f\right|^{2} P_{t} v_{N} \frac{d y d t}{t} \\
& =\delta I_{\varepsilon, N}+C(\delta) \int_{\mathbb{R}^{n}} \int_{\varepsilon}^{1 / \varepsilon}\left|\Theta_{t, m+1} f\right|^{2} P_{t} \nu_{N} \frac{d y d t}{t}
\end{aligned}
$$

Choosing $\delta$ small enough we can hide the first term on the right hand side of (5.20).

Finally, we rewrite $I I I_{\varepsilon, N}$ in the following way, using the Cauchy-Schwarz inequality,

$$
\begin{aligned}
I I I_{\varepsilon, N} & =\int_{\mathbb{R}^{n}} \int_{\varepsilon}^{1 / \varepsilon}\left|\Theta_{t, m} f\right| \frac{\left|Q_{t} v_{N}\right|}{\left|P_{t} \nu_{N}\right|} \sqrt{P_{t} v_{N}}\left|\Theta_{t, m} f\right| \sqrt{P_{t} v_{N}} \frac{d y d t}{t} \\
& \leq I_{\varepsilon, N} \int_{\mathbb{R}^{n}} \int_{\varepsilon}^{1 / \varepsilon}\left|\Theta_{t, m} f\right|^{2} d \mu_{N}(y, t),
\end{aligned}
$$

where we have defined

$$
d \mu_{N}(x, t):=\frac{\left|Q_{t} \nu_{N}(x)\right|^{2}}{\left|P_{t} v_{N}(x)\right|^{2}} P_{t} \nu_{N}(x) \frac{d x d t}{t}
$$


By Proposition 2.4.64 and the modified Carleson's lemma (Lemma 2.4.44) we obtain

$$
\int_{\mathbb{R}^{n}} \int_{\varepsilon}^{1 / \varepsilon}\left|\Theta_{t, m} f\right|^{2} d \mu_{N}(y, t) \lesssim \int_{\mathbb{R}^{n}} \tilde{\mathcal{N}}\left(\Theta_{t, m}\right)^{2} v_{N} d y=\int_{\mathbb{R}^{n}} h_{m} v_{N} d y \lesssim \int_{\mathbb{R}^{n}} h_{m}^{p} d y
$$

Therefore, applying once again Cauchy's inequality with a parameter, we see that

$$
I I I_{\varepsilon, N} \lesssim \delta I_{\varepsilon, N}+C(\delta) \int_{\mathbb{R}^{n}} h_{m}^{p} d y
$$

Combining the estimates (5.26), (5.30) we arrive at

$$
I_{\varepsilon, N} \lesssim \int_{\mathbb{R}^{n}} \int_{\varepsilon}^{1 / \varepsilon}\left|\Theta_{t, m+1} f\right|^{2} P_{t} \nu_{N} \frac{d y d t}{t}+\int_{\mathbb{R}^{n}} H_{m}^{p} d y+I V_{\varepsilon, N}+V_{\varepsilon, N},
$$

where we notice that $I_{\varepsilon, N}<\infty$ owing to the fact that $v_{N} \leq N$ and $\sup _{t>0}\left\|\Theta_{t, m} f\right\|_{L^{2}\left(\mathbb{R}^{n}\right)}<\infty$. We now use Proposition 2.4.64 to get that $\left|P_{t} v_{N}(y)\right| \lesssim f_{|x-y|<t} v_{N} d x$ and so

$$
\int_{\mathbb{R}^{n}} \int_{\varepsilon}^{1 / \varepsilon}\left|\Theta_{t, m+1} f(y)\right|^{2} P_{t} \nu_{N}(y) \frac{d y d t}{t} \lesssim \int_{\mathbb{R}^{n}} \int_{\varepsilon}^{1 / \varepsilon} f_{|x-y|<t}\left|\Theta_{t, m+1} f(y)\right|^{2} \frac{d y d t}{t} v(x) d x
$$

Now taking first the limit as $\varepsilon \rightarrow 0$ and then as $N \rightarrow \infty$, and using (5.23), (5.25) and the previous equation we see

$$
I \lesssim \int_{\mathbb{R}^{n}} \mathbb{S}\left(\Theta_{t, m+1}\right)^{2} v d x+\int_{\mathbb{R}^{n}} H_{m}^{p} d x \lesssim \int_{\mathbb{R}^{n}} H_{m}^{p} d x
$$

The result now follows from the definition of $H_{m}$, more specifically (5.15).

Remark 5.4.34. As far as the hypotheses of the previous result are concerned, we note that we have good control on the quantities involving $\mathbb{V}$, by Theorem 5.4.1. Moreover by Theorem 4.7.12 (see also Hypothesis A in Definition 5.1.1), the conditions on the quantities 
$\left\|\Theta_{t, m} f\right\|_{L^{2}\left(\mathbb{R}^{n}\right)}$ are also satisfied. Therefore, under the same hypotheses as Theorem 5.1.5, we may rewrite the above as

$$
\left\|\mathbb{S}\left(\Theta_{t, m} f\right)\right\|_{L^{p}\left(\mathbb{R}^{n}\right)} \lesssim\left\|\mathbb{S}\left(\Theta_{t, m+1} f\right)\right\|_{L^{p}\left(\mathbb{R}^{n}\right)}+\left\|\tilde{\mathcal{N}}_{2}\left(\Theta_{t, m} f\right)\right\|_{L^{p}\left(\mathbb{R}^{n}\right)}
$$

As a Corollary of this result and Lemma 5.3.17 on the boundedness of the nontangential maximal function we have the following

Theorem 5.4.36. Suppose $\mathcal{L}$ satisfies the hypotheses of Theorem 5.1.5. Let $p \in\left(2-\varepsilon_{0}, 2+\right.$ $\left.\varepsilon_{0}\right)$, with $\varepsilon_{0}$ as in Theorem 5.1.5, then for every $f \in C_{c}^{\infty}\left(\mathbb{R}^{n}\right)$

$$
\left\|\mathbb{S}\left(t \partial_{t} \nabla \mathcal{S}_{t}^{\mathcal{L}} f\right)\right\|_{L^{p}\left(\mathbb{R}^{n}\right)} \lesssim\|f\|_{L^{p}\left(\mathbb{R}^{n}\right)}, \quad\left\|\tilde{\mathcal{N}}_{2}\left(\nabla \mathcal{S}_{t}^{\mathcal{L}} f\right)\right\|_{L^{p}\left(\mathbb{R}^{n}\right)} \lesssim\|f\|_{L^{p}\left(\mathbb{R}^{n}\right)}
$$

In addition

$$
\left\|\mathbb{S}\left(t \nabla \mathcal{D}_{t}^{\mathcal{L}} f\right)\right\|_{L^{p}\left(\mathbb{R}^{n}\right)} \lesssim\|f\|_{L^{p}\left(\mathbb{R}^{n}\right)}, \quad\left\|\tilde{\mathcal{N}}_{2}\left(\mathcal{D}_{t}^{\mathcal{L}} u\right)\right\|_{L^{p}\left(\mathbb{R}^{n}\right)} \lesssim\|f\|_{L^{p}\left(\mathbb{R}^{n}\right)}
$$

Proof. We define $\Theta_{t, m}$ to be either $t^{m} \partial_{t}^{m-1} \nabla\left(\mathcal{S}_{t}^{\mathcal{L}} \nabla\right)$ or $t^{m} \partial_{t}^{m-1} \nabla \mathcal{D}_{t}^{\mathcal{L}}$ (in case $m=1$ in the single layer, the inner gradient is also replaced by a $t$-derivative). For $p>2$ we have by Proposition 2.4.6 and Theorem 5.4.1

$$
\left\|\mathbb{S}\left(\Theta_{t, 1} f\right)\right\|_{L^{p}\left(\mathbb{R}^{n}\right)}+\left\|\mathbb{V}\left(\Theta_{t, 1} f\right)\right\|_{L^{p}\left(\mathbb{R}^{n}\right)} \lesssim\|f\|_{L^{p}\left(\mathbb{R}^{n}\right)}
$$

It remains to show the nontangential maximal function bound when $p \neq 2$, and the conical square function bound when $p<2$. We will show both the square function and nontangential maximal function bounds in the case $p<2$. The case of the non-tangential maximal 
function bounds when $p>2$ can be obtained directly from Lemma 5.3.17, owing to the fact that both the vertical and conical square functions with any amount of $t$-derivatives are under control in this range of $p$.

We treat the case of the single layer first. By Theorem 5.1.5, together with the traveling down for vertical square functions in Theorem 5.4.1 and Corollary 5.3.52 we see that for some $m_{0} \geq 1$,

$$
\left\|\mathbb{S}\left(\Theta_{t, m_{0}} f\right)\right\|_{L^{p}\left(\mathbb{R}^{n}\right)}+\left\|\tilde{\mathcal{N}}_{2}\left(\Theta_{t, m_{0}} f\right)\right\|_{L^{p}\left(\mathbb{R}^{n}\right)} \lesssim\|f\|_{L^{p}\left(\mathbb{R}^{n}\right)}
$$

We shall show that (5.40) holds with $m_{0}$ replaced by $m_{0}-1$, as long as $m_{0}-1 \geq 1$.

To treat the nontangential maximal function we appeal to Corollary 5.3 .52 to obtain

$$
\left\|\tilde{\mathcal{N}}_{2}\left(\Theta_{t, m_{0}-1}\right)\right\|_{L^{p}\left(\mathbb{R}^{n}\right)} \lesssim\left\|\mathbb{S}\left(\Theta_{t, m_{0}} f\right)\right\|_{L^{p}\left(\mathbb{R}^{n}\right)}+\sup _{t>0}\left\|\Theta_{t, m_{0}-1} f\right\|_{L^{p}\left(\mathbb{R}^{n}\right)}+\|f\|_{L^{p}\left(\mathbb{R}^{n}\right)} \lesssim\|f\|_{L^{p}\left(\mathbb{R}^{n}\right)}
$$

This gives the desired bound as long as $m_{0}-1 \geq 1$.

By the traveling down procedure for the conical square function (Lemma 5.4.7) we have (recall that the vertical square function is under control for any $m$ by Theorem 5.4.1)

$$
\begin{aligned}
\left\|S\left(\Theta_{t, m_{0}-1} f\right)\right\|_{L^{p}\left(\mathbb{R}^{n}\right)} \lesssim & \left\|\mathbb{S}\left(\Theta_{t, m_{0}} f\right)\right\|_{L^{p}\left(\mathbb{R}^{n}\right)}+\left\|\tilde{\mathcal{N}}\left(\Theta_{t, m_{0}-1} f\right)\right\|_{L^{p}\left(\mathbb{R}^{n}\right)} \\
& +\sup _{t>0}\left\|\Theta_{t, m_{0}-1}\right\|_{L^{p}\left(\mathbb{R}^{n}\right)}+\|f\|_{L^{p}\left(\mathbb{R}^{n}\right)} \lesssim\|f\|_{L^{p}\left(\mathbb{R}^{n}\right)},
\end{aligned}
$$

and this gives the desired square function bound for $m_{0}-1 \geq 1$. Therefore we have shown, by induction,

$$
\left\|\mathbb{S}\left(\Theta_{t, 1} f\right)\right\|_{L^{p}\left(\mathbb{R}^{n}\right)},\left\|\tilde{\mathcal{N}}_{2}\left(\Theta_{t, 1}\right)\right\|_{L^{p}\left(\mathbb{R}^{n}\right)} \lesssim\|f\|_{L^{p}\left(\mathbb{R}^{n}\right)}
$$


To get the bound for $\tilde{\mathcal{N}}_{2}\left(\nabla \mathcal{S}_{t}^{\mathcal{L}}\right)$ we use Proposition 5.3.1 to get

$$
\left\|\tilde{\mathcal{N}}_{2}\left(\nabla \mathcal{S}_{t}^{\mathcal{L}} f\right)\right\|_{L^{p}\left(\mathbb{R}^{n}\right)} \lesssim\left\|\tilde{\mathcal{N}}_{q}\left(\partial_{t} \mathcal{S}_{t}^{\mathcal{L}} f\right)\right\|_{L^{p}\left(\mathbb{R}^{n}\right)}+\sup _{t>0}\left\|\nabla \mathcal{S}_{t}^{\mathcal{L}} f\right\|_{L^{p}\left(\mathbb{R}^{n}\right)}
$$

for any $1 \leq q$. In particular, choosing $q<p$ we can apply directly Lemma 5.3.17 and interpolation to obtain the result.

The double layer is handled in the same way, owing to the appropriate estimates from Theorem 5.1.77, Theorem 5.2.8 and Corollary 5.3.52. 


\section{Chapter 6}

\section{Existence and Uniqueness}

In this chapter we deal with the questions of existence and uniqueness of solutions for the Dirichlet, Neumann, and Regularity problems in $L^{2}\left(\mathbb{R}^{n}\right)$ for the operator $\mathcal{L}$, starting from the assumption that $\mathcal{L}$ is a small perturbation of an operator $\mathcal{L}_{0}$ for which these results are available.

As far as existence goes, we show that, under the above perturbative assumption, we can always construct layer potential solutions with appropriate square and nontangential maximal function estimates. These results seem to be easily generalized to $L^{p}\left(\mathbb{R}^{n}\right)$ for $p$ in a window around 2 (see chapter 5), however we don't pursue such results here.

Regarding uniqueness, we prove this among a wider class of solutions; more specifically, we show that solutions to the Dirichlet problem are unique among good $\mathcal{D}$ solutions, and similarly for the Neumann and Regularity problems, among good $\mathcal{N} / \mathcal{R}$ solutions. In contrast with the existence arguments, it's not immediately clear if the methods of this section generalize to $L^{p}\left(\mathbb{R}^{n}\right)$ results. 


\subsection{Existence}

Proposition 6.1.1 (Mapping Properties, Part I). The operators $\mathcal{S}^{\mathcal{L}}: C_{c}^{\infty}\left(\mathbb{R}^{n}\right) \rightarrow S_{+}^{2}, \mathcal{D}^{\mathcal{L},+}$ : $C_{c}^{\infty}\left(\mathbb{R}^{n}\right) \rightarrow D_{+}^{2}$ both have unique continuous extensions to $L^{2}\left(\mathbb{R}^{n}\right)$; that is,

$$
\mathcal{S}^{\mathcal{L}}: L^{2}\left(\mathbb{R}^{n}\right) \rightarrow S_{+}^{2}, \quad \mathcal{D}^{\mathcal{L},+}: L^{2}\left(\mathbb{R}^{n}\right) \rightarrow D_{+}^{2}
$$

Moreover, for $f, g \in L^{2}\left(\mathbb{R}^{n}\right)$ we have that $\mathcal{S}^{\mathcal{L}} g, \mathcal{D}^{\mathcal{L},+} f \in W_{\text {loc }}^{1,2}\left(\mathbb{R}_{+}^{n+1}\right)$ are solutions of $\mathcal{L} w=0$ in $\mathbb{R}_{+}^{n+1}$, and we have the square function estimates

$$
\left\|\mathbb{S}\left(t \partial_{t} \nabla \mathcal{S}_{t}^{\mathcal{L}} g\right)\right\|_{L^{2}\left(\mathbb{R}^{n}\right)} \lesssim\|g\|_{L^{2}\left(\mathbb{R}^{n}\right)}, \quad\left\|\mathbb{S}\left(t \nabla \mathcal{D}_{t}^{\mathcal{L},+} f\right)\right\|_{L^{2}\left(\mathbb{R}^{n}\right)} \lesssim\|f\|_{L^{2}\left(\mathbb{R}^{n}\right)}
$$

Similar considerations also hold in the lower half space (in this case we work with $\mathcal{D}^{\mathcal{L},-}$ ).

The proof is a simple density argument (using the fact that $S_{+}^{2}$ and $D_{+}^{2}$ are Banach spaces), and as such is omitted.

The next result is a statement about Sobolev functions that will allow us to eventually assign boundary values to the extensions of the layer potentials defined above.

Proposition 6.1.2. Let $u \in Y^{1,2}\left(\mathbb{R}_{+}^{n+1}\right)$. The following statements hold.

(i) If $u \in D_{+}^{2}$ then $u_{0}:=\lim _{t \rightarrow 0^{+}} u(t)$ exists as a weak limit in $L^{2}\left(\mathbb{R}^{n}\right)$. Moreover $u_{0}$ agrees with the trace of $u$ in the sense that for every $\Phi \in C_{c}^{\infty}\left(\mathbb{R}^{n+1}\right)$

$$
\left(u_{0}, \Phi(\cdot, 0)\right)=\iint_{\mathbb{R}_{+}^{n+1}}\left(u \partial_{t} \Phi+\partial_{t} u \Phi\right)
$$

where $(\cdot, \cdot)$ denotes the inner product in $L^{2}\left(\mathbb{R}^{n}\right)$. 
(ii) If $u \in S_{+}^{2}$ then $U_{0}:=\lim _{t \rightarrow 0^{+}} u(t)$ exists as a weak limit in $Y^{1,2}\left(\mathbb{R}^{n}\right)$. Moreover, $U_{0}$ agrees with the trace of $u$ in the sense described in $i$ ).

Proof. To prove i), we start by noticing that since $u \in D_{+}^{2}$, there exists a subsequence $t_{k} \rightarrow 0^{+}$and a function $u_{0} \in L^{2}\left(\mathbb{R}^{n}\right)$ such that $u_{t_{k}} \rightarrow u_{0}$ weakly in $L^{2}\left(\mathbb{R}^{n}\right)$ as $k \rightarrow \infty$. Now, again since $u \in D_{+}^{2}$, we only need to show that

$$
\lim _{t \rightarrow 0^{+}}(u(t), \phi)=\left(u_{0}, \phi\right), \quad \text { for each } \phi \in C_{c}^{\infty}\left(\mathbb{R}^{n}\right) .
$$

Consider $\Phi(x, t)=\phi(x) \eta(t)$, where $\eta \in C_{c}^{\infty}(-2,2)$ is such that $\eta \equiv 1$ in $(-1,1)$, so that $\Phi \in C_{c}^{\infty}\left(\mathbb{R}^{n+1}\right)$. Now, the hypotheses imply that, for fixed $t>0$, the identity

$$
(u(t), \Phi(\cdot, t))=\iint_{\mathbb{R}^{n+1} t}\left(u \partial_{t} \Phi+\partial_{t} u \Phi\right),
$$

holds, which, for our particular choice of $\Phi$ and $t<1$, implies that

$$
(u(t), \phi)=\iint_{\mathbb{R}^{n+1} t_{t}}\left(u \partial_{t} \Phi+\partial_{t} u \Phi\right) .
$$

Hence, an application of the Dominated Convergence Theorem yields the desired result since $u \in L^{2(n+1) /(n-1)}\left(\mathbb{R}_{+}^{n+1}\right)$ and $\nabla u \in L^{2}\left(\mathbb{R}_{+}^{n+1}\right)$. The second part of the statement in i) now follows by the fact that $\lim _{t \rightarrow 0} \Phi(\cdot, t)=\Phi(\cdot, 0)$ strongly in $L^{2}\left(\mathbb{R}^{n}\right)$ for a given $\Phi \in C_{c}^{\infty}\left(\mathbb{R}^{n+1}\right)$.

The proof of ii) follows similar ideas. Arguing as before, we can prove that there exists a weak limit $U_{0} \in L^{2 n /(n-2)}\left(\mathbb{R}^{n}\right)$, and that it agrees with the usual trace in the sense described in i). Similarly, along a subsequence $t_{k} \rightarrow 0$, we have that $\nabla_{\|} u\left(t_{k}\right) \rightarrow v \in L^{2}\left(\mathbb{R}^{n}\right)$ weakly 
for some $v \in L^{2}\left(\mathbb{R}^{n}\right)^{n}$. If we can show that $v=\nabla_{\|} U_{0}$, then the result would follow from the uniqueness of the limit. To this end, we fix $\phi \in C_{c}^{\infty}\left(\mathbb{R}^{n} ; \mathbb{C}^{n}\right)$ and compute that

$$
(v, \phi)=\lim _{k \rightarrow \infty}\left(\nabla_{\|} u\left(t_{k}\right), \phi\right)=-\lim _{k \rightarrow \infty}\left(u\left(t_{k}\right), \operatorname{div}_{\|} \phi\right)=-\left(U_{0}, \operatorname{div}_{\|} \phi\right),
$$

as desired.

Combining the two previous propositions, we arrive at the following corollary whose proof is standard and thus omitted.

Corollary 6.1.3. For every $f, g \in L^{2}\left(\mathbb{R}^{n}\right)$ we can define the bounded linear operators

$$
\mathcal{S}_{0}^{\mathcal{L}}: L^{2}\left(\mathbb{R}^{n}\right) \rightarrow Y^{1,2}\left(\mathbb{R}^{n}\right), \quad \mathcal{D}_{0}^{\mathcal{L},+}: L^{2}\left(\mathbb{R}^{n}\right) \rightarrow L^{2}\left(\mathbb{R}^{n}\right)
$$

given by

$$
\mathcal{S}_{0}^{\mathcal{L}} g:=\lim _{t \rightarrow 0^{+}} \mathcal{S}_{t}^{\mathcal{L}} g, \quad \mathcal{D}_{0}^{\mathcal{L},+} f:=\lim _{t \rightarrow 0+} \mathcal{D}_{t}^{\mathcal{L},+} f
$$

where both are weak limits, the first being in $Y^{1,2}\left(\mathbb{R}^{n}\right)$ and the second in $L^{2}\left(\mathbb{R}^{n}\right)$.

We may remove the condition that $u \in Y^{1,2}\left(\mathbb{R}_{+}^{n+1}\right)$ for solutions satisfying trace decay at infinity.

Proposition 6.1.4. Suppose that $u \in W_{\mathrm{loc}}^{1,2}\left(\mathbb{R}_{+}^{n+1}\right) \cap S_{+}^{2}$ satisfies that $\mathcal{L} u=0$ in $\mathbb{R}_{+}^{n+1}$. Then there exists $u_{0} \in Y^{1,2}\left(\mathbb{R}^{n}\right)$ such that $\lim _{t \rightarrow 0^{+}} u(t)=u_{0}$ exists weakly in $Y^{1,2}\left(\mathbb{R}^{n}\right)$. Moreover, since $u \in W_{\text {loc }}^{1,2}\left(\mathbb{R}_{+}^{n+1}\right) \cap S_{+}^{2} \subset W^{1,2}\left(I_{R}^{+}\right)$for any $R>0$, the trace $\operatorname{Tr}_{0} u$ exists as an element of $L_{\text {loc }}^{2}\left(\mathbb{R}^{n}\right)$, and $\operatorname{Tr}_{0} u=u_{0}$ as distributions. In particular, the conclusion holds for $u=\mathcal{S}^{\mathcal{L}} g$ for $g \in L^{2}\left(\mathbb{R}^{n}\right)$ or $u=\mathcal{D}^{\mathcal{L},+} f$ with $f \in Y^{1,2}\left(\mathbb{R}^{n}\right)$ (see Corollary 6.1.9). 
Proof. Since $u \in S_{+}^{2}$ there exists a subsequence $t_{k} \rightarrow 0^{+}$and $u_{0} \in L^{2 n /(n-2)}\left(\mathbb{R}^{n}\right)$ such that $\lim _{k \rightarrow \infty} u\left(t_{k}\right)=u_{0}$ weakly in $L^{2 n /(n-2)}\left(\mathbb{R}^{n}\right)$. Now, since $u \in Y^{1,2}\left(\Sigma_{0}^{b}\right)$ for any $b>0$, we have that for each $\Phi \in C_{c}^{\infty}\left(\mathbb{R}^{n+1}\right)$, there exists $b>0$ such that

$$
\left(u\left(t_{k}\right), \Phi\left(t_{k}\right)\right)=-\iint_{\Sigma_{t_{k}}^{b}}\left(u D_{n+1} \Phi+D_{n+1} u \Phi\right) .
$$

Fixing $\phi \in C_{c}^{\infty}\left(\mathbb{R}^{n}\right)$ and extending it to $\mathbb{R}^{n+1}$ so that $\Phi(\cdot, t) \equiv \phi(\cdot)$ in a neighborhood of $t=0$, we see that

$$
\left(u_{0}, \phi\right)=-\iint_{\Sigma_{0}^{b}}\left(u D_{n+1} \Phi+D_{n+1} u \Phi\right),
$$

which gives the uniqueness of the limit $u_{0}$. Therefore, $\lim _{t \rightarrow 0} u(t)=u_{0}$ exists as a weak limit in $L^{2 n /(n-2)}\left(\mathbb{R}^{n}\right)$. To see that $u_{0} \in Y^{1,2}\left(\mathbb{R}^{n}\right)$, we proceed as follows: Since for any weak limit $v$ in $L^{2}\left(\mathbb{R}^{n}\right)$ of $\nabla_{\|} u$, we have that for any $\phi \in C_{c}^{\infty}\left(\mathbb{R}^{n} ; \mathbb{C}^{n}\right)$, the identity

$$
(v, \phi)=\lim _{k \rightarrow \infty}\left(\nabla_{\|} u\left(t_{k}\right), \phi\right)=-\lim _{k \rightarrow \infty}\left(u\left(t_{k}\right), \operatorname{div}_{\|} \phi\right)=-\left(u_{0}, \operatorname{div}_{\|} \phi\right),
$$

holds, we conclude that $v=\nabla_{\|} u_{0}$. This shows that the weak limits are unique and thus, since $u \in S_{+}^{2}$, the full limit exists; that is, $\lim _{t \rightarrow 0} \nabla_{\|} u(t)=\nabla_{\|} u_{0}$ weakly in $L^{2}\left(\mathbb{R}^{n}\right)$. Now, we recall that every element $\ell \in Y^{1,2}\left(\mathbb{R}^{n}\right)^{*}$ can be written in the form

$$
\ell(w)=\int_{\mathbb{R}^{n}}\left(\psi_{0} w+\psi \cdot \nabla_{\|} w\right), \quad \text { for all } w \in Y^{1,2}\left(\mathbb{R}^{n}\right),
$$

for some $\psi_{0} \in L^{2 n /(n+2)}\left(\mathbb{R}^{n}\right)$ and $\psi=\left(\psi_{1}, \ldots, \psi_{n}\right) \in L^{2}\left(\mathbb{R}^{n}\right)^{n}$. This gives that $u(t) \rightarrow u_{0}$ weakly in $Y^{1,2}\left(\mathbb{R}^{n}\right)$. 
We now turn to the proof of the final statement in the proposition. Since for every $\Phi(\cdot, t) \in C_{c}^{\infty}\left(\mathbb{R}^{n+1}\right)$ we have that $\Phi(\cdot, t) \rightarrow \Phi(\cdot, 0)$ strongly in $L^{2}\left(\mathbb{R}^{n}\right)$, we need only check that $\lim _{t \rightarrow 0}(u(t), \Phi(t))=\left(u_{0}, \Phi(0)\right)$. Along these lines it is enough to prove that

$$
(u(t), \Phi(t))=-\iint_{\mathbb{R}^{n+1}>t}\left(u D_{n+1} \Phi+D_{n+1} u \Phi\right), \quad \text { for all } t>0,
$$

but this in turn follows from Proposition 2.2.13, since $u \in W_{\mathrm{loc}}^{1,2}\left(\Sigma_{t / 2}^{\infty}\right)$.

Proposition 6.1.5 (Conormal derivative of solutions in slice spaces). Suppose that $u \in$ $W_{\text {loc }}^{1,2}\left(\mathbb{R}_{+}^{n+1}\right) \cap S_{+}^{2}$ satisifies $\mathcal{L} u=0$ in $\mathbb{R}_{+}^{n+1}$. Then there exists $g$ in $L^{2}\left(\mathbb{R}^{n}\right)$ such that

$$
(g, \phi)=\iint_{\mathbb{R}_{+}^{n+1}}\left(\left(A \nabla u+B_{1}\right) \overline{\nabla \Phi}+B_{2} \cdot \nabla u \bar{\Phi}\right), \quad \text { for all } \Phi \in C_{c}^{\infty}\left(\mathbb{R}^{n+1}\right),
$$

where $\phi(\cdot)=\Phi(\cdot, 0)$. We write $g=\partial_{\mathcal{L}^{\mathcal{L}},+} u$. Moreover, $g=\lim _{t \rightarrow 0^{+}}-e_{n+1} \cdot \operatorname{Tr}_{t}\left(A \nabla u+B_{1} u\right)$, where the limit is taken in the weak sense in the space $L^{2}\left(\mathbb{R}^{n}\right)$. In particular, this notion of the conormal derivative agrees with our previous definition in $Y^{1,2}\left(\mathbb{R}_{+}^{n+1}\right)$ whenever both exist.

Proof. We follow the proof of $\left[\mathrm{AAA}^{+} 11\right.$, Lemma 4.3 (iii)]. We will first show that for any $R>0$, there exists $g_{R} \in\left(C_{c}^{\infty}\left(\Delta_{R}\right)\right)^{*}$ such that for any $\Phi \in C_{c}^{\infty}\left(I_{R}\right)$,

$$
\left\langle g_{R}, \Phi(\cdot, 0)\right\rangle=\iint_{\mathbb{R}_{+}^{n+1}}\left(\left(A \nabla u+B_{1}\right) \cdot \bar{\nabla} \Phi+B_{2} \cdot \nabla u \bar{\Phi}\right)
$$

In particular, this allows us to define $g \in\left(C_{c}^{\infty}\left(\mathbb{R}^{n}\right)\right)^{*}$ such that $g=\lim _{R \uparrow \infty} g_{R}$ in the sense of distributions and (6.6) holds for any $\Phi \in C_{c}^{\infty}\left(\mathbb{R}^{n+1}\right)$ and $g$ in place of $g_{R}$. Thus, fix $R>0$, 
$\phi \in C_{c}^{\infty}\left(\Delta_{R}\right)$, and $\Phi \in W_{0}^{1,2}\left(I_{R}\right)$ any extension of $\phi$ (that is, $\operatorname{Tr}_{0} \Phi=\phi$ ). We define the (anti-)linear functional $\Lambda_{R}: C_{c}^{\infty}\left(\Delta_{R}\right) \rightarrow \mathbb{C}$ by

$$
\Lambda_{R}(\phi)=\iint_{\mathbb{R}_{+}^{n+1}}\left(\left(A \nabla u+B_{1} u\right) \cdot \overline{\nabla \Phi}+B_{2} \cdot \nabla u \bar{\Phi}\right) .
$$

To see this is indeed well-defined, that is, it does not depend on the extension $\Phi$, we simply note that for any two extensions $\Phi_{1}, \Phi_{2}$, we have that $\Phi_{1}-\Phi_{2} \in W_{0}^{1,2}\left(I_{R}^{+}\right)$, and $u \in S_{+}^{2}$ solves $\mathcal{L} u=0$ in $\mathbb{R}_{+}^{n+1}$. Now, as in the proof of the Lax-Milgram theorem, we have that $\left|\Lambda_{R}(\phi)\right| \lesssim$ $\|\nabla u\|_{L^{2}\left(\Sigma_{0}^{R}\right)}\|\nabla \Phi\|_{L^{2}\left(I_{R}^{+}\right)}$. Construct $\Phi$ to satisfy that $\Delta \Phi=0$ in $I_{R}^{+}, \Phi=\varphi$ on $\Delta_{R}$, and $\Phi=0$ on $\partial I_{R} \cap \mathbb{R}_{+}^{n+1}$. In this case, we have that $\|\nabla \Phi\|_{L^{2}\left(I_{R}^{+}\right)} \lesssim\|\phi\|_{\dot{H}^{1 / 2}\left(\Delta_{R}\right)}$ by the usual extension theorem. Combining these last two estimates, we arrive at $\left|\Lambda_{R}(\phi)\right| \lesssim\|\nabla u\|_{L^{2}\left(\Sigma_{0}^{R}\right)}\|\phi\|_{\dot{H}^{1 / 2}\left(\Delta_{R}\right)}$, whence via the Riesz representation theorem there exists $g_{R} \in\left(\dot{H}^{1 / 2}\left(\Delta_{R}\right)\right)^{*}$ such that $\left\langle g_{R}, \phi\right\rangle=\Lambda_{R}(\phi)$ for each $\phi \in C_{c}^{\infty}\left(\Delta_{R}\right)$. From the definition of $\Lambda_{R}$, we see that the restriction of $g_{R}$ to $\Delta_{R^{\prime}}$ equals $g_{R^{\prime}}$ whenever $R^{\prime}<R$. In particular, this allows us to define a distribution $g$ such that

$$
\langle g, \phi\rangle=\iint_{\mathbb{R}_{+}^{n+1}}\left(\left(A \nabla u+B_{1}\right) \overline{\nabla \Phi}+B_{2} \cdot \nabla u \bar{\Phi}\right),
$$

for all $\Phi \in C_{c}^{\infty}\left(\mathbb{R}^{n+1}\right)$ with $\Phi(\cdot, 0)=\phi$. It remains to show that $g \in L^{2}\left(\mathbb{R}^{n}\right)$. For this, note that via the previous procedure we can define a conormal at height $t \geq 0$, which we denote as $g^{t}$, as the distribution which satisfies

$$
\left\langle g^{t}, \Phi^{t}\right\rangle=\iint_{\mathbb{R}_{t}^{n+1}}\left(\left(A \nabla u+B_{1}\right) \overline{\nabla \Phi}+B_{2} \cdot \nabla u \bar{\Phi}\right),
$$

for all $\Phi \in C_{c}^{\infty}\left(\mathbb{R}^{n+1}\right)$ where $\Phi^{t}(\cdot)=\Phi(\cdot, t)$. This formula shows that the conormal $\partial_{v, t}^{\mathcal{L},+} u$ in Definition 3.1.9 agrees with $g^{t}$, as distributions in $\mathbb{R}^{n}$. In particular, from the proof of 
Lemma 3.1.10 (i) we see that, for any $t>0, g^{t} \in L^{2}\left(\mathbb{R}^{n}\right)$ and $g^{t}=-e_{n+1} \cdot \operatorname{Tr}_{t}\left(A \nabla u+B_{1} u\right)$. Moreover, since $u \in S_{+}^{2}$, we have that $\left\|g^{t}\right\|_{L^{2}\left(\mathbb{R}^{n}\right)} \lesssim\|u\|_{S_{+}^{2}}$. By weak compactness, we can extract a subsequence $t_{k} \rightarrow 0$ and $\tilde{g}$ such that $g^{t_{k}} \rightarrow \tilde{g}$ weakly in $L^{2}\left(\mathbb{R}^{n}\right)$. From (6.7) it is then easy to see that $\tilde{g}=g^{0}=g$ as distributions, and the result follows.

We now take the first step towards proving existence of layer potential solutions, by proving the appropriate so-called jump relations for the Double Layer and the conormal derivative of the Single Layer.

Lemma 6.1.8 (Jump Relations). There exist bounded linear operators $K, \tilde{K}: L^{2}\left(\mathbb{R}^{n}\right) \rightarrow$ $L^{2}\left(\mathbb{R}^{n}\right)$ such that for every $f, g \in L^{2}\left(\mathbb{R}^{n}\right)$ we have that $\left( \pm \frac{1}{2} I+\tilde{K}\right) g=\partial_{v}^{\mathcal{L}, \pm}\left(\mathcal{S}^{\mathcal{L}} g\right)$, and $\left(\mp \frac{1}{2} I+K\right) f=\mathcal{D}_{0}^{\mathcal{L}, \pm} f$.

Proof. First, we note that by Proposition 3.2.7, we can define operators $K: H_{0}^{1 / 2}\left(\mathbb{R}^{n}\right) \rightarrow$ $H_{0}^{1 / 2}\left(\mathbb{R}^{n}\right), \tilde{K}: H^{-1 / 2}\left(\mathbb{R}^{n}\right) \rightarrow H^{-1 / 2}\left(\mathbb{R}^{n}\right)$, such that the identities in the lemma are satisfied for $f, g \in C_{c}^{\infty}\left(\mathbb{R}^{n}\right)$. Moreover, by Propositions 6.1.1 and 6.1.5, we obtain that $K, \tilde{K}$ are $L^{2}\left(\mathbb{R}^{n}\right)$ bounded (that is, admit a unique linear, continuous extension to $L^{2}\left(\mathbb{R}^{n}\right)$ ); the result now follows via a density argument.

Corollary 6.1.9 (Additional mapping property of $\mathcal{D})$. Suppose $\mathcal{L}$ satisfies Hypothesis A (see Definition 5.1.1). Assume further that the operator $\left(\mathcal{S}_{0}^{\mathcal{L}}\right)^{-1}: Y^{1,2}\left(\mathbb{R}^{n}\right) \rightarrow L^{2}\left(\mathbb{R}^{n}\right)$ exists and is bounded. Then we have

$$
\sup _{t>0}\left\|\mathcal{D}_{t}^{\mathcal{L},+} f\right\|_{Y^{1,2}\left(\mathbb{R}^{n}\right)} \lesssim\|f\|_{Y^{1,2}\left(\mathbb{R}^{n}\right)}
$$


with implicit constants depending on dimension, ellipticity of $\mathcal{L}$, and the norm of $\left(\mathcal{S}_{0}^{\mathcal{L}}\right)^{-1}$.

Proof. We know by Theorem 6.1.34 that the map

$$
\mathcal{S}_{0}^{\mathcal{L}}: L^{2}\left(\mathbb{R}^{n}\right) \rightarrow Y^{1,2}\left(\mathbb{R}^{n}\right)
$$

is bounded and invertible. In particular we have that the set

$$
\mathcal{F}:=\left\{f \in Y^{1,2}\left(\mathbb{R}^{n}\right): f=\mathcal{S}_{0}^{\mathcal{L}} \psi, \psi \in C_{c}^{\infty}\left(\mathbb{R}^{n}\right)\right\}
$$

is dense in $Y^{1,2}\left(\mathbb{R}^{n}\right)$. We note that for $f \in \mathcal{F}$ we have $f \in H_{0}^{1 / 2}\left(\mathbb{R}^{n}\right) \cap Y^{1,2}\left(\mathbb{R}^{n}\right)$ by $\left[\mathrm{BHL}^{+} \mathrm{a}\right.$, Proposition 4.7 (iii)]. For such an $f$ and $\psi:=\left(\mathcal{S}_{0}^{\mathcal{L}}\right)^{-1} f$ we set

$$
u(\cdot, \tau):=\mathcal{S}_{\tau}^{\mathcal{L}} \psi, \quad \tau<0
$$

Then by Theorem 3.2.1 (iv), applied to $u$ in $\mathbb{R}^{n+1}$, we have

$$
\mathcal{D}^{\mathcal{L},+}(f)=-\mathcal{S}^{\mathcal{L}}\left(\partial_{\nu} \mathcal{L}, u\right), \quad \text { in } \mathbb{R}_{+}^{n+1} .
$$

Now recall from the jump relations (see Proposition 3.2.7(ii)) that

$$
\partial_{\nu^{\mathcal{L}},-}=\left(-\frac{1}{2} I+\tilde{K}\right) \psi, \quad \text { in } H^{-1 / 2}\left(\mathbb{R}^{n}\right),
$$

so that, using the definition of $u$, (6.14) becomes

$$
\mathcal{D}^{\mathcal{L},+}(f)=-\mathcal{S}^{\mathcal{L}}\left(\left(-\frac{1}{2} I+\tilde{K}\right) \psi\right)=-\mathcal{S}^{\mathcal{L}}\left(\left(-\frac{1}{2} I+\tilde{K}\right)\left(\mathcal{S}_{0}^{\mathcal{L}}\right)^{-1} f\right) .
$$

Finally from Proposition 6.1.1 and Theorem 6.1.34 we know the following maps are bounded

$$
\begin{gathered}
\mathcal{S}^{\mathcal{L}}: L^{2}\left(\mathbb{R}^{n}\right) \rightarrow S_{+}^{2}, \quad\left(-\frac{1}{2} I+\tilde{K}\right): L^{2}\left(\mathbb{R}^{n}\right) \rightarrow L^{2}\left(\mathbb{R}^{n}\right), \\
\left(\mathcal{S}_{0}^{\mathcal{L}}\right)^{-1}: Y^{1,2}\left(\mathbb{R}^{n}\right) \rightarrow L^{2}\left(\mathbb{R}^{n}\right),
\end{gathered}
$$


which gives the desired bound

$$
\left\|\mathcal{D}^{\mathcal{L},+}(f)\right\|_{S_{+}^{2}} \lesssim\|f\|_{Y^{1,2}\left(\mathbb{R}^{n}\right)}, \quad f \in \mathcal{F} .
$$

We conclude the claimed inequality from the density of $\mathcal{F}$ in $Y^{1,2}\left(\mathbb{R}^{n}\right)$.

Proposition 6.1.19. Suppose $\mathcal{L}$ satisfies Hypothesis A (see Definition 5.1.1). Assume further that $\left(\mathcal{S}_{0}^{\mathcal{L}}\right)^{-1}: Y^{1,2}\left(\mathbb{R}^{n}\right) \rightarrow L^{2}\left(\mathbb{R}^{n}\right)$ exists and is bounded. Let $f \in Y^{1,2}\left(\mathbb{R}^{n}\right)$, then the operator K from Lemma 6.1.8 extends to a bounded operator from $Y^{1,2}\left(\mathbb{R}^{n}\right)$ to itself. Moreover we retain the jump relation

$$
\left(-\frac{1}{2} I+K\right) f=\lim _{t \rightarrow 0} \mathcal{D}_{t}^{\mathcal{L},+}(f)
$$

where the limit on the right is a weak limit in $Y^{1,2}\left(\mathbb{R}^{n}\right)$.

Proof. The proof of the first statement follows from Corollary 6.1.9, which together with Proposition 6.1.4 guarantees the existence of a weak limit in $Y^{1,2}\left(\mathbb{R}^{n}\right)$ for $f \in C_{c}^{\infty}\left(\mathbb{R}^{n}\right)$, and Lemma 6.1.8 which gives the desired identity.

Lemma 6.1.21 (Additional mapping property of $\mathcal{S}$ ). Suppose $\mathcal{L}$ satisfies Hypothesis A (see Definition 5.1.1). Assume further that the inverse operators $\left(\mathcal{S}_{0}^{\mathcal{L}^{*}}\right)^{-1},\left(\mathcal{S}_{0}^{\mathcal{L}}\right)^{-1}: L^{2}\left(\mathbb{R}^{n}\right) \rightarrow$ $\left[Y^{1,2}\left(\mathbb{R}^{n}\right)\right]^{*}$ and

$$
\left(-\frac{1}{2}+K\right)^{-1}: L^{2}\left(\mathbb{R}^{n}\right) \rightarrow L^{2}\left(\mathbb{R}^{n}\right)
$$


exist and are bounded. Then the operator $\mathcal{S}$ extends as a bounded operator $\mathcal{S}:\left[Y^{1,2}\left(\mathbb{R}^{n}\right)\right]^{*} \rightarrow$ $D_{+}^{2}$, that is,

$$
\sup _{t>0}\left\|\mathcal{S}_{t}^{\mathcal{L}} g\right\|_{L^{2}\left(\mathbb{R}^{n}\right)} \lesssim\|g\|_{\left[Y^{1,2}\left(\mathbb{R}^{n}\right)\right]^{*}},
$$

with implicit constants depending on dimension, ellipticity of $\mathcal{L}$ and the norm of $\left(\mathcal{S}_{0}^{\mathcal{L}}\right)^{-1},\left(\mathcal{S}_{0}^{\mathcal{L}}\right)^{-1}$.

Proof. Notice that, by the mapping properties of $-\frac{1}{2} I+K$ (see Corollary 6.1.9), and using the smallness of $\left\|B_{i}\right\|_{L^{n}\left(\mathbb{R}^{n}\right)}$, we obtain that

$$
-\frac{1}{2} I+\tilde{K}: Y^{1,2}\left(\mathbb{R}^{n}\right) \rightarrow Y^{1,2}\left(\mathbb{R}^{n}\right)
$$

is bounded and invertible. From this and the Green's Formula (see Theorem 3.2.1(iv)) we have that for any $g \in C_{c}^{\infty}\left(\mathbb{R}^{n}\right)$

$$
\mathcal{D}^{\mathcal{L},+}\left(\mathcal{S}_{0}^{\mathcal{L}} g\right)=-\mathcal{S}^{\mathcal{L}}\left(\left(-\frac{1}{2} I+\tilde{K}\right) g\right) .
$$

By Proposition 6.1.19 and Corollary 6.1.3 we have that, taking weak limits in $Y^{1,2}\left(\mathbb{R}^{n}\right)$ as $t \rightarrow 0$

$$
\left(-\frac{1}{2} I+K\right)\left(\mathcal{S}_{0}^{\mathcal{L}} g\right)=-\mathcal{S}_{0}^{\mathcal{L}}\left(-\frac{1}{2} I+\tilde{K}\right) g,
$$

or equivalently

$$
-\left(\mathcal{S}_{0}^{\mathcal{L}}\right)^{-1}\left(-\frac{1}{2} I+K\right) \mathcal{S}_{0}^{\mathcal{L}} g=\left(-\frac{1}{2} I+\tilde{K}\right) g=: h,
$$

which means, using the corresponding mapping properties for $-(1 / 2) I+K$ and $\mathcal{S}_{0}^{\mathcal{L}}$ (see Proposition 6.1.19 and the fact that $\left.\operatorname{adj}\left(\mathcal{S}_{0}^{\mathcal{L}}\right)=\mathcal{S}_{0}^{\mathcal{L}^{*}}\right)$, that we can extend

$$
-\frac{1}{2} I+\tilde{K}: Y^{1,2}\left(\mathbb{R}^{n}\right)^{*} \rightarrow Y^{1,2}\left(\mathbb{R}^{n}\right)^{*}
$$


as a bounded and, with smallness of $\left\|B_{i}\right\|_{L^{n}\left(\mathbb{R}^{n}\right)}$, invertible operator. In particular $\|g\|_{Y^{1,2}\left(\mathbb{R}^{n}\right)^{*}} \approx$ $\|h\|_{Y^{1,2}\left(\mathbb{R}^{n}\right)^{*}}$. Using this in (6.14) we arrive at the fact that, for $g \in C_{c}^{\infty}\left(\mathbb{R}^{n}\right)$ it holds $\mathcal{S}^{\mathcal{L}} h \in D_{+}^{2}$ and

$$
\left\|\mathcal{S}_{t}^{\mathcal{L}} h\right\|_{D_{+}^{2}} \lesssim\left\|\mathcal{S}_{0}^{\mathcal{L}} g\right\|_{L^{2}\left(\mathbb{R}^{n}\right)} \lesssim\|g\|_{Y^{1,2}\left(\mathbb{R}^{n}\right)^{*}} \approx\|h\|_{Y^{1,2}\left(\mathbb{R}^{n}\right)^{*}}
$$

Since the set

$$
\left\{h=\left(-\frac{1}{2} I+\tilde{K}\right) g: g \in C_{c}^{\infty}\left(\mathbb{R}^{n}\right)\right\}
$$

is dense in $Y^{1,2}\left(\mathbb{R}^{n}\right)^{*}$ we conclude the desired property by a density argument.

Definition 6.1.30 (Hypothesis B). We will say $\mathcal{L}$ satisfies Hypothesis B if the following properties hold.

1. $\mathcal{L}$ satisfies Hypothesis A, along with the hypotheses of Theorem 5.4.1 and Theorem 5.4 .36 .

2. The following operators are invertible

$$
\mathcal{S}_{0}^{\mathcal{L}^{*}}, \mathcal{S}_{0}^{\mathcal{L}}: L^{2}\left(\mathbb{R}^{n}\right) \rightarrow Y^{1,2}\left(\mathbb{R}^{n}\right), \quad-\frac{1}{2}+K: L^{2}\left(\mathbb{R}^{n}\right) \rightarrow L^{2}\left(\mathbb{R}^{n}\right) .
$$

3. The following operators are invertible

$$
\begin{aligned}
& \pm \frac{1}{2}+K: Y^{1,2}\left(\mathbb{R}^{n}\right) \rightarrow Y^{1,2}\left(\mathbb{R}^{n}\right), \\
& \pm \frac{1}{2}+\tilde{K}: L^{2}\left(\mathbb{R}^{n}\right) \rightarrow L^{2}\left(\mathbb{R}^{n}\right) .
\end{aligned}
$$


The first condition in Hypothesis B ensures that we have the right square and nontangential maximal function estimates in $L^{p}\left(\mathbb{R}^{n}\right)$ for the layer potentials associated to $\mathcal{L}$ and $\mathcal{L}^{*}$. In particular the first condition implies that the objects in item (2) are well-defined and bounded (not necessarily invertible in general). The objects in item (2), more specifically their inverses, are used in the previous Propositions to define the objects in (3); this is the reason for the statement to be written in this way.

Theorem 6.1.34 (Invertibility of Layer Potentials). Suppose $\mathcal{L}_{0}$ satisfies hypothesis B (see Definition 6.1.30), with coefficients $A^{0}, B_{i}^{0}$ for $i=1,2$, and let $\mathcal{L}_{1}$ be defined by

$$
\mathcal{L}_{1}=-\operatorname{div}\left(\left(A^{0}+M\right) \nabla+\left(B_{1}^{0}+B_{1}\right)\right)+\left(B_{2}^{0}+B_{2}\right) \cdot \nabla
$$

There exists $\rho>0$ depending on dimension, ellipticity of $\mathcal{L}_{0}$, and the norms of the inverse operators in item (2) of Hypothesis B with the property that if

$$
\max \left\{\|M\|_{L^{\infty}\left(\mathbb{R}^{n}\right)},\left\|B_{1}\right\|_{L^{n}\left(\mathbb{R}^{n}\right)},\left\|B_{2}\right\|_{L^{n}\left(\mathbb{R}^{n}\right)}\right\}<\rho
$$

then $\mathcal{L}_{1}$ satisfies Hypothesis $B$.

Proof. Set $\|M\|_{\infty}=1$ and $\left\|B_{i}\right\|_{n}=1, i=1,2$, and then define the operator

$$
\mathcal{L}_{z} u:=-\operatorname{div}\left((A+z M) \nabla u+\left(B_{1}^{0}+z B_{1}\right) u\right)+\left(B_{2}^{0}+z B_{2}\right) \cdot \nabla u, \quad z \in \mathbb{C}, u \in Y^{1,2}\left(\mathbb{R}^{n+1}\right) .
$$

We write $\mathcal{L}_{z}=\mathcal{L}_{0}-z \mathcal{M}$. The idea will be to show that $K_{z}, \tilde{K}_{z}$ and $\mathcal{S}_{0}^{\mathcal{L}_{z}}$ are analytic in $z$ in a neighborhood of the origin. Note that, by Lax-Milgram, $\mathcal{L}_{0}$ is always invertible, and thus there exists $\varepsilon_{0}$ such that if $z \in B_{\varepsilon_{0}}=B\left(0, \varepsilon_{0}\right)$, then $\mathcal{L}_{z}$ is also invertible, 
and moreover $\mathcal{L}_{z}^{-1}=\mathcal{L}_{0}^{-1} \sum_{k=0}^{\infty}\left(z \mathcal{M} \mathcal{L}_{0}^{-1}\right)^{k}$, the series converging in the operator norm of $\mathcal{B}\left(Y^{1,2}\left(\mathbb{R}^{n+1}\right)^{*} ; Y^{1,2}\left(\mathbb{R}^{n+1}\right)\right)$. In particular, the map $z \mapsto \mathcal{L}_{z}^{-1}$ is analytic in $B_{\varepsilon_{0}}$. Now fix $t \geq 0$. By definition of the single layer, we conclude that $\mathcal{S}_{t}^{\mathcal{L}_{z}}$ is also analytic with values in $\mathcal{B}\left(H^{-1 / 2}\left(\mathbb{R}^{n}\right) ; H_{0}^{1 / 2}\left(\mathbb{R}^{n}\right)\right)$. Since $\nabla_{\|}: H_{0}^{1 / 2}\left(\mathbb{R}^{n}\right) \rightarrow H^{-1 / 2}\left(\mathbb{R}^{n}\right)$, we have that $\nabla_{\|} \mathcal{S}_{t}^{\mathcal{L}_{z}}$ is analytic in $B_{\varepsilon_{0}}$ with values in $\mathcal{B}\left(H^{-1 / 2}\left(\mathbb{R}^{n}\right) ; H^{-1 / 2}\left(\mathbb{R}^{n}\right)\right)$. Thus, for $f \in C_{c}^{\infty}\left(\mathbb{R}^{n}\right)$ and $g \in C_{c}^{\infty}\left(\mathbb{R}^{n} ; \mathbb{C}^{n}\right)$, we have that the map $z \mapsto\left(\nabla_{\|} \mathcal{S}_{t}^{\mathcal{L}_{z}} f, g\right)$ is analytic, and

$$
\sup _{z \in B_{\varepsilon_{0}}} \sup _{t \geq 0}\left\|\mathcal{S}_{t}^{\mathcal{L}_{z}}\right\|_{L^{2}\left(\mathbb{R}^{n}\right) \rightarrow Y^{1,2}\left(\mathbb{R}^{n}\right)} \lesssim 1
$$

It follows by [Kat95, Theorem 3.12] that the map $z \mapsto \mathcal{S}_{t}^{\mathcal{L}_{z}}$ is holomorphic with values in $\mathcal{B}\left(L^{2}\left(\mathbb{R}^{n}\right) ; Y^{1,2}\left(\mathbb{R}^{n}\right)\right)$, for any $t \geq 0$. In particular, we have that $\mathcal{S}_{0}^{\mathcal{L}_{z}}$ is analytic. Similarly, $\partial_{v}^{\mathcal{L}_{z},+} \mathcal{S}^{\mathcal{L}_{z}}$ is analytic with values in $\mathcal{B}\left(H^{-1 / 2}\left(\mathbb{R}^{n}\right) ; H^{-1 / 2}\left(\mathbb{R}^{n}\right)\right)$, and for $f, g \in C_{c}^{\infty}\left(\mathbb{R}^{n}\right)$, we have that

$$
\sup _{z \in B_{\varepsilon_{0}}}\left\|\partial_{v}^{\mathcal{L}_{2},+} \mathcal{S}^{\mathcal{L}_{2}}\right\|_{L^{2}\left(\mathbb{R}^{n}\right) \rightarrow L^{2}\left(\mathbb{R}^{n}\right)} \lesssim 1,
$$

and the map $z \mapsto\left(\partial_{\nu} \mathcal{L}_{z}, \mathcal{S}^{\mathcal{L}_{z}} f, g\right)=\lim _{t \rightarrow 0}\left(A \nabla \mathcal{S}_{t}^{\mathcal{L}_{z}} f+z B_{1} \mathcal{S}_{t}^{\mathcal{L}_{z}} f, g\right)$ is analytic. Thus we obtain that $\partial_{v}^{\mathcal{L}_{z},+}\left(\mathcal{S}^{\mathcal{L}_{z}}\right)$ is analytic with values in $\mathcal{B}\left(L^{2}\left(\mathbb{R}^{n}\right)\right)$. By the jump relations in Lemma 6.1.8, $\tilde{K}_{z}$ is also analytic with values in $\mathcal{B}\left(L^{2}\left(\mathbb{R}^{n}\right)\right)$. The analyticity of $K_{z}$ follows from that of $\tilde{K}_{z}$ by noting that $\left\langle\mathcal{D}_{0}^{\mathcal{L}_{z},+} f, g\right\rangle=\left\langle f, \partial_{\nu}^{\mathcal{L}_{2}^{*},+} \mathcal{S}_{z}^{\mathcal{L}_{z}^{*}} g\right\rangle-\langle f, g\rangle$ for $f, g \in C_{c}^{\infty}\left(\mathbb{R}^{n}\right)$ (see Proposition 3.2.3(ii)).

We have thus shown that the maps $z \mapsto \mathcal{S}_{0}^{\mathcal{L}_{z}}, z \mapsto K_{z}, z \mapsto \tilde{K}_{z}$ are all analytic. Therefore, 
by the Cauchy integral formula, we obtain that

$$
\sup _{z \in B_{\varepsilon_{0}} / 2}\left\|\frac{d}{d z} \tilde{K}_{z}\right\|_{L^{2}\left(\mathbb{R}^{n}\right) \rightarrow L^{2}\left(\mathbb{R}^{n}\right)} \lesssim_{\varepsilon_{0}} \sup _{z \in B_{\varepsilon_{0}}}\left\|\tilde{K}_{z}\right\|_{L^{2}\left(\mathbb{R}^{n}\right) \rightarrow L^{2}\left(\mathbb{R}^{n}\right)} \lesssim_{\varepsilon_{0}} 1
$$

where we used (6.37). Consequently, for any $z, w \in B_{\varepsilon_{0} / 2}$, we have that $\left\|\tilde{K}_{z}-\tilde{K}_{w}\right\|_{L^{2}\left(\mathbb{R}^{n}\right) \rightarrow L^{2}\left(\mathbb{R}^{n}\right)} \lesssim|z-w|$. This implies that for all $z$ small enough, $\tilde{K}_{z}$ is invertible. The other boundary operators are treated similarly.

Theorem 6.1.38 (Existence of Solutions). Suppose $\mathcal{L}$ satisfies Hypothesis B (see Definition 6.1.30). Then (D2), (R2), (N2), as in the introduction, admit a solution.

Proof. To solve the Dirichlet problem, we fix $f \in L^{2}\left(\mathbb{R}^{n}\right)$ and set $F=\left(-\frac{1}{2} I+K\right)^{-1} f$, which is well-defined by Theorem 6.1 .34 as an element of $L^{2}\left(\mathbb{R}^{n}\right)$. Let $u:=\mathcal{D}^{\mathcal{L},+} F$. Then the fact that $u \in D_{+}^{2}$ follows from Proposition 6.1.1, the nontangential maximal function estimate follows from Theorem 5.4.36, while Lemma 6.1.8 gives the weak convergence to $f$.

To upgrade the convergence of $\mathcal{D}_{t}^{\mathcal{L},+} F$ to strong convergence in $L^{2}\left(\mathbb{R}^{n}\right)$, we mimmick the proof of $\left[\mathrm{AAA}^{+} 11\right.$, Lemma 4.23]. First, we note that by Theorem 6.1 .34 we have that $\mathcal{A}:=\left\{\mathcal{S}_{0}^{\mathcal{L}} \operatorname{div}_{\|} g: g \in C_{c}^{\infty}\left(\mathbb{R}^{n}\right)\right\}$ is dense in $L^{2}\left(\mathbb{R}^{n}\right)$. Indeed, since $\operatorname{adj}\left(\mathcal{S}_{0}^{\mathcal{L}}\right)=\mathcal{S}_{0}^{\mathcal{L}^{*}}$, we have that $\mathcal{S}_{0}^{\mathcal{L}}: Y^{1,2}\left(\mathbb{R}^{n}\right)^{*} \rightarrow L^{2}\left(\mathbb{R}^{n}\right)$ is invertible, and therefore any $h \in L^{2}\left(\mathbb{R}^{n}\right)$ may be written as $h=\mathcal{S}_{0}^{\mathcal{L}} H$ for some $H \in L^{2}\left(\mathbb{R}^{n}\right)$. Moreover, any $H \in Y^{1,2}\left(\mathbb{R}^{n}\right)^{*}$ can be written as $H=\operatorname{div}_{\|} g$ for some $g \in L^{2}\left(\mathbb{R}^{n}\right)^{n}$ (as can be seen for instance by embedding $Y^{1,2}\left(\mathbb{R}^{n}\right) \rightarrow L^{2}\left(\mathbb{R}^{n}\right)^{n}$ via $u \mapsto \nabla_{\|} u$ and using the Hahn-Banach and Riesz Representation Theorems). These 
observations yield the claim.

Now fix $F=\mathcal{S}_{0}^{\mathcal{L}}\left(\operatorname{div}_{\|} g\right)$ for some $g \in C_{c}^{\infty}\left(\mathbb{R}^{n}\right)$ and define $v=\mathcal{S}_{s}^{\mathcal{L}}\left(\operatorname{div}_{\|} g\right)$ for $s<0$. By Theorem 3.2.1 (iv), we have that $\mathcal{D}^{\mathcal{L},+} F=-\mathcal{S}^{\mathcal{L}}\left(\partial_{v}^{\mathcal{L},-} v\right)$ in $\mathbb{R}_{+}^{n+1}$. Therefore, for any $0<t^{\prime}<t$, we have that

$$
\left\|\mathcal{D}_{t}^{\mathcal{L},+} F-\mathcal{D}_{t^{\prime}}^{\mathcal{L},+} F\right\|_{L^{2}\left(\mathbb{R}^{n}\right)}=\left\|\int_{t^{\prime}}^{t} \partial_{\tau} \mathcal{S}_{\tau}^{\mathcal{L}}\left(\partial_{v}^{\mathcal{L},+} v\right) d \tau\right\|_{L^{2}\left(\mathbb{R}^{n}\right)} \lesssim\left(t-t^{\prime}\right)\left\|\partial_{v}^{\mathcal{L},+} v\right\|_{L^{2}\left(\mathbb{R}^{n}\right)},
$$

where we used the estimates on slices from Theorem 5.2.1. Thus $\left\{\mathcal{D}_{t}^{\mathcal{L},+} F\right\}_{t}$ is a Cauchy sequence in $L^{2}\left(\mathbb{R}^{n}\right)$ as $t \rightarrow 0^{+}$.

For the Neumann problem we proceed in a similar way, with $w:=\mathcal{S}^{\mathcal{L}}(1 / 2 I+\tilde{K})^{-1} h$, and we appeal to Lemma 6.1.8, Theorem 6.1.34, Proposition 6.1.1, and Theorem 5.4.36.

Finally for the Regularity problem we set $v:=\mathcal{S}^{\mathcal{L}}\left(S_{0}^{\mathcal{L}}\right)^{-1} g$, and make use of Corollary

\subsection{3, Theorem 6.1.34, and Theorem 5.4.36.}

It remains to show the nontangential convergence statements, to which we now turn.

The convergence for the Regularity Problem goes as follows: By Proposition 2.5.29 we have that the solution $v$ has a nontangential limit, call it $g_{0}$, so we only need to show $g=g_{0}$. We know that $v(\cdot, t)$ converges weakly to $g$ in $L^{2^{*}}\left(\mathbb{R}^{n}\right)$ as $t \rightarrow 0^{+}$. Define

$$
v^{\prime}(\cdot, t)=\int_{t / 2}^{3 t / 2} v(\cdot, s) d s
$$

then $w^{\prime}(\cdot, t)$ converges weakly to $g$ in $L^{2^{*}}\left(\mathbb{R}^{n}\right)$ as $t \rightarrow 0^{+}$. Indeed, for fixed $\varphi \in L^{2_{*}}$ we have

$$
\left|\int_{\mathbb{R}^{n}} v(x, t) \varphi(x)-\int_{\mathbb{R}^{n}} g(x) \varphi(x)\right| \leq \epsilon_{\varphi}(t)
$$


where $\epsilon_{\varphi}(t) \downarrow 0$ as $t \rightarrow 0^{+}$. From this one may establish $v^{\prime}(\cdot, t)$ converges weakly to $g$ in $L^{2^{*}}\left(\mathbb{R}^{n}\right)$. Moreover, if for $f \in L_{\text {loc }}^{1}\left(\mathbb{R}^{n}\right)$ we define

$$
\left(A_{t} f\right)(x):=f_{|y-x|_{\infty}<t} f(y) d y
$$

then $\tilde{v}(x, t)=\left(A_{t} v^{\prime}\right)(x)$ and it follows that $\tilde{v}(x, t)$ converges weakly to $g$ in $L^{2^{*}}\left(\mathbb{R}^{n}\right)$ as $t \rightarrow 0^{+}$. Indeed, for if $\varphi \in L^{2 *}$ then

$$
\int_{\mathbb{R}^{n}}\left(A_{t} v^{\prime}\right)(x) \varphi(x) d x=\int_{\mathbb{R}^{n}} v^{\prime}(x, t)\left(A_{t} \varphi\right)(x) d x
$$

and since $v^{\prime}(\cdot, t)$ converges weakly to $g$ in $L^{2^{*}}\left(\mathbb{R}^{n}\right)$ as $t \rightarrow 0^{+}$and $\left(A_{t} \varphi\right)(x)$ converges strongly in $L^{2 *}$ as $t \rightarrow 0^{+}$we have that

$$
\int_{\mathbb{R}^{n}} v^{\prime}(x, t)\left(A_{t} \varphi\right)(x) d x \rightarrow \int_{\mathbb{R}^{n}} g(x) \varphi(x) d x, \quad \text { as } t \rightarrow 0^{+} .
$$

It follows that $g_{0}(x)=g(x)$ for a.e. $x \in \mathbb{R}^{n}$.

For the Dirichlet problem, we use compatible well-posedness (see below in the proof) to get that for smooth initial data $f \in C_{c}^{\infty}\left(\mathbb{R}^{n}\right)$ the solutions to the Dirichlet and Regularity problems obtained via layer potentials agree. In particular, if $u_{f}=\mathcal{D}^{\mathcal{L},+}(-1 / 2+K)^{-1} f$, then $u_{f}$ has a nontangential limit. Since $C_{c}^{\infty}\left(\mathbb{R}^{n}\right)$ is dense in $L^{2}\left(\mathbb{R}^{n}\right)$ and we have the maximal function estimate

$$
\left\|\tilde{\mathcal{N}}_{2}\left(u_{f}\right)\right\|_{L^{2}\left(\mathbb{R}^{n}\right)} \lesssim\|f\|_{L^{2}\left(\mathbb{R}^{n}\right)}
$$

the existence of a limit for general $f \in L^{2}\left(\mathbb{R}^{n}\right)$ follows a standard argument. 
Now we turn to the compatible well-posedness statement: If $f \in C_{c}^{\infty}\left(\mathbb{R}^{n}\right)$ and we set

$$
u_{f}:=\mathcal{D}^{\mathcal{L}}\left(-\frac{1}{2}+K\right)^{-1} f, \quad v_{f}:=\mathcal{S}^{\mathcal{L}}\left(\mathcal{S}_{0}^{\mathcal{L}}\right)^{-1} f
$$

the layer potential solutions of the Dirichlet and Regularity problems with data $f$ respectively; we claim then $u_{f}=v_{f}$ and both agree with the solution furnished via Lax-Milgram with Dirichlet data $f$.

We first prove that $u_{f}$ agrees with the Lax-Milgram solution. For this, by the mapping properties of the double layer (see Definition 3.1.6) it's enough to show that

$$
T f:=\left(-\frac{1}{2}+K\right)^{-1} f \in H_{0}^{1 / 2}\left(\mathbb{R}^{n}\right) \text {. }
$$

We know (see Theorem 6.1.34 and Proposition 6.1.19) that $T$ maps $L^{2}\left(\mathbb{R}^{n}\right)$ and $Y^{1,2}\left(\mathbb{R}^{n}\right)$ to itself, so in particular $T f \in W^{1,2}\left(\mathbb{R}^{n}\right) \subset H_{0}^{1 / 2}$.

For $v_{f}$ we proceed similarly, noting that $\left(\mathcal{S}_{0}^{\mathcal{L}}\right)^{-1}$ maps $Y^{1,2}\left(\mathbb{R}^{n}\right)$ to $L^{2}\left(\mathbb{R}^{n}\right)$ and $L^{2}\left(\mathbb{R}^{n}\right) \rightarrow$ $\left[Y^{1,2}\left(\mathbb{R}^{n}\right)\right]^{*}$ (see Theorem 6.1.34 and Lemma 6.1.21). It's thus enough, by the mapping properties of the single layer (see Proposition 3.1.2), to prove that

$$
\left[Y^{1,2}\left(\mathbb{R}^{n}\right)\right]^{*} \cap L^{2}\left(\mathbb{R}^{n}\right) \subset H^{-1 / 2}\left(\mathbb{R}^{n}\right)
$$

This follows from the fact that elements of the first space are of the form $G \in L^{2}\left(\mathbb{R}^{n}\right)$ such that $G=\operatorname{div} H$ for some $H \in L^{2}\left(\mathbb{R}^{n} ; \mathbb{C}^{n}\right)$, while the second space contains all elements of the form $(-\Delta)^{1 / 2} F$ with $F \in H_{0}^{1 / 2}\left(\mathbb{R}^{n}\right)$. Fix $G, H$ as above. By the Riesz representation theorem in $Y^{1,2}\left(\mathbb{R}^{n}\right)$ there exists a weak solution $F_{1} \in Y^{1,2}\left(\mathbb{R}^{n}\right)$ of the problem $G=\operatorname{div} H=-\Delta F_{1}$; 
set $F:=(-\Delta)^{1 / 2} F_{1}$, so that it's enough to prove $F \in H^{1 / 2}\left(\mathbb{R}^{n}\right)$. First, clearly $F \in L^{2}\left(\mathbb{R}^{n}\right)$ by Plancherel's Theorem, since $\nabla F_{1} \in L^{2}\left(\mathbb{R}^{n}\right)$; moreover, since $(-\Delta)^{1 / 2} F=G \in L^{2}\left(\mathbb{R}^{n}\right)$, we have that $F \in W^{1,2}\left(\mathbb{R}^{n}\right)$, and by interpolation, $F \in H^{1 / 2}\left(\mathbb{R}^{n}\right)$ as desired.

\subsection{Uniqueness}

Lemma 6.2.1. Suppose $\mathcal{L}$ satisfies Hypothesis B (see Definition 6.1.30). Assume u is a good $\mathcal{D}$ solution. Then for every $\tau>0, \partial_{\nu_{\tau}} u \in\left[Y^{1,2}\left(\mathbb{R}^{n}\right)\right]^{*}$, with the bound

$$
\sup _{\tau>0}\left\|\partial_{v_{\tau}} u\right\|_{\left[Y^{1,2}\left(\mathbb{R}^{n}\right)\right]^{*}} \leq C \sup _{t>0}\|u\|_{L^{2}\left(\mathbb{R}^{n}\right)}
$$

Proof. By symmetry of hypotheses and Lemma 6.1.21 and Theorem 6.1.34 the operator $S_{0}^{\mathcal{L}^{*}}: L^{2}\left(\mathbb{R}^{n}\right) \rightarrow Y^{1,2}\left(\mathbb{R}^{n}\right)$ is bounded and invertible. Then the collection of functions $\mathcal{F}:=$ $\left\{\varphi \in Y^{1,2}\left(\mathbb{R}^{n}\right): \varphi=S_{0}^{\mathcal{L}^{*}} f, f \in C_{c}^{\infty}\right\}$ is dense in $Y^{1,2}\left(\mathbb{R}^{n}\right)$. Notice that $v_{\varphi}=\mathcal{S}^{\mathcal{L}^{*}}\left(\left[S_{0}^{\mathcal{L}^{*}}\right]^{-1} \varphi\right)=$ $S^{\mathcal{L}^{*}} f \in Y^{1,2}\left(\mathbb{R}^{n+1}\right)$ since $f \in C_{c}^{\infty}\left(\mathbb{R}^{n}\right) \subset H^{-1 / 2}\left(\mathbb{R}^{n}\right)$. Also, $\operatorname{Tr}_{0} u_{\tau} \in H_{0}^{1 / 2}\left(\mathbb{R}^{n}\right)$ since $u_{\tau} \in$ $Y^{1,2}\left(\mathbb{R}^{n+1}{ }_{+}\right)$, where, as above $u_{\tau}(\cdot, \cdot):=u(\cdot, \cdot+\tau)$. Then by definition of $\partial_{v^{*}} v_{\varphi} \in H^{-1 / 2}\left(\mathbb{R}^{n}\right)$ (see Definition 3.1.9) with

$$
\begin{aligned}
\left(\operatorname{Tr}_{0} u_{\tau}, \partial_{v^{*}} v_{\varphi}\right) & =\overline{\left(\partial_{v^{*}} v_{\varphi}, \operatorname{Tr}_{0} u_{\tau}\right)} \\
& =\overline{B_{\mathcal{L}^{*}}\left[v_{\varphi}, u_{\tau}\right]}=B_{\mathcal{L}}\left[u_{\tau}, v_{\varphi}\right] .
\end{aligned}
$$

Now $B_{\mathcal{L}}\left[u_{\tau}, v_{\varphi}\right]=\left(\partial_{v_{\tau}} u, \varphi\right)$, since $v_{\varphi}$ solves the regularity problem with data $\varphi$ by Theorem 6.1.38. In particular $\varphi$ is the weak limit of $v_{\varphi}(\cdot, t)$ in $Y^{1,2}\left(\mathbb{R}^{n}\right)$ as $t \rightarrow 0$. 
Having established

$$
\left(\partial_{v_{\tau}} u, \varphi\right)=\left(\operatorname{Tr}_{0} u_{\tau}, \partial_{v^{*}} v_{\varphi}\right)
$$

for $\varphi$ in $\mathcal{F}$ we see that $\partial_{v_{\tau}} u \in\left[Y^{1,2}\left(\mathbb{R}^{n}\right)\right]^{*}$ by the fact that the map

$$
F_{\varphi}:=\partial_{v^{*}} v_{\varphi}=\partial_{v^{*}} \mathcal{S}^{\mathcal{L}^{*}}\left(\left[S_{0}^{\mathcal{L}^{*}}\right]^{-1} \varphi\right)
$$

maps $Y^{1,2}\left(\mathbb{R}^{n}\right) \rightarrow L^{2}\left(\mathbb{R}^{n}\right)$, by Proposition 6.1 .5 , the mapping property mentioned at the start of the proof for $\mathcal{S}_{0}^{\mathcal{L}^{*}}$, and the density of $\mathcal{F}$ in $Y^{1,2}\left(\mathbb{R}^{n}\right)$.

Finally we state a technical lemma that will allow us to prove a representation formula for solutions to the regularity and Neumann problems

Proposition 6.2.2. Suppose $\mathcal{L}$ satisfies Hypothesis $B$ (see Definition 6.1.30). Let $u \in$ $W_{\text {loc }}^{1,2}\left(\mathbb{R}_{+}^{n+1}\right) \cap S_{+}^{2}$ be a solution of $\mathcal{L} u=0$ in $\mathbb{R}_{+}^{n+1}$. Then for every $\tau_{0}>0$ and every $t>0$ we have

$$
\left.\partial_{\tau}\right|_{\tau=\tau_{0}} \mathcal{D}_{t}^{\mathcal{L},+}\left(\operatorname{Tr}_{0} u_{\tau}\right)=\mathcal{D}_{t}^{\mathcal{L},+}\left(\operatorname{Tr}_{0}\left(D_{n+1} u_{\tau_{0}}\right)\right)
$$

and

$$
\left.\partial_{\tau}\right|_{\tau=\tau_{0}} \mathcal{S}_{t}^{\mathcal{L}}\left(\partial_{v^{\mathcal{L},+}} u_{\tau}\right)=\mathcal{S}_{t}^{\mathcal{L}}\left(\partial_{\nu^{\mathcal{L},+}}\left(D_{n+1} u_{\tau_{0}}\right)\right)
$$

Proof. We work with the double layer first. For this we consider, for $t>0$ fixed, the following functions:

$$
f(\tau):=\operatorname{Tr}_{0} u_{\tau}=\operatorname{Tr}_{\tau} u, \quad H(\tau):=\mathcal{D}_{t}^{\mathcal{L},+}(f(\tau))
$$


We note that, by hypothesis and Corollary 6.1.9, we have

$$
f \in C\left((0, \infty) ; Y^{1,2}\left(\mathbb{R}^{n}\right)\right), \quad H \in C\left((0, \infty) ; Y^{1,2}\left(\mathbb{R}^{n}\right)\right) .
$$

The idea is now to use Theorem 2.2.11 to get the desired differentiability of $f$. For this purpose define

$$
\varphi(\tau):=\left\|\operatorname{Tr}_{\tau}\left(D_{n+1} u\right)\right\|_{Y^{1,2}\left(\mathbb{R}^{n}\right)}=\left\|\nabla_{\|} \operatorname{Tr}_{\tau}\left(D_{n+1} u\right)\right\|_{L^{2}\left(\mathbb{R}^{n}\right)} \in L_{\mathrm{loc}}^{2}((0, \infty) ; \mathbb{R})
$$

First we note that by Lemma 2.2.2 we have that $f:(0, \infty) \rightarrow Y^{1,2}\left(\mathbb{R}^{n}\right)$ and $\varphi:(0, \infty) \rightarrow \mathbb{R}$ are continuous functions. By the Lebesgue Differentiation Theorem it's then enough to show

$$
\left(f_{-\varepsilon}^{\varepsilon}\left\|f\left(\tau_{2}+s\right)-f\left(\tau_{1}+s\right)\right\|_{Y^{1,2}\left(\mathbb{R}^{n}\right)}^{2} d s\right)^{1 / 2} \leq \int_{\tau_{1}}^{\tau_{2}}\left(f_{-\varepsilon}^{\varepsilon} \varphi^{2}(s+\tau) d s\right)^{1 / 2} d \tau,
$$

for all $\varepsilon$ small enough (depending on $\tau_{1}$ and $\tau_{2}$ ). For this purpose we compute, calling $I$ the left hand side of (6.6),

$$
\begin{aligned}
I & =\left(f_{-\varepsilon}^{\varepsilon} \int_{\mathbb{R}^{n}}\left|\nabla_{\|} \operatorname{Tr}_{\tau_{2}+s} u(x)-\nabla_{\|} \operatorname{Tr}_{\tau_{1}+s} u(x)\right|^{2} d x d s\right)^{1 / 2} \\
& =\left(f_{-\varepsilon}^{\varepsilon} \int_{\mathbb{R}^{n}}\left|\nabla_{\|} u_{\tau_{2}}(x, s)-\nabla_{\|} u_{\tau_{1}}(x, s)\right|^{2} d x d s\right)^{1 / 2} \\
& =\left(f_{-\varepsilon}^{\varepsilon} \int_{\mathbb{R}^{n}}\left|\int_{\tau_{1}}^{\tau_{2}} \nabla_{\|} \partial_{\tau} u(x, \tau+s) d \tau\right|^{2} d x d s\right)^{1 / 2} \\
& \leq \int_{\tau_{1}}^{\tau_{2}}\left(f_{-\varepsilon}^{\varepsilon} \int_{\mathbb{R}^{n}}\left|\nabla_{\|} \partial_{\tau} u(x, \tau+s)\right|^{2} d x d s\right)^{1 / 2} d \tau \\
& =\int_{\tau_{1}}^{\tau_{2}}\left(f_{-\varepsilon}^{\varepsilon} \varphi^{2}(s+\tau) d s\right)^{1 / 2} d \tau,
\end{aligned}
$$

where we used the Fundamental Theorem of Calculus in the third line and Minkowski's inequality in the fourth. 
As mentioned above this shows that $f \in W_{\mathrm{loc}}^{1,2}\left((0, \infty) ; Y^{1,2}\left(\mathbb{R}^{n}\right)\right)$. Now we will show that

$$
f^{\prime}(\tau)=\operatorname{Tr}_{\tau}\left(D_{n+1} u\right), \quad \forall \tau>0
$$

and moreover the difference quotients converge weakly

$$
\Delta^{h} f(\tau) \rightarrow f^{\prime}(\tau), \quad \text { for every } \tau>0
$$

For this fix $\psi \in C_{c}^{\infty}(0, \infty), \phi \in C_{c}^{\infty}\left(\mathbb{R}^{n} ; \mathbb{C}^{n}\right)$ and let $\ell:=-\operatorname{div}_{\|} \phi \in Y^{1,2}\left(\mathbb{R}^{n}\right)^{*}$. Using that the function $\tau \mapsto f(\tau) \psi^{\prime}(\tau) \in Y^{1,2}\left(\mathbb{R}^{n}\right)$ is continuous (see again Lemma 2.2.2) and compactly supported on $(0, \infty)$ and properties of the Bochner integral (see for instance [CH98, Proposition 1.4.22]) we obtain

$$
\begin{aligned}
\left\langle\int_{0}^{\infty} f(\tau) \psi^{\prime}(\tau) d \tau, \ell\right\rangle & =\int_{0}^{\infty}\left\langle f(\tau) \psi^{\prime}(\tau), \ell\right\rangle d \tau \\
& =\int_{0}^{\infty} \int_{\mathbb{R}^{n}} \nabla_{\|} \operatorname{Tr}_{\tau} u(x) \psi^{\prime}(\tau) \phi(x) d x d \tau \\
& =\int_{0}^{\infty} \int_{\mathbb{R}^{n}} \nabla_{\|} u(x, \tau) \psi^{\prime}(\tau) \phi(x) d x d \tau \\
& =-\int_{0}^{\infty} \int_{\mathbb{R}^{n}} \nabla_{\|} D_{n+1} u(x, \tau) \psi(\tau) \phi(x) d x d \tau \\
& =-\int_{0}^{\infty} \int_{\mathbb{R}^{n}}^{\infty} \nabla_{\|} \operatorname{Tr}_{\tau}\left(D_{n+1} u\right)(x) \psi(\tau) \phi(x) d x d \tau \\
& =-\int_{0}^{\infty}\left\langle\operatorname{Tr}_{\tau}\left(D_{n+1} u\right) \psi(\tau), \ell\right\rangle d \tau \\
& =\left\langle-\int_{0}^{\infty} \operatorname{Tr}_{\tau}\left(D_{n+1} u\right) \psi(\tau) d \tau, \ell\right\rangle
\end{aligned}
$$

where we used integration by parts in the fourth line. Now we conclude, since the collection $\left\{\operatorname{div}_{\|} \phi: \phi \in C_{c}^{\infty}\left(\mathbb{R}^{n} ; \mathbb{C}^{n}\right)\right\}$ is dense in $Y^{1,2}\left(\mathbb{R}^{n}\right)^{*}$, that indeed (6.8) holds.

The convergence of the difference quotients is a consequence of the fact that $f^{\prime} \in$ 
$C\left((0, \infty) ; Y^{1,2}\left(\mathbb{R}^{n}\right)\right)$ and the Fundamental Theorem of Calculus. In fact we get strong convergence in $Y^{1,2}\left(\mathbb{R}^{n}\right)$ as $h \rightarrow 0$ of $\Delta^{h} f(\tau)$ for every $\tau>0$.

With this we can conclude the argument for the Double Layer: Define

$$
H(\tau):=\mathcal{D}_{t}^{\mathcal{L},+} \operatorname{Tr}_{0} u_{\tau}=\mathcal{D}_{t}^{\mathcal{L},+} \operatorname{Tr}_{\tau} u
$$

We claim that $H \in C^{1}\left((0, \infty) ; Y^{1,2}\left(\mathbb{R}^{n}\right)\right)$ and

$$
H^{\prime}(\tau)=\mathcal{D}_{t}^{\mathcal{L},+}\left(\operatorname{Tr}_{0}\left(D_{n+1} u_{\tau}\right)\right)=\mathcal{D}_{t}^{\mathcal{L},+}\left(\operatorname{Tr}_{\tau}\left(D_{n+1} u\right)\right)
$$

Notice first that $H \in C\left((0, \infty) ; Y^{1,2}\left(\mathbb{R}^{n}\right)\right)$ by the mapping properties of the Double Layer (see Corollary 6.1.9) and the fact that $H(\tau)=\mathcal{D}_{t}^{\mathcal{L},+}(f(\tau))$ (recall that $t>0$ is fixed throughout). Morever, using these two facts again we see

$$
\left\|H\left(\tau_{1}\right)-H\left(\tau_{2}\right)\right\|_{Y^{1,2}\left(\mathbb{R}^{n}\right)} \lesssim\left\|f\left(\tau_{1}\right)-f\left(\tau_{2}\right)\right\|_{Y^{1,2}\left(\mathbb{R}^{n}\right)} \leq\left|\int_{\tau_{1}}^{\tau_{2}} \varphi(s) d s\right|
$$

where $\varphi$ is defined in (6.5). This shows that $H \in W_{\text {loc }}^{1,2}\left((0, \infty) ; Y^{1,2}\left(\mathbb{R}^{n}\right)\right)$. Moreover we have

$$
\Delta^{h} H(\tau)=\mathcal{D}_{t}^{\mathcal{L},+}\left(\Delta^{h} f(\tau)\right)
$$

so that, by the linearity and continuity of $\mathcal{D}_{t}^{\mathcal{L},+}$ in $Y^{1,2}\left(\mathbb{R}^{n}\right)$ and the weak convergence of $\Delta^{h} f(\tau)$ in $Y^{1,2}\left(\mathbb{R}^{n}\right)$, we obtain for every $\tau>0$

$$
\Delta^{h} H(\tau) \rightarrow \mathcal{D}^{\mathcal{L},+}\left(f^{\prime}(\tau)\right)=\mathcal{D}_{t}^{\mathcal{L},+}\left(\operatorname{Tr}_{0}\left(D_{n+1} u_{\tau}\right)\right)
$$

weakly in $Y^{1,2}\left(\mathbb{R}^{n}\right)$ as $h \rightarrow 0$ 
The proof for the Single Layer follows the same lines. Define, for $t>0$ fixed and $\tau>0$,

$$
g(\tau):=\partial_{v^{\mathcal{L}},+} u_{\tau}=\partial_{v_{\tau}^{\mathcal{L}},+} u
$$

where the second equality follows from Lemma 3.1.10 (i). As before we first claim that $g \in C^{1}\left((0, \infty) ; L^{2}\left(\mathbb{R}^{n}\right)\right)$ and we have

$$
g^{\prime}(\tau)=\partial_{v^{\mathcal{L},+}}\left(D_{n+1} u_{\tau}\right)=\partial_{v_{\tau}^{\mathcal{L},+}}\left(D_{n+1} u\right)
$$

For this purpose we use the $L^{2}$ characterization of the conormal derivative (see again Lemma 3.1.10) so that

$$
\begin{aligned}
g(\tau) & =N \cdot \operatorname{Tr}_{0}\left(A \nabla u_{\tau}+B_{1} u_{\tau}\right)=N \cdot \operatorname{Tr}_{\tau}\left(A \nabla u+B_{1} u\right) \\
& =N \cdot \operatorname{Tr}_{\tau}\left(\tilde{A} \nabla_{\|} u+B_{1} u\right)+N \cdot \operatorname{Tr}_{\tau}\left(\vec{a} D_{n+1} u\right) \\
& =: g_{1}(\tau)+g_{2}(\tau),
\end{aligned}
$$

where $\tilde{A}:=\left(a_{i j}\right)_{1 \leq i \leq n+1,1 \leq j \leq n}$ and $\vec{a}:=\left(a_{i, n+1}\right)_{1 \leq i \leq n+1}$. We note that by Hölder's and Sobolev's inequalities

$$
\left\|g_{1}\left(\tau_{2}\right)-g_{1}\left(\tau_{1}\right)\right\|_{L^{2}\left(\mathbb{R}^{n}\right)} \lesssim\left\|f\left(\tau_{2}\right)-f\left(\tau_{1}\right)\right\|_{Y^{1,2}\left(\mathbb{R}^{n}\right)} \leq\left|\int_{\tau_{1}}^{\tau_{2}} \varphi(s) d s\right|,
$$

where $f, \varphi$ are as in the proof for the Double Layer. Therefore it's enough to control $g_{2}$, and for this we can proceed exactly in the same way as we did for $f$ : For fixed $\tau_{2}, \tau_{1}$ and 
$\varepsilon>0$ small

$$
\begin{aligned}
f_{-\varepsilon}^{\varepsilon}\left\|g\left(\tau_{2}+s\right)-g\left(\tau_{1}+s\right)\right\|_{L^{2}\left(\mathbb{R}^{n}\right)}^{2} d s & =f_{-\varepsilon}^{\varepsilon} \int_{\mathbb{R}^{n}}\left|D_{n+1}\left(u_{\tau_{2}}(x, s)-u_{\tau_{1}}(x, s)\right)\right|^{2} d x d s \\
& =f_{-\varepsilon}^{\varepsilon} \int_{\mathbb{R}^{n}}\left|\int_{\tau_{1}}^{\tau_{2}} D_{n+1}^{2} u(s+\tau) d \tau\right|^{2} d x d s \\
& \lesssim f_{-\varepsilon}^{\varepsilon} \int_{\tau_{1}}^{\tau_{2}}\left\|\operatorname{Tr}_{\tau+s}\left(D_{n+1}^{2} u\right)\right\|_{L^{2}\left(\mathbb{R}^{n}\right)} d \tau d s,
\end{aligned}
$$

and

$$
\tilde{\varphi}(\tau):=\left\|\operatorname{Tr}_{\tau}\left(D_{n+1}^{2} u\right)\right\|_{L^{2}\left(\mathbb{R}^{n}\right)} \in L_{\mathrm{loc}}^{2}(0, \infty)
$$

Therefore by Theorem 2.2.11 we get that $g \in W_{\text {loc }}^{1,2}\left((0, \infty) ; L^{2}\left(\mathbb{R}^{n}\right)\right)$ and the difference quotients converge a.e. to $g^{\prime}$. To verify the formula for $g^{\prime}$ we compute, for $\phi \in C_{c}^{\infty}\left(\mathbb{R}^{n}\right)$ and $\psi \in C_{c}^{\infty}(0, \infty)$

$$
\begin{aligned}
\left\langle\int_{0}^{\infty} g(\tau) \psi^{\prime}(\tau) d \tau, \phi\right\rangle_{L^{2}\left(\mathbb{R}^{n}\right)} & =\int_{0}^{\infty} \int_{\mathbb{R}^{n}} N \cdot\left(A \nabla u(x, \tau)+B_{1} u(x, \tau)\right) \psi^{\prime}(\tau) \phi(x) d x d \tau \\
& =-\int_{0}^{\infty} \int_{\mathbb{R}^{n}} N \cdot\left(A \nabla D_{n+1} u(x, \tau)+B_{1} D_{n+1} u(x, \tau)\right) \psi(\tau) \phi(x) d x d \tau \\
& =\left\langle\int_{0}^{\infty} \partial_{v_{\tau}^{\mathcal{\tau}}+}\left(D_{n+1} u\right) \psi(\tau), \phi\right\rangle_{L^{2}\left(\mathbb{R}^{n}\right)} .
\end{aligned}
$$

This gives the desired representation for $g^{\prime}(\tau)$. Moreover, using this representation we see that $g^{\prime} \in C\left((0, \infty) ; L^{2}\left(\mathbb{R}^{n}\right)\right)$ and so the difference quotients satisfy $\Delta^{h} g(\tau) \rightarrow g^{\prime}(\tau)$ weakly for every $\tau>0$. The result now follows from the mapping properties of the single layer (see Proposition 6.1.1). 


\subsubsection{Neumann and Regularity Problems}

We begin with a lemma that gives a representation of $\operatorname{good} \mathcal{N} / \mathcal{R}$ solutions above a positive height.

Lemma 6.2.23. Suppose $\mathcal{L}$ satisfies Hypothesis B (see Definition 6.1.30). Let $u$ be a good $\mathcal{N} / \mathcal{R}$ solution and $u_{\tau}(\cdot, \cdot)=u(\cdot, \cdot+\tau)$, as above. Then

$$
u_{\tau}=-\mathcal{D}\left(T r_{0} u_{\tau}\right)+\mathcal{S}\left(\partial_{\nu} u_{\tau}\right)
$$

where $\operatorname{Tr}_{0} u_{\tau} \in Y^{1,2}\left(\mathbb{R}^{n}\right), \partial_{\nu} u_{\tau} \in L^{2}\left(\mathbb{R}^{n}\right)$, and $\mathcal{D}$ and $\mathcal{S}$ are viewed (as their natural extensions) from these spaces mapping into $S_{+}^{2}$.

Proof. We have $\operatorname{Tr}_{0} u_{\tau}(\cdot)=u(\cdot, \tau) \in Y^{1,2}\left(\mathbb{R}^{n}\right)$ (by the fact that $u \in S_{+}^{2}$ ) and $\partial_{\nu} u_{\tau} \in L^{2}\left(\mathbb{R}^{n}\right)$ (by Proposition 6.1.5). For the latter we may consider $u_{\tau / 2} \in Y^{1,2}\left(\mathbb{R}^{n+1}{ }_{+}\right)$, a solution in $\mathbb{R}^{n+1}{ }_{+}$, since the operator $\mathcal{L}$ is $t$-independent.

By Proposition 6.2.2 together with the Green's formula for $\partial_{t} u_{\tau} \in Y^{1,2}\left(\mathbb{R}^{n+1}\right)$ (see Theorem 3.2.1 (ii)) we know that for $\tau_{0}>0$

$$
\left.\partial_{\tau} u_{\tau}\right|_{\tau=\tau_{0}}(x, t)=-\left.\partial_{\tau}\left[\mathcal{D}\left(\operatorname{Tr}_{0} u_{\tau}\right)+\mathcal{S}\left(\partial_{\nu} u_{\tau}\right)\right]\right|_{\tau=\tau_{0}}(x, t)
$$

as functions in $S_{+}^{2}$. We may now integrate in $\tau_{0}$ to obtain

$$
u_{\tau}=-\mathcal{D}\left(\operatorname{Tr}_{0} u_{\tau}\right)+\mathcal{S}\left(\partial_{\nu} u_{\tau}\right)
$$

where we must use the decay at infinity hypothesis in the definition of $S_{+}^{2}$. 
Now we push the representation above down to the boundary.

Lemma 6.2.25. Suppose $\mathcal{L}$ satisfies Hypothesis $B$ (see Definition 6.1.30). Suppose that $u$ is $a \operatorname{good} \mathcal{N} / \mathcal{R}$ solution, then

$$
u=-\mathcal{D} f+\mathcal{S} g
$$

where $f \in Y^{1,2}\left(\mathbb{R}^{n}\right)$ and $g \in L^{2}\left(\mathbb{R}^{n}\right)$ are as in Propositions 6.1 .4 and 6.1 .5 respectively.

Proof. By Propositions 6.1.4 and 6.1.5, $u_{\tau}(\cdot, 0) \rightarrow f \in Y^{1,2}\left(\mathbb{R}^{n}\right)$ and $\partial_{\nu} u_{\tau} \rightarrow g \in L^{2}\left(\mathbb{R}^{n}\right)$ weakly in $Y^{1,2}\left(\mathbb{R}^{n}\right)$ and $L^{2}\left(\mathbb{R}^{n}\right)$ respectively as $\tau \rightarrow 0$. Set $u_{\tau}(\cdot, 0)=f_{\tau}$ and $\partial_{\nu} u_{\tau}=g_{\tau}$, then rephrasing the above, we have $\vec{h}_{\tau}=\left(f_{\tau}, g_{\tau}\right)$ converges to $(f, g)=: \vec{h}$ weakly in $Y^{1,2}\left(\mathbb{R}^{n}\right) \times$ $L^{2}\left(\mathbb{R}^{n}\right)$. Let $\tau_{k} \downarrow 0$ then by Mazur's lemma there exists a sequence $\left\{\tilde{h}_{l}\right\}_{l=1}^{\infty} \subset Y^{1,2}\left(\mathbb{R}^{n}\right) \times L^{2}\left(\mathbb{R}^{n}\right)$ such that $\tilde{h}_{l} \rightarrow \vec{h}$ strongly in $Y^{1,2}\left(\mathbb{R}^{n}\right) \times L^{2}\left(\mathbb{R}^{n}\right)$ with

$$
\tilde{h}_{l}=\sum_{k=l}^{N(l)} \lambda_{k, l} \vec{h}_{\tau_{k}},
$$

where $l \leq N(l)<\infty, \lambda_{k, l} \in[0,1]$ and $\sum_{k=l}^{N(l)} \lambda_{k, l}=1$. Set

$$
\tilde{u}:=\mathcal{D} f+\mathcal{S} g
$$

To prove the lemma it is enough to show for $t>0, \tilde{u}(\cdot, t)=u(\cdot, t)$ as functions in $Y^{1,2}\left(\mathbb{R}^{n}\right)$.

We have from Lemma 6.2.23 that

$$
u_{\tau}=-\mathcal{D}\left(f_{\tau}\right)+S\left(g_{\tau}\right)
$$

Set

$$
u_{l}:=\sum_{k=l}^{N(l)} \lambda_{k, l} u_{\tau_{k}}
$$


We show $u_{l}(\cdot, t)$ converges strongly to both $u(\cdot, t)$ and $\tilde{u}(\cdot, t)$ in $Y^{1,2}\left(\mathbb{R}^{n}\right)$. From the bounded mappings $\mathcal{D}: Y^{1,2}\left(\mathbb{R}^{n}\right) \rightarrow S_{+}^{2}$ and $\mathcal{S}: L^{2}\left(\mathbb{R}^{n}\right) \rightarrow S_{+}^{2}$ we have

$$
\left\|\nabla\left[\tilde{u}(\cdot, t)-u_{l}(\cdot, t)\right]\right\|_{L^{2}\left(\mathbb{R}^{n}\right)} \leq\left\|\vec{h}-\tilde{h}_{l}\right\|_{Y^{1,2}\left(\mathbb{R}^{n}\right) \times L^{2}\left(\mathbb{R}^{n}\right)} \rightarrow 0 \text { as } l \rightarrow \infty,
$$

where we used the strong convergence of $\tilde{h}_{l}$ to $\vec{h}$. To show $u_{l}(\cdot, t)$ converges strongly to $u(\cdot, t)$ in $Y^{1,2}\left(\mathbb{R}^{n}\right)$ we write for $l \geq 0$,

$$
\begin{aligned}
\left\|\nabla u_{l}(\cdot, t)-\nabla u(\cdot, t)\right\|_{L^{2}\left(\mathbb{R}^{n}\right)} & =\left\|\sum_{k=l}^{N(l)} \lambda_{k, l} \nabla\left[u_{\tau_{k}}-u\right](\cdot, t)\right\|_{L^{2}\left(\mathbb{R}^{n}\right)} \\
& \leq \sum_{k=l}^{N(l)} \lambda_{k, l}\left\|\nabla\left[u_{\tau_{k}}-u\right](\cdot, t)\right\|_{L^{2}\left(\mathbb{R}^{n}\right)} \\
& \leq \sup _{k \geq l}\left\|\nabla\left[u\left(\cdot, t+\tau_{k}\right)-u(\cdot, t)\right]\right\|_{L^{2}\left(\mathbb{R}^{n}\right)},
\end{aligned}
$$

where we used $\sum_{k=l}^{N(l)} \lambda_{k, l}=1$ and $u(\cdot, \cdot+\tau)=u_{\tau}(\cdot, \cdot)=\mathcal{D}\left(f_{\tau}\right)+S\left(g_{\tau}\right)$. We can then use the continuity of $\nabla u(\cdot, t)$ in $L^{2}\left(\mathbb{R}^{n}\right)$ (see Lemma 2.2.2) along with $\tau_{k} \downarrow 0$ to obtain $\left\|\nabla u_{l}(\cdot, t)-\nabla u(\cdot, t)\right\|_{L^{2}\left(\mathbb{R}^{n}\right)} \rightarrow 0$ as $l$ tends to infinity.

Theorem 6.2.27 (Uniqueness of the Regularity Problem Among Good $\mathcal{N} / \mathcal{R}$ solutions). Suppose $\mathcal{L}$ satisfies Hypothesis B (see Definition 6.1.30). Suppose u is a good $\mathcal{N} / \mathcal{R}$ solution, with $u(\cdot, 0)=0$, interpreted in the sense of Proposition 6.1 .4 (i.e. $\lim _{t \rightarrow 0} u(t)=0$ weakly in $\left.Y^{1,2}\left(\mathbb{R}^{n}\right)\right)$. Then $u \equiv 0$ in $\mathbb{R}_{+}^{n+1}$

Proof. By Lemma 6.2.25, we have $u=-\mathcal{D} f+\mathcal{S}^{\mathcal{L}} g$, where $f$ and $g$ are as in Lemma 6.2.25. It follows that $u=\mathcal{S}^{\mathcal{L}} g$, since $f=0$ (see the proof of Lemma 6.2.25). Moreover, by taking 
traces (in the sense of Proposition 6.1.4) in $Y^{1,2}\left(\mathbb{R}^{n}\right)$ we obtain $0=\mathcal{S}_{0}^{\mathcal{L}} g$, for $g \in L^{2}\left(\mathbb{R}^{n}\right)$. It follows from the invertibility of $\mathcal{S}_{0}^{\mathcal{L}}: L^{2}\left(\mathbb{R}^{n}\right) \rightarrow Y^{1,2}\left(\mathbb{R}^{n}\right)$ that $g=0$. This gives $u \equiv 0$.

Theorem 6.2.28 (Uniqueness of the Neumann problem among good $\mathcal{N} / \mathcal{R}$ solutions). Suppose $\mathcal{L}$ satisfies Hypothesis B (see Definition 6.1.30). Suppose $u$ is a good $\mathcal{N} / \mathcal{R}$ solution, with $\partial_{v} u=0$, interpreted in the sense of Proposition 6.1.5. Then $u \equiv 0$ in $\mathbb{R}_{+}^{n+1}$.

Proof. By Lemma 6.2.25, we have $u=-\mathcal{D} f+\mathcal{S} g$, where $f$ and $g$ are as in Lemma 6.2.25. It follows that $u=-\mathcal{D} f$, since $g=0$ (see the proof of Lemma 6.2.25), where $f \in Y^{1,2}\left(\mathbb{R}^{n}\right)$. From (6.16), we have after taking conormal derivatives, in the sense of Proposition 6.1.5 and using the jump relations for the conormal of the single layer potential

$$
0=\partial_{v^{\mathcal{L}},+} u=-\partial_{v^{\mathcal{L},+}} \mathcal{D} f=-\left(-\frac{1}{2} I+\widetilde{K}\right)\left(\frac{1}{2} I+\widetilde{K}\right) S_{0}^{-1} f \quad \text { in } L^{2}\left(\mathbb{R}^{n}\right)
$$

The invertibility of $\pm \frac{1}{2} I+\widetilde{K}: L^{2}\left(\mathbb{R}^{n}\right) \rightarrow L^{2}\left(\mathbb{R}^{n}\right)$ and $S_{0}^{-1}: Y^{1,2}\left(\mathbb{R}^{n}\right) \rightarrow L^{2}\left(\mathbb{R}^{n}\right)$ yields that $f=0$ and hence $u \equiv 0$.

\subsubsection{The Dirichlet Problem}

Lemma 6.2.29. Suppose $\mathcal{L}$ satisfies Hypothesis B (see Definition 6.1.30). Let u be a good $\mathcal{D}$ solution. For $\tau>0$, set $\left(f_{\tau}, g_{\tau}\right):=\left(\operatorname{Tr}_{0} u_{\tau}, \partial_{\nu} u_{\tau}\right)=\left(T r_{0} u_{\tau}, \partial_{\nu_{\tau}} u\right) \in L^{2}\left(\mathbb{R}^{n}\right) \times\left[Y^{1,2}\left(\mathbb{R}^{n}\right)\right]^{*}$, where we use Lemma 6.2.1 to identify $\partial_{v_{\tau}}$ u as an element of $\left[Y^{1,2}\left(\mathbb{R}^{n}\right)\right]^{*}$. Then

$$
u=-\mathcal{D} f+\mathcal{S} g
$$

\footnotetext{
${ }^{1}$ We note that, having obtained the mapping property $\mathcal{D} \rightarrow S_{+}^{2}$, the equality of (6.16) holds on every $t$-slice in the space $Y^{1,2}\left(\mathbb{R}^{n}\right)$ therefore the weak $L^{2}\left(\mathbb{R}^{n}\right)$ limits, in $t$, of the co-normal derivatives $\partial_{v_{t}}$ are the same.
} 
where the pair $(f, g) \in L^{2}\left(\mathbb{R}^{n}\right) \times\left[Y^{1,2}\left(\mathbb{R}^{n}\right)\right]^{*}$ is any convergent weak limit of $\left(f_{\tau_{k}}, g_{\tau_{k}}\right), \tau_{k} \downarrow 0$ in the space $L^{2}\left(\mathbb{R}^{n}\right) \times\left[Y^{1,2}\left(\mathbb{R}^{n}\right)\right]^{*}$.

Remark 6.2.30. We note that the existence of at least one such limiting pair $(f, g)$ is guaranteed by the fact that $f_{\tau}, g_{\tau}$ are uniformly bounded in $L^{2}\left(\mathbb{R}^{n}\right)$ and $Y^{1,2}\left(\mathbb{R}^{n}\right)^{*}$ respectively (the first by the hypothesis $u \in D_{+}^{2}$ and the second by Lemma 6.2.1) together with the fact that both of these spaces are reflexive.

Unlike the case of good $\mathcal{N} / \mathcal{R}$ solutions, here we make no assertion about the uniqueness of such a limiting pair.

Proof. The proof is quite similar to Lemma 6.2.25, but we provide the details here. We have that $u_{\tau} \in Y^{1,2}\left(\mathbb{R}^{n+1}{ }_{+}\right)$with $\mathcal{L} u_{\tau}=0$ we have

$$
u_{\tau}=-\mathcal{D}\left(\operatorname{Tr}_{0} u_{\tau}\right)+S\left(\partial_{\nu} u_{\tau}\right)
$$

for all $\tau>0$. Let $\vec{h}_{\tau_{k}}:=\left(f_{\tau_{k}}, g_{\tau_{k}}\right) \rightarrow(f, g)=: \vec{h} \in L^{2}\left(\mathbb{R}^{n}\right) \times\left[Y^{1,2}\left(\mathbb{R}^{n}\right)\right]^{*}$ be as in the statement of the lemma. Using Mazur's lemma there exists a sequence $\left\{\tilde{h}_{l}\right\}_{l=1}^{\infty} \subset L^{2}\left(\mathbb{R}^{n}\right) \times\left[Y^{1,2}\left(\mathbb{R}^{n}\right)\right]^{*}$ such that $\tilde{h}_{l} \rightarrow \vec{h}$ strongly in $L^{2}\left(\mathbb{R}^{n}\right) \times\left[Y^{1,2}\left(\mathbb{R}^{n}\right)\right]^{*}$ with

$$
\tilde{h}_{l}=\sum_{k=l}^{N(l)} \lambda_{k, l} \vec{h}_{\tau_{k}}
$$

where $l \leq N(l)<\infty, \lambda_{k, l} \in[0,1]$ and $\sum_{k=l}^{N(l)} \lambda_{k, l}=1$. Set

$$
\tilde{u}:=\mathcal{D} f+\mathcal{S} g
$$


and

$$
u_{l}:=\sum_{k=l}^{N(l)} \lambda_{k, l} u_{\tau_{k}} .
$$

We show $u_{l}(\cdot, t)$ converges strongly to both $u(\cdot, t)$ and $\tilde{u}(\cdot, t)$ in $L^{2}\left(\mathbb{R}^{n}\right)$. From the bounded mappings $\mathcal{D}: L^{2}\left(\mathbb{R}^{n}\right) \rightarrow D_{+}^{2}$ and $\mathcal{S}:\left[Y^{1,2}\left(\mathbb{R}^{n}\right)\right]^{*} \rightarrow D_{+}^{2}$ we have

$$
\left\|\tilde{u}(\cdot, t)-u_{l}(\cdot, t)\right\|_{L^{2}\left(\mathbb{R}^{n}\right)} \leq\left\|\vec{h}-\tilde{h}_{l}\right\|_{L^{2}\left(\mathbb{R}^{n}\right) \times\left[Y^{1,2}\left(\mathbb{R}^{n}\right)\right]^{*}} \rightarrow 0 \text { as } l \rightarrow \infty .
$$

To show $u_{l}(\cdot, t)$ converges strongly to $u(\cdot, t)$ in $L^{2}\left(\mathbb{R}^{n}\right)$ we write for $l \geq 0$,

$$
\begin{aligned}
\left\|u_{l}(\cdot, t)-u(\cdot, t)\right\|_{L^{2}\left(\mathbb{R}^{n}\right)} & =\left\|\sum_{k=l}^{N(l)} \lambda_{k, l}\left[u_{\tau_{k}}-u\right](\cdot, t)\right\|_{L^{2}\left(\mathbb{R}^{n}\right)} \\
& \leq \sum_{k=l}^{N(l)} \lambda_{k, l}\left\|\left[u_{\tau_{k}}-u\right](\cdot, t)\right\|_{L^{2}\left(\mathbb{R}^{n}\right)} \\
& \leq \sup _{k \geq l}\left\|u\left(\cdot, t+\tau_{k}\right)-u(\cdot, t)\right\|_{L^{2}\left(\mathbb{R}^{n}\right)},
\end{aligned}
$$

where we used $\sum_{k=l}^{N(l)} \lambda_{k, l}=1$ and $u(\cdot, \cdot+\tau)=u_{\tau}(\cdot, \cdot)=\mathcal{D}\left(f_{\tau}\right)+S\left(g_{\tau}\right)$. We can then use the continuity of $u(\cdot, t)$ in $L^{2}\left(\mathbb{R}^{n}\right)$ (see Lemma 2.2.2) $)^{2}$ along with $\tau_{k} \downarrow 0$ to obtain $\left\|u_{n}(\cdot, t)-u(\cdot, t)\right\|_{L^{2}\left(\mathbb{R}^{n}\right)} \rightarrow 0$ as $n$ tends to infinity. Therefore $u=\tilde{u}$ in $D_{+}^{2}$ and the lemma is shown.

Theorem 6.2.31 (Uniqueness of the Dirichlet problem among good $\mathcal{D}$ solutions). Suppose $\mathcal{L}$ satisfies Hypothesis B (see Definition 6.1.30). Suppose u is a good $\mathcal{D}$ solution, with $u(\cdot, t) \rightarrow 0$ weakly in $L^{2}\left(\mathbb{R}^{n}\right)$. Then $u \equiv 0$.

\footnotetext{
${ }^{2}$ We may modify this Lemma, using now the function space $W^{1,2}\left(\Sigma_{a}^{b}\right)$ instead of $Y^{1,2}\left(\Sigma_{a}^{b}\right)$ to obtain the desired continuity in $L^{2}\left(\mathbb{R}^{n}\right)$ instead of $L^{2^{*}}\left(\mathbb{R}^{n}\right)$.
} 
Proof. By Lemma 6.2.29, we have that $u=\mathcal{S} g$ for some $g \in\left[Y^{1,2}\left(\mathbb{R}^{n}\right)\right]^{*}$, where $g \in$ $\left[Y^{1,2}\left(\mathbb{R}^{n}\right)\right]^{*}$ is any weak limit of $g_{\tau_{k}}=\partial_{\nu \mathcal{L}} u_{\tau_{k}}, \tau_{k} \downarrow 0$ as in Lemma 6.2.29. We also have $(\operatorname{see}(6.24))$

$$
\mathcal{S}_{t} g=\mathcal{D}_{t}\left(S_{0}\left[-\frac{1}{2} I+\tilde{K}\right]^{-1} g\right)
$$

where we used $\left[-\frac{1}{2} I+\tilde{K}\right]^{-1}:\left[Y^{1,2}\left(\mathbb{R}^{n}\right)\right]^{*} \rightarrow\left[Y^{1,2}\left(\mathbb{R}^{n}\right)\right]^{*}$ and $S_{0}:\left[Y^{1,2}\left(\mathbb{R}^{n}\right)\right]^{*} \rightarrow L^{2}\left(\mathbb{R}^{n}\right)$. Taking weak limits in $L^{2}\left(\mathbb{R}^{n}\right)$ we obtain

$$
0=\left[-\frac{1}{2} I+K\right] S_{0}\left[-\frac{1}{2} I+\tilde{K}\right]^{-1} g
$$

The invertibility of the mappings $-\frac{1}{2} I+K: L^{2}\left(\mathbb{R}^{n}\right) \rightarrow L^{2}\left(\mathbb{R}^{n}\right), S_{0}:\left[Y^{1,2}\left(\mathbb{R}^{n}\right)\right]^{*} \rightarrow L^{2}\left(\mathbb{R}^{n}\right)$ and $\left[-\frac{1}{2} I+\tilde{K}\right]^{-1}:\left[Y^{1,2}\left(\mathbb{R}^{n}\right)\right]^{*} \rightarrow\left[Y^{1,2}\left(\mathbb{R}^{n}\right)\right]^{*}$ give that $g=0$ in $\left[Y^{1,2}\left(\mathbb{R}^{n}\right)\right]^{*}$ and hence $u \equiv 0$. 


\section{Bibliography}

[AA11] Pascal Auscher and Andreas Axelsson. Weighted maximal regularity estimates and solvability of non-smooth elliptic systems I. Invent. Math., 184(1):47-115, 2011. (p. 15)

[AAA $\left.{ }^{+} 11\right]$ M. Angeles Alfonseca, Pascal Auscher, Andreas Axelsson, Steve Hofmann, and Seick Kim. Analyticity of layer potentials and $L^{2}$ solvability of boundary value problems for divergence form elliptic equations with complex $L^{\infty}$ coefficients. Adv. Math., 226(5):4533-4606, 2011. (p. 3, 11, 12, 13, 14, 36, $37,38,64,101,126,128,141,201,210,239,248)$

[AAH08] Pascal Auscher, Andreas Axelsson, and Steve Hofmann. Functional calculus of Dirac operators and complex perturbations of Neumann and Dirichlet problems. J. Funct. Anal., 255(2):374-448, 2008. (p. 12, 13, 14)

[AAM10] Pascal Auscher, Andreas Axelsson, and Alan McIntosh. On a quadratic estimate related to the Kato conjecture and boundary value problems. In Harmonic analysis and partial differential equations, volume 505 of Contemp. 
Math., pages 105-129. Amer. Math. Soc., Providence, RI, 2010. (p. 12, 13, 14)

[ABES19] Pascal Auscher, Simon Bortz, Moritz Egert, and Olli Saari. Nonlocal selfimproving properties: a functional analytic approach. Tunis. J. Math., $1(2): 151-183,2019 .($ p. 85,86$)$

$\left[\mathrm{AHL}^{+}\right.$02] Pascal Auscher, Steve Hofmann, Michael Lacey, Alan McIntosh, and Ph. Tchamitchian. The solution of the Kato square root problem for second order elliptic operators on $\mathbb{R}^{n}$. Ann. of Math. (2), 156(2):633-654, 2002. (p. 12, 14, $162,166)$

[AHLT01] Pascal Auscher, Steve Hofmann, John L. Lewis, and Philippe Tchamitchian. Extrapolation of Carleson measures and the analyticity of Kato's square-root operators. Acta Math., 187(2):161-190, 2001. (p. 13)

[AHM12] Pascal Auscher, Steve Hofmann, and José-María Martell. Vertical versus conical square functions. Trans. Amer. Math. Soc., 364(10):5469-5489, 2012. (p. $34,63)$

[AM19] Pascal Auscher and Mihalis Mourgoglou. Representation and uniqueness for boundary value elliptic problems via first order systems. Rev. Mat. Iberoam., 35(1):241-315, 2019. (p. 5) 
[AP17] Pascal Auscher and Cruz Prisuelos Arribas. Tent space boundedness via extrapolation. Math. Z., 286(3-4):1575-1604, 2017. (p. 198)

[AT98] Pascal Auscher and Philippe Tchamitchian. Square root problem for divergence operators and related topics. Astérisque, (249):viii+172, 1998. (p. 167)

[Axe10] Andreas Axelsson. Non-unique solutions to boundary value problems for non-symmetric divergence form equations. Trans. Amer. Math. Soc., 362(2):661-672, 2010. (p. 5, 13)

[Bar13] Ariel Barton. Elliptic partial differential equations with almost-real coefficients. Mem. Amer. Math. Soc., 223(1051):vi+108, 2013. (p. 13, 16)

[Bar17] Ariel Barton. Layer potentials for general linear elliptic systems. Electron. J. Differential Equations, pages Paper No. 309, 23, 2017. (p. 4, 109)

[BES19] Simon Bortz, Moritz Egert, and Olli Saari. Sobolev contractivity of gradient flow maximal functions, 2019. (p. 142)

$\left[\mathrm{BHL}^{+} \mathrm{a}\right]$ Simon Bortz, Steve Hofmann, José Luis Luna García, Svitlana Mayboroda, and Bruno Poggi. Critical perturbations for second order elliptic operators. part 1: Square function bounds for layer potentials. Preprint. March 2020. arXiv:2003.02703. (p. vii, 16, 91, 144, 199, 223, 242) 
$\left[\mathrm{BHL}^{+} \mathrm{b}\right]$ Simon Bortz, Steve Hofmann, José Luis Luna García, Svitlana Mayboroda, and Bruno Poggi. Critical perturbations for second order elliptic operators. part 2: Existence, uniqueness, and bounds on the nontangential maximal function. In preparation. (p. vii, 17)

[BL76] Jöran Bergh and Jörgen Löfström. Interpolation spaces. An introduction. Springer-Verlag, Berlin-New York, 1976. Grundlehren der Mathematischen Wissenschaften, No. 223. (p. 85)

[CF74] R. R. Coifman and C. Fefferman. Weighted norm inequalities for maximal functions and singular integrals. Studia Math., 51:241-250, 1974. (p. 46)

[CFK81] Luis A. Caffarelli, Eugene B. Fabes, and Carlos E. Kenig. Completely singular elliptic-harmonic measures. Indiana Univ. Math. J., 30(6):917-924, 1981. (p. 14)

[CH98] Thierry Cazenave and Alain Haraux. An introduction to semilinear evolution equations, volume 13 of Oxford Lecture Series in Mathematics and its Applications. The Clarendon Press, Oxford University Press, New York, 1998. Translated from the 1990 French original by Yvan Martel and revised by the authors. (p. 28, 255) 
[CJ87] Michael Christ and Jean-Lin Journé. Polynomial growth estimates for multilinear singular integral operators. Acta Math., 159(1-2):51-80, 1987. (p. 36, 38)

[CLMS93] R. Coifman, P.-L. Lions, Y. Meyer, and S. Semmes. Compensated compactness and Hardy spaces. J. Math. Pures Appl. (9), 72(3):247-286, 1993. (p. 2)

[CM86] R. R. Coifman and Yves Meyer. Nonlinear harmonic analysis, operator theory and P.D.E. In Beijing lectures in harmonic analysis (Beijing, 1984), volume 112 of Ann. of Math. Stud., pages 3-45. Princeton Univ. Press, Princeton, NJ, 1986. (p. 167, 200)

[CMM82] R. R. Coifman, A. McIntosh, and Y. Meyer. L'intégrale de Cauchy définit un opérateur borné sur $L^{2}$ pour les courbes lipschitziennes. Ann. of Math. (2), 116(2):361-387, 1982. (p. 11, 12)

[CMP11] David V. Cruz-Uribe, José Maria Martell, and Carlos Pérez. Weights, extrapolation and the theory of Rubio de Francia, volume 215 of Operator Theory: Advances and Applications. Birkhäuser/Springer Basel AG, Basel, 2011. (p. $9,46,49,197)$ 
[CMP20] Li Chen, José María Martell, and Cruz Prisuelos Arribas. Conical square functions for degenerate elliptic operators. Adv. Calc. Var., 13(1):75-113, 2020. (p. 34)

[CMR18] David Cruz-Uribe, José María Martell, and Cristian Rios. On the Kato problem and extensions for degenerate elliptic operators. Anal. PDE, 11(3):609660, 2018. (p. 60)

[CMS85] R. R. Coifman, Y. Meyer, and E. M. Stein. Some new function spaces and their applications to harmonic analysis. J. Funct. Anal., 62(2):304-335, 1985. (p. 34)

[CR80] R. R. Coifman and R. Rochberg. Another characterization of BMO. Proc. Amer. Math. Soc., 79(2):249-254, 1980. (p. 45)

[CZ56] A. P. Calderón and A. Zygmund. A note on the interpolation of sublinear operations. Amer. J. Math., 78:282-288, 1956. (p. 48)

[Dah77] Björn E. J. Dahlberg. Estimates of harmonic measure. Arch. Rational Mech. Anal., 65(3):275-288, 1977. (p. 11)

[Dah86] Björn E. J. Dahlberg. On the absolute continuity of elliptic measures. Amer. J. Math., 108(5):1119-1138, 1986. (p. 15) 
[DG57] Ennio De Giorgi. Sulla differenziabilità e l'analiticità delle estremali degli integrali multipli regolari. Mem. Accad. Sci. Torino. Cl. Sci. Fis. Mat. Nat. (3), 3:25-43, 1957. (p. 11)

[DHM18] Blair Davey, Jonathan Hill, and Svitlana Mayboroda. Fundamental matrices and Green matrices for non-homogeneous elliptic systems. Publ. Mat., 62(2):537-614, 2018. (p. 4)

[DNPV12] Eleonora Di Nezza, Giampiero Palatucci, and Enrico Valdinoci. Hitchhiker's guide to the fractional Sobolev spaces. Bull. Sci. Math., 136(5):521-573, 2012. (p. 26)

[DP19] Martin Dindos and Jill Pipher. Perturbation theory for solutions to second order elliptic operators with complex coefficients and the $L^{p}$ Dirichlet problem. Acta Math. Sin. (Engl. Ser.), 35(6):749-770, 2019. (p. 15)

[DPP07] Martin Dindos, Stefanie Petermichl, and Jill Pipher. The $L^{p}$ Dirichlet problem for second order elliptic operators and a $p$-adapted square function. $J$. Funct. Anal., 249(2):372-392, 2007. (p. 15, 16)

[DPR17] Martin Dindos, Jill Pipher, and David Rule. Boundary value problems for second-order elliptic operators satisfying a Carleson condition. Comm. Pure Appl. Math., 70(7):1316-1365, 2017. (p. 15) 
[DR86] Javier Duoandikoetxea and José L. Rubio de Francia. Maximal and singular integral operators via Fourier transform estimates. Invent. Math., 84(3):541561, 1986. (p. 47)

[FJK84] Eugene B. Fabes, David S. Jerison, and Carlos E. Kenig. Necessary and sufficient conditions for absolute continuity of elliptic-harmonic measure. Ann. of Math. (2), 119(1):121-141, 1984. (p. 12, 15)

[FKP91] R. A. Fefferman, C. E. Kenig, and J. Pipher. The theory of weights and the Dirichlet problem for elliptic equations. Ann. of Math. (2), 134(1):65-124, 1991. (p. 15)

[FS72] C. Fefferman and E. M. Stein. $H^{p}$ spaces of several variables. Acta Math., 129(3-4):137-193, 1972. (p. 37)

[Geh73] F. W. Gehring. The $L^{p}$-integrability of the partial derivatives of a quasiconformal mapping. Acta Math., 130:265-277, 1973. (p. 3)

[GH16] Ana Grau de la Herrán and Steve Hofmann. A local $T b$ theorem with vectorvalued testing functions. In Some topics in harmonic analysis and applications, volume 34 of Adv. Lect. Math. (ALM), pages 203-229. Int. Press, Somerville, MA, 2016. (p. 8, 14, 36, 145, 151, 169, 170, 172) 
[GH17] Ana Grau De La Herrán and Steve Hofmann. Generalized local $T b$ theorems for square functions. Mathematika, 63(1):1-28, 2017. (p. 62)

[GHN16] Fritz Gesztesy, Steve Hofmann, and Roger Nichols. On stability of square root domains for non-self-adjoint operators under additive perturbations. Mathematika, 62(1):111-182, 2016. (p. 2, 14, 145)

[Gia83] Mariano Giaquinta. Multiple integrals in the calculus of variations and nonlinear elliptic systems, volume 105 of Annals of Mathematics Studies. Princeton University Press, Princeton, NJ, 1983. (p. 3)

[GR85] José García Cuerva and José L. Rubio de Francia. Weighted norm inequalities and related topics, volume 116 of North-Holland Mathematics Studies. North-Holland Publishing Co., Amsterdam, 1985. Notas de Matemática [Mathematical Notes], 104. (p. 45)

[Gra14a] Loukas Grafakos. Classical Fourier analysis, volume 249 of Graduate Texts in Mathematics. Springer, New York, third edition, 2014. (p. 23)

[Gra14b] Loukas Grafakos. Modern Fourier analysis, volume 250 of Graduate Texts in Mathematics. Springer, New York, third edition, 2014. (p. 48) 
[HK07] Steve Hofmann and Seick Kim. The Green function estimates for strongly elliptic systems of second order. Manuscripta Math., 124(2):139-172, 2007. (p. 3)

[HKMP15a] Steve Hofmann, Carlos Kenig, Svitlana Mayboroda, and Jill Pipher. The regularity problem for second order elliptic operators with complex-valued bounded measurable coefficients. Math. Ann., 361(3-4):863-907, 2015. (p. $5,13)$

[HKMP15b] Steve Hofmann, Carlos Kenig, Svitlana Mayboroda, and Jill Pipher. Square function/non-tangential maximal function estimates and the Dirichlet problem for non-symmetric elliptic operators. J. Amer. Math. Soc., 28(2):483529, 2015. (p. 13)

[HL97] Qing Han and Fanghua Lin. Elliptic partial differential equations, volume 1 of Courant Lecture Notes in Mathematics. New York University, Courant Institute of Mathematical Sciences, New York; American Mathematical Society, Providence, RI, 1997. (p. 92)

[HL01] Steve Hofmann and John L. Lewis. The Dirichlet problem for parabolic operators with singular drift terms. Mem. Amer. Math. Soc., 151(719):viii+113, 2001. (p. 15) 
[HLM02] Steve Hofmann, Michael Lacey, and Alan McIntosh. The solution of the Kato problem for divergence form elliptic operators with Gaussian heat kernel bounds. Ann. of Math. (2), 156(2):623-631, 2002. (p. 13)

[HM02] Steve Hofmann and Alan McIntosh. The solution of the Kato problem in two dimensions. In Proceedings of the 6th International Conference on Harmonic Analysis and Partial Differential Equations (El Escorial, 2000), number Vol. Extra, pages 143-160, 2002. (p. 13)

[HMM15] Steve Hofmann, Svitlana Mayboroda, and Mihalis Mourgoglou. Layer potentials and boundary value problems for elliptic equations with complex $L^{\infty}$ coefficients satisfying the small Carleson measure norm condition. Adv. Math., 270:480-564, 2015. (p. 14, 15, 145, 161, 166, 199)

[Hof10] Steve Hofmann. Local $T(b)$ theorems and applications in PDE. In Harmonic analysis and partial differential equations, volume 505 of Contemp. Math., pages 29-52. Amer. Math. Soc., Providence, RI, 2010. (p. 173)

[JK81a] David S. Jerison and Carlos E. Kenig. The Dirichlet problem in nonsmooth domains. Ann. of Math. (2), 113(2):367-382, 1981. (p. 11)

[JK81b] David S. Jerison and Carlos E. Kenig. The Neumann problem on Lipschitz domains. Bull. Amer. Math. Soc. (N.S.), 4(2):203-207, 1981. (p. 11) 
[Jon80] Peter W. Jones. Factorization of $A_{p}$ weights. Ann. of Math. (2), 111(3):511530, 1980. (p. 50)

[Kat95] Tosio Kato. Perturbation theory for linear operators. Classics in Mathematics. Springer-Verlag, Berlin, 1995. Reprint of the 1980 edition. (p. 247)

[Ken94] Carlos E. Kenig. Harmonic analysis techniques for second order elliptic boundary value problems, volume 83 of CBMS Regional Conference Series in Mathematics. Published for the Conference Board of the Mathematical Sciences, Washington, DC; by the American Mathematical Society, Providence, RI, 1994. (p. 15)

[KKPT00] C. Kenig, H. Koch, J. Pipher, and T. Toro. A new approach to absolute continuity of elliptic measure, with applications to non-symmetric equations. Adv. Math., 153(2):231-298, 2000. (p. 13)

[KMM07] Nigel Kalton, Svitlana Mayboroda, and Marius Mitrea. Interpolation of Hardy-Sobolev-Besov-Triebel-Lizorkin spaces and applications to problems in partial differential equations. In Interpolation theory and applications, volume 445 of Contemp. Math., pages 121-177. Amer. Math. Soc., Providence, RI, 2007. (p. 85) 
[KP93] Carlos E. Kenig and Jill Pipher. The Neumann problem for elliptic equations with nonsmooth coefficients. Invent. Math., 113(3):447-509, 1993. (p. 11, 93, 94)

[KP95] Carlos E. Kenig and Jill Pipher. The Neumann problem for elliptic equations with nonsmooth coefficients. II. Duke Math. J., 81(1):227-250 (1996), 1995. A celebration of John F. Nash, Jr. (p. 15)

[KP01] Carlos E. Kenig and Jill Pipher. The Dirichlet problem for elliptic equations with drift terms. Publ. Mat., 45(1):199-217, 2001. (p. 15, 16)

[KR09] Carlos E. Kenig and David J. Rule. The regularity and Neumann problem for non-symmetric elliptic operators. Trans. Amer. Math. Soc., 361(1):125-160, 2009. (p. 13)

[KS19] Seick Kim and Georgios Sakellaris. Green's function for second order elliptic equations with singular lower order coefficients. Comm. Partial Differential Equations, 44(3):228-270, 2019. (p. 4)

[Leo17] Giovanni Leoni. A first course in Sobolev spaces, volume 181 of Graduate Studies in Mathematics. American Mathematical Society, Providence, RI, second edition, 2017. (p. 23, 30) 
[LMPT10] Michael T. Lacey, Kabe Moen, Carlos Pérez, and Rodolfo H. Torres. Sharp weighted bounds for fractional integral operators. J. Funct. Anal., 259(5):1073-1097, 2010. (p. 198)

[May10] Svitlana Mayboroda. The connections between Dirichlet, regularity and Neumann problems for second order elliptic operators with complex bounded measurable coefficients. Adv. Math., 225(4):1786-1819, 2010. (p. 15)

[Mey63] Norman G. Meyers. An $L^{p}$ e-estimate for the gradient of solutions of second order elliptic divergence equations. Ann. Scuola Norm. Sup. Pisa Cl. Sci. (3), 17:189-206, 1963. (p. 3)

[MMT01] Dorina Mitrea, Marius Mitrea, and Michael Taylor. Layer potentials, the Hodge Laplacian, and global boundary problems in nonsmooth Riemannian manifolds. Mem. Amer. Math. Soc., 150(713):x+120, 2001. (p. 12)

[Mos61] Jürgen Moser. On Harnack's theorem for elliptic differential equations. Comm. Pure Appl. Math., 14:577-591, 1961. (p. 11)

[Mou] Mihalis Mourgoglou. Regularity theory and green's function for elliptic equations with lower order terms in unbounded domains. Preprint. April 2019. arXiv:1904.04722. (p. 16) 
[Muc72] Benjamin Muckenhoupt. Weighted norm inequalities for the Hardy maximal function. Trans. Amer. Math. Soc., 165:207-226, 1972. (p. 45)

[MW74] Benjamin Muckenhoupt and Richard Wheeden. Weighted norm inequalities for fractional integrals. Trans. Amer. Math. Soc., 192:261-274, 1974. (p. 52)

[MZ97] Jan Malý and William P. Ziemer. Fine regularity of solutions of elliptic partial differential equations, volume 51 of Mathematical Surveys and Monographs. American Mathematical Society, Providence, RI, 1997. (p. 24, 185)

[Nas58] J. Nash. Continuity of solutions of parabolic and elliptic equations. Amer. J. Math., 80:931-954, 1958. (p. 11)

[Pet08] Stefanie Petermichl. The sharp weighted bound for the Riesz transforms. Proc. Amer. Math. Soc., 136(4):1237-1249, 2008. (p. 198)

[Pri19] Cruz Prisuelos Arribas. Vertical square functions and other operators associated with an elliptic operator. J. Funct. Anal., 277(12):108296, 63, 2019. (p. $9,189)$

[Ros13] Andreas Rosén. Layer potentials beyond singular integral operators. Publ. Mat., 57(2):429-454, 2013. (p. 8, 14, 109) 
[Rub83] José Luis Rubio de Francia. A new technique in the theory of $A_{p}$ weights. In Topics in modern harmonic analysis, Vol. I, II (Turin/Milan, 1982), pages 571-579. Ist. Naz. Alta Mat. Francesco Severi, Rome, 1983. (p. 46)

[Rub84] José L. Rubio de Francia. Factorization theory and $A_{p}$ weights. Amer. J. Math., 106(3):533-547, 1984. (p. 46)

[Sak19] Georgios Sakellaris. Boundary value problems in Lipschitz domains for equations with lower order coefficients. Trans. Amer. Math. Soc., 372(8):5947-5989, 2019. (p. 16)

[She94] Zhongwei Shen. On the Neumann problem for Schrödinger operators in Lipschitz domains. Indiana Univ. Math. J., 43(1):143-176, 1994. (p. 16)

[Sne74] I. Ja. Sneiberg. Spectral properties of linear operators in interpolation families of Banach spaces. Mat. Issled., 9(2(32)):214-229, 254-255, 1974. (p. $85,86)$

[Ste70] Elias M. Stein. Singular integrals and differentiability properties of functions. Princeton Mathematical Series, No. 30. Princeton University Press, Princeton, N.J., 1970. (p. 31)

[Ste93] Elias M. Stein. Harmonic analysis: real-variable methods, orthogonality, and oscillatory integrals, volume 43 of Princeton Mathematical Series. 
Princeton University Press, Princeton, NJ, 1993. With the assistance of Timothy S. Murphy, Monographs in Harmonic Analysis, III. (p. 67)

[Str16] Robert S. Strichartz. "Graph paper" trace characterizations of functions of finite energy. J. Anal. Math., 128:239-260, 2016. (p. 27)

[SW58] E. M. Stein and G. Weiss. Interpolation of operators with change of measures. Trans. Amer. Math. Soc., 87:159-172, 1958. (p. 47, 48)

[Tao12] Xiangxing Tao. The regularity problems with data in Hardy-Sobolev spaces for singular Schrödinger equation in Lipschitz domains. Potential Anal., 36(3):405-428, 2012. (p. 16)

[Tri95] Hans Triebel. Interpolation theory, function spaces, differential operators. Johann Ambrosius Barth, Heidelberg, second edition, 1995. (p. 85)

[TW01] Xiang Xing Tao and Si Lei Wang. $H^{p}$-boundary value problems for the Schrödinger equation in domains with non-smooth boundaries. Chinese Ann. Math. Ser. A, 22(3):307-318, 2001. (p. 16)

[Ver84] Gregory Verchota. Layer potentials and regularity for the Dirichlet problem for Laplace's equation in Lipschitz domains. J. Funct. Anal., 59(3):572-611, 1984. (p. 11) 


\section{VITA}

Jose Luis was born in Mexico City, Mexico one November 27, 1987. He attended school there his whole life, culminating in a B.Sc degree at the National Autonomous University of Mexico (UNAM), obtained in 2013. Shortly after he joined the Math Department at UM-Columbia, in the fall of 2014. 